Andreas de Bruin

ACHTSAMKEIT

UND MEDITATION IM

HOCHSCHULKONTEXT

10 Jahre Münchner Modell

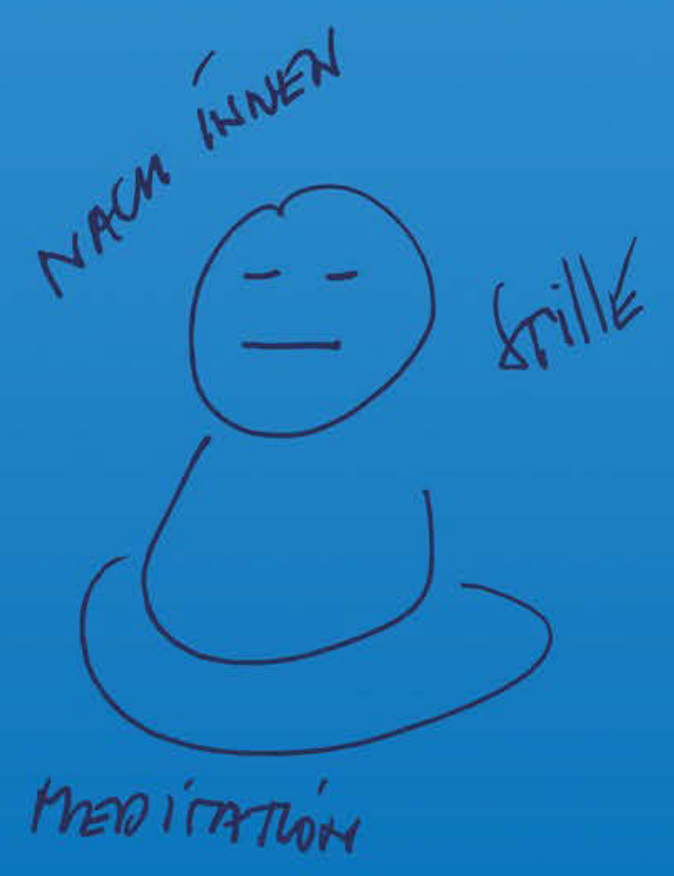

[transcript] ACHTSAMKEIT - bLLOUNG - MEOIEN 
Andreas de Bruin

\section{ACHTSAMKEIT UND MEDITATION im Hochschulkontext}

10 Jahre Münchner Modell

\section{[transcript]}


Das Buchprojekt „Achtsamkeit und Meditation im Hochschulkontext” wurde gefördert von dem Verein für Achtsamkeit in Osterloh e.V. (www.achtsamkeit-osterloh.org) und der Edith-Haberland-Wagner Stiftung (www.ehw-stiftung.de).

Bibliografische Information der Deutschen Nationalbibliothek

Die Deutsche Nationalbibliothek verzeichnet diese Publikation in der Deutschen Nationalbibliografie; detaillierte bibliografische Daten sind im Internet über http://dnb.d-nb.de abrufbar.

\section{(c) $(1 \otimes \Theta$}

Dieses Werk ist lizenziert unter der Creative Commons Attribution-NonCommercial-No-Derivs 4.0 Lizenz (BY-NC-ND). Diese Lizenz erlaubt die private Nutzung, gestattet aber keine Bearbeitung und keine kommerzielle Nutzung. Weitere Informationen finden Sie unter https://creativecommons.org/licenses/by-nc-nd/4.0/deed.de Um Genehmigungen für Adaptionen, Übersetzungen, Derivate oder Wiederverwendung zu kommerziellen Zwecken einzuholen, wenden Sie sich bitte an rights@transcript-publishing.com Die Bedingungen der Creative-Commons-Lizenz gelten nur für Originalmaterial. Die Wiederverwendung von Material aus anderen Quellen (gekennzeichnet mit Quellenangabe) wie z.B. Schaubilder, Abbildungen, Fotos und Textauszüge erfordert ggf. weitere Nutzungsgenehmigungen durch den jeweiligen Rechteinhaber.

\section{(C) 2021 transcript Verlag, Bielefeld}

1. Auflage 2021

(C) Andreas de Bruin (Text- und Bildrechte)

Buchgestaltung: Tanja Renz, Andreas de Bruin

Layout: Tanja Renz (www.1hundert8.de)

Fotografie: Stefan Zinsbacher

Zeichnungen: Andreas de Bruin, außer Zeichnungen

in den Tagebuchnotizen der Studierenden

Lektorat: Andrea Bistrich (andrea.bistrich@gmail.com)

Umschlaggestaltung: Maria Arndt, Bielefeld

Druck: Friedrich Pustet GmbH \& Co. KG, Regensburg

Print-ISBN 978-3-8376-5638-1

PDF-ISBN 978-3-8394-5638-5

https://doi.org/10.14361/9783839456385

Gedruckt auf alterungsbeständigem Papier mit chlorfrei gebleichtem Zellstoff.

Besuchen Sie uns im Internet: https://www.transcript-verlag.de

Unsere aktuelle Vorschau finden Sie unter www.transcript-verlag.de/vorschau-download

(c) Münchner Modell | www.hm.edu/meditationsmodell 


\author{
Allen spirituellen \\ Lehrerinnen und Lehrern gewidmet, \\ die seit Jahrtausenden \\ ihre Weisheit in die Welt bringen.
}




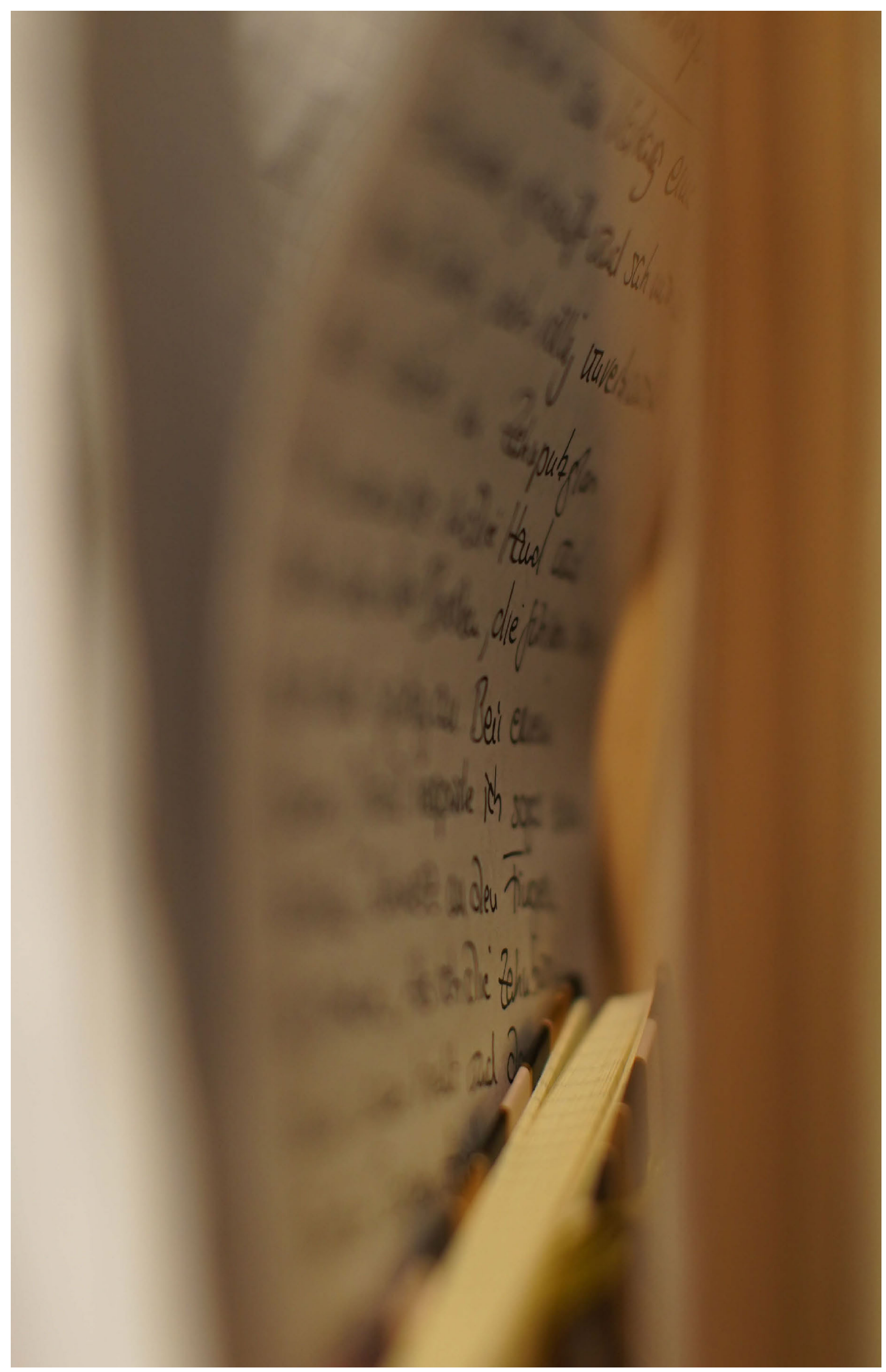




\section{DANK I}

Die Realisierung dieses Buches wäre ohne die wertvolle Hilfe anderer nicht zustande gekommen.

So danke ich herzlich an dieser Stelle Maria Kluge, die mir bei der Sichtung und Auswahl der Einträge aus den Meditationstagebüchern behilflich war und die Finanzierung des Buchlayouts in deutscher und englischer Sprache ermöglichte.

Mein aufrichtiger Dank gilt der Edith-Haberland-Wagner Stiftung, insbesondere dem Vorstand, Catherine Demeter und Martin Liebhäuser, für die Finanzierung der Open-Access-E-Book-Versionen in deutscher und englischer Sprache. Durch diese Förderung wird ermöglicht, dass der vorliegende Band sowohl auf nationaler als auch internationaler Ebene Studierenden, Lehrpersonal und weiteren Interessierten auf zahlreichen Online-Plattformen kostenlos zur Verfügung gestellt werden kann.

Besonders danken möchte ich Andrea Bistrich, die mir als zuverlässige und erfahrene Lektorin zur Seite stand und mir wichtige sachliche Hinweise gab.

In diesem Zusammenhang danke ich auch Kirstine Rasmussen für ihre wertvolle Unterstützung bei der Abrundung einiger Textpassagen. Durch ihre aufmerksame Durchsicht erhielten diese einen letzten Feinschliff.

Ein großes Dankeschön geht auch an Tanja Renz für die gut durchdachte und ästhetisch schöne Gestaltung, die sicherlich viele Leserinnen und Leser inspirieren wird.

Für ihre einfühlsame Übersetzungsarbeit möchte ich Elisa Graf danken. Sie hat es geschafft, dass der "Geist“ der deutschen Buchtexte voll und ganz in die englische Übersetzung übertragen wurde.

Stefan Zinsbacher hat die Fotografien der Skizzen, Zeichnungen und der Auszüge aus den Meditationstagebüchern mit großer Sorgfalt hergestellt. Auch an ihn geht ein warmherziges Dankeschön.

Nicht zuletzt möchte ich den Herausgebern Mike Sandbothe und Reyk Albrecht meinen Dank aussprechen, dass sie dieses Buch in die von ihnen herausgegebene Buchreihe „Achtsamkeit - Bildung - Medien“ aufgenommen haben. Dem transcript Verlag, insbesondere Karin Werner, Roswitha Gost, Jennifer Niediek, Katharina Kotschurin und Dagmar Buchwald danke ich für die professionelle Zusammenarbeit. 


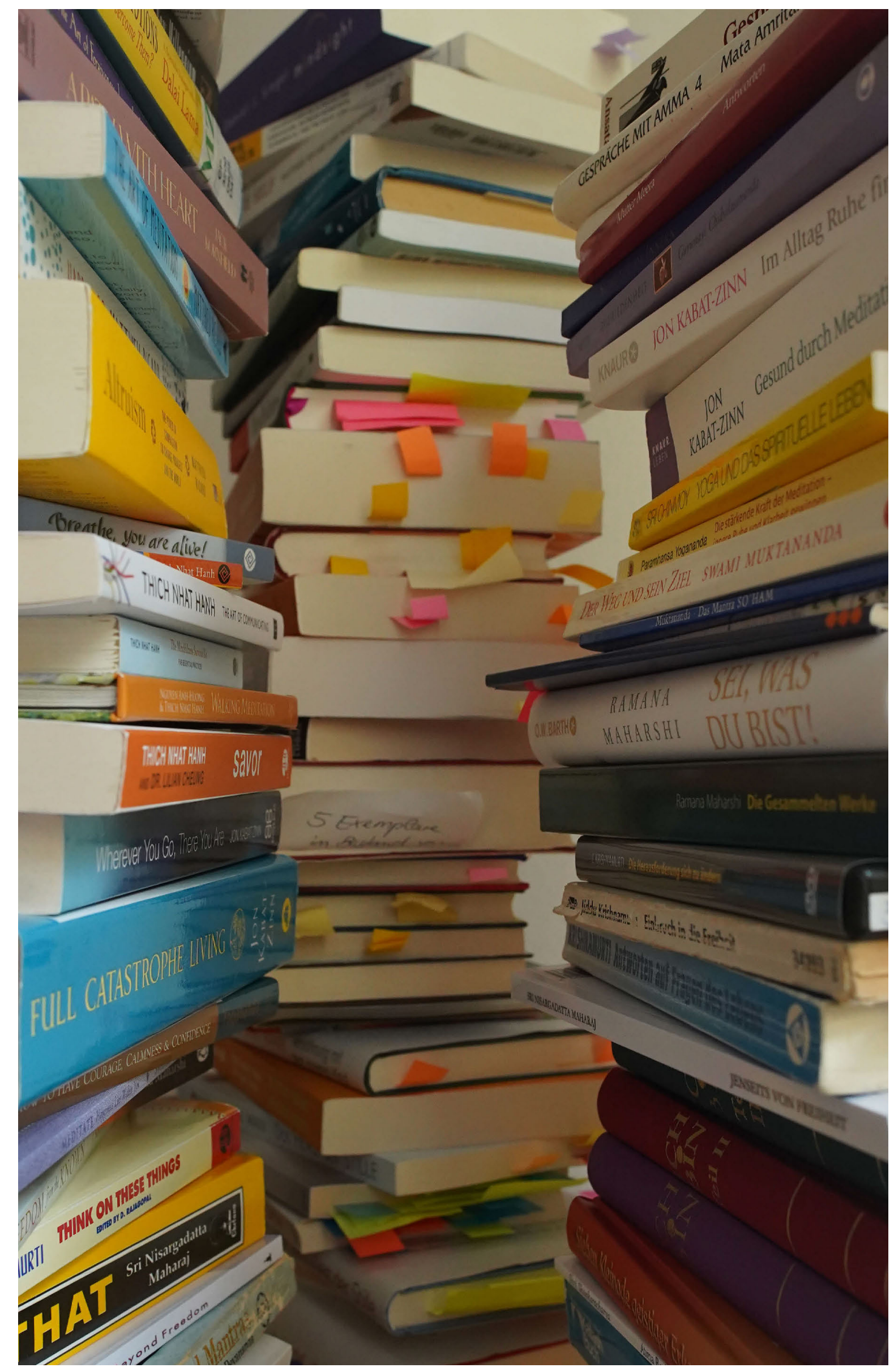




\section{DANK II}

Mein Dank gilt allen Studierenden,

für ihr großes Interesse an Achtsamkeits- und Meditationsangeboten, für ihre bereichernde Mitarbeit, für ihr Vertrauen und ihre unvoreingenommene Offenheit, für die Schilderung ihrer Eindrücke, Erlebnisse und Erfahrungen, für ihre Fragen und Rückmeldungen.

Besonders danken möchte ich allen Studierenden, die für dieses Buch ihre persönlichen Notizen aus den Meditationstagebüchern zur Verfügung gestellt haben.

Dem Paritätischen Ausschuss der Hochschule München möchte ich ebenfalls meinen Dank aussprechen, da er das Münchner Modell von Anfang an finanziell unterstützt hat.

Ich danke zudem den vielen Kooperationspartnern für den unkomplizierten Austausch von Erfahrungen und Informationen und für die gegenseitige Unterstützung, Achtsamkeits- und Meditationsangebote an Hochschulen nachhaltig zu verankern. Insbesondere danke ich in diesem Zusammenhang Britta Hölzel, Ulrich Ott, Harald Piron, Niko Kohls, Ortwin Lüers, Mike Sandbothe, Günter Hudasch und Antaranga Gressenich für ihre fachlichen Anmerkungen. 

INHALT

Vorwort

\section{Einführung}

Achtsamkeit und Meditation im Bildungssystem - ein Paradigmenwechsel? ...... 15

Selbst, Seele und Geist ......................................................................................... 25

Herz, Liebe und Selbst sind auch Bildungsthemen - ein Erfahrungsbericht ......... 31

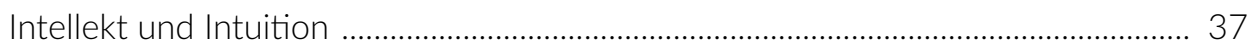

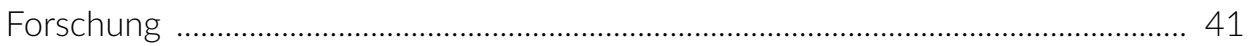

\section{Aufbau}

Das Münchner Modell „Achtsamkeit und Meditation im Hochschulkontext" ....... 57

Abschlussarbeiten im Rahmen des Münchner Modells ............................................. 69

Veranstaltungen im Rahmen der Vortrags- und Filmreihe

„Spirituelle Lehrer und ihre Unterweisungen“

\section{Übungen und Tagebuchnotizen}

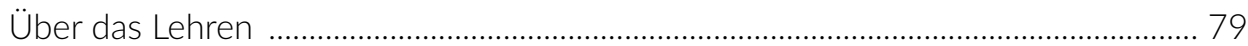

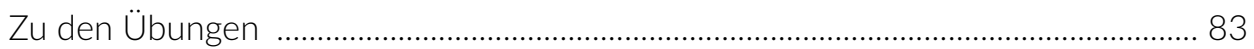

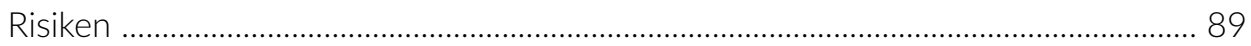

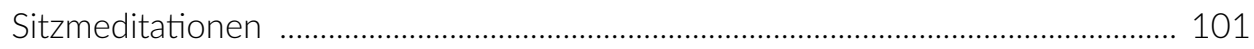

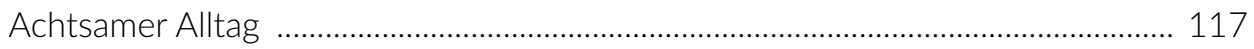

Interview: „Den wichtigen Dingen mehr Raum geben“ ............................................ 122

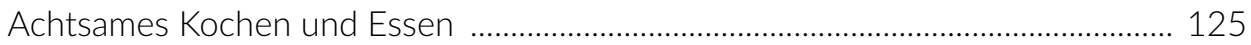

Achtsame Kommunikation ................................................................................... 131

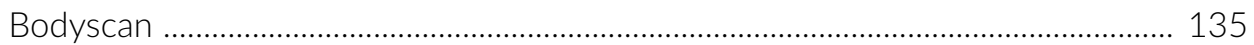

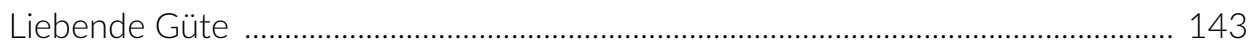

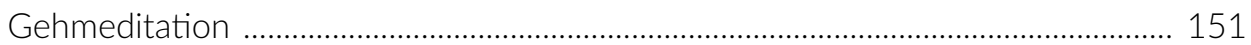

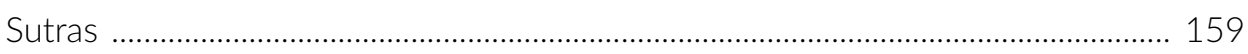

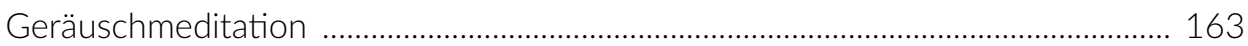

\section{Fazit}

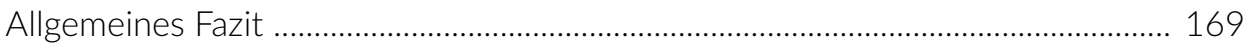

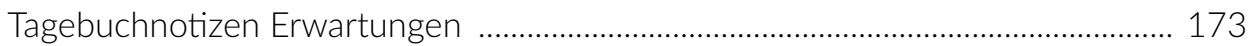

Tagebuchnotizen Lernen und Prüfungen ............................................................... 175

Tagebuchnotizen Rückblick ...................................................................................... 177

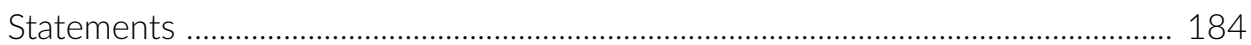

Feedback von Studierenden aus dem Film „Shanti“ ............................................... 187

Implementierungserfahrungen aus der Praxis ........................................................ 189

\section{Appendix}

Nachwort

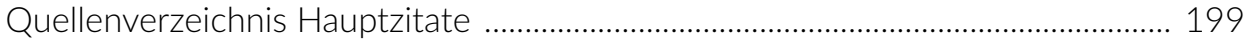

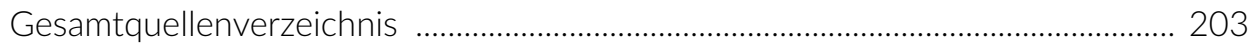

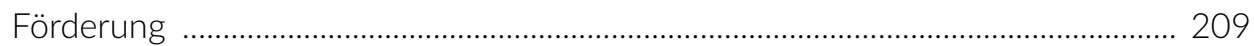

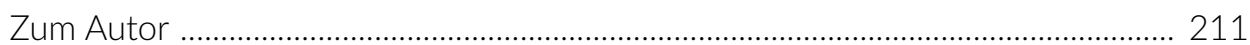



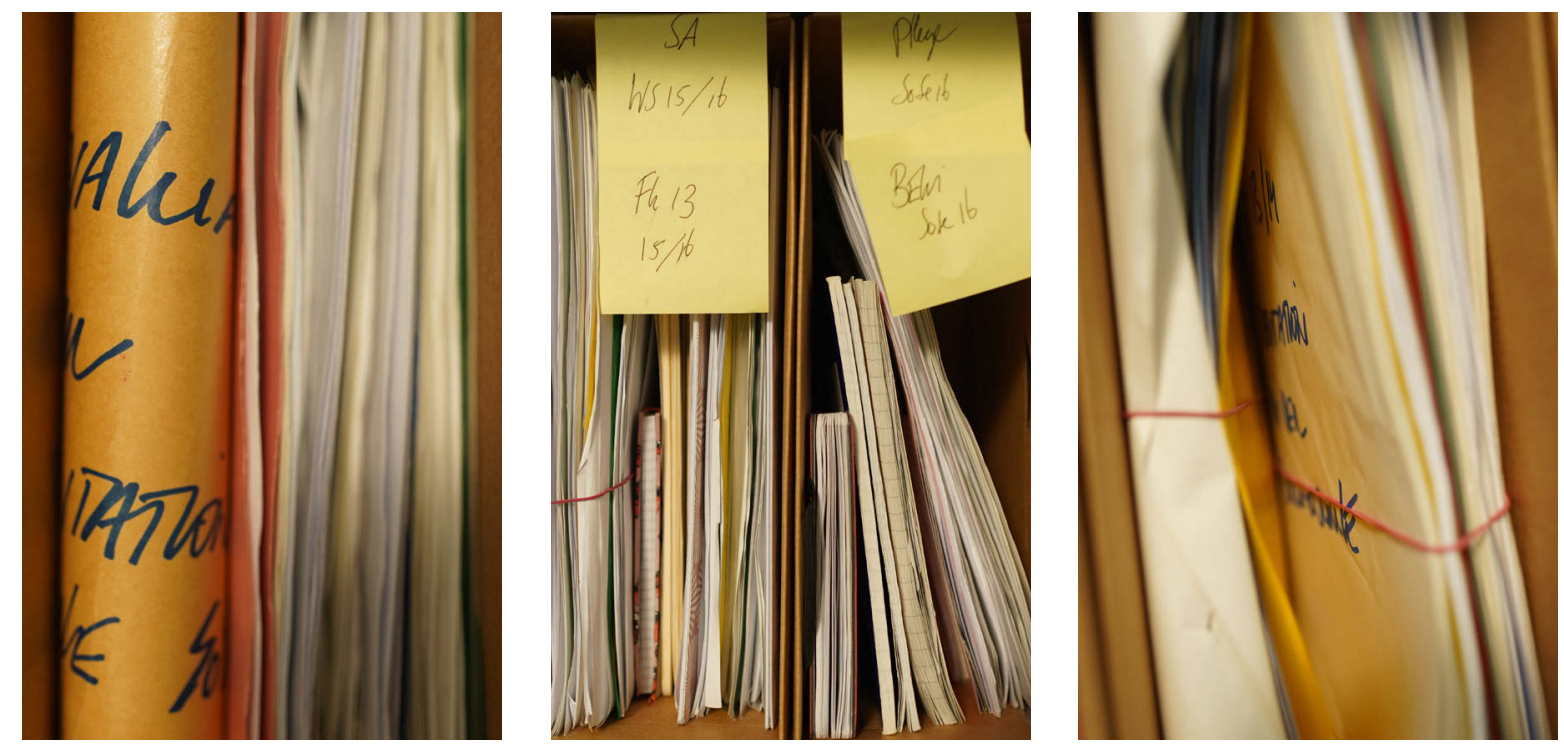


\section{VORWORT}

Das vorliegende Buch gibt einen Einblick in die ersten zehn Jahre des „Münchner Modells - Achtsamkeit und Meditation im Hochschulkontext" (März 2010 bis März 2020). Es beschreibt die Beweggründe, ein solches Angebot an Hochschulen zu verankern, und gibt Auskunft über die Struktur und die Implementierung des Konzepts in diesem Umfeld.

Seit der Einführung des Münchner Modells im Jahr 2010 mit einer Meditationslehrveranstaltung für 15 Kursteilnehmer im Studiengang Soziale Arbeit an der Hochschule München hat sich dieses Programm stetig weiterentwickelt. Mittlerweile nehmen pro Semester über 150 Studierende aus 21 Studiengängen teil.

Obwohl das Münchner Modell neben Theorie und Praxis für Studierende inzwischen auch Zusatzangebote für Lehrende und Hochschulbeschäftigte beinhaltet, sollen in diesem Buch in erster Linie die Stimmen der Studierenden im Mittelpunkt stehen, die an den Lehrveranstaltungen teilgenommen haben. Sie kommen zu Wort mittels ihrer Meditationstagebücher, in denen sie über ihre Erfahrungen während der Achtsamkeits- und Meditationsübungen berichten und auch darüber, welche Bedeutung das Praktizieren von Achtsamkeit und Meditation in ihrem Studium und im täglichen Leben erhalten hat.

Ursprünglich sollten die Meditationstagebücher, die als Teil eines Leistungsnachweises geführt wurden, lediglich archiviert werden. Doch mit der Idee der Entstehung dieses Buches stand plötzlich auch die Überlegung im Raum, einige sorgfältig ausgewählte Kommentare aus den Tagebüchern miteinzubeziehen, um zu veranschaulichen, welche Bedeutung Achtsamkeits- und Meditationsübungen im Hochschulkontext für Studierende haben und was sie bewirken können.

Von den 2000 Studierenden, die bisher an den Lehrveranstaltungen teilgenommen haben, wurden etwa 100 angefragt, ob sie einer Veröffentlichung von Auszügen aus ihren Tagebucheinträgen in anonymisierter Form zustimmen. Die Zusagen, die von den Studierenden eintrafen, waren einstimmig; das Vorhaben wurde als „wichtig“, „super", „genial“ begrüßt.

Das Buch ist durch diese Beiträge sehr lebendig geworden. Es entstand eine Art „Arbeitsbuch“, das sowohl im privaten Bereich genutzt werden kann als auch Anregung und Beispiel bietet, das Thema „Achtsamkeit und Meditation“ an anderen Hochschulen und weiteren Bildungsinstitutionen erfolgreich einzuführen.

„Aller Anfang ist schwer“, so lautet ein deutsches Sprichwort - was bedeutet, dass neue Situationen oder Herausforderungen zu Beginn oft schwierig sein können. Das mag zuweilen richtig sein, dennoch ist diese Aussage restriktiv. Denn genauso könnte man sagen: „Aller Anfang ist aufregend, inspirierend, begeisternd.“ Vielleicht sollte man es daher mit Konfuzius halten, von dem überliefert ist: „Auch der weiteste Weg beginnt mit dem ersten Schritt." Geht man den ersten Schritt oder die ersten Schritte, entfaltet sich oftmals vieles „wie von selbst“. Man trifft zum richtigen Zeitpunkt die richtigen Leute, die sich mit ihren Fähigkeiten einbringen. Benötigte Räumlichkeiten werden gefunden, Materialien und Gelder freigestellt dank überzeugender Argumente. Das Münchner Modell hat sich so über die Jahre entwickelt.

Seit Beginn des Münchner Modells haben sich 75 Studierende in ihrer Abschlussarbeit mit Achtsamkeit und Meditation befasst - jeweils mit unterschiedlichen Themenstellungen, entsprechend dem jeweiligen Studiengang, wie beispielsweise Soziale Ar- 
beit, Schulpädagogik, Pflege oder Bildung und Erziehung im Kindesalter. Das spricht für ein großes Interesse seitens der Studierenden. Viele von ihnen führen inzwischen in ihrem beruflichen Handlungsfeld Achtsamkeits- und Meditationsprojekte durch. Einige sind sogar Lehrbeauftragte geworden und leiten seit Kurzem selbst Lehrveranstaltungen im Rahmen des Münchner Modells.

Es gibt national und international zahlreiche gute Ansätze, Achtsamkeit und Meditation im Bildungssystem zu verankern. Das Münchner Modell ist ein Beispiel dafür, wie diese Implementierung im Hochschulbereich erfolgreich gelingen kann. 
$$
\text { . }
$$

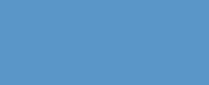$$
\text { - }
$$ 
Seite

\section{Achtsamkeit und Meditation}

im Bildungssystem -

ein Paradigmenwechsel?

Seite $\quad 25 \quad$ Selbst, Seele und Geist

Seite $\quad 31 \quad$ Herz, Liebe und Selbst

sind auch Bildungsthemen ein Erfahrungsbericht

Seite $\quad 37 \quad$ Intellekt und Intuition

Seite $\quad 41 \quad$ Forschung 


\section{Achtsamkeit und Meditation im Bildungssystem - ein Paradigmenwechsel? ${ }^{1}$}

Über Jahrtausende hat sich die Praxis der Achtsamkeit und Meditation als sehr wertvoll erwiesen, um mehr über den eigenen Geist in Erfahrung zu bringen. Seit den 1970er-Jahren haben sich vor allem die Neurowissenschaften mit diesem Thema beschäftigt und herausgefunden, dass Achtsamkeit und Meditation nicht nur Geist und Körper entspannen, sondern Funktionen des Gehirns insgesamt verändern können. Das Interesse an Achtsamkeit und Meditation hat insbesondere in jüngerer Zeit auch gesellschaftlich zugenommen; Achtsamkeit und Meditation haben in Volkshochschulen, Yogazentren, Therapieeinrichtungen, Kliniken, Gefängnissen und Unternehmen Einzug gehalten. Zahlreiche Angebote finden sich inzwischen auch an Schulen und Hochschu$l^{2}{ }^{2}$. Außerdem werden in Kindertagesstätten bereits einfache Achtsamkeitsübungen durchgeführt.

Der nachfolgende Text möchte dazu anregen, in diesem Zusammenhang das Konzept des Selbst-Gewahrseins näher zu betrachten und sieht darin auch für die Wissenschaft ein wichtiges Aufgabenfeld.

\section{Wissenschaft in Bewegung}

Der Begriff WISSENSCHAFT ist vielschichtig. Insbesondere strebt Wissenschaft nach Erkenntnisgewinn. Dafür nutzt sie anerkannte und nachvollziehbare Vorgehensweisen, Methoden und Ansätze, sie sammelt Daten und erstellt Theorien. Sie setzt sich zusammen aus vielen Teildisziplinen und Einzelwissenschaften, die eigene Gegenstandsbereiche erforschen. Auch die systematische Aufbewahrung und Archivierung und das Vermitteln von Wissen sind Aufgaben der Wissenschaft. Der Begriff steht zudem für die zahlreichen Menschen, die im Wissenschaftsbetrieb tätig sind, und ebenso für die unterschiedlichsten Institutionen und Settings, in denen Wissenschaft stattfindet. Wissenschaft kann als guter Kompass dienen, das Leben besser zu verstehen, und verhilft in Zusammenklang mit Ethik und Moral zu einem besseren gesellschaftlichen Wohl. ${ }^{3}$
Ein Blick auf die Wissenschaftsgeschichte zeigt die vielseitigen Errungenschaften, welche die Menschheit in dieser Hinsicht über die Jahrtausende bereits vollbringen konnte. Unsere Wissenschaftsgeschichte zeigt auch, dass die Wissenschaft nicht statisch, sondern immer in Bewegung ist. Ging man früher beispielsweise davon aus, dass die Erde flach ist, wurde irgendwann wissenschaftlich belegt, dass die Erde rund ist - und dass sich die Erde um die Sonne dreht, nicht umgekehrt.

Der Philosoph Thomas S. Kuhn (1922-1996) prägte in diesem Zusammenhang den Begriff PARADIGMA. Für Kuhn umfasst das Paradigma ein Konglomerat von festen Regeln und Kriterien, wie Welt und Wissenschaft zu interpretieren sind. Ein grundlegender Perspektivenwechsel innerhalb der Wissenschaft, ein sogenannter wissenschaftlicher PARADIGMENWECHSEL, kann Kuhn zufolge erst stattfinden, wenn ein alternatives Paradigma sich genügend stark durchsetzen kann und das bisher gültige Paradigma ablöst. Diese Ablösung ist aus der Sicht Kuhns keine kompromissbereite, gemäßigte Veränderung, sondern ein kompletter Wandel bis auf die wissenschaftlichen Grundlagen - eine „Revolution“ also. ${ }^{4}$

Umbruchzeiten sind für die Wissenschaft eine Herausforderung. Veraltete gesellschaftliche Strukturen stagnieren und müssen verändert oder komplett ersetzt werden. Neue werden erst noch erprobt, was ein gewisses Vakuum und eine Unsicherheit entstehen lässt. Eine solche Zeit erleben wir auch heute. In den Bereichen Politik, Wirtschaft und Religion scheint ein bequemes „Weiter-so“ nicht mehr zu funktionieren. Der bisherige materielle Lebensstil, vor allem in den Industrieländern, erscheint nicht mehr zeitgemäß. ${ }^{5}$ Es zeigt sich, dass wir zu weit über das Ziel hinausgeschossen sind: zu viel Individualismus und Eigeninteressen auf Kosten anderer, zu viel Konsum, zu viel Geschwindigkeit. Und auch unsere Verbindung zu Natur und Umwelt ist aus dem Gleichgewicht geraten, wofür der Klimawandel das unübersehbare Zeichen ist. ${ }^{6}$ 


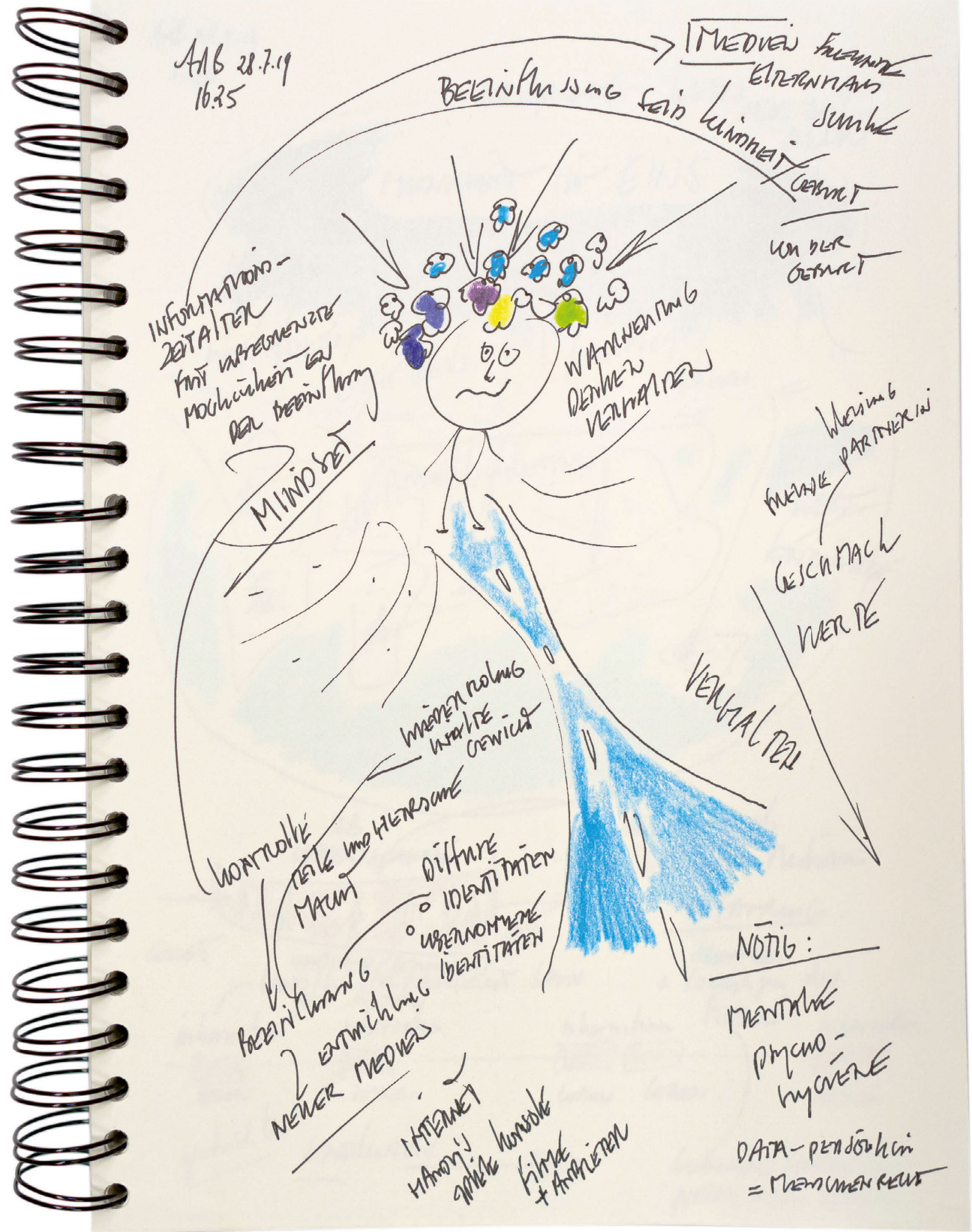

Gedanken prägen Lebenswege. 
Dein spirituelles Selbst ruft jeden Tag nach dir.

Du musst nur erkennen, dass du nicht dein Körper bist, sondern das unendliche Selbst, das in ihm wohnt.

Paramahansa Yogananda (1893-1952)

\section{Glück und Erfolg - oder was ist der Sinn des Lebens?}

Glück und Erfolg werden derzeit noch stark verknüpft mit materiellem Wohlstand. Insbesondere Geld hat einen immens hohen Stellenwert erhalten. Anhand von materiellen Gütern und der symbolischen Bedeutung, die wir ihnen beimessen, stärken wir unser Selbstwertgefühl und Selbstbewusstsein. Es zeigt sich aber auch, dass das Erlangen von Glück und Erfolg auf diese Art und Weise nicht von Dauer sein kann. Das Streben nach Geld und Macht entfacht destruktive Emotionen wie Neid, Hass und Gier, und diese führen letztendlich zu Unzufriedenheit, zu Konflikten und im Extremfall zu Kriegen. Dabei ist längst offensichtlich, dass unser Glück nicht in der äußeren, materiellen Welt liegt, und dass unsere Bestimmung als Menschen nicht das Streben nach Wohlstand und Besitz sein kann. ${ }^{7}$ - Aber was ist dann der Sinn des Lebens? Worauf kommt es an im Leben?

\section{Freiheit des Geistes}

Der indische spirituelle Lehrer und Philosoph Jiddu Krishnamurti (1895-1986) zielt in seinem Buch Antworten auf Fragen des Lebens genau auf diesen Aspekt, als er am Anfang den Leser vor die Frage stellt, ob wir uns jemals gefragt haben, wozu Erziehung eigentlich da ist - „Nur, um irgendwelche Prüfungen zu bestehen und Arbeit zu bekommen?" - und gleich darauf auf ihre eigentliche Aufgabe hinweist: „Oder ist es die Aufgabe der Erziehung, uns, solange wir noch jung sind, darauf vorzubereiten, den ganzen Vorgang des Lebens zu verstehen? Arbeit zu haben und den eigenen Lebensunterhalt zu verdienen ist notwendig - aber ist das alles?" ${ }^{\text {"8 }}$
Eine wesentliche Voraussetzung, das Leben zu verstehen, ist für Krishnamurti die „Freiheit des Geistes“ und damit die Abwesenheit von Furcht. "Aber wie können wir frei sein, um zu schauen und zu lernen, wenn unser Verstand von dem Augenblick unserer Geburt an bis zu dem Augenblick, da wir sterben, durch eine bestimmte Kultur in den engen Grenzen der Ichbezogenheit geformt wird?" ${ }^{10}$ Erst ein freier Geist ohne Konditionierung ist nach Krishnamurti in der Lage, das Denken und Handeln zu beobachten und zu ändern: „Um die Regung Ihres Geistes und Herzens, Ihres ganzen Seins aufmerksam zu beobachten, müssen Sie einen ungebundenen, freien Geist haben [...]. ${ }^{“ 11}$

\section{Forschung über den Geist}

Der 14. Dalai Lama und Friedensnobelpreisträger, Tenzin Gyatso, sieht in der Forschung über den Geist ebenfalls eine Aufgabe für die Wissenschaft: „Auf akademischer Ebene brauchen wir weitere Forschung über die inneren Abläufe und Zusammenhänge, über den Geist. " ${ }^{12}$ Nennenswert sind hier insbesondere die „Mind and Life Dialogues“, die Mitte der 1980er-Jahre von dem chilenischen Neurowissenschaftler und Philosoph Francisco Varela (1946-2001), dem US-amerikanischen Sozialunternehmer R. Adam Engle und dem Dalai Lama initiiert wurden. Zusammen mit anderen westlichen Wissenschaftlern und buddhistischen Mönchen war es das Ziel, einen Austausch zwischen moderner Wissenschaft und Buddhismus anzuregen, um herauszufinden, ob es gemeinsame Schnittstellen gibt. ${ }^{13}$ Im Rahmen dieser Zusammenarbeit haben Achtsamkeits- und Meditationspraktiken mehr und mehr Beachtung in den (Neuro-)Wissenschaften erhalten. ${ }^{14}$

Für den amerikanischen Neurowissenschaftler und Achtsamkeitsforscher Richard Davidson, der zu den Pionieren der Achtsamkeits- und Meditationsforschung ${ }^{15}$ zählt und auch an den „Mind and Life“-Dialogen beteiligt ist, sind für einen gesunden Geist vier Komponenten bedeutend: erstens das grundlegende Erkennen des Gewahrseins, zweitens Verbindung, d.h. über Emotionen wie Mitgefühl, Güte, Dankbarkeit zu verfügen und dadurch mit seiner Umwelt in Beziehung zu treten, drittens Einsicht in das eigene Selbst und viertens die eigene Bestimmung im Leben zu erkennen und das eigene Verhalten daran auszurichten. ${ }^{16}$ In seinem Center for Healthy Minds ${ }^{17}$ in Wisconsin forschen über 100 Personen im Rahmen von zahlreichen wissenschaftlichen Projekten daran, wie ein gesunder Geist und 
insbesondere Wohlbefinden kultiviert werden kann. Dabei stehen unter anderem Achtsamkeits- und Meditationsansätze im Mittelpunkt.

Der Molekularbiologe und buddhistische Mönch Matthieu Ricard, dessen Gehirn in der Achtsamkeitsund Meditationsforschung bislang als eines der am meisten untersuchen gilt ${ }^{18}$, sieht die Meditation als wesentliches Instrument, um Einsicht in den eigenen Geist zu erlangen: „Meditation ist nicht nur ein Luxus, sondern der beste Weg, herauszufinden, wie unser Geist funktioniert. In der Essenz ist Meditation die Arbeit an unserem eigenen Geist. Es geht darum, den Geist zu transformieren, denn er ist es, der die Welt erfährt. Er kann unser bester Freund sein, aber auch unser größter Feind. Darum müssen wir ihn kultivieren. Und das hat nicht nur Auswirkungen auf unser inneres Erleben, sondern auch auf unsere Beziehungen und unser Umfeld.“" ${ }^{19}$ Meditation und eine „mitfühlende Achtsamkeit ${ }^{\text {"20 }}$ können vor allem auch wichtige Eigenschaften wie Mitgefühl und Altruismus fördern, die für ein glückliches und friedliches Zusammenleben essenziell sind. ${ }^{21}$

\section{Achtsamkeit als Haltung}

Eine eher allgemeinere Deutung von ACHTSAMKEIT, die heute viel Verbreitung findet, beinhaltet, den aktuellen Zustand bewusst wahrzunehmen, nur zu beobachten, nicht zu bewerten. „Achtsamkeit besteht im Wesentlichen in nichts anderem als einer besonderen Art von Aufmerksamkeit, die wir Gewahrsein nennen. " ${ }^{22}$ Viele Alltagsaktivitäten eignen sich dafür gut: zum Beispiel Essen, Spazierengehen, mit jemandem im Gespräch sein, Putzen, Bügeln, Geschirrspülen, der Natur lauschen und vieles mehr ${ }^{23}$. Beim Geschirrspülen beispielsweise sind die meisten Menschen mit den Gedanken woanders. Achtsames Geschirrspülen bedeutet, dass ich sehe, was ich spüle. Dass ich spüre, ist das Wasser warm, ist es kalt; wie fühlt sich der Teller oder das Glas an. Wenn wir lernen, diese Dinge wieder bewusster zu tun, dann trainieren wir unseren „Achtsamkeitsmuskel“", sodass wir bei allen Dingen, die wir machen, mehr im Jetzt sind. Entscheidend ist, dass man dies alles nicht zu sehr verkopft und mechanisch tut. Nach dem Motto: Ich muss jetzt achtsam essen, ich muss jetzt achtsam atmen. Der Wunsch, achtsam zu sein, sollte von innen kommen. Achtsamkeit ist eine Haltung.

\section{Achtsamkeits- und Einsichtsmeditation}

Bei der ACHTSAMKEITS- und EINSICHTSMEDITATION stehen das achtsame Beobachten physischer Zustände, Gedanken und Emotionen im Vordergrund. Ziel ist es, den Fluss der Gedanken und Emotionen zu beobachten und auf diese Weise mehr Einsicht in ihre Mechanismen zu erlangen. Dafür kann man beispielsweise strukturiertere Übungen praktizieren, wie täglich 15 bis 20 Minuten in Stille zu sitzen und entspannt den Atem zu beobachten, oder den „Bodyscan“ - eine Entspannungsmethode, bei der man mit seiner Aufmerksamkeit schrittweise durch den eigenen Körper „wandert" und in die Körperteile hineinspürt. Auch Sutras (Leitfäden) wie: „Ich atme ein und lasse die Aktivitäten meines Geistes ruhig und friedvoll werden. Ich atme aus und lasse die Aktivitäten meines Geistes ruhig und friedvoll werden " ${ }^{24}$ zielen darauf $\mathrm{ab}$, den Geist gelassener werden zu lassen.

\section{Metta-Meditation}

Unter den Meditationstechniken bilden die METTAMEDITATIONEN oder MEDITATIONEN DER LIEBENDEN GÜTE eine eigene Kategorie. Sie stellen insbesondere das Fördern von MITGEFÜHL und VERBUNDENHEIT in den Mittelpunkt. Bei diesen Meditationformen werden in der Regel liebevollen Sätze für sich selbst oder andere Personen innerlich rezitiert oder ausgesprochen, wie beispielsweise: „Möge ich sicher und geborgen sein. Möge ich glücklich sein. Möge ich gesund sein.“ Und bezogen auf andere: „Möge diese Person sicher und geborgen sein. Möge sie glücklich sein. Möge sie gesund sein. "Dabei kann man sich eine Person vorstellen, die man mag, oder eine Person, zu der man kaum eine Beziehung hat, bis hin zu Menschen, die man ablehnt oder nicht wertschätzt. Eine andere Variante ist, sich ein liebevolles wohlwollendes Gefühl vorzustellen und diese Herzenswärme immer weiter auszudehnen und neben Menschen beispielsweise auch die Natur darin einzuschließen. Der Radius kann sich auf eine direkte Umgebung beziehen, bis hin zur gesamten Erde oder noch weiter darüber hinaus. ${ }^{25}$ 


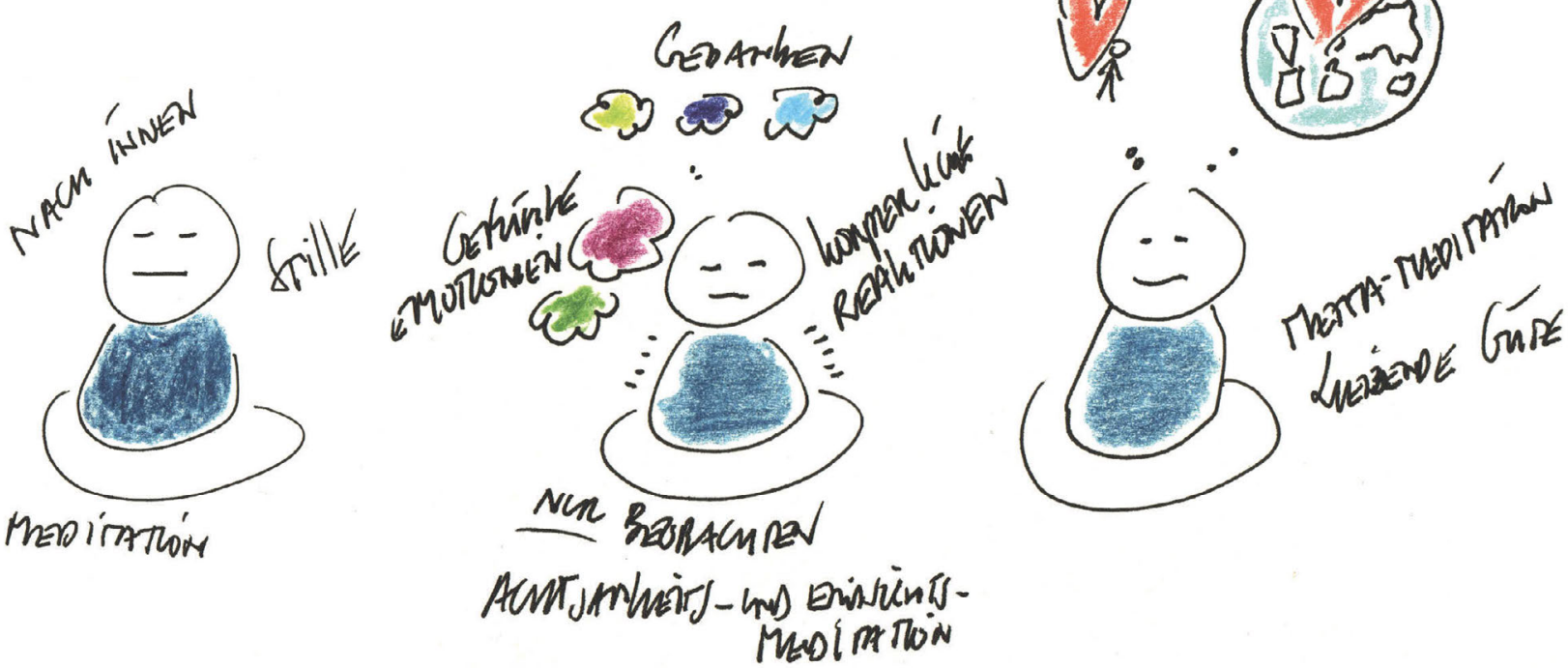

Meditation, Achtsamkeits- und Einsichtsmeditation und Metta-Meditation.

\section{Problematik des Achtsamkeitstrainings}

Ein Problem der rasanten Verbreitung von Achtsamkeitstechniken ist, dass sie inzwischen auch für Optimierungen genutzt werden, die Profit- und Leistungsmaximierung als vorrangiges Ziel haben. Ein Beispiel sind Achtsamkeitsangebote in Unternehmen, die Arbeitnehmerinnen und Arbeitnehmer stressresistenter machen sollen, ohne aber die stressverursachenden Strukturen zu verändern. Eine andere Schwierigkeit ist, dass Achtsamkeit auch die Selbstgefälligkeit verstärken kann, sodass man sich vorrangig auf seine eigenen Belange konzentriert. ${ }^{26}$

Und nicht zuletzt gibt es die moralische Frage. Dürfen beispielsweise Scharfschützen Achtsamkeitstrainings durchlaufen, um anschließend konzentrierter und gezielter töten zu können? Richard Davidson betont in diesem Zusammenhang, dass die Achtsamkeitspraxis immer auch mit einer ethischen Haltung einhergehen muss. ${ }^{27}$ Der Pionier der Achtsamkeitsbewegung und Gründer des Center for Mindfulness in Medicine, Health Care and Society an der University of Massachusetts Medical School, Jon Kabat-Zinn, weist ebenfalls auf diesen Aspekt hin. Für ihn muss bei der Achtsamkeitspraxis stets auch die Herzqualität berücksichtigt werden. ${ }^{28}$ Matthieu Ricard spricht von einer „mitfühlenden Achtsamkeit“29. Das Mitgefühl sorgt seiner Meinung nach dafür, dass die Achtsamkeit aufrichtig gelebt wird. ${ }^{30}$

Eine weitere Komplexität, mit der sich Achtsamkeit konfrontiert sieht, ist die Frage des Dualismus. Kann ich in meiner Rolle als bewusster Beobachter das Geschehen an sich überhaupt achtsam erleben? Beispielsweise, wenn ich achtsam esse und versuche, mir dessen bewusst zu sein. Ich merke, wie ich esse, was ich esse, wie es aussieht, wie es riecht etc. Aber die Verbindung zu mir als Referenzpunkt schafft eine Trennung zwischen mir und der Handlung „essen“ - eine Dualität. Ist es möglich, diese Dualität aufzuheben? Dies würde beinhalten, den Referenzpunkt „Ich“ aufzulösen. Statt „Ich esse einen Salat“, heißt es dann: „Salat essen“.

\section{Der innere Beobachter}

Der indische spirituelle Lehrer Nisargadatta Maharaj (1897-1981) greift diesen Aspekt auf: „Der Beobachter, die Beobachtung und die beobachtete Welt erscheinen gemeinsam und lösen sich gemeinsam auf. Hinter all dem steht die Leere. Die Leere ist alles, was ist. ${ }^{\text {" }}{ }^{31}$ Es stellt sich vor allem die Frage, was unter dem Beobachter verstanden wird. „Wer ist es, der behauptet, lebendig 
zu sein? Finde heraus, wer der Beobachter ist, der weiß, dass er lebendig ist. Dies ist das Gewahrsein der eigenen Existenz, das allen Gedanken vorausgehende ,Ich bin'. Wer sagt, ,Ich bin lebendig', wer sagt ,Ich bin nicht lebendig'? Was ist das? Das ,Ich bin' kann nicht in Worte gefasst werden; es ist das Wissen, das Gewahrsein, noch vor der Entstehung von Gedanken. Du musst einfach nur sein. " ${ }^{32}$ Ein wesentlicher Punkt in der Lehre von Nisargadatta Maharaj ist die Aufteilung in ein verbales „Ich bin“ und in ein nonverbales „Ich bin“, das nur ohne Gedanken, Erinnerungen, Emotionen, Assoziationen und Wahrnehmungen erfahrbar ist. Will man $\mathrm{zu}$ seinem wahren inneren Wesen gelangen, muss man sich an das nonverbale "Ich bin“ halten. ${ }^{33}$ Gelingt es uns, auf dieser Ebene ohne irgendwelche Zuschreibungen zu bleiben, kommen wir in Berührung mit dem reinen Bewusstsein. „Die Wahrheit ist ewig, die Realität unveränderlich. Was sich verändert, ist nicht real; was real ist, verändert sich nicht. Also, was ist es in Ihnen, das sich nicht verändert? Solange es Nahrung gibt, gibt es Körper und Verstand. Gibt es keine Nahrung mehr, stirbt der Körper, und der Verstand löst sich auf. Doch löst sich auch der Beobachter auf?“34

\section{Um still zu sein, musst du den Geist völlig im Selbst versenken.}

Ramana Maharshi (1879-1950)

\section{Praxis der Selbstforschung}

Bereits im 8. Jahrhundert hat der indische Philosoph und Lehrer des Vedanta, Shankaracharya (788-820), die Bedeutung der Selbstforschung hervorgehoben: „Da ich stets das Eine, Subtile, der Kenner, der Zeuge, das immer Existierende und Unbewegliche bin, kann es keinerlei Zweifel daran geben, dass ich DAS (Brahman) bin. So sollte Selbstforschung betrieben werden. ${ }^{\text {" }}{ }^{35}$

Der bekannte indische spirituelle Lehrer Ramana Maharshi (1879-1950) greift diesen Aspekt auf und beschreibt, wie die Praxis der Selbstforschung betrieben werden könnte - indem man sich die folgenden einfachen Fragen stellt und diesen gewissenhaft auf den Grund geht. „Wer bin ich? Wer sieht, wenn ich sehe? Wer hört, wenn ich höre? Wer weiß, dass ich wahrnehme? Wer bin ich?" ${ }^{36}$ Diese Fragen führen an einen Punkt, der unveränderlich ist durch Zeit. Betrachtet man sich beispielsweise über die Jahre regelmäßig im
Spiegel, sieht man, dass man älter wird. Aber verändert sich auch der innere Beobachter?

Im Grundlagenwerk Die drei Pfeiler des Zen von Philip Kapleau (1912-2004) heißt es dazu: „Um ein Buddha zu werden, muss man erkennen, wer es ist, der ein Buddha werden will. Um diesen Meister zu erkennen, muss man eben hier und jetzt entschlossenen Willens danach forschen: ,Was ist es, das alles als Gut-und-Böse auffasst, das Farben sieht und Laute hört? ‘“37

Ein solcher Prozess der Selbstforschung erfordert die radikale Aufgabe alter Konditionierungen und der Identifizierung damit. Der buddhistische Lehrer Jack Kornfield weist in seinem Buch Frag den Buddha und geh den Weg des Herzens darauf hin: „Unsere Welt und unser Gefühl von Ich oder Selbst sind ein Spiel von Mustern. Jegliche Identität, an der wir festhalten können, ist flüchtig, vorübergehend. Das lässt sich aus Begriffen wie ,Ichlosigkeit" oder ,Leerheit von Selbst schwer entnehmen. Mein Lehrer Achaan Chah sagte: ,Wenn du versuchst, es intellektuell zu verstehen, wird dein Kopf wahrscheinlich platzen.' Doch die Erfahrung der Ichlosigkeit kann uns zu großer Freiheit führen. “"

Kurz: Es geht darum, sich vom Verstand zu lösen und mit ganzem Wesen zu sein. Und dies kann in jedem Augenblick praktiziert werden. „Um zum eigenen Selbst zu gelangen, zur Verwirklichung des unpersönlichen Gottes, des allgegenwärtigen Seins, ist kein Weg denkbar; denn schon der bloße Begriff eines Weges entfernt das Selbst vom eigenen Sein. Die Idee des Weges trägt eine Vorstellung von etwas in sich, das weit entfernt ist, während doch Sein das eigentliche Selbst ist.

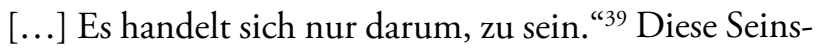
Form ist eine tiefe Form des achtsamen Lebens.

Das bedeutet selbstverständlich nicht, dass das Denken im Rahmen des achtsamen Lebens gar keine Funktion mehr hat. Das Denken kann als Werkzeug äußerst wichtig sein, um den täglichen Alltag zu meistern. Das benötigte Wissen für die jeweiligen Handlungsabläufe kann das Denken mithilfe des Gedächtnisses speichern und, wenn benötigt, passend abrufen. Aber die zahlreichen Gedanken auf der psychologischen Ebene, die sich mit Vergangenheit und Zukunft beschäftigen, mit Ängsten und Wünschen, halten uns fortwährend vom Erleben des jetzigen Moments ab. ${ }^{40}$ Zudem ist unser Denken kein fixer, stabiler Zustand, sondern ändert sich ständig.

Die in England geborene Dozentin und Psychotherapeutin Phyllis Krystal (1914-2016) schreibt in ihrem Buch Monkey Mind. Den Verstand bändigen: „Das Denken ist demzufolge nicht immer ein verlässlicher Indikator dafür, was richtig oder falsch ist. Es stattet uns lediglich mit einer Sichtweise aus, in die unsere persönli- 
chen Vorlieben und Abneigungen einfließen. Diese sind wiederum von den Bindungen an bestimmte Objekte, Ideen, Meinungen, Glaubenssätze, an Aberglauben und eine Menge menschlicher Gedanken geprägt [...].“ ${ }^{41}$

Wollen wir unsere Aufmerksamkeit nach innen richten, benötigen wir Stille: „Wenn der Geist sehr still, vollkommen still ist, wenn es also keine Gedankenbewegungen und deshalb keinen Erfahrenden, keinen Beobachter gibt, dann besitzt gerade diese Stille ihr eigenes kreatives Verstehen. In dieser Stille wird der Geist in etwas anderes verwandelt. ${ }^{\text {" }}{ }^{22}$ In diesem Fall sprechen wir über ein Verständnis von Achtsamkeit, das weit über eine eher allgemeinere Auffassung von Achtsamkeit hinausgeht. Achtsamkeit in ihrer ganzen Tiefe ist ein meditativer Zustand, ist MEDITATION. „Achtsamkeit kann uns dabei helfen, wieder zu kommunizieren, vor allem mit uns selbst. “43 Oder mit den Worten von Jon Kabat-Zinn gesprochen: „Doch das wichtigste Ziel der Achtsamkeitspraxis ist, in Kontakt zu kommen mit sich selbst. ${ }^{44}$

\section{Meditation und Selbst}

„Die Quelle der Weisheit ist das Selbst“, sagt der indische spirituelle Lehrer und Friedensaktivist Vishnu Devananda (1927-1993). Und weiter: „Das Selbst ist nicht der individuelle Körper oder der Geist, sondern vielmehr der Aspekt tief innen in jedem Menschen, der die Wahrheit kennt. “45

Und um zu dieser Wahrheit, zu diesem tiefen inneren Seins- und Wesenszustand zu gelangen, bedarf es der meditativen Versenkung. Denn: „Ohne die Hilfe der Meditation kann man das Wissen um das Selbst nicht erlangen. Ohne ihre Hilfe kann man nicht in den göttlichen Zustand hineinwachsen." ${ }^{46}$ Und andersherum: Wenn es gelingt, das SELBST-GEWAHRSEIN fortwährend aufrechtzuerhalten, so wird dies als „wahre Meditation" bezeichnet ${ }^{47}$.

\section{Ausblick}

Um nochmals mit Krishnamurti zu sprechen: „Wie können wir lernen, das eigene Leben zu verstehen? Wie kann jeder herausfinden, wer er selber wirklich ist, anstelle andere zu imitieren ${ }^{48}$, und wie können wir ohne Angst in ,vollständiger Freiheit wachsen und eine andere Gesellschaft, eine neue Welt erschaffen "“? ${ }^{49}$. Um dies zu erreichen, ist es wichtig, dass wir auch im Bildungssystem lernen, SELBST-GEWAHRSEIN zu fördern und zu pflegen..$^{50}$ Und dafür soll der Geist ungebunden und frei sein. Achtsamkeits- und Meditationsangebote können hier einen wichtigen Beitrag leisten. „In der Meditation geht es darum, den Geist zu erforschen und die positiven Qualitäten zu stärken. Wofür aber ist die Meditation gut? Sie dient der inneren Entwicklung, damit wir mehr auf der Basis heilsamer Geisteszustände handeln." ${ }^{\text {1 }}$

Vergleicht man die Anfänge der neurowissenschaftlichen Achtsamkeits- und Meditationsforschung in den 1970er-Jahren mit heute, hat sich bereits sehr viel getan. Da die Forschungsinstrumentarien und Vorgehensweisen immer feiner und genauer werden, sind sicherlich weiterhin neue Erkenntnisse zu erwarten. ${ }^{52}$ Inwiefern sich die Existenz und das Wirken des Selbst dabei wissenschaftlich erfassen lassen, bleibt abzuwarten. ${ }^{53}$ Voraussetzung dafür wäre dann eine Verschiebung der Perspektive von der materiellen Ebene (zum Beispiel das Gehirn) zur immateriellen Ebene (das Selbst). In diesem Fall könnte man wohl auch von einem Paradigmenwechsel sprechen.

\section{QUELLEN}

Dalai Lama XIV. (2015a). „Achtsam sein heißt, den Geist bewusst zu gebrauchen“, in: Zimmermann, Michael / Spitz, Christof / Schmidt, Stefan (Hrsg.), Achtsamkeit. Ein buddhistisches Konzept erobert die Wissenschaft. Bern: Verlag Hans Huber, S. 211-216

Dalai Lama XIV. (2015b). „Anstelle eines Schlussworts: Gespräch mit S. H. dem Dalai Lama“, in: Zimmermann, Michael / Spitz, Christof / Schmidt, Stefan (Hrsg.). Achtsamkeit. Ein buddhistisches Konzept erobert die Wissenschaft. Bern: Verlag Hans Huber, S. 337-345.

Dalai Lama XIV. (2000). Das Buch der Menschlichkeit. Eine neue Ethik für unsere Zeit. Köln: Bastei Lübbe AG.

Davidson, Richard J. / Goleman, Daniel (2017). Altered Traits. Science Reveals How Meditation Changes Your Mind, Brain, and Body. New York: Avery. de Vries, Gerard (1985). De ontwikkeling van wetenschap. Een inleiding in de wetenschapsfilosopfie. Groningen: Wolters-Noordhoff.

Goleman, Daniel (2003). Dialog mit dem Dalai Lama. Wie wir destruktive Emotionen überwinden können. München / Wien: Carl Hanser Verlag.

Kabat-Zinn, Jon (2019). Gesund durch Meditation. München: Knaur Verlag. 
Kabat-Zinn, Jon (2015). Im Alltag Rube finden. Meditationen für ein gelassenes Leben. München: Knaur Verlag.

Kapleau, Philip (2012). Die drei Pfeiler des Zen. Lehre - Übung - Erleuchtung. München:

O. W. Barth Verlag.

Kornfield, Jack (2017). Frag den Buddha und geh den Weg des Herzens. Was uns bei der spirituellen Suche unterstützt. München: Kösel-Verlag.

Kornfield, Jack (2010). Meditationen, die unser Herz öffnen. München: Arkana.

Krishnamurti, Jiddu (1993). Einbruch in die Freiheit. Berlin / Frankfurt am Main: Ullstein.

Krishnamurti, Jiddu (1992). Antworten auf Fragen des Lebens. Freiburg im Breisgau: Verlag Hermann Bauer KG.

Krystal, Phyllis (2017). Monkey Mind. Den Verstand bändigen. Dietzenbach: Sathya Sai Vereinigung e.V.

Kuhn, Thomas, S. (1967). Die Struktur wissenschaftlicher Revolutionen. Frankfurt: Suhrkamp.

Maharishi Mahesh Yogi (2010). Die Wissenschaft vom Sein und die Kunst des Lebens. Bielefeld: J. Kamphausen Verlag und Distribution GmbH.

Maharshi, Ramana (2011a). „Wer bin ich?“ Der Übungsweg der Selbstergründung. Norderstedt: BoD.

Maharshi, Ramana (2011b). Sei, was Du bist! Die wichtigsten Lehren des großen indischen Weisen. München: O.W. Barth Verlag.

Nisargadatta Maharaj (2017). Ich Bin. Teil I. Gespräche mit Sri Nisargadatta Maharaj. Bielefeld: J. Kamphausen Mediengruppe GmbH.

Nisargadatta Maharaj (2014b). Jenseits von Freiheit. Gespräche mit Sri Nisargadatta Maharaj. Lohne: Lotus Press.

Ott, Ulrich (2015). Meditation für Skeptiker. Ein Neurowissenschaftler erklärt den Weg zum Selbst. München: Droemer Knaur.

Ricard, Matthieu (2020). Glück. München: Knaur Verlag.

Ricard, Matthieu (2018a). Allumfassende Nächstenliebe. Hamburg: Edition Blumenau.

Ricard, Matthieu (2015). „Achtsamkeit - ein Zustand vollkommener Einfachheit“, in: Zimmermann, Michael / Spitz, Christof / Schmidt, Stefan (Hrsg.), Achtsamkeit. Ein buddhistisches Konzept erobert die Wissenschaft. Bern: Verlag Hans Huber, S. 49-55. Sedlmeier, Peter (2016). Die Kraft der Meditation. Was die Wissenschaft darüber weiß. Reinbek bei Hamburg: Rowohlt Taschenbuch Verlag.
Shankaracharya (2016). Aparoksha Anubhuti. Die direkte Verwirklichung des Selbst. Horn-Bad Meinberg: Yoga Vidya Verlag.

Singer, Michael A. (2019). Die Seele will frei sein. Eine Reise zu sich selbst. Berlin: Ullstein Buchverlage $\mathrm{GmbH}$.

Tang, Yi-Yuan (2019). Die Wissenschaft der Achtsamkeit. Wie Meditation die Biologie von Körper und Geist verändert. Paderborn: Junfermann Verlag.

Thich Nhat Hanh (2000). Das Wunder des bewussten Atmens. Stuttgart: Theseus Verlag.

Vishnu Devananda (1986). Meditation und Mantras. Eine Darstellung der großen Yoga-Systeme in Theorie und Praxis. München: Heinrich Hugendubel Verlag.

Wolinsky, Stephen H. (2002). Ich bin dieses Eine: Begegnungen mit Sri Nisargadatta Maharaj. Kirchzarten bei Freiburg: VAK Verlags GmbH.

\section{Zeitschrift}

Davidson, Richard J. (2018). „Interview“, in: Moment by Moment, Ausgabe 03. Freiburg: Hammer Solutions Media, S. 56-63.

Ricard, Matthieu (2018b). „Interview“, in: Moment by Moment, Ausgabe 03, Freiburg: Hammer Solutions Media, S. 36-42.

\section{DVD}

Engle, Dawn Gifford. (2019). The Dalai LamaScientist. Arvada / Colorado: PeaceJam Foundation. Mendiza, Michael (2008). Jiddu KrishnamurtiDie Herausforderung sich zu ändern. Eine Dokumentation über Krishnamurtis Werdegang und einige seiner wichtigsten Aussagen. Sulzbach: Arbeitskreis für freie Erziehung e.V.

Wolinsky, Stephen H. (2009). Ich bin das Ich bin. Erfahre die Lehren des Sri Nisargadatta MaharajTeil 1. Stuttgart: Mouna GmbH.

\section{Internet}

Davidson, Richard J. (2019a). Vortrag und Podiumsdiskussion: „We can change the brain, by changing the mind“ am 19. März 2019 in München beim Forum für den Wandel der Edith-Haberland-Wagner Stiftung, www.ehw-stiftung.de (letzter Zugriff: 13.09.2020)

Kornfield, Jack (2014). „Jack Kornfleld on Nisargadatta Maharaj (2)“, www.youtube.com/watch? $v=b X u F 8 q m v 5 \mathrm{Nc}$ (letzter Zugriff: 13.09.2020) 
Morel, Delphine (2016). Mönche im Labor,

www.youtube.com/watch?v=3qpLUpkdbKE (letzter

Zugriff 13.09.2020)

Singer, Michael A. (2018). „What Do You Really

Want?", www.youtube.com/watch?v=73-2PggJJWO

(letzter Zugriff: 13.09.2020)

Wolinsky, Stephen H. (2010). „Nisargadatta Maharaj /

Experiential Meditation",

www.youtube.com/watch?v=rE6kS7vVsXw (letzter

Zugriff: 13.09.2020)

1 Dieser Text wurde auch veröffentlicht in: Frey, Reiner (Hrsg.) (2020). Meditation und die Zukunft der Bildung: Spiritualität und Wissenschaft. Weinhein / Basel: Beltz-Juventa.

2 Für konkrete Beispiele, wie Achtsamkeit und Meditation erfolgreich an Hochschulen implementiert werden können, sowie eine Übersicht zahlreicher Hochschulen, die auf diesem Gebiet bereits aktiv sind, siehe www.hm.edu/meditationsmodell (letzter Zugriff: 13.09.2020). Siehe auch https://achtsamehochschulen.de (S. 65) sowie www.netzwerk-achtsamkeit-in-der-bildung.de. (S. 66) (letzter Zugriff: 13.09.2020).

3 Vgl. de Vries 1985.

${ }^{4}$ Vgl. Kuhn 1967, S. 119.

5 Vgl. Dalai Lama 2000.

6 Vgl. Dalai Lama 2000, S. 204ff.

7 Vgl. Dalai Lama 2000, S. 14; Ricard 2020.

8 Krishnamurti 1992, S. 11. Für eine Zusammenfassung über Krishnamurtis Werdegang und einige seiner wichtigsten Aussagen siehe der Dokumentarfilm Jiddu Krishnamurti - Die Herausforderung sich zu ändern von Michael Mendiza.

9 Vgl. Krishnamurti 1992, S. 13.

10 Krishnamurti 1993, S. 23.

11 Krishnamurti 1993, S. 22.

12 Dalai Lama 2015a, S. 213.

${ }^{13}$ Weitere Informationen siehe www.mindandlife.org (letzter Zugriff: 13.09.2020). Siehe auch den Film Dalai Lama - Scientist (2019).

${ }^{14}$ Vgl. Goleman 2003; Davidson / Goleman 2017.

15 Vgl. Davidson / Goleman 2017; Ott 2015; Sedlmeier 2016; Tang 2019.

${ }^{16}$ Vgl. Davidson 2018, S. 61. Siehe auch Vortrag und Podiumsdiskussion "We can change the brain, by changing the mind" am 19. März 2019a, Prof. Dr. Richard Davidson in München beim Forum für den Wandel der Edith-Haberland-Wagner Stiftung, www.ehw-stiftung.de (letzter Zugriff: 13.09.2020).

17 Siehe www.centerhealthyminds.org (letzter Zugriff: 13.09.2020).

18 Vgl. Ricard 2018b, S. 38.

19 Ricard 2018b, S. 38.

20 Ricard 2018b, S. 42.

${ }^{21} \mathrm{Vgl}$. Ricard 2015, S. 53ff. und 2018a.

22 Kabat-Zinn 2019, S. 43.

${ }^{23}$ Vgl. Kabat-Zinn 2019, S. $169 \mathrm{ff}$.

24 Thich Nhat Hanh 2000, S. 9 und S. 72.

25 In dem Buch Meditationen, die unser Herz öffnen (2010) von Jack Kornfield sind eine Reihe von Übungen zur Metta-Meditation oder Liebenden Güte beschrieben. Für weitere Übungsbeispiele siehe auch Kornfield 2017.

${ }^{26}$ Vgl. Davidson 2018, S. 61.
${ }^{27}$ Siehe Vortrag und Podiumsdiskussion am 19. März 2019a, Prof. Dr. Richard Davidson, wWW.ehw-stiftung.de (letzter Zugriff: 13.09.2020).

${ }^{28} \mathrm{Vgl}$. Kabat-Zinn 2019, S. 23.

29 Ricard 2018b, S. 42.

$30 \mathrm{Vgl}$. Ricard 2018a.

31 Wolinksky 2002, S. 22.

32 Nisargadatta 2014, S. 27f. Dass die Benennung des Wahrnehmenden eine Schaffung des eigenen Denkens ist, findet sich auch bei Krishnamurti 1992, S. 192.

${ }^{33}$ Vgl. Wolinksky 2002, S. 31. Nisargadatta Maharaj unterscheidet in seinen Lehren zwischen dem verbalen und dem norverbalen Ich bin. Eine Übung zum „nonverbalen Ich bin“ zeigt der langjährige Schüler von Nisargadatta Maharaj und Begründer der Quantenpsychologie, Dr. Stephen H. Wolinsky, unter "What is the I am?", „Nisargadatta Maharaj / Experiential Meditation", www.youtube.com/watch?v=rE6kS7vVsXw (letzter Zugriff: 13.09.2020). Die Übung ist ebenfalls Teil des Films Ich bin das Ich bin. Erfahre die Lehren des Sri Nisargadatta Maharaj - Teil 1 (2009, 7:33-16:39 Min.). Vertiefend dazu siehe den Kurzbeitrag von Jack Kornfield über seine Erfahrung mit Nisargadatta: „Jack Kornfield on Nisargadatta Maharaj (2)",

www.youtube.com/watch?v=bXuF8qmv5Nc (letzter Zugriff: 13.09.2020).

34 Nisargadatta 2017, S. 104.

35 Shankaracharya 2016, S. 39.

36 Singer 2019, S. 35; siehe auch Maharshi 2011a, S. 18f. sowie Maharshi 2011b, S. 142f. Auch der US-amerikanische spirituelle Lehrer und Wirtschaftsprofessor Michael A. Singer betont die Ausrichtung auf das Selbst: „Wenn Sie über die Natur des Selbst nachdenken, meditieren Sie. Deshalb ist die Meditation der höchste Zustand. Es ist die Rückkehr zu den Wurzeln Ihres Wesens, zum einfachen Gewahrsein dessen, dass Sie gewahr sind." (Singer 2019, S. 53) Siehe auch Interview „What do you really want?",

www.youtube.com/watch?v=73-2PggJJW0 (letzter Zugriff: 13.09.2020).

37 Kapleau 2012, S. 236.

38 Kornfield 2017, S. $262 f$.

39 Maharishi Mahesh Yogi 2010, S. 323.

40 Vgl. Krishnamurti 1993, S. 41 und S. $87 f$.

${ }^{41}$ Krystal 2017, S. 171.

42 Krishnamurti 1992, S. 192.

${ }^{43}$ Thich Nhat Hanh. Zitat in: Glogowski, Dieter (2016). Buddhistische Ansichten. 365 Weisheiten aus dem Himalaya. München: Frederking \& Thaler.

${ }^{44}$ Kabat-Zinn 2015, S. 19.

45 Vishnu Devananda 1986, S. 18.

46 Swami Sivananda in: Vishnu Devananda 1986, S. 13.

47 Vgl. Krishnamurti 1993, S. $101 \mathrm{f}$.

${ }^{48} \mathrm{Vgl}$. Krishnamurti 1992, S. 13.

49 Krishnamurti 1992, S. 14.

50 Vgl. auch Maharshi 2011b, S. 17.

51 Dalai Lama 2015b, S. 344f.

52 Vgl. Goleman / Davidson 2017, S. 288ff.; Morel 2016.

${ }^{53}$ Vgl. Morel 2016, 17:51 Min. 
(24) Einführung

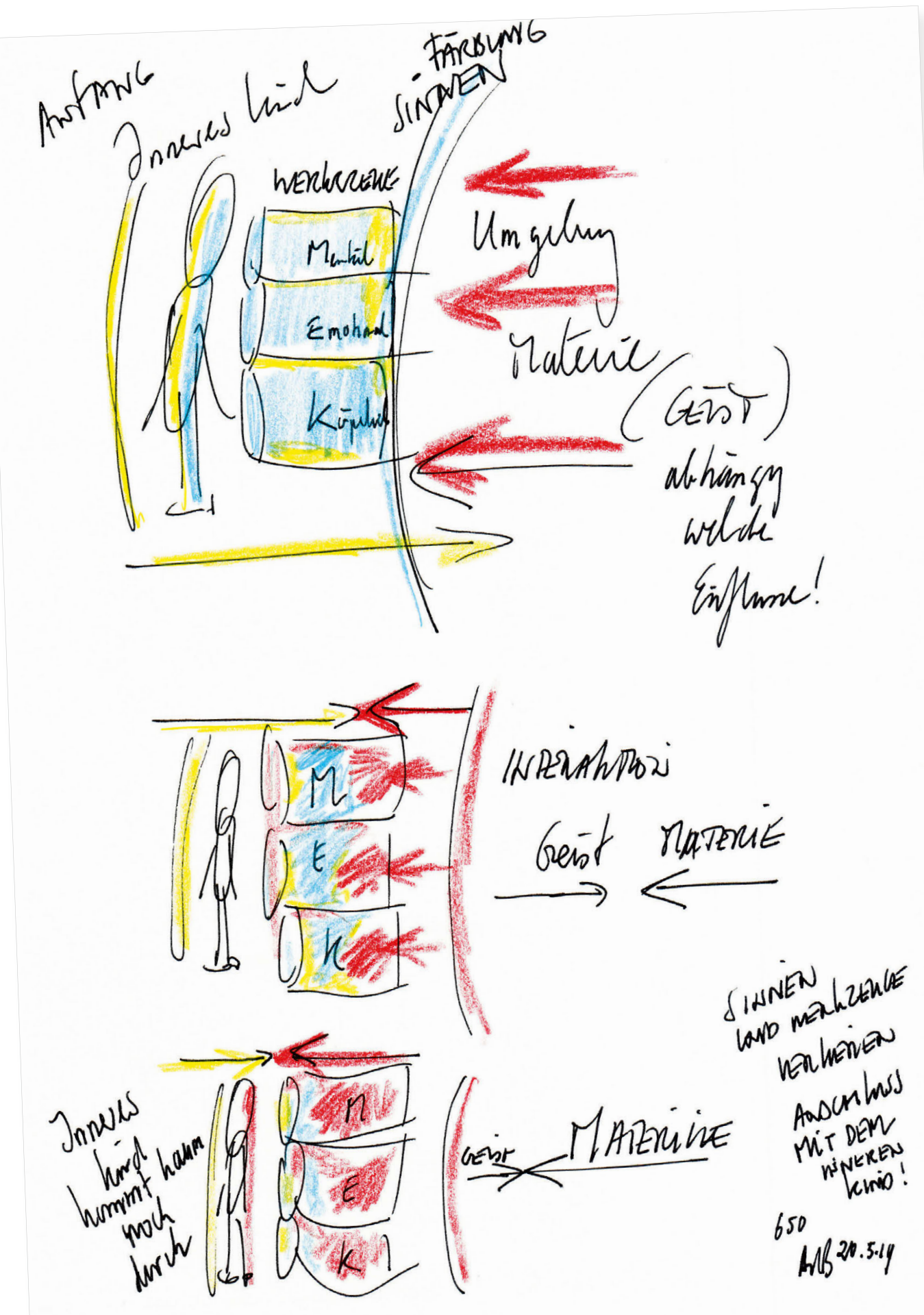

Interaktion zwischen Geist und Materie. Die Träger des Selbst, der Seele, sind zunächst gefüllt mit Materie.

Durch die Vergeistigung der Materie werden die Träger wieder durchlässig und klar.

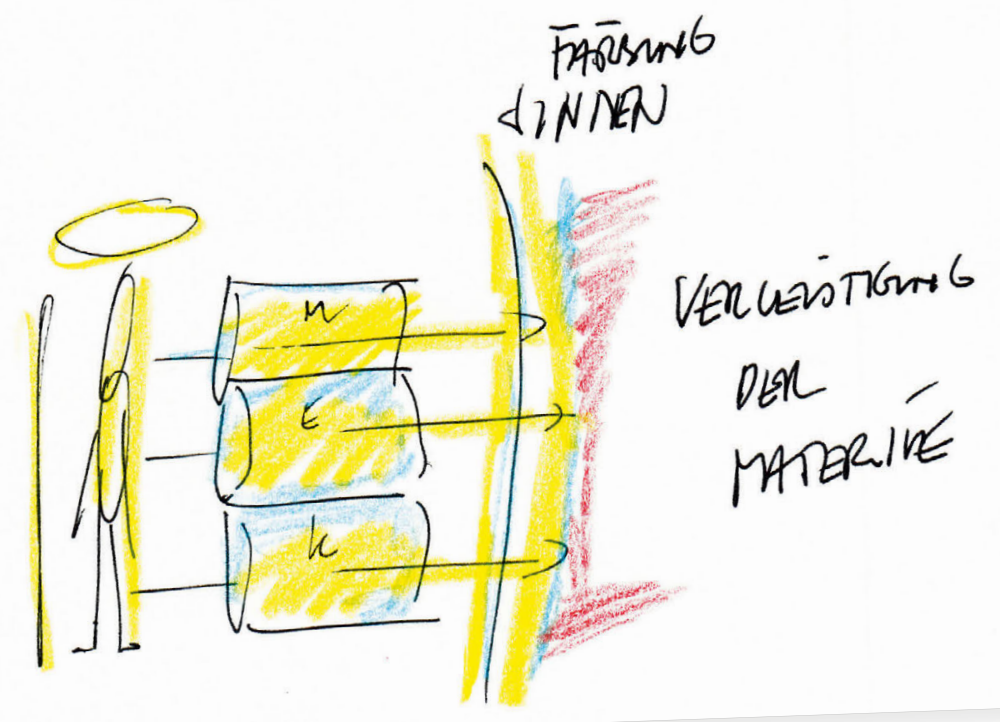

(C) Münchner Modell | www.hm.edu/meditationsmodell 


\section{Selbst, Seele und Geist}

Wenn man sich mit Achtsamkeit und Meditation auseinandersetzt, wird man schnell feststellen, dass der Begriff des SELBST in der themenbezogenen Literatur eine wesentliche Rolle spielt und häufig in unterschiedlicher Bedeutung verwendet wird. Was aber bedeutet „das Selbst“?

Unterschieden werden im Allgemeinen zwei Ebenen des SELBST: ${ }^{1}$

- Das NIEDERE SELBST: Das Ich, die Person, unser sogenanntes Ego, das, was uns vom HÖHEREN SELBST trennt. Es betrifft die Identifizierung mit unserem Körper, mit unseren schwankenden Gefühlen und Gedanken, d.h. mit dem, was wir unsere Persönlichkeit nennen.

- Das HÖHERE SELBST: In den Lehren über Achtsamkeit und Meditation bezieht sich der Begriff auf eine höhere Ebene, die über das IchKonstrukt der Persönlichkeit hinausgeht. Dieses SELBST betrifft den inneren Kern des Menschen, auch SEELE genannt.

In diesem Textabschnitt wird neben den beiden Ebenen und Bedeutungen des SELBST auch der Begriff EGO beleuchtet, da die durch das EGO hervorgerufene Begrenzung des menschlichen Geistes den Weg zum HÖHEREN SELBST verschleiert.

Des Weiteren werden zwei Bedeutungen des Wortes GEIST aufgezeigt und erläutert.

\section{Selbst, Ich und Persönlichkeit - das NIEDERE SELBST}

Vor allem in der spirituellen Tradition Indiens wird postuliert, dass der Mensch im Kern das Selbst (die Seele) und somit göttlich ist. Aber diese Göttlichkeit wird weder erkannt noch gelebt, weil der menschliche Geist mehrheitlich nach außen gerichtet und im Materiellen verhaftet ist. Swami Muktananda (1908-1982), einer der großen indischen spirituellen Lehrer unserer Zeit, sagt in seinem Buch Der Weg und sein Ziel: „Es gibt ein großes Hindernis, das uns davon abhält, das Selbst zu erkennen, und das ist der menschliche Geist. Der Geist verhüllt das innere Selbst und versteckt es vor uns. “2

\section{Das wesentliche Merkmal des}

\section{Selbst-Bewusstseins ist daher die}

Kontinuität, die Dauerhaftigkeit.

Die des bewussten Ich ist jedoch nur

eine blasse Reflexion der ewigen, unsterblichen Essenz des

spirituellen Ich, des Selbst.

Roberto Assagioli (1888-1974)

Folgen wir einer traditionellen Meditationstechnik, kann uns aber dieser Geist - unter der Voraussetzung, dass er gesund ist - das HÖHERE SELBST erfahren lassen. Swami Muktananda beschreibt das wie folgt: „Derselbe Geist jedoch, der uns vom Selbst trennt, hilft uns, uns wieder mit ihm zu vereinen. Aus diesem Grund zogen die Weisen der Vergangenheit, die wahre Psychologen waren, den Schluss, dass der Geist der Ursprung, sowohl von Abhängigkeit als auch von Befreiung, von Leid als auch von Freude ist, dass er sowohl unser schlimmster Feind als auch unser bester Freund ist. ${ }^{\text {"3 }}$

In der Psychologie, der „Wissenschaft von der Seele, vom Seelenleben " ${ }^{4}$, finden sich eine Menge Fachbegriffe, die im Zusammenhang mit dem Wort Selbst gebraucht werden, wie z.B. SELBSTKONZEPT oder SELBSTBILD. Diese Bezeichnungen beziehen sich in vielen Teilgebieten der Psychologie eher auf konkrete Vorstellungen, die Menschen von sich selbst haben, und stehen in einem engen Zusammenhang mit ihrer Persönlichkeit.

Inwiefern wir mit unserem Selbstbild zufrieden sind, hängt vom Ideal- oder Wunschbild ab, das wir von uns haben. Wer möchte ich sein? Was möchte ich im Leben erreichen? Entscheidend an dieser Stelle ist, dass dieses Verständnis vom persönlichen Selbst in der Regel an äußere Objekte und Verhaltensformen gekoppelt ist und nichts mit dem Höheren Selbst oder der Seele zu tun hat. 


\section{Höheres Selbst und Seele}

Es gibt aber Richtungen in der Psychologie, die eine transzendierende Ebene des Selbst schon angenommen und fest in ihre Theorien integriert haben. ${ }^{5}$ So sprach der Schweizer Psychiater und Gründer der analytischen Psychologie, Carl Gustav Jung (1875-1961), über das Selbst als inneres Zentrum: „Ich habe diesen Mittelpunkt als das Selbst bezeichnet, den Mittelpunkt der Persönlichkeit, es könnte eben sowohl als der Gott in uns bezeichnet werden. Die Anfänge unseres ganzen seelischen Lebens scheinen unentwirrbar aus diesem Punkt zu entspringen. Und alle höchsten und letzten Ziele scheinen auf ihn hinzulaufen." 6

Ein weiteres Beispiel ist die von dem italienischen Arzt, Psychiater und Psychotherapeuten Roberto Assagioli (1888-1974) gegründete Psychosynthese. ${ }^{7}$ „Die Psychosynthese ist nicht nur Therapie, sie ist eine Lebensphilosophie, eine Psychologie für den Menschen, der wachsen will. Eine Methode, um alle psychischen Funktionen zu harmonisieren, wobei das Unbewusste wieder eingegliedert und ein Zentrum der Harmonie geschaffen wird, auf dass es zum Höheren Selbst werde: die ausgedrückte, bezeugte, gelebte, manifestierte Seele. ${ }^{\text {"8 }}$

Assagioli sprach anstelle eines Höheren Selbst auch vom transpersonalen bzw. spirituellen Selbst. ${ }^{9}$ Dieses transpersonale Selbst verleiht „Dauerhaftigkeit“ und „Stabilität“ im Gegensatz zum persönlichen, bewussten Ich, was Assagioli zufolge, nur eine „abgeschwächte und verschleierte Form" vom transpersonalen Selbst ist. ${ }^{10}$

In seinem Buch „Wer bin ich?" Der Übungsweg der Selbstergründung geht der bekannte indische spirituelle Lehrer Ramana Maharshi (1879-1950) auf das „Selbst“ und das „Ich“ ein: „Das wirkliche Selbst ist das unendliche ,Ich-Ich'. Es ist vollkommen und ewig. Es hat keinen Anfang und kein Ende. Das andere Ich wurde geboren und stirbt. "11 Maharshi zufolge sorgen Anhaftungen an das „andere Ich“ dafür, dass wir das unendliche „Ich-Ich“ als Ausrichtungspunkt verlieren: „Nachdem der Ich-Gedanke aufgetaucht ist, geschieht die falsche Identifikation des Ich mit dem Körper, den Sinnen, dem Geist usw. Das Ich wird fälschlich mit ihnen in Verbindung gebracht, und das wahre Ich gerät dabei aus dem Blick. Um das reine Ich vom verunreinigten Ich zu lösen, spricht man davon, es auszulöschen. Aber es bedeutet nicht wirklich, dass man das Nicht-Selbst auslöschen soll, sondern man das wahre Selbst finden muss. “12
Für ihn ist an dieser Stelle die Frage „Wer bin ich?“ der zentrale Punkt. Dieser Frage aufrichtig nachzugehen, führt schließlich zur Aufhebung der Identifizierung mit eigenen Sinneseindrücken, Emotionen und Gedanken und letztendlich zum tiefen Gewahrsein, zum reinen Ich, zum Selbst. ${ }^{13}$

\section{Jenseits des Höheren Selbst und der Seele - das Göttliche Selbst}

In der indischen Tradition wird für die Begriffe „Höheres Selbst“ bzw. „Seele“ der Begriff „Atman“ benutzt. ${ }^{14}$ Auf die Frage, was Atman, die Seele ist, antwortet die indische spirituelle Lehrerin Mutter Meera: „Der Atman kommt von Paramatman. So wie der Atman für den Körper unentbehrlich ist, so ist Paramatman notwendig für den Atman. ${ }^{\text {"15 }}$

Dabei ist für Mutter Meera Paramatman „das Höchste“ und „der Ursprung und die Essenz des gesamten Universums. Er ist allgegenwärtig, ohne Namen und Form"16.

Auch der spirituelle Lehrer Sri Chinmoy (19312007), der von 1970 bis zu seinem Lebensende zweimal wöchentlich Friedensmeditationen für Delegierte und Angestellte am Hauptsitz der UNO in New York leitete, sieht die Seele als Bindungsglied zum Höchsten: „Der Innere Lenker ist unser Lord Supreme [Gott], und die Seele ist die Repräsentantin des Inneren Lenkers, unseres geliebten Lord Supreme. [...] In gleicher Weise wie

\section{Die Seele agiert als Beschützer} während unserer Leben und ist immer bei uns. Sie hat keine eigenen Wünsche. Sie ist nicht nur Zeuge oder Lenker, sondern unterstützt auch unsere Entwicklung. Frei vom Einfluss unserer Handlungen, bleibt sie während unserer Leben ständig bei uns, bis wir mit dem Göttlichen eins werden. 
die Seele die Repräsentantin Gottes ist, so sollten auch der Körper, das Vitale, der Verstand und das Herz die Repräsentanten der Seele sein. ${ }^{\text {17 }}$

\section{Das Ego}

Grund dafür, dass diese oben zitierten Repräsentanten nicht als Stellvertreter der Seele agieren können, ist die Existenz des Egos. „Das Ego ist das, was uns in allen Lebensbereichen beschränkt. [...] Das gewöhnliche, menschliche Ego gibt uns das Gefühl eigenständiger Identität, eigenständigen Bewusstseins. Zweifellos ist das Gefühl von Individualität, von eigener Bedeutung in einem bestimmten Entwicklungsstadium des Menschen notwendig. Doch das Ego trennt unser individuelles Bewusstsein vom universellen Bewusstsein." ${ }^{18}$
In dem Buch Mut und Zufriedenheit der indischen spirituellen Lehrerin Swami Chidvilasananda wird das Ego definiert als „begrenztes Ich-Bewusstsein, das sich mit Körper, Geist und Sinnen identifiziert. Manchmal auch als ,Schleier des Leidens" beschrieben“" ${ }^{19}$

Für den indischen spirituellen Lehrer und Friedensaktivist Vishnu Devananda (1927-1993) bewirkt die Identifizierung mit dem Ego Leid und verhindert zudem die Ausrichtung auf das Höhere Selbst: „Die Ursache für diese missliche Lage ist das Ahamkara oder Ego. [...] Dieses Ahamkara ist die Ursache aller Bindung und ist das Haupthindernis zur Erfahrung der inneren Wirklichkeit. ${ }^{20}$

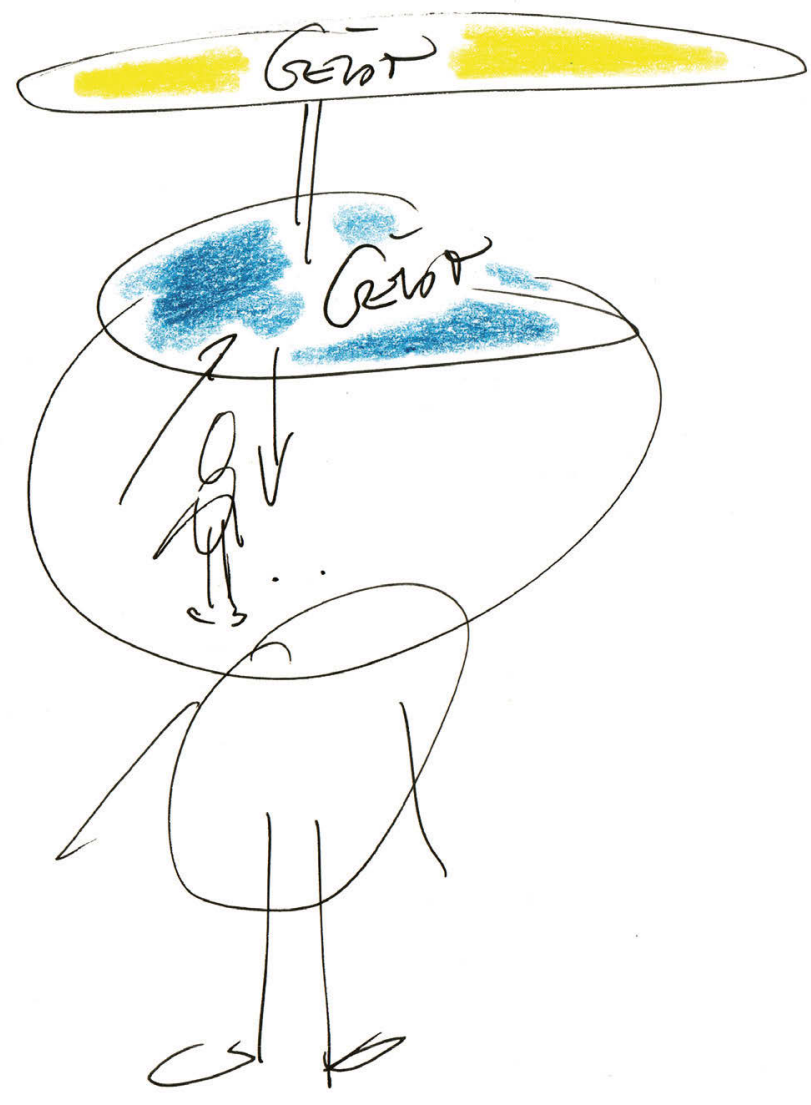

Göttlicher Geist und menschlicher Geist 
Um sich aus den Fängen des Egos zu befreien, ist Mutter Meera zufolge der Blick auf das Göttliche zu verlagern: "Jeder Mensch hat ein Ego. Es ist besser, wenn man seinem Ego oder seinem Namen oder Ansehen nicht so viel Beachtung schenkt. Versucht zu erkennen, dass Paramatman der Größte ist, dann gibt es kein Ego mehr. “21

Lernen Menschen sich auf das Göttliche auszurichten und die Bindung zum Höheren Selbst bzw. zur Seele zu festigen, werden sie weniger anfällig für Einflüsse und Vorbilder, die das Ego bzw. das Ich-bezogene Selbstkonzept und Selbstbild stärken. Dann führt diese göttliche Ausrichtung letztendlich zur inneren Harmonie und Zufriedenheit. ${ }^{22}$

Nur schon aus den wenigen im obigen Text erwähnten Quellen ist ersichtlich, dass viele Bezeichnungen für das Selbst in den verschiedenen Schriften existieren. Nachfolgend - für ein besseres Verständnis und eine klarere Übersicht - sind die genannten unterschiedlichen Benennungen ihrer Bedeutung nach in die auf S. 25 beschriebenen zwei Ebenen des Selbst eingeordnet:

- Das NIEDERE SELBST: Nicht-Selbst, Ego ${ }^{23}$, Ich, anderes Ich, bewusstes Ich, persönliches Ich, Persönlichkeit

- Das HÖHERE SELBST: Selbst, inneres Selbst, spirituelles Selbst, transpersonales Selbst, wahres Selbst, wirkliches Selbst, Atman, Seele, spirituelles Ich, unendliches Ich-Ich

\section{Die zwei verschiedenen Aspekte des Geistes}

Wie beim Begriff Selbst, so finden wir auch beim Wort GEIST in der Fachliteratur mehrere Bedeutungsebenen. ${ }^{24}$ In Bezug auf die Themen „Achtsamkeit und Meditation" sind vor allem zwei dieser Ebenen wichtig. Mit dem Begriff Geist kann erstens die PSYCHE gemeint sein und bezieht sich dann auf das eigene subjektive Fühlen und Denken. Dieser „menschliche Geist ${ }^{\text {“25 ist }}$ auf der Ebene des Ichs, der Persönlichkeit, angesiedelt. ${ }^{26}$

Zweitens kann aber Geist auch das ABSOLUTE, das GÖTTLICHE bedeuten. Assagioli beschreibt diese Ebene folgendermaßen: „Der Geist an und für sich ist die höchste Realität, in ihrem transzendenten, also absoluten Aspekt, frei von jeder Begrenzung und konkreten Bestimmung. Der Geist transzendiert alle Grenzen von Zeit und Raum, sprengt alle materiel- len Fesseln. Er ist in seinem Wesen ewig, unendlich, frei und universell. Diese höchste, absolute Realität kann intellektuell nicht erfasst werden, da sie über den menschlichen Verstand hinausgeht; sie kann aber rational postuliert, intuitiv erfasst und in gewissem Maße mystisch erfahren werden. ${ }^{\text {"27 }}$

Damit dieser göttliche Geist als reines Bewusstsein, als Funken Gottes, sich in der physischen Welt, in der Materie, manifestieren kann, wird ein Intermediär benötigt: das Höhere Selbst bzw. die Seele, die dann wiederum ihrerseits auf die Persönlichkeit und ihre Träger, das Denken, die Emotionen und den Körper, einwirkt.

Diese Träger sind aber lange Zeit über die Sinne nur auf die physische Welt ausgerichtet, was ein - wie im Textabschnitt Ego erwähnt - identitätsbezogenes und in der Begrenzung geführtes Leben zur Folge hat.

Das wahre Ziel der Seele ist jedoch, die Begrenztheit der Persönlichkeitsträger aufzulösen und diese auf ihre Ebene anzuheben, damit letztendlich die „höchste Wahrheit ${ }^{\text {" } 28}$ in der physischen Welt verwirklicht werden kann. ${ }^{29}$

Mutter Meera beschreibt dies wie folgt: „Der Atman lenkt unsere Entwicklung und ist die Basis oder der Ursprung unserer physischen und feinstofflichen Körper. Er wirkt durch die verschiedenen Körper, um alles zu erfahren, und dann bringt er bei der Verwirklichung die gesamten Erfahrungen zurück zu Paramatman. “30

\section{QUELLEN}

Assagioli, Roberto (2008). Psychosynthese und transpersonale Entwicklung. Rümlang / Zürich: Nawo Verlag $\mathrm{GmbH}$.

Chidvilasananda, Swami (Gurumayi) (2004). Mut und Zufriedenheit. Telgte: Siddha Yoga Verlag GmbH.

Fürst, Maria (1985). Philosophie - Band 1: Psychologie. Wien: Verlag Carl Ueberreuter.

Giovetti, Paola (2007). Roberto Assagioli. Leben und Werk des Begründers der Psychosynthese. Rümlang / Zürich: Nawo Verlag GmbH.

Haug, Achim (2018). Das kleine Buch von der Seele. Ein Reiseführer durch unsere Psyche und ihre Erkrankungen. München: Verlag C. H. Beck oHG.

Hazrat Inayat Khan (2018). Die Sufi-Botschaft von Hazrat Inayat Khan. Jubiläumsausgabe Band 1. Das Innere Leben. Polling: Verlag Heilbronn. Kahler, Thomas / Nitsch, Thomas (1994). Neues großes Wörterbuch. Fremdwörter. Karlsruhe: Trautwein. 
Maharshi, Ramana (2019). Die Gesammelten Werke. Norderstedt: BoD.

Maharshi, Ramana (2011a). „Wer bin ich?“ Der Übungsweg der Selbstergründung. Norderstedt: BoD. Muktananda, Swami (1996). Der Weg und sein Ziel. Ein Handbuch für die spirituelle Reise. München: Siddha Yoga Verlag GmbH.

Mutter Meera (2007). Antworten. Teil II. DornburgThalheim: Verlag \& Buchvertrieb Adilakshmi.

Mutter Meera (1994). Antworten. Teil I. DornburgThalheim: Verlag \& Buchvertrieb Adilakshmi.

Piron, Harald (2020). Meditationstiefe. Grundlagen, Forschung, Training, Psychotherapie. Berlin: Springer-Verlag.

Singer, Michael A. (2019). Die Seele will frei sein. Eine Reise zu sich selbst. Berlin: Ullstein Buchverlage GmbH.

Sri Chinmoy (2015). Answers, Volume I, Book 19. Oxford: Ganapati Press.

Sri Chinmoy (2012). Beyond likes and dislikes, Illumining questions and answers. New York: Agni Press.

Sri Chinmoy (2004). Der Körper, die Festung der Menschheit. Nürnberg: The Golden Shore Verlagsges.mbH.

Vishnu Devananda (1986). Meditation und Mantras. Eine Darstellung der großen Yoga-Systeme in Theorie und Praxis. München: Heinrich Hugendubel Verlag. Yogananda, Paramahansa (2018). Die stärkende Kraft der Meditation - innere Ruhe und Klarheit gewinnen. Petersberg: Verlag Via Nova.

\section{Zeitschrift}

Sri Chinmoy (1966). „Questions and Answers“, in: AUM, Vol. 2, No. 4/5, Nov.- Dec. 27. Brooklyn / New York: Boro Park Printers, S. 12-15.

\footnotetext{
1 In manchen Schriften wird noch eine dritte, feinere Ebene des Selbst unterschieden: das unpersönliche Selbst, die Monade.

2 Muktananda 1996, S. 45.

Ebd.

4 Kahler / Nitsch 1994, S. 426. Für eine kurze Einführung zum Thema „Seele" siehe Haug 2018, S. 10ff. und $197 \mathrm{ff}$.

5 Vgl. Piron 2020, S. $9 f f$.

6 Fürst 1985, S. 100.

7 Für eine detaillierte Beschreibung des Lebens und Werks von Roberto Assagioli siehe Giovetti 2007.

8 Giovetti 2007, S. 110.

9 Assagioli bevorzugte den Begriff „transpersonal“ anstelle von „spirituell", da er diesen als neutraler und wissenschaftlicher erachtete (vgl. Giovetti 2007, S. 182).

${ }^{10}$ Assagioli 2008, S. 31.
}

11 Maharshi 2011a, S. 43.

12 Maharshi 2011a, S. 43; siehe auch Singer 2019, S. $42 f$.

${ }^{13}$ Vgl. Maharshi 2011a, S. 18f.; siehe auch Singer 2019, S. 43. sowie Hazrat Inayat Khan 2018, S. $256 f$.

14 Vgl. Singer 2019, S. 43; siehe auch Giovetti 2007, S. $104 f$.

15 Mutter Meera 1994, S. 135f.; siehe auch Yogananda 2018, S. 59.

16 Mutter Meera 2007, S. 14; siehe auch Mutter Meera 2011, S. 13.

17 Sri Chinmoy 2015, S. 755.

18 Sri Chinmoy 1966, S. 12; Maharshi zufolge ist dies auch der Moment, in dem das Ego entsteht: „Der Ich-Gedanke ist der erste Gedanke des Geistes. Er ist das Ego (das individuelle Ich-Gefühl, Ahamkara)." Maharshi 2011a, S. $20 f$.

19 Chidvilasananda 2004, S. 186; Maharshi sieht den Geist in diesem Zusammenhang als „inneres Sinnesorgan", siehe Maharshi 2019, S. 25.

20 Vishnu Devananda 1986, S. $15 f$.

21 Mutter Meera 2007, S. 31f.; siehe auch Sri Chinmoy 1966, S. 13.

22 Vgl. Sri Chinmoy 2012, S. 6.

${ }^{23}$ In manchen Schriften steht der Begriff Ego für die Seele. In der Regel ist aber mit dem Ego das Ich, das niedere Selbst, gemeint.

24 Vgl. Assagioli 2008, S. 204.

25 Muktananda 1996, S. 45.

26 Siehe auch das erste Zitat von Swami Muktananda auf S. 25. Ein Beispiel zur Funktionsweise des menschlichen Geistes gibt Swami Vishnu Devananda 1986, S. 15.

27 Assagioli 2008, S. 204.

28 Sri Chinmoy 2004, S. 7.

29 Vgl. Sri Chinmoy 2015, S. 755; siehe auch Hazrat Inayat Khan 2018, S. 305.

30 Mutter Meera 1994, S. 136. 
$800^{25 \cdot 11.19}$
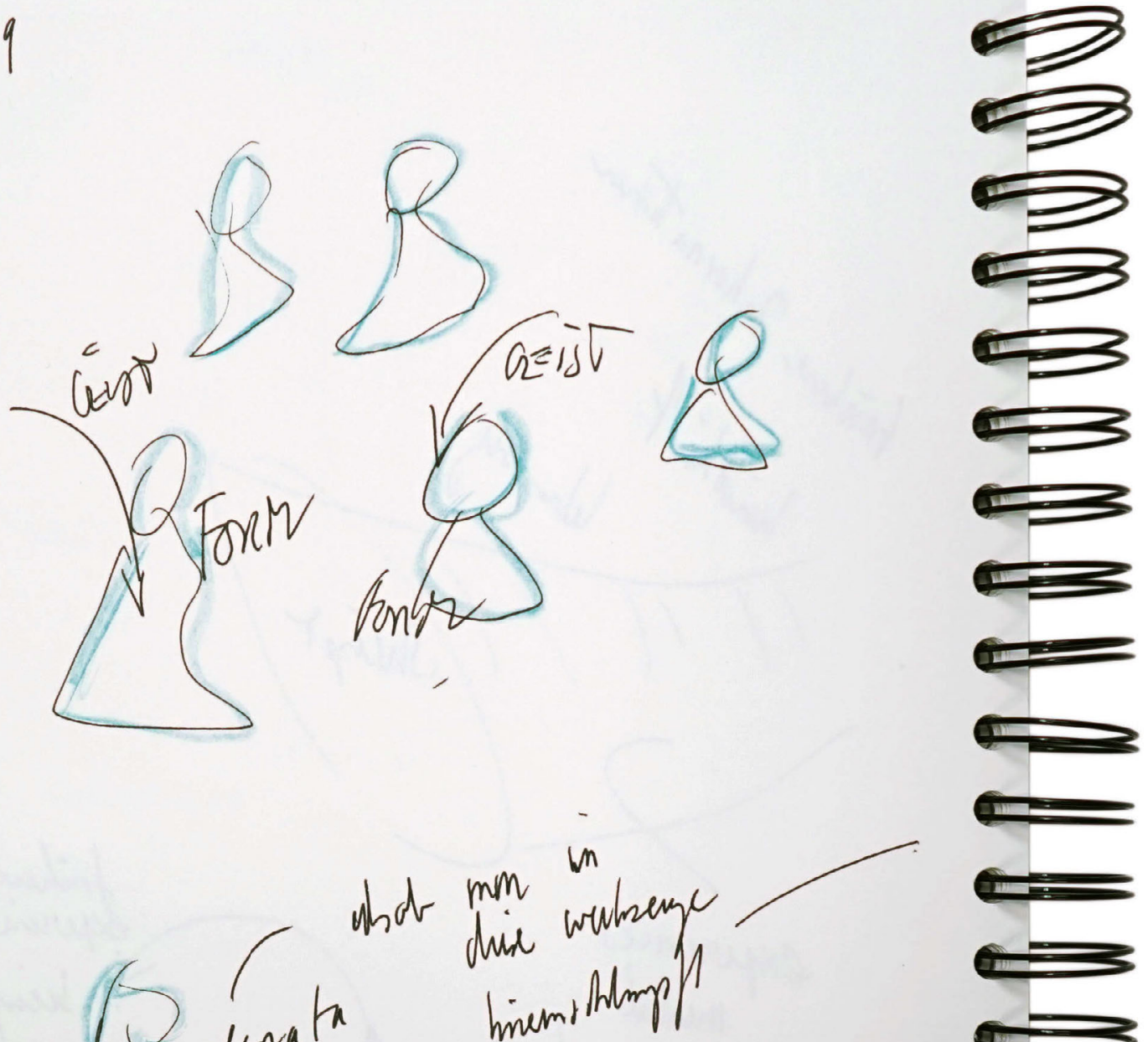
schorata hiemethlompl

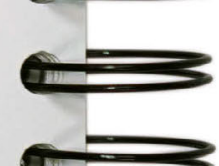
Wílue die

$\int$ time

subithwes tised

un do undil hid

Jhrim

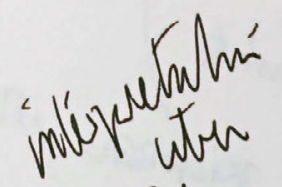

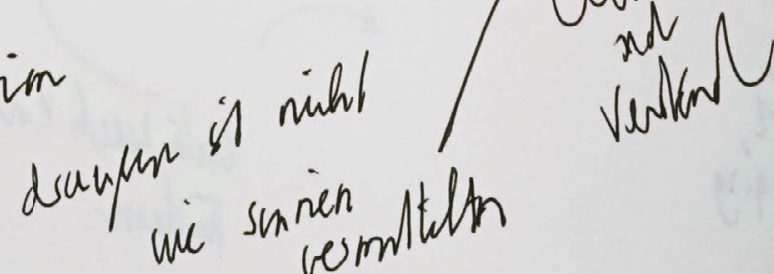

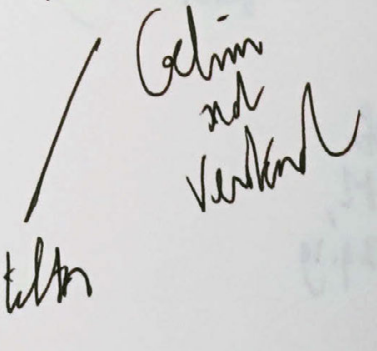

Geist tritt in die Form. Die äußere Form des Menschen ist nur die Hülle. 


\section{Herz, Liebe und Selbst sind auch Bildungsthemen - ein Erfahrungsbericht ${ }^{1}$}

\section{Eine Herzerfahrung}

Kennen Sie Delft? Entstehungsort des weltbekannten blauen Porzellans und zusammen mit Amsterdam bedeutende Handelsdrehscheibe im Goldenen Zeitalter der Niederlande. Es ist auch der Ort, wo die Mitglieder des Königshauses über die Jahrhunderte ihre letzte Ruhe finden. Die wunderschöne Delfter Altstadt, mit den vielen Grachten und geschichtsträchtigen Herrenhäusern, ist bekannt für ihr magisches Licht - das vor allem der berühmte Maler Johannes Vermeer perfekt einzufangen wusste. In dieser Stadt bin ich geboren.

Als ich noch jung war, etwa 13 Jahre alt, habe ich mit meiner zwei Jahre älteren Schwester die Morgenzeitung ausgetragen. Meine Mutter weckte uns täglich um 5 Uhr früh, dann ging es mit den Fahrrädern zum Zeitungslager, wo wir unsere Fahrradtaschen voll mit Zeitungen stopften. Anschließend fuhren wir in die Innenstadt, und jeder von uns nahm sich die uns zugewiesenen Abonnentenadressen vor. Gegen 6.30 Uhr kamen wir wieder nach Hause, wo unsere Mutter uns bereits mit einer großen Portion Brinta, einem warmen Haferbrei, erwartete. Gleich nach dem Frühstück machten wir uns auf dem Weg in die Schule.

Während eines Meditationsretreats für Hochschullehrende am Benediktushof - Zentrum für Meditation und Achtsamkeit in Holzkirchen bei Würzburg im Oktober 2019 wurde diese Erinnerung an damals mit einem Schlag wieder lebendig. Es war am Dienstag, dem zweiten Tag des Retreats, genau um 6 Uhr früh während der ersten Gehmeditation. Zusammen mit den anderen Seminarteilnehmerinnen und -teilnehmern hatte ich mich im Innenhof eingefunden. Da es zu dieser Jahreszeit bereits ziemlich kalt war, waren die meisten von uns warm eingepackt und trugen Mützen, Jacken und Schals. In einem großen Kreis gingen wir nun Runde um Runde; achtsam, schweigend, aber dennoch in zügigem Tempo. Der Boden war aus kleinen Kopfsteinpflastern, ein Material, mit dem auch viele Grachtenstraßen in Delft bestückt sind. Als die nahe gelegene Kirchturmuhr läutete, erinnerte mich das mit einem Mal an die Kirchenglocken in Delft. Dort schlagen die Glocken jede halbe Stunde, und es gibt in der
Nieuwe Kerk auf dem Marktplatz ein Glockenspiel, das Carillon, das sogar jede Viertelstunde eine kleine Melodie erklingen lässt. Während ich so Schritt für Schritt und in Stille versenkt vor mich her ging, war plötzlich meine Heimat präsent. Ich konnte den Frühnebel über den Grachten und in den engen Gassen sehen; die Kälte während der Wintertage an den Fingern spüren und den Haferbrei riechen, als stünde er vor mir.

Vor allem aber war meine Mutter da. Ich sah, wie sie meine Schwester und mich liebevoll umarmte, bevor wir morgens aus dem Haus gingen. Die Liebe, die sie für uns empfand, war für mich plötzlich sichtbar und spürbar. Ich sah meine Mutter umgeben von dieser Liebe, die durch sie hindurchging und von ihr ausströmte; diese Liebesenergie bewegte sich von hinten über den Kopf zu den Schultern und nach vorne zum Brustbereich. Die Liebe war völlig präsent, ich konnte sie als Essenz wahrnehmen, was mich zutiefst berührte.

Aber inmitten dieser völlig unerwarteten Erfahrung kamen mir gleichzeitig auch rationale Gedanken in den Sinn: Was hat sie wohl gedacht, als sie meiner Schwester und mir zuwinkte, bevor wir mit den Fahrrädern am Ende der Straße abbogen? Wir waren ja noch jung, alleine so früh unterwegs zu sein, war nicht ungefährlich. Würde alles gut gehen? Ein anderer Gedanke war: Täglich ist sie so früh für uns aufgestanden, immer hat sie warmen Haferbrei für uns zubereitet.

Als die Gedanken allmählich nachließen, entstand ein weiteres Bild. Ich sah bei meiner Schwester und mir nun auch ein solches Feld aus Liebesenergie, das mit dem von meiner Mutter interagierte. Gleichzeitig waren wir alle drei umgeben von einem noch größeren, übergeordneten Kraftfeld. Wir befanden uns inmitten dieses Feldes, das sich um uns herum auszudehnen schien. Während ich dieses Bild vor Augen hatte, so präsent und real, als wäre es gestern gewesen, wurde mir klar: Diese Kraft ist gegenwärtig, immerwährend und überall. Sie war damals da und ist es noch. Sie hält alles zusammen.

Während ich langsam meine Gehmeditationsrunden drehte, spürte ich mehr und mehr mein Herz. Es war eine starke, außergewöhnliche Kraft, die mich erfüllte. Ein tiefes Gefühl der Geborgenheit und Ruhe durchdrang mich mit der dankbaren Gewissheit: Alles ist gut. 
Plötzlich ertönte das helle Schellen von Handglocken: Die Gehmeditation war beendet. Die Teilnehmerinnen und Teilnehmer unserer Seminargruppe machten sich auf den Weg in Richtung Meditationsraum. Ich holte mir rasch noch einen heißen Tee und versuchte derweil, das Geschehene etwas einzuordnen.

War diese Erfahrung aus dem Nichts entstanden oder gab es womöglich einen erklärbaren Grund dafür? Hing sie mit dem Tag zuvor zusammen, wo wir nach der Anreise eine Zen-Einführung erhalten hatten, bei der auch eine rege Diskussion über die Konditionierung des Menschen und die Bedeutung der Leere entstanden war? Mich plagte die Frage, ob man sich bei der Beobachtung und Analyse des Denkens nicht zu sehr in rationale Erklärungen verstrickt. Wäre es nicht sinnvoller, direkt die Stille zu suchen, um dort auf direktem Wege die Kraft zu erfahren, worüber Mystiker aus Ost und West immer wieder berichtet haben? Und ist es womöglich diese Kraft, die mir während der Gehmeditation gezeigt wurde, die in der Stille auf einen wartet? Ist es das, was wir Liebe nennen? Und ist diese lenkende Kraft unser Wesenskern? Unsere Seele? Unser Selbst?

In diesem Kontext fügte sich auch eine Anmerkung unseres Zen-Meisters Alexander Poraj während der gemeinsamen Sitzmeditation: „Es gibt den Atem nicht, weil ich atme, sondern es gibt mich, weil es den Atem gibt." So betrachtet stellt sich die Frage, welche innere Essenz für uns atmet. Was wirkt durch uns? Auch die Philosophie hinter dem im Benediktushof vorhandenen Zen-Garten passte dazu: In einem Zen-Garten, geht es darum, die wahre Essenz jeder Pflanze und jedes Baumes sichtbar zu machen.

Die unerwartete und intensive Erfahrung während der Gehmeditation hat mich die ganze Woche über begleitet. Obwohl wir die Gehmeditation danach noch häufig durchgeführt haben, kam sie in dieser Intensität nicht mehr vor. Während der frühen Gehmeditation am Donnerstag schien sich allerdings der Raum um mich herum zu verändern. Die Schritte der anderen Seminarteilnehmerinnen und -teilnehmer erschienen mir leiser, auch ihre Gestalt änderte sich; sie war eher schattenartig. Mir fiel spontan das Höhlengleichnis von Platon ein, bei dem angekettete, langjährige Gefangene sich in einer Höhle befinden und auf der Höhlenwand Schatten wahrnehmen. Da sie die Träger der Schatten aber nicht sehen können, gehen sie davon aus, dass die Schatten die Lebewesen selbst sind. Ich fragte mich: Sehen wir die Menschen um uns herum eigentlich wirklich? Was wäre, wenn wir ihre wahre Essenz stärker wahrnehmen könnten? Vergleichbar mit der Einzigartigkeit der Pflanzen und Bäume in einem Zen-Garten.

\section{Achtsam sein}

Als wir am Donnerstagnachmittag über die Stille-Erfahrung der Tage zuvor Resümee zogen, sagte ein Teilnehmer: „Alles ist mehr: mehr Wind, mehr Licht, Vögel, Schmetterlinge; alles ist stärker geworden!" Ich konnte mich darin wiederfinden und fand die Aussage ganz zutreffend. Gleichzeitig wurde mir auch bewusst, dass bei Achtsamkeitspraktiken bezogen auf Alltagshandlungen generell die Gefahr besteht, zu viel zu wollen, und dass diese dann zu verkopft und zu mechanisch ausgeführt werden: „Ich muss jetzt achtsam essen, ich muss jetzt achtsam gehen usw."

In der christlichen Tradition werden verschiedene Formen des Sehens unterschieden: das Sehen über die Sinneswahrnehmung, das Sehen durch den Verstand und das Sehen mit dem Herzen. ${ }^{2}$ Es geht um die letzte Ebene. Wenn man Achtsamkeit mit dem Herzen verbindet, wird das Praktizieren ganz natürlich. Im Rückblick auf meine Erfahrung erweist sich dieser Aspekt als bedeutungsvoll. Es waren unsere Herzen, die verbunden wurden, und die Liebe, die uns umhüllte. $\mathrm{Ob}$ ich das damals bemerkt habe oder nicht, es hat dennoch stattgefunden.

Ich frage mich, was wäre, wenn wir auch im Alltag eine solche Herzverbundenheit bewusster wahrnehmen und ausdrücken könnten? Der Verstand würde möglicherweise nicht mehr die Position einnehmen, wie er sie sonst innehat, was bedeuten würde, dass wir den Verstand nur dann benutzen, wenn es eine bestimmte Situation verlangt.

Der indische spirituelle Lehrer Nisargadatta Maharaj (1897-1981) sagte über den Verstand: „Wenn du vollständig erkennst, dass du bist, allerdings ohne von diesem Körper-Verstand konditioniert zu sein, wirst du mit der ganzen Welt eins sein. “"

Und der Benediktinermönch, Zen-Meister und Gründer des Benediktushofes Willigis Jäger (19252020) schreibt in seinem Buch Kontemplation - ein spiritueller Weg: „Es ist richtig: Unser Verstand macht uns zu Menschen. Aber wir müssen aufhören, uns mit ihm $\mathrm{zu}$ identifizieren. Er ist nur das Werkzeug, ein Instrument, auf dem unser wahres Wesen spielt."

Damit dieses „wahre Wesen“ spielen und frei wirken kann, müssten wir vielleicht gar nicht so viel machen. Vielleicht genügt es bereits, ganz einfach zu sein. Hinsichtlich der Achtsamkeitspraxis stellt sich allerdings die Frage, ob ich wirklich im Moment sein kann, wenn ich mich als Beobachter beim Beobachten des Beobachtenden wahrnehme? Der indische spirituelle Lehrer Jiddu Krishnamurti (1895-1986) hat in seinen Vorträgen 


\section{Einsamkeit und Alleinsein}

Aber vom Augenblick an, in dem Sie in Ihrem Herzen dieses Außergewöhnliche haben, das Liebe genannt wird, und Sie ihre Tiefe spüren, ihr Entzücken, ihre Ekstase, werden Sie entdecken, dass für Sie die Welt verwandelt ist.

Jiddu Krishnamurti (1895-1986)

oft über Wahrnehmung und die Auflösung des Ichs gesprochen. Auf die Frage „Wie können wir unseren eigenen Geist wahrnehmen, wenn der Geist sowohl der Wahrnehmende ist als auch das, was er wahrnimmt?" beleuchtet Krishnamurti zunächst das Denken, das das Ich, den Denker, den Wahrnehmenden, den Beobachter, erzeugt und damit eine Spaltung hervorbringt. Zwar scheint der Wahrnehmende auf den ersten Blick eine unabhängige Instanz zu sein, ist aber letztendlich ebenfalls vom Denken erschaffen. ${ }^{5}$ Es geht darum, eine Ebene weiterzugehen. „Wenn Sie eine Blume ansehen, wenn Sie sie einfach sehen, gibt es in diesem Augenblick eine Wesenheit, die sieht? Oder gibt es nur sehen?" ${ }^{6}$

Für Nisargadatta Maharaj liegt die Schwierigkeit in der Verbalisierung des Beobachters: „Zu sagen ,Ich bin nur der Beobachter' ist sowohl falsch als auch richtig. Falsch in Bezug auf das ,Ich bin' und richtig in Bezug auf den Beobachter. Es ist besser zu sagen, ,Die Beobachtung findet statt'. Sobald Sie sagen: ,Ich bin' entsteht ein ganzes Universum zusammen mit seinem Schöpfer. "7 Worum es seiner Meinung nach geht, ist die Auflösung dieser Benennung: „Du musst eins werden mit dem Selbst, dem Ich bin. Wenn du von, Wissen' sprichst, dann handelt es sich dabei lediglich um Informationen. Lass vielleicht die Worte ,Ich bin`weg, denn selbst ohne diese Worte weißt du, dass du bist. Sprich es noch nicht einmal aus oder denke, dass du bist. Sei dir lediglich der Präsenz gewahr, ohne darüber nachzudenken. ${ }^{\text {"8 }}$
Die Verbalisierung des Beobachters aufzugeben, kann ein Gefühl der Einsamkeit und des Alleinseins mit sich bringen. Es fehlt dann der eigene Referenzpunkt: „Ich gehe, ich sehe, ich ..." wird „gehen, sehen“. Für Krishnamurti besteht allerdings ein deutlicher Unterschied zwischen der Einsamkeit und dem Alleinsein. Krishnamurti: „Sehen Sie, es gibt einen riesigen Unterschied zwischen Einsamkeit und Alleinsein. [...] Der Geist erkennt diese Angst, wenn er einen Augenblick lang realisiert, dass er sich auf nichts verlassen kann, dass ihm keine Ablenkung das Gefühl einer ihn selbst umschließenden Leere nehmen kann. Das ist Einsamkeit. Aber Alleinsein ist etwas völlig anderes: es ist ein Zustand von Freiheit, der entsteht, nachdem Sie durch die Einsamkeit hindurchgegangen sind und sie verstanden haben."9

Krishnamurti betont, dass es wichtig ist, sich mit dieser Einsamkeit auseinanderzusetzen: „Dies alles gehört zur Erziehung: sich dem Schmerz der Einsamkeit, diesem ungewöhnlichen Gefühl von Leere, das wir alle kennen, zu stellen, und wenn es kommt, nicht zu erschrecken, nicht das Radio anzustellen, sich nicht in Arbeit zu vertiefen, nicht ins Kino zu laufen, sondern das Gefühl anzusehen, hineinzugehen und es zu verstehen. " ${ }^{10}$ Sich nicht ständig ablenken lassen und stattdessen die Stille zu suchen, ist wesentlich, um den ,inneren Raum“ zu erkunden. ${ }^{11}$ Doch: „Sehr wenige gehen über diese außerordentliche Angst vor Einsamkeit hinaus. Man muss aber darüber hinausgehen, weil der wahre Schatz jenseits von ihr liegt. " ${ }^{12}$

Der Benediktinermönch David Steindl-Rast sieht das Herz als Weg, die Einsamkeit aufzulösen. „Im Innersten unseres Herzens finden wir uns in einem Bereich, in dem wir nicht nur auf das Innigste mit uns selbst, sondern ebenso mit anderen vereint sind, mit allen anderen. Das Herz ist kein einsamer Ort. Es ist der Bereich, in dem Alleinsein und Beisammensein zusammentreffen. " ${ }^{13}$ Für ihn sind es die besonderen Momente des Alleinseins, etwa ein schöner Spaziergang, eine Bergwanderung, ein Sonnenaufgang, bei denen wir uns mit allem verbunden fühlen können: „Das würde sich anfühlen, als weite sich Ihr Herz, als würde Ihr ganzes Wesen sich weiten, um alles zu umarmen, und als würden irgendwie alle Schranken abgebrochen oder aufgelöst und Sie wären mit allem eins. ${ }^{\text {14 }}$ 
(34) Einführung
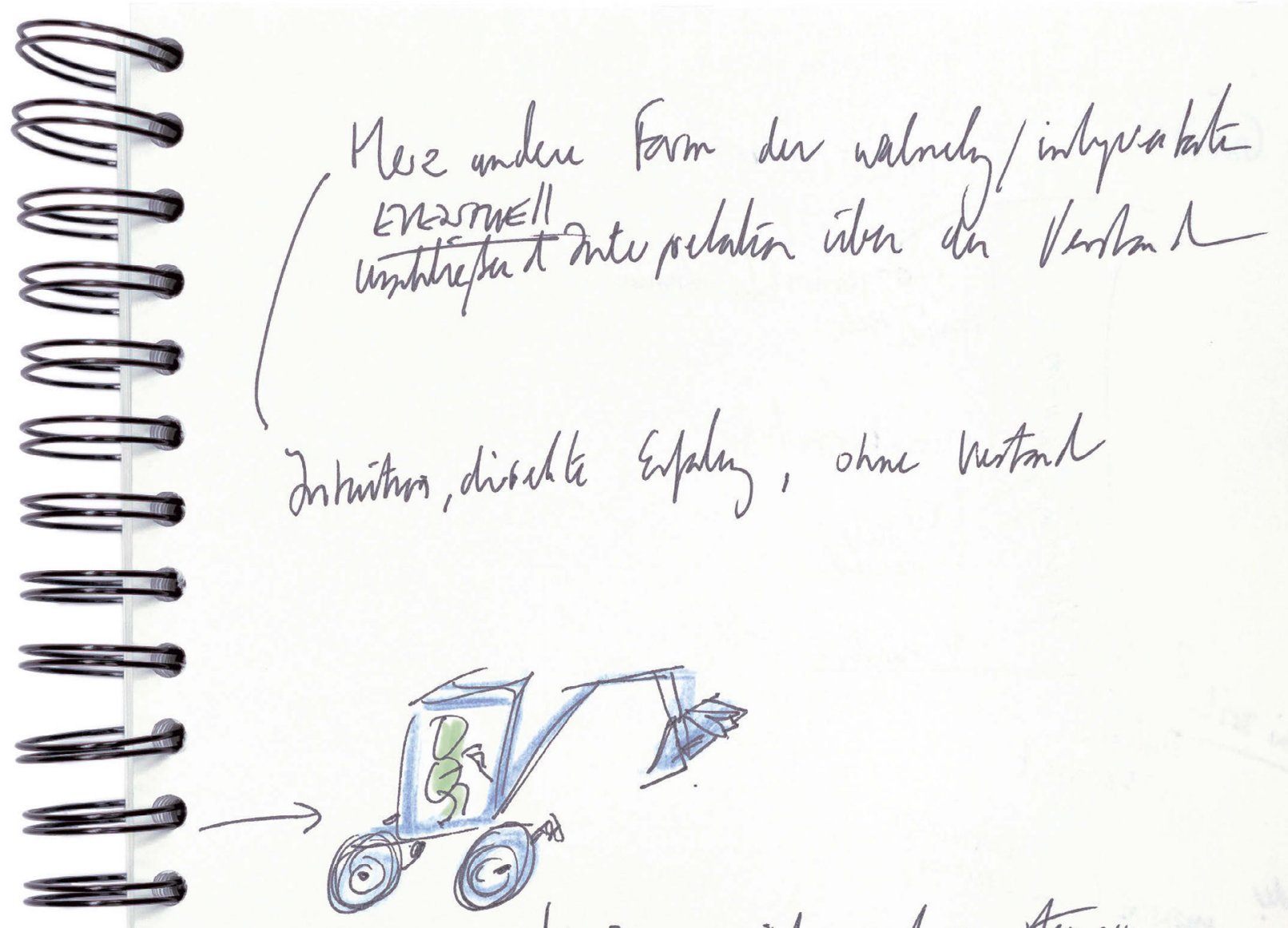

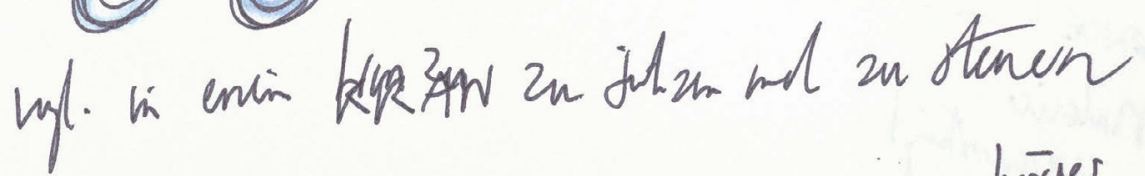
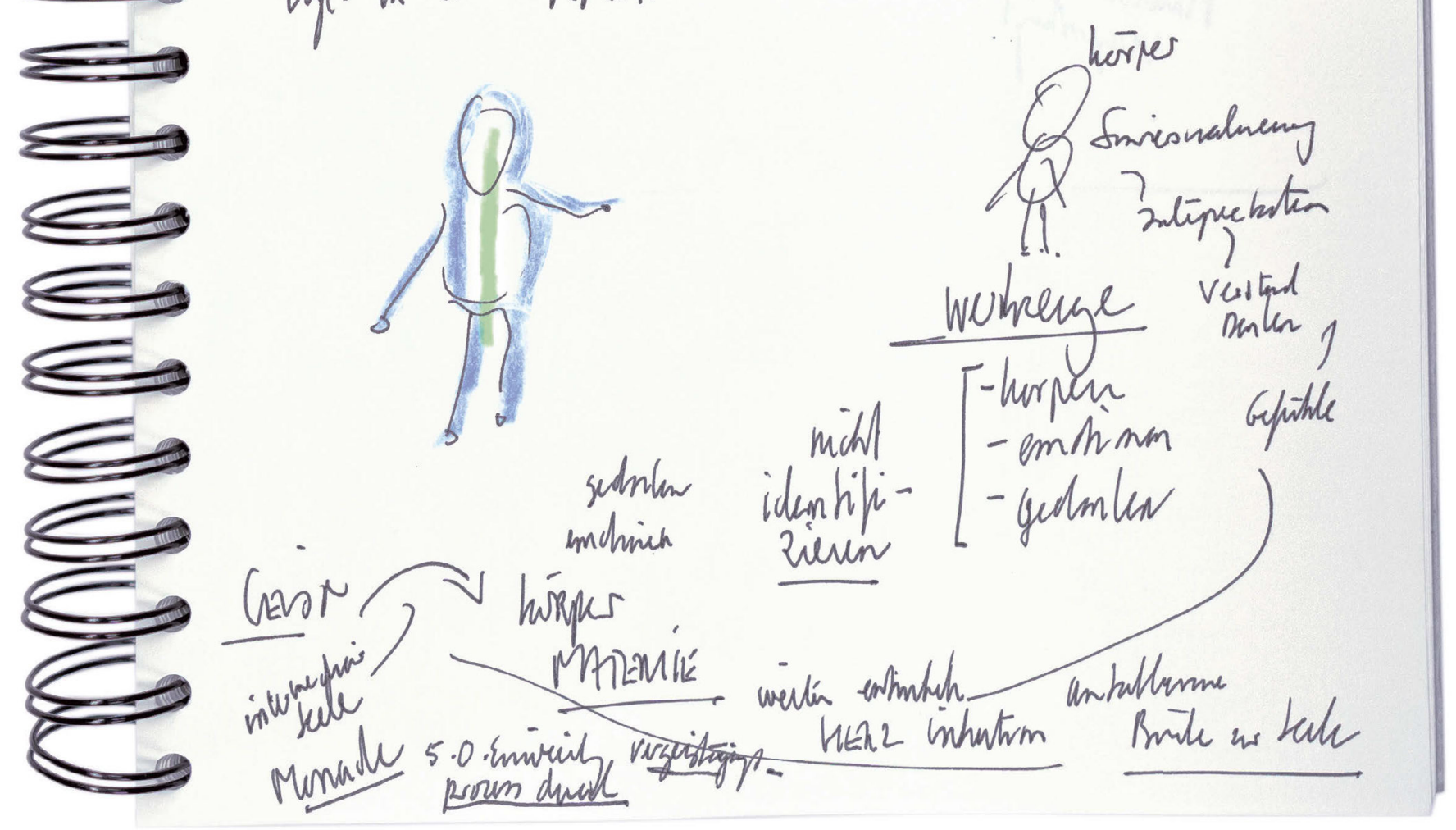

Wir sind in einem Körper - unser Werkzeug. Wie das Sitzen in einem Bagger.

(C) Münchner Modell | www.hm.edu/meditationsmodell 
Für Willigis Jäger ist es die Liebe, die Geborgenheit verschafft: „Es ist die Liebe, die Vertrauen und Kraft gibt und damit die Gewissheit, aufgefangen zu werden und getragen zu sein. " ${ }^{15}$ Auch der buddhistische Lehrer Jack Kornfield hat diesen Aspekt der Liebe beschrieben: „Die Liebe ist ein großartiges Geheimnis. Unermesslich, unsichtbar wie die Schwerkraft verknüpft sie unaufhaltsam alle Dinge. "16 Diese Liebe ist auch in uns, sie ist unsere Essenz. Nisargadatta betont: „Die wahre Natur des Selbst ist Liebe “17; und an anderer Stelle: „Ich bin-Sein, Gewahrsein ohne Gedanken, ist Liebe." ${ }^{18}$

\section{Menschen, welche die Schriften}

wirklich kennen und erfahren, gewinnen ihr Verständnis nicht nur aus Büchern, sondern aus

$$
\begin{gathered}
\text { ihrem Herzen. Das Herz ist } \\
\text { die größte Bibliothek. }
\end{gathered}
$$

Swami Chidvilasananda

\section{Ausblick}

Die Verbindung zum inneren Selbst hilft uns, klarer zu sehen, was im Leben von Bedeutung ist, und entsprechend zu handeln. Fragt man Sterbende, was sie im Rückblick auf ihr Leben am meisten bedauern, zeigt sich, dass sie gerne mehr Gefühle ausgedrückt hätten, den Kontakt zu Freunden stärker gepflegt hätten, nicht so viel gearbeitet und mehr den Mut gehabt hätten, das Leben so zu leben, wie sie es gerne gewollt hätten. ${ }^{19}$

Als Nisargadatta Maharaj einmal von einer Frau gefragt wurde, warum sie ihr ganzes Leben lang unglücklich gewesen ist, antwortete er: „Weil Sie nicht zu den Wurzeln Ihres Seins vorgedrungen sind. Es ist die völlige Unwissenheit Ihrer selbst, die Ihre Liebe und Ihr Glück verschleiert haben und Sie etwas suchen lassen, dass Sie niemals verloren haben. Liebe ist Wille, der Wille, Ihr Glück mit allen zu teilen. Glücklich zu sein, glücklich zu machen - das ist der Rhythmus des Lebens. " 20

Willigis Jäger beschreibt in seinem Buch Über die Liebe, welche Wirkung seine Nahtoderfahrung auf ihn gehabt hat: „Zurück blieb nach dieser Erfahrung die Gewissheit: Wenn ich sterbe, kehre ich zurück in die- se unendliche Liebe, ohne jede Ich-Eingrenzung. Und diese Liebe ist der Urgrund allen Seins. Unser Ich mit all seinen Verkrustungen und egoistischen Prägungen verdeckt es nur ständig. Mir wurde klar, dass wir als Menschen nicht weiterkommen, wenn es uns nicht gelingt, in diese Erfahrungsebene der bedingungslosen Liebe hineinzuwachsen." ${ }^{21}$

Als ich nach dem fünftägigen Retreat am Freitagnachmittag am Hauptbahnhof München eintreffe, ist das eine ziemliche Umstellung. Viele Eindrücke, Hektik, alles ist laut. Die Menschen, die an mir vorüberjagen, wie Schatten. Sofort ist das Höhlengleichnis Platons wieder präsent. Und in mir kommt erneut die Frage auf: Sehen wir einander wirklich? Erkennen wir unsere wahre Essenz? Und gleichzeitig spüre ich das Gefühl der Verbundenheit - dass in uns allen diese Essenz vorhanden ist.

Das Retreat im Benediktushof hatte auch das Ziel, den Austausch unter Hochschullehrenden über die Implementierung von Achtsamkeits- und Meditationsansätzen an Hochschulen zu intensivieren. Rückblickend schließt sich für mich der Kreis. Mir ist nach dieser tiefen Herzerfahrung noch einmal mehr klargeworden, wie wichtig es ist, den Themen Herz und Liebe sowie der Verbindung zum inneren Selbst im täglichen Leben und daher auch an Hochschulen genügend Raum zu geben.

\section{QUELLEN}

Hazrat Inayat Khan (2019). Heilung aus der Seele.

Die Mystik der Sufis. Polling: Verlag Heilbron

Jäger, Willigis (2017). Über die Liebe. München:

Penguin Verlag.

Jäger, Willigis (2015). Kontemplation - ein spiritueller

Weg. Freiburg im Breisgau: Verlag Herder.

Kornfield, Jack (2010). Meditationen, die unser Herz öffnen. München: Arkana.

Krishnamurti, Jiddu (1993). Einbruch in die Freiheit.

Berlin / Frankfurt am Main: Ullstein.

Krishnamurti, Jiddu (1992). Antworten auf Fragen des Lebens. Freiburg im Breisgau: Verlag Hermann Bauer KG.

Nisargadatta Maharaj (2017). Ich Bin. Teil I. Gespräche mit Sri Nisargadatta Maharaj. Bielefeld: J. Kamphausen Mediengruppe $\mathrm{GmbH}$.

Nisargadatta Maharaj (2016). Ich Bin. Teil II. Gespräche mit Sri Nisargadatta Maharaj. Bielefeld: J. Kamphausen Mediengruppe GmbH. 
Nisargadatta Maharaj (2014a). Ich Bin. Teil III.

Gespräche mit Sri Nisargadatta Maharaj. Bielefeld: J. Kamphausen Mediengruppe GmbH.

Nisargadatta Maharaj (2014b). Jenseits von Freiheit. Gespräche mit Sri Nisargadatta Maharaj. Lohne:

Lotus Press.

Steindl-Rast, David (2018). Dankbarkeit.

Das Herz allen Betens. Freiburg im Breisgau:

Verlag Herder GmbH.

Steindl-Rast, David (2016). Auf dem Weg der Stille.

Das Heilige im Alltag leben. Freiburg im Breisgau:

Verlag Herder GmbH.

Ware, Bronnie (2015). 5 Dinge, die Sterbende am meisten bereuen. Einsichten, die Ihr Leben verändern werden. München: Wilhelm Goldmann Verlag.

DVD

Wolinsky, Stephen H. (2009). Ich bin das Ich bin. Erfahre die Lehren des Sri Nisargadatta Maharaj - Teil 1. Stuttgart: Mouna GmbH.

Internet

Kornfield, Jack (2014). „Jack Kornfield on

Nisargadatta Maharaj (2)“,

www.youtube.com/watch?v=bXuF8qmv5Nc (letzter

Zugriff: 13.09 .2020$)$

Wolinsky, Stephen H. (2010). „Nisargadatta Maharaj / Experiential Meditation“, www.youtube.com/watch?v=rE6kS7VVsXw (letzter Zugriff: 13.09 .2020$)$

\footnotetext{
1 Die in diesem Textabschnitt beschriebenen Erfahrungen wurden im Rahmen des Inkubationsworkshops Achtsame Hochschulen Innehalten und Vorwärtsschreiten gemacht, zu dem meine Kollegen Mike Sandbothe und Reyk Albrecht mich eingeladen hatten. Die Veranstaltung war ein Angebot des Thüringer Modells Achtsame Hochschulen und fand vom 7. bis 11. Oktober 2019 am Benediktushof - Zentrum für Meditation und Achtsamkeit in Holzkirchen bei Würzburg, www.benediktushof-holzkirchen.de (letzter Zugriff: 13.09.2020), statt.

2 Vgl. Jäger 2015, S. $125 f$.

3 Nisargadatta 2014b, S. 70.

4 Jäger 2015, S. $9 f$.

5 Vgl. Krishnamurti 1992, S. 192.

6 Krishnamurti 1992, S. 192.

7 Nisargadatta 2014a, S. 104.
}

8 Nisargadatta 2014b, S. 27. Nisargadatta Maharaj unterscheidet in seinen Lehren zwischen dem verbalen und dem norverbalen $\mathrm{ICH}$ BIN. Eine Übung zum „nonverbalen ICH BIN" zeigt der langjährige Schüler von Nisargadatta Maharaj und Begründer der Quantenpsychologie, Dr. Stephen H. Wolinsky, unter "What is the I am?",

"Nisargadatta Maharaj / Experiential Meditation", www.youtube.com/watch?v=rE6kS7vVsXw (letzter Zugriff: 13.09.2020). Die Übung ist ebenfalls Teil des Films Ich bin das Ich bin. Erfahre die Lehren des Sri Nisargadatta Maharaj - Teil 1 (2009,

7:33-16:39 Min.). Vertiefend dazu siehe den Kurzbeitrag von Jack Kornfield über seine Erfahrung mit Nisargadatta: „Jack Kornfield on Nisargadatta Maharaj (2)",

www.youtube.com/watch?v=bXuF8qmv5Nc (letzter Zugriff:

13.09.2020)

9 Krishnamurti 1992, S. 198; Vgl. auch Krishnamurti 1993, S. 62.

10 Krishnamurti 1992, S. 198.

11 Vgl. Krishnamurti 1992, S. $196 f$.

12 Krishnamurti 1992, S. 197.

${ }^{13}$ Steindl-Rast 2018, S. 33

${ }^{14}$ Steindl-Rast 2016, S. 53.

15 Jäger 2017, S. 115.

16 Kornfield 2010, S. 78.

17 Nisargadatta 2017, S. 104

18 Nisargadatta 2014b, S. 94 ; siehe auch Hazrat Inayat Khan 2019, S. 60.

19 Vgl. Ware 2015.

20 Nisargadatta 2016, S. 235.

21 Jäger 2017, S. 133. 


\section{Intellekt und Intuition}

Das heutige Hochschulsystem konzentriert sich vor allem auf eine kognitive Ausrichtung, um Studierende auf ein künftiges Arbeitsleben vorzubereiten. Was bislang gelehrt wird, bezieht sich fast ausschließlich auf die Schulung des INTELLEKTS. Durch rationales Denken sollen Zusammenhänge vom Verstand erfasst werden. Gelehrt werden Fähigkeiten wie Analysieren, Einschätzen, Argumentieren, Schlussfolgern und Auswählen; diese ermöglichen es, zu schlüssigen Urteilsbildungen, zur Erkenntnisgewinnung und zu neuem Wissen zu gelangen.

Es gibt aber auch eine andere Art von Wissenserwerb, die allerdings wenig mit dem oben genannten intellektuellen Vorgehen zu tun hat: die INTUITION. Sie erschließt uns einen Zugang zum Wissen, der nicht über eine diskursive Herangehensweise erreicht wird. Mit den Worten von Albert Einstein (1879-1955) gesprochen: „Auf dem Weg zu einer Entdeckung hat der Intellekt wenig zu tun. Es kommt zu einem Bewusstseinssprung, nenne es Intuition oder was du willst, und die Lösung kommt zu dir und du weißt nicht wie oder

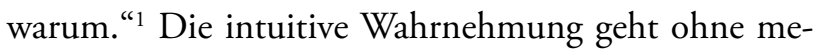
thodische und bewusste Schlussfolgerungen vor und entsteht unmittelbar; sie ist unfehlbar und in der Lage die „Wahrheit“ ganzheitlich zu erfassen.

Die Intuition inspiriert (lat. inspiratio, „das Einhauchen") und beseelt (spiritus) uns. Eine passende Definition von Intuition, welche die Verbindung zum Selbst, zur Seele, miteinschließt, findet sich bei dem indischen Gründer der Self-Realization Fellowship, Paramahansa Yogananda (1893-1952), der in den 1920er-Jahren Yoga und Meditation im Westen verbreitete: „Intuition ist diejenige Eigenschaft der Seele, die unmittelbar und direkt die Wahrheit einer jeden Sache und eines jeden Wesens wahrnimmt. Ohne die Macht der Intuition kannst du die Wahrheit nicht erkennen. Intuition bedeutet ,Seelenwahrnehmung' und ist die erkennende Seelenkraft, ohne Einsatz der Sinne oder des Geistes. Intuition kann dir Wissen über Dinge vermitteln, die deine Sinne und dein Denken dir nie vermitteln können. ${ }^{\text {"2 }}$ Und weil die Intuition diese Verbindung zu einer anderen Inspirationsquelle, einer anderen BEWUSSTSEINSEBENE beinhaltet, ist sie nicht selten Grundlage für kreative Entdeckungen geistiger und/ oder materieller Art.

Betrachtet man beispielsweise die Entstehung der Werke der großen Meister in der Malerei oder in der

\section{Die Intuition ist ein göttliches}

Geschenk, der denkende Verstand

ein treuer Diener. Es ist paradox, dass wir heutzutage angefangen haben, den Diener zu verehren und die
göttliche Gabe zu entweihen.

Albert Einstein (1879-1955)

Musik, so wird als Inspirationsquelle für den Schaffungsprozess nicht selten eine Verbindung mit dem "Göttlichen“" genannt. Von dem Komponisten Johannes Brahms (1833-1897) ist bekannt, dass er einmal, als er gefragt wurde, woher er seine Inspiration erhalte, zur Antwort gab: „Nicht ich, sondern der Vater, der in mir wohnt, der tut die Werke. ${ }^{\text {3 }}$ Er sagte, dass er sich sehr gut fokussieren müsste, um keinen einzigen Teil der Inspiration zu verpassen. „Sofort strömen die Ideen auf mich ein, direkt von Gott; ich sehe nicht nur bestimmte Themen vor meinem geistigen Auge, sondern auch die richtige Form, in die sie gekleidet sind, die Harmonien und die Orchestrierung. "“ Brahms fährt fort: „Ich muss mich im Zustand der Halbtrance befinden, um solche Ergebnisse zu erzielen - ein Zustand, in welchem das bewusste Denken vorübergehend herrenlos ist und das Unterbewusstsein herrscht, denn durch dieses, als einem Teil der Allmacht, geschieht die Inspiration. "5 Dabei betont er, dass dieser Zustand keineswegs mit Träumen vergleichbar ist, sondern einem ständigen Ringen gleicht: „Ich muss jedoch darauf achten, dass ich das Bewusstsein nicht verliere, sonst entschwinden die Ideen. [...] Um sich zu entwickeln und zu wachsen, muss deshalb der Mensch erst lernen, wie er seine eigenen seelischen Kräfte gebrauchen und ausbilden soll. Alle großen schöpferischen Geister tun dies, obgleich einige von ihnen sich dieses Vorgangs nicht so bewusst zu sein scheinen wie andere. " ${ }^{\text {"U Und obwohl }}$ viele fortgeschrittene Denker, wie Brahms angibt, sich nicht unbedingt ihrer intuitiven Vorgehensweise gewahr sind, stellt sich die Frage, wie der Mensch lernen kann, bewusst mit seinen seelischen Kräften in Verbindung zu treten. 


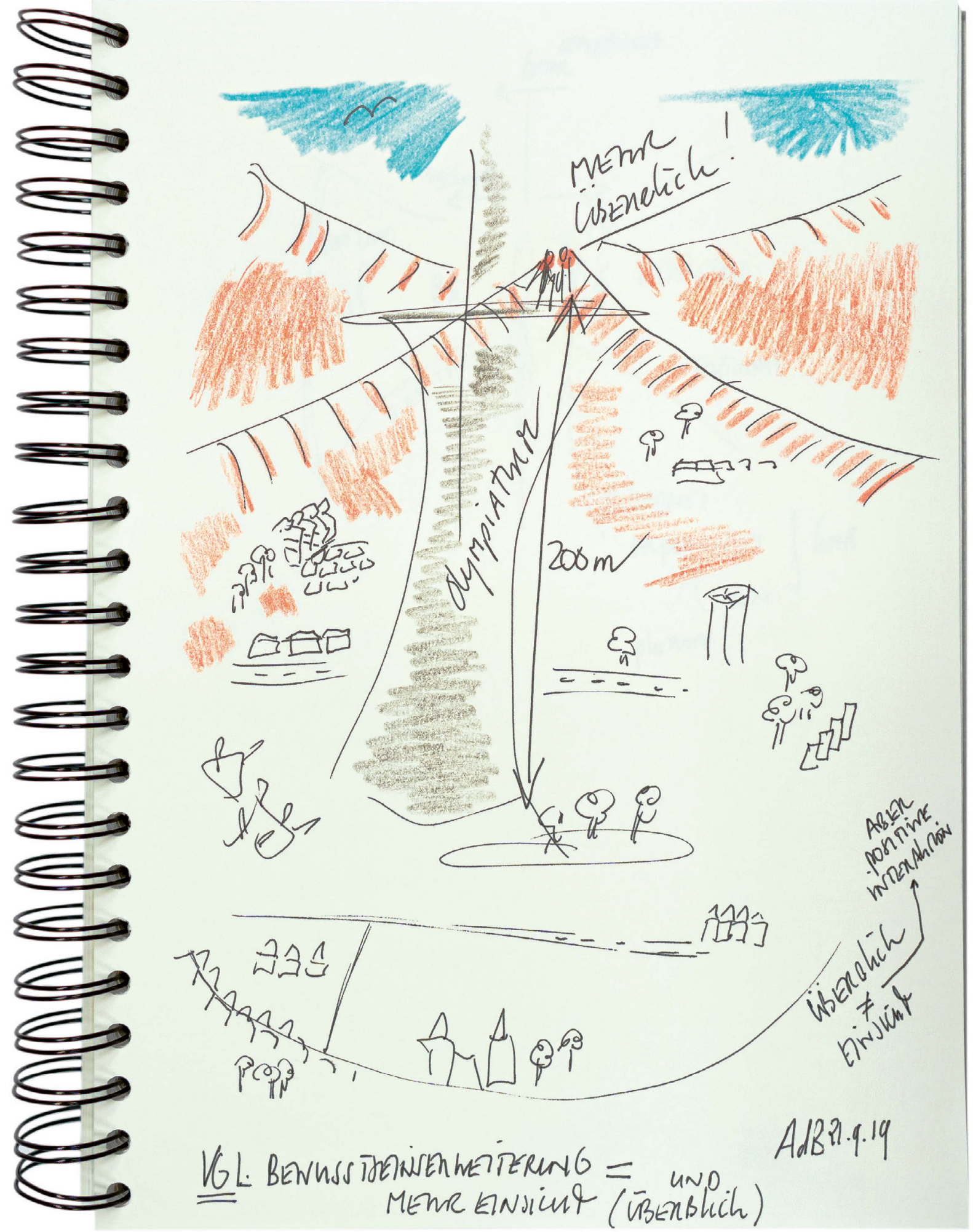

Meine Nichte und ich auf dem Olympiaturm in München. Von oben sieht man die Dinge ganz anders. Man hat einen Überblick und sieht horizontale Verbindungen.
Etwa so könnte sich eine Bewusstseinserweiterung anfühlen. Aber dazu müsste streng genommen noch eine weitere Dimension dazukommen: vertikal die Tiefe zu erfassen. 


\section{Die Ausrichtung des Denkvermögens}

Betrachten wir die intellektuelle und intuitive Erkenntnisgewinnung, so liegt ein ausschlaggebender Unterschied in der Ausrichtung des DENKVERMÖGENS. Beim intellektuellen Weg ist das Denkvermögen nach außen gerichtet und registriert die äußeren Informationen und Reize, die über die Sinne an das Gehirn weitergegeben werden. Es erkennt zudem die damit einhergehenden Empfindungen und mentalen Vorstellungen und setzt alles in Relation.

Beim intuitiven Weg wendet sich das Denkvermögen in eine andere Richtung, und zwar zur inneren Welt. Während der intuitiven Wahrnehmung werden seelische Kräfte in das jeweilige Erfahrungsgebiet des Denkers gelenkt.

Die Entwicklung dieser Fähigkeit bedarf einer mentalen Schulung, die zur Seelenverbindung führt.

\section{Konzentration, Meditation und Intuition}

Eine bewusste Ausrichtung des Denkvermögens erfordert KONZENTRATION. Der indische spirituelle Lehrer und Friedensaktivist Vishnu Devananda (19271993) weist darauf hin, dass Konzentration uns hilft, den Geist in eine Richtung zu lenken. „Das hauptsächliche Ziel der Konzentration ist es, den Geist immer wieder auf denselben Punkt oder Gegenstand zurückzuführen, indem man am Anfang seine Bewegungen auf einen kleinen Kreis begrenzt. "7 Schafft man es, die Konzentration länger zu halten und fokussiert zu bleiben, geht man - Devananda zufolge - in das Stadium der Meditation über: „Es wird eine Zeit kommen, in der der Geist auf einen Punkt allein konzentriert bleibt, wie der ununterbrochene Klang einer Kirchenglocke. Das ist Meditation, das Resultat ständiger und fortgesetzter Übung der Konzentration. “8

Die Stille, die durch die MEDITATION entsteht, ermöglicht die Kontaktaufnahme mit dem Inneren, mit der Seele. „Die Ruhe ist in der Meditation gesichert, wenn der Geist voll konzentriert ist, weit weg von den Objekten und nahe dem Atman, dem Selbst." Es ist die Ebene, wo Gedanken schweigen und der Geist verwandelt wird. ${ }^{10}$ „Wahre Meditation bedeutet, frei vom Denken zu sein. In dem Moment, in dem die Gedanken ruhig werden, wird das Licht des Selbst von innen heraus erstrahlen. " 11
Der spirituelle Lehrer Sri Chinmoy (1931-2007), der als Schriftsteller, Dichter, Komponist, Musiker, Künstler und Sportler wirkte, sagte: „Was wir beim Meditieren tatsächlich tun, ist, dass wir in den tieferen Bereich unseres Wesens eintreten. $\mathrm{Zu}$ diesem Zeitpunkt sind wir in der Lage, den Reichtum, den wir tief in uns haben, zum Vorschein zu bringen. " 12

Diese Tiefe zu erreichen, ist durch den Intellekt nicht möglich, da er nach außen gerichtet ist und dementsprechend an der Oberfläche agiert. Aus dieser Sicht ist der Intellekt der INTUITION untergeordnet. ${ }^{13}$ Dennoch hat auch der Intellekt eine wichtige Funktion. Um das innere Potenzial letztendlich in der Welt zu manifestieren, wird je nach Themenbereich das jeweilige „Handwerk“, der Umgang mit dem entsprechenden Wissen und somit auch der Intellekt, benötigt. Im Falle von Brahms war das seine Fähigkeit zu komponieren. Hätte er nicht über diese Gabe verfügt, hätte er die $\mathrm{Mu}$ sik zwar innerlich gehört, aber der Menschheit wäre sie vorenthalten geblieben.

Da bis heute an Hochschulen der Schwerpunkt der Ausbildung auf der Schulung des Intellekts beruht, bleibt der Welt eine Fülle von Erkenntnissen und Inspirationen, die sie aus der Intuition schöpfen könnten, nicht zugänglich.

Darum wäre es empfehlenswert, im Rahmen einer ganzheitlichen Erkenntnisgewinnung an Hochschulen und anderen Bildungsstätten neben dem Intellekt auch die Intuition bewusst zu fördern. Regelmäßige Konzentrations- und Meditationsübungen könnten dazu einen wertvollen Beitrag leisten.

\section{QUELLEN}

Abell, Arthur M. (1981): Gespräche mit berühmten Komponisten. Über die Entstehung ihrer unsterblichen Meisterwerke, Inspiration und Genius. Kleinjörl bei Flensburg: G. E. Schroeder-Verlag. Hazrat Inayat Khan (2018). Die Sufi-Botschaft von Hazrat Inayat Khan. Jubiläumsausgabe Band 1. Das Innere Leben. Polling: Verlag Heilbronn.

Krishnamurti, Jiddu (1992). Antworten auf Fragen des Lebens. Freiburg im Breisgau: Verlag Hermann Bauer KG.

Muktananda, Swami (2001). Meditiere. Das Glück liegt in Dir. Telgte: Siddha Yoga Verlag GmbH.

Sri Chinmoy (1974). Meditation: God's Duty and man's beauty. New York: Agni Press. 
Vishnu Devananda (1986). Meditation und Mantras.

Eine Darstellung der großen Yoga-Systeme in Theorie und Praxis. München: Heinrich Hugendubel Verlag. Yogananda, Paramahansa (2018). Die stärkende Kraft der Meditation - innere Ruhe und Klarheit gewinnen. Petersberg: Verlag Via Nova.

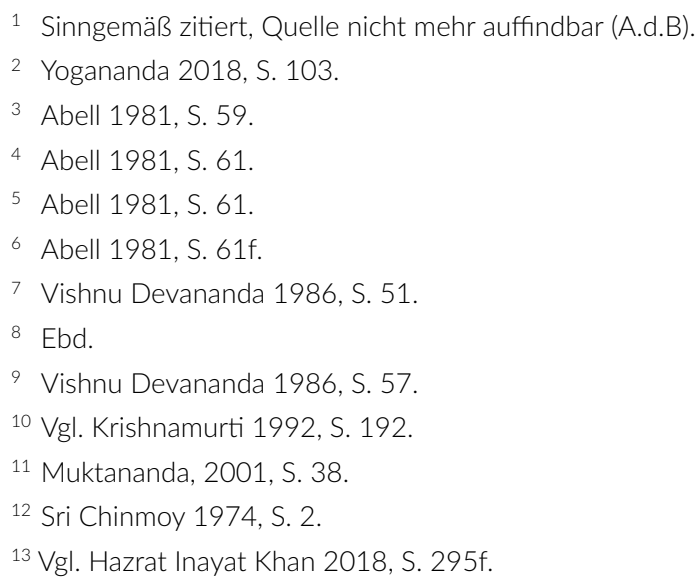

${ }^{13}$ Vgl. Hazrat Inayat Khan 2018, S. $295 f$.

Wenn das Denkvermögen auf die innere Stille

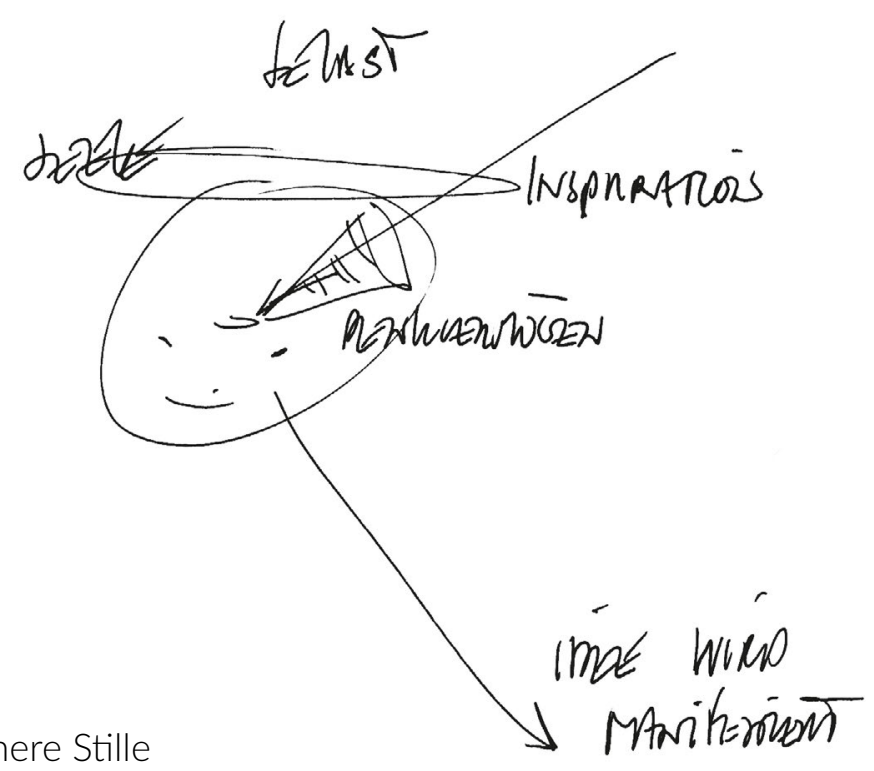
ausgerichtet wird, können Inspirationen über die seelischen Impulse als Ideen erkannt werden. Wenn das Denkvermögen anschließend auf die Außenwelt gelenkt wird, können diese Ideen in der Welt manifestiert werden. 


\section{Forschung}

\section{Wenn ihr den Geist verstehen wollt, dann meditiert.}

Buddhistischer Mönch

\section{Erforschung des Geistes}

Forschungen auf dem Gebiet der Achtsamkeit und Meditation haben in den letzten Jahrzehnten enorm zugenommen. Waren es in den 1970er-Jahren einige wenige Veröffentlichungen ${ }^{1}$, in denen das Thema wissenschaftlich behandelt wurde, sind es seit 2010 bis heute mehrere Hundert Publikationen jährlich. ${ }^{2}$ Insbesondere die Neurowissenschaften haben dieses Forschungsgebiet für sich entdeckt und untersuchen, auf welche Weise Achtsamkeit und Meditation Körper und Geist beeinflussen. Anhand von wissenschaftlichen Messungen haben Hirnforscherinnen und -forscher beispielsweise festgestellt, dass Meditation nachweislich bestimmte Bereiche des Gehirns trainiert und verändert. Wenngleich schon zahlreiche wichtige Erkenntnisse gesammelt werden konnten, bleiben noch viele Fragen unbeantwortet. Kann der menschliche Geist überhaupt erfasst werden? Ist Bewusstsein messbar?

Als der bekannte indische Lehrer Swami Muktananda (1908-1982) während seiner Welttournee im Jahr 1974 gefragt wurde, ob er als selbstverwirklichter Meister seine Gehirnwellen erforschen lassen wolle, antwortete er: „Das Bewusstsein ist viel zu subtil, es lässt sich nicht von einer Maschine einfangen. Wenn Sie wissen möchten, welche Erfahrungen ein Meditierender macht, müssen Sie in mein Labor kommen und es selbst erleben." 3

Auch im Dokumentarfilm Mönche im Labor von Delphine Morel wird auf die Schwierigkeit des Messens tiefer Meditationsstadien eingegangen. Berichtet wird über Hirnforscher, die zu sehr erfahrenen Yogis, die seit Jahren in völliger Abgeschiedenheit lebten, reisen mussten, da diese nicht in ihre Labore gehen würden. Man musste die Labore sozusagen zu ihnen bringen. Die Yogis waren durchaus skeptisch, ob die Forschungsvorhaben etwas bewirken würden und konterten mit Anmerkungen wie: „Der Geist hat weder Geruch noch Farbe, sondern ist einfach ein Raum, in dem verschiedene Phänomene existieren. Wie wollt ihr das messen?" 4
Dennoch konnte die Forschung bisher einiges über den Geist herausfinden. Dabei bezieht sich die Erforschung des Geistes hier auf die Psyche, auf die Ebene unseres Denkens und Fühlens und damit zusammenhängend unseres Verhaltens. Insbesondere handelt es sich um die Auswirkungen von Achtsamkeit und Meditation auf das Gehirn. Diese Effekte können mit modernsten Geräten sichtbar gemacht werden. Im vorliegenden Text sollen die bisher wichtigsten Erkenntnisse auf diesem Gebiet beleuchtet werden. Ebenso wird auf die Herausforderungen eingegangen, mit denen sich die Achtsamkeits- und Meditationsforschung konfrontiert sieht.

Im Mittelpunkt des oben genannten Films Mönche im Labor steht der Austausch (Mind and Life Dialogu$\left.e^{5}{ }^{5}\right)$ zwischen dem 14. Dalai Lama und Wissenschaftlerinnen und Wissenschaftlern unterschiedlicher Fachdisziplinen (siehe „Achtsamkeit und Meditation im Bildungssystem - ein Paradigmenwechsel?“, S. 17). Ein Thema, das im Rahmen des Dialogs aufgegriffen wurde, war der Umgang mit destruktiven Emotionen wie Leid, Hass, Eifersucht, Habgier und Angst. Das Verständnis dieser Emotionen und ihre Erforschung sind aus Sicht des Mind-and-Life-Teams eine der bedeutendsten Herausforderungen des 21. Jahrhunderts. Laut den Forschern würde eine bessere Einsicht in sowohl konstruktive als destruktive Emotionen jedem Menschen, aber auch der Gesellschaft und letztendlich der gesamten Menschheit von großem Nutzen sein. Um das Thema der destruktiven Emotionen zu vertiefen, fand im Jahr 2000 ein Treffen in Dharamsala (Indien) statt, bei dem der Dalai Lama und einige Mönche sich zusammen mit westlichen Wissenschaftlerinnen und Wissenschaftlern eine Woche lang intensiv austauschten. Diese Dialoge sind festgehalten in dem Buch: Dialog mit dem Dalai Lama. Wie wir destruktive Emotionen überwinden können, verfasst von Daniel Goleman, der auch der Autor des Buches Emotionale Intelligenz ist und diesen Begriff zum Mainstream machte. Das Mind-and-Life-Team konnte belegen, dass Achtsamkeits- und Meditationsansätze nachweisbare positive Effekte hinsichtlich des Umgangs mit Emotionen haben. Zahlreiche wissenschaftliche Untersuchungen sind inzwischen in den Laboren der Mind-and-Life-Forscherinnen und -Forscher durchgeführt worden. Herausgefunden hat man zudem, 
dass Achtsamkeit und Meditation nicht nur Geist und Körper entspannen, sondern Hirnbereiche beeinflussen und teilweise sogar umstrukturieren können.

\section{Wissenschaftliche Befunde und Forschungsfragen}

Die oben genannten Befunde haben maßgeblich dazu beigetragen, dass Achtsamkeit und Meditation immer mehr an gesellschaftlichem Interesse gewonnen und in vielen Handlungsfeldern bereits Einzug gefunden haben. Auch ist im Bereich der Neurowissenschaften ein neues Forschungsgebiet entstanden, die KONTEMPLATIVE NEUROWISSENSCHAFT. ${ }^{6}$ Ein wichtiger Baustein der kontemplativen Neurowissenschaft ist die NEUROPLASTIZITÄT, die besagt, dass „das Gehirn ein lebenslang lernfähiges Organ ist, das bis ins hohe Alter hinein und in Anpassung an äußere Einflüsse ständig weiter ausreift, sich wandelt und neu strukturiert" ${ }^{\text {"7 }}$.

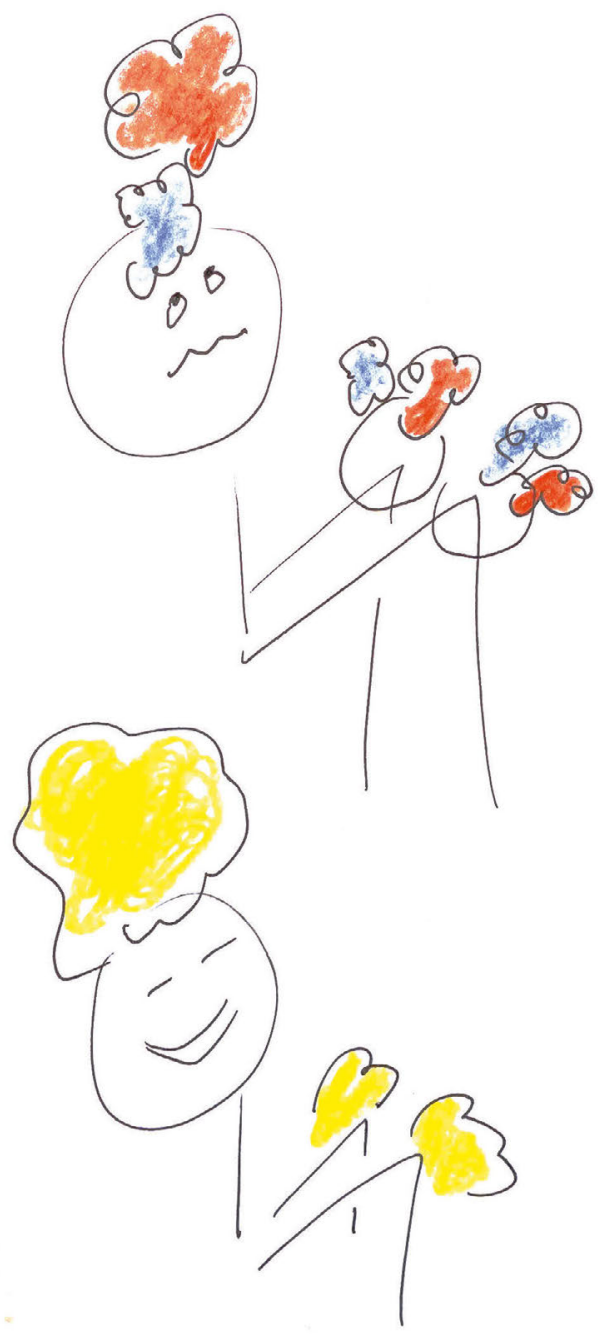

Richard Davidson, einer der Pioniere der kontemplativen Neurowissenschaft und Experte auf dem Gebiet der Emotionsforschung, geht davon aus, dass Menschen glücklicher sein könnten, wenn sie mehr Kontrolle über ihren Geist erlangen würden. Dies beinhaltet Davidson zufolge, selbst mehr Verantwortung für die eigene Psyche zu übernehmen und zu mehr Einsicht in das eigene Denken und Fühlen zu gelangen. ${ }^{8}$ Er weist darauf hin, dass Achtsamkeits- und Meditationspraktiken hier einen wesentlichen Beitrag leisten können.

Davidson sieht vier Grundvoraussetzungen für einen gesunden Geist, und zwar das grundlegende Erkennen des Gewahrseins (awareness), die Verbindung zu uns selbst und anderen (connection), d.h. über Emotionen wie Mitgefühl, Güte, Dankbarkeit zu verfügen und dadurch mit der Umwelt in Beziehung treten zu können, drittens Einsicht in das eigene Selbst (insight) und viertens die Bestimmung im Leben (purpose) zu erkennen und das Verhalten daran auszurichten. ${ }^{9}$ Zudem nennt Davidson vier wichtige Bausteine für das Wohlbefinden: Resilienz/Widerstandskraft (resilience), positive Lebenseinstellung (outlook), Aufmerksamkeit (attention) und Großzügigkeit (generosity). ${ }^{10}$

Davidson sieht die Erforschung der Wirkmechanismen von Achtsamkeit und Meditation als einen wichtigen Schritt zu einem besseren Verständnis davon, wie wir einen gesunden Geist kultivieren und ein zufriedenes und erfülltes Leben führen können. ${ }^{11}$

Welche Auswirkungen das Praktizieren von Meditation auf das Gehirn hat, ist für Davidson eine vielschichtige Frage. Unter anderem hängt dies zunächst von der Meditationsform ab, da die verschiedenen Ansätze, zumeist unterschiedliche Effekte bewirken. Zudem geht Davidson davon aus, dass die Auswirkungen der jeweiligen Meditationsformen auf das Gehirn nicht bei jedem gleich sind, da die Menschen unterschiedlich sind und daher auch unterschiedlich auf die verschiedenen Ansätze ansprechen. ${ }^{12}$
Grübeln und negative Gedanken wirken sich auch physisch aus. Optimismus und positive Gedanken ebenfalls. 
Auch die Neurowissenschaftlerin, Psychologin und wissenschaftliche Leiterin der Forschungsgruppe Soziale Neurowissenschaften in Berlin, Tania Singer, kommt hinsichtlich der Auswirkungen von Achtsamkeit und Meditation zu dem gleichen Schluss. Als Initiatorin und Leiterin des ReSource-Projekts, des bislang umfangreichsten Forschungsprojekts auf diesem Gebiet, weist sie darauf hin, dass unterschiedliche Achtsamkeits- und Meditationsübungen unterschiedliche Befunde ergeben. ${ }^{13}$ Wichtig ist es Singer zufolge, diese Ergebnisse differenzierter zu betrachten, inklusive die sogenannten Nullbefunde, bei denen keine Effekte festgestellt werden. ${ }^{14}$ Zudem muss die Konstitution der jeweiligen Testperson berücksichtigt werden: „Man sollte nicht einfach sagen: Bei jedem Menschen, der meditiert, wird das Immunsystem gestärkt und das Altern verlangsamt. [...] Es hängt auch davon ab, wie alt oder wie krank du bist. “15

Das ReSource-Projekt wurde im Jahr 2011 ins Leben gerufen und soll neben der aufmerksamkeitsbasierten Achtsamkeit vor allem neue Kenntnisse in Bezug auf Mitgefühl und Ethik sowie mehr Einsicht in Emotionsregulation, Stresslinderung und mentale Gesundheit liefern. ${ }^{16}$ Die 300 Testpersonen nahmen an drei Trainingsmodulen von jeweils drei Monaten im Zeitraum von Juni 2013 bis November 2014 teil. ${ }^{17}$

Die Module hatten die folgenden Schwerpunkte:

- Präsenz-Modul: klassische aufmerksamkeitsbasierte Achtsamkeitsübungen

- Affekt-Modul: Herzqualitäten, aber über die Selbstfürsorge hinaus - auch Dankbarkeit, Akzeptanz, Mitgefühl für andere und prosoziale Motivation

- Perspektiv-Modul: kognitiv, soziokognitiv. Schult die Vogelperspektive auf Gedanken, auf eigene Persönlichkeitsanteile und auf die Gedanken und Glaubenssätze anderer. ${ }^{18}$

Erste Auswertungen haben ergeben, dass bei den Teilnehmenden aller drei Module plastische Veränderungen in neuronalen Netzwerken sichtbar geworden sind. Wegen der umfangreichen und vielschichtigen Daten, über 90 Messparameter (Gehirn, Chromosomen, Verhalten usw.), dauern die Auswertungen weiterhin an. ${ }^{19}$

Für Singer ist es naheliegend, dass unterschiedliche Übungen unterschiedliche Effekte ergeben, sie vergleicht dies mit der Differenzierung bei Sportarten, die unterschiedlich auf den Körper wirken. „Aber dadurch, dass der Geist versteckt im Innern ist und nicht sichtbar wie Muskeln, ist es schwieriger, die Komplexität und Differenziertheit unserer Geistesfunktionen $\mathrm{zu}$ verstehen und $\mathrm{zu}$ verstehen, dass man die genauso kultivieren, verändern und üben kann wie im Sport bestimmte Muskelgruppen. “20

Was die Praxis anbelangt, bleiben noch viele Fragen offen. Unklar ist beispielsweise, ob Übungen, damit sie wirksam sind, zeitlich länger, beispielsweise über 20 Minuten lang, dauern sollten oder ob besser mehrere Kurzeinheiten, beispielsweise viermal fünf Minuten angeboten werden sollten. Bisher geht man davon aus, dass es hier keine Pauschalantworten geben wird, sondern dies individuell unterschiedlich ist. ${ }^{21}$

Ein weiterer Aspekt, der bei der Wirkung von Achtsamkeits- und Meditationstechniken in Betracht gezogen werden muss, ist, wie entscheidend die Reihenfolge der Übungen ist, falls mehrere nacheinander praktiziert werden. Und ebenso die Frage, welche Folgen es hat, wenn Übungen ausgelassen oder hinzugefügt werden. ${ }^{22}$ Bedeutend ist ebenfalls zu prüfen, welche Übungsart in welcher Situation geeignet ist (siehe „Risiken“, S. 89ff.). ${ }^{23}$

Das ist eine Erfahrung, die alle machen, die anfangen zu meditieren [...], dass diese Praktiken viel mehr bewirken, dass sie das Leben auf unerklärliche Art bereichern und einem in Dingen helfen, bei denen man vorher gar nicht wusste, dass man Hilfe braucht.

Sara Lazar

\section{Weitere Fragen und Herausforderungen}

Peter Sedlmeier, Professor für Forschungsmethodik und Evaluation, der sich in seinem Buch Die Kraft der Meditation. Was die Wissenschaft darüber weiß auf Metaanalysen und Metastudien ${ }^{24}$ sowie die Auswertung allgemeiner Reviews ${ }^{25}$ beruft, sieht systematische Auswirkungen von Meditation auf das Gehirn eindeutig belegt. Er führt allerdings kritisch an, dass bislang viele Studien nicht in einem fundierten theoretischen Rahmen eingebettet sind und oftmals erst nachwirkend 
geprüft wird, welche Befunde womöglich zum Modell passen könnten. ${ }^{26}$ Sedlmeier weist dabei auf ein wichtiges Problem hin, mit der sich die Forschung konfrontiert sieht: „Das, was im Gehirn passiert und was man beobachten kann, sind jedoch keine Gedanken oder Gefühle, sondern gehirnphysiologische Prozesse, die auf noch überwiegend ungeklärte Art und Weise mit Gedanken und Gefühlen zusammenhängen. “ ${ }^{27}$

In dem Film Free the Mind. Kann ein Atemzug dein Denken verändern? von Phie Ambo berichtet Richard Davidson über eine Studie mit Meditationsanfängern, die über einen Zeitraum von zwei Monaten regelmäßig meditierten. Im Anschluss daran wurde den Teilnehmern eine Grippeimpfung verabreicht. Im Vergleich mit einer Kontrollgruppe, die ebenfalls eine Grippeimpfung erhielt, zuvor aber nicht zwei Monate regelmäßig meditiert hatte, zeigte sich, dass die Meditierenden viel besser mit der Grippeimpfung zurechtkamen als die Kontrollgruppe, die nicht meditiert hatte. ${ }^{28}$ Trotz bisheriger positiver Befunde räumt Davidson aber ein, dass die Forschung noch nicht herausgefunden hat, warum dies letztendlich so ist. Für ihn bleibt eine Reihe an Fragen offen:

"Wachsen ihnen buchstäblich neue Neuronen, in speziellen Bereichen, die dafür wichtig sind? Werden neue Verbindungen dadurch verstärkt? Werden Verbindungen beschnitten, die sonst zu Störungen geführt hätten? Wie bei einem Bildhauer, der einen Marmorblock nimmt und daraus etwas Schönes schafft, indem er Dinge wegnimmt und nicht hinzufügt? Bislang ist es noch nicht klar, wie der Mechanismus wirkt. Einer der Faktoren kann stimmen, womöglich auch alle. " 29

Sara Lazar, Neurowissenschaftlerin in der Psychiatrie des Massachusetts General Hospital und Dozentin an der medizinischen Fakultät der Universität Harvard, sieht hinsichtlich einer genauen Zuschreibung von Ursachen auch Schwierigkeiten bezüglich der Interpretation von Veränderungen in der GRAUEN SUBSTANZ: „Da wir mit dem MRT nicht zwischen diesen verschiedenen Typen neuraler Veränderungen unterscheiden können, ist unklar, ob die von uns im Gehirn beobachteten Veränderungen auf neue Verbindungen, auf Helferzellen oder auf Blutgefäße zurückgehen. Wir wissen nur, dass es Unterschiede in der grauen Substanz gibt. “ 30

Mit grauer Substanz im Gehirn sind die Gehirnregionen gemeint, wo unter anderem die Kerne der Nervenzellen liegen und die Neuronen miteinander kommunizieren. Hier spielt sich Lazar zufolge „das Denken“" 31 ab. Die WEISSE SUBSTANZ umfasst die Teile im Gehirn, die aus langen Fasern bestehen, die die verschiedenen Hirnregionen miteinander verbinden und den Informationsaustausch gewährleisten. ${ }^{32}$

\section{Meditationspraktiken - der "tiefe“ und der "breite Weg"}

Davidson und Goleman unterscheiden bei der Differenzierung von Meditationspraktiken zwei unterschiedliche Wege: erstens den „tiefen Weg“, der sich in zwei Levels aufteilen lässt, und zweitens den „breiten Weg", der aus drei Levels besteht.

Beim ersten Level des „tiefen Weges“ handelt sich um Meditationsarten in ihrer reinen Form, sie bilden die intensivste Art der Praxis (z.B. Theravada-Buddhismus). Zum zweiten Level gehören die Formen, die nah an der reinen Form sind, jedoch ohne einige nicht übertragbare interkulturelle Aspekte.

Auf dem „breiten Weg“ besteht der erste Level aus Meditationsformen, die sich aus ihrem ursprünglichen spirituellen Kontext herausgelöst haben und mehr in die breite Masse wirken (z.B. MBSR). Spezielle Achtsamkeits- und Meditations-Apps für das Smartphone, kurze Übungen am Schreibtisch sind Beispiele von Ansätzen des zweiten Levels. Um mehr Menschen und vor allem die junge Generation zu erreichen, werden derzeit noch niedrigschwelliger angelegte Übungen anhand von Apps entwickelt. Diese werden dem dritten Level des „breiten Weges“ zugerechnet. ${ }^{33}$

\section{Tiefenbereiche der Meditation}

Der Psychologe und Psychotherapeut Dr. Harald Piron geht davon aus, dass die Bewusstseinserfahrungen von Meditierenden sich in der Regel entlang einer Tiefendimension entfalten ${ }^{34}$ und dass diese mit der bisherigen Erfahrungsdauer regelmäßigen Praktizierens korrelieren. ${ }^{35}$

Er unterscheidet fünf Tiefenbereiche: Hindernisse, Entspannung und Beruhigung, die Kunst der Aufmerksamkeitssteuerung, essenzielle Qualitäten sowie Non-Dualität. ${ }^{36}$

Der Psychologe und Neurowissenschaftler Dr. Ulrich Ott, der sich auf die Forschungen über Meditationstiefenbereiche von Piron beruft, hat die fünf Tiefenbereiche folgendermaßen zusammengefasst:

- „Hindernisse: Unruhe, Langeweile, Motivations-/ Konzentrationsprobleme

- Entspannung und Beruhigung: Wohlbefinden, ruhige Atmung, wachsende Geduld, Ruhe

- Die Kunst der Aufmerksamkeitssteuerung (Konzentration): Achtsamkeit, kein Anhaften an Gedanken, innere Mitte, Energiefeld, Leichtigkeit, Einsichten, Gleichmut, Frieden 
- Essenzielle Qualitäten: Klarheit, Wachheit, Liebe, Hingabe, Verbundenheit, Demut, Gnade, Dankbarkeit, Selbstakzeptanz

- Non-Dualität: Gedankenstille, Einssein, Leerheit, Grenzenlosigkeit, Transzendenz von Subjekt und Objekt.“ ${ }^{37}$

\section{Messverfahren}

Insbesondere die Neurowissenschaften haben in der bisherigen Achtsamkeits- und Meditationsforschung eine führende Rolle eingenommen. Je nachdem, welche Effekte gemessen werden sollen, gibt es dazu verschiedene wissenschaftliche Verfahren. Ott nennt in seinem Buch Meditation für Skeptiker. Ein Neurowissenschaftler erklärt den Weg zum Selbst die Messung von elektrischer Hirnaktivität (EEG) sowie bildgebende Verfahren, die zeigen, wo Effekte im Gehirn stattfinden (u.a. MRT). ${ }^{38}$

Beim EEG werden die folgenden Frequenzbereiche im Gehirn unterschieden:

- Delta $(0,1$ bis $<4 \mathrm{~Hz})$ : traumlose Tiefschlafphase

- Theta (4 bis $<8 \mathrm{~Hz}$ ): Schläfrigkeit, leichte Schlafphasen

- Alpha ( 8 bis < $13 \mathrm{~Hz}$ ): leichte Entspannung bzw. entspannte Wachheit bei geschlossenen Augen

- Beta (13 bis < $30 \mathrm{~Hz})$ : verschiedene Ursachen und Bedeutungen

- Gamma (ab $30 \mathrm{~Hz}$ ): bei starker Konzentration, Lernprozessen oder beim Meditieren ${ }^{39}$

Sedlmeier fasst den bisherigen Forschungsstand zusammen und sieht insbesondere durch Meditation Veränderungen im Theta- und Alpha-Bereich. ${ }^{40}$ Zudem weist er auf eine Zunahme von Gamma-Frequenzen hin: „Diese Effekte können als Anzeichen zunehmender Entspannung interpretiert werden, die dennoch (für gewöhnlich) nicht Hand in Hand geht mit zunehmender Schläfrigkeit, sondern im Gegenteil mit erhöhter Aufmerksamkeit und Konzentration. " ${ }^{41}$

EEG-Messungen liefern zwar wertvolle Erkenntnisse, vor allem eine hohe zeitliche Auflösung, doch für die Feststellung, wo Veränderungen während Achtsamkeits- und Meditationspraktiken im Gehirn genau lokalisiert sind, reicht diese Methode nicht aus. Ein Verfahren, mit dem Hirnstrukturen, Hirnfunktionen und Hirnprozesse in dreidimensionalen Bildern dargestellt und untersucht werden können, bietet die Magnetresonanztomografie (MRT). Die untersuchte Person befindet sich dabei in einer großen Röhre, die von einem starken Magneten umschlossen ist; mithilfe von Funkwellen können die Wasserstoffkerne im Gewebe dazu gebracht werden, sich kurzfristig gegen das Magnetfeld auszurichten; wenn sie in die ursprüngliche Ausrichtung zurück klappen, geben sie die aufgenommene Energie wieder als Funksignal ab. Dadurch wird es möglich, die verschiedene Gewebearten und Durchblutungsänderungen im Gehirn sichtbar zu machen. Letzteres kann beispielsweise der Fall sein, wenn zwischen Aufgaben gewechselt wird. Funktionelle MRTBilder (fMRT) liefern Informationen über Veränderungen der Durchblutung in Gehirnregionen in Echtzeit. Strukturelle MRT-Bilder zeigen die Menge an grauer und weißer Substanz in den verschiedenen Gehirnregionen. ${ }^{42}$ Der Vergleich zwischen MRT-Bildern vor dem Erlernen der Meditation und MRT-Bildern nach einigen Wochen Meditationspraxis zeigt die durch das Meditieren verursachten Veränderungen im Gehirn. ${ }^{43}$

\section{Forschungsergebnisse}

Ein wichtiges Ergebnis der Achtsamkeits- und Meditationsforschung insgesamt ist, dass Achtsamkeit und Meditation nachweisbare positive Auswirkungen auf unsere Einstellung und Verhaltensweisen haben - und dass das im Prinzip jeder erreichen kann. Wie der Mönch und Wissenschaftler Matthieu Ricard im Film Die Revolution der Selbstlosen betont: „Mein Gehirn ist kein bisschen außergewöhnlich, genau das hat mich beruhigt. Die Studie wäre uninteressant, wenn sie sich nur mit einigen außergewöhnlichen Leuten befasst hätte. Das Ermutigende und Faszinierende an dieser Studie ist, dass Menschen aus dem Himalaja, wie Tibeter und Bhutaner, aber auch Engländer, Franzosen, Amerikaner, Männer wie Frauen, Mönche, Nonnen und Laien, daran teilgenommen hatten und das Einzige, was zählte war, wie lange jemand schon Meditation praktiziert hatte. “ ${ }^{44}$

Neben der Tatsache, dass Achtsamkeit und Meditation in Prinzip bei allen Menschen eine positive Wirkung erzielen können, ist es auch bemerkenswert, wie schnell sich erste Ergebnisse zeigen. Beispielsweise weist Davidson darauf hin, dass sich bei ganz normalen Menschen, die für nur zwei Wochen 30 Minuten am Tag Achtsamkeits- und Meditationsübungen praktizieren, im Gehirn bereits Änderungen erkennen lassen. ${ }^{45}$

Sara Lazar widmet sich in ihren Forschungen intensiv der Frage, wie die Meditationspraxis das Gehirn beeinflusst und wie Veränderungen zu einem nachhaltigen positiven Nutzen werden können. Verhalten definiert 


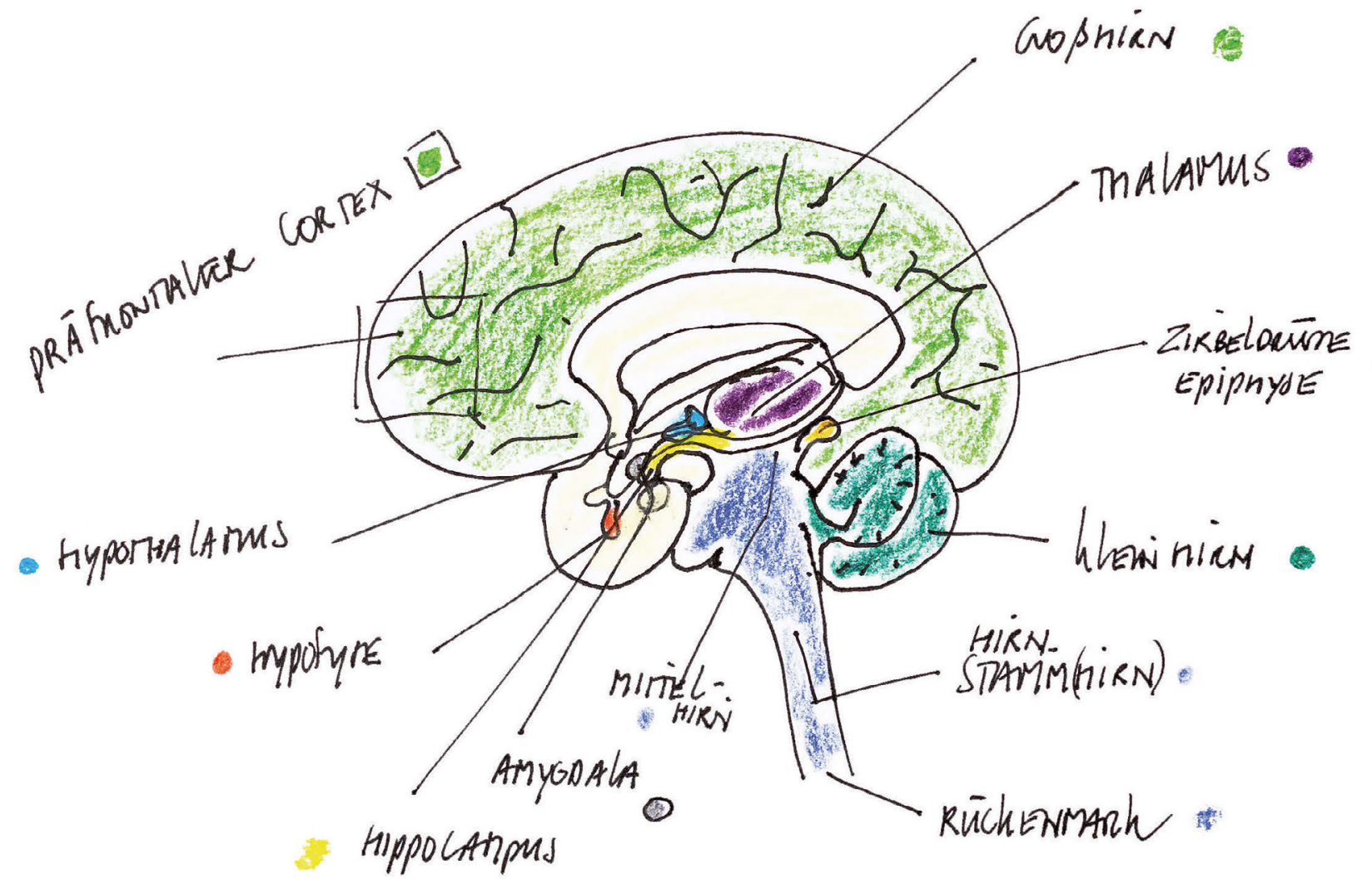

Das eigene Gehirn auch mal zeichnen.

sie sowohl als eine Handlung des Körpers als auch des Geistes. Lazar zufolge hängt Verhalten mit der Hirntätigkeit zusammen und diese wiederum mit der Hirnstruktur. Wollen Menschen ihr Verhalten dauerhaft umstellen, setzt dies für Lazar auch eine entsprechende Umstrukturierung in der Hirnstruktur voraus. ${ }^{46}$ Lazar beschreibt die Hirnstruktur als ,alles, was mit der Art und Weise zu tun hat, wie Neuronen miteinander kommunizieren, angefangen bei der Anzahl der Verbindungen zwischen Neuronen bis hin zur Menge der Neurotransmitter, die an den Verbindungsstellen (Synapsen) ausgeschüttet werden “ ${ }^{47}$.

Die Psychologin Dr. Britta Hölzel, die an der Harvard Medical School in Boston, am Bender Institute of Neuroimaging in Gießen und an der Charité in Berlin Forschungsprojekte zum Thema „Achtsamkeit und Meditation" durchgeführt hat, differenziert in ihrem Artikel „Mechanismen der Achtsamkeit. Psychologisch-neurowissenschaftliche Perspektiven" drei unterschiedliche, aber aufeinander einwirkende Bereiche, in denen die Effekte von Achtsamkeitsmeditation anhand wissenschaftlicher Befunde bislang belegt werden konnten, und zwar im Bereich der Aufmerksamkeitsregulation, der Emotionsregulation sowie des Selbsterlebens. ${ }^{48}$
In Anlehnung insbesondere an Richard Davidson, Britta Hölzel, Sara Lazar und Ulrich Ott werden im Folgenden eine Reihe an bedeutenden ForschungsBefunden beleuchtet:

- Default-Mode-Netzwerk

- „Attentional blink“-Effekt

- Vigilanz, selektive und exekutive Aufmerksamkeit

- Körpergewahrsein

- Emotionsregulation

- Mitgefühl und Altruismus

- Konditionierungsabbau

- Zellalterung

Dabei wird auch auf die Hirnregionen eingegangen, die durch die Achtsamkeits- und Meditationspraxis verändert werden. ${ }^{49}$ 
Die Erde wäre ein anderer Ort,

würden wir für die Pflege unseres

Geistes auch nur die gleiche kurze Zeit

wie fürs Zähneputzen aufwenden.

Richard Davidson

\section{Default-Mode-Netzwerk}

Neurowissenschaftliche Forschungen haben ergeben, dass manche Hirnregionen gerade dann eine höhere Aktivität ausweisen, wenn Testpersonen keine Aufgabe ausführen und sie sich in einer Ruhephase befinden. Diese im Ruhezustand aktiven Hirnregionen werden zusammen als DEFAULT-MODE-Netzwerk (Ruhezustandsnetzwerk) bezeichnet; dieses Netzewerk „umfasst unter anderem Regionen des MEDIALEN PRÄFRONTALEN und des POSTERIOREN CINGULÄREN CORTEX und den PRECUNEUS (Regionen, die auf der Mittelebene des Gehirns liegen) sowie den im TEMPORALLAPPEN gelegenen HIPPOCAMPUS und auch die TEMPORO-PARIETALE VERBINDUNGSREGION. “" ${ }^{50}$ Laut Ott weist die Aktivität des Default-Mode-Netzwerks unter anderem auf das sogenannte MINDWANDERING, das Abschweifen der Gedanken, hin: „Immer dann, wenn uns eine Situation keine Reaktionen abverlangt oder nur Routinehandlungen erfordert, so dass geistige Ressourcen zur Verfügung stehen, nutzen wir diese zum Erinnern, Nachdenken, Planen. "51 Hölzel sieht die Funktion des Default-Mode-Netzwerks ebenfalls als Möglichkeit, Erfahrungen zu reflektieren: „Wenn wir uns also überlegen, welche Relevanz bestimmte Ereignisse für uns selbst haben, oder wenn wir uns selbst in einem anderen räumlichen oder zeitlichen Kontext vorstellen - uns also in die Zukunft versetzen oder uns an Vergangenes erinnern -, dann werden diese Vorgänge vom DefaultMode-Netzwerk ermöglicht." 52

Da beim Mindwandering die ganze Psyche beteiligt ist, werden neben Gedanken auch Gefühle aktiviert. Im Falle von belastenden Gedanken und Gefühlen, hat dies eine negative Auswirkung auf unsere Psychohygiene. Ein aktives Default-Mode-Netzwerk führt grundsätzlich auch dazu, dass sich die Aufmerksamkeit auf das jetzige Tun verringert und man nicht wirklich im Moment ist. Die Forscher Matthew A. Killings- worth und Daniel T. Gilbert weisen darauf hin, dass Menschen oftmals Schwierigkeiten haben, wirklich präsent zu sein, bei dem, was sie gerade tun. In ihrer Studie zum Thema „A Wandering Mind Is an Unhappy Mind" fanden sie heraus, dass die befragten Personen sich im Schnitt zu 47 Prozent geistig nicht im jetzigen Moment befanden..$^{53}$ Davidson betont, dass es möglich und wichtig ist, dieses Aufmerksamkeitsdefizit zu verringern; ihm zufolge können vor allem auch Achtsamkeits- und Meditationsübungen dazu beitragen. Indem man nur beobachtet und nicht bewertet, lernt man, aufkommende Gedanken über Vergangenheit und $\mathrm{Zu}$ kunft nicht weiterzuverfolgen. Eine richtige Präsenz im Hier und Jetzt sorgt zudem dafür, dass Gedanken viel weniger aufkommen. ${ }^{54}$

Forschungen belegen, dass bei Meditierenden die Aktivität im Default-Mode-Netzwerk verändert ist. Forscher schließen daraus, dass Menschen die Achtsamkeit und Meditation praktizieren, generell weniger durch Gedanken abgelenkt werden und zudem weniger mit sich selbst beschäftigt sind. Hölzel weist in diesem Zusammenhang auf eine Veränderung im SELBSTERLEBEN bei Meditierenden hin: „Wir identifizieren uns weniger starr mit uns und den Dingen in unserem Leben - mit unseren Besitztümern, unserem Äußeren, aber auch mit Gedanken, Gefühlen, mit unserer Persönlichkeit, Gewohnheit und Eigenarten. An die Stelle, wo vorher eine feste Identifikation mit einer Persönlichkeit stand, tritt ein Empfinden, dass wir alle in unserer menschlichen Erfahrung miteinander - mit einem größeren Ganzen - verbunden sind. "55 Durch die Beobachterrolle und Nichtanhaftung sowie eine stärkere Verbindung mit dem eigenen Wesen, entstehen VERTRAUEN und GELASSENHEIT, der Welt zu begegnen. In Forschungen wurde eine positive Auswirkung unter anderem auf die SELBSTWAHRNEHMUNG, SELBSTWERTSCHÄTZUNG und SELBSTWIRKSAMKEIT sowie auf das SELBSTBILD im Allgemeinen festgestellt. ${ }^{56}$ 


\section{Aufmerksamkeitsblinzeln („,attentional blink-Effekt")}

Um eine mögliche Veränderung im Bereich der Aufmerksamkeit zu prüfen, kann der ATTENTIONALBLINK-EFFEKT, auch „Aufmerksamkeitsblinzeln“"57 genannt, herangezogen werden. Er bezieht sich auf das Phänomen, dass während der Verarbeitung eines spezifischen Reizes keine weiteren Reize zusätzlich verarbeitet werden können. Dieser kurzfristige „blinde Fleck“ (Aufmerksamkeitsdefizit) in der Wahrnehmung sorgt dafür, dass wir einen darauffolgenden Reiz verpassen, wenn dieser zu kurzfristig auf den ersten Reiz erfolgt oder wenn der erste Reiz zu viel Aufmerksamkeitszuwendung verlangt. ${ }^{58}$ Studien zeigen, dass die Meditationspraxis dazu führt, den ersten Reiz effizienter zu verarbeiten, sodass das Gehirn rechtzeitig bereit ist, den darauffolgenden Reiz zu erfassen. ${ }^{59}$

Eine erhöhte Erkennungsrate wäre beispielsweise auch sehr wertvoll bei der Wahrnehmung von Gesichtsausdrücken. ${ }^{60}$ Gerade flüchtige Gesichtsausdrücke, die sogenannten Mikroausdrücke (micro-expressions), sagen sehr viel über Menschen aus, da sie die Gefühle völlig unzensiert zeigen. Sie wahrzunehmen ist nicht leicht, aber trainierbar. In einer Studie mit erfahrenen Meditierenden zeigte sich eine im Vergleich bessere Erkennungsrate der Gefühlsausdrücke als bei Testpersonen ohne Meditationserfahrung. ${ }^{61}$

\section{Vigilanz, selektive und exekutive Aufmerksamkeit}

In drei Bereichen der Aufmerksamkeit sieht Hölzel Veränderungen aufgrund von Achtsamkeits- und Meditationspraktiken: VIGILANZ, SELEKTIVE AUFMERKSAMKEIT und EXEKUTIVE AUFMERKSAMKEIT.

Vigilanz, eine lang anhaltende Aufmerksamkeit, „bezeichnet die Bereitschaft, in monotonen und lange andauernden Situationen auf seltene Reize angemessen zu reagieren" ${ }^{\text {62 }}$. Die selektive Aufmerksamkeit „bezeichnet die Auswahl einer spezifischen Information aus einer Vielzahl von Informationen. Hierbei geht es um die Auswahl des Objekts oder Gedankengangs, worauf die Aufmerksamkeit gerichtet wird" ${ }^{\text {“63. }}$.
Die exekutive Aufmerksamkeit „bezeichnet das Überwachen und Lösen von Konflikten der Aufmerksamkeit durch ablenkende Reize. Hierbei geht es also darum, sich bewusst einem Stimulus zuzuwenden auch wenn dabei anderes ausgeblendet werden muss" ${ }^{\text {"64. }}$

Laut Hölzel zeigen Studien, dass sich die selektive und exekutive Aufmerksamkeit durch Achtsamkeitsund Meditationsübungen schnell verbessern lassen, wogegen ein positiver Effekt auf die Vigilanz eine lange Praxiserfahrung voraussetzt. ${ }^{65}$ Als Hirnregion, die bei der Regulierung der exekutiven Aufmerksamkeit deutlich involviert ist, nennt sie den sogenannten ANTERIOREN CINGULÄREN CORTEX (ACC). Studien konnten hier als Effekt von Achtsamkeit und Meditation sowohl Veränderungen in der GRAUEN SUBSTANZ (dickeren Cortex) und in der WEISSEN SUBSTANZ (erhöhte Integrität der Verbindungsfasern) belegen. ${ }^{66}$

\section{Körpergewahrsein}

Mittels unseres Körpergewahrseins sind wir in der Lage, Körperempfindungen zu erkennen. Achtsamkeits- und Einsichtsmeditationstechniken, die solche Empfindungen als Aufmerksamkeitsobjekt in den Mittelpunkt stellen, stärken die Verbindung zwischen Körper und Geist. Eine bedeutende Übung diesbezüglich ist der Bodyscan (siehe S. 135ff.). Studien zeigen, dass solche Techniken zu einer Veränderung im vorderen INSELCORTEX der RECHTEN GEHIRNHÄLFTE führen. Lazar weist diesbezüglich auf eine Zunahme der Dichte der GRAUEN SUBSTANZ hin. ${ }^{67}$ Diese Hirnregion ermöglicht es Ott zufolge, eine sogenannte „META-REPRÄSENTATION des GEFÜHLTEN LEIBES $^{\text {“68 }}$ zu erstellen, da hier zahlreiche Informationen aus dem Körperinneren zusammenfinden. Neben der Körperempfindung kann dadurch zudem der individuelle emotionale Zustand registriert werden. ${ }^{69}$ Ein verbessertes Leibgefühl hat auch eine positive Auswirkung auf unser Denken und auf Entscheidungen, die wir treffen. ${ }^{70}$ Mehr Kenntnisse über die eigene Gefühlswelt zu erlangen, trägt dazu bei, sich auch in Mitmenschen besser hineinversetzen zu können. Hölzel weist darauf hin, dass durch die Achtsamkeits- und Meditationspraxis neben der EMPATHIE auch die AFFEKTREGULATION, insbesondere das Registrieren und angemessene Reagieren auf eigene Gefühle, positiv beeinflusst werden. ${ }^{71}$ 


\section{Emotionsregulation}

Emotionen wahrzunehmen, diesen aber nicht ausgeliefert zu sein, gibt uns mehr Handlungsspielraum, um in bestimmten Situationen passend zu reagieren. Aus Forschungen geht hervor, dass die Achtsamkeits- und Meditationspraxis eine positive Auswirkung auf die EMOTIONSREGULATION hat. Zudem konnte ebenfalls gezeigt werden, dass sich negative Gefühle qua Häufigkeit und Intensität reduzieren lassen und positive Gefühlszustände sich verbessern. ${ }^{72}$

Als Gründe für diese Verschiebung zu positiven Gefühlszuständen nennt Hölzel, dass sich durch Achtsamkeits- und Meditationsübungen das Umdeuten von emotionalen Situationen, das sogenannte REAPPRAISAL, leichter realisieren lässt. Auch tragen die Akzeptanz und das Nichtbewerten einer Situation, das sogenannte NON-APPRAISAL, dazu bei, dass man sich weniger mit destruktiven Emotionen identifiziert.
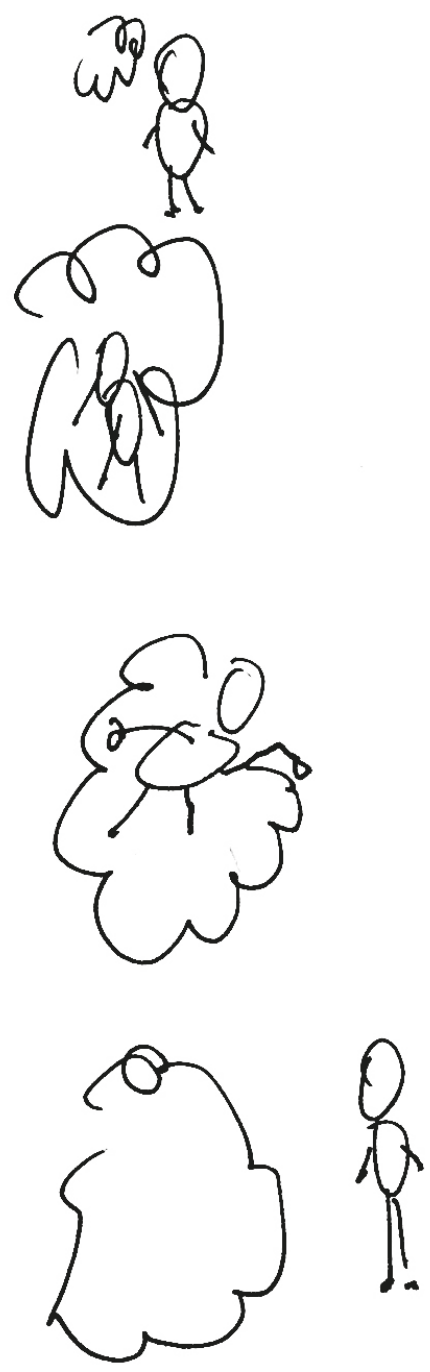

Darüber hinaus können Achtsamkeit und Meditation generell bewirken, dass KONDITIONIERUNGEN besser aufgelöst werden können. Dadurch lassen sich auch Gefühlsreaktionen leichter modellieren. ${ }^{73}$

Wie bei der Steuerung von Aufmerksamkeit ist der PRÄFRONTALE CORTEX auch bei der Steuerung von Emotionen wichtig. ${ }^{74}$ Unter anderem wirkt er regulierend auf limbische Regionen wie etwa die AMYGDALA (MANDELKERN). Dieser Bereich im Gehirn wird aktiviert, wenn wir uns mit angstauslösenden Reizen konfrontiert sehen. ${ }^{75}$ Ein Teil der Amygdala ist wichtig dafür, Anzeichen von Gefahr überhaupt zu erkennen. Sind diese einmal erkannt, löst ein anderer Teil der Amygdala anschließend eine Kaskade von Reaktionen aus. Davidson zufolge besteht diese aus drei zentralen Komponenten: „Die erste betrifft das Verhalten und sorgt z.B. dafür, zu erstarren oder wegzulaufen oder zu fliehen. Es kann sich vegetativ auswirken, das wäre die zweite Komponente, was zu Veränderungen der Herzfrequenz und des Blutdrucks führen kann, um den Organismus aufs Handeln vorzubereiten. Und die dritte Komponente betrifft hormonelle Veränderungen. Und das Schlüsselhormon hierbei ist CORTISOL, ein Stresshormon. "76

Studien haben ergeben, dass die Achtsamkeits- und Meditationspraxis zu einer Reduzierung der Amygdala-Aktivität führt. ${ }^{77}$ Diese Veränderung hat auch einen Effekt auf den HIPPOCAMPUS. Dieser Bereich im Gehirn ist für die Emotionsregulation bedeutend und sorgt unter anderem dafür, emotional angemessen auf eine Situation zu reagieren. Ist die Aktivität des Hippocampus geschwächt, fällt uns diese Steuerungsfähigkeit weitaus schwerer. ${ }^{78}$ Die Zellen im Hippocampus werden durch Cortisol geschädigt. Ist im Falle von andauerndem Stress der Cortisolspiegel länger erhöht, wirkt sich dies schädlich auf den Hippocampus und seine Funktionen aus. Sichtbar ist in diesem Hirnbereich eine Abnahme an grauer Masse. Aus Studien geht hervor, dass Achtsamkeit und Meditation Stress und dadurch unter anderem die Aktivität der Amygdala reduzieren. Die Abnahme des Cortisolspiegels hat einen positiven Effekt auf den Hippocampus und zeigt sich unter anderem in einer Zunahme der grauen Masse in diesem Bereich. ${ }^{79}$

Emotionsregulation heißt: kein Spielball eigener Emotionen zu sein und diese auch von außen beobachten zu können. Wir sind nicht unsere Emotionen! 


\section{Mitgefühl und Altruismus}

Kombiniert man Meditationstechniken mit Empathie und Mitgefühl, sogenannte Metta-Meditationsformen oder Meditationen der Liebenden Güte, sind in der Regel als Effekte eine Intensivierung des Mitgefühls und altruistischen Verhaltens feststellbar. ${ }^{80}$ Tania Singer hat zusammen mit Matthieu Ricard das Buch Mitgefühl in der Wirtschaft herausgegeben. Darin berichten mehrere Autorinnen und Autoren über die dem Menschen angeborene Anlage des Mitgefühls und warum wir diese Fähigkeit anstelle von Individualismus und Wettbewerb fördern sollten. ${ }^{81}$ Davidson und Begley schreiben in ihrem Buch Warum wir fühlen, wie wir füblen. Wie die Gehirnstruktur unsere Emotionen bestimmt - und wie wir darauf Einfluss nehmen können unter anderem über Teilnehmerinnen und Teilnehmer eines Forschungsprojekts, die nach einem Training in Metta-Meditation bei Risikosimulationsspielen wesentlich altruistischere Entscheidungen trafen als vor dem Training. ${ }^{82}$

Generell bewirkt die Zunahme der Verbindungen zwischen den betroffenen Hirnregionen eine Verbesserung der Empathie und der sozialen Intuition. ${ }^{83}$ Davidson weist im Film Free the Mind darauf hin, dass bereits nach drei Monaten des Praktizierens von Mitgefühlsmeditation die Teilnehmerinnen und Teilnehmer in der Lage waren, ihre Umgebung subtiler wahrzunehmen und andere Menschen besser einschätzen zu können. ${ }^{84}$

Bisherige Studien zeigen, dass Menschen grundsätzlich über eine angeborene BASIC INNER GOODNESS verfügen. ${ }^{85}$ Um diese Fähigkeit bei Kindern im Alter von vier bis sieben Jahren zu fördern, hat Davidsons Center für Healthy Minds dafür unter anderem ein achtsamkeitsbasiertes Kindness Curriculum (Freundlichkeitslehrplan) für den Kernunterricht entwickelt. ${ }^{86}$ Anhand der Übungen lernen die Kinder unter anderem, „wie sie mit sich selbst und den Menschen um sich herum verbunden und freundlich zu ihnen sein können"87. Da in diesem Alter das Gehirn einen hohen Grad an Neuroplastizität aufweist, setzt das Team um Davidson auf diesen Bereich. Gerade in einer solchen Phase finden im Gehirn zahlreiche Umstrukturierungen statt und können Interventionen bedeutenden Einfluss haben. ${ }^{88}$

\section{Konditionierungsabbau}

Die Achtsamkeitspraxis lehrt uns, mehr im Hier und Jetzt zu sein, den jeweiligen Moment bewusst wahrzunehmen und ihm mit Neugierde zu begegnen. Es geht dabei auch darum, zunächst zu beobachten und nicht sofort auf die jeweiligen Sinnesreize zu reagieren und Situationen nicht gleich zu beurteilen. ${ }^{89}$ Diese Haltung führt automatisch zu einer offenen, unvoreingenommenen Grundeinstellung im Leben und zu mehr Gelassenheit und Akzeptanz. Achtsamkeits- und Einsichtsmeditationsformen ermöglichen darüber hinaus, AUTOMATISIERTE WAHRNEHMUNGS-, GEDANKEN- und VERHALTENSMUSTER neu zu prüfen und, wenn gewünscht, zu verändern. Situationen können konstruktiv umgedeutet und dadurch Vermeidungsstrategien reduziert werden. Meditationen der tiefen Versenkung, die auf eine umfassende innere Stille ausgerichtet sind, führen sogar dazu, dass KONDITIONIERUNGEN generell an Bedeutung verlieren (siehe „Achtsamkeit und Meditation im Bildungssystem - ein Paradigmenwechsel?“, S. 20).

Für den Abbau von Konditionierungen und das Umlernen von emotionalen Reaktionen sind insbesondere der ventromediale Präfrontalcortex, die Amygdala und der Hippocampus wichtig. Diese Hirnregionen lassen sich, wie bereits erwähnt, durch Achtsamkeitsund Meditationspraxis positiv verändern. ${ }^{90}$

\section{Zellalterung}

Die EPIGENETIK, ein Gebiet der Biologie, das sich mit den Mechanismen der Genregulation und ihrer Vererbung befasst, beschäftigt sich seit Kurzem auch mit den Effekten von Achtsamkeits- und Meditationsinterventionen auf unsere Chromosomen.

Bei der Zellteilung wird die Länge der Schutzkappen unserer Chromosomen, der TELOMERE, verringert. Teilt die Zelle sich immer weiter, haben die Zellen irgendwann keinen Schutz mehr und sterben ab. ${ }^{91}$

Die Molekularbiologin und Nobelpreisträgerin für Physiologie/Medizin, Elizabeth Blackburn, konnte belegen, dass Stress diesen Verkürzungsprozess beschleunigt. Umgekehrt kann eine Stressreduzierung, aber auch bereits ein konstruktiver Umgang mit Stress, einer solchen Degeneration entgegenwirken. Erste Studien zeigen, dass Achtsamkeits- und Meditationsübungen die Konzentration des Enzyms TELOMERASE, das für die Produktion der Telomere zuständig ist, erhöhen können. Dadurch werden die 
Telomere regeneriert und Abnutzungsprozesse verlangsamt. ${ }^{92}$ Wie lange eine Steigerung der Telomerase-Werte anhält, konnte noch nicht eindeutig geklärt werden. Erhofft wird, dass weitere Studien künftig mehr Aufschluss darüber geben werden.

\section{QUELLEN}

Blackburn, Elizabeth / Epel, Elissa (2019). Die Entschlüsselung des Alterns. Der Telomer-Effekt. München: Wilhelm Goldmann Verlag.

Davidson, Richard J. / Goleman, Daniel (2017). Altered Traits. Science Reveals How Meditation Changes Your Mind, Brain, and Body. New York: Avery.

Davidson, Richard J. / Begley, Sharon (2012). Warum wir fühlen, wie wir fühlen. Wie die Gehirnstruktur unsere Emotionen bestimmt - und wie wir darauf Einfluss nehmen können. München: Arkana.

Goleman, Daniel (2003). Dialog mit dem Dalai Lama. Wie wir destruktive Emotionen überwinden können. München / Wien: Carl Hanser Verlag.

Goleman, Daniel (1997). Emotionale Intelligenz. München: dtv Verlagsgesellschaft mbH \& Co. KG. Hölzel, Britta (2015). „Mechanismen der Achtsamkeit. Psychologisch-neurowissenschaftliche Perspektiven“, in: Hölzel, Britta / Brähler, Christine (Hrsg.), Achtsamkeit mitten im Leben. Anwendungsgebiete und wissenschaftliche Perspektiven. München: O. W. Barth, S. 43-77.

Kabat-Zinn, Jon (2019). Gesund durch Meditation. München: Knaur Verlag.

Langer, Ellen J. (1989). Mindfulness. Boston: AddisonWesley Publishing Company, Inc.

Lazar, Sara (2015). „Die neurowissenschaftliche Erforschung der Meditation “, in: Zimmermann, Michael / Spitz, Christof / Schmidt, Stefan (Hrsg.), Achtsamkeit. Ein buddhistisches Konzept erobert die Wissenschaft. Bern: Verlag Hans Huber, S. 71-81.

Muktananda, Swami (2001). Meditiere. Das Glück liegt in Dir. Telgte: Siddha Yoga Verlag GmbH.

Ott, Ulrich (2015). Meditation für Skeptiker. Ein Neurowissenschaftler erklärt den Weg zum Selbst. München: Droemer Knaur.

Piron, Harald (2020). Meditationstiefe. Grundlagen, Forschung, Training, Psychotherapie. Berlin: Springer-Verlag.

Ricard, Matthieu (2018a). Allumfassende Nächstenliebe. Hamburg: Edition Blumenau.
Sedlmeier, Peter (2016). Die Kraft der Meditation. Was die Wissenschaft darüber weiß. Reinbek bei Hamburg: Rowohlt Taschenbuch Verlag.

Siegel, Daniel J. (2017). Das achtsame Gehirn. Freiburg im Breisgau: Arbor Verlag.

Singer, Tania (2019b). „Perspektiven der kontemplativen Neurowissenschaften zu Macht und Fürsorge: wie man sich in Fürsorge und Mitgefühl übt", in: Ricard, Matthieu / Singer, Tania / Karius, Kate (Hrsg.), Die Macht der Fürsorge. Für eine gemeinsame Zukunft. Wissenschaft und Buddhismus im Dialog mit dem Dalai Lama. München: Knaur Verlag, S. 86-99.

Singer, Tania / Ricard, Matthieu (2015). Mitgefühl in der Wirtschaft. München: Albrecht Knaus Verlag. Singer, Wolf / Ricard, Matthieu (2018). Jenseits des Selbst. Dialoge zwischen einem Hirnforscher und einem buddhistischen Mönch. Berlin: Suhrkamp Taschenbuch Verlag.

Singer, Wolf / Ricard, Matthieu (2008). Hirnforschung und Meditation. Ein Dialog. Frankfurt am Main: Suhrkamp Verlag.

Tang, Yi-Yuan (2019). Die Wissenschaft der Achtsamkeit. Wie Meditation die Biologie von Körper und Geist verändert. Paderborn: Junfermann Verlag.

\section{Zeitschrift}

Davidson, Richard J. (2019c). „Interview“, in: Moment by Moment, Ausgabe 01. Freiburg: Hammer Solutions Media, S. 48-52.

Davidson, Richard J. (2018). „Interview“, in: Moment by Moment, Ausgabe 03. Freiburg: Hammer Solutions Media, S. 56-63.

Singer, Tania (2019a). „Interview“, in: Moment by Moment, Ausgabe 04. Freiburg: Hammer Solutions Media, S. 58-64.

\section{DVD}

Ambo, Phie (2013). Free the Mind. Kann ein Atemzug dein Denken verändern? Köln: mindjazz pictures.

Engle, Dawn Gifford (2019). The Dalai Lama - Scientist. Arvada / Colorado: PeaceJam Foundation.

Gilman, Sylvie / Lestrade, Thierry de (2016). Die Revolution der Selbstlosen. Köln: mindjazz pictures. 
Internet

Center for Healthy Minds (2017). Kindness Curriculum, https://centerhealthyminds.org/join-the-movement/ sign-up-to-receive-the-kindness-curriculum (letzter Zugriff: 13.09.2020)

Davidson, Richard J. (2019a). Vortrag und Podiumsdiskussion: „We can change the brain, by changing the mind“" am 19. März 2019 in München beim Forum für den Wandel der Edith-Haberland-Wagner Stiftung, www.ehw-stiftung.de (letzter Zugriff: 13.09.2020)

Davidson, Richard J. (2019b). „How mindfulness changes the emotional life of our brains. TEDxSanFrancisco", www.youtube.com/watch?v=7CBfCW67xT8 (letzter Zugriff: 13.09.2020)

Davidson, Richard J. (2016). „The Four Constituents of Well-Being“, www.youtube.com/watch?v=HeBpsiFQiTI (letzter Zugriff: 13.09.2020)

Killingsworth, Matthew A. / Gilbert, Daniel T. (2010). A Wandering Mind Is an Unhappy Mind, www. danielgilbert.com/KILLINGSWORTH\%20\&\%20GILBERT\%20(2010).pdf (letzter Zugriff: 13.09.2020)

Lazar, Sara (2011). „How Meditation Can Reshape Our Brains: Sara Lazar at TEDxCambridge“, www. youtube.com/watch?v=m8rRzTtP7Tc (letzter Zugriff: 13.09.2020)

Magiera, Birgit (2020). BR2 IQ - Wissenschaft und Forschung „Achtsamkeitsforschung - Frieden für die Welt oder Wellness fürs Ich?“, https://www.br.de/ mediathek/podcast/iq-wissenschaft-und-forschung/ achtsamkeitsforschung-frieden-fuer-die-weltoder-wellness-fuers-ich/1793281 (letzter Zugriff: 13.09.2020)

Morel, Delphine (2016). Mönche im Labor, www.youtube.com/watch? $v=3 q p L U p k d b K E$ (letzter Zugriff 13.09.2020)

Singer, Tania / Bolz, Matthias (Hrsg.) (2013). eBook Mitgefühl. In Alltag und Forschung, www.compassion-training.org/?lang=de (letzter Zugriff: 13.09.2020)

\footnotetext{
1 Siehe u.a. Langer 1989.

2 Siehe „Mindfulness Journal Publications by Year, 1980-2015", in: American Mindfulness Research Association (2016): goAMRA.org. Vgl. auch Sedlmeier 2016, S. 59f.; Hölzel / Brähler 2015, S. 9; Ott 2015, S. $167 f$.

3 Muktananda 2001, S. xiii.

4 Morel 2016, 17:51 Min.
}

5 Weitere Informationen: www.mindandlife.org; siehe auch Goleman 2003, S. 11ff. sowie der Film Dalai Lama - Scientist (2019) von Dawn Gifford Engle.

6 Vgl. Kabat-Zinn 2019, S. 220.

7 Kabat-Zinn 2019, S. 220.

8 In Bezug auf Verantwortung für unseren Geist übernehmen siehe auch Singer / Ricard 2018, S. 233ff.; vgl. auch Gilman / Lestrade 2016, 51:55-52:22 Min.

9 Vgl. Davidson 2018, S. 61. Siehe auch Davidsons TED-Vortrag (2019b). „How mindfulness changes the emotional life of our brains", www.youtube.com/watch?v=7CBfCW67xT8 (letzter Zugriff: 13.09.2020); sowie Vortrag und Podiumsdiskussion „We can change the brain, by changing the mind" am 19. März 2019a, Prof. Dr. Richard Davidson in München beim Forum für den Wandel der Edith-Haberland-Wagner Stiftung, www.ehw-stiftung.de (letzter Zugriff: 13.09.2020).

${ }^{10}$ Richard Davidson Vortrag (2016) „The Four Constituents of WellBeing", www.youtube.com/watch? v=HeBpsiFQiTI (letzter Zugriff: 13.09.2020).

11 In dem von Richard Davidson gegründeten Center for Healthy Minds werden zahlreiche Forschungen diesbezüglich durchgeführt. Weitere Informationen: www.centerhealthyminds.org (letzter Zugriff: 13.09.2020)

12 Vgl. Davidson 2018, S. 58; vgl. Ott 2015, S. 167.

${ }^{13} \mathrm{Vgl}$. Singer 2019a, S. 60f.

14 Vgl. Singer 2019a, S. 64.

15 Singer 2019a, S. 64

$16 \mathrm{Vgl}$. Singer 2019b, S. $86 f f$.

17 Das kostenlose E-Book Mitgefühl. In Alltag und Forschung von Tania Singer und Matthias Bolz beschreibt Trainingsprogramme, den aktuellen Stand der Wissenschaft sowie Erfahrungsberichte aus der Praxis: www.compassion-training.org/?lang=de.

18 Vgl. Singer 2019a, S. 58ff.; siehe auch Singer 2019b, S, 87ff.

19 Vgl. Singer 2019a, S. $63 f$.

20 Singer 2019a, S. 63.

21 Vgl. Davidson / Goleman 2017, S. 276.

$22 \mathrm{Vgl}$. Sedlmeier 2016, S. $168 \mathrm{f}$.

${ }^{23} \mathrm{Vgl}$. Sedlmeier 2016, S. 169f.; siehe auch Singer 2019a, S. $61 \mathrm{f}$.

${ }^{24} \mathrm{Vgl}$. Sedlmeier 2016, S. $74 \mathrm{ff}$.

$25 \mathrm{Vgl}$. Sedlmeier 2016, S. 80ff.

26 Vgl. Sedlmeier 2016, S. 172

27 Sedlmeier 2016, S. 103; siehe auch Siegel 2017, S. $76 f f$.

$28 \mathrm{Vgl}$. Ambo 2013, 1.04:10-1.04:43 Min.

29 Ambo 2013, 1.04:44-1.05:22 Min.

30 Lazar 2015, S. 79; siehe auch Hölzel 2015, S. 70.

31 Lazar 2015, S. 74

32 Vgl. Lazar 2015, S. 74

${ }^{33} \mathrm{Vgl}$. Davidson / Goleman 2017, S. 3ff.

${ }^{34} \mathrm{Vgl}$. Piron 2020, S. 27ff.

$35 \mathrm{Vgl}$. Piron 2020, S. 29.

36 Vgl. Piron 2020, S. $27 f f$.

37 Ott 2015, S. 17

$38 \mathrm{Vgl}$. Ott 2015, S. $169 \mathrm{ff}$.

39 Vgl. Ott 2015, S. 169f.; siehe auch Sedlmeier 2016, S. 104ff. und Singer / Ricard 2008, S. 67ff. und S. $116 f$.

40 Vgl. Sedlmeier 2016, S. 109; siehe auch Ott 2015, S. 170.

41 Sedlmeier 2016, S. 109.

42 Vgl. Lazar 2015, S. 73.

${ }^{43}$ Für eine Beschreibung der beiden Verfahren vgl. Ott 2015,

S. 175ff.; siehe auch Sedlmeier 2016, S. 107ff. 
${ }^{44}$ Gilman / Lestrade 2016, 49:12-49.52 Min.

${ }^{45} \mathrm{Vgl}$. Gilman/Lestrade 2016, 50:12-50:24 Min.

${ }^{46}$ Vgl. Lazar 2015, S. 71f.; siehe auch Lazar, Sara (2011). „How Meditation Can Reshape Our Brains: Sara Lazar at TEDxCambridge", www.youtube.com/watch?v=m8rRzTtP7Tc, 0:13-8:33 Min. (letzter Zugriff: 13.09.2020).

${ }^{47}$ Lazar 2015, S. 72.

${ }^{48}$ Vgl. Hölzel 2015, S. $43 f$

49 Das Buch Die Wissenschaft der Achtsamkeit von Yi-Yuan Tang erläutert bei den neurowissenschaftlichen Befunden auch die betroffenen spezifischen Hirnregionen. Ebenso enthalten ist ein Glossar mit einer Erläuterung vieler Gehirnregionen, siehe Tang 2019, S. 24ff. und S. 115ff.; für eine Übersicht, welche Gehirnveränderungen durch Achtsamkeits- und Meditationsübungen bislang wissenschaftlich nachweisbar sind, siehe auch Ott 2015, S. $178 f$.

50 Hölzel 2015, S. 68.

51 Ott 2015, S. $98 f$.

52 Hölzel 2015, S. 68.

${ }^{53}$ Vgl. Killingsworth, Matthew A. / Gilbert, Daniel T. (2010). A Wandering Mind Is an Unhappy Mind., www.danielgilbert.com/ KILLINGSWORTH\%20\&\%20GILBERT\%20(2010).pdf (letzter Zugriff: 13.09.2020).

${ }^{54} \mathrm{Vgl}$. Ott 2015, S. $99 \mathrm{ff}$.

55 Hölzel 2015, S. 60

56 Vgl. Hölzel 2015, S. 43f. und S. $67 f$.

57 Bei Singer / Ricard 2008, S. 66: „Blinzeln der Aufmerksamkeit”.

${ }^{58}$ Vgl. Singer / Ricard 2008, S. 66ff.; siehe auch Hölzel 2015,

S. 49f. sowie Ott 2015, S. 102.

59 Vgl. Singer / Ricard 2008, S. 66ff.; siehe auch Hölzel 2015,

S. 49f. sowie Ott 2015, S. 102

60 Zur Wahrnehmung von Gesichtsausdrücken siehe Vortrag „Die Universalität der Emotion“ von Paul Ekman, Professor der Psychologie und Direktors des Human Interaction Laboratory an der Medical School der University of California in Goleman 2003, S. 183ff.

${ }^{61} \mathrm{Vgl}$. Singer / Ricard 2008, S. $73 \mathrm{f}$.

62 Hölzel 2015, S. 46

${ }^{63}$ Hölzel 2015, S. 47; siehe auch Morel 2016, 4:07-5:38 Min.

${ }^{64} \mathrm{Ebd}$.

${ }^{65}$ Vgl. Hölzel 2015, S. $47 f$.

${ }^{66}$ Vgl. Hölzel 2015, S. $48 f$.

${ }^{67}$ Vgl. Lazar 2015, S. 75f.; siehe auch Hölzel 2015, S. 62f. und Ott 2015, S. 65.

${ }^{68} \mathrm{Ott} 2015$, S. 47

${ }^{69} \mathrm{Vgl}$. Ott 2015, S. 65.

70 Vgl. Ott 2015, S. 65ff.; siehe auch Sedlmeier 2016, S. $66 f$.

${ }^{71}$ Vgl. Hölzel 2015, S. $63 f$.

72 Vgl. Hölzel 2015, S. 51.

${ }^{73}$ Vgl. Hölzel 2015, S. 52.

${ }^{74} \mathrm{Vgl}$. Ambo 2013, 1.03:28-1.04:10 Min.

75 Vgl. Hölzel 2015, S. 53; siehe auch Lazar 2015, S. 74; Ott 2015,

S. 72. und S. 181.

76 Ambo 2013, 37:54-38:41 Min.

$77 \mathrm{Vgl}$. auch den Beitrag in Bayern 2 von der Moderatorin Birgit Magiera „Achtsamkeitsforschung - Frieden für die Welt oder Wellness fürs Ich?" am 05. März 2020, https://www.br.de/mediathek/podcast/ iq-wissenschaft-und-forschung/achtsamkeitsforschung-frieden-fuer-diewelt-oder-wellness-fuers-ich/1793281.

$78 \mathrm{Vgl}$. Ambo 2013, 37:22-37:54 Min.

79 Vgl. Hölzel 2015, S. 58f.; siehe auch Lazar 2015, S. 77f. sowie Ott 2015, S. 71f. und S. 178
80 Vgl. Ambo 2013, 12:25-14:15 Min.

${ }^{81}$ Siehe Singer / Ricard 2015.

82 Vgl. Davidson / Begley 2012, S. 343ff.; vgl. Sedlmeier 2016, S. $67 f$.

${ }^{83}$ Vgl. Davidson / Begley 2012, S. 345.

$84 \mathrm{Vgl}$. Ambo 2013, 12:25-14:15 Min.

${ }^{85}$ Vgl. Gilman / Lestrade 2016; vgl. auch Ricard 2018a, S. 224ff. sowie Vortrag und Podiumsdiskussion „We can change the brain, by changing the mind" am 19. März 2019a, Prof. Dr. Richard Davidson in München beim Forum für den Wandel der Edith-HaberlandWagner Stiftung, www.ehw-stiftung.de (letzter Zugriff: 13.09.2020).

86 Center for Healthy Minds (2017). Kindness Curriculum. https://centerhealthyminds.org/science/studies/kindness-curriculum-studywith-pre-kindergarten-students (letzter Zugriff: 13.09.2020).

87 Davidson 2019c, S. 50.

${ }^{88}$ Vgl. Davidson 2019c, S. 50; siehe auch Gilman / Lestrade 2016, 52:25-56:08 Min

${ }^{89} \mathrm{Vgl}$. Kabat-Zinn 2019, S. 67ff.

90 Vgl. Hölzel 2015, S. 58f.

91 Vgl. Kabat-Zinn 2019, S. 221.

92 Vgl. Blackburn / Epel 2017, S. 27 und S. 190ff.; siehe auch Kabat-Zinn 2019, S. 221 sowie Davidson / Goleman 2017, S. 177. 

AUFBAU J

(

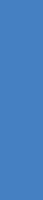

AUFBAU 
Seite
57 Das Münchner Modell
„Achtsamkeit und Meditation
im Hochschulkontext"

Seite

69 Abschlussarbeiten im Rahmen des

Münchner Modells

Seite
$74 \quad$ Veranstaltungen im Rahmen der Vortrags- und Filmreihe „Spirituelle Lehrer und ihre Unterweisungen" 


\section{Das Münchner Modell „Achtsamkeit und Meditation im Hochschulkontext"}

Auf den folgenden Seiten sollen die verschiedenen Angebote, die Entwicklung und Implementierung sowie die bisherigen Ergebnisse des Münchner Modells vorgestellt werden. Dabei werden auch die Teilnahmebedingungen, Leistungskriterien und Lehrinhalte der Lehrveranstaltungen zum Thema „Achtsamkeit und Meditation" beleuchtet. Insbesondere die regulären Lehrveranstaltungsangebote haben das Modell national und auch international bekannt gemacht, da Lehrveranstaltungen dieser Art als fester Bestandteil des Curriculums inklusive Notenvergabe und ECTS ${ }^{2}$ an Hochschulen bislang kaum anzutreffen sind.

\section{Reguläre Lehrveranstaltungen "Achtsamkeit und Meditation"}

Im SoSe 2010 habe ich zum ersten Mal an der Fakultät für Angewandte Sozialwissenschaften der Hochschule München eine Lehrveranstaltung zum Thema „Meditation“ angeboten. Auf 15 Plätze bewarben sich im Studiengang Soziale Arbeit 65 Studierende. Das war der Anfang des Münchner Modells. Als die Lehrveranstaltung im Schwerpunktbereich Kreative Medien/1. Fachsemester Mitte Juli 2010 beendet war, hatten 14 Teilnehmerinnen und Teilnehmer Interesse an einer Fortsetzung des Angebots im darauffolgenden Semester. Die Teilnehmeranzahl würde für eine neue Lehrveranstaltung reichen. Darüber hinaus gab es noch 50 Studierende auf der Warteliste.

Leider konnte aber kurzfristig für das 2. Fachsemester keine weitere Lehrveranstaltung in den Lehrplan aufgenommen werden. Stattdessen bestand die Möglichkeit, eine weitere Lehrveranstaltung zum Thema „Meditation“ im WiSe 2010/11 im Rahmen des Hauptstudiums zu implementieren. Diese Lehrveranstaltung mit dem Titel „Meditation II“ im Schwerpunktbereich Kreative Medien/Vertiefung/5. Fachsemester behandelte neben Praxisübungen im Vergleich zum Angebot im 1. Semester vor allem eine größere Reihe an Forschungsthemen sowie Handlungsfeldern der Sozialen Arbeit, in denen Achtsamkeit und Meditation bereits Anwendung finden. Im WiSe 2011/12 folgte erstmals eine Veranstaltung an der Ludwig-Maximilians-Universität (LMU) München ${ }^{3}$ mit dem Titel „Meditation in der Grundschule“ im Studiengang Grundschulpädagogik.

Im Semester darauf, SoSe 2012, folgte im Studiengang Schulpädagogik die Lehrveranstaltung „Meditation in der Schule“. Beide Lehrveranstaltungen waren auch für Studierende des Studiengangs Sonderpädagogik zugänglich. Im WiSe 2013/14 entstand an der Hochschule München ein weiteres Angebot, an der Fakultät Studium Generale und Interdisziplinäre Studien im Schwerpunktbereich „Personenbezogene Kompetenz“, zunächst unter dem Titel „Stressbewältigung und Stressprävention" und ab dem WiSe 2014/15 mit der Bezeichnung "Stressmanagement und Meditation“" An dieser Fakultät werden sogenannte Wahlpflichtfächer angeboten, welche Studierende aller Fakultäten belegen können. Die Resonanz bei den Studierenden war von Anfang an groß. Auf 15 Plätze bewarben sich weit über 100 Studierende. Im SoSe 2014 folgte ein zusätzliches Angebot. Mit zunehmender Bekanntheit der beiden Lehrveranstaltungen unter den Studierenden stieg auch die Bewerberzahl; nach einigen Semestern waren es bereits über 700 Bewerberinnen und Bewerber auf jeweils 15 Plätze.

Im Teilzeitstudium Soziale Arbeit wurde im SoSe 2014 eine Lehrveranstaltung „Meditation“ für das 2. Fachsemester eingeführt, ebenso im dualen Studiengang Pflege, hier im Schwerpunktbereich „Einübung in die Körperwahrnehmung“. Im WiSe 2014/15 wurde im Studiengang Musikpädagogik an der Fakultät für Kunstwissenschaften (LMU München) die Lehrveranstaltung „Klassische Musik ganzheitlich vermitteln“ im Lehrplan angeboten. Mittelpunkt der Veranstaltung bildet ein Ansatz, der vollständig auf Musikmeditation basiert. Die Veranstaltung ist im Münchner Modell Teil des Randschwerpunktes: „Meditation and Art“.

Im WiSe 2015/16 folgte im Studiengang Bildung und Erziehung im Kindesalter im Schwerpunktbereich "Methoden der Stressbewältigung" die Lehrveranstaltung „Achtsamkeit und Meditation“. Dies war eine 
wichtige Ergänzung zu den bereits vorhandenen Studiengängen im pädagogischen Bereich, da diese Studienrichtung sich auf Kinder von 0-12 Jahre bezieht, und so konnten auch die Studierenden, die sich mit Vorschulkindern befassen, in das Münchner Modell einbezogen werden.

Ab dem WiSe 2017/18 startete im Studiengang Soziale Arbeit die vertiefende Lehrveranstaltung „Meditation III" im Hauptstudium. Ebenso wurde das Angebot an der Fakultät Studium Generale und Interdisziplinäre Studien durch die auf Englisch abgehaltene Lehrveranstaltung „Stress Management and Meditation“ ergänzt.

Zum Randschwerpunkt „Meditation and Art“ wurden im gleichen Semester an der Hochschule München weitere Lehrbausteine, hier aber zum Thema „Malerei der alten Meister" ${ }^{\text {"5 }}$ im Studiengang Soziale Arbeit sowie im Studiengang Bildung und Erziehung im Kindesalter integriert. Dieses Angebot wurde ab dem SoSe 2018 zusätzlich mit einer Lehrveranstaltung im Bereich „Courses in English“ ergänzt und bietet insbesondere internationalen Studierenden der Hochschule München die Möglichkeit, den Ansatz „Meditation and Art" kennenzulernen.

Das Lehrveranstaltungsprogramm hat sich im Zeitfenster SoSe 2010 bis SoSe 2020 fortwährend erweitert; mittlerweile nehmen pro Semester über 150 Studierende daran teil. Die Teilnehmerinnen und Teilnehmer kommen bislang aus folgenden Fakultäten und Studiengängen:

\section{Hochschule München}

Fakultät für Angewandte Sozialwissenschaften

- BA Soziale Arbeit

- BA Soziale Arbeit Teilzeit

- BA Bildung und Erziehung im Kindesalter

- BA Pflege

Fakultät für Studium Generale und

Interdisziplinäre Studien

- Fakultät für Architektur

- Fakultät für Bauingenieurwesen

- Fakultät für Maschinenbau, Fahrzeugtechnik, Flugzeugtechnik

- Fakultät für Elektrotechnik und Informationstechnik

- Fakultät für Versorgungs- und Gebäudetechnik, Verfahrenstechnik Papier und Verpackung,

Druck- und Medientechnik
- Fakultät für Angewandte Naturwissenschaften und Mechatronik

- Fakultät für Informatik und Mathematik

- Fakultät für Geoinformation

- Fakultät für Wirtschaftsingenieurswesen

- Fakultät für Betriebswirtschaft

- Fakultät für Angewandte Sozialwissenschaften

- Fakultät für Design

- Fakultät für Tourismus

\section{Ludwig-Maximilians-Universität (LMU) München}

Fakultät für Psychologie und Pädagogik

- Lehramt für Grundschulpädagogik und -didaktik

- Lehramt für Schulpädagogik

- Lehramt für Sonderpädagogik

Auf der Website des Münchner Modells www.hm.edu/ meditationsmodell sind die verschiedenen Angebote erfasst. Ebenso angegeben sind die Semesterwochenstunden, die ECTS, das Fachsemester, die Bewerber- und Teilnehmerzahlen, die Fachspezifizierung, der Implementierungsbeginn sowie der Leistungsnachweis.

\section{Teilnahmebedingungen}

Voraussetzung für die Teilnahme an den Lehrveranstaltungen und Meditationszusatzangeboten im Rahmen des Münchner Modells ist eine stabile psychische Verfassung. Vorkenntnisse in Meditation, Stressmanagement oder Entspannungstechniken sind nicht erforderlich. Einzig für die Vertiefungslehrveranstaltungen "Meditation II" und „Meditation III“ im Studiengang Soziale Arbeit werden Vorkenntnisse im Bereich Achtsamkeit und Meditation vorausgesetzt. Diese können nachgewiesen werden durch die bestandene Teilnahme an der Basislehrveranstaltung „Meditation I“ oder anhand von absolvierten externen Meditationsangeboten. Für fast alle Lehrveranstaltungen besteht ein Online-Anmeldeverfahren. Auf www.hm.edu/meditationsmodell ist nachzulesen, wie viele Bewerber auf die verfügbaren Plätze pro Lehrveranstaltung kommen und wie viele einen Platz erhalten. 


\section{Lehrveranstaltungsinhalte, Leistungsnachweise und Benotung}

Im Praxisteil der Lehrveranstaltungen lernen die Studierenden verschiedene Achtsamkeits- und Meditationsübungen. Theoretische Bausteine der Lehrveranstaltung sind insbesondere die wissenschaftliche Forschung, Hintergrundwissen über die verschiedenen Meditationsansätze und Anwendungsmöglichkeiten von Achtsamkeit und Meditation in den späteren Berufsfeldern.

\section{Ablauf}

In der Regel dauern die Lehrveranstaltungseinheiten zwei Semesterwochenstunden (SWS), also insgesamt 90 Minuten ( 2 x 45 Minuten). Wir beginnen mit einer Sitzmeditation, die beispielsweise die Fokussierung auf den Atem, auf den Bereich zwischen den Augenbrauen oder das innerliche Rezitieren eines Mantras (Wort, Vers oder Silbe) beinhaltet. Zu Beginn des Semesters dauert diese Übung einige wenige Minuten, im Laufe des Semesters wird sie dann bis auf circa 15-20 Minuten ausgedehnt. Im Anschluss daran folgt eine kurze Feedbackrunde: Die Studierenden berichten, wie sie die Meditation erfahren haben: Was ging gut und wo gab es Schwierigkeiten?

Im weiteren Unterrichtsverlauf wird je Lehrveranstaltungseinheit und abhängig vom Studiengang ein spezifisches Thema behandelt. Das können teils praktische Übungen und teils theoretische Bausteine sein. Am Ende der Lehrveranstaltung folgt nochmals eine kurze Sitzmeditation. Ich benutze eine Klangschale, um die praktischen Übungen anzuleiten. Einmal schlagen: Aufmerksamkeit zurückholen, falls man abschweift; dreimal schlagen: die Übung ist zu Ende.

Im Studiengang Soziale Arbeit ist die Dauer der Meditationslehrveranstaltungen auf insgesamt drei SWS ausgelegt. Aus diesem Grund haben die Teilnehmerinnen und Teilnehmer zusätzlich einen Achtsamkeitstag (Blockveranstaltung). Neben theoretischen Unterrichtsinhalten gehört bei dieser Veranstaltung beispielsweise auch dazu, schweigend und achtsam zu kochen und zu essen.

\section{Lehrinhalte}

Im Rahmen der Lehrveranstaltungen wurden die folgenden Lehrinhalte vermittelt. Für eine ausführliche Beschreibung der praktischen Übungen siehe S. 83ff. und S. 101ff.

\section{Praktische Übungen (Auswahl)}

- Achtsamkeits- und Atemübungen

- Mantra-Meditation

- Achtsames Kochen und Essen

- Achtsame Kommunikation

- Bodyscan

- Liebende Güte / Metta-Meditation

- Gehmeditation

- Sutras

- Geräuschmeditation

\section{Theoretische Bausteine}

- Achtsamkeits- und Meditationsforschung (insbesondere Neurowissenschaft)

- Anwendungsbereiche von Achtsamkeit und Meditation (spezifiziert je nach Studiengang)

- Hintergrundwissen über Achtsamkeits- und Meditationsansätze (beispielsweise: Vipassana, MBSR, Christliche Meditation, Zen-Meditation, Transzendentale Meditation, Transmissionsmeditation etc.)

- Spirituelle Lehrer und ihre Unterweisungen

\section{Sonstige Themen (Auswahl)}

- Gedanken und Emotionen

- Gesundheit und Stress

- Glück

- Religion und Spiritualität

- Zeit (Vergangenheit, Gegenwart/Augenblick, Zukunft) 


\section{Filmbeispiele (Auswahl)}

- Mönche im Labor

- Revolution der Selbstlosen

- Free the Mind

- The Dhamma Brothers

- InnSaei - die Kraft der Intuition

- Zeit für Stille

Weitere Themen- und Filmbeispiele siehe Abschnitt „Zusatzangebote (Vortrags- und Filmprogramm)“(S. 62 und S. 74f.). Für Literaturhinweise zu den verschiedenen Lehrinhalten: www.hm.edu/meditationsmodell (Rubrik „Literatur und Filme“).

\section{Kompetenzerwerb}

Die in den Lehrplänen angegebenen Hauptziele:

- Förderung von Selbst-Gewahrsein

- Bewusstwerden der eigenen Gedankenaktivitäten

- Erhöhung der Konzentration und Aufmerksamkeit

- Stärkung von Selbstvertrauen, Selbstwirksamkeit, Wohlbefinden und Emotionsregulation

- Konstruktiver Umgang mit Stress

- Förderung von Kreativität und Intuition

Im Abschnitt „Bisherige Ergebnisse“ wird auf die Effekte der Lehrveranstaltungseinheiten eingegangen (S. 64ff.).

\section{Leistungsnachweis und Benotung}

Benotet wird die erbrachte Leistung aufgrund von Präsenz, regelmäßiger Mitarbeit, dem Führen eines persönlichen Meditationstagebuchs und einer schriftlichen Reflexion darüber, wie Achtsamkeit und Meditation im späteren beruflichen Handlungsfeld sinnvoll angeboten bzw. realisiert werden können.

Die Studierenden sind angehalten, die im Unterricht vorgestellten Übungen zu Hause zu praktizieren. Im Meditationstagebuch werden die entsprechenden Erfahrungen festgehalten. Notiert wird dabei immer das jeweilige Setting:
- Was: welche Übung

- Wann: Tag/Uhrzeit

- Wo: zu Hause, im Park, im Zug, an der Bushaltestelle etc.

- Sonstiges: eventuell ergänzende Materialien (zum Beispiel Timer/Handy-App, Sitzkissen)

- Dauer: flexibel

- Persönliches Befinden vorher/nachher

Ziel der Tagebücher ist vor allem, dass die Teilnehmerinnen und Teilnehmer mehr Einsicht in die eigene Meditationspraxis erlangen: Wann und wo meditiere ich gut? Welche Uhrzeit passt mir am besten? Warum? Welche Übung ist wann geeignet? Es ist nicht notwendig, dass die verschiedenen Übungen zu Hause gleich oft ausgeführt werden. Meistens kristallisieren sich einige Übungen heraus, die man am liebsten mag und mit denen man sich am wohlsten fühlt. Ebenso geht es nicht darum, so lange wie möglich eine Übung zu wiederholen. Wichtig ist, nicht zu übertreiben und den zeitlichen Rahmen nicht zu überspannen. $\mathrm{Zu}$ viel des Guten ist nicht unbedingt immer zielführend. Empfehlenswerte Sitzmeditationszeiten: 15-20 Minuten, 1- bis 2-mal täglich.

Zur Mitte des Semesters hin werden alle Meditationstagebücher eingereicht. Ich sehe sie dann durch und habe auf diese Weise die Möglichkeit, nochmals zu prüfen, wie es den Studierenden bei den Übungen geht und ob sie diese richtig durchführen; falls erforderlich, werden Korrekturen im Unterricht besprochen. Die in den Meditationstagebüchern enthaltenen Informationen werden selbstverständlich vertraulich behandelt.

Nach der Einführung im Unterricht sind die Übungen für die Studierenden zu Hause gut durchführbar. Es gibt aber zudem Meditationsformen, die ausschließlich über einen längeren Zeitraum unter Anleitung eines erfahrenen Lehrers oder einer Lehrerin praktiziert werden sollten. Studierenden, die sich solche Formen auswählen, wird empfohlen, sich mit seriösen Meditationsschulen in Verbindung zu setzen.

Im Studiengang Bildung und Erziehung im Kindesalter findet neben dem oben beschriebenen Leistungsnachweis zusätzlich noch eine 20-minütige mündliche Prüfung statt. Diese besteht in einem ersten Teil aus einer kurzen Präsentation eines selbstständig erarbeiteten Praxisbeispiels, z.B. wie Achtsamkeit mit Kindern in Kindertagesstätten durchführbar ist. In einem zweiten Teil wird das Thema Achtsamkeit im Allgemeinen sowie Achtsamkeits- und Meditationsforschung im Besonderen geprüft. 


\section{Zusatzangebote \\ im Rahmen des \\ Münchner Modells}

Fünf wichtige ergänzende Bereiche sind bislang im Münchner Modell verankert:

- Zusatzangebote für Studierende

- Zusatzangebote für Hochschulbeschäftigte

- Zusatzangebote für Lehrpersonal

- Netzwerktreffen

- Öffentliches Vortrags- und Filmprogramm

Für aktuelle Informationen zu den Zusatzangeboten: www.hm.edu/meditationsmodell (Rubrik „Angebote“).

\section{Zusatzangebote für Studierende}

\section{Seminare und Workshops}

Zusätzlich zu den regulären Lehrveranstaltungen an der LMU München werden seit dem WiSe 2013/14 pro Semester weitere vier- sowie achtstündige Workshops mit dem Titel „Achtsamkeit und Meditation in der Schule“ am Münchener Zentrum für Lehrerbildung (MZL) der LMU München angeboten. Ab dem WiSe 2015/16 und in den darauffolgenden WiSe bis einschließlich 2018/19 wurde an der Fakultät Psychologie der dreistündige Workshop „Achtsamkeit und Meditation in der Beratung" angeboten. Im WiSe 2020/21 soll die Kooperation mit neuen Angeboten fortgesetzt werden.

\section{Meditationsprojekt für (ehemalige) Studierende}

Seit dem WiSe 2016/17 besteht ein Meditationsangebot für (ehemalige) Studierende der Hochschule München, LMU München, TU München, Akademie der Bildenden Künste München sowie der Hochschule für Musik und Theater München. Für die Teilnahme sind keine Vorkenntnisse erforderlich, eine stabile psychische Verfassung ist aber Voraussetzung. Die Meditation wird dienstags von 17.15 bis 18.15 Uhr angeboten. Eine kurze Einführung findet jeweils vorab um $17 \mathrm{Uhr}$ statt. Veranstaltungsort ist der "Raum der Stille“ an der Hochschule München.

\section{Veranstaltung „Gelassen in die Prüfung - Entspannungstechniken vor Prüfungssituationen"}

In Zusammenarbeit mit der Studienberatung der Fakultät für Angewandte Sozialwissenschaften werden seit dem SoSe 2019 Workshops zum Thema Prüfungsangst durchgeführt.

\section{Coaching-Seminare und -Retreats}

Ab dem SoSe 2020 werden semesterweise CoachingSeminare und Coaching-Retreats für (ehemalige) Studierende angeboten, die im Rahmen des Studiums bzw. in ihren Berufsfeldern Achtsamkeits- und Meditationsprojekte durchführen.

\section{Teilnahme an „The Toolbox is You"-Retreats}

Für (ehemalige) Studierende besteht zudem die Möglichkeit, an „The Toolbox is You“-Retreats unter Leitung von der Achtsamkeitstrainerin und Körpertherapeutin Maria Kluge im Achtsamkeitszentrum Osterloh teilzunehmen (www.achtsamkeit-osterloh.org).

\section{Zusatzangebote für Hochschulbeschäftigte}

Als Angehörige des Hochschulapparats erfuhren, dass es ein Meditationsangebot für Studierende gibt, wurde der Wunsch geäußert, etwas Ähnliches auch für Hochschulbeschäftigte zu haben. Im SoSe 2012 gab es erstmals eine Meditations-Schnupperstunde im Rahmen des Tags der Gesundheit an der Hochschule München. Im Anschluss daran fragten die 15 Teilnehmenden, ob es auch einen Wochenkurs geben könnte - woraufhin 10 Einheiten freitags von 7.30 bis $8.30 \mathrm{Uhr}$ angeboten wurden. Der Aufbau war damals: 20 Minuten Meditation, 30 Minuten Vertiefung eines Themas und anschließend nochmals 10 Minuten Meditation. Nach dem Kurs haben 14 Teilnehmerinnen und Teilnehmer dieses Angebot zwei Jahre fortgesetzt. Anschließend wurde das Angebot auf donnerstags von 8.00 bis 8.30 Uhr gelegt und von den Hochschulbeschäftigten selbst weitergeführt. Am Hochschulstandort Campus Pasing ist ab dem SoSe 2020 ein Angebot geplant. 


\section{Zusatzangebote für Lehrpersonal}

Im WiSe 2017/18 sind im Rahmen des Programms auch Workshops für Lehrende zum Thema „Achtsamkeit und Meditation in der Lehre" eingeführt worden. Hier geht es neben der Theorie und den Übungen verstärkt um die Forschung zu Achtsamkeit und Meditation sowie um die Anwendungsmöglichkeiten im jeweiligen Fach. Im Mittelpunkt steht die Praxis, damit die Lehrenden selber Erfahrungen machen und das Gelernte anschließend authentisch vermitteln können. Während man für die Vermittlung von Meditationstechniken, insbesondere die der inneren Versenkung, eine lange und fundierte Meditationserfahrung benötigt, ist für eine Reihe von einfachen, aber bereits wirksamen Achtsamkeitsübungen nicht viel Vorerfahrung erforderlich (siehe S. 87 und S. 169f.). Das macht kurze Anwendungen im regulären Unterricht so vielversprechend. Rückmeldungen von Dozentinnen und Dozenten zeigen, dass sich die Gesamtatmosphäre des Unterrichts verändert, vor allem entspannter wird. Neben dem Lehrstoff werden den Studierenden jetzt auch Tools vermittelt, die das SelbstGewahrsein betreffen.

Im WiSe 2019/20 wurde mit dem Zentrum für Hochschuldidaktik (DiZ) in Ingolstadt eine Vereinbarung getroffen, Seminare zum Thema „Achtsamkeit und Meditation in der Lehre" in das DiZ-Kursprogramm aufzunehmen. Im SoSe 2020 sollen die ersten zweitägigen Seminare stattfinden und bei entsprechend positiver Resonanz semesterweise angeboten werden. Auch ist in diesem Fall der Aufbau einer Arbeitsgruppe anvisiert.

\section{Netzwerktreffen}

Im Rahmen des Münchner Modells finden seit dem SoSe 2017 Netzwerktreffen statt. Diese sind vor allem für Studierende und ehemalige Studierende gedacht, die an den Meditationslehrveranstaltungen des Münchner Modells teilnehmen bzw. teilgenommen haben. Ebenso können Personen, die im Bildungsbereich tätig sind und Interesse am Thema haben, nach Voranmeldung teilnehmen. Im Vordergrund steht der Austausch von Erfahrungen über eigenständig durchgeführte Achtsamkeits- und Meditationsprojekte (beispielsweise im Rahmen von Praktika und Abschlussarbeiten) und über neue Erkenntnisse aus der Achtsamkeits- und Meditationsforschung. Die Treffen dauern maximal zwei Stunden und sind wie folgt strukturiert: 20 Minuten gemeinsame Meditation, 30 Minuten Vorstellung aktueller
Entwicklungen (u.a. „Neues aus der Wissenschaft“, kurze Projektvorstellungen, Literaturtipps/Internetlinks). Anschließend Austausch/Diskussion. Die Treffen finden mehrmals pro Semester statt. Auf www.hm.edu/meditationsmodell werden die Termine bekannt gegeben.

\section{Vortrags- und Filmprogramm}

Seit dem WiSe 2014/15 finden im Rahmen des Münchner Modells Vortrags- und Filmabende zum Thema „Spirituelle Lehrer und ihre Unterweisungen“ statt. Die ursprüngliche Idee war, den Teilnehmerinnen und Teilnehmern über die Lehrveranstaltungen mehr Fachwissen über die verschiedenen Meditationsansätze, über Hintergründe, spirituelle Lehrer und auch über die Ansätze in der Neurowissenschaft zu vermitteln. Daraus hat sich - aufgrund des großen Interesses - eine öffentliche und kostenlose Veranstaltungsreihe entwickelt. Auf www.hm.edu/meditationsmodell werden die Veranstaltungen und Themenschwerpunkte des Programms angekündigt; der aktuelle Programmflyer steht als PDF-Download zur Verfügung. Für eine Übersicht über die bisher behandelten Themen siehe Vortragsund Filmreihe „Spirituelle Lehrer und ihre Unterweisungen" (S. 74f.).

\section{Infrastruktur}

\section{Räume}

Die Lehrveranstaltungen an der Hochschule München finden größtenteils in den Räumen der Katholischen und Evangelischen Hochschulgemeinde Paoso statt. Nur die Fakultät Studium Generale und Interdisziplinäre Studien wird an einem anderen Ort, im „Raum der Stille“ der Fakultät für Elektrotechnik, durchgeführt. An der LMU München werden die praktischen Übungen an der Katholischen Hochschulgemeinde (KHG) angeboten und die theoretischen Bausteine der Lehrveranstaltungen in den regulären Unterrichtsräumen der LMU München.

Workshops zum Thema „Gelassen in die Prüfung“ werden in Unterrichtsräumen der Hochschule München durchgeführt. Das Zusatzprojekt für Hochschulbeschäftigte findet im „Raum der Stille“ der Fakultät für Elektrotechnik statt. Zusatzangebote für Lehrpersonal werden vor allem im Zentrum für Hochschuldidaktik (DiZ) durchgeführt. 
Netzwerktreffen werden im Paoso bzw. der KHG angeboten. Die öffentliche Film- und Vortragsreihe findet im Hörsaal „Roter Würfel“ der Fakultät für Informatik und Mathematik der Hochschule München statt. Einige Veranstaltungen wurden an der Fakultät für Design durchgeführt.

\section{Materialien}

Für die Meditationslehrveranstaltungen wurden Kissen, Matten, Decken sowie passende Schränke für die Lagerung angeschafft. Die Finanzierung erfolgte zunächst aus den damaligen Studienbeitragsgeldern, und als diese abgeschafft wurden, aus Zusatzmitteln. Dafür wurden entsprechende Anträge eingereicht. ${ }^{6}$

\section{Fachliteratur und Filme}

Im Rahmen des Münchner Modells befindet sich ein umfangreiches Buch- und Filmsortiment in der Bibliothek am Campus Pasing der Hochschule München. Zugriff darauf hat man auch von anderen Zweigstellen der Hochschule aus sowie deutschlandweit von anderen Hochschulen. Darüber hinaus befindet sich am Campus Pasing ein ausführlicher Handapparat mit einem festen Bestand an Büchern. Alle Bücher und Filme haben neben der regulären Bibliothekscodierung noch eine zusätzliche, und zwar die Abkürzung „MZ“, die für „Meditationszentrum“ steht. Anhand dieser Codierung können auf der Internetseite der Hochschulbibliothek über das Feld „Freie Suche" alle Werke rund um das Thema „Achtsamkeit und Meditation", die an der Hochschule München vorhanden sind, aufgerufen werden. Unter der Rubrik „Treffer eingrenzen“ können zudem die verschiedenen Erscheinungsformen, wie zum Beispiel Druckschrift oder Video (DVD), ausgewählt werden.

Das Sortiment besteht inzwischen aus über 700 Buchtiteln zum Thema „Achtsamkeit und Meditation“ sowie zahlreichen audiovisuellen Medien (CDs und DVDs). Von den meisten Titeln sind mehrere Exemplare vorhanden. Von den Büchern, die im Rahmen einiger Lehrveranstaltungen Primärliteratur sind, gibt es sogar jeweils 35 Exemplare. Diese können von den Kursteilnehmerinnen und Kursteilnehmern semesterweise ausgeliehen werden.

Auch an der LMU München wurde ein kleiner Bestand an Büchern zum Thema „Achtsamkeit und Meditation" aufgebaut. Die Bücher sind aber normal in der Bibliothek (Zweigstelle Pädagogik/Psychologie) registriert und nicht direkt an das Münchner Modell gekoppelt. Auf www.hm.edu/meditationsmodell sind in der Rubrik „Literatur und Filme“ ausführliche Literaturempfehlungen als PDF-Downloads verfügbar. Interessanterweise hat sich bislang gezeigt, dass neben den Studierenden der Hochschule München und LMU München auch Studierende anderer Hochschulen in Deutschland auf das Sortiment aufmerksam geworden sind und Bücher sowie Filme aus dem Münchner Modell über die Fernleihe bestellen.

\section{Kooperationspartner}

Neben den Kooperationen mit Paoso und der KHG, die seit dem WiSe 2010/11 bestehen, sind inzwischen zahlreiche weitere Kooperationen entstanden. Insbesondere mit Hochschulen, aber auch mit einigen Institutionen, die Meditationsrichtungen anbieten. Im Abschnitt „Bisherige Ergebnisse“ sind einige der Kooperationspartner beschrieben; weitere sind auf www.hm.edu/meditationsmodell angegeben.

\section{Kommunikation der Angebote}

Die Lehrveranstaltungen sind im Online-Vorlesungsverzeichnis der jeweiligen Fakultät aufgeführt. Die Fakultät Studium Generale und Interdisziplinäre Studien hat zudem ein gedrucktes Vorlesungsverzeichnis, in dem die Lehrveranstaltungen „Stressmanagement und Meditation“ (Deutsch) bzw. „Stress Management and Meditation" (Englisch) ausgeschrieben sind. Über diese Veranstaltungen wird ebenfalls in einem Flyer des Programms „Gesunde Hochschule “ informiert, in dem auch die Meditationszusatzangebote für Studierende aufgeführt sind.

Die öffentlichen und kostenlosen Vorträge und Filmvorführungen werden über die Veranstaltungskalender der Internetseiten der Hochschule München und LMU München sowie auf Flyern und Plakaten bekannt gemacht. Zudem existiert ein Verteiler mit Kontaktadressen von Interessentinnen und Interessenten. Alle Veranstaltungen stehen auch auf www.hm.edu/meditationsmodell (Rubrik „Vortrags- und Filmreihe“). 


\section{Bisherige Ergebnisse}

\section{Feedback von Studierenden}

Im März 2016 hat die Studentin Isabel Braunger im Rahmen ihres Studiums Integrative Gesundheitsförderung unter der Leitung von Professor Dr. Niko Kohls, Hochschule Coburg, die Teilnehmerinnen und Teilnehmer aus den Meditationslehrveranstal-

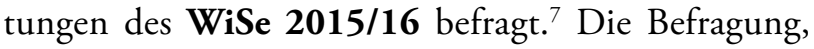
die mittels eines Online-Fragebogens durchgeführt wurde, zeigt unter anderem signifikante Unterschiede zwischen denjenigen Teilnehmenden, die bereits über Meditationserfahrung verfügen, im Vergleich zu „Neulingen“. Je gestresster eine Person sich fühlte, umso weniger achtsam und selbstwirksam konnte sie sich selbst erfahren. Je achtsamer sich eine Person empfand, umso selbstwirksamer erfuhr sie sich.

In der Studie wurde unter anderem auch gefragt, wie die teilnehmenden Studierenden zu der folgenden Aussage stehen: „Meditation im universitären und Hochschulkontext ist eine sinnvolle und gewinnbringende Bereicherung für Studierende und sollte daher vermehrt angeboten werden." - 86,1 Prozent der befragten Studierenden gaben hier an: „stimme ich voll zu“.

Weiteres Feedback über die Meditationslehrveranstaltungen kommt auch von Studierenden, die im Film Shanti - Meditation im Hochschulkontext am Beispiel des Münchner Modells von Katharina Nowack, Ramona Wegele und David Kutschi, zu Wort kommen (S. 186ff.). Die drei Studierenden des Studiengangs Soziale Arbeit haben im Rahmen ihrer Abschlussarbeit den Film fertiggestellt und ein Semester lang alle Kurse mit der Kamera begleitet.

Auch aus eigener Beobachtung in den zahlreichen Lehrveranstaltungen seit SoSe 2010 und dem Lesen von bislang circa 2000 Meditationstagebüchern à 2540 Seiten wird deutlich erkennbar, dass die Angebote Studierenden helfen, besser mit der Hektik der heutigen Zeit, mit unsicheren Zukunftsperspektiven, dem allgemeinen Leistungsdruck und der teils hohen Belastung im Studium umzugehen. Aus den Berichten und den Feedbackgesprächen im Unterricht ist auch zu entnehmen, dass insbesondere die Übungen der Liebenden Güte zu einem besseren zwischenmenschlichen Verständnis und zu mehr Gelassenheit bei den Studierenden führen. (Siehe diesbezüglich auch die Tagebuchnotizen zu den Übungen, S. 147ff., sowie die Fazits, S. 177ff.)

Als weiteres Ergebnis kann genannt werden, dass bereits über 75 Abschlussarbeiten zum Thema „Achtsam- keit und Meditation" erstellt wurden, unter anderem über die Anwendung und Durchführung eigener Achtsamkeits- und Meditationsprojekte. Da diese im Rahmen unterschiedlicher Studiengänge verfasst wurden, sind dabei auch viele unterschiedliche Anwendungsbereiche und Berufsfelder beleuchtet worden. Dabei ist die Anzahl der Studierenden, die ihre Abschlussarbeit über diese Themen schreiben möchten, in den letzten Semestern kontinuierlich gestiegen. Aufgrund dessen ist davon auszugehen, dass dies auch in nächster Zeit zu erwarten ist. Für eine Auswahl der bisher eingereichten Arbeiten siehe Übersicht „Abschlussarbeiten im Rahmen des Münchner Modells“ (S. 69ff.). Alle Abschlussarbeiten sind auf www.hm.edu/meditationsmodell (Rubrik „Abschlussarbeiten“) aufgeführt.

Nicht zuletzt ist zu nennen, dass einige ehemalige Kursteilnehmerinnen und Kursteilnehmer nach Abschluss ihres Studiums bereits selbst Lehrbeauftragte geworden sind und damit begonnen haben, Lehrveranstaltungen im Rahmen des Münchner Modells zu übernehmen. Siehe www.hm.edu/meditationsmodell (Rubrik „Angebote“).

\section{Kooperationen}

Mit einer Reihe von Kooperationspartnern ist ein dynamisches Netzwerk entstanden. Zu nennen sind in erster Linie die Kooperationspartner Paoso und die KHG, die ihre Räume für die Achtsamkeits- und Meditationsangebote zur Verfügung stellen (siehe Abschnitt „Infrastruktur“", S. 62f.).

\section{Hochschulen}

Im Folgenden folgt eine Auflistung wichtiger Kooperationspartner des Münchner Modells. Für eine Übersicht aller aktuellen Kooperationspartner samt Kontaktdaten siehe www.hm.edu/meditationsmodell.

Mit Drs. Anna Caspersen und Drs. Maaike Rijken der Fakultät Social Sciences and Law der Amsterdam University of Applied Sciences besteht seit 2016 eine enge Zusammenarbeit. Im Rahmen des ERASMUS-Angebots wird jedes Jahr im internationalen Minor-Programm Positive Psychology den Studierenden das Münchner Modell vorgestellt. Auch wird zusätzlich zu den Themen Meditationsforschung, Meditationstechniken sowie Anwendungsbereiche von Achtsamkeit und Meditation referiert. Zudem können jährlich zwei Studierende der Hochschule München an dem Studiengang teilnehmen und so von dieser Kooperation profitieren. 
Mit Prof. Dr. Niko Kohls der Hochschule Coburg besteht seit 2015 eine fruchtbare Zusammenarbeit, insbesondere beim Austausch über aktuelle Ergebnisse der Achtsamkeits- und Meditationsforschung und der gemeinsamen Betreuung von Abschlussarbeiten.

Eine Kooperation mit Dr. Susanne Krämer vom Zentrum für Lehrerbildung und Schulforschung der Universität Leipzig begann im Mai 2015 anlässlich eines Gastvortrags: „Möglichkeiten der Geistesschulung: Meditation im universitären Kontext? - Das Münchner Modell“. Hier ist auch erstmals der Name „Münchner Modell“" entstanden.

Auf Einladung vom Dr. Daniel Holt wurde im gleichen Monat das Münchner Modell in den Fachbereichen Psychologie und Medizin an der Universität Heidelberg vorgestellt. Inzwischen sind dort erste Lehrveranstaltungseinheiten durchgeführt worden.

Ein intensiver Austausch besteht seit 2016 mit der Ernst-Abbe-Hochschule Jena sowie mit der FriedrichSchiller-Universität Jena. Diese beiden Hochschulen haben unter Leitung von Prof. Dr. Mike Sandbothe und PD Dr. Reyk Albrecht gemeinsam mit vier weiteren Thüringer Hochschulen, dem Thüringer Wissenschaftsministerium und der AOK PLUS das Thüringer Modell „Achtsame Hochschulen“ konzipiert und erprobt. An der Weiterentwicklung dieses bislang einmaligen Programmangebots zur Implementierung von Achtsamkeitstrainings im Hochschulkontext sind derzeit Hochschulangehörige aus mehreren Bundesländern involviert. Im Rahmen des transdisziplinär evaluierten Modells, dessen Angebote für Studierende, Lehrende, Mitarbeitende und Führungskräfte an Hochschulen im Zeitraum 2015-2019 mehr als 10000 Teilnehmerinnen und Teilnehmer erreicht haben, konnte unter anderem die in Jena entwickelte Zertifikatsausbildung „Achtsame Hochschullehrende" etabliert werden.

An der im Rahmen des Thüringer Modells entwickelten überregionalen Kooperationsplattform Achtsame Hochschulen, https://achtsamehochschulen.de, nehmen bereits mehr als 300 Hochschulangehörige aus Deutschland, Österreich und der Schweiz teil.

Die Plattform erforscht die Wirkungskraft von Achtsamkeit und Meditation in einer sich wandelnden, digitalisierten Hochschullandschaft. Es soll geklärt werden, wie zielgruppenspezifische Achtsamkeitstrainings die Fähigkeit zu einem umsichtigen, zukunftsfähigen und sozial ausbalancierten Umgang mit disruptiven Transformationsprozessen (wie z.B. Digitalisierung, Globalisierung, Corona- und Klimakrise) verbessern und die Motivation zur Förderung salutogener Persönlichkeitsbildung an Hochschulen stärken können. Auf europäi- scher Ebene ist die Kooperationsplattform im Erasmus + Programm Training Embodied Critical Thinking (20202023) aktiv. Weitere Informationen unter: http://ect. hi.is/uncategorized/tect-training-embodied-criticalthinking-erasmus-funded-project-2020-2023/. ${ }^{8}$

Ebenso wurde 2016 an der Hochschule in Wien (FH Campus Wien) das Münchner Modell vorgestellt; und im gleichen Jahr hat eine erfolgreiche Zusammenarbeit mit Santino Güntert und Dr. Silke Vlecken der Züricher Hochschule für Angewandte Wissenschaften (ZHAW) begonnen.

Im Oktober 2016 begann ein fruchtbarer Austausch mit Dr. Reiner Frey von der Frankfurt University of Applied Sciences. Ein Jahr darauf, 2017, wurden hier die ersten Achtsamkeits- und Meditationsangebote zusammen mit Prof. Dr. Gerd Döben-Henisch implementiert. Mit Unterstützung des Präsidenten Prof. Dr. Frank E. P. Dievernich wurde 2018 der Kongress "Meditation und die Zukunft der Bildung" ausgetragen und im Jahr darauf ein weiterer Kongress unter dem Titel „Meditation und die Zukunft der Bildung 2019: Spiritualität und Wissenschaft“. Die Inhalte der Vorträge und Workshops wurden beide Male in Buchform veröffentlicht. ${ }^{9}$ Im Januar 2020 wurde an der Frankfurt University of Applied Sciences das Wissenschaftliche Zentrum für Persönlichkeitsbildung und gesellschaftliche Verantwortung (ZPG) gegründet, das drei Arbeitsbereiche umfasst, nämlich Persönlichkeitsbildung (durch Meditation und Selbstreflexion), gesellschaftliche Verantwortung (u.a. Social Learning) und Hochschuldidaktik. Diese drei Säulen sollen als integrierter Zusammenschluss in die Curricula einfließen und Basis für Forschungsaktivitäten sein.

Auch an der Hochschule Osnabrück hat das Präsidium das Vorhaben, Achtsamkeits- und Meditationsangebote zu implementieren, unterstützt. Auf Initiative des Präsidenten Prof. Dr. Andreas Bertram wurde zur Entwicklung, Implementierung und Koordinierung solcher Angebote bereits im Vorfeld eine Vollzeitstelle, „Mindful Leadership“ (Besetzung: Christiane Leiste), geschaffen. Die Kooperation mit der Hochschule Osnabrück begann 2018 mit einem Austauschtreffen in München. Kurz darauf erfolgte ein mehrtägiges Hospitieren in München durch Christiane Leiste, wo sie an einigen Lehrveranstaltungen des Münchner Modells teilnahm. Im Herbst des gleichen Jahres konnten die Durchführung eines öffentlichen Gastvortrags und verschiedener Workshops mit Studierenden und Hochschulpersonal in Osnabrück realisiert werden. Inzwischen wurde an der Hochschule Osnabrück ein umfangsreiches Programm zum Thema „Achtsamkeit und Meditation“ 
implementiert. Darunter eine Reihe von Achtsamkeitstrainings für Studierende und Lehrende sowie der in Jena bereits durchgeführte Zertifikatskurs „Achtsame Hochschullehrende“. Weitere Informationen unter: www.hs-osnabrueck.de/de/mindful-leadership.

Seit 2018 besteht auch eine Kooperation mit Dr. Nils Altner von der Universität Duisburg-Essen. Neben einem Austausch über Forschung sowie die Anwendung von Achtsamkeits- und Meditationsansätzen in Kindertagesstätten und Schulen gab es inzwischen auch eine gemeinsame Veröffentlichung zum Thema „Achtsamkeit in der Grundschule“.

Mit Kooperationspartner Dr. Ulrich Ott am Bender Institute of Neuroimaging der Justus-Liebig-Universität Gießen besteht ebenfalls eine Zusammenarbeit in Bezug auf Forschungsfragen, insbesondere bei der Betreuung von Studierenden, die Abschlussarbeiten schreiben.

Ebenfalls 2018 wurde eine Zusammenarbeit mit Dr. Karlheinz Vaitl vom Zentrum für Lehrer*innenbildung an der Universität in Wien ins Leben gerufen.

Die Kooperationspartner-Universitäten Leipzig und Tübingen haben das „(Forschungs-) Netzwerk Achtsamkeit in der Bildung" initiiert. Es ist eine Austauschplattform für Hochschulen, Schulen und andere Bildungsinstitutionen. Dabei geht es darum, wie Achtsamkeit in die jeweiligen Handlungsfelder integriert werden kann. Die Website www.netzwerk-achtsamkeit-in-der-bildung.de bietet einen Überblick der Projekte im deutschsprachigen Raum, die Kontaktpersonen sind an den Universitäten Leipzig und Tübingen verortet.

An den Universitäten Bayreuth, Würzburg, Tübingen, St. Gallen und Konstanz wurde eine erste Zusammenarbeit durch die Initiativen von Studierenden bewirkt.

\section{MBSR-MBCT-Verband}

Mit dem Vorsitzenden des MBSR-MBCT-Verbandes, Günter Hudasch, besteht eine fruchtbare Zusammenarbeit, insbesondere beim Austausch über aktuelle Ergebnisse der Achtsamkeits- und Meditationsforschung und die Anwendung von Achtsamkeit und Meditation in verschiedenen Handlungsfeldern.

\section{Fortbildungsorganisationen}

Im Zentrum für Hochschuldidaktik (DiZ) beginnen ab SoSe 2020 zweitägige Seminare zum Thema „Achtsamkeit und Meditation". Bei entsprechend positiver Resonanz ist es das Ziel, dieses Angebot regelmäßig semesterweise anzubieten und darüber hinaus eine Arbeitsgruppe aufzubauen.
In Zusammenarbeit mit dem Pädagogischen Institut in München finden Tagesseminare für Erzieherinnen/Erzieher und Lehrkräfte statt. Ein erstes Seminar zum Thema „Achtsamkeit für Lehrkräfte" wurde an der Akademie für Lehrerfortbildung und Personalführung in Dillingen (Bayern) durchgeführt.

Im Achtsamkeitszentrum in Osterloh können (ehemalige) Studierende „Toolbox is You“-Retreats wahrnehmen (S. 61).

\section{Schulen und Kindertagesstätten}

In München und Umgebung bestehen zahlreiche Kooperationen mit Schulen und Kindertagesstätten. Studierende führen hier Achtsamkeitsprojekte im Rahmen ihres Studiums durch. Darüber hinaus setzen sich zahlreiche Studierende auch nach Anschluss ihres Studiums mit Achtsamkeit und Meditation weiterhin auseinander, beispielsweise auch in ihren Berufsfeldern Schule und Kindertagestätten.

\section{Offene Kinder- und Jugendarbeit}

Nach einem ersten Kontakt mit dem Kreisjugendring München-Land im WiSe 2019/20 ist für das SoSe 2020 ein Praxisworkshop über die Anwendung von Achtsamkeits- und Meditationsansätzen in der offenen Kinder- und Jugendarbeit geplant. Anschließend sind entsprechende Seminare für das reguläre Ausbildungsprogramm anvisiert.

\section{Spirituelle Gruppen, Vereine und} Informationszentren

Insbesondere durch die Vortrags- und Filmreihe „Spirituelle Lehrer und ihre Unterweisungen", die im Rahmen des Münchner Modell stattfindet, besteht eine Zusammenarbeit mit einer Vielfalt an spirituellen Gruppen, Vereinen und Informationszentren, wie beispielsweise Thich Nhat Hanh, Krishnamurti, Mutter Meera und Sri Chinmoy. Für weitere Informationen und Links siehe www.hm.edu/meditationsmodell (Rubrik „Weiterführende Links“).

\section{Vorträge und Workshops}

Mit zahlreichen Gastvorträgen und Workshops an Hochschulen sowie auf Fachtagungen und Kongressen im In- und Ausland ist die Idee des Münchner Modells national und international bekannter geworden. Die vielen unterschiedlichen Zielgruppen, an welche die Veranstaltungen gerichtet waren, umfassen Sozialpädagoginnen/Sozialpädagogen, Jugend- 
arbeiterinnen/Jugendarbeiter, Erzieherinnen/Erzieher, Lehrkräfte, Hochschuldozentinnen/Hochschuldozenten, Achtsamkeitstrainerinnen/Achtsamkeitstrainer, Medizinerinnen/Mediziner, Künstlerinnen/Künstler, Führungskräfte in Unternehmen sowie Richterinnen/ Richter. Insbesondere zu erwähnen sind in diesem Zusammenhang die im Folgenden genannten Vorträge und Workshops:

- Im Juni 2017 wurde auf dem World Forum for Ethics in Business im Panelbereich „Continuing Education for Ethics in Innovations" das Münchner Modell unter dem Titel „Possibilities of Training the Mind: Meditation at University? The Munich Model" vorgestellt, bei der insbesondere auch auf die Bedeutung der Förderung von Intuition im Hochschulkontext diskutiert wurde.

- Im November 2017 konnte auf dem internationalen Symposium „Museum Guides Now!“ des Amsterdamer Rijksmuseums anhand eines Workshops und Gastvortrags der neue Ansatz „Meditation and Art" vorgestellt werden. Im gleichen Monat folgte in London auf dem internationalen World Goodwill Seminar „From the Unreal to the Real. Discerning Truth in Our Times" der Vortrag: „The Revelation of Meaning: Meditation and Higher Education“. Durch die Parallelübertragung zu zwei weiteren Standorten, Genf und New York, wurde ein internationales Publikum über das Münchner Modell informiert.

- Im März 2018 folgten ein Workshop und Vortrag „Possibilities of training the mind: Meditation at university? - The Munich Model“ im Rahmen des Symposiums "Studenten hebben dorst" in Delft, Niederlande. Im gleichen Monat wurde das Münchner Modell mit zwei Vorträgen auf den internationalen Konferenzen über Achtsamkeit und Meditation in Bern und Wien vorgestellt, wobei als Hauptredner der Neurowissenschaftler und Pionier der Achtsamkeits- und Meditationsforschung Richard Davidson auftrat.

- Vortrag: „The Munich Model brings Mindfulness and Meditation to University Students" auf der internationalen Konferenz „The Future of Education - Edition 8“ in Florenz im Juni 2018. Im Oktober 2018 Vortrag: „Vom Intellekt zur Intuition. Meditation im Hochschulkontext - das Münchner Modell“ sowie Workshop „Praxisbox - Meditation und Intuition“ auf dem Frankfurter Kongress „Meditation und die Zukunft der Bildung“.
- Auf Einladung von Studierenden der Stiftung des Deutschen Volkes fanden im April 2019 auf der Veranstaltung „Meditation von A bis Zen - theoretische Perspektiven einer kontemplativen Methode“ der Vortrag und Workshop „Vom Intellekt zur Intuition: Achtsamkeit und Meditation im Hochschulkontext - das Münchner Modell“ statt.

- Vortrag: „Meditation and Art - The Conscious Perception of the Great Works of Painting " auf der internationalen Konferenz „The 9th Edition of The Future of Education“ in Florenz im Juni 2019.

Alle Vorträge, Workshops und Seminare siehe www. hm.edu/meditationsmodell (Rubrik „Veranstaltungen“).

\section{Presseresonanz}

Das Münchner Modell hat bereits eine hohe Presseresonanz erfahren. Für alle in den Medien veröffentlichten Beiträge siehe www.hm.edu/meditationsmodell (Rubrik „Presse“).

\section{QUELLEN}

Braunger, Isabel (2016). Evaluation des Münchner Modells: Meditation an der Hochschule - ein zukunftsweisendes Konzept? Coburg / München.

de Bruin, Andreas (2019a). „Spiritualität im säkularen Raum am Beispiel des Münchner Modells - Achtsamkeit und Meditation im Hochschulkontext", in: Rötting, Martin / Hackbarth-Johnson, Christian (Hrsg.), Spiritualität der Zukunft. Suchbewegungen in einer multireligiösen Welt. Sankt Ottilien: EOS Editions, S. 341-350.

Dievernich, Frank, E. P. / Döben-Henisch, Gerd-Dietrich / Frey, Reiner (2019). Bildung 5.0: Wissenschaft, Hochschulen und Meditation. Das Selbstprojekt.

Weinheim / Basel: Beltz Juventa.

Frey, Reiner (Hrsg.) (2020). Meditation und die Zukunft der Bildung: Spiritualität und Wissenschaft. Weinheim / Basel: Beltz Juventa.

Kluge, Maria (2019). The Toolbox is You. 4. überarbeitete Auflage. Osterloh: Verein für Achtsamkeit in Osterloh e.V.

Sandbothe, Mike / Albrecht, Reyk (Hrsg.) (2021). Achtsame Hochschulen in der digitalen Gesellschaft. Bielefeld: transcript Verlag. 


\section{Zeitschrift}

de Bruin, Andreas (2019b). „Meditation and Art -

Große Werke der Malerei bewusst wahrnehmen“, in: Zeitschrift für Bewusstseinswissenschaften. Transpersonale Psychologie und Psychotherapie, 25. Jahrgang 1. Petersberg: Verlag Via Nova, S. 10-20.

de Bruin, Andreas (2017). „Möglichkeiten der Geistesschulung: Meditation im universitären Kontext? Das Münchener-Modell“, in: Zeitschrift für Bewusstseinswissenschaften. Transpersonale Psychologie und Psychotherapie, 23. Jahrgang 2. Petersberg: Verlag Via Nova, S. 68-84.

\section{Internet}

Kutschi, David / Nowack, Katharina / Wegele, Ramona (2017). Shanti - Meditation im Hochschulkontext am Beispiel des Münchner Modells, www.sw.hm.edu/die_fakultaet/personen/ professoren/bruin/muenchner_modell/film.de.html (letzter Zugriff 13.09.2020)
1 Der Text ist teils entnommen aus: de Bruin 2017 und de Bruin 2019a.

2 European Credit Transfer System: Arbeitsaufwand in Form von Leistungspunkten.

3 Im weiteren Verlauf des Textes: LMU München.

4 Ab dem Wintersemester 2014/15 wurde der Kurstitel geändert in „Stressmanagement und Meditation“. Größtenteils auf Bitten der Teilnehmer, da Stressmanagement im Unterschied zu Stressbewältigung mehr auf eine Kompetenz hinweist. „Meditation“ wurde als Begriff in den Titel mit aufgenommen, da die Veranstaltung fast ausschließlich Achtsamkeits- und Meditationsthemen behandelte.

5 Für eine Beschreibung des Ansatzes "Meditation and Art" siehe de Bruin 2019b.

6 Dem Paritätischen Ausschuss der Hochschule München ist an dieser Stelle ein Dank auszusprechen, da er das Münchner Modell von Anfang an finanziell unterstützt hat.

7 Für Details zur Befragung und weitere bisherige Ergebnisse im Rahmen des Münchner Modells siehe Braunger 2016.

8 Für eine Übersicht der bisherigen Entwicklungen siehe: Sandbothe, Mike / Albrecht, Reyk (Hrsg.) (2021). Achtsame Hochschulen in der digitalen Gesellschaft. Bielefeld: transcript Verlag.

9 Dievernich / Döben-Henisch / Frey (2019) und Frey (Hrsg.) (2020).

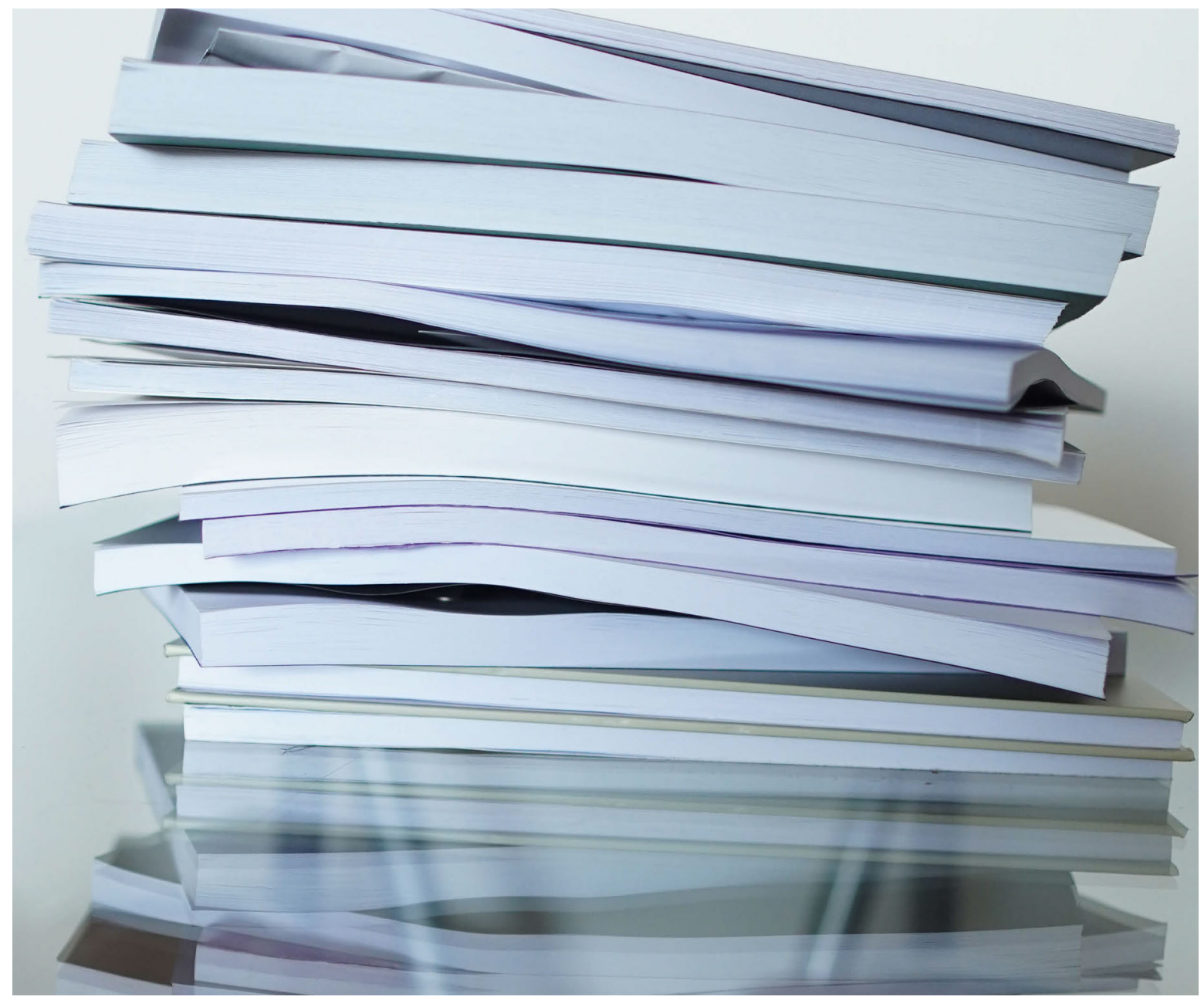




\section{Abschlussarbeiten im Rahmen des Münchner Modells}

Für eine Auswahl der bisher eingereichten Abschlussarbeiten siehe die folgende Übersicht. Alle weiteren Arbeiten sind auf www.hm.edu/meditationsmodell (Rubrik „Abschlussarbeiten“) aufgeführt. Im Wintersemester 2019/20 fand aufgrund eines Forschungssemesters keine Betreuung von Abschlussarbeiten statt.

\section{Bachelorarbeiten (Hochschule München)}

Böker, A. A. Achtsamkeit im Kindergarten. Theoretische und praktische Überlegungen am

(2019) Beispiel der Projektgruppe „Die achtsame Adlerbande“

Held, A. M. Achtsamkeit in Kindertageseinrichtungen

(2019) zur Stärkung personaler Resilienzfaktoren

Nöprick, L. Traumasensitives Yoga in der Sozialen Arbeit -

(2019) Chancen und Risiken

Kühl, B. Achtsamkeit mit Kindern

(2019) in der Grundschule

Strobl, I. Achtsamkeit im Kindergarten - Chancen, Risiken und

(2018) Implementierungsmöglichkeiten

Emberger, F. „Learning Emotion“ - Achtsamkeit und Meditation zur Förderung

(2018) emotionaler Kompetenzen am Beispiel der Schulsozialarbeit

Ernst, A. Meditation und Achtsamkeit bei Menschen mit psychischen Traumata? -

(2018) Grenzen und Möglichkeiten

Linner, M. Achtsamkeit in Kindertagesstätten - Chancen, Risiken und

(2018) Implementierungsmöglichkeiten

Völker, H. Das Erleben von Meditations- und Achtsamkeitsinterventionen in der

(2018) Schwangerschaft und ihre Wirksamkeit in Bezug auf pränatalen Stress.

Eine qualitative Studie

Skiebe, K. Das Konzept der Achtsamkeit

(2018) in Beratungsprozessen der Sozialen Arbeit

Lexhaller, C. Achtsamkeit in der Grundschule als Maßnahme zur Stressprävention

(2018) und Stressbewältigung 
Kutschi, D. Shanti - Meditation im Hochschulkontext

Nowack, K. am Beipiel des Münchner Modells

Wegele, R. (Film)

(2017)

Stoßberger, A. M. Achtsamkeit und Meditation mit Jugendlichen

(2017) am Beispiel des Jugendtreffs Cosimapark des Kreisjugendrings München-Stadt

Fischbacher, M. Achtsamkeit in der Behindertenhilfe. Ein Projekt mit geistig behinderten

(2017) und lernbehinderten jungen Frauen

Steer, A. L. Achtsamkeit und Meditation mit Kindern und Jugendlichen

(2017) am Beispiel der offenen Ganztagsschule der Mittelschule Augsburg Herrenbach

Wahlandt, P. Achtsamkeit und Meditation als Maßnahmen

(2017) zur Prävention und Behandlung von Burnout

Grimm, S. Auswirkungen von Meditations- und Achtsamkeitsübungen

(2017) auf das schulische Wohlbefinden von Kindern

Maier, J. „Ich raste gleich aus!“ Chancen und Implementierungsmöglichkeiten

(2017) von Achtsamkeit für verhaltensauffällige Kinder und Jugendliche

Hofmann, N. Achtsamkeit mit Jugendlichen im Rahmen der offenen Jugendarbeit

(2017) am Beispiel eines Kooperationsprojektes zwischen Jugendhaus „Come In“ und dem sonderpädagogischen Förderzentrum Weilheim

Gut, L. V. Soziale Arbeit und Stress.

(2017) Achtsamkeitsmeditation als Möglichkeit zur Stressreduktion

Keppeler, C. Meditation als Maßnahme zur Entwicklung eigener Gesundheitskompetenzen

(2017) am Beispiel von Gesundheits- und KrankenpflegerInnen in der stationären Pflege

Dukas, M. Meditation und Achtsamkeit in der Zentralen Notaufnahme.

(2017) Ein Konzept für Gesundheits- und KrankenpflegerInnen

Schuster, M. Entspannungs- und Achtsamkeitsmethoden als

(2017) eine pädagogische Intervention für verhaltensauffällige Kinder

Käfer, M. Achtsamkeit in Heilpädagogischen Tagesstätten -

(2017) Theoretische und konzeptionelle Überlegungen für den Gruppenalltag

Maier, M. Achtsamkeit mit begleiteten Flüchtlingskindern -

(2017) Theoretische Ansätze und Umsetzungsmöglichkeiten am Beispiel der Gemeinschaftsunterkunft des AGDW e.V. Stuttgart-Hofen

Lemppenau, M. Achtsamkeit -

Schmelzer, A. ein Projekt zur Stressbewältigung für Grundschüler 
Zenker, A. Meditation und Soziale Arbeit.

(2016) Ein Weg zur Stärkung professionellen Handelns?

Uhl, A. Die Relevanz von Meditation für Kinder und Jugendliche

(2016) in der Sozialen Arbeit

Schimon, A. "Willst du was erleben, dann musst du dich bewegen!“

(2016) Kinderyoga als gesundheitsfördernde Maßnahme am Beispiel Kindergarten

Schröck, R. Die progressive Muskelrelaxation nach Jacobson - ein präventives Angebot

(2016) zur Förderung der Entspannung für Kinder zwischen sechs und zehn Jahren

Htagkonikou, D. Religion als Teil des Menschseins

(2016) und ihre Bedeutung für die Soziale Arbeit

Jaster, I. Kann Achtsamkeitsmeditation

(2016) bei stressbedingten Beschwerden helfen?

Hornung, J. Meditation als ästhetisches Medium in der Sozialen Arbeit

(2015) am Beispiel der Transzendentalen Meditation (TM)

Penning, N. Die Wirkungsdimensionen von Kampfkunst in der Sozialen Arbeit

(2015) am Beispiel der koreanischen Kampfkunst Tang Soo Do

Maierbacher, R. Mediation im Handlungsfeld

(2015) der Sozialpsychiatrie

Beck, H. Achtsamkeitsmeditation als ästhetisches Medium in der Hospizarbeit.

(2014) Ein Projektbeispiel im Johannes-Hospiz der Barmherzigen Brüder in München

Gäbler, M. Daoistische Lebenspflege

(2014) als mögliche Ressource in der Sozialen Arbeit

Jiwa, J. Meditation als ästhetisches Medium

(2013) am Beispiel der Behandlung von chronischem Schmerz

Nachbar, B. V. Kinderyoga - ein Aspekt der Gesundheitserziehung in der Grundschule.

(2013) Wirkungsdimensionen und Implementierungsansätze

Hick, N. Kinderyoga als gesundheitsfördernde Maßnahme

(2012) in der Sozialen Arbeit

Hobbit, M. Lebensqualität und Gesundheit

(2012) durch Meditation 


\section{Masterarbeiten (Hochschule München)}

Gut, L. V. Achtsamkeit und Meditation im Hochschulkontext (Arbeit noch nicht Chancen, Grenzen und Implementierungsmöglichkeiten! abgeschlossen) Eine qualitative Befragung von Hochschullehrenden

\section{Zulassungsarbeiten (LMU München Lehramt)}

Wittmann, D. Meditation und Achtsamkeit mit Kindern

(2017) am Beispiel einer praktischen Untersuchung in der Grundschule

Friedrich, A. Christliche Meditation

(2017) im Schulkontext

Koster, K. Können Meditationspraktiken einen positiven Beitrag zur Stressbewältigung (2016) im Schul- und Lebensalltag von Kindern leisten?

Markwardt, S. Vipassana-Meditation nach S. Goenka - Einführung in die Tradition (2016) sowie Überlegungen zum Einsatz in der Schule

Holz auf der Heide, E. Meditation in der Grundschule -

(2016) Auswirkungen von Achtsamkeitsübungen auf Prüfungsangst bei Kindern

Schaule, C. M. Achtsamkeit in der Schulpsychologie -

(2016) Relevanz und Umsetzungsmöglichkeiten

Tschernov, M. Kinderyoga als pädagogisches Angebot

(2016) ästhetischer Bildung

Schmid, L. Meditation in der Grundschule -

(2015) eine Antwort auf die veränderte Lebenssituation von Kindern?

Czajka, L. Auswirkungen von Meditation

(2015) bei Verhaltensauffälligkeiten am Beispiel Gefängnis

Brzakovic, J. Achtsamkeit in der Schule -

(2015) das Fünf-Phasen-Modell

Henkel, J. S. Wie kann der Soziale Buddhismus Lernen in der Schule fördern?

(2015) Ein Projekt in einer Ü1-Klasse in München

Hofbauer, C. Meditation in der Schule.

(2014) Meditative Elemente als wichtiger Bestandteil der gymnasialen Oberstufe

Reinecke, J. Achtsamkeit bei Kindern mit Verhaltensauffälligkeiten.

(2014) Möglichkeiten und Grenzen einer Umsetzung in der Grundschule

ReckerzügI, M. Ein pädagogisches Konzept der Achtsamkeit -

(2013) mit wachem Geist und Körper die Welt verändern 


\section{Promotionen}

Stauss, J. F. Zur Professionalisierung durch Achtsamkeit - Entwicklung eines

(Arbeit noch nicht systematisierten Achtsamkeitskonzepts für die Lehrer/-innenbildung.

Schramm, A. MAIDS (Meditation und Achtsamkeit in der Schule).

(Arbeit noch nicht Auswirkungen und Aufmerksamkeit und Wohlbefinden von Schülerinnen abgeschlossen) und Schülern sowie Klassenklima - eine quantitativ-qualitative Studie. Betreuung: Prof. Dr. E. Kiel (LMU), Prof. Dr. A. de Bruin (HM)

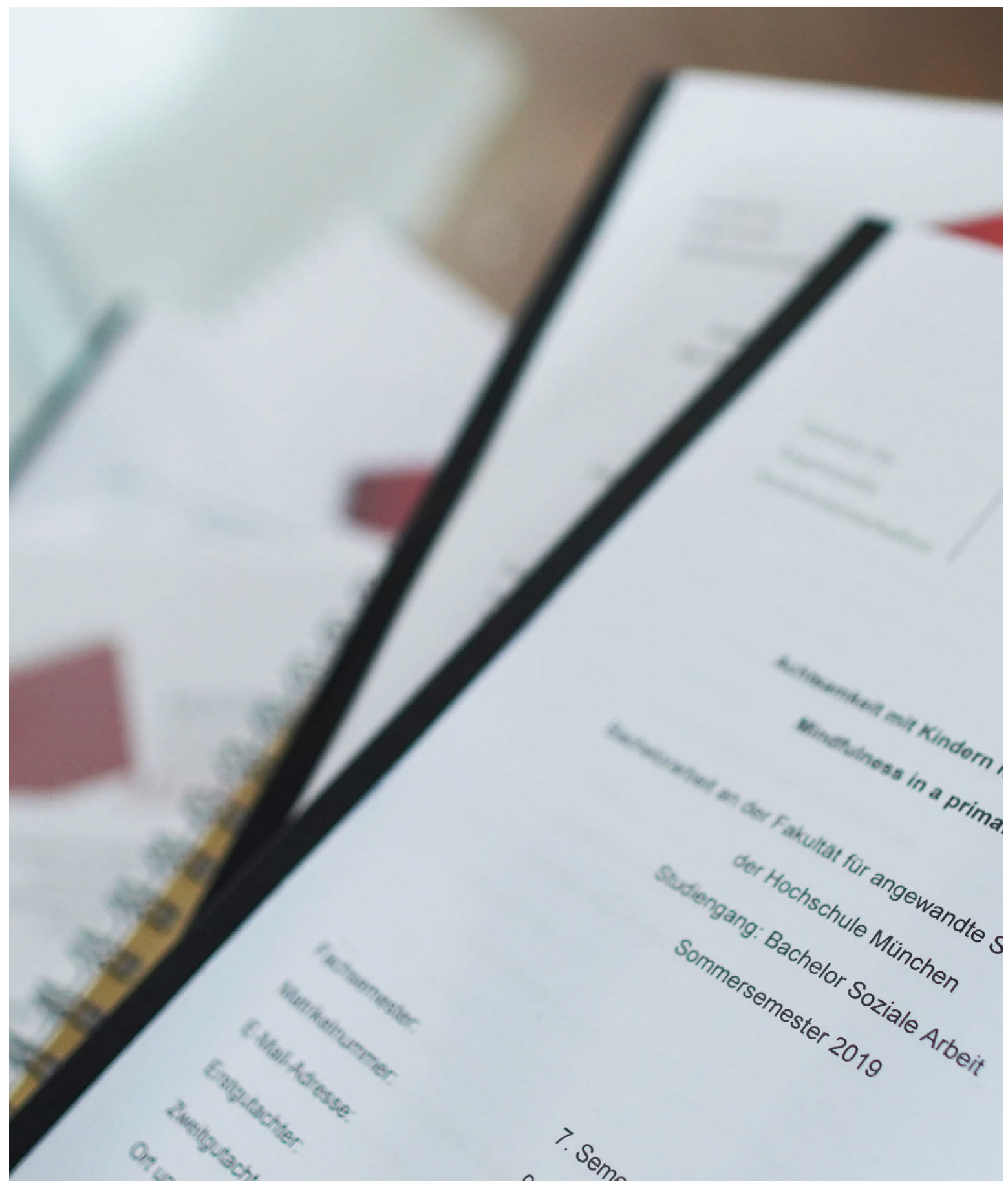




\section{Veranstaltungen im Rahmen der Vortrags- und Filmreihe „Spirituelle Lehrer und ihre Unterweisungen“}

Donnerstag | $19 \mathrm{Ubr}$

11. Juli 2019

6. Juni 2019

23. Mai 2019

11. April 2019

31. Januar 2019

13. Dezember 2018

22. November 2018

11. Oktober 2018

7. Juni 2018

12. Juli 2018

19. April 2018

22. März 2018

18. Januar 2018

6. Juli 2017

18. Mai 2017

20. April 2017

30. März 2017
Auf www.hm.edu/meditationsmodell stehen die Programmflyer der Vortrags- und Filmreihe als PDF-Download zur Verfügung. In den Flyern sind auch die Kurzbeschreibungen der jeweiligen Veranstaltungsthemen aufgeführt. Im Sommersemester 2015 sowie im Wintersemester 2019/20 fanden wegen eines Forschungssemesters keine Veranstaltungen statt.

Die stille Revolution

From Business to Being

Zeit für Stille

Speed - Auf der Suche nach der verlorenen Zeit

Athos - Im Jenseits dieser Welt

Meditation and Art - die Malerei der großen Meister bewusst wahrnehmen (Workshop)

Mind \& Life - early dialogues

Buddha begegnen - An den heiligen Orten in Nepal und Indien

Nisargadatta Maharaj - I Am That

Mutter Meera - Darshan in Silence

Schule der Achtsamkeit

Shanti - Meditation im Hochschulkontext am Beispiel des Münchner Modells

Walk With Me - Eine Reise zur Achtsamkeit

Facetten des Erwachens: Das Wissen der Meister

InnSaei - Die Kraft der Intuition

My Reincarnation - Wiederkehr

Mind \& Life - early dialogues 
26. Januar 2017

12. Januar 2017

8. Dezember 2016

24. November 2016

13. Oktober 2016

7. Juli 2016

23. Juni 2016

12. Mai 2016

14. April 2016

28. Januar 2016

14. Januar 2016

10. Dezember 2015

26. November 2015

29. Oktober 2015

Dienstag | $19 \mathrm{Uhr}$

27. Januar 2015

13. Januar 2015

16. Dezember 2014

2. Dezember 2014

25. November 2014

11. November 2014

28. Oktober 2014
Morgenröte im Aufgang - Hommage à Jacob Böhme

Die große Stille

Musicosophia - klassische Musik bewusst hören (Workshop)

Die Revolution der Selbstlosen

10 Fragen an den Dalai Lama

Der atmende Gott

Awake - Das Leben des Yogananda

Meditation im Hochschulkontext - Das Münchner Modell

Auf der Suche nach dem Gedächtnis

Maitreya und die Meister der Weisheit

Sathya Sai Baba: Gespräche mit dem Westen

Mata Amritanandamayi - Amma: Darshan - Die Umarmung

The Dhamma Brothers

Meditation im Hochsicherheitsgefängnis

Free the Mind

Kann ein Atemzug dein Denken verändern?

Krishnamurti: Die Herausforderung sich zu ändern

Sri Chinmoy

Das Unmögliche herausfordern/Interviews (Ausschnitte)

Benjamin Creme

Die Lehren Maitreyas - Das Selbst entdecken / Zur Lage der Welt

Helena Petrovna Blavatsky - Alice A. Bailey

Die Lehren der zeitlosen Weisheit

Francisco Varela

Monte Grande - Was ist Leben?

Dalai Lama

Renaissance - Der wahre Wandel beginnt in dir

Thich Nhat Hanh

Mein Leben ist meine Botschaft 



$$
\begin{gathered}
\text { ÜBUNGEN } \\
\text { UND } \\
\text { TAGEBUCH- } \\
\text { NOTIZEN }
\end{gathered}
$$


Seite $\quad 79$ Über das Lehren

Seite $\quad 83 \quad$ Zu den Übungen

Seite $\quad 89 \quad$ Risiken

Seite $101 \quad$ Sitzmeditationen

Seite 117 Achtsamer Alltag

Seite 122 Interview: „Den wichtigen Dingen mehr Raum geben"

Seite 125 Achtsames Kochen und Essen

Seite 131 Achtsame Kommunikation

Seite 135 Bodyscan

Seite 143 Liebende Güte

Seite 151 Gehmeditation

Seite $159 \quad$ Sutras

Seite 163 Geräuschmeditation 


\section{Über das Lehren}

Bevor die im Rahmen des Münchner Modells durchgeführten Übungen in Detail erläutert werden, wird in diesem Abschnitt auf die Art und Weise des Unterrichtens eingegangen. Dabei werde ich zunächst meine frühere Referententätigkeit mit arbeitslosen Jugendlichen beleuchten, da diese meine weitere Lehrtätigkeit nachhaltig geprägt hat.

\section{Eine erste Lehrerfahrung}

Als ich im November 1996 eine dreitägige Referententätigkeit an einer berufsvorbereitenden Bildungsmaßnahme aufnahm, war dies meine erste Lehrerfahrung mit jungen Menschen. Die Gruppe bestand aus 15 arbeitslosen Jugendlichen, teils mit Hauptschulabschluss, teils auch ohne. Einige von ihnen waren bereits mehrmals von der Schule „geflogen“, und die berufsvorbereitende Bildungsmaßnahme bedeutete eine allerletzte Chance für sie.

Die Maßnahme diente zur Orientierung auf dem Arbeitsmarkt und sollte die Teilnehmenden darin unterstützen, Berufspraktika zu finden und eventuell von einem der Betriebe übernommen zu werden. Es war einer der vielen Ansätze der Bundesregierung, um die Jugendarbeitslosigkeit in den Griff zu bekommen.

Ich hatte mir vorgenommen, den Jugendlichen in den nächsten drei Tagen zu vermitteln, wie die eigene Wahrnehmung (Tag 1), das eigene Denken (Tag 2) und daran gekoppelt das Verhalten (Tag 3) funktionieren. Am Tag 3 würde ich am Nachmittag noch den Bogen zum Thema „Bewerbungsgespräche“ spannen und glaubte tatsächlich, ich würde am Ende meines Referenteneinsatzes „musterreife“ Bewerberinnen und Bewerber präsentieren können! Ein bis in die Minute durchgeplantes Konzept sollte mir dabei als Kompass dienen.

Doch es kam alles ganz anders! Mein Konzept erwies sich als nutzlos und landete innerhalb kürzester Zeit im Mülleimer. Den Unterricht musste ich am ersten Tag bereits früh abbrechen. Ich hatte mehrmals einige Kursteilnehmer aufgrund ihres Benehmens „vor die Tür gesetzt“, und die Gruppe wurde allmählich sehr klein. Zu meiner Überraschung waren sie mehr daran interessiert, mich zu provozieren oder eigene Sachen zu machen, als mein gut gemeintes „Hilfsangebot“ anzunehmen.

Erst als ich am Abend wieder zu Hause war, wurde mir klar, dass ich komplett anders vorgehen muss; dass ich eigentlich gar nichts über diese jungen Menschen weiß; dass ich nur meine eigenen Vorstellungen darüber, wie der Unterricht ablaufen sollte, im Kopf gehabt hatte.

Mir wurde ebenfalls bewusst, dass ich die Provokationen auch anders deuten kann, statt als Angriffe auf meine Persönlichkeit. Es ist anzunehmen, dass die Jugendlichen bereits zahlreiche negativen Erfahrungen mit Lehrkräften gemacht hatten. Offenbar wollten sie mich testen und für sich ausloten, ob sie mich letztendlich als Lehrer akzeptieren würden.

Den nächsten Tag kam ich sozusagen blanko, ohne irgendwelche Pläne, in den Unterricht und fragte lediglich, wozu sie Lust hätten. Einige meinten, wir sollten einen Film drehen. „Aber keine Aufnahmen zum Üben von Bewerbungsgesprächen oder so, etwas über Gangster, das wäre cool!“ Die anderen aus der Gruppe stimmten zu. Ich war so dankbar, dass sie überhaupt mitmachen wollten, sodass mir nahezu alles recht 
gewesen wäre. Also legten wir gleich los und begannen zunächst damit, ein Drehbuch zu schreiben. Irgendwann hatten wir eine Story und eine Rollenverteilung. Die benötigte Videokamera bekamen wir von der Bildungseinrichtung. Ich wusste wenig über Kameratechnik, aber zwei Teilnehmer kannten sich aus. Einige Aufnahmen schafften wir noch am gleichen Tag! Den Rest drehten wir an Tag 3. Es war das perfekte Chaos und es hat mich einiges an Nerven gekostet! Als völlig Unerfahrener sowohl hinsichtlich des Unterrichtens als auch im Umgang mit Jugendlichen musste ich nicht nur das Filmprojekt an sich „stemmen“, sondern auch die Jugendlichen im Auge behalten.

Statt passende Bewerberinnen und Bewerber für den Arbeitsmarkt zu präsentieren, hatte ich am Ende der drei Unterrichtstage eine Videokassette mit mehreren aneinandergereihten Filmsequenzen. Mit viel Wohlwollen konnte man darin eine „Gangsterstory" entdecken.

„Hey Andy, wann kommst du wieder?“, ist ein Satz den ich nicht vergessen werde. Ich dachte in dem Moment: „Mein Gott, nie wieder!“, und ich war mir bewusst, dass von meinem ursprünglichen Vorhaben nichts umgesetzt worden war. Dies würde die Kursleiterin mit Sicherheit veranlassen, mich nicht mehr als Referent anzufragen.

Aber nochmals kam alles anders! Zu meiner völligen Überraschung fragte die Kursleiterin sogleich, wann ich wiederkommen würde. Hatte sie mich mit jemandem verwechselt? Ich stimmte schließlich zu, aber nur unter der Bedingung, dass ich meinen Unterricht komplett selbst gestalten kann. Zwar könnten Themen vorgegeben werden, aber die Art, wie ich diese mit der jeweiligen Teilnehmergruppe bearbeiten würde, wollte ich selbst entscheiden können. Sie hatte keine Einwände und stimmte zu.

Mit der Zeit bekam ich zahlreiche weitere Aufträge auch von anderen Trägern und Bildungsinstitutionen in München und Umgebung. Es hatte sich herumgesprochen, dass es da einen „Holländer“ gibt, der mit schwierigen Jugendlichen klarkommt. Aus den drei Tagen Referententätigkeit wurden fast zehn Jahre, von Ende 1996 bis Mitte 2006 (mit einer 7-monatigen Unterbrechung im Jahr 1998).

Diese Jahre haben mich stark geprägt, und ich habe viel darüber gelernt, wie Menschen unterschiedlicher Herkunft und mit unterschiedlichen Bildungsvoraussetzungen aufrichtig und achtsam zusammenarbeiten können. Und vor allem ist in der Arbeit mit den Jugendlichen klargeworden, dass sie alle, wie schwierig und verhaltensauffällig sie auch sind, ein Potenzial haben, das herausgearbeitet werden kann. Diese Aufgabe kommt insbesondere den Lehrenden zu. Sie können den Raum schaffen, der für die Entfaltung der jungen Menschen notwendig ist. Das eigene Potenzial entdecken und entfalten zu können, hat einen positiven Effekt auf das Selbstwertgefühl und die Selbstwirksamkeit der jungen Menschen und ist auch bestimmend dafür, inwiefern sie sich in der Gesellschaft akzeptiert fühlen.

Die Tätigkeit mit den Jugendlichen machte ich dann auch zum Thema meiner Promotion im Fach Ethnologie an der LMU München. Es war klargeworden, dass meine pädagogische Arbeit mit den jungen Menschen sehr viele Parallelen aufwies zum sogenannten Fox-Projekt - aus diesem Projekt war in den 1960er-Jahren letztendlich ein neuer Ansatz in der Ethnologie entstanden, die Aktionsethnologie. ${ }^{1}$

Die Erfahrungen aus meiner jahrelangen Referententätigkeit mit arbeitslosen Jugendlichen im Vergleich mit dem Fox-Projekt ist in meiner Dissertation Jugendliche ein fremder Stamm? ausführlich dargestellt. ${ }^{2}$ An dieser Stelle möchte ich nur noch kurz auf die Anforderungen und Prinzipien der Aktionsethnologie eingehen, die ein sogenanntes System of Values, einen Wertekanon, postuliert, der auch im Rahmen meiner damaligen Arbeit mit den Jugendlichen sowie in meiner späteren Professur an der Hochschule München einen bedeutsamen Stellenwert eingenommen hat und noch immer einnimmt. 


\section{Aktionsethnologie - System of Values}

In der Aktionsethnologie gibt es keine starren Vorgaben, keine festgelegten Methoden, wie man im „Forschungsfeld“ zu agieren hat. Stattdessen gibt es bestimmte Werte, wie VERSTEHEN, INTERAKTION, VERANTWORTUNG und ERGÄNZENDE TUGENDEN, die als Handlungsleitfaden dienen. ${ }^{3}$ Diese können meines Erachtens auch für Lehrende in Unterrichtssituationen sehr wertvoll und hilfreich sein und die Art und Weise, wie Lehrinhalte vermittelt werden, enorm bereichern. Übertragen auf die Lehrsituation in Bildungseinrichtungen könnte der aktionsethnologische Wertekanon folgendermaßen ausgelegt werden.

Beim VERSTEHEN geht es für Lehrende darum, das Denken und Verhalten der Teilnehmerinnen und Teilnehmer aus der „Innenperspektive“ wahrzunehmen. Bei diesem sogenannten emischen Verstehen geht es auch um das Aufdecken und Bewusstwerden der eigenen subjektiven Wahrnehmungsfilter und Konditionierungen. Denn erst wenn ich mir dieser bewusst bin, kann es mir gelingen, mich für die Sichtweisen meiner Teilnehmenden zu öffnen.

Der INTERAKTION beinhaltet für Lehrende einen Rahmen zu schaffen, in dem alle Teilnehmerinnen und Teilnehmer respektiert und wertgeschätzt werden und sich mit ihren eigenen Fähigkeiten im Unterricht einbringen können.

VERANTWORTUNG impliziert für Lehrende, dass die Teilnehmenden darin unterstützt werden, mehr Selbstbestimmung und Selbstwirksamkeit zu erlangen. Dies beinhaltet, den Teilnehmenden Wissen und Fähigkeiten zu vermitteln und ihr Selbstvertrauen zu stärken.

$\mathrm{Zu}$ den ERGÄNZENDEN TUGENDEN werden noch GEDULD, BESCHEIDENHEIT und LERNEN UND REFLEKTIEREN gerechnet. Diese Tugenden geben Lehrenden die Möglichkeit, sich mehr in einen Dialog auf Augenhöhe mit den Teilnehmerinnen und Teilnehmern zu begeben. Indem sie signalisieren, dass Lernen und Gestaltung von Unterricht wechselseitig stattfindet und sie auch von den Teilnehmenden lernen können, wird eine Atmosphäre der gegenseitigen Akzeptanz und des Respekts geschaffen, was die Teilnehmenden motiviert, sich in den Diskursen einzubringen.

Eine nicht unwichtige Rolle in der Aktionsethnologie spielen nicht zuletzt HUMOR und AUTHENTIZITÄT. Beim Lehren darf auch „Humor“ nicht fehlen. Er trägt zu einer entspannten Atmosphäre bei. Als Lehrende ist es darüber hinaus wichtig, authentisch zu sein. Im Rahmen des Münchner Modells bedeutet für mich authentisch zu sein, auch zu zeigen, dass man sich genauso wie die Teilnehmerinnen und Teilnehmer mit den Übungen auseinandersetzt und neue Erfahrungen macht. Hin und wieder erzähle ich deshalb im Unterricht kurze Anekdoten aus dem täglichen Leben, um bestimmte Themen bezüglich der Achtsamkeits- und Meditationspraxis lebensweltnah zu verdeutlichen. Nicht nur wird der Unterricht dadurch lebendiger, es hat sich zudem gezeigt, dass diese Beispiele den Studierenden helfen, die Ansätze auch im eigenen Leben besser zu integrieren.

\section{QUELLE}

de Bruin, Andreas (2004). Jugendliche - ein fremder Stamm? Jugendarbeitslosigkeit aus aktionsethnologischer Sicht. Zur kritischen Reflexion von Lehrkräften und Unterrichtskonzepten im deutschen Schul- und Ausbildungssystem. Münster: LIT Verlag. 
Gedanken „wegfegen“ ist wie das Ausmisten einer Wohnung.
1 Entwickelt wurde der aktionsethnologische Ansatz von dem amerikanischen Ethnologen Sol Tax (1907-1995) zusammen mit einem Team von Studenten an der Universität Chicago. Sie hatten ursprünglich versucht, den Mesquakie, einer Gruppe von Native Americans in lowa, zu helfen, sich in den „American Way of Live“ zu integrieren. Die von den Studierenden entwickelten Hilfsprogramme schlugen allerdings komplett fehl. Sie hatten völlig übersehen, dass die Mesquakie ihre sozialen Probleme wie Armut, Arbeitslosigkeit und soziale Deklassierung bewusst in Kauf nahmen, weil sie ihre eigene Kultur nicht für die Integration in die amerikanische Gesellschaft aufgeben wollten. Sie hatten die Anliegen der Mesquakie bei der Entwicklung der Programme gar nicht miteinbezogen, sondern bloß ihre eigene Sichtweise im Projekt berücksichtigt. Eine aufrichtige Fehleranalyse des Vorgehens aufseiten der Studierenden und erste Versuche, einen echten Dialog mit den Mesquakie zu initiieren, führten letztendlich unter der Leitung von Sol Tax zu diesem neuen Verstehensansatz in der Ethnologie. (Vgl. de Bruin 2004, S. 21f.)

2 Siehe de Bruin 2004

3 Vgl. de Bruin 2004, S. 22.

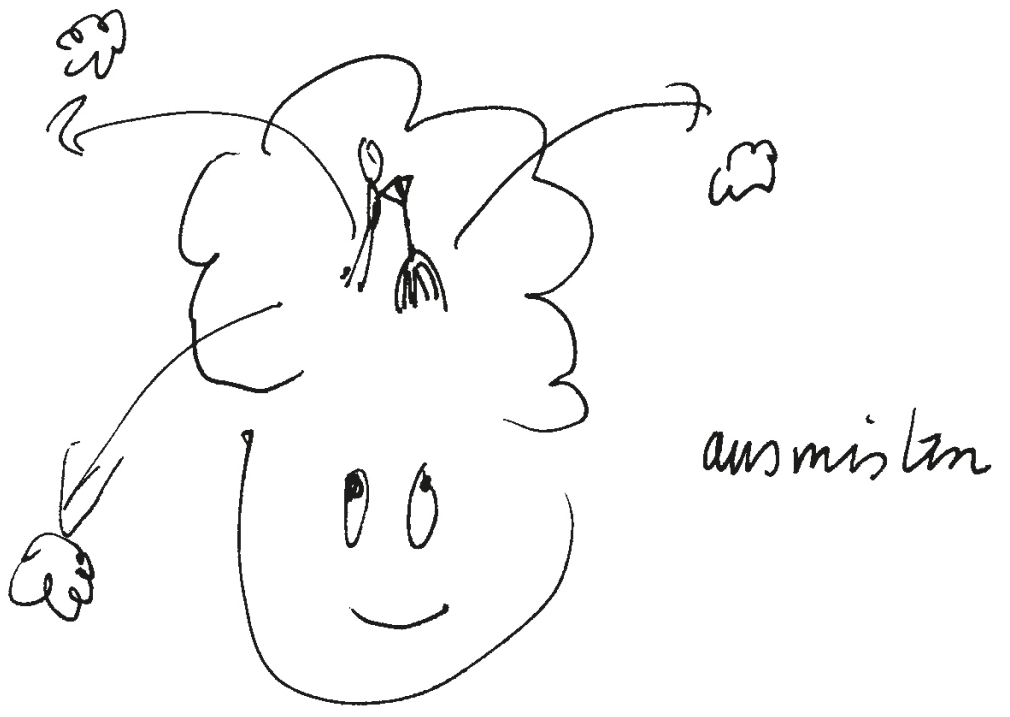




\section{Zu den Übungen}

Die im Folgenden genannten Übungen stammen aus den Lehrveranstaltungen des Münchner Modells und wurden meistens in ebendieser Reihenfolge angeboten. Ergänzend dazu wurden auch Themen über Achtsamkeits- und Meditationsforschung sowie Implementierungsbeispiele für diverse Berufsfelder vermittelt (siehe „Münchner Modell“, S. 59f.).

Einen besonderen Stellenwert in den Lehrveranstaltungen nehmen die verschiedenen Formen der Sitzmeditation ein, die nacheinander in den Unterrichtseinheiten vorgestellt und praktiziert werden. Nachdem alle Sitzmeditationstechniken eingeführt wurden, können die Teilnehmerinnen und Teilnehmer eine oder zwei Sitzmeditationsformen auswählen, die ihnen am besten zusagen. In den weiteren Unterrichtseinheiten, die immer mit einer Sitzmeditation beginnen, wenden die Teilnehmenden diese dann an.

Es soll hier erwähnt werden, dass die in dem vorliegenden Buch aufgeführten Meditationsanleitungen Studierenden erste Einblicke in verschiedene Meditationsformen und -techniken vermitteln wollen. Zur weiteren Vertiefung wird empfohlen, sich an die in der Literatur der entsprechenden Meditationen angegebenen Richtlinien und Anweisungen zu halten. Darüber hinaus besteht immer die Möglichkeit, Meditationsschulen zu besuchen und sich in entsprechenden Seminaren und Kursen weiterzubilden (siehe „Risiken“, S. 95f.).

Es stehen vier Formen zur Auswahl:

- Atem

- Maranatha

- Fokussierung zwischen den Augenbrauen

- So’ham / Ich bin Das

Darüber hinaus ist den Teilnehmenden, wenn sie bereits Meditationserfahrung haben, freigestellt, ob sie lieber ihre eigene Sitzmeditation anwenden oder aus den genannten vier Formen eine auswählen. Allerdings gilt es in diesem Fall vom Lehrenden zu prüfen, ob die jeweilige Technik aus einer anerkannten Meditationsschule stammt und für den jeweiligen Teilnehmer, die jeweilige Teilnehmerin förderlich ist (siehe „Risiken“, S. 89ff.).

Die Sitzmeditationen sind deshalb so wichtig, da sie den am Kurs Teilnehmenden die Möglichkeit geben, den Fokus nach innen zu richten und mehr in die Stille zu gehen.

Eine Übung, die wir in der Regel in der Mitte der Lehrveranstaltungsreihe praktiziert haben, kommt aus den Lehren von Nisargadatta Maharaj (1897-1981). Die Übung kann eine tiefe Erfahrung vermitteln, die über die Gedankenebene hinausgeht (siehe Übung „Was ist das ,Ich bin'?“', S. 108f.).

Neben den Sitzmeditationen werden in den Unterrichtseinheiten die folgenden

Achtsamkeitsübungen praktiziert:

- Achtsamer Alltag

- Achtsames Kochen und Essen

- Achtsame Kommunikation

- Bodyscan 
- Liebende Güte / Metta-Meditation

- Gehmeditation

- Sutras

- Geräuschmeditation

Diese Übungen sind hilfreich, um mehr Einsicht in die eigenen körperlichen, emotionalen und mentalen Prozesse und deren Wechselwirkungen zu erlangen. Obwohl sie sehr alltagsbezogen und zumeist nach außen gerichtet sind, vermitteln sie Gelassenheit und innere Ruhe. Sie wirken auch unterstützend im Hinblick auf die mehr nach innen gerichteten Sitzmeditationen.

\section{Prüfungsangst}

In Zusammenarbeit mit der Studienberatung der Fakultät für angewandte Sozialwissenschaften werden seit dem SoSe 2019 Workshops gegen Prüfungsangst angeboten (siehe „Münchner Modell“, S. 61). Unter anderem wurde auch eine wirksame Übung von dem vietnamesischen buddhistischen Mönch und Achtsamkeitslehrer Thich Nhat Hanh vorgestellt, wie man mit den eigenen Ängsten konstruktiv umgehen kann. Die Übung heißt: „Das eigene Wohnzimmer betreten“. Da diese Übung bislang jedoch noch nicht in die Lehrveranstaltungen aufgenommen wurde und somit auch nicht in den Meditationstagebüchern erscheint, wird sie im vorliegenden Buch nicht weiter ausgearbeitet.

Die Übung ist zu finden in: Thich Nhat Hanh (2011). Versöhnung mit dem inneren Kind. Von der heilenden Kraft der Achtsamkeit. München: O.W. Barth Verlag, S. 16ff.

\section{Wo und wann wird die Meditation durchgeführt?}

Die Sitzmeditation sollte möglichst an einem ungestörten Ort durchgeführt werden. Nach Möglichkeit kann man sich speziell dafür einen Platz einrichten. Der katholische Priester und Benediktinermönch John Main (1926-1982) schreibt in seinem Buch Das Herz der Stille. Einführung ins Herzensgebet dazu: „Es ist auch hilfreich, regelmäßig am selben Platz und auch immer zur gleichen Zeit zu meditieren; denn das hilft, sich in seinem Leben einen schöpferischen Rhythmus zu schaffen, der zum Reifungsprozess wirksam beiträgt. Die Meditation wird dann zu einer Art regelmäßigen Pulsschlag jedes Tages. "1 Allerdings ist es aufgrund von beengtem Wohnraum oder unregelmäßigen Arbeitszeiten nicht immer möglich, einen solchen festen Rahmen einzuhalten. Manchmal gibt es auch geeignete Zeitfenster für die Meditation, zum Beispiel in der Arbeitspause, während längerer Zug- oder S-Bahn-Fahrten oder während Wartezeiten vor Terminen, je nachdem, wo man sich gerade befindet. Wichtig ist, dass man an dem jeweiligen Ort während des Meditierens nicht gestört wird, zum Beispiel durch zu viel Lärm oder Menschen, die einen ansprechen. ${ }^{2}$

\section{Körperhaltung}

Für die Körperhaltung während der Sitzmeditation gibt es eine Reihe an Möglichkeiten. Entscheidend ist, dass die Haltung ungezwungen und der Körper während der Meditation ruhig und ausgeglichen ist. Diejenigen, die Schwierigkeiten haben auf einem Meditationskissen oder Meditationsbänkchen zu sitzen, können sich auch auf einen Stuhl setzen. Die Füße sollten in diesem Fall Kontakt mit dem Boden haben. 
Die Hände liegen gefaltet im Schoß. Das Kinn ist etwas nach unten gesenkt, damit der Nacken entspannt bleibt. Wichtig ist, dass der Rücken, die Wirbelsäule aufrecht ist. Ausnahmsweise kann man, wenn sitzen nicht möglich ist, auch liegen. Allerdings ist es beim Liegen viel schwieriger, konzentriert und fokussiert zu bleiben, und die Gefahr des Einschlafens ist groß.

Beim Sitzen auf dem Meditationsbänkchen können die Beine seitlich gelegt oder unter das Bänkchen geschoben werden. Die seitliche Haltung funktioniert auch, wenn man auf einem Meditationskissen sitzt.

Was die Positionierung der Beine anbelangt, gibt es die halbe Lotushaltung sowie den ganzen Lotussitz. Bei Letzterem werden die Beine gekreuzt. Erst wird das rechte über das linke Bein gelegt und anschließend das linke über das rechte Bein. Beim halben Lotussitz schiebt man das rechte Bein unter die linke Wade und anschließend das linke Bein unter die rechte Wade. Die Hände werden in den Schoß gelegt. Wird die rechte äußere Handfläche auf die linke Innenhandfläche gelegt, so hat man die „Haltung des Gleichmuts“3. Dabei berühren sich die Daumenspitzen beider Hände. Das Kinn wird leicht zur Kehle gesenkt. Die Haltung des Gleichmuts ist für eine Sitzmeditation jedoch nicht unbedingt erforderlich. ${ }^{4}$

Im Unterricht haben wir die Sitzmeditation immer mit geschlossenen Augen praktiziert. Bei manchen Meditationsformen wird allerdings auch mit halb geöffneten Augen meditiert. In einigen Fällen haben Studierende berichtet, dass sie sich beim Meditieren mit geschlossenen Augen nicht wohlfühlen und diese lieber etwas öffnen. Dazu haben sie einen äußeren Fixierungspunkt circa 1,5 Meter vor ihnen auf dem Boden gewählt.

Abgesehen von der Gehmeditation ist der Aspekt der Körperbewegung im gesamten Übungsspektrum nur wenig vorhanden. Dies hat zwei Gründe: Erstens ist die im Unterricht zur Verfügung stehende Zeit ohnehin knapp bemessen, um die verschiedenen Themen und Achtsamkeits- und Meditationsübungen hinreichend zu vermitteln; zweitens finden an der Hochschule bereits einige meditativ-körperbetonte Lehrveranstaltungen statt, beispielsweise Taiji und Yoga.

\section{Konzentriert, aber nicht verkrampft}

Als ein Meister einmal seine Schüler fragte, wie sie die Meditation erlebt haben, antwortete ein Schüler, dass er sich kaum konzentrieren konnte, weil er einen Juckreiz am Bein hatte. Daraufhin sagte der Meister: „Dann kratzt halt!“

Diese Kurzgeschichte erzähle ich meinen Teilnehmerinnen und Teilnehmern immer. Anschließend kläre ich auf, dass es natürlich nicht heißt, dass man immer kratzen soll, wenn man während der Meditation Juckreiz verspürt. Es geht hier mehr darum, nicht verkrampft zu sein. Wichtig ist es, herauszufiltern, ob der Körper sich nur „meldet" und Aufmerksamkeit verlangt oder wirklich ein gesundheitliches Problem hat. So wie Gedanken ablenken können, kann der Körper das genauso. Ist der Körper nur „unruhig“, gilt es zu lernen, dies mehr und mehr zu ignorieren. Es kann aber erforderlich sein, dass wir dennoch kurz die Augen öffnen oder die Sitzhaltung etwas ändern. Treten jedoch tatsächlich Kopfschmerzen, Übelkeit, Schwindel auf, müssen wir die Meditation abbrechen. Ebenso kann es sein, dass wir die Meditationspraxis für eine Weile aussetzen müssen (siehe „Risiken“, S. 89ff.). ${ }^{5}$

Innere Anspannung und Unruhe kann entstehen, wenn wir beispielsweise Husten oder Niesen müssen, aber dies zurückhalten, weil wir die anderen Teilnehmerinnen und Teilnehmer nicht stören wollen. Muss man öfter Husten oder Niesen, ist es besser, vorübergehend den Raum zu verlassen. 
Auch ein intensiver Schluckreiz kann für die betroffene Person störend sein. In der Regel lässt dieses Schlucken aber schnell nach. Wichtig ist, sich keine Sorgen/Gedanken darüber zu machen.

Der an anderer Stelle bereits angesprochene Humor spielt auch hier wieder eine wichtige Rolle. Für mich ist es im Unterricht wichtig, dass wir uns wohlfühlen und hier und da auch mal lachen können. Es hilft, die Atmosphäre zu lockern und eventuell vorhandene Spannungen bei den Teilnehmenden abzubauen. Die Durchführung der Übungen nehmen wir aber sehr ernst. Wir sind im Moment der Durchführung diszipliniert, aber nicht verkrampft bzw. angespannt.

In seinem Buch Die Praxis der Achtsamkeit. Eine Einführung in die Vipassana-Meditation behandelt der buddhistische Mönch und Präsident der Bhavana Society, Mahathera Henepola Gunarantana, zahlreiche Probleme und Ablenkungen, die während der Meditation auftreten können. Ebenso geht er darauf ein, wie man diese umgehen kann. ${ }^{6}$ Im Abschnitt „Atemmeditation“ (siehe S. 102f.) werden einige seiner Hinweise bezüglich Konzentrationsverlust bei Atemmeditationen beschrieben.

\section{Meditation und Zeit}

Das Thema "Zeit" spielt in der Achtsamkeits- und Meditationspraxis eine wesentliche Rolle. Aus den Rückmeldungen im Anschluss an die Sitzmeditationen wird ersichtlich, dass das Zeitempfinden der Teilnehmerinnen und Teilnehmer sehr unterschiedlich sein kann. Obwohl die objektive Zeit beispielsweise 10 Minuten betraf, war das subjektive Zeitempfinden oft ein völlig anderes. Manchen erschien „das Sitzen“ viel kürzer als 10 Minuten, für andere ging die Zeit kaum vorüber.

Ein weiterer Zeitaspekt bezieht sich darauf, dass viele Teilnehmende Schwierigkeiten haben, im normalen Alltag für die Achtsamkeits- und Meditationsübungen Zeit frei zu machen. Obwohl die meisten Teilnehmerinnen und Teilnehmer die wohltuende und entspannende Wirkung der Meditation bereits selbst erfahren haben, sind viele bislang noch geneigt, andere Alltagstätigkeiten zu priorisieren.

Der Moment der Meditation ist etwas sehr Kostbares. Es geht nicht darum, wie viel Zeit verstreicht. Es ist eine Ebene, wo Vergangenheit und Zukunft keine Rolle mehr spielen; wo wir in Kontakt mit unserem inneren Raum kommen - unabhängig von (Zeit-)Konstrukten und Konditionierung. Sobald einem dies mehr bewusst wird, wird es auch leichter, sich „Zeit“ für die Meditationspraxis zu nehmen.

Zeit hat auch viel mit Mindwandering zu tun. Wir sind so oft mit unseren Gedanken in der Vergangenheit bzw. Zukunft. Durch Achtsamkeit und Meditation kann das Mindwandering reduziert werden, und wir lernen wieder mehr im Hier und Jetzt zu sein.

Die in diesem Abschnitt genannten Aspekte zeigen die Verbindung zwischen Zeit und die Achtsamkeits- und Meditationspraxis. Und deshalb ist die Auseinandersetzung mit dem Thema "Zeit" auch immer ein fester Bestandteil in einer der Lehrveranstaltungen. Es werden dabei viele Fragen diskutiert, beispielsweise: Was ist Zeit? Existiert Zeit überhaupt? Was sind Vergangenheit und Zukunft?

Der indische spirituelle Lehrer Jiddu Krishnamurti (1895-1986) unterscheidet zwischen chronologischer Zeit und psychologischer Zeit. Für letztere gilt: „Sie ist das Intervall zwischen Idee und Handlung. "7 Setzt man sein Vorhaben direkt um, so gibt es keine psychologische Zeit. Das Wichtigste ist für Krishnamurti das Jetzt, das augenblickliche Sein. Vergangenheit und Zukunft spielen somit eine untergeordnete Rolle. ${ }^{8}$ Zeit in diesem Sinne ist letztendlich ein menschliches Konstrukt, und spätestens als Kinder in der Grundschule werden wir ausführlich damit bekannt gemacht. 
Insbesondere in den Wahlpflichtfächern „Stressmanagement und Meditation“ im Rahmen des Studiengangs Studium Generale und Interdisziplinäre Studien ist die Auseinandersetzung mit dem Thema "Zeit“ sehr anregend. Da sich für dieses Angebot alle Studierende der Hochschule München bewerben können, kommen die Kursteilnehmerinnen und Kursteilnehmer aus unterschiedlichen Studiengängen, etwa Flugzeugtechnik, Maschinebau, Informatik, Tourismus, Design, Elektrotechnik etc. Durch die teils ganz unterschiedlichen Blickwinkel der Fachrichtungen und Sichtweisen der Teilnehmenden, findet jedes Mal ein fruchtbarer Diskurs statt.

In meiner eigenen langjährigen Meditationspraxis habe ich die Erfahrung gemacht, dass es insbesondere bei längeren Meditationseinheiten oft schwierig ist, bis zum Ende durchzuhalten. So viele Gedanken gingen mir manche Male durch den Kopf! Insbesondere dachte ich darüber nach, was ich alles noch tun müsste und ob ich die Zeit nicht anders nutzen könnte, statt in der Meditation zu sitzen. Ich blieb dennoch sitzen, aber die Gedanken waren oft sehr hartnäckig. Manchmal habe ich auch an eine Tafel Schokolade als „Belohnung“ gedacht, die ich am Ende der Meditation dann meist auch gekauft habe. Irgendwann habe ich verstanden, dass es möglich ist, diese Gedanken loszuwerden und einfach sitzen zu bleiben: nicht mit dem Denken verhandeln! Ich habe mir ab diesem Moment innerlich vor jeder Meditation gesagt: „Ich bleibe sowieso bis zum Ende sitzen." Am Anfang hat das Denken versucht mich herauszulocken, mir Vorschläge zu machen, was ich alles in der Zeit des Sitzens erledigen könnte, später ließen diese Gedanken nach. Wie ein kleines Kind, das irgendwann aufhört, Süßigkeiten zu erbetteln, weil es gemerkt hat, dass es sowieso keine bekommt!

\section{Wie lange wird meditiert?}

Sitzmeditationen können von einigen Minuten bis zu mehreren Stunden (etwa während eines Retreats) durchgeführt werden. Wie ausführlich unter „Risiken“ (S. 89ff.) beschrieben, sollte man nicht übertreiben und im Falle von längeren Retreats Vorerfahrung mitbringen. Für die eigene Praxis zu Hause empfehle ich in der Regel 15 bis 20 Minuten ein- oder zweimal täglich. Selbstverständlich richtet sich die Meditationsdauer auch nach der jeweiligen Meditationstechnik. Weitere Informationen bieten Fachliteratur und Meditationsschulen (siehe auch „Zu den Übungen“, S. 83).

\section{Als Lehrende selbst mitmachen}

Wichtig ist, dass Lehrende mit den verschiedenen Achtsamkeits- und Meditationsübungen gut vertraut sind und bevor sie Anleiten, diese selbst verinnerlicht haben. Die eigene regelmäßige Übungspraxis ist somit eine Voraussetzung. Sind die Übungsabläufe verinnerlicht, wird es möglich, beim Anleiten sowohl die Übung selbst mitzumachen, als auch die Aufmerksamkeit auf die Teilnehmerinnen und Teilnehmer und das gemeinsame Übungsfeld zu richten. Diese beidseitige Präsenz hat eine authentische, achtsame Vermittlung zur Folge und sorgt dafür, dass ein GEMEINSAMES SEIN und ERFAHREN entstehen kann.

Vor allem die Sitzmeditationen sind an dieser Stelle hervorzuheben. Meine eigene Erfahrung hat gezeigt, dass das gemeinsame Meditieren Zusammengehörigkeit vermittelt und die Gruppe stärkt. 


\section{QUELLEN}

Gunaratana, Mahathera Henepola (2010). Von der Achtsamkeit zur Sammlung. Eine Einführung in die tieferen Stadien der Meditation. Heidelberg: Werner Kristkeitz Verlag.

Gunaratana, Mahathera Henepola (1996). Die Praxis der Achtsamkeit. Eine Einführung in die Vipassana-Meditation. Heidelberg: Werner Kristkeitz Verlag.

Kabat-Zinn, Jon (2019). Gesund durch Meditation. München: Knaur Verlag. Krishnamurti, Jiddu (1993). Einbruch in die Freiheit. Berlin / Frankfurt am Main: Ullstein.

Main, John (2015). Das Herz der Stille. Einführung ins Herzensgebet. München: Claudius Verlag.

Ott, Ulrich (2015). Meditation für Skeptiker. Ein Neurowissenschaftler erklärt den Weg zum Selbst. München: Droemer Knaur.

Ricard, Matthieu (2009). Meditation. München: nymphenburger.

Thich Nhat Hanh (2011). Versöhnung mit dem inneren Kind. Von der heilenden Kraft der Achtsamkeit. München: O.W. Barth Verlag.

\footnotetext{
1 Main 2015, S. 49; siehe auch Muktananda 1996, S. $122 f$.

2 Vgl. Gunaratana 1996, S. $91 \mathrm{f}$

3 Vgl. Ricard 2009, S. 48

4 Für Einzelheiten zur Körperhaltung siehe Gunaratana 1996, S. 73ff.; Kabat-Zinn 2019, S. $95 \mathrm{ff.}$ Main 2015. S. 44; Ott 2015, S. 27ff.; Ricard 2009, S. 46ff.; Muktananda 2001, S. xv und S. 27.

5 Für Hinweise, wie mit physischem Schmerz und mit Körperablenkungen während des Meditierens umgegangen werden kann, siehe Gunaratana 1996, S. 109ff.

6 Vgl. Gunaratana 1996, S. 106ff. und 125ff.; Gunarantana 2010, S. 77ff.; Muktananda 1996, S. $87 f f$. und Muktananda 2001, S. 23ff.

7 Krishnamurti 1993, S. 65

8 Vgl. ebd.
} 


\section{Risiken}

Neben den im Forschungsabschnitt (siehe S. 41ff.) beschriebenen positiven Auswirkungen von Achtsamkeits- und Meditationsübungen gibt es auch einige Risiken, die bei nicht korrekter Anwendung sowie im Zusammenhang mit einer problematischen psychischen Disposition auftreten können. Obwohl dies im Hochschulkontext erfahrungsgemäß nicht allzu häufig vorkommt, ist es dennoch sinnvoll und verantwortlich, sich systematisch mit diesen Fragen auseinanderzusetzen. Auch sollten dabei zentrale Aspekte vor Beginn des Programms mit den Teilnehmerinnen und Teilnehmern in geeigneter Form diskutiert werden. Nachfolgend werden dazu einige wichtige Punkte beleuchtet.

Die wichtigsten Risiken sind: ${ }^{1}$

- Psychiatrische, psychosomatische oder somatische Vorerkrankungen/ Vorbelastungen

- Psychische, emotionale Instabilität

- Zu ehrgeizige Zielsetzungen/Zu anspruchsvolle Meditationstechniken

- Praktizieren von Meditation ohne Begleitung durch Lehrer oder Gruppe

- Praktizieren von Meditation mit Begleitung durch unerfahrene Lehrer

\section{Psychiatrische Vorerkrankung/Vorbelastung}

Als „psychiatrische Erkrankungen“ werden klinische Störungen mit eindeutigem Krankheitswert wie beispielsweise Schizophrenie, Psychosen, bipolare Störungen, Angststörungen und Depression sowie Traumafolgestörungen, aber auch Persönlichkeitsstörungen wie die Borderline-Persönlichkeitsstörung oder die Narzisstische PS verstanden. Darüber hinaus ist es sinnvoll in diesem Zusammenhang auch potenziell vorhandene „Vorbelastungen“ im Sinne von körperlichen, psychosomatischen oder psychosozialen Vulnerabilitäten wie beispielsweise epileptische Symptome, ADHS, Migräne oder Sucht-/Abhängigkeitsprobleme zu berücksichtigen. Inwiefern die Anwendung von Achtsamkeits- und Meditationsübungen bei dieser Art Vorerkrankungen und Vorbelastungen überhaupt möglich ist, hängt von dem Schwergrad der Beeinträchtigung sowie der potenziellen Gefährdung durch das Praktizieren von Meditation ab. Dabei hat es sich bewährt, nach einem „Ampelmodell“ zu verfahren, das Meditationsinteressierte in die Kategorien „unbedenklich“, „potenziell gefährdet“ und „höchstwahrscheinlich gefährdet und daher Teilnahme am Programm nicht empfehlenswert" einteilt. Im Zweifelsfall ist dies jedoch nur durch eine medizinisch oder psychologisch geschulte Kursleitung möglich. Kursleiterinnen und Kursleiter ohne diesen Hintergrund sollten sich daher nicht scheuen, gegenüber den Teilnehmenden Vorbehalte offen anzusprechen, wenn sie selbst unsicher sind, und empfehlen, dass Rücksprache mit medizinisch/psychologisch geschulten Expertinnen und Experten geschieht, die auch hinreichend vertraut mit meditativen Praktiken sind. 
Niko Kohls, Medizinpsychologe und Professor für Gesundheitswissenschaften und Gesundheitsförderung sowie Vorstandsmitglied der Gesellschaft für Bewusstseinswissenschaften und Bewusstseinskultur weist darauf hin, dass ein wichtiges Kriterium für die Anwendung von achtsamkeitsbasierten Interventionen bei psychiatrischen Erkrankungen ist, inwiefern eine hinreichend stabile Ich-Struktur bzw. Ich-Stabilität bei der betroffenen Person noch vorhanden ist. Ist diese nicht gegeben, können die Interventionen nicht wirken und sollten nicht durchgeführt werden. In Einzelfällen kann es sogar zur Symptomverstärkung, wie beispielsweise dissoziativen Prozessen, Niedergeschlagenheit oder Ängsten, oder bei Traumafolgestörungen das Auftreten von sogenannten belastenden Erinnerungen, den sogenannten Intrusionen, kommen. ${ }^{2}$

Kohls, der neben der Durchführung und Evaluierung zahlreicher Achtsamkeitsprogramme im Schul-, Hochschul- und Arbeitskontext auch mit Soldatinnen und Soldaten auf dem Gebiet der einsatzbedingten Stressbewältigung gearbeitet hat, berichtet, dass bei hochbelasteten Gruppen/Patienten häufig zunächst nur basale Vertrauensübungen sinnvoll angewendet werden können. Nach solchen Übungen, wie beispielsweise das Getragenwerden im Wasser, sind anschließend achtsamkeitsbasierte Interventionen sinnvoll, die niederschwellig, alltagsbezogen und bevorzugt körperbetont sind. Auf dieser Weise wird sichergestellt, dass Meditationsdauer, -tiefe, und -qualität den psychoemotionalen Kompetenzen und Ressourcen der betreffenden Person entsprechen. So wird vermieden, dass eine zu frühe Innenausrichtung und ungewollte Auseinandersetzung mit belastenden Gedanken und Gefühlen entstehen kann, die im Extremfall zu einer Re-Traumatisierung führen könnte.

Der Facharzt für Psychiatrie und Psychotherapie und Spezialist auf dem Gebiet der achtsamkeitsbasierten Behandlung von Traumata, Dr. Ortwin Lüers, betont in diesem Zusammenhang, dass bei Traumata durch die bewusste oder unbewusste Lenkung auf belastende Themen verdrängte Verletzungen wieder ungewollt an die Oberfläche gelangen und plötzliche Flashbacks ausgelöst werden können. Durch das Trauma gelingt eine Neubewertung der traumatischen Situation in der jetzigen Gegenwart in der Regel nicht und Dissoziation ${ }^{3}$ und Re-Traumatisierung können die Folge sein. ${ }^{4}$

Ein weiterer Punkt, der nach Lüers berücksichtigt werden muss, ist, dass insbesondere Menschen mit chronischen psychiatrischen Erkrankungen, wie beispielsweise lang anhaltenden Depressionen, häufig unter tiefgreifenden körperlichen Erschöpfungszuständen leiden. Solche chronischen psychiatrischen Erkrankungen sind emotional, mental und vor allem biologisch determiniert. Aus diesem Grund ist es bei der Anwendung von Achtsamkeitsübungen entscheidend, nicht über das zur Verfügung stehende Energielevel hinauszugehen, da es sonst eher zu einem Scheitern des Vorhabens kommt. Ein solches Misslingen der Übung kann schnell zu einer negativen Selbstattribution führen, welche die betroffene Person zusätzlich belastet. ${ }^{5}$ Eine weitere Folge einer zu hohen Intensität zu Beginn, kann auch eine vollständige Demotivation sein.

Ausschlaggebend für eine gelungene Anwendung von Achtsamkeitsübungen sind in erster Linie eine angenehme Atmosphäre basierend auf Herzenswärme, Akzeptanz und Mitgefühl, damit in keiner Weise Erfolgsdruck entsteht. Es geht also nicht darum, ein betontes Ziel der Übung in den Vordergrund zu stellen, wie etwa die Verbesserung der Aufmerksamkeit, Stressreduzierung, dies soll nur indirekt gefördert werden.

Wie Kohls spricht auch Lüers die Bedeutung der Körperbetonung an. Zudem empfiehlt er, die Übungspraxis bei Menschen mit psychiatrischen Vorerkrankungen so zu gestalten, dass die Übungen kurz gehalten werden, dass keine große Anstrengung damit verbunden ist und nur eine geringe Konzentrationsspanne benötigt wird. Falls die therapeutische Arbeit mit mehreren Personen gleichzeitig stattfindet, sollten es kleine Gruppen sein. Es sollte angenehm bequem und warm genug sein, und die Augen soll- 
ten am besten nicht geschlossen werden. Atemübungen sollten sehr einfach gehalten und nur kurz ausgeführt werden. ${ }^{6}$

Um zu vermeiden, dass Symptome sich durch die achtsamkeitsbasierten Interventionen verstärken und es zu einer Re-Traumatisierung kommt, ist Lüers zufolge bei Menschen mit Traumata eine professionelle Betreuung durch Therapeutinnen und Therapeuten mit eigener Achtsamkeits- und Meditationserfahrung unabdingbar. ${ }^{7}$

David Treleaven, promovierter Psychologe, Traumatherapeut und Achtsamkeitsspezialist, betont in seinem Buch Traumasensitive Achtsamkeit. Posttraumatischen Stress erkennen und vermindern, dass das Praktizieren einfacher Meditationsübungen automatisch dazu führe, dass traumatische Stimuli wachgerufen werden. Für die Betroffenen ist es seiner Meinung nach wichtig, „dass sie ihren Fokus während der Achtsamkeitsübungen von den traumatischen Stimuli weglenken können“8. Dabei sollten „Aufmerksamkeitsanker" ausgesucht werden, wie beispielsweise Objekte im Raum oder das Gefühl der Füße auf dem Boden. ${ }^{9}$ Treleaven empfiehlt daher, nur kurze Übungen anzubieten. Bodyscans, beispielsweise, die in den regulären MBSRProgrammen ${ }^{10}$ nicht selten 30 bis 45 Minuten dauern, sind für Traumaüberlebende zu lang und können statt Entspannung, Beängstigung und ein Gefühl der Entfremdung auslösen. ${ }^{11}$

Dr. Brigitte Fuchs, Meditationslehrerin und Privatdozentin an der Universität Würzburg, warnt vor einem zu schnellen Heranführen an Meditationstechniken bei psychisch kranken Menschen. Sie hat für diese Zielgruppe das Konzept „Therapeutische Meditation" entwickelt, welches aus acht aufeinanderfolgenden Stufen besteht und die Teilnehmenden schrittweise an Achtsamkeits- und Meditationstechniken heranführt. ${ }^{12}$ Auch Fuchs plädiert für eine intensive und individuell abgestimmte Achtsamkeits- und Meditationsbegleitung der Betroffenen.

Der Professor für Psychologische Wissenschaften und Innere Medizin an der Texas University (USA), Yi-Yuan Tang, weist ebenfalls auf mögliche negative Folgen von Achtsamkeitsübungen bei verschiedenen Erkrankungen hin: „Hinsichtlich der Patientenpopulationen mit PTBS, Schizophrenie und Epilepsie ${ }^{13}$ oder bei Individuen mit einem Risiko für Psychosen oder Krampfanfälle wurden einige Bedenken geäußert, dass diese Individuen durch Achtsamkeitspraxis einem erhöhten Risiko für eine Verschlimmerung ihrer Symptome ausgesetzt seien oder dass Achtsamkeitspraxis unerwünschte Ereignisse oder Erfahrungen wie Träume oder depressive Episoden auslösen könnte. "14 Tang sieht wegen der geringen Menge an empirischen Studien die Notwendigkeit für weitere Forschungen, um die Anwendungsmöglichkeiten auf diesem Gebiet und die damit verbundenen Risiken besser auszuloten. ${ }^{15}$

Peter Sedlmeier, Professor für Forschungsmethodik und Evaluation, schreibt in seinem Buch Die Kraft der Meditation. Was die Wissenschaft darüber weiß: „Während mittlerweile weitgehend Einigkeit darüber herrscht, dass Meditation bei der Behandlung von Depression und Ängsten sowie weiteren emotionalen und psychischen Problemen hilfreich sein kann, wird nach wie vor kontrovers diskutiert, ob sie sich zur Therapie von Psychosen wie der Schizophrenie eignet. " ${ }^{16}$

Dr. Harald Piron, Psychologischer Psychotherapeut und Mitgründer der Society for Meditation and Meditation Research (SMMR) sowie des Qualitätszirkels für meditationsbasierte Psychotherapie, stellt in seinem Buch Meditationstiefe. Grundlagen, Forschung, Training, Psychotherapie eine Reihe an Übungen vor, die im psychotherapeutischen Kontext angewendet werden können; unter anderem für die Bereiche Angststörungen und Depressionen. ${ }^{17}$ Allerdings sieht Piron auch die Möglichkeit von Nebenwirkungen und Kontraindikationen einer Meditationspraxis im therapeutischen Kontext und warnt, dass in keinem Fall der Betroffene alleine gelassen werden darf, wenn belastende Elemente aus dem Unbewussten auftauchen. ${ }^{18}$ 
Hinsichtlich geeigneter meditativer Übungen bei Depression unterscheidet Piron in seinen detaillierten Beschreibungen zwischen motorischen, physiologischen, emotionalen und kognitiven Effekten. ${ }^{19}$ Insbesondere durch die Förderung eines Bewusstseins für Selbstverantwortlichkeit kann die Meditation den Betroffenen unterstützen, einen Weg aus der Depression zu finden. ${ }^{20}$

Niko Kohls weist darauf hin, dass achtsamkeitsbasierte Interventionen auf jeden Fall wirkmächtige Programme darstellen, mit denen höchst relevante gesundheitsförderliche Effekte erzielt werden können. Beispielsweise können standardisierte Achtsamkeitsprogramme bei Depressionen, mit Ausnahme von schweren Depressionen, sogar die Wirkung von Psychopharmaka übertreffen. ${ }^{21}$ Sie unterstützen die jeweilige Person unter anderem darin, präsenter im Hier und Jetzt zu sein und den aktuellen Moment bewusster wahrzunehmen und weniger auf negative Gedanken zu fokussieren. Indem das sogenannte Mindwandering, das Abschweifen der Gedanken, durch die Achtsamkeitspraxis reduziert wird, nimmt in der Regel auch das Grübeln und Sorgenmachen ab.

Ein wichtiger Faktor ist zudem, dass die Achtsamkeitspraxis die Selbstregulierung und Selbstwirksamkeit positiv beeinflusst und die Betroffenen besser aus eigener Kraft gegensteuern können, anstatt von Medikamenten abhängig zu sein. ${ }^{22}$ Durch die Achtsamkeitspraxis lässt sich zudem die Psychohygiene insgesamt verbessern. ${ }^{23}$

Was die Behandlung von Depressionen anbelangt, können auch die Meditationen der Liebenden Güte oder Metta-Meditationen unterstützend wirken. Diese Meditationstechniken stärken die Verbundenheit mit der sozialen Umwelt und wirken dem subjektiven Empfinden, isoliert zu sein, entgegen. Das Erleben der sozialen Verbundenheit ist laut dem Neurowissenschaftler Richard Davidson, einer der Pioniere der Achtsamkeits- und Meditationsforschung, ein wichtiger Parameter für eine gesunde Psyche. ${ }^{24}$ Menschen mit Depressionen haben im Gegensatz dazu oft eine niedergedrückte Stimmung und leiden nicht selten unter einem Gefühl der Einsamkeit und Ausgeschlossenheit. ${ }^{25}$ „Die Öffnung des Herzens belebt die Beziehungsfähigkeit. Das Erleben, geben zu können (und als Kranker nicht nur nehmen zu müssen), vermittelt ein Gefühl inneren Reichtums. "26

Bei der Anwendung von Achtsamkeits- und Meditationstechniken mit Personen mit akuten sowie nicht akuten psychiatrischen Erkrankungen ist es wichtig, dass diese Techniken nicht die Psychotherapie ersetzen. Werden sie ohne professionelle psychologische und psychotherapeutische Erfahrung des Vermittelnden angewendet, besteht eine große Gefahr, dass insbesondere bei schweren Störungen wie Schizophrenie und Borderline-Erkrankungen die Ansätze eher schädliche Auswirkungen haben. ${ }^{27}$ Piron betont: „Psychotherapie und Meditation schließen sich ja nicht aus, sondern können gleichberechtigt nebeneinander laufen. Sie lassen sich sogar aufeinander abstimmen. ${ }^{\text {"28 }}$

Dies beinhalt für Dr. med Ulrike Anderssen-Reuster, leitende Ärztin einer Klinik für Psychosomatik und Psychotherapie in Dresden-Neustadt, dass Achtsamkeits- und Meditationslehrerinnen und -lehrer erkennen sollten, wann Teilnehmende mit psychischen Störungen an Psychotherapeutinnen/Psychotherapeuten weiterverwiesen werden müssten, und umgekehrt, dass Psychotherapeutinnen/Psychotherapeuten Personen solche Ansätze ermöglichen, wenn dies aus gesundheitlicher Sicht möglich ist und diese Personen bei Vorschlägen Interesse dafür zeigen und/oder von sich aus den Wunsch äußern, solche Angebote wahrzunehmen. ${ }^{29}$ 


\section{Gesundheitliche Probleme ohne psychiatrische Vorerkrankung/Vorbelastung}

Auch wenn keine psychiatrische Vorerkrankung vorliegt, kann die Ausübung von Achtsamkeits- und Meditationstechniken dennoch zu Problemen führen. Da diese Übungen auf die persönliche Entwicklung der jeweiligen Person einwirken, können teils völlig neue Erfahrungen gemacht werden, die nicht immer positiv sind. Beispielweise das Erfahren einer gewissen Leere und Einsamkeit oder die Relativierung bisheriger Lebensgewohnheiten und Sichtweisen. Ebenso kann eine stärkere Empfindsamkeit in der Sinneswahrnehmung auftreten, die belastend sein kann. ${ }^{30}$

Missbrauchsmöglichkeiten und Gefahren, die bei der Ausübung einer Achtsamkeits- und Meditationspraxis vonseiten des Meditierenden entstehen können, sieht Piron unter anderem darin, wenn die Ausübung die eigene Ich-Zentrierung verstärkt; wenn sie zu sehr als Selbstoptimierung genutzt wird; wenn sie als Verdrängung für eigene Defizite genutzt wird, oder wenn die ausübende Person sich zu devot an einen spirituellen Lehrer bindet. ${ }^{31}$

Auch Sedlmeier warnt vor einem solchen Ausweichen vor den eigenen Defiziten: „Man sollte jedoch keinesfalls versuchen, seine emotionalen Schwierigkeiten und psychischen Probleme zu ignorieren und sie auf der Meditations-Überholspur zu lösen: Spiritual Bypassing wird nicht funktionieren." ${ }^{2}$

Um mehr Einsicht in das Praktizieren von Meditation und das mögliche Auftreten von spirituellen Krisen zu bekommen, plädieren Michael Tremmel und Dr. Ulrich Ott, Psychologen und Achtsamkeits- und Meditationsforscher am Bender Institute of Neuroimaging an der Justus-Liebig-Universität Gießen, für eine professionelle Systematisierung und Kategorisierung: „Auf längere Sicht bedarf es neuer Diagnosekategorien, um der Vielfalt meditativer Entwicklungsprozesse und möglicherweise krisenhafter Übergangsphänomene gerecht zu werden. ${ }^{\text {"33 }}$

\section{Münchner Modell: Umgang mit psychiatrischer Vorerkrankung/Vorbelastung}

Bei der Anwendung von Achtsamkeits- und Meditationsübungen im Hochschulkontext ist es oberste Priorität, dass mögliche Risiken aufgefangen werden, damit Teilnehmende nicht zu Schaden kommen. Es ist vorab zu klären, ob akute oder nicht akute psychiatrische Vorerkrankungen und Vorbelastungen vorliegen.

Der Dozentin, dem Dozenten kommt hier eine hohe Verantwortung zu. Falls dieser nicht medizinisch, psychologisch oder psychotherapeutisch geschult ist, sollte bei ersten Anzeichen einer psychiatrischen Vorerkrankung/Vorbelastung mit der psychosozialen Beratung vor Ort/an der Hochschule Kontakt aufgenommen werden.

Beim Münchner Modell wird die Auswahl der Teilnehmerinnen und -teilnehmer für die jeweiligen Achtsamkeits- und Meditationsangebote über ein Online-Bewerbungsverfahren durchgeführt. Aus diesem Grund wird bei allen Kursbeschreibungen als verbindliche Voraussetzung für die Teilnahme angegeben, dass die Teilnehmenden über eine stabile psychische Verfassung verfügen müssen.

Bei den Vertiefungsangeboten gilt es für die Teilnahme zudem nachzuweisen, dass man bereits Meditationsvorerfahrung hat. Dies kann der Besuch einer Einführungslehrveranstaltung zum Thema „Achtsamkeit und Meditation“ bzw. ein weiteres Angebot im Rahmen des Münchner Modells sein oder das eigene Praktizieren an einer anerkannten Meditationsschule. 
Seit dem SoSe 2020 wird den Teilnehmerinnen und -teilnehmern zur Klärung der emotionalen und psychischen Gesundheit in der ersten Unterrichtsstunde zusätzlich ein Merkblatt ausgehändigt, auf dem die Achtsamkeits- und Meditationspraxis in Kurzform erklärt wird. Auf dem Merkblatt unterschreiben die Teilnehmenden, dass sie nicht in psychiatrischer bzw. psychologischer Behandlung sind und keine Psychopharmaka einnehmen. Im Fall einer psychiatrischen Vorerkrankung/Vorbelastung müssen sie erst Rücksprache mit dem behandelnden Therapeuten bzw. der Therapeutin halten und eine Bescheinigung vorlegen, um an dem jeweiligen Kurs teilnehmen zu können. ${ }^{34}$

Um mehr über die psychische Verfassung der Teilnehmenden herauszufinden, gibt es bei allen Angeboten im Rahmen des Münchner Modells grundsätzlich zwei Möglichkeiten: zum einen in der Fragerunde der Einführungsstunde und zum anderen in den Gruppendiskussionen, die jeweils im Anschluss an die Übungen stattfinden. Bei den Lehrveranstaltungen gibt es zudem noch eine dritte Gelegenheit: bei der Durchsicht der Meditationstagebücher.

In der ersten Unterrichtseinheit findet eine Fragerunde statt: Warum haben sich die Teilnehmenden für das Angebot angemeldet? Was erwarten sie vom Angebot? Haben sie bereits Erfahrungen mit Achtsamkeits- und Meditationstechniken gemacht? Es wird auch darauf eingegangen, was Achtsamkeit und Meditation beinhalten und insbesondere auch, was Meditation nicht ist und welche Missverständnisse darüber kursieren. ${ }^{35}$ Betont wird vor allem, dass es nicht um Ehrgeiz oder ambitioniertes Wollen und Leistung geht. Auch die Bedeutung von Gesundheit und einer stabilen psychischen Verfassung wird erläutert. Wer sich hier unsicher ist und persönliche Fragen hat, kann nach dem Unterricht noch ausführlich mit mir darüber sprechen.

Zweitens finden nach den Übungen im Rahmen der Angebote regelmäßig Feedbackgespräche in Kleingruppen und anschließend in der großen Runde statt. Auf diese Weise kann beobachtet werden, wie die Teilnehmenden mit den jeweiligen Übungen zurechtkommen. Zudem können sie voneinander lernen, wie Übungen richtig praktiziert werden und wie mit eventuellen Schwierigkeiten umgegangen werden kann.

Im Rahmen der regulären Lehrveranstaltungen werden Mitte des Semesters die Meditationstagebücher für eine Woche abgegeben. Ich lese sie dann und habe damit eine zusätzliche Möglichkeit zu prüfen, wie die Teilnehmerinnen und Teilnehmer mit den jeweiligen Übungen zurechtkommen. Eventuelle Übungskorrekturen werden anschließend als Feedback im Rahmen der Lehrveranstaltungen besprochen, dies geschieht anonym; durch das Behandeln der einzelnen Schwierigkeiten in der ganzen Gruppe können auch die anderen Teilnehmenden davon lernen. Bei gravierenden Problemen wird mit den jeweiligen Teilnehmerinnen und Teilnehmern ein persönliches Einzelgespräch geführt, etwa direkt nach dem Unterricht oder in der Sprechstunde.

Das persönliche Gespräch steht den Teilnehmerinnen und Teilnehmern grundsätzlich immer offen. Daher ist neben Fachkompetenz das Vertrauen zwischen Lehrenden und den Teilnehmenden sehr wichtig (siehe S. 81).

Bislang gab es unter den 2000 Teilnehmerinnen und Teilnehmern an den Lehrveranstaltungen im Rahmen des Münchner Modells nur eine Studentin, die wegen eines akuten Traumas abgelehnt werden musste.

Auch bei den offenen Angeboten, die für alle Studierende frei zugänglich sind, mussten bislang nur wenige Interessentinnen und Interessenten abgewiesen werden. Diese waren nicht richtig "geerdet“. Nach einem persönlichen Gespräch wurde ihnen geraten, für eine gewisse Zeit weniger oder gar nicht zu meditieren. Ihnen wurden Anleitungen für alltagsbezogene Achtsamkeitsübungen mitgegeben, um sich im normalen (Alltags-)Leben mehr zu festigen. Die Möglichkeit, sich nach einer gewissen Zeit erneut für die Angebote anzumelden, wurden ihnen immer gegeben. 
In den letzten Jahren haben sich verstärkt Teilnehmerinnen und Teilnehmer mit körperlichen Einschränkungen, unter anderem Rollstuhlfahrer, für die Lehrveranstaltungen beworben. Weil eine stabile mentale und emotionale Verfassung gegeben war, konnten sie selbstverständlich teilnehmen. Dies hat sich als enorme Bereicherung für den Unterricht erwiesen. Manche Übungen mussten für sie etwas angepasst werden, beispielsweise wurde aus einer Gehmeditation eine „Rollmeditation“, was einen berührenden Effekt auf die anderen Teilnehmenden hatte. Auch die Gruppendiskussionen über den Umgang mit Problemen und belastenden Gedanken wurden tiefgehender. Einmal hat auch eine blinde Studentin an einer Lehrveranstaltung teilgenommen. Es war für alle Beteiligten beeindruckend, wie sie ihre Erfahrungen beim Meditieren geschildert hat.

\section{Zu ehrgeizige Zielsetzungen/ Zu anspruchsvolle Meditationstechniken}

Ein anderes Risiko ist, dass Teilnehmerinnen und Teilnehmer zu viel auf einmal erreichen möchten und sich zu schnell und extrem in die Übungen hineinsteigern. Bei den regulären Lehrveranstaltungen kann dieses Problem noch verstärkt werden, weil hier auch Leistungsnachweise samt Noten und ECTS vergeben werden. Das Risiko der Übermotivation versuche ich zu entschärfen, indem ich den Teilnehmenden verdeutliche, dass es um Qualität und nicht um Quantität geht. Es geht darum, sich selbst durch die Achtsamkeits- und Meditationspraxis besser kennenzulernen und gut auf die eigene innere Stimme zu hören. Vor allem geht es darum, mehr Einsicht in die eigenen mentalen, emotionalen und körperlichen Prozesse und deren Wechselwirkungen zu erhalten. $-\mathrm{Zu}$ welcher Tageszeit meditiere ich beispielsweise gerne, wann eher nicht? Warum läuft das Meditieren manchmal besser und manchmal weniger gut?

Ulrich Ott weist daraufhin, dass bei Personen mit zu ehrgeiziger Zielsetzung die Gefahr besteht, sich zu stark von der Realität zu entfernen, abzuheben und „zwei Zentimeter über dem Boden zu schweben“" ${ }^{36}$. Im Extremfall könnte dies zum Depersonalisations- und Derealisationssyndrom führen, wobei die jeweilige Person den Bezug zu sich selbst und zur Umwelt verliert. Ott zufolge ist es in diesem Fall ratsam, die Meditation zu reduzieren bzw. für eine gewisse Zeit auszusetzen. In erster Linie geht es dann darum, wieder bodenständiger zu werden, beispielsweise durch eine körperliche Tätigkeit wie Gartenarbeit. Auch eine balancierte, gesunde Ernährung ist dabei wichtig. Sogar eine "deftige Mahlzeit“ kann in manchen Fällen hilfreich sein. ${ }^{37}$

Neben der Gefahr, dass die Übungsmenge viel zu hoch ist, besteht das Risiko auch darin, zu anspruchsvolle und überfordernde Techniken auszuwählen. Dies ist nicht selten der Fall, wenn Meditationsanfänger sich für längere Schweige-Retreats anmelden. Insbesondere wenn auch noch zu wenig Schlaf und zu wenig Nahrung hinzukommen, besteht die Gefahr einer Überforderung. Ott vergleicht den Meditationsübungsweg mit dem Bergsteigen: „Wer oben glücklich ankommen will, muss den Weg gut planen." ${ }^{38}$

Im Rahmen des Münchner Modells sind die praktizierten Achtsamkeitsübungen gut und sicher durchführbar, ohne dass die Gefahr einer Überanstrengung besteht. Was die intensiveren Sitzmeditationen anbelangt, werden die Meditationszeiten schrittweise und entsprechend dem Erfahrungsstand der Teilnehmerinnen und Teilnehmer erhöht. Sind es zunächst nur ein paar Minuten, dauert die Sitzmeditation ab Mitte der Lehrveranstaltungsreihe 15 bis 20 Minuten. Auch für die Übungspraxis zu Hause wird empfohlen, nicht über 20 Minuten hinauszugehen. Wenn sich die Teilnehmenden vertiefend mit einem bestimmten Meditationsansatz aus- 
einandersetzen möchten, werden ihnen anerkannte, zertifizierte Meditationsschulen empfohlen, wo sie sich unter Leitung professioneller Lehrerinnen und Lehrer mit fortgeschritteneren Meditationstechniken vertraut machen können.

Beispielsweise haben sich inzwischen mindestens 50 Studierende aus den Lehrveranstaltungen des Münchner Modells in externen Meditationsangeboten mit der „Vipassana"-Meditationsform befasst und an 10-tägigen Retreats teilgenommen. Andere Teilnehmerinnen und Teilnehmer haben sich mittels MBSR-Kursen weiter geschult oder sich im Rahmen von Klosteraufenthalten mit christlichen Meditationsformen und Zen-Techniken auseinandergesetzt. Manche haben auch Zentren wie das InterSein von Thich Nhat Hanh oder das Münchner Sri-Chinmoy-Zentrum besucht.

\section{Praktizieren von Meditation ohne Begleitung durch Lehrer oder Gruppe}

Ein weiteres Risiko bei der Beschäftigung mit Meditation kann auch darin bestehen, dass man versucht, sich bestimmte Meditationsübungen selbst anzueignen und dadurch kein Feedback erhält, ob man die Übungen richtig ausführt. Auch der wichtige Austausch darüber fehlt in diesem Fall. Problematisch wird es insbesondere, wenn zudem noch Risiken wie psychiatrische Vorerkrankungen, zu ambitionierte Zielsetzungen und weitere nicht förderliche Persönlichkeitsmerkmale vorliegen. Wie oben bereits erläutert, ist in diesem Fall die Anleitung eines Lehrers erforderlich.

Allgemein gilt, dass eine professionelle Begleitung wünschenswert ist, insbesondere wenn man die Achtsamkeits- und Meditationspraxis intensivieren und vertiefen möchte. ${ }^{39}$ Praktiziert man dazu auch noch regelmäßig in einer Gruppe oder Gemeinschaft $\left(\right.$ Sangha ${ }^{40}$ ), hat man die Möglichkeit, Erfahrungen auszutauschen. ${ }^{41}$

Im Rahmen der Angebote des Münchner Modells wird viel Wert auf den Gruppenaustausch gelegt. Dies ist insbesondere bei den Lehrveranstaltungen der Fall, wo man sich wöchentlich begegnet. Im Gespräch mit der vertrauten Gruppe können die Teilnehmerinnen und Teilnehmer mehr über die Erfolge und Schwierigkeiten der anderen erfahren.

\section{Praktizieren von Meditation mit Begleitung durch unerfahrene Lehrer}

Harald Piron beschreibt in seinem bereits erwähnten Handbuch eine Anzahl von Qualitätskriterien, die bei der Suche nach einem geeigneten Meditationsangebot hilfreich sein können; unter anderem nennt er: die Qualifikation des Lehrers, die Authentizität der Schule und Übermittlung, die Organisationsstruktur und Selbstdarstellung, die Offenheit für Wissenschaft und Forschung sowie Eintrittsgelder und Teilnahmegebühren. ${ }^{42}$

Diese Kriterien sind deshalb so bedeutend, da Meditationstechniken eine starke Auswirkung auf die gesamte Psychohygiene des Menschen haben. „Aus einleuchtenden Gründen ist also äußerste Umsicht geboten, wenn man auf instinktive körperliche Prozesse, wie Atmung oder Herzschlag einzuwirken versucht. Ein Missbrauch des physischen Verfahrens kann die Gesundheit eines Menschen ruinieren und sein geistiges Gleichgewicht erheblich stören, von daher auch die hohe Bedeutung eines zuverlässigen Meisters. “43 
Wie im Forschungsbeitrag (S. 41ff.) und unter Fazit (S. 169ff.) erläutert, ist ein wesentlicher Punkt für eine erfolgreiche Implementierung von Achtsamkeits- und Meditationsangeboten die Art und Weise der Vermittlung. Dabei ist zu unterscheiden zwischen der Vermittlung von Basisachtsamkeitsübungen und der Vermittlung von Meditationstechniken.

Für Lehrerinnen/Lehrer und Dozentinnen/Dozenten bestehen diverse Ausbildungs- und Weiterbildungsmöglichkeiten, zum Beispiel die MBSR-Ausbildungen oder die Zertifikatsausbildungen zum Thema „Achtsamkeit in der Lehre“, wie sie beispielsweise die Hochschule und Universität Jena sowie die Hochschule Osnabrück anbieten (siehe S. 65f. und S. 171). Werden allerdings mehr nach innen gerichtete Sitzmeditationen unterrichtet, ist darüber hinaus eine langjährige Erfahrungspraxis des Meditationslehrers erforderlich. Piron schreibt dazu: „Erst nachdem er den meditativen Weg bei sich und anderen Weggefährten gründlich studiert und überprüft hat, und dann zu dem Ergebnis gelangt, dass dieser niemanden ernsthaft schadet, sondern in vielfacher Hinsicht hilft, wenn er in den rechten Art und Weise gegangen wird, kann er mit gutem Gewissen diese Art der Praxis unterrichten. “44

Im Rahmen des Münchner Modells benötigen Dozentinnen und Dozenten für die Vermittlung der Sitzmeditationen eine mehrjährige Meditationserfahrung sowie die nachweisliche Teilnahme an Schweige-Retreats. Mehrmals im Semester finden unter den Lehrenden Austauschtreffen statt, bei denen über die Lehrerfahrungen und verwandte Themen gesprochen wird.

Ein guter Lehrer soll vor allem authentisch sein. „Worauf es ankommt ist, dass etwas von dem Weg, den er selbst zum überwiegenden Teil seines bisherigen Lebens gegangen ist, durch ihn hindurch scheint bzw. in ihm auf authentische Weise zum Ausdruck kommt. “45

\section{Fazit}

Trotz der in diesem Abschnitt beleuchteten Risiken, die bei nicht korrekter Anwendung von Achtsamkeits- und Meditationsansätzen sowie im Zusammenhang mit einer problematischen psychischen Disposition auftreten können, sprechen die zahlreichen positiven Effekte für sich. Insbesondere in den letzten zwei Jahrzehnten hat die Achtsamkeits- und Meditationsforschung wissenschaftlich belegt, dass Achtsamkeit und Meditation beispielsweise die Psychohygiene, die Konzentration und Aufmerksamkeit, die Emotionsregulierung sowie einen konstruktiven Umgang mit Stress fördern (siehe S. 46ff.).

Auch in den zehn Jahren des Münchner Modells haben sich die bisherigen wissenschaftlichen Forschungsergebnisse bestätigt. Ich habe zahlreiche positive Auswirkungen von Achtsamkeit und Meditation feststellen können, die sich bei der Beobachtung im Unterricht, bei den Diskursen mit den Teilnehmerinnen und -teilnehmern sowie beim Lesen der Meditationstagebücher zeigten. Werden die oben genannten Punkte bezüglich Risiken berücksichtigt, spricht meines Erachtens nichts dagegen, mit Achtsamkeits- und Meditationstechniken zu beginnen. Es wäre bedauerlich, wenn Studierenden, die ein aufrichtiges Interesse dafür zeigen, diese Möglichkeit vorenthalten wird. ${ }^{46}$ 


\section{QUELLEN}

Anderssen-Reuster, Ulrike (2015). „Achtsamkeit in Psychosomatik und Psychotherapie“, in: Zimmermann, Michael / Spitz, Christof / Schmidt, Stefan (Hrsg.). Achtsamkeit. Ein buddhistisches Konzept erobert die Wissenschaft. Bern: Verlag Hans Huber, S. 103-114.

Fuchs, Brigitte (2011). „Sitzen in Stille, was kann das schon bewegen? Meditieren mit kranken Menschen“, in: Büssing, Arndt / Kohls, Niko (Hrsg.), Spiritualität transdisziplinär. Wissenschaftliche Grundlagen im Zusammenhang mit Gesundheit und Krankheit. Berlin / Heidelberg: Springer-Verlag, S. 223-229.

Gunaratana, Mahathera Henepola (1996). Die Praxis der Achtsamkeit. Eine Einführung in die Vipassana-Meditation. Heidelberg: Werner Kristkeitz Verlag.

Hölzel, Britta / Brähler, Christine (2015). „Achtsamkeit mitten im Leben“, in: Hölzel, Britta / Brähler, Christine (Hrsg.), Achtsamkeit mitten im Leben. Anwendungsgebiete und wissenschaftliche Perspektiven. München: O. W. Barth Verlag, S. 7-19.

Hofmann, Liane / Heise, Patrizia (2017). „Spirituelle Krisen: allgemeine Kennzeichen, Auslöser und Gefährdungsfaktoren“, in: Hofmann, Liane / Heise, Patrizia (Hrsg.), Spiritualität und spirituelle Krisen. Handbuch zu Theorie, Forschung und Praxis. Stuttgart: Schattauer GmbH, S. 141-155.

Kabat-Zinn, Jon (2019). Gesund durch Meditation. München: Knaur Verlag.

Kohls, Niko (2017). „Spiritualität und außergewöhnliche Erfahrungen im Kontext der akademischen Psychologie“, in: Hofmann, Liane / Heise, Patrizia (Hrsg.), Spiritualität und spirituelle Krisen. Handbuch zu Theorie, Forschung und Praxis. Stuttgart, S. 3-14.

Piron, Harald (2020). Meditationstiefe. Grundlagen, Forschung, Training, Psychotherapie. Berlin: Springer-Verlag.

Ricard, Matthieu (2009). Meditation. München: nymphenburger.

Sedlmeier, Peter (2016). Die Kraft der Meditation. Was die Wissenschaft darüber weiß. Reinbek bei Hamburg: Rowohlt Taschenbuch Verlag.

Segal, Zindel V. / Williams, J. Mark G. I Teasdale, John D. (2015). Die Achtsamkeitsbasierte Kognitive Therapie der Depression. Tübingen: dgvt-Verlag.

Tang, Yi-Yuan (2019). Die Wissenschaft der Achtsamkeit. Wie Meditation die Biologie von Körper und Geist verändert. Paderborn: Junfermann Verlag.

Thich Nhat Hanh (2005). Friedlich miteinander leben. Handbuch für harmonische Gemeinschaft. München: Lotos Verlag.

Treleaven, David (2019). Traumasensitive Achtsamkeit. Posttraumatischen Stress erkennen und vermindern. Freiburg im Breisgau: Arbor Verlag.

Tremmel, Michael / Ott, Ulrich (2017). „Negative Wirkungen von Meditation“, in: Hofmann, Liane / Heise, Patrizia (Hrsg.), Spiritualität und spirituelle Krisen. Handbuch zu Theorie, Forschung und Praxis. Stuttgart: Schattauer Gmb, S. 233-243.

Ware, Kallistos / Jungclaussen, Emmanuel (2004). Hinführung zum Herzensgebet. Freiburg im Breisgau: Verlag Herder.

\section{Zeitschrift}

Davidson, Richard (2018). „Interview“, in: Moment by Moment, Ausgabe 03. Freiburg: Hammer Solutions Media, S. 56-63.

Ott, Ulrich (2018). „Meditation auf dem Prüfstand“, in: Moment by Moment, Ausgabe 03, Freiburg: Hammer Solutions Media, S. 64-67. 
DVD

Ambo, Phie (2013). Free the Mind. Kann ein Atemzug dein Denken verändern? Köln: mindjazz pictures.

\section{Internet}

Brown University (USA). Meditation Safety Toolbox: https://www.brown.edu/research/labs/britton/meditation-safety-toolbox (letzter Zugriff: 13.09.2020)

Davidson, Richard J. (2019a). Vortrag und Podiumsdiskussion: „We can change the brain, by changing the mind“ am 19. März 2019 in München beim Forum für den Wandel der Edith-Haberland-Wagner Stiftung, www.ehw-stiftung.de (letzter Zugriff: 13.09.2020)

Ott, Ulrich (2016). Vortrag: „Ist Achtsamkeit für jeden gut? Neue Forschungsergebnisse.“ im Rahmen der Vortragsreihe „Achtsamkeit - kritischer Blick auf einen Trend“ der Universität Hamburg am 17. November 2016, https://lecture2go.unihamburg.de/I2go/-/get/v/20619 (letzter Zugriff: 13.09.2020).

1 Vgl. Piron 2020, S. 214ff.; Hofmann / Heise 2017; Tremmel / Ott 2017; Ott 2018, S. 64-67; Fuchs 2011, 223ff.; Sedlmeier 2016, S. 174ff.; siehe auch den Vortrag „Ist Achtsamkeit für jeden gut? Neue Forschungsergebnisse." von Dr. Ulrich Ott im Rahmen der Vortragsreihe „Achtsamkeit - kritischer Blick auf einen Trend" der Universität Hamburg am 17. November 2016. Der Vortrag ist im Internet abrufbar unter: https://lecture2go.uni-hamburg.de/l2go/-/get/v/20619 (letzter Zugriff: 13.09.2020). Was die Forschung bezüglich Risiken anbelangt, hat die Brown University (USA) eine Meditation Safety Toolbox erstellt mit einer Reihe an Dokumenten, Protokollen und Best-Practice-Richtlinien des UMass Memorial Center for Mindfulness, der Achtsamkeitszentren in Bangor und Oxford sowie weiterer Achtsamkeitsforscher. Sie ist erhältlich unter: https://www.brown.edu/research/labs/britton/ meditation-safety-toolbox (letzter Zugriff: 13.09.2020). Zum Thema „Spirituelle Krisen und Krisenbegleitung" siehe auch Tremmel / Ott 2017, S. 242.

2 Telefoninterview mit Prof. Dr. Niko Kohls am 5. April 2020. Siehe auch Piron 2020, S. 217.

3 Für mögliche Anzeichen einer Dissoziation und abfedernde Maßnahmen siehe Treleaven 2019,

S. $206 f f$.

4 Skype-Interview mit Dr. Ortwin Lüers am 5. April 2020.

5 Vgl. Treleaven 2019, S. 209ff.

6 Skype-Interview mit Dr. Ortwin Lüers am 5. April 2020. Für Kriterien achtsamkeitsbasierter Methoden als Therapie für Menschen mit psychotischen Störungen siehe auch Sedlmeier 2016, S. $187 \mathrm{f}$.

7 Vortrag „Meditation bei Traumafolgestörungen. Grenzen und Möglichkeiten“ auf der 16. SMMR-Tagung „Meditation und Mitgefühl“, 7.-9. Oktober 2016. Zudem Skype-Interview mit Dr. Ortwin Lüers am 5. April 2020

8 Treleaven 2019, S. $170 f$

9 Vgl. Treleaven 2019, S. 171.

10 Für eine Einführung in Mindfulness-Based Stress Reduction (MBSR) siehe Kabat-Zinn 2019.

11 Vgl. Treleaven 2019, S. 209f.

12 Vgl. Fuchs 2011, S. 226ff.

${ }^{13}$ Im Fall von Epilepsie wird zum Teil auch über eine Reduktion von Anfällen berichtet, allerdings liegen bislang noch keine ausreichenden Forschungsergebnisse vor (vgl. Tremmel / Ott 2017, S. 235).

14 Tang 2019, S. 105.

15 Vgl. Tang 2019, S. 105. Eine Kurzstudie der University of Wiscon-Madison und des Center for Healthy Minds unter Leitung von Richard Davidson zeigte beispielsweise, dass spezielle Atemübungen bei Kriegsveteranen mit PTBS für mehrere Studienteilnehmer unter anderem Schlafstörungen sowie die Abhängigkeit von Psychopharmaka reduzierten (siehe Ambo 2013).

16 Sedlmeier 2016, S. $186 f$

17 Siehe Piron 2020, S. $177 f f$.

18 Vgl. Piron 2020, S. $214 f f$

19 Vgl. Piron 2020 188ff.

20 Vgl. Piron 2020, S. $194 f$.

${ }^{21}$ Vortrag „Achtsamkeit - Konzept, Forschungsstand und Messung“ an der LMU München, November 2013.

${ }^{22}$ Für die Auswirkung von Selbstkontrolle auf Depressionen siehe Tang 2019, S. $86 f f$. 
${ }^{23}$ Für eine auf Achtsamkeitsinterventionen basierende kognitive Therapie der Depression siehe Segal / Williams / Teasdale 2015.

${ }^{24}$ Siehe Vortrag und Podiumsdiskussion „We can change the brain, by changing the mind“ am

19. März 2019a, Prof. Dr. Richard Davidson in München beim Forum für den Wandel der Edith-Haberland-Wagner Stiftung, www.ehw-stiftung.de (letzter Zugriff: 13.09.2020); vgl. auch Davidson 2018.

${ }^{25} \mathrm{Vgl}$. Anderssen-Reuster 2015, S. 111; siehe auch Podiumsdiskussion „We can change the brain, by changing the mind" am 19. März 2019a, Erick Rinner MBA, in München beim Forum für den Wandel der Edith-Haberland-Wagner Stiftung, www.ehw-stiftung.de (letzter Zugriff: 13.09.2020).

${ }^{26}$ Fuchs 2011, S. 228.

${ }^{27}$ Vgl. Sedlmeier 2016, S. $102 f$.

28 Piron 2020, S. 221f.; siehe auch Hölzel / Brähler 2015, S. $12 f$.

29 Vgl. Anderssen-Reuster 2015, S. 112; siehe auch Treleaven 2019, S. $25 f$.

30 Vgl. Tremmel / Ott 2017, S. 235f.; siehe auch Kohls 2017, S. 10ff.

31 Vgl. Piron 2020, S. 220ff.; siehe auch Hofmann / Heise 2017, S. 146ff.

32 Sedlmeier 2016, S. 193.

33 Tremmel / Ott 2017, S. $242 f$.

34 Diese Vorgehensweise wird von Prof. Dr. Niko Kohls im Rahmen seiner Achtsamkeits- und Meditationslehrveranstaltungen an der Hochschule Coburg seit Jahren erfolgreich gehandhabt. Auch bei MBSRKursen erhalten Interessenten bei der Anmeldung zunächst einen Fragebogen, mit dem unter anderem geprüft wird, ob man schon einmal suizidale Gedanken und/oder Depressionen hatte.

${ }^{35} \mathrm{Vgl}$. Gunaratana 1996, S. 26ff; siehe auch Ricard 2009, S. 32f.; für Missverständnisse bezüglich Achtsamkeitsmeditation siehe auch Tang 2019, S. 89ff.

36 Ott 2018, S. 67

$37 \mathrm{Vgl}$. Ott 2018, S. 67.

38 Ott 2018, S. 66; siehe auch Tremmel / Ott 2017, S. 241.

39 Vgl. Sedlmeier 2016, S. 193.

40 Vgl. Thich Nhat Hanh 2005, S. $11 \mathrm{ff}$.

${ }^{41} \mathrm{Vgl}$. Ott 2018, S. $66 f$

${ }^{42}$ Vgl. Piron 2020, S. $226 f f$.

${ }^{43}$ Ware / Jungclaussen 2002, S. 60f; vgl. Hofmann / Heise 2017, S. 151.

44 Piron 2020, S. 227

45 Piron 2020, S. 226.

${ }^{46}$ Vgl. Sedlmeier 2016, S. 194; siehe auch Ott 2018, S. 67 und Piron 2020, S. 217.

Wie fängt man einen Elefanten?

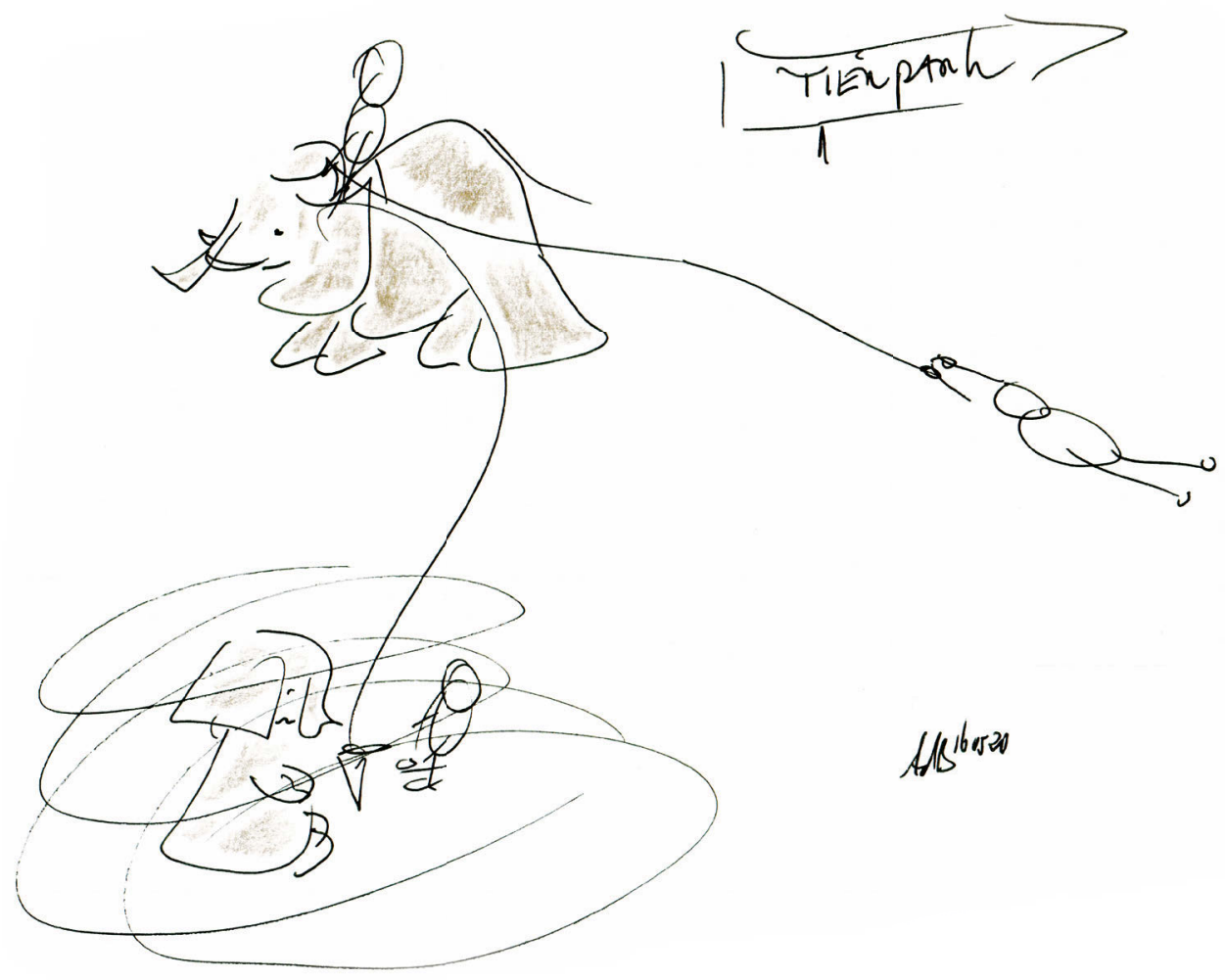




\title{
Sitzmeditationen
}

\author{
Wenn der Mensch ein inwendiges Werk werden will, \\ so muss er all seine Kräfte in sich ziehen, wie in einen \\ Winkel seiner Seele, und muss sich verbergen vor allen \\ Bildern und Formen, und da kann er dann wirken. \\ Da muss er in ein Vergessen und \\ in ein Nichtwissen kommen.
}

Meister Eckhart (1260-1327)

„Hat jemand schon mal einen Elefanten eingefangen?“ Ich stelle diese Frage oft zu Beginn der ersten Lehrveranstaltung. - Bislang noch niemand.

Stellen Sie sich einen Elefanten vor, der aus dem Münchner Tierpark Hellabrunn entkommen ist und sich nun auf dem Weg zum Marienplatz befindet. Er ist neugierig und will sich einfach einmal umsehen.

Und stellen wir uns vor, dass wir ihn davon abhalten und ihn zurückbringen wollen. - Wie könnte uns das gelingen? Wie können wir ihn fangen? Was wird passieren, wenn wir beispielsweise versuchten, auf ihn zu klettern, um ihn zu stoppen?

Es würde nicht funktionieren! Der Elefant ist zu stark und würde seine Reise einfach fortsetzen.

Wir könnten auch versuchen, ihn mit einem Seil festzuhalten. - Was wird passieren? Sie würden sicherlich mitgezogen werden. Das funktioniert also auch nicht.

Wir könnten das Seil aber an etwas besonders Festes und Stabiles binden, zum Beispiel an einen fest verankerten Brückenpfahl oder einen großen Baum. Dazu müssten wir sicher wissen, dass das Seil sehr reißfest ist und viel aushalten kann. - Was würde passieren? Der Elefant wird am Seil ziehen und sich vielleicht auch um unseren stabilen Anker mehrfach im Kreis drehen, bis er letztendlich müde wird. Und währenddessen können wir uns entspannen.

Der Grund, warum ich diese Kurzgeschichte erzähle, ist, dass der Elefant hier eine Metapher für unser Denken und unsere Gedanken ist. Wenn wir meditieren, werden wir mit dem Denken und den Gedanken konfrontiert. Wenn wir dabei nicht achtsam sind, zerren diese uns mit und wir verlieren uns darin. Es ist beim Meditieren wichtig zu lernen, Abstand von den Gedanken zu nehmen. Um dies zu erleichtern, kann man sich auf etwas anderes konzentrieren. In unserer Geschichte ist es ein Brückenpfahl oder Baum. Diesen Fokus zu halten, ist ein wesentlicher Teil aller Meditationsformen. ${ }^{1}$

Im Unterricht wurden vier verschiedene Meditationsobjekte für die Sitzmeditation vorgestellt, und zwar die Fokussierung auf:

- den Atem

- das Mantra „Maranatha“

- den Punkt zwischen den Augenbrauen

- das Mantra „So’ham“ / „Ich bin Das“ 


\section{Atemmeditation}

Die Fokussierung auf den Atem wird in zahlreichen Meditationsschulen praktiziert. Bei manchen Traditionslinien wie der Vipassana-Tradition geht es vor allem um Einsicht in Gedanken, Emotionen und körperlichen Prozesse und ihre Wechselwirkungen. „Wenn Sie eine schlechte Zeit haben, untersuchen Sie, worin dieses Übel besteht, beobachten Sie es achtsam, studieren Sie das Phänomen und lernen Sie seine Mechanismen kennen. Der Weg aus der Falle besteht darin, die Falle selbst zu erforschen, herauszufinden, wie sie gebaut ist. Sie schaffen dies, indem Sie das Ding Stück für Stück auseinandernehmen. Die Falle kann nicht zuschnappen, wenn sie in Stücke

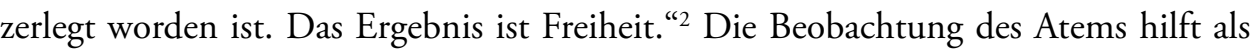
Ankerpunkt, sich bei diesem Unterfangen nicht in den verschiedenen Gedanken, Emotionen und körperlichen Reaktionen zu verlieren.

Auch bei den Sitzmeditationen im MBSR-Programm nutzt man den Atem vor allem als Ankerpunkt, um „bei sich zu bleiben“ und von hier aus Gedanken zu beobachten, ohne sie zu bewerten.

Wir haben uns bei der Atemmeditation auf den eingehenden und ausgehenden Atem bei den Nasenflügeln konzentriert und versucht, Gedanken nicht zu betrachten. Es ging darum, in Stille zu verweilen und zu versuchen, sich mehr nach innen zu wenden. ${ }^{3}$

\section{Anleitung}

Klangschale 1x anschlagen

\section{Entspannt und aufrecht sitzen}

\section{Fokussierung bei den Nasenflügeln}

Der Atem geht ein

Der Atem geht wieder raus

Ganz entspannt, jeder in seinem eigenen Tempo; wenn man abschweift, entspannt zurück zu den Nasenflügeln.

Wenn man möchte, kann man auch dabei zählen, beispielsweise auf 1 einatmen, auf 1 ausatmen; auf 2 einatmen und ausatmen, auf $3 \ldots$ und so weiter bis 5 und dann wieder von vorne.

Oder Sie können auch innerlich beim Einatmen „ein“ und beim Ausatmen „aus“ sagen. ${ }^{4}$

\section{Ab und zu Klangschale 1x anschlagen}

\section{$\mathrm{Ab}$ und zu erinnern an Fokussierung bei den Nasenflügeln ...}

\section{Am Schluss Klangschale 3x anschlagen}

Wenn man diese Meditationstechnik regelmäßig praktizieren möchte, sollte man sie je nach Bedarf 15 bis 20 Minuten ein- oder zweimal täglich durchführen.

Im Unterricht wird die Meditationszeit schrittweise gesteigert. In der ersten Sitzung sind es nur wenige Minuten. In jeder weiteren Unterrichtseinheit kommen einige Minuten hinzu, bis wir am Ende bei 15 bis 20 Minuten angelangt sind. 
Für den Unterricht benutzte Primärliteratur

Gunaratana, Mahathera Henepola (1996). Die Praxis der Achtsamkeit. Eine Einführung in die Vipassana-Meditation. Heidelberg: Werner Kristkeitz Verlag.

Kabat-Zinn, Jon (2019). Gesund durch Meditation. München: Knaur Verlag.

Ott, Ulrich (2015). Meditation für Skeptiker. Ein Neurowissenschaftler erklärt den Weg zum Selbst. München: Droemer Knaur.

Ricard, Matthieu (2009). Meditation. München: nymphenburger.

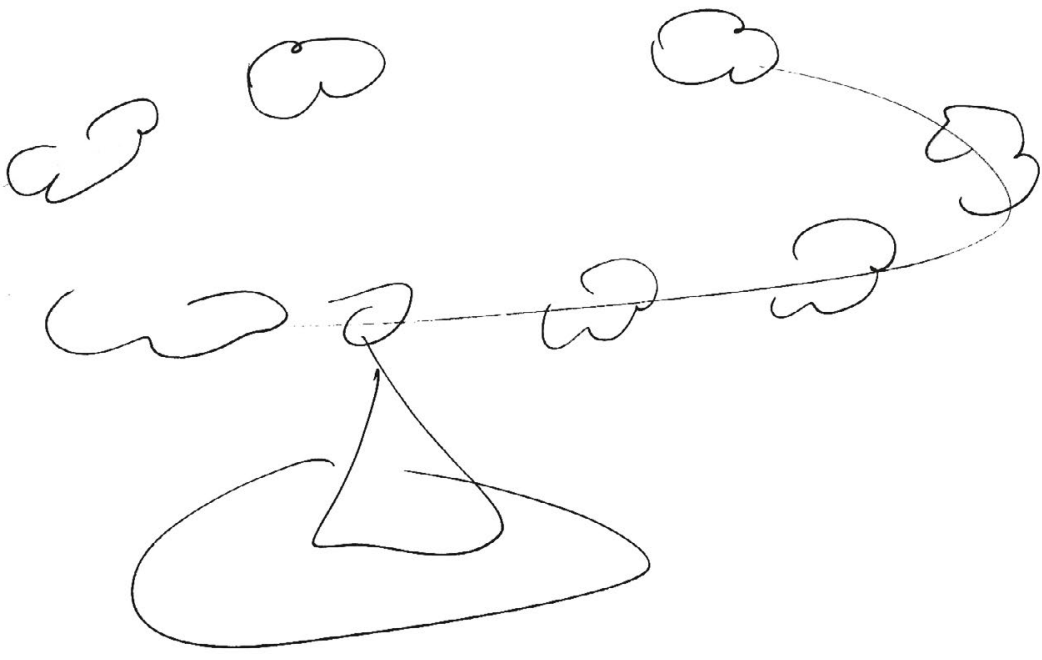

Adb

Das Denken denkt, aber wir sind nicht das Denken!

18.5 .19

\section{Mantra-Meditation „Maranatha"}

Während bei den bisherigen Angeboten etwa die Hälfte der Teilnehmerinnen und Teilnehmer die Atemmeditation bevorzugt, hat sich für fast ein Drittel der Teilnehmenden herausgestellt, dass für sie das Meditieren mithilfe eines Mantras („Maranatha“) einfacher ist. Auf diese Weise sind sie nicht so sehr mit aufkommenden Gedanken beschäftigt und gelangen leichter in einen Zustand der Stille. ${ }^{5}$ Mit der Einführung eines weiteren Mantras, „So'ham“ (siehe S. 107), im SoSe 2019 hat die Zahl der Teilnehmenden, die ein Mantra bevorzugt, weiter zugenommen.

Der Begriff „Mantra“ stammt aus dem Sanskrit und bezeichnet eine heilige Silbe, ein heiliges Wort oder einen heiligen Vers als „Klangkörper“ einer spirituellen Kraft, die durch Rezitieren (laut, leise oder lautlos, innerlich) manifestiert werden soll. Das Wiederholen des Mantras bietet einen Fokussierungspunkt und dient zur Ausrichtung des Geistes. ${ }^{6}$

Im Hinduismus und Buddhismus haben Mantras eine lange Tradition und werden seit Jahrtausenden benutzt. Aber auch im Christentum gibt es Mantras, wie beispielsweise das Mantra „Maranatha“, das wir im Unterricht angewendet haben. Es ist ein urchristliches Mantra und stammt aus dem Aramäischen, der Sprache Jesu. Bekannt 
wurde das Beten und Meditieren mithilfe dieses Mantras vor allem durch die Arbeit des katholischen Priesters und Benediktinermönchs John Main (1926-1982). Während einer Beamtenarbeit für den britischen Kolonialdienst in Kuala Lumpur, Malaysia, lernte er Swami Satyananda (1923-2009) kennen, der ihn in die Meditationspraxis mit dem Mantra „Maranatha“ einwies. Main hat diesen Ansatz daraufhin in christlichen Kreisen verbreitet. Daraus entstanden christliche Meditationsgruppen, die sich zu der Weltgemeinschaft für Christliche Meditation (WCCM) ${ }^{7}$ zusammengeschlossen haben.

„Bei der Meditation ist es einfach die beharrliche Treue zum Sprechen des Mantra, was uns dieser Wahrnehmung des in uns betenden Heiligen Geistes immer näherbringt. Indem wir unermüdlich treu unser Mantra wiederholen, webt es allmählich unser gesamtes Wesen zur Ganzheit zusammen. Dies bewirkt es dadurch, dass es uns ins Schweigen und in die Konzentration führt und somit auf jene Bewusstseinsebene, die wir erreichen müssen, um Geist und Herz für das Wirken der Liebe Gottes in der Tiefe unseres Wesens zu öffnen."

Das Wort steht für mehrere Bedeutungen: „Unser Herr ist gekommen“ oder „Unser Herr wird kommen“ oder - am wahrscheinlichsten - „Komm, Herr. Komm, Herr Jesus, komm!“9

Bedeutungsmöglichkeiten sind auch „Jesu sei bei mir“ oder „Ich rufe das Göttliche in mir an". Insbesondere die letzte Übersetzung eignet sich für Menschen, die sich nicht direkt mit dem christlichen Glauben identifizieren können.

Die Teilnehmerinnen und Teilnehmer können bei dieser Übung selbst entscheiden, welche Übersetzung für das Mantra „Maranatha“ sie für sich am geeignetsten finden.

\section{Anleitung}

Klangschale $1 \mathrm{x}$ anschlagen

Entspannt und aufrecht sitzen

Sie können die Hände in den Schoß oder auf die Oberschenkel legen.

Augen geschlossen, ganz entspannt

Atmen Sie bewusst und ruhig ein und aus. Der Atem kommt und geht von alleine, ganz entspannt.

Und jetzt können Sie das Mantra innerlich sprechen.

Sie können das Mantra in vier Silben aufteilen: MA-RA-NA-THA.

Sprechen Sie das Mantra innerlich aus und hören Sie dabei auf den innerlichen Klang.

Der Atem fließt dabei auf natürliche Weise. Sie können den Atem auch bewusster mit dem Aussprechen des Mantras verbinden. Zum Beispiel beim Einatmen sprechen Sie MARANATHA, beim Ausatmen sprechen Sie nicht und bleiben in Stille. Oder Sie atmen ein mit den ersten beiden Silben MA-RA und atmen aus mit den Silben NA-THA. ${ }^{10}$ Wichtig ist, dass der Rhythmus fließend und natürlich bliebt und nicht mechanisch geschieht.

Am Schluss Klangschale 3x anschlagen

Was die Dauer für eine regelmäßige Meditationspraxis anbelangt, schlägt John Main zumindest 20 Minuten, besser aber 25 oder 30 Minuten, pro Meditationssitzung vor. ${ }^{11}$ 
Für den Unterricht benutzte Primärliteratur

Main, John (2015). Das Herz der Stille. Einführung ins Herzensgebet. München: Claudius Verlag.

Ng, Peter (Hrsg.) (2008). Die Sehnsucht nach Tiefe und Sinn. Meditieren mit John Main. Bielefeld: Aurum.

Ware, Kallistos / Jungclaussen, Emmanuel (2004). Hinführung zum Herzensgebet. Freiburg im Breisgau: Verlag Herder.

Die Höchste Meditation ist der Zustand der völligen inneren Stille. In diesem Zustand taucht nicht ein einziger Gedanke im Geist auf. Allerdings können die meisten Menschen diese innere Stille nicht sofort erlangen. Aus diesem Grunde ist es für den Meditierenden von größter Wichtigkeit zu wissen, wie er mit dem Geist umgehen soll.

Swami Muktananda (1908-1982)

\section{Fokussierung zwischen den Augenbrauen}

Eine weitere Meditationsform ist die Fokussierung zwischen den Augenbrauen.

Anleitung

Klangschale $1 \mathrm{x}$ anschlagen

Entspannt die Augen schließen

Fokussierung zwischen den Augenbrauen

Aber ganz entspannt, ohne Druck

Augen nicht innerlich dorthin richten

Es ist eine mentale Ausrichtung.

Entspannt sein, aufmerksam und präsent sein

$\mathrm{Ab}$ und zu Klangschale anschlagen

Ab und zu sagen: „Fokussierung zwischen den Augenbrauen halten“

Am Ende Klangschale 3x anschlagen

Für den Unterricht benutzte Primärliteratur

Keine für den Unterricht benutzte Primärliteratur. 


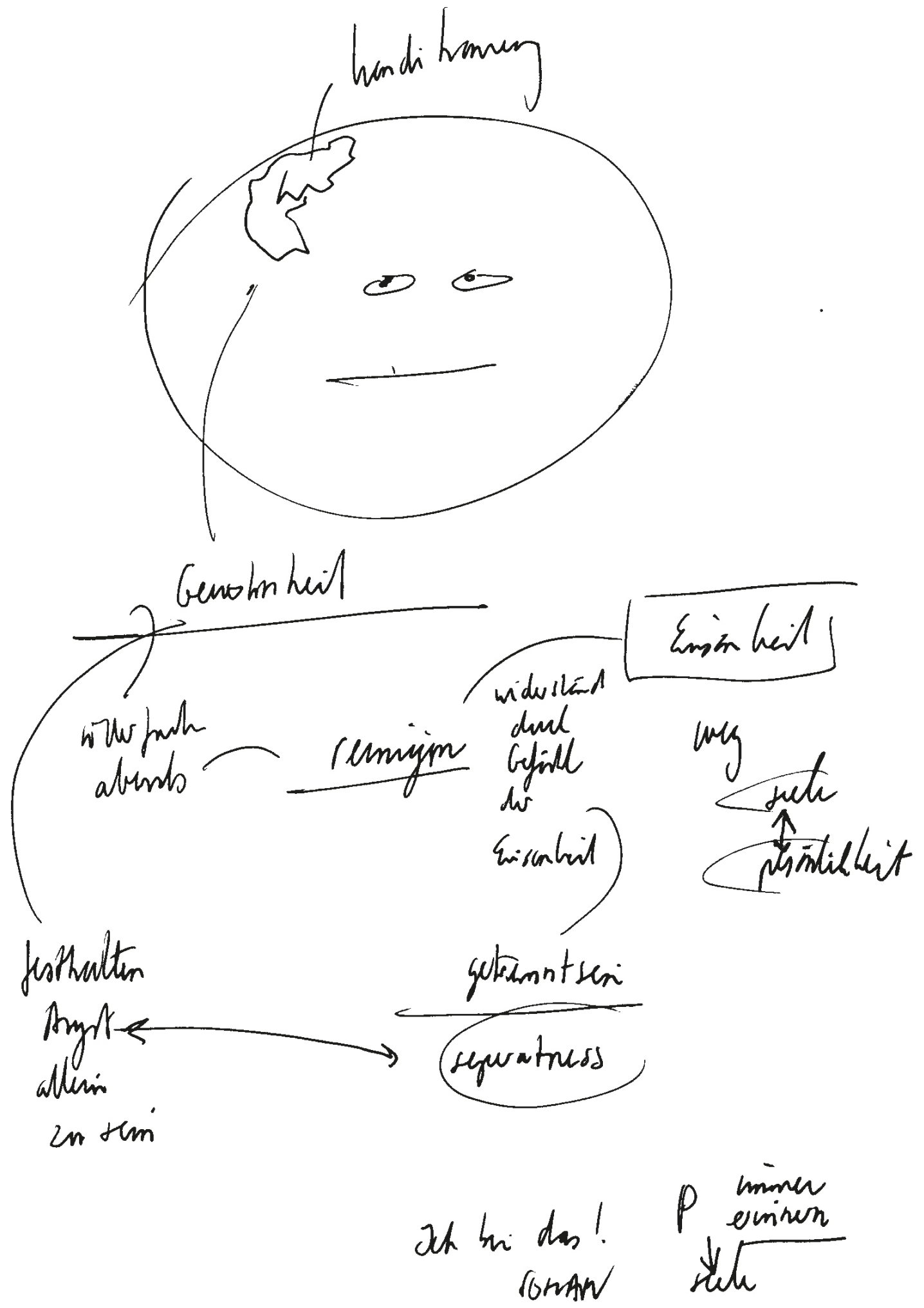

Wir sind konditioniert, aber wir können uns auch wieder davon befreien. 


\section{Mantra-Meditation „So'ham" / „Ich bin Das"}

Eine Meditationsform, die erst viel später ins Lehrprogramm aufgenommen wurde und daher kaum in den bisherigen Tagebuchnotizen der Studierenden auftaucht, ist die Meditation mit dem Mantra "So'ham“, das eines der Hauptmantras der SiddhaYoga-Tradition ist und „Ich bin Das“12 bedeutet.

"So'ham ist das natürliche Mantra, das Mantra des Selbst. Es gehört nicht zum Osten oder zum Westen oder zu irgendeiner Religion. So'ham ist Teil unserer Natur; es wiederholt sich ständig von selbst, zusammen mit unserem Atem. (...) Der Atem fließt aus mit Klang So und fließt ein mit dem Klang Ham. Bei jedem Ein- und Ausatmen findet eine Wiederholung des So'ham-Mantras statt. "'13 Wichtig dabei ist auch auf die Augenblicke der Stille zu achten, die während des Atmens entstehen. „Zwischen dem Ein- und Ausatmen und zwischen dem Aus- und Einatmen gibt es jeweils einen Augenblick, der absolut still und frei von Gedanken ist. Jener Raum ist der Raum der höchsten Wahrheit. Wenn du ein- und ausatmest und dabei dem Ham und So zuhörst, solltest du dich auf jenen inneren Raum konzentrieren, solange er andauert. Wenn du diese Technik übst, wird sich dieser Raum allmählich ausdehnen. " ${ }^{14}$

Diese Mantra-Meditation wurde vor allem durch den indischen spirituellen Lehrer Swami Muktananda (1908-1982) im Westen bekanntgemacht und seine Lehren werden heute durch seine Nachfolgerin Swami Chidvilasananda weitergegeben.

\section{Anleitung}

Klangschale $1 \mathrm{x}$ anschlagen

Entspannt und aufrecht sitzen, Augen geschlossen

Atmen Sie bewusst und ruhig ein und aus.

Der Atem kommt und geht von alleine, ganz entspannt.

Wiederholen Sie nun lautlos das Mantra.

Beim Ausatmen So, beim Einatmen Ham

Lassen Sie den Klang So mit dem Atem ausfließen.

Lassen Sie den Klang Ham mit dem Atem einfließen.

Achten Sie dabei auch auf die Momente der Ruhe und Stille

zwischen dem Aus- und Einatmen und dem Ein- und Ausatmen.

$\mathrm{Ab}$ und zu Klangschale anschlagen

Ab und zu wiederholen: „Beim Ausatmen So, beim Einatmen Ham“.

Zum Schluss Klangschale 3x anschlagen

Für den Unterricht benutzte Primärliteratur

Chidvilasananda, Swami (2003). Schätze im Innern. Telgte: Siddha Yoga Verlag GmbH. Muktananda, Swami (2001). Meditiere. Das Glück liegt in Dir. Telgte: Siddha Yoga Verlag GmbH.

Muktananda, Swami (1996). Der Weg und sein Ziel. Ein Handbuch für die spirituelle Reise. München: Siddha Yoga Verlag GmbH. 


\section{Über Gedanken hinaus}

Hier folgt eine Übung aus den Lehren von Nisargadatta Maharaj (1897-1981), vorgestellt von seinem langjährigen Schüler und Begründer der Quantenpsychologie Dr. Stephen H. Wolinksy im Film Ich bin das Ich bin. Erfahre die Lehren des Sri Nisargadatta Maharaj. ${ }^{15}$

\section{Anleitung}

(Originaltext Dr. Stephen H. Wolinsky)

\section{Eventuell 1x Klangschale}

Was ist das „Ich bin“?

Um eine Erfahrung zu vermitteln von dem, was das „Ich bin“ ist, möchte ich dich als Zuhörer bitten: Lass deine Augen sich für einen Moment schließen.

Ohne deine Gedanken, Erinnerungen, Emotionen, Assoziationen oder Wahrnehmungen zu gebrauchen, bist du ein Mann, eine Frau oder weder noch?

Wenn du deine Gedanken, Erinnerungen, Emotionen, Assoziationen oder Wahrnehmungen nicht gebrauchst, bist du definiert, undefiniert oder weder noch?

Ohne deine Gedanken, Erinnerungen, Emotionen, Assoziationen oder Wahrnehmungen zu gebrauchen, bist du begrenzt, unbegrenzt oder weder noch?

Wenn du deine Gedanken, Erinnerungen, Emotionen, Assoziationen oder Wahrnehmungen nicht gebrauchst, bist du in einem Körper, außerhalb eines Körpers oder weder noch?

Wenn du deine Gedanken, Erinnerungen, Emotionen, Assoziationen oder Wahrnehmungen nicht gebrauchst, merkst du den zustandslosen Zustand des „Ich bin“. Ohne Gedanken, Erinnerungen, Emotionen, Assoziationen und Wahrnehmungen. Gleich werde ich dich bitten, öffne deine Augen, kehre in den Raum zurück und halte einen Teil deines Gewahrseins dort hinten, in dem zustandslosen Zustand, ohne Gedanken, Erinnerungen, Emotionen, Assoziationen und Wahrnehmungen.

Und wenn du deine Augen sich öffnen lässt, kann ein Teil deines Gewahrseins wieder hier im Raum sein, also du teilst deine Aufmerksamkeit in zwei Richtungen.

Maharaj sprach vom „Ich bin“ als verbalem „Ich bin“ und nonverbalem „Ich bin“. Zum Beispiel: das verbale „Ich bin“ wäre „Ich bin gut. Ich bin schlecht. Ich bin klug. Ich bin dumm, was auch immer!“

Er sagte, schneid das erst mal ab und bleib einfach im „Ich bin“; lass „schlecht, gut, was immer sich zeigt, los und halte einfach am verbalen „Ich bin“ fest.

Ohne deine Gedanken, Erinnerungen, Emotionen, Assoziationen oder Wahrnehmungen hast du, was er das nonverbale „Ich bin“ genannt hätte.

Vertiefen wir es nochmals und gehen es erneut durch.

Erlaube es deinen Augen, sich zu schließen. 
Wenn du deine Gedanken, Erinnerungen, Emotionen, Assoziationen oder Wahrnehmungen nicht gebrauchst, bist du perfekt, unperfekt oder weder noch?

Wenn du deine Gedanken, Erinnerungen, Emotionen, Assoziationen oder Wahrnehmungen nicht gebrauchst, was bedeutet das Wort perfekt, unperfekt dann überhaupt?

Wenn du deine Gedanken, Erinnerungen, Emotionen, Assoziationen oder Wahrnehmungen nicht gebrauchst, bist du wertvoll, wertlos oder weder noch?

Wenn du deine Gedanken, Erinnerungen, Emotionen, Assoziationen oder Wahrnehmungen nicht gebrauchst, was bedeutet überhaupt wertvoll oder wertlos?

Wenn du deine Gedanken, Erinnerungen, Emotionen, Assoziationen oder Wahrnehmungen nicht gebrauchst, bist du allein, in Verbindung oder weder noch?

Wenn du deine Gedanken, Erinnerungen, Emotionen, Assoziationen oder Wahrnehmungen nicht gebrauchst, was bedeutet dann überhaupt allein oder Verbindung?

Wenn du deine Gedanken, Erinnerungen, Emotionen, Assoziationen oder Wahrnehmungen nicht gebrauchst, bist du machtvoll, machtlos oder weder noch?

Wenn du deine Gedanken, Erinnerungen, Emotionen, Assoziationen oder Wahrnehmungen nicht gebrauchst, was bedeutet dann überhaupt machtvoll oder machtlos?

Langsam öffnen wir wieder die Augen ...

Eventuell 3x Klangschale

Nach einem kurzen Moment der Stille kann sich jetzt ein reflektierendes Gespräch anschließen, wie die Teilnehmerinnen und Teilnehmer die Übung erfahren haben. Zunächst in Zweiergruppen, dann im Gesamtplenum.

Nisardgadatta Maharaj sagte immer wieder, dass alles, was du über dich weißt, von außen kommt und definiert wird, und plädierte dafür, den Weg nach innen zu begehen, um dem eigenen wahren Selbst zu begegnen.

Eine Studentin sagte einmal nach der Übung in Tränen, dass sie durch die Übung plötzlich erfahren hat, wie sehr sie sich bisher im Leben so von anderen hat lenken lassen. Durch so viele Auffassungen von außen, beispielsweise wie man aussehen sollte, wie man sich benimmt oder was man später werden sollte.

Als ich diese Übung mal im Rahmen eines Seminars für Führungskräfte gemacht habe, saß ein Teilnehmer neben mir und antwortete auf meine Frage, wie man die Übung empfunden hat: „Es war komplett still.“ Er hatte anschließend das ganze Seminar fast gar nichts mehr gesagt, so tief beeindruckt war er von diesem Moment. Auch manche Studierende sind überwältigt und positiv überrascht, dass sie für einen kurzen Moment über die Gedanken hinausgelangen konnten. 
Für den Unterricht benutzte Primärliteratur

Maharshi, Ramana (2011a). „Wer bin ich?“ Der Übungsweg der Selbstergründung. Norderstedt: BoD.

Nisargadatta Maharaj (2017). Ich Bin. Teil I. Gespräche mit Sri Nisargadatta Maharaj.

Bielefeld: J. Kamphausen Mediengruppe GmbH.

Nisargadatta Maharaj (2016). Ich Bin. Teil II. Gespräche mit Sri Nisargadatta

Maharaj. Bielefeld: J. Kamphausen Mediengruppe GmbH.

Nisargadatta Maharaj (2014a). Ich Bin. Teil III. Gespräche mit Sri Nisargadatta

Maharaj. Bielefeld: J. Kamphausen Mediengruppe GmbH.

Nisargadatta Maharaj (2014b). Jenseits von Freiheit. Gespräche mit Sri Nisargadatta

Maharaj. Lohne: Lotus Press.

Wolinsky, Stephen H. (2002). Ich bin dieses Eine: Begegnungen mit Sri Nisargadatta Maharaj. Kirchzarten bei Freiburg: VAK Verlags GmbH.

DVD

Wolinsky, Stephen H. (2009). Ich bin das Ich bin. Erfahre die Lehren des Sri Nisargadatta Maharaj-Teil 1. Stuttgart: Mouna GmbH.

Internet

Kornfield, Jack (2014). „Jack Kornfleld on Nisargadatta Maharaj (2)“, www.youtube.com/watch?v=bXuF8qmv5Nc (letzter Zugriff: 13.09.2020)

Wolinsky, Stephen H. (2010). „Nisargadatta Maharaj / Experiential Meditation“, www.youtube.com/watch?v=rE6kS7vVsXw (letzter Zugriff: 13.09.2020)

Vgl. Gunaratana 1996, S. 39 und S. $135 f$.

Gunaratana 1996, S. 107; eine Einführung in die Vipassana-Meditation gibt Gunaratana 1996.

3 Eine kurze Beschreibung von Atemmeditationsübungen findet sich bei Ricard 2009, S. 76ff.; Ott 2015

S. 49ff. und Kabat-Zinn 2019, S. 84ff. Für kurze Meditationsformen in Verbindung mit Atem (siehe auch

Sutras, S. 159ff.).

${ }^{4}$ Vgl. Gunaratana 1996, S. $126 f$.

5 Vgl. Main 2015, S. 8f.

6 Vgl. Vishnu Devananda 1986, S. 67f.; „Der Kern , man' im Wort Mantra ist die erste Silbe dieses Wortes und bedeutet, denken' und ,tra' kommt von ,trai' - ,beschützen' oder ,befreien' von der Bindung an die Erscheinungswelt. Ein Mantra erzeugt schöpferische Kraft und schenkt ewige Wonne. Ein Mantra erweckt, wenn es ständig wiederholt wird, das Bewusstsein." (Swami Sivananda in Devananda 1986, S. $67 f$

7 Siehe Ng 2008, S. 211f.; siehe auch www.wccm.de.

8 Main 2015, S. 51

9 Main 2015, S. 47; siehe auch Ng 2008, S. 98.

10 Vgl. Main 2015, S. 8f.; siehe auch Ng. 2008, S. 81ff.

11 Vgl. Main 2015, S. 49.

12 Muktananda 1996, S. 99 sowie S. 135 und S. 204. Siehe auch Chidvilasananda 2003, S. 79f.; „Ham, die Silbe, mit der man einatmet, ist das höchste ,Ich'-Bewusstsein, das vollkommene ,Ich bin', das Gott ist. So, ist die Silbe, mit der man ausatmet, ist die Kraft Gottes, die Shakti, welche die Gestalt des Universums annimmt." (Muktananda 1996, S. 100)

${ }_{13}$ Muktananda 1996, S. 99. Hier findet sich auch eine Anleitung zur So'ham-Praxis.

14 Muktananda 1996, 100f.

15 Siehe den Film: Ich bin das Ich bin. Erfahre die Lehren des Sri Nisargadatta Maharaj - Teil 1 (2009, 7:33-16:39 Min.). Siehe ebenfalls: Wolinsky, Stephen H. (2010). „Nisargadatta Maharaj / Experiential Meditation", www.youtube.com/watch?v=rE6kS7vVsXw (letzter Zugriff: 13.09.2020). Der Abdruck dieser

Übung erfolgt mit freundlicher Genehmigung von Dr. Stephen H. Wolinsky. 


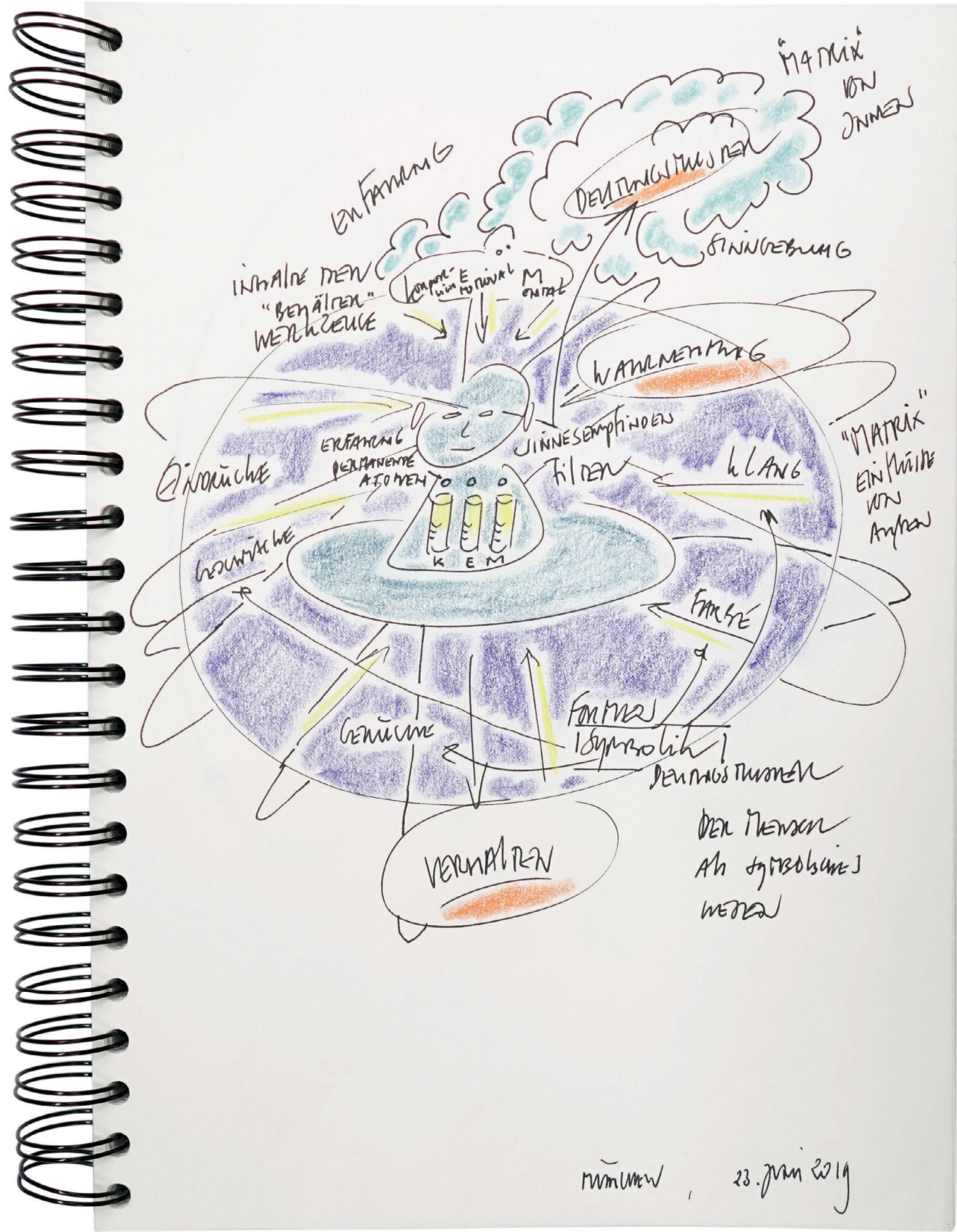

Einflüsse, die uns konditionieren. 


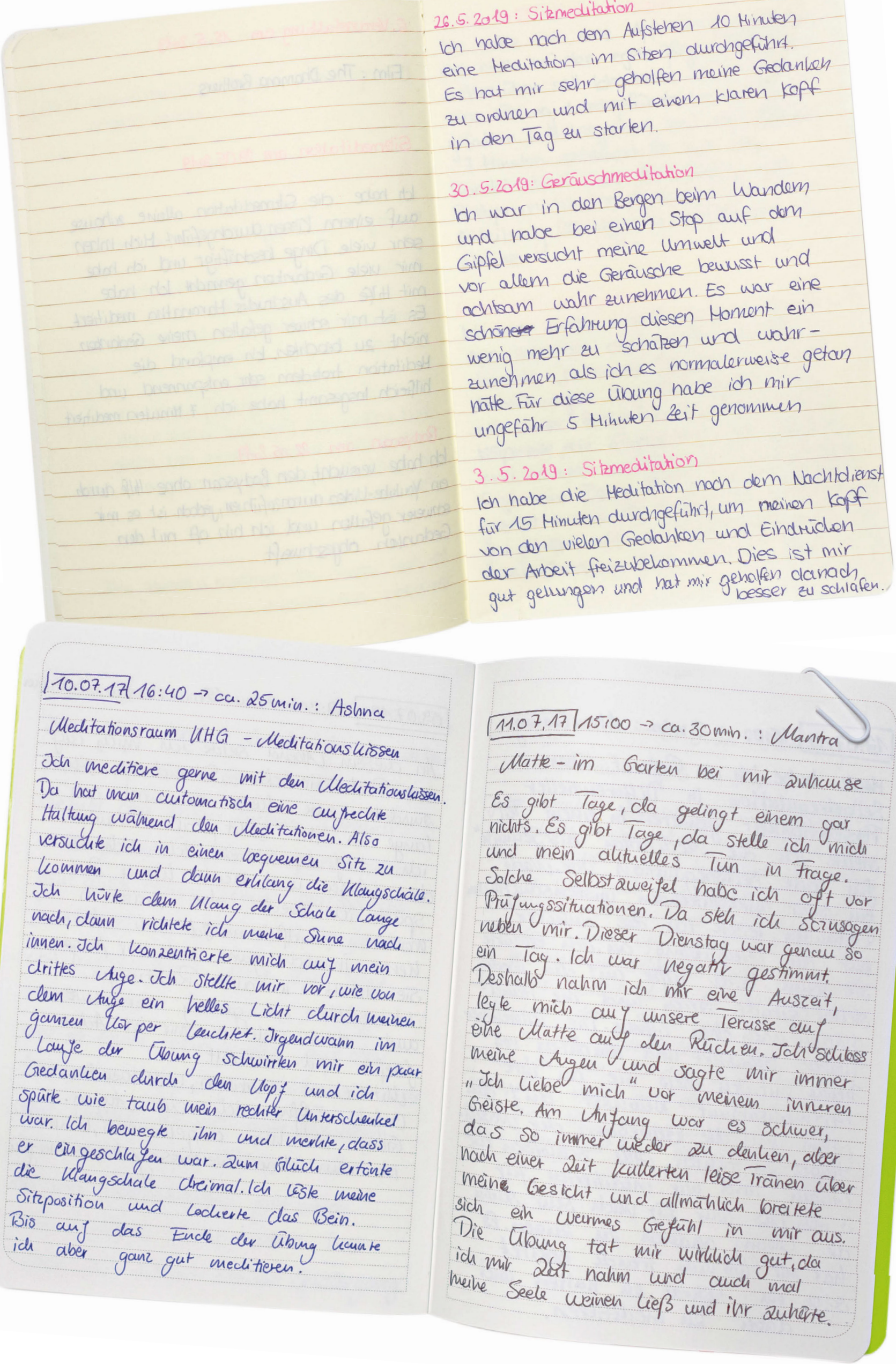




\section{Tagebuchnotizen Sitzmeditationen}

A. B.

Zeit: 14.15-15.45 Uhr (10+5+5 Minuten)

Ort: Meditationsraum der KHG (sitzend)

Methode: MA-RA-NA-THA, Grübelfabrik, Erste

Hilfe bei schwierigen Gedanken

Utensilien: Klangschale, Gedanke: „Wellen“, CD

$\mathrm{Zu}$ Beginn unserer heutigen Sitzung haben wir $10 \mathrm{Mi}$ nuten lang jeder auf seine Weise meditiert. Mir gelang es gut, meinen Kopf frei von Gedanken zu bekommen, und ich dachte nur zweimal ganz kurz an Essen und an die Geräusche von draußen in Form von Vogelgezwitscher und Straßenlärm. Mir ist außerdem der Geruch im Meditationsraum sehr stark aufgefallen. Es roch nach den Matten, auf denen wir saßen. Ich habe diesen Geruch zuvor nie wirklich wahrgenommen. Während ich das Mantra mir selbst vorsagte, dachte ich an Wellen, die beim Einatmen hoch- und beim Ausatmen runtergingen.

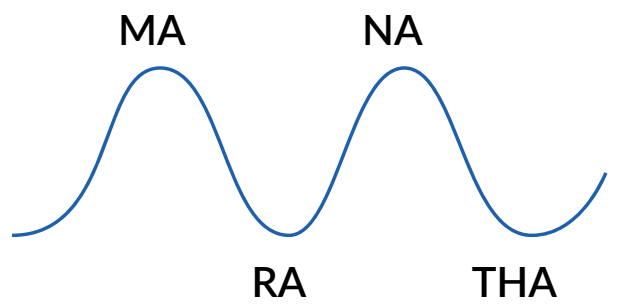

Nach der Meditation haben wir noch zwei kleinere Übungen von der CD aus dem Meditationsbuch für Kinder gemacht. Die Übungen waren für mich total ungeeignet, da die Sprecherin zu schnell sprach und ich keine Zeit hatte, einen klaren Gedanken zu fassen.

\section{A. v. W.}

\section{4.-30.10.2019}

Ich habe meine Mutter gefragt, ob sie mit mir zusammen meditieren möchte, und sie hat sich darauf eingelassen. Am Nachmittag gegen halb fünf haben wir uns im Wohnzimmer auf den Boden gesetzt. Als Unterlage haben wir Decken genommen. Ich dachte mir, es wäre schön, eine Kerze anzuzünden. Die Kerze haben wir zwischen uns in die Mitte auf den Couchtisch gestellt. Da meine Mutter noch nie zuvor meditiert hat, habe ich ihr die Übung mit der Konzentration auf die Atmung bei der Nase erklärt. Ich selbst habe mich auf

die Stirnregion in der Mitte konzentriert. Wir haben fünf Minuten lang meditiert. Anstelle einer Klangschale habe ich einfach gesagt: „So, wir fangen jetzt an und schließen unsere Augen."

Als ich meine Mutter danach fragte, wie es bei ihr geklappt hat, meinte sie, dass es ihr anfangs schwer gefallen sei, an nichts zu denken und sie sich deswegen oft „an nichts denken“ gesagt hat. Aber gegen Ende hin hat sie sich ausschließlich auf ihre Atmung konzentriert, wobei sie eher auf die Brustregion geachtet hat.

Bei mir ist es ganz gut gelaufen, nur war ich, glaube ich, etwas aufgeregt, weil ich mir mehrmals Gedanken darüber gemacht habe, wie es wohl bei meiner Mutter läuft. Vielleicht war ich auch einfach nicht ruhig und gelassen. Davor waren wir Kaffee trinken und spazieren, weshalb ich vielleicht nicht ganz zur Ruhe kommen konnte. Auf jeden Fall habe ich mich mehrmals dabei ertappt, an meine Mutter zu denken. Das könnte ich mir auch bei einigen Schülerinnen vorstellen, dass sie sich über ihre Mitschüler Gedanken machen beim gemeinsamen Meditieren in der Klasse.

$$
\begin{aligned}
& \text { 17.04. 19: Hör- und Atemmediation } \\
& \text { ich hab: ca. 10-15 Min. } \\
& \text { Hügel einen bestirn } \\
& \text { mich an einelen Bäumen und Platz, den ich nur allene a } \\
& \text { mich auf meine Baum an. Dort fühteren. ich setzte mich aufsuche. Er liegt auf eh } \\
& \text { und hören kane Atmung zu konzon ich mich, als wäre dann gerne auf die einem kleinen }
\end{aligned}
$$


C. $M$.

\subsubsection{9 - Sitzmeditation}

Ich habe nach dem Aufstehen 10 Minuten lang eine Meditation im Sitzen durchgeführt. Es hat mir sehr gut geholfen, meine Gedanken zu ordnen und mit einem klaren Kopf in den Tag zu starten.

\subsubsection{9 - Sitzmeditation}

Ich habe die Meditation nach dem Nachtdienst $15 \mathrm{Mi}$ nuten lang durchgeführt, um meinen Kopf von den vielen Gedanken und Eindrücken der Arbeit freizubekommen. Dies ist mir gut gelungen und hat mir geholfen, danach besser zu schlafen.

\section{F. E.}

Am besten gefällt mir eigentlich die Atem-Meditation, da sie sich am einfachsten ausführen lässt und auch überall angewandt werden kann (Bus, Bahn, Fahrrad, Gehen ...). Man kann sie auch schnell zwischendurch einbauen, um kurz abzuschalten und sich danach wieder auf eine Sache konzentrieren zu können. Manchmal ist es schon schwer, seine Gedanken nicht zu beachten, daher finde ich es eher leichter, abends zu meditieren, wenn man den Tag schon einigermaßen hinter sich hat, als früher am Tag, wenn man an all die Sachen denkt, die man vielleicht vorhat oder erledigen muss.

Auch die Ajnazentrum-Meditation habe ich öfters gemacht [...]. Als Mantra habe ich mir anstatt MARA-NA-THA immer eigene Motivationssprüche ausgedacht, die ich mir vorgesagt habe, zum Beispiel: „Heute ist ein schöner Tag“, „Ich schaffe heute alles, was ich vorhabe". Das hat man dann verinnerlicht, und die Tage verliefen wirklich gut!

\section{F. S.}

\subsubsection{9, MA-RA-NA-THA}

Dauer: ca. 10 Min.

Seit Tagen bin ich sehr nachdenklich und eher traurig gestimmt. Ich versuche, mehr auf mich und meine Bedürfnisse (psychisch und seelisch) zu hören. Ich bin innerlich sehr unruhig - andauernd. Meine Gedanken fangen an zu kreisen, und es gibt kein Ende. Ich komme nicht mal auf eine Lösung, sondern drehe mich dann nur im Kreis, was mich zusätzlich anspannt und unzufrieden macht.

Deshalb versuche ich, mich auf die Couch zu legen und die Meditation mit einem Mantra durchzuführen. $\mathrm{Zu}$ Beginn konnte ich mich nicht darauf einlassen, und das MA-RA-NA-THA lief eher im Hintergrund - meine Gedanken im Vordergrund. Nach einigen Minuten fiel mir dies auf und ich versuchte, meine Gedanken weiter- zuschieben. Dies gelang mir nicht so gut. Deshalb versuchte ich es umgekehrt. Ich versuchte nun nicht mehr, zwanghaft die Gedanken wegzuschieben, sondern erlaubte mir, sie zuzulassen. Das lief wesentlich besser.

Die letzten Minuten konnte ich mich sehr gut auf das Mantra konzentrieren, und die Gedanken waren weg. Ich war stolz, eine Möglichkeit gefunden zu haben, um die Gedanken für ein paar Minuten ausschalten zu können, und fühlte mich geordneter.

\subsubsection{9, MA-RA-NA-THA + Atem}

\section{Dauer: ca. 10-15 Min.}

Da es gestern so gut lief, dachte ich mir, ich probiere die Mantra-Meditation erneut. Mal wieder zum Einschlafen, denn da fangen die Gedanken gerne an zu kreisen. Ich lag also im Bett und sagte das Mantra innerlich auf. Dies kombinierte ich mit einer gleichmäßigen Atmung. Eine Silbe einatmen und eine ausatmen. Zwischenzeitlich verlor ich mich doch wieder in Gedanken. Dies fiel mir schnell auf und ich konzentrierte mich wieder auf Mantra und Atmung. Auch dieses Mal hat es geklappt, da ich eingeschlafen bin und sehr gut schlafen konnte.

L. K. M.

Für 10 Minuten habe ich auf einer Bank auf dem Nordfriedhof meditiert. Dabei habe ich die Atemmeditation angewendet und mich auf meine Nasenflügel konzentriert. $\mathrm{Zu}$ Beginn der Meditation schweifte ich ab - ich merkte, dass ich die freie Zeit zu nutzen versuchte, um meine Gedanken zu ordnen. Mit der Zeit gelang es mir jedoch besser, meine Gedanken vorbeiziehen zu lassen.

Im Nachhinein fiel mir auf, dass ich mich weniger auf die Atmung, sondern auf die Naturgeräusche (das Zwitschern, Rascheln) um mich herum konzentriert habe. Zuvor war ich zum Joggen auf dem Friedhof. Deshalb ging ich angestrengt in die Meditation. Im Anschluss hatte ich ein sehr wohliges Gefühl und merkte, dass ich ruhig und entspannt war.

Ich habe mich für 10 Minuten in mein Zimmer gesetzt und die Tür hinter mir geschlossen. Dieses Mal habe ich mich auf das Mantra MA-RA-NA-THA konzentriert. Dies gelang mir zu Beginn der Meditation auch. Ich konnte mir das Wort vor meinen Augen vorstellen und mich darauf konzentrieren. Im Verlauf der Meditation schweifte ich allerdings immer wieder $a b$. Ich merkte, wie ich die Zeit der Ruhe nutzte, um meine Gedanken zu ordnen und runterzukommen. Für die nächste Meditation nahm ich mir deshalb vor, schon zu Wochenbeginn feste Zeiten für die Meditation einzuplanen, sodass ich nicht das Gefühl habe, dass mir die Zeit an einer anderen Stelle fehlt. 


\section{$\Longrightarrow 10 \mathrm{~min}$}

Gedanken lch habe mich fur ptated

bessor mene ulasen in men ammer gese corhasin

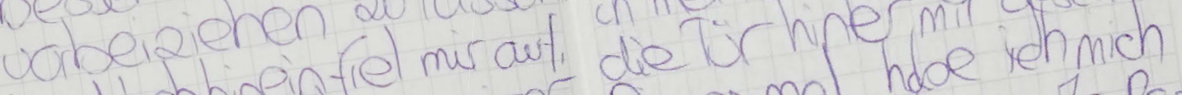

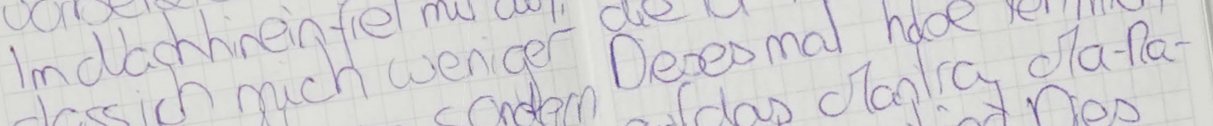

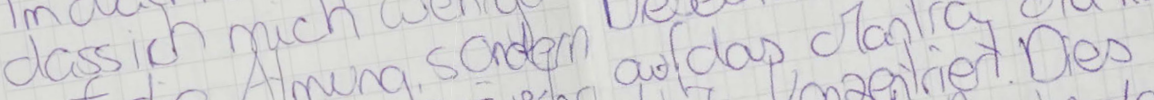

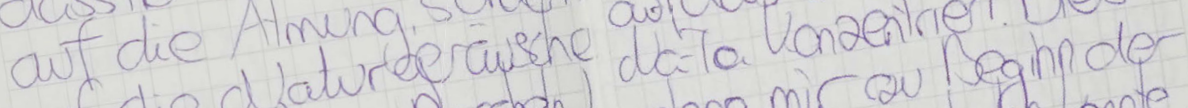

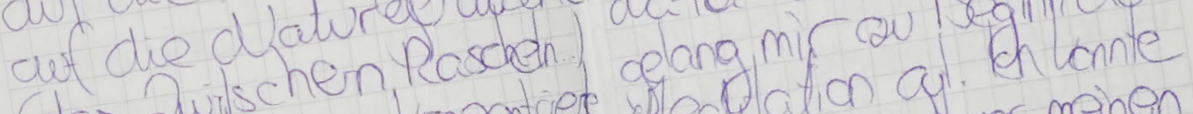

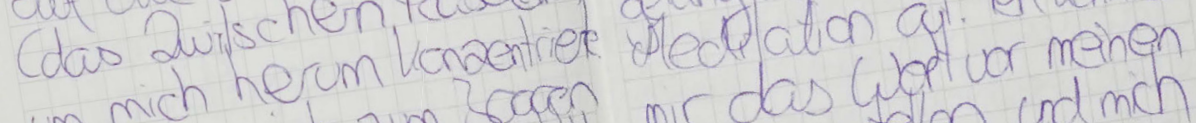
un mor war ith aum gagh mir das walen undmen

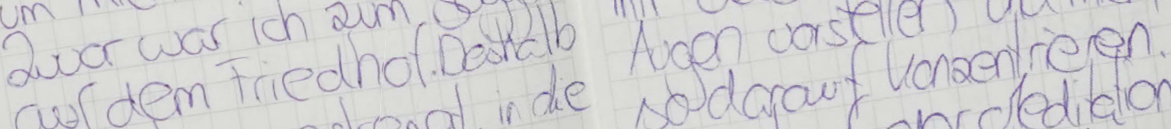

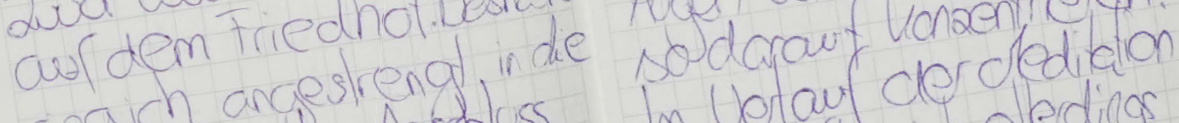

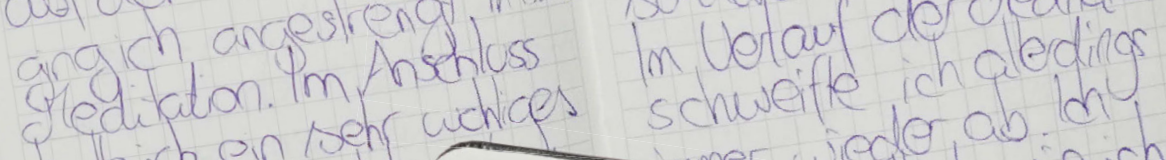
habich en sen cefon und merke ichrohigund ents un meine cooknke nuab

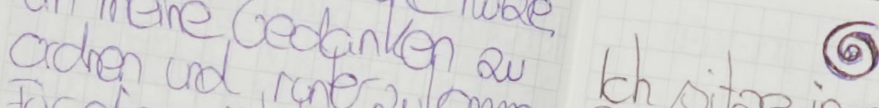

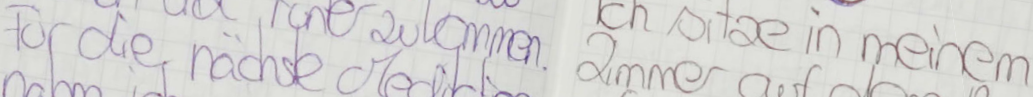

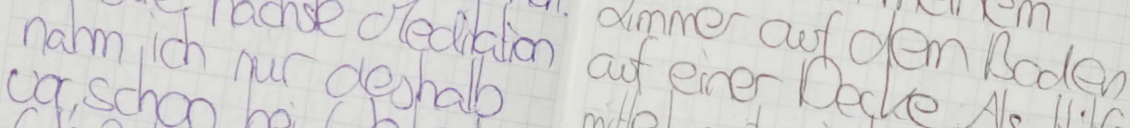

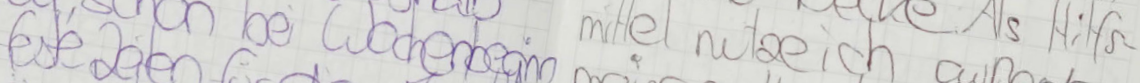
endevanon or de olediam meinen tandy awpeotem

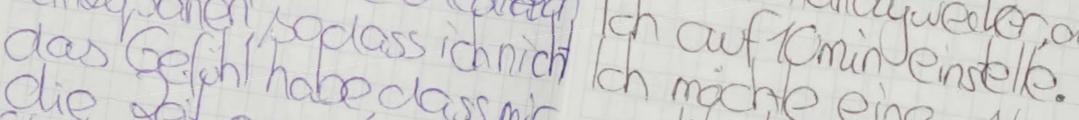
dip setian classmir meditate eine Alom.

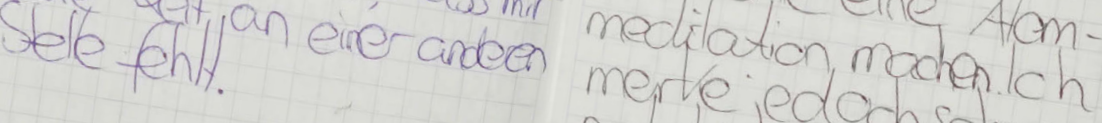
toer habe iondie. emmeditation angewender, und mich ayt ansentrieft

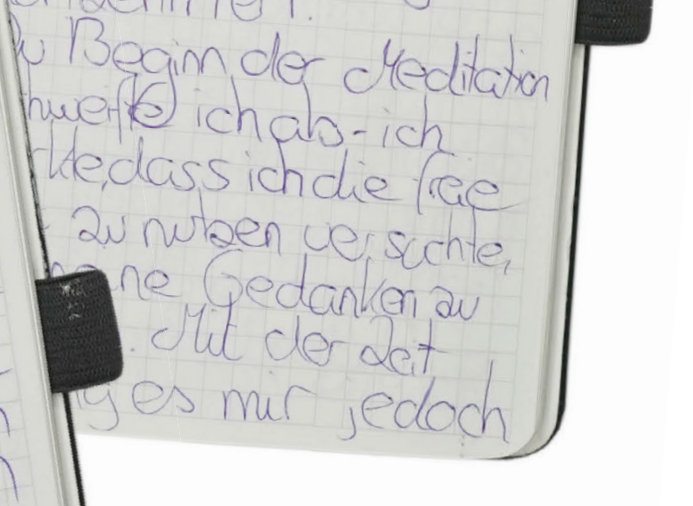




\section{R.}

\subsubsection{7, 15-15.30 Uhr, Mantra}

\section{Matte im Garten bei mir zu Hause}

Es gibt Tage, da gelingt einem gar nichts. Es gibt Tage, da stelle ich mich und mein aktuelles Tun infrage. Solche Selbstzweifel habe ich oft vor Prüfungssituationen. Da stehe ich sozusagen neben mir. Dieser Dienstag war genau so ein Tag. Ich war negativ gestimmt. Deshalb nahm ich mir eine Auszeit und legte mich auf unserer Terrasse auf eine Matte auf den Rücken. Ich schloss meine Augen und sagte mir immer „Ich liebe mich“ vor meinem inneren Geiste. Am Anfang war es schwer, das so immer wieder zu denken, aber nach einer Zeit kullerten leise Tränen über mein Gesicht und allmählich breitete sich ein warmes Gefühl in mir aus. Die Übung tat mir wirklich gut, da ich mir Zeit nahm und auch mal meine Seele weinen ließ und ihr zuhörte.

\section{N.}

\subsubsection{9}

\section{Dauer ca. 30 Min. insgesamt}

Zum Ausklang der Woche habe ich mir heute einmal viel Zeit genommen zum Meditieren.

Zuerst habe ich mit der Atemmeditation begonnen, wurde aber schnell müde und bin nach ca. 5 Min. kurz für ein paar Sekunden eingeschlafen.

Danach war mir klar: Wenn ich müde bin - Auf alle Fälle mit Mantram meditieren. Also machte ich mit "Maranatha“ weiter, was mir half zu entspannen ohne einzuschlafen. Ich merkte, wie ich mehr zur Ruhe kam und die Gedanken wie bei schwachem Regen auf mich herabnieselten, mich leicht streiften, aber nie ganz trafen.

\section{P. S.}

\subsubsection{6, ca. 10 Min., bei mir zu Hause Ergebnis: Sitzmeditation (Mantra)}

Ich habe mich heute total müde und unentschlossen gefühlt. Ich hatte noch so viele Gedanken im Kopf wegen den Weihnachtsgeschenken. Wem schenke ich was? Wie soll ich die Geschenke einpacken? Was fehlt noch? Ich habe mir noch 10 Minuten genommen und habe mich vom Alltag zurückgezogen. Das Mantra ist bei mir irgendwie im Unterbewusstsein so gespeichert, dass ich es relativ schnell abrufen kann. Auch der Rhythmus, die einzelnen Silben zu sprechen, pendelt sich sehr gut ein. Die einzelnen Silben erscheinen vor meinen Augen wie beim Karaoke-Singen. Wie erhofft haben mir die Minuten (kleine Auszeit) gutgetan und ich konnte die restlichen Geschenke noch einpacken.

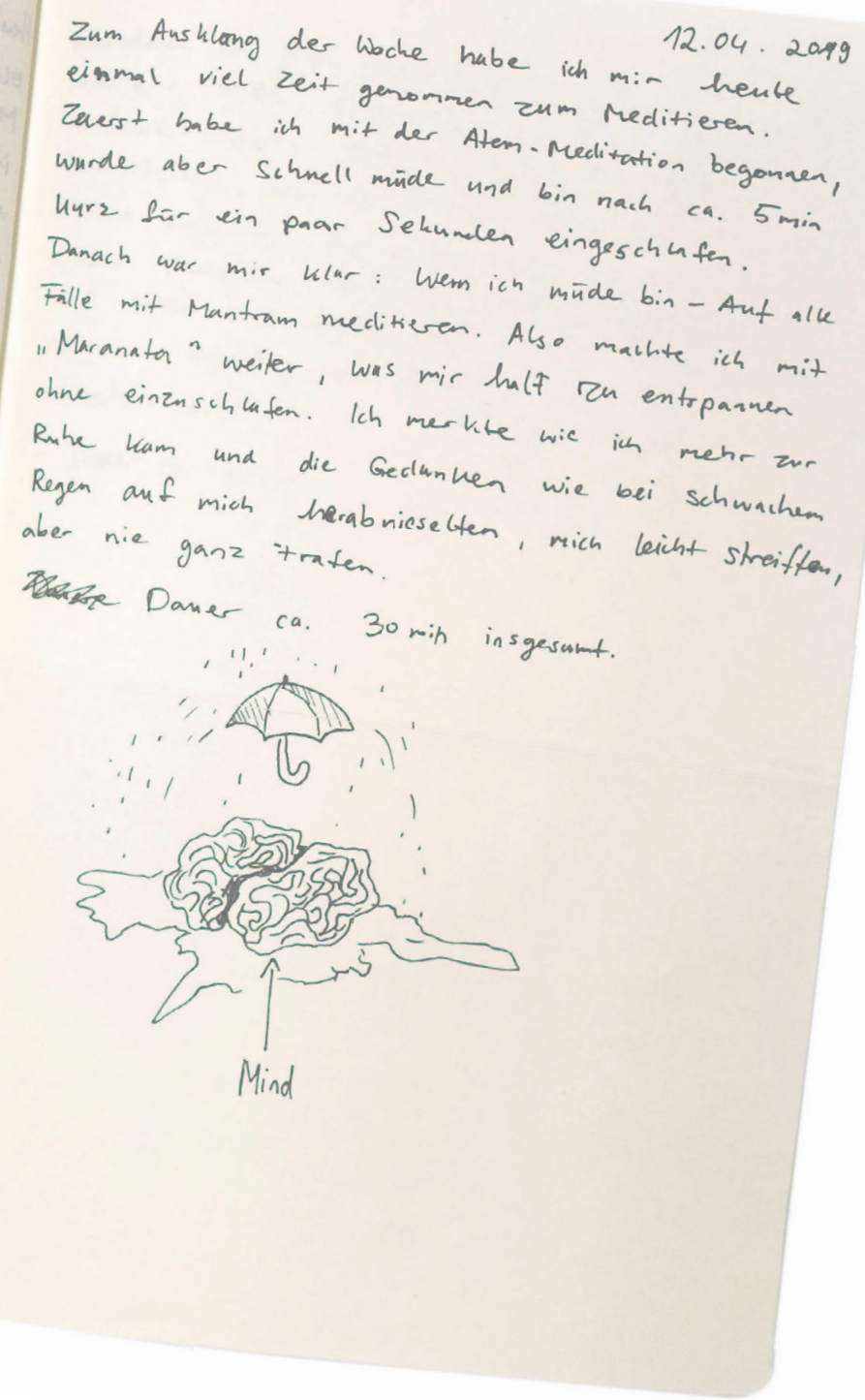




\section{Achtsamer Alltag}
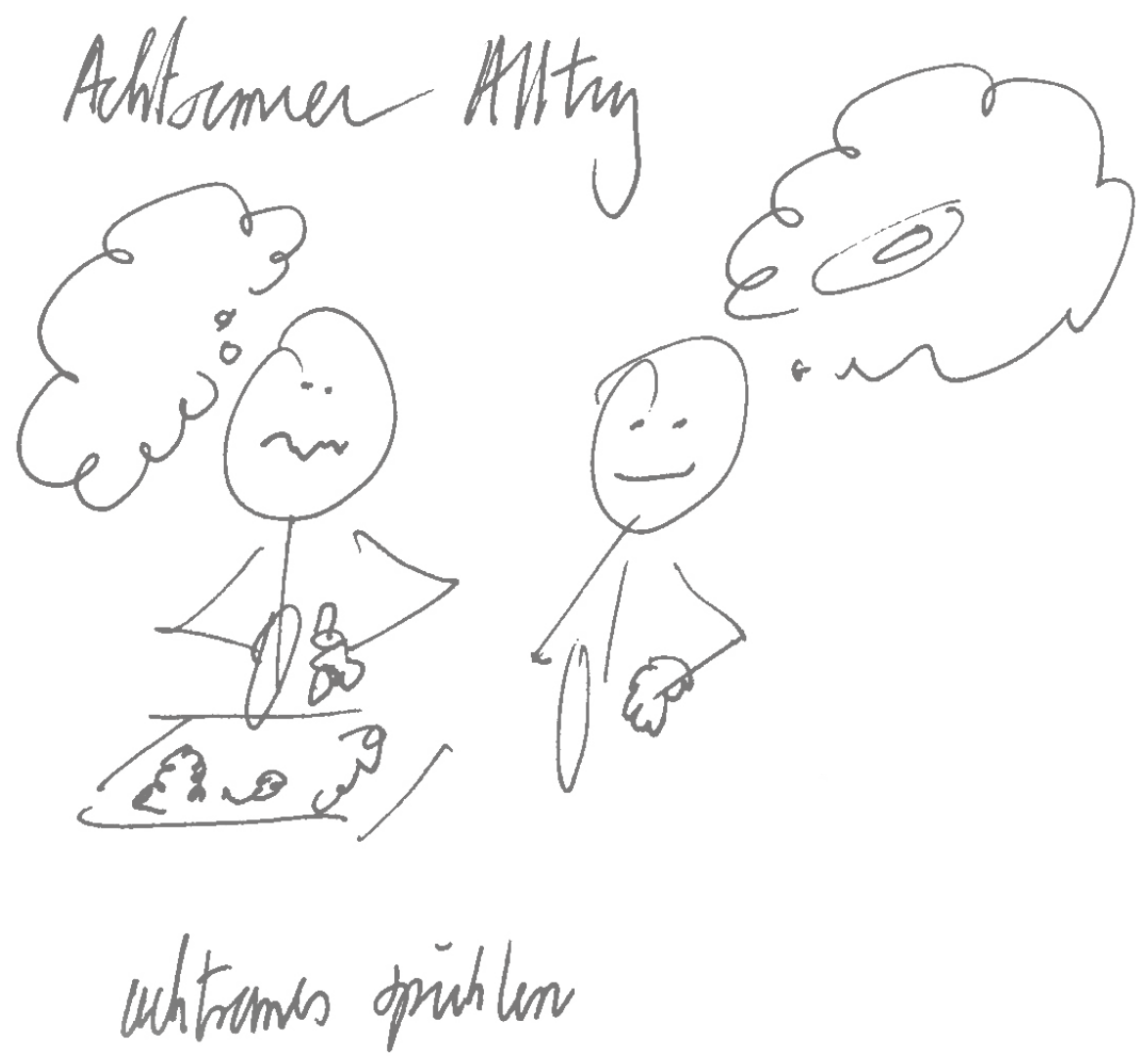

Achtsam

Geschirr

spülen.

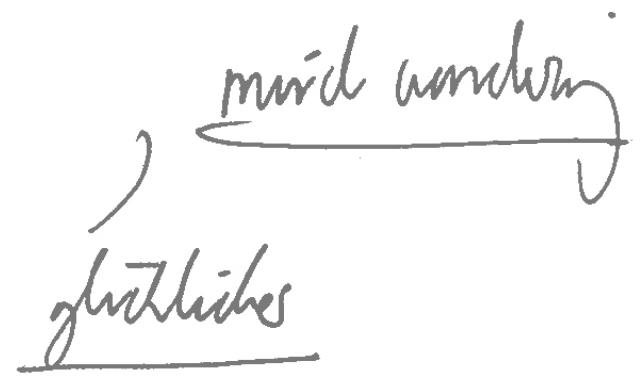

Bei der Achtsamkeit geht es insbesondere darum, aus einer inneren authentischen $\mathrm{Hal}$ tung heraus den gegenwärtigen Moment bewusst zu erleben und mit Offenheit wahrzunehmen statt das Geschehen kognitiv zu bewerten.

Um das Thema Achtsamkeit zu verdeutlichen, vermittle ich zu Beginn des Unterrichts eine praktische Erfahrung. Die Übung zeigte mir mein Kollege Niko Kohls während eines Gastvortrags, und ich habe sie seitdem immer in meinen Unterricht eingebaut. Die Übung heißt: „Das eigene Gesicht mit der ,verkehrten“ Hand malen/ zeichen" und wird folgendermaßen durchgeführt:

Moderation: „Nehmen Sie ein Blatt Papier und einen Stift zum Schreiben." Moderation: „Wer ist Rechtshänder? Wer ist Linkshänder? Diejenigen, die Rechtshänder sind, zeichnen mit der linken Hand und die Linkshänder zeichnen mit der rechten Hand.

Und was Sie zeichnen, ist das eigene Gesicht." 
Um wirklich in Kontakt mit unserem Hier und Jetzt zu sein

- wo auch immer das sein mag -, müssen wir so lange in unserem Wahrnehmen innehalten, bis der gegenwärtige Augenblick in uns einsinken kann - so lange, bis wir den gegenwärtigen Augenblick wirklich spüren, bis wir ihn in seinem ganzen Ausmaß sehen, bis wir seiner gewahr werden und ihn dadurch besser kennenlernen und verstehen.

Jon Kabat-Zinn

Normalerweise wird meistens zunächst gelacht und gekichert, dann aber folgt eine tiefe Konzentration bei den Teilnehmerinnen und Teilnehmern, und es wird sehr ruhig im Raum.

Irgendwann sind die ersten fertig, und es wird wieder lauter.

Nach einer Weile wird die Übung beendet.

Moderation: „Sind Sie zufrieden mit Ihrem Kunstwerk? Wenn Sie möchten, können Sie es auch Ihrem Sitznachbarn zeigen."

[Gelächter bei den Studierenden]

Moderation: „Was haben wir jetzt eigentlich gemacht? Haben Sie bemerkt, wie ruhig es plötzlich im Raum war? Warum?"

Wortmeldung 1: „Man musste sich richtig konzentrieren.“

Wortmeldung 2: „Es war etwas Neues.“

Moderation: „Haben Sie während der Übung auch an anderes gedacht? Wie konzentriert waren Sie?"

Danach folgt eine Überleitung zur Beschreibung von Achtsamkeit. Unter anderem stelle ich eine Definition von dem Pionier der Achtsamkeitsbewegung Jon KabatZinn vor. Ebenso habe ich an dieser Stelle das Thema „Mindwandering“ miteinfließen lassen (siehe KNA-Interview, S. 123, und Abschnitt „Forschung“', S. 47).

Achtsamkeit im Alltag kann geübt werden, zum Beispiel indem man sich ab und zu selbst fragt oder daran erinnert, ob man in diesem Moment präsent ist. Im Unterricht habe ich diesen Aspekt der Präsenz im Alltag manchmal zu Beginn eingebracht: „Was haben Sie heute gesehen oder wahrgenommen, worauf Sie sonst nicht geachtet haben?"

Es gibt so viele Tätigkeiten, die wir bewusster machen können, wie beispielsweise Essen, Kochen, Geschirrspülen, Aufräumen, Putzen, Spazierengehen, Zähneputzen, Bügeln und vieles mehr. Wenn wir das Mindwandering reduzieren, sind wir präsenter im Augenblick, und die Qualität der Erfahrung wird intensiver. An dieser Stelle habe ich häufig auf das Buch 5 Dinge, die Sterbende am meisten bereuen von Bronnie Ware hingewiesen und warum es so wichtig ist, diese wache Präsenz jetzt schon zu pflegen. 
Für den Unterricht benutzte Primärliteratur

Kabat-Zinn, Jon (2019). Gesund durch Meditation. München: Knaur Verlag.

Kabat-Zinn, Jon (2015). Im Alltag Ruhe finden. Meditationen für ein gelassenes Leben. München: Knaur Verlag.

Marti, Lorenz ((2016). Mystik an der Leine des Alltäglichen. Freiburg im Breisgau: Verlag Herder GmbH.

Thich Nhat Hanh (2015). Achtsamkeits-Survival-Kit. Fünf grundlegende Übungen. München: O. W. Barth Verlag.
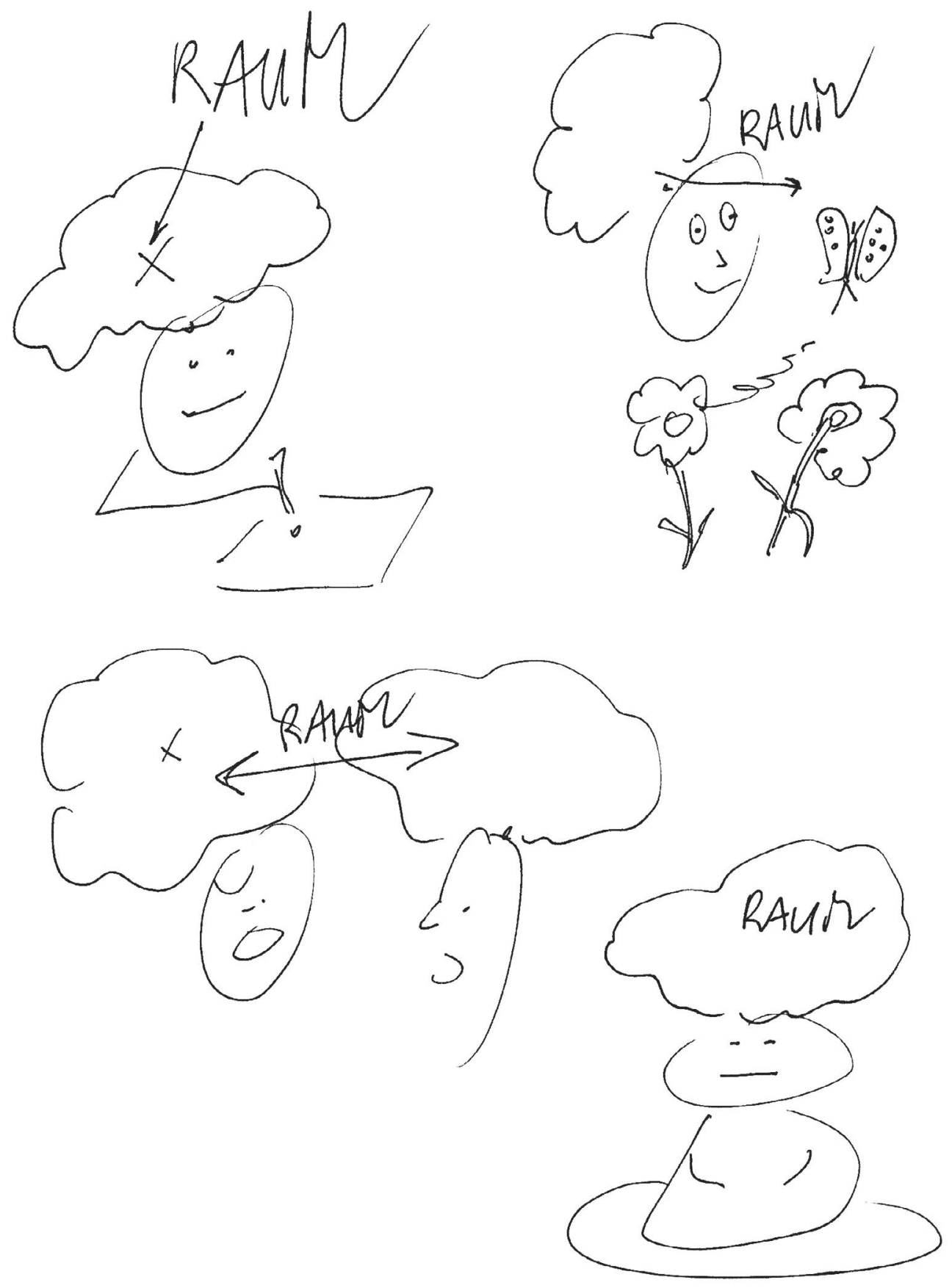

Im Moment zu sein, heißt Raum zu schaffen. 
Panta puischen meinen Augenorawen su kanentrieren. Hier merke ich aledings dass der Puck nacla einer Weile inmer sairke wird. Den - Eotder zeit sitae ich nur da und lasse die Gedanen schueiten. Darach fühle ich mich trotadem entspannter und beruhiger, lch merke jedoen dasses wrchtig fir mich is einen wecker a haben de mur die deit corgibt

Ich meditere in der S-Bamn auf der Srecke un Pasing an

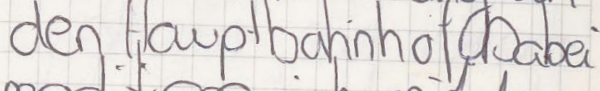
meditiefe ich mit der Alemneditation. Ich merke dass mir die Meditation 2u Beginn gut gelingt. In Vanp mich. dut mene vasenfiugel ronzentriesen und meine Gedanien beiscile schicren Dam beginne ich mich doer auf qie Msagen in de S.ban av

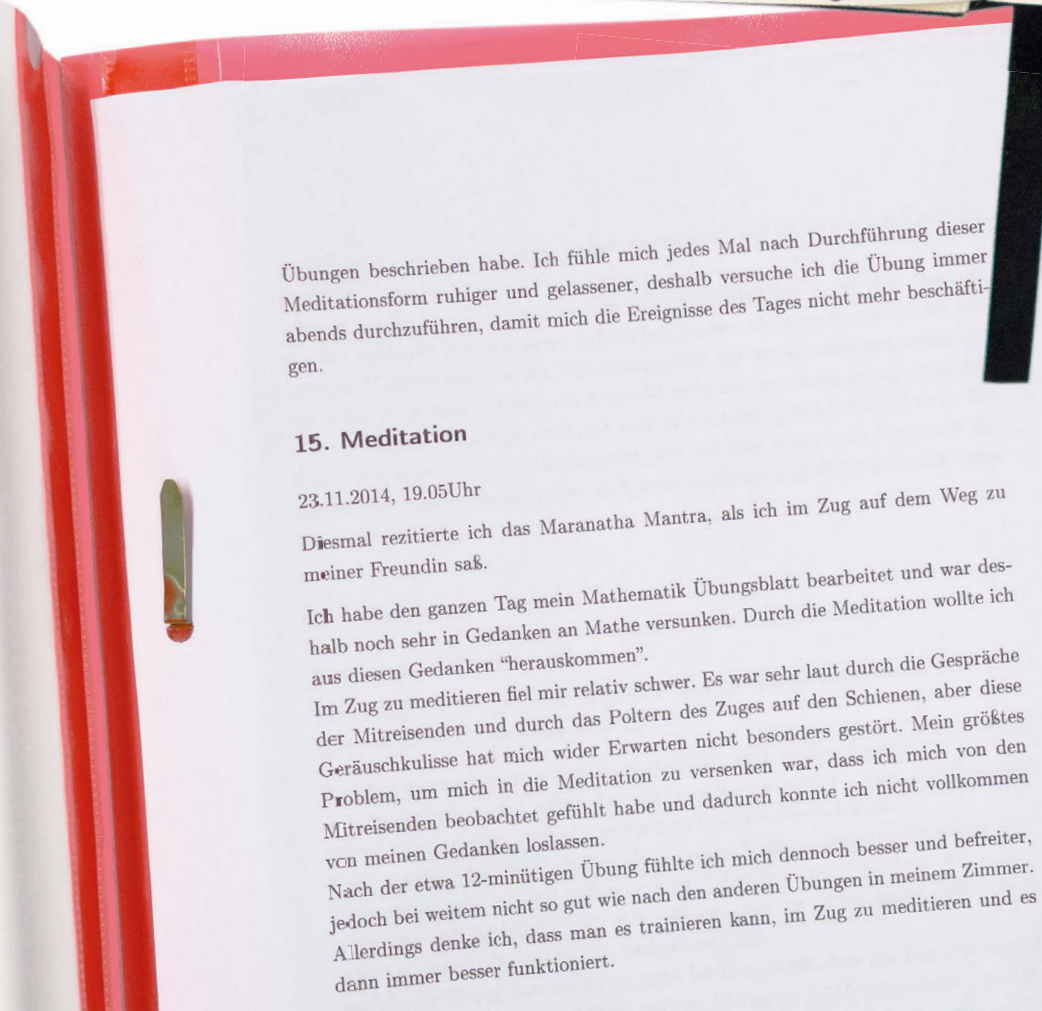

\section{Meditation}

24.11.2014, im Seminar

. In dieser Sitzung vom Seminar daben Boden und Herr de Bruin hat uns durch die bei lagen wir auf Matten aul Übung geführt, indem er vesentlich leichter mich auf die einzelnen Sann die Öbung ren sollen. Mir fallt es wesen, wenn eine Person dies vorgibt. Tch kann, auch dieses Badysean zu konzent tneren, waihren und werde dadurch sehr get. Was noch auffallig dann sehr konzentriert durchfulire nonspannt und müde gewesen. Was noch aufrall Mal bin ich nach der Übung sehr entos

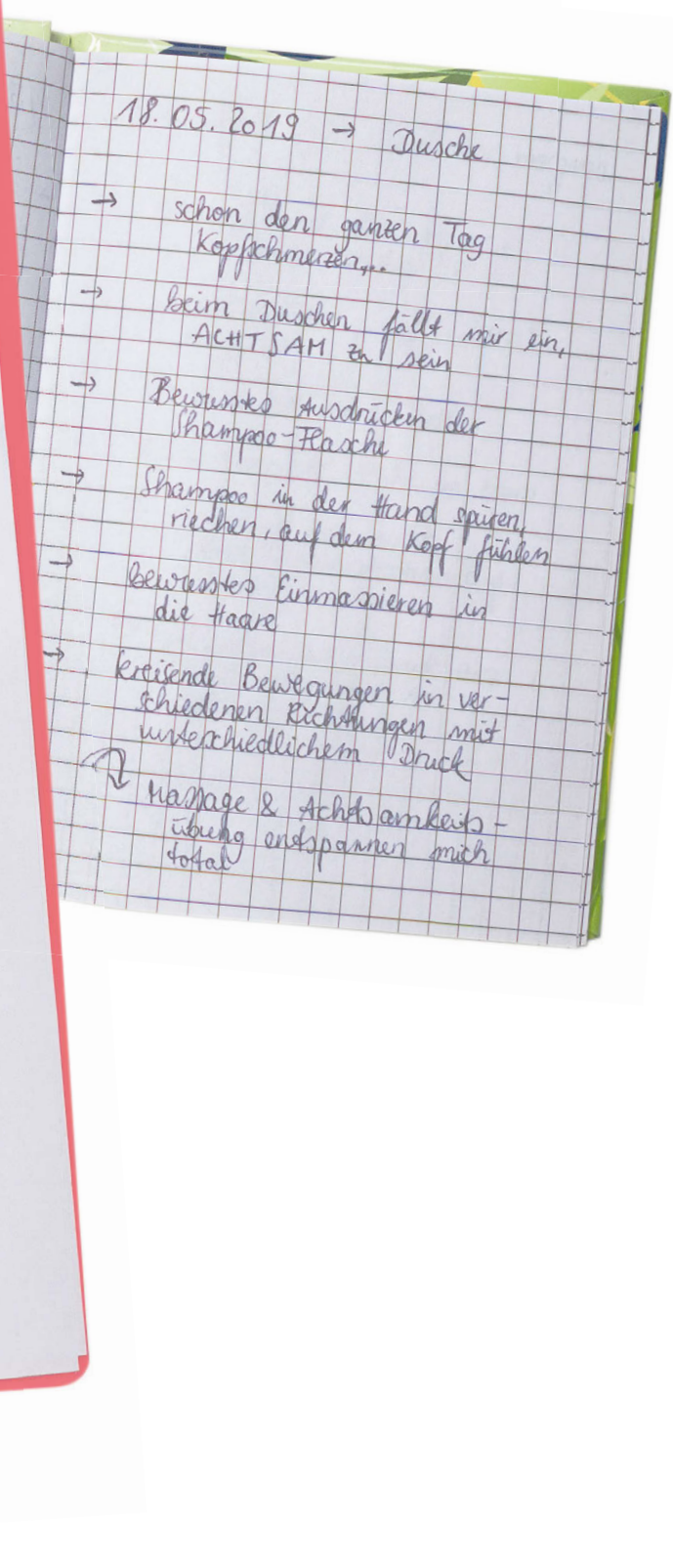




\section{Tagebuchnotizen Achtsamer Alltag}

\section{H. V.}

\section{Meditieren in der Kirche}

Es ist Ostern. Ich war in der Kirche, allerdings fand kein Gottesdienst statt. Ich war dort alleine. Es war wunderschön. Ich setzte mich in die Bank und fühlte mich sehr wohl.

Ich schloss meine Augen und meditierte ein paar Minuten mit - Maranatha. Es war eines der gelungensten Meditationsversuche von mir. Ich war total bei mir, nicht abgelenkt, und hatte das Gefühl, ich wurde noch zusätzlich von der Umgebung unterstützt.

Als ich aus der Kirche kam, war ich so erfüllt, und zwar von mir selbst! Das fühl ich nicht oft.

\section{J. H.}

Wann: 15.5 .2017

\section{Wo: Wohnung von einem Freund Was: Abspülen \\ Wie lange: 15-20 Min.}

Heute war meine Chance! Ich war bei einem Freund eingeladen und wir wollten eigentlich kochen. Seine Küche war aber noch voll mit nicht abgewaschenem Geschirr. Erst haben sich alle kurz beschwert, aber dann habe ich gesagt, dass ich das übernehme und jetzt achtsam abspülen werde. Sie haben mich zwar sehr überrascht angeschaut, aber hatten natürlich nichts dagegen, weil sie es ja dann nicht machen mussten. Ich muss sagen, das Abspülen gefällt mir von den Achtsamkeitsübungen im Haushalt am besten. Irgendwann hat die eine mich gefragt, ob ich ernsthaft immer noch dabei bin. Aber man verliert sich total darin! Und was mir gefällt - man hat eine „direkte“ Wirkung, die man beobachten kann. Also man sieht am Ende, was man "geschafft" hat, wenn das Geschirr sauber ist.

Alle waren zufrieden, und dann haben wir angefangen zu kochen und das Geschirr wieder dreckig zu machen ;-)

\section{J.}

\subsubsection{9, Dusche}

- Schon den ganzen Tag Kopfschmerzen.

- Beim Duschen fällt mir ein, achtsam zu sein.

- Bewusstes Ausdrücken der Shampoo-Flasche.

- Shampoo in der Hand spüren, riechen, auf dem Kopf fühlen.
- Bewusstes Einmassieren in die Haare.

- Kreisende Bewegungen in verschiedenen Richtungen mit unterschiedlichem Druck.

- Massage und Achtsamkeitsübungen entspannen mich total.

\section{K. M.}

Ich meditiere in der S-Bahn auf der Strecke von Pasing zum Hauptbahnhof. Dabei meditiere ich mit der Atemmeditation. Ich merke, dass mir die Meditation zu Beginn gut gelingt. Ich kann mich auf meine $\mathrm{Na}-$ senflügel konzentrieren und meine Gedanken beiseiteschieben.

Dann beginne ich mich aber auf die Ansagen in der S-Bahn zu konzentrieren, um meinen Ausstieg nicht zu verpassen. Ab dann ist meine Konzentration weg. Ich merke hier wieder, dass ich einen Zeitrahmen brauche, den ich mir bewusst für die Meditation nehme. Trotzdem fährt mich der kurze Fokus auf mich selbst schon runter.

\section{H.}

\subsubsection{4, 19.05 Uhr}

Diesmal rezitierte ich das Maranatha-Mantra, als ich im Zug auf dem Weg zu meiner Freundin war.

Ich habe den ganzen Tag mein Mathematik-Übungsblatt bearbeitet und war deshalb noch sehr in Gedanken an Mathe versunken. Durch die Meditation wollte ich aus diesen Gedanken „herauskommen“.

Im Zug zu meditieren fiel mir relativ schwer. Es war sehr laut durch die Gespräche der Mitreisenden und durch das Poltern des Zuges auf den Schienen, aber diese Geräuschkulisse hat mich wider Erwarten nicht besonders gestört. Mein größtes Problem, um mich in die Meditation zu versenken, war, dass ich mich von den Mitreisenden beobachtet gefühlt habe, und dadurch konnte ich nicht vollkommen von meinen Gedanken loslassen.

Nach der 12-minütigen Übung fühlte ich mich dennoch besser und befreiter, jedoch bei Weitem nicht so gut wie nach den anderen Übungen in meinem Zimmer. Allerdings denke ich, dass man es trainieren kann, im Zug zu meditieren, und dass es dann immer besser funktioniert. 


\section{„Den wichtigen Dingen mehr Raum geben"}

Interview Katholische Nachrichten-Agentur

Unter anderem veröffentlicht in: Altöttinger Liebfrauenbote, Nr. 2 - 12. Januar 2020, S. 5. Interview: Denise Thomas (KNA)

Während die Welt immer schnelllebiger wird, gibt es einen Trend, der dem entgegenwirken soll: der Trend zur Achtsamkeit. Andreas de Bruin hat das achtsame Leben zum Beruf gemacht. Er unterrichtet und forscht an der Hochschule München sowie Ludwig-Maximilians-Universität (LMU) München zu der Wirkung von Achtsamkeit und Meditation und ist Initiator des "Münchner Modells", einer Initiative zur Integration von achtsamen, meditativen Inhalten in den Hochschulkontext. Im Interview erklärt er das Phänomen Achtsamkeit.

Herr de Bruin, was bedeutet Achtsamkeit genau?

Warum, glauben Sie, gibt es diesen Achtsamkeitsboom?

Welche Effekte denn zum Beispiel?
Achtsamkeit bedeutet, den aktuellen Zustand bewusst wahrzunehmen - nur zu beobachten, nicht zu bewerten, Dinge neu zu sehen. Dies kann bezogen sein auf körperliche Reaktionen, Gedanken, Emotionen oder unsere direkte Umwelt, wie beispielsweise andere Personen und die Natur.

Wir sind weit über das Ziel hinausgeschossen: zu viel Individualismus und eigene Ideale auf Kosten anderer, zu viel Konsum, zu viel Geschwindigkeit, keine Verbindung mehr zur Umwelt. Auch der Verstand und Intellekt haben eine zu große Bedeutung. Auf der Strecke geblieben sind das Herz und die Liebe. Die Achtsamkeit hilft vielen Menschen, wieder gelassener zu werden und mehr zu sich zu kommen. Auch rückt das Gemeinwohl wieder mehr in den Vordergrund. Außerdem sind Achtsamkeitsübungen leicht praktizierbar. Es gibt eine Vielfalt an Übungsvarianten, sodass jeder ausprobieren kann, was zu ihm passt. Es sind keine Kosten damit verbunden, sie stehen somit jedem zur Verfügung. Und nicht zuletzt sind die Effekte sogar bei einer nicht umfangreichen Praxis ziemlich schnell spürbar.

Zum Beispiel die Verbesserung der Psychohygiene, der Körperwahrnehmung, der Konzentration und selektiven Wahrnehmung, der Selbstwirksamkeit, des Wohlbefindens und der Emotionsregulation. Verbindet man die Achtsamkeit noch mit Mitgefühlsübungen, verbessert sich auch der Umgang mit einem selbst und der Umgebung. Der berühmte Neurowissenschaftler und Achtsamkeitsforscher Richard Davidson sagt, dass „die Erde ein anderer Ort wäre, wenn wir für die Pflege unseres Geistes auch nur die gleiche kurze Zeit wie für das Zähneputzen aufwenden würden“. Intensiviert man die eigene Praxis und übt man regelmäßig, festigen sich die Effekte nachhaltig. 
Achtsamkeit kann man leicht in den Alltag einbauen. Viele Alltagsaktivitäten eignen sich dafür gut: zum Beispiel Essen, Spazierengehen, mit jemandem im Gespräch sein, Putzen, Kleiderbügeln, Geschirrspülen, der Natur lauschen und vieles mehr. Man kann aber auch strukturiertere Übungen praktizieren, wie zum Beispiel täglich 15 bis 20 Minuten in Stille zu sitzen und entspannt den Atem zu beobachten, oder den „Bodyscan“ - eine Entspannungsmethode, bei der man mit seiner Aufmerksamkeit schrittweise durch den eigenen Körper „wandert“ und in die Körperteile hineinspürt. Entscheidend ist, dass man dies alles nicht zu sehr verkopft und mechanisch tut. Nach dem Motto: Ich muss jetzt achtsam essen, ich muss jetzt achtsam atmen. Der Wunsch achtsam zu sein, sollte von innen kommen. Achtsamkeit ist eine Haltung. In der christlichen Tradition zum Beispiel werden verschiedene Formen des Sehens unterschieden: das Sehen über die Sinneswahrnehmung, das Sehen durch den Verstand und das Sehen mit dem Herzen. Es geht um die letzte Ebene. Wenn man Achtsamkeit mit dem Herzen verbindet, wird das Praktizieren ganz natürlich.

Wenn die meisten Menschen Geschirr spülen, sind sie mit dem Kopf woanders. Achtsames Geschirrspülen ist, dass ich sehe, was ich spüle. Dass ich spüre, ist das Wasser warm, ist es kalt, wie fühlt sich der Teller oder das Glas an. Wenn wir lernen, diese Dinge wieder bewusster zu tun, dann trainieren wir unseren „Achtsamkeitsmuskel“, sodass wir bei allen Dingen, die wir tun, mehr im Jetzt sind.

Forschungen zeigen, dass wir im Alltag knapp die Hälfte der Zeit nicht wirklich bei der Sache sind. Weil man durch bewusste Achtsamkeit mehr im Jetzt ist und sich das sogenannte Mindwandering - also das Abschweifen der Gedanken - reduziert, wird man weniger abgelenkt, erkennt klarer, was im Leben bedeutend ist und handelt entsprechend. Fragt man Sterbende, was sie im Rückblick auf ihr Leben am meisten bedauern, zeigt sich, dass sie gerne mehr Gefühle ausgedrückt hätten, den Kontakt zu Freunden stärker gepflegt hätten, nicht so viel gearbeitet und mehr den Mut gehabt hätten, das Leben so zu leben, wie sie es gerne gewollt hätten. Achtsamkeit hilft, den wichtigen Dingen mehr Raum zu geben. Durch das Nichtbewerten lernen wir zudem, in Situationen zunächst innezuhalten und nicht zu impulsiv zu reagieren.

Ich meditiere zweimal täglich 20 Minuten - eine Sitzmeditation direkt nach dem Aufstehen und die andere spätnachmittags, meist um 17 Uhr. Dies mache ich seit 1991. Und es ist, wie beispielsweise das Zähneputzen, zum Standardprogramm geworden. Auch versuche ich, viele Alltagsaktivitäten achtsamer auszuführen, wie etwa meinen täglichen Gang zur Arbeit oder die Gespräche mit meinen Studierenden.
Sie haben gesagt, die Übungen kann man leicht praktizieren. Wie sieht dies denn konkret im Alltag aus?

Wie zum Beispiel funktioniert denn achtsames Geschirrspülen?

Warum sollte man seinen Alltag achtsamer begehen?

Haben Sie selbst tägliche Achtsamkeitsrituale? 
(124) Übungen

Eine Rosine achtsam essen.

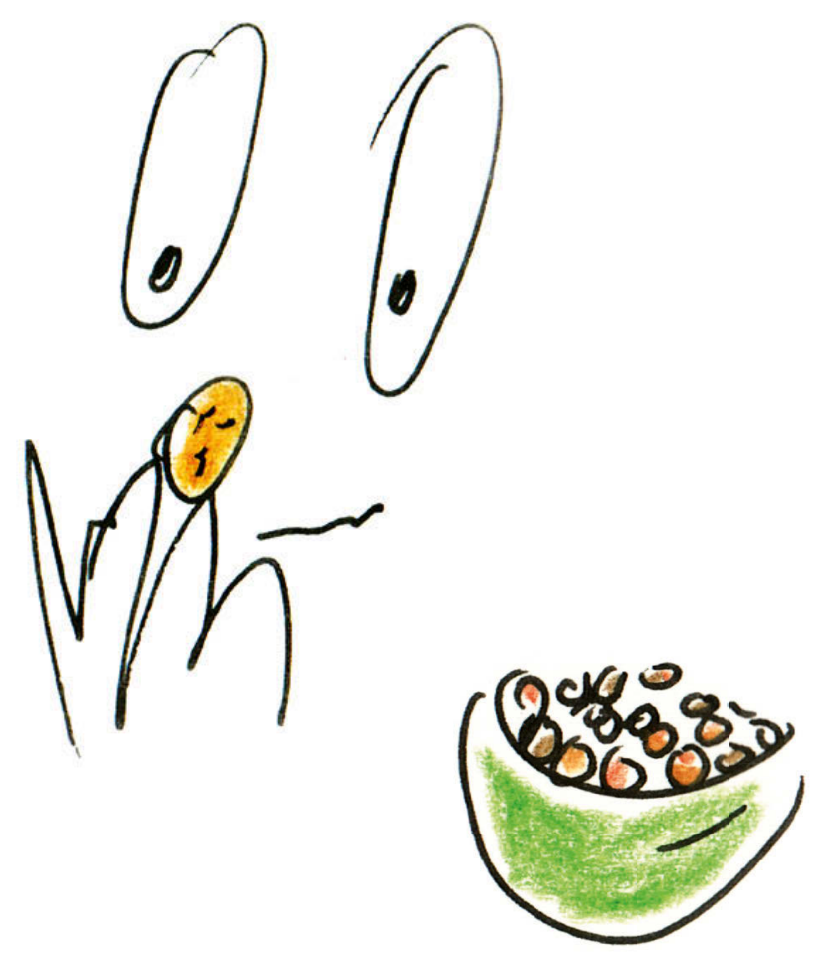




\title{
Achtsames Kochen und Essen
}

\author{
Der Schlüssel zu Gesundheit und innerem Frieden \\ liegt darin, zu lernen, \\ wie man achtsam isst und lebt. \\ Thich Nhat Hanh / Dr. Lilian Cheung
}

Beim achtsamen Kochen und Essen als Achtsamkeitspraxis geht es in erster Linie darum, innezuhalten und im aktuellen Moment präsent zu sein, das heißt in diesem Fall, sich voll und ganz auf das Zubereiten der Mahlzeit und das anschließende Essen zu konzentrieren. Dies beinhaltet auch eine Neugierde an den Abläufen zu entwickeln und ihnen unsere volle Aufmerksamkeit zu widmen.

Wir können beispielswiese beim Schneiden des Gemüses auf die Scheiben achten, die Stück für Stück geschnitten werden, auf die Form und Struktur sowie die jeweiligen Farben, die dabei sichtbar werden. Wir können auch die Geräusche beim Schneiden wahrnehmen oder den Geruch, der womöglich intensiver wird. Kochen wir beispielsweise Reis, so können wir den Dampf im Topf beobachten und das Wasser köcheln hören. Beim achtsamen Essen können wir ebenfalls alle unsere Sinne nutzen.

Bevor wir mit dem eigentlichen Essen beginnen, können wir auch bei der Gestaltung des Tischdeckens und beim Servieren der Mahlzeit aufmerksam und achtsam sein.

Da Achtsamkeit generell auch einen achtsamen Umgang mit sich selbst einschließt, ist die Frage, was wir essen, ebenfalls bedeutend. Nährt es uns ausreichend? Fühlen wir uns vital und gesund mit den Nahrungsmitteln, die wir zu uns nehmen?

Achtsames Kochen und Essen kann zudem auch beinhalten, über die Entstehung, den Transport und Verkauf von Nahrungsmitteln bewusster nachzudenken. Welche Wege legen die Lebensmittel ab, bis sie am Ende auf unserem Teller liegen? Wie sind die Produktionsbedingungen für die Beteiligten? Ist die Herstellung der Nahrungsmittel umweltgerecht und fair?

Um die Idee des achtsamen Essens erfahrbar zu machen, habe ich für diese Lehrveranstaltungseinheit oft die Übung „Der Klang einer Rosine“ aus dem Buch Achtsam mit Kindern leben. Wie wir uns die Freude am Lernen erhalten. Ein Entdeckungsbuch von Dr. Nils Altner, Achtsamkeitsforscher an der Universität Duisburg/Essen, gewählt. 


\section{Der Klang einer Rosine}

(Originaltext Dr. Nils Altner)

Lass dich zu einem sinnlichen Wahrnehmungsexperiment einladen:

Such dir eine Rosine, ohne sie gleich zu essen, und nimm sie zwischen die Finger.

Ich habe hier auch eine. Schau dir dieses kleine Etwas an und tue so, als sähest du ein solches Ding zum ersten Mal.

Was siehst du?

Wenn du willst, beschreibe laut, was sich dir da zeigt.

Ich sehe etwas Schrumpeliges, Braunes mit Rillen. Das Licht scheint zum Teil hindurch. Der Vergleich mit Bernstein liegt nahe. Hier ist der Stielansatz zu sehen.

Siehst du noch was anderes?

Wie fühlt sich das Ding an?

Meins hier ist weich und formbar. Es fühlt sich rau an und wird immer weicher zwischen meinen Fingern und klebriger.

Was spürst du?

Meinst du, dass das Früchtchen, wenn wir es ans Ohr halten und leicht drücken, ein Geräusch macht? Probier mal. Ja, meins knistert! Und deins?

Hättest du das gedacht?

Schnuppern wir mal dran. Wie riecht das? Ich rieche etwas Fruchtiges, Süßes.

Irgendwie auch ledrig herb, gar nicht nur einfach süß. Wie riecht deine Rosine? Spürst du was im Mund? Bei mir fängt schon der Speichel an zu fließen.

Mein Mund ist bereit zum Kauen und Verspeisen. Aber noch sind wir nicht so weit.

Halten wir das Früchtchen erst mal an die Lippen. Wie fühlt sich das an?

Mich kitzelt es. Und meine Lippen spüren die rauen Rillen der getrockneten Frucht. Spüren die Lippen diese Rillen deutlicher als die Finger?

Stecken wir die Rosine jetzt zwischen die Lippen und stupsen mit der Zunge dagegen.

Was passiert? Schmeckst du was? Meine Zunge spürt schon die Süße. Spürst du das auch? Wo schmeckt deine Zunge den süßen Saft, vorne an der Zungenspitze, weiter hinten oder an den Seiten?

Jetzt nehmen wir die Rosine in den Mund und bewegen sie hin und her. Was spürst du nun? Mir scheinen die Rillen immer größer zu werden. Auch wird die ganze

Rosine größer und größer im Mund. Wie ist das bei dir?

Ich habe immer mehr Spucke. Ich glaube jetzt ist es so weit.

Legen wir das Früchtchen zwischen zwei Zähne und schließen die Augen.

Die Kaumuskeln drücken zu und, und was spürst du? Bei mir füllt sich der Mund über und über mit Süße. Diese eine kleine Rosine schmeckt so süß! ${ }^{1}$

Nach der Übung folgt ein Gespräch:

Moderation: „Was haben wir gerade gemacht?"

Wortmeldung 1: „Achtsam gegessen.“

Wortmeldung 2: „Mit allen Sinnen gegessen.“

Moderation: „Essen Sie normalerweise auch auf diese Art eine Rosine? Nein, wie dann?

Haben Sie in der Zeit des Essens an anderes gedacht? Haben Sie etwas Neues während des Essens entdeckt?" 
Den Kursteilnehmerinnen und Kursteilnehmern wird als Aufgabe mitgegeben, das achtsame Essen im Alltag einige Male auszuprobieren. Egal was gegessen wird und wie viel und wie man Lust und Zeit hat. Ebenso kann auch das Zubereiten achtsam durchgeführt werden. Bei Salat beispielsweise: das Schneiden der Gurke, der Saft, der aus den Gurkenscheiben herauskommt, die verschiedenen Grüntöne ...

Da es im Studiengang Soziale Arbeit Lehrveranstaltungen gibt, die im Vergleich zu den anderen Kursen mehr Semesterwochenstunden haben, wurden diese in einen Achtsamkeitstag (Blockveranstaltung) umgewandelt. Im Mittelpunkt steht an diesem Tag das gemeinsame achtsame Kochen und Essen. In einem relativ kleinen Raum samt Küche bewegen sich dann circa 20 Personen und versuchen, ohne zu sprechen Gerichte zu kochen. Es ist immer wieder erstaunlich, wie gut das funktioniert, und das Essen schmeckt (meistens) ausgezeichnet ;-) Das Eindecken der Tische geschieht ebenso schweigend, und auch die ersten 10 Minuten wird beim Essen nicht gesprochen. Für viele ist das Schweigen über eine so lange Zeit - vom Kochen bis zum ersten Biss sind es meistens 90 Minuten - eine völlig neue Erfahrung. Nach den ersten 10 Minuten am Tisch sprechen einige sofort, andere haben so Gefallen an der Stille gefunden, dass sie am liebsten die weitere Zeit beim Essen so weitermachen würden.

Wortmeldung 1: „Super! Werde ich auch zu Hause ausprobieren.“

Wortmeldung 2: „Ich hätte nie gedacht, dass wir das Kochen so hinbekommen würden.“ Wortmeldung 3: „Mir war es peinlich, andere anzuschauen und nichts zu sagen.“

Wortmeldung 4: „Ich habe viel mehr auf mein Essen geachtet.“

Das Thema des achtsamen Kochens und Essens kann auch weiter ausgebaut werden. Zum Beispiel: gesundes und nachhaltiges Essen. ${ }^{2}$

Da so viele Studierende das Schweigen beim Essen als positiv bewertet haben, könnte das ein interessanter Anstoß sein, in den Mensen auch einen Bereich einzurichten, wo dies möglich ist.

Für den Unterricht benutzte Primärliteratur

Thich Nhat Hanh / Cheung, Lilian (2016). achtsam essen - achtsam leben:

Der buddhistische Weg zum gesunden Gewicht. München: O. W. Barth Verlag.

Altner 2009, S. 60f. Der Abdruck dieser Übung erfolgt mit freundlicher Genehmigung des Autors.

2 An der University of Applied Sciences in Amsterdam, ein Kooperationspartner des Münchner Modells, besteht beispielsweise seit mehreren Jahren bereits eine Zusammenarbeit zwischen dem Studiengang Positive Psychology und dem Europäischen Institut für Makrobiotik (Kushi). 
(128) Übungen

oluch habe ich die Konsistenz des gorichts (Semmellencider mit Bilzsauce) noch wie so bewusst wahrgenommen. Gine Freundin von mir meinte allerdiugs, ils sei das Essen zu. schleimid wenn sie fich so darauf kanzentilest.

vAuch wenn es sparnend wor, sich über vie Erfochrung auszutauschen, queine ausprobiesen. Wir siud dann doch recht schneu abgeschweift und uaben
uns unterhattent.

$30 \cdot 10$.

Achtsam essen: Alleine.

Was ich sehr angenehm fand:

Jch watte dan Eindruce a'us ich ruirklich oufgenōrt uabe, zu. essen als ich sut war. Whodrs als sonst luabe ich nicht, mels nackgefasst weil es mir so geschmecter hat Dacturctay dass ich eh total bei der Sache wiar und den Gescilhmack ansgekostet" habe hat mir eine Bortion völig gereiclot.

Worperscan im situn auf dum sofa.

20 min, war ein bisschen Romisch wcil ich mich immur wieder umsehen musst und so war meine Uonzentration richt to put $14.05 \cdot 19$

Ich habe gerade auf Gund mein Sehwanjerscheat extrem Gelusts out Schabe Caele. Alsc habe ith ein Schoko scichiohen aufmerksam refessen. Ung
1. 11

20 Minuten Meditation am Morgen.

$\rightarrow$ Itat get getan. Konnte mich gut forlessieren... wahrscheivilich auch, weil ich heute frei habe.

orbends: bodyscan zum Einschlafen (weiter als bis zu cien vien bin ich nicht gerommen)

8. 11 .

- liebende gäte fiude ich tiemlich cool. Jich glaube, dass. das. line Gibung ist, auf die man supe zurrickgreifen tamniduenns mal nicht so läufa. Heute war ein blöder Trödel. Tag an dem ich wicht ansakweise. clas an dem ich wicht ansatch mir vorgenommen
geschafft habe, was ich wabh abens
hatle. Deswegen war ich fiemlich geknicket und ein bisschen rover auf mich selbst.

damit habe ich en feschallt bel einum w bleiben.

17.05 .19

Genmeditalior, 20 min Dabej bann man super absrialun do man so schr damit beschatti.t. ist auf sein schuite us achten. 20.05 .19

Meditation mil einum Slein. lich habe mireitur Slein genommen und in ganz gendu beluthet. Ic habe probient all Uonbun noukenfabun und in mi.

(c) Münchner Modell | www.hm.edu/meditationsmodel 


\section{Tagebuchnotizen Achtsames Kochen und Essen}

F. S.

\subsubsection{9, Atemmeditation + MA-RA-NA-THA Dauer: 15 Min.}

Heute war ein heißer Tag und ich war gerade bei einem Klienten, den ich 24 Stunden lang betreue. Wir waren Einkaufen und danach fing ich mit dem Kochen an. Ich denke, es war die Anstrengung vom Einkaufstaschetragen in der Hitze und die Dämpfe beim Kochen, denn unerwartet streikte mein Kreislauf. Ich schwitze, mir wurde übel und ich konnte mich fast nicht mehr auf den Beinen halten. Ich setzte mich hin, doch leichte Panik machte sich in mir breit. Es wurde nicht besser. Deshalb versuchte ich, gleichmäßig zu atmen und mich auf meine Atmung zu konzentrieren. Meine Gedanken waren wirr und ich fühlte mich sehr unwohl. Deshalb versuchte ich zusätzlich die Maranatha-Methode, um die Panik zu mildern. Dies machte ich einige Minuten, bis ich imstande war, aufzustehen und mir Wasser zu holen. Ich setzte mich wieder hin und führte die Meditation weiter. Das Maranatha hat mir sehr geholfen, Ruhe zu bewahren und diesen Zustand zu überwinden. Nach $15 \mathrm{Mi}-$ nuten ging es mir besser und ich konnte weiterkochen.

M. M.

\section{Achtsames Essen einer Kiwi}

Wann: Freitag, 14.11.2014, 15.25-15.35 Uhr Wo: In der Küche

Warum: Ich bin von den vielen Eindrücken dieser Woche überwältigt, deshalb tut es mir jetzt gut, mich auf nur einen Sinn zu konzentrieren.

Recherche: Nach Jon Kabat-Zinn ist Achtsamkeit

- auf den Augenblick ausgerichtet. Das heißt, ich nehme den gegenwärtigen Moment ganz bewusst wahr und achte darauf, was in mir selbst vorgeht oder auch um mich herum geschieht

- ein bewusster, absichtlicher Zustand. Das heißt, dass ich alles, was ich tue, mit voller Aufmerksamkeit tue

- wohlwollend und wertfrei. Das heißt, ich lerne mich selbst, andere Menschen und Situationen so anzunehmen, wie sie sind

\section{Achtsamkeitsübung:}

- Ich schneide die Kiwi auseinander, sodass ich 2 Hälften habe.

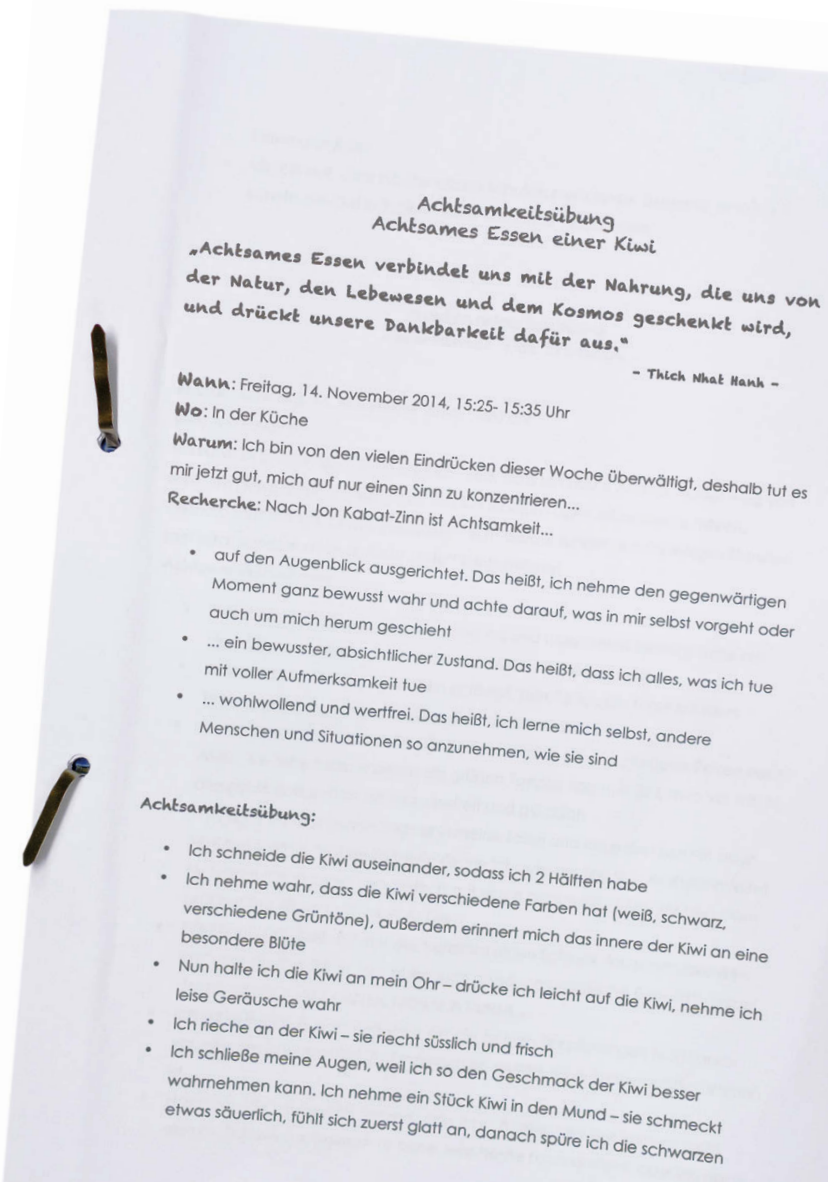

- Ich nehme wahr, dass die Kiwi verschiedene Farben hat (weiß, schwarz, verschiedene Grüntöne), außerdem erinnert mich das Innere der Kiwi an eine besondere Blüte.

- Nun halte ich die Kiwi an mein Ohr - drücke ich leicht auf die Kiwi, nehme ich leise Geräusche wahr.

- Ich rieche an der Kiwi - sie riecht süßlich und frisch.

- Ich schließe meine Augen, weil ich so den Geschmack der Kiwi besser wahrnehmen kann. Ich nehme ein Stück Kiwi in den Mund - sie schmeckt etwas säuerlich, fühlt sich zuerst glatt an, danach spüre ich die schwarzen Kerne und Rillen.

- Mir fällt auf, dass ich die letzten Minuten mehr in der Gegenwart war; ich konnte die Kiwi in Ruhe und ohne Hektik essen. 


\section{Achtsames Teetrinken}

\section{Wann: Sonntag, 16.11.2014, 14.20 Uhr Wo: Am Esstisch}

Warum: Es ist Sonntagmittag und ich weiß, dass ich heute Abend wieder weg von meinem gewohnten Umfeld muss, um fürs Studium nach München zu fahren. Deshalb möchte ich meine Gedanken nicht darauf lenken, was in einigen Stunden sein wird, sondern mich auf das „Jetzt“ konzentrieren.

\section{Achtsamkeitsübung:}

- Zuerst schalte ich den Wasserkocher ein und hole meine Lieblingstasse mit vielen bunten Blüten und Punkten aus dem Küchenschrank.

- Während das Wasser zu kochen anfängt, bereite ich die Tasse mit dem Teebeutel eines leckeren ayurvedischen Gewürztees vor.

- Ich rieche am Teebeutel. Der Duft erinnert mich an verschiedene Reisen nach Asien; ich sehe beispielsweise die grünen Teeplantagen in Sri Lanka vor mir. In diesem Moment fühle ich mich befreit und glücklich.

- Ich gieße das kochende Wasser in meine Tasse und lasse den Tee ein paar Minuten ziehen. Währenddessen rieche ich, wie der Tee sein Aroma entfaltet.

- Nun setze ich mich an den Esstisch auf einen bequemen Stuhl vor den Ofen und nehme einen Schluck Tee.

- Er ist noch sehr heiß; ich nehme trotzdem einen Schluck. Ich schmecke den Zimt und die Vanille im Tee, aber auch andere orientalische Gewürze. Dabei denke ich an bunte Farben, Märkte in Indien ...

- Ich schließe die Augen und stelle mir die grünen Teeplantagen in Sri Lanka vor, wie der Tee geerntet und transportiert wurde, bis er bei uns angekommen ist.

- Nach ca. 10 Minuten fällt mir auf, dass ich während des Teetrinkens nicht einmal darüber nachgedacht habe, was heute noch ansteht, dass ich nach München fahren und mich hier verabschieden muss ...

\section{N. S.}

\subsubsection{9}

Ich habe gerade aufgrund meiner Schwangerschaft extreme Gelüste auf Schokolade. Also habe ich ein Schokostückchen aufmerksam gegessen. Und damit habe ich es geschafft, bei mir zu bleiben.

\section{P. A.}

Seit einiger Zeit versuche ich stärker, achtsam zu essen. Während des Kochens und während des Essens nicht zu sprechen, ist sehr angenehm. Man nimmt das Essen und die Umgebung besser, stärker wahr.

Während des Essens kann man sich genau auf den Geschmack und die Konsistenz in seinem Mund fokussieren. Man schmeckt die einzelnen Geschmacksrichtungen viel intensiver, und die einzelnen "Teile“ des Essens werden erst nach und nach zu einem.

Ich mag es sehr, wenn für das Essen viel Zeit verwendet wird und man es langsam und bedächtigt tut und auch hin und wieder mal eine Pause macht. Leider ist das im Alltag nicht immer möglich, vor allem wenn man oft mit anderen zusammen isst (was ja auch schön ist). Leider kann man nicht immer jeden dazu anregen, auch achtsam zu essen, und der/die andere muss warten. Aber das ist ja vielleicht auch ganz gut so.

\section{S. L.}

\section{Achtsam essen: alleine}

Was ich sehr angenehm fand: Ich hatte den Eindruck, dass ich wirklich aufgehört habe zu essen, als ich satt war. Anders als sonst habe ich nicht mehr nachgefasst, weil es mir so geschmeckt hat. Dadurch dass ich eh total bei der Sache war und den Geschmack ,ausgekostet“ habe, hat mir eine Portion völlig gereicht.

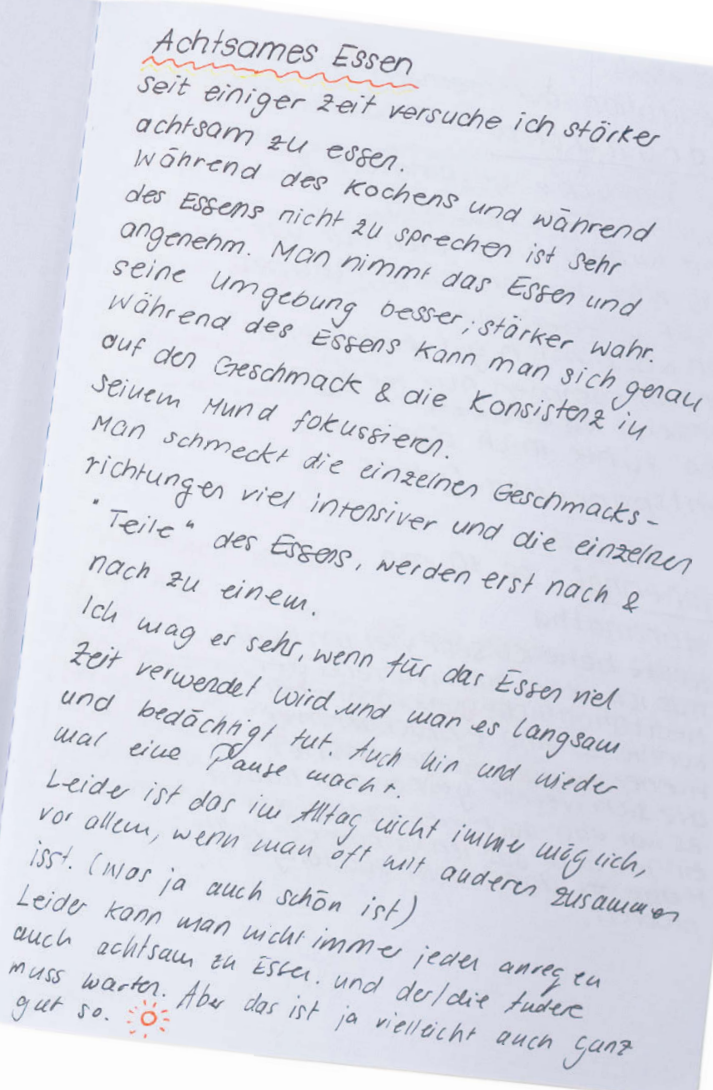




\section{Achtsame Kommunikation}

Als ich im Zug zu Verwandten nach Freising fuhr, fielen mir zwei jungen Frauen ein paar Sitze von mir entfernt auf. Ich bemerkte sie deshalb, weil sie so laut redeten. Nicht beide, nur eine redete und die andere hörte fast ausschließlich zu. Immer wenn die Zuhörerin selbst etwas einbringen wollte, hat die andere gleich wieder das Wort ergriffen. Es war ein permanenter Monolog - und das fast 35 Minuten lang. Irgendwann wollte ich nur noch meine Ohren abschirmen, damit ich diesem fortwährenden Geplapper nicht weiter ausgesetzt war.

Achtsame Kommunikation ist etwas völlig Gegenteiliges als das einseitige „Reden“, das sich im Zug abgespielt hat. Es geht darum, im Gespräch Raum zu kreieren, damit Menschen sich wirklich begegnen können.

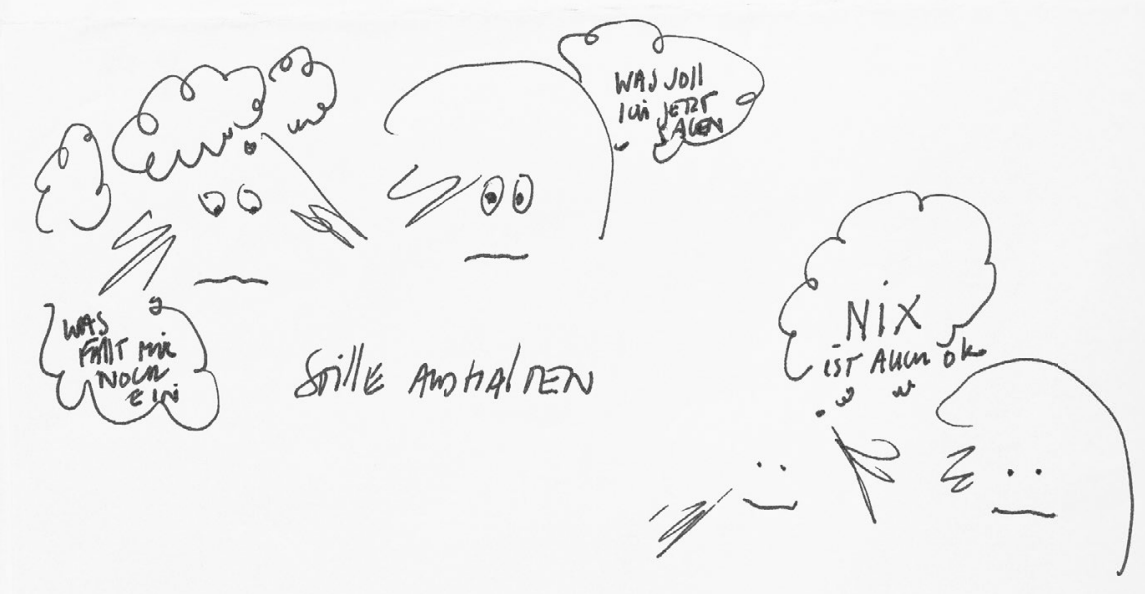

Eine Übung zur achtsamen Kommunikation, die wir im Unterricht durchgeführt haben, ist folgendermaßen aufgebaut:

Es werden Zweiergruppen gebildet.

Eine Person spricht, die andere hört nur zu. Kleine Feedbacks zum Gesagten mit Mimik sind möglich, aber keine verbalen Reaktionen.

Blickkontakt soll wie im normalen Gespräch weiterhin stattfinden.

Die zuhörende Person kann neben dem Inhalt auch darauf achten, wie etwas gesagt wird. Insbesondere auf die Stimme, wie beispielsweise die Betonung ist, die Sprechmelodie, die Tonhöhe und die Klangfarbe.

Die sprechende Person wiederum kann während des Sprechens auf die Mimik und Gestik des Gegenübers achten.

Nach circa 5 Minuten wird gewechselt.

Danach folgt eine Feedbackrunde zwischen beiden Personen.

"Was wurde beobachtet, erfahren?"

"War es leicht, dem anderen zuzuhören?“

„Wie ist es für den Sprecher, die Sprecherin, wenn die andere Person sich nicht am Gespräch beteiligt?“ 


\title{
Am Anfang einer Kommunikation mit einem anderen Menschen ist es hilfreich, sich daran zu erinnern, dass es in jedem von uns einen Buddha gibt.
}

\author{
Thich Nhat Hanh
}

Abschließend folgt eine Gruppendiskussion über die gemachten Erfahrungen.

Das Gesprächsthema ist offen. Im Unterricht nahmen wir meistens „Was bedeutet Glück für mich?" als Thema.

Beispiele aus der Feedbackrunde:

Wortmeldung: „Irgendwann wusste ich nicht mehr, was ich sagen soll. Das war irgendwie peinlich."

Moderation: „Ich habe nicht gesagt, dass man permanent reden soll."

Wortmeldung: „Ja, aber es ist schwierig, man erwartet eine Rückantwort.“

Moderation: „Warum ist es peinlich, wenn man einmal nichts sagt? Haben wir Angst vor Stille?“

Wortmeldung 1: „Schön ist, dass man einfach ausreden kann. Man hat die Zeit, einen Gedanken aufzubauen und auszuformulieren."

Wortmeldung 2: „Da kein anderer Input hinzukommt, kann das Gespräch auch nicht ungewollt in eine andere Richtung gehen."

Wortmeldung 3: „Es ist spannend, wirklich genau zuzuhören, was die andere Person sagen will. Man erfährt dadurch auch Neues."

Wortmeldung 4: „Und beim Hören braucht man auch nicht gleich darüber nachzudenken, was man auf manche Sätze sagen soll.“

Wortmeldung 5: „Es wird alles viel entspannter!“

In einer weiteren Einheit haben wir die Übung noch etwas intensiviert.

Wieder spricht eine Person über eine festgelegte Zeit und die andere hört zu.

Jetzt soll aber die sprechende Person gleichzeitig auch auf die eigenen körperlichen und emotionalen Reaktionen achten. Es geht nun um die Selbstwahrnehmung. Zum Beispiel um die eigene Stimme, die Betonung des Gesagten, die Klangfarbe, die Gefühle beim Sprechen etc. Auch die zuhörende Person achtet jetzt auf ihre Reaktionen.

Nach Ablauf der festgelegten Zeit wird gewechselt. Anschließend gibt es wieder ein Feedback in Zweiergruppen, danach in der Gesamtgruppe.

Im Alltag ist es oft so, dass immer die gleichen Personen reden und andere kaum zu Wort kommen. Wenn Menschen eher introvertiert sind oder länger brauchen, um einen Gedankengang richtig aufzubauen, haben sie es in unserer schnelllebigen Gesellschaft nicht leicht. Es kann aufschlussreich sein, gerade denjenigen Menschen zuzuhören, die in der Regel eher wenig sagen.

Achtsame Kommunikation erfordert eine respektvolle und interessierte Haltung aller Gesprächsteilnehmerinnen und -teilnehmer. Bleiben die Gesprächspartner dabei auch authentisch und aufrichtig, kann ein tieferer Austausch stattfinden, der auch die Herzebene miteinschließt.

Für den Unterricht benutzte Primärliteratur

Thich Nhat Hanh (2019). achtsam sprechen - achtsam zuhören. Die Kunst der bewussten Kommunikation. München: Knaur Verlag. 


\section{Tagebuchnotizen \\ Achtsame Kommunikation}

J. $\mathrm{H}$.

15.6.2017

\section{Wo: Zu Hause}

\section{Was: Achtsames Zuhören}

Ich habe heute zwar nicht meditiert, aber ein Freund von mir hat mir etwas erzählt, und ich habe wirklich bewusst zugehört und nicht darauf gewartet, selbst sprechen zu können. Das ist ihm auch aufgefallen, und er hat mir gesagt, dass er es als angenehm empfindet, mit mir zu sprechen. Und das eben genau deswegen weil er das Gefühl hat, dass mich wirklich interessiert, was er sagt. Diese Rückmeldung gibt einem natürlich positive Bestätigung.

\section{N.S.}

1.5.2019

Ich habe seit dem letzten Seminar versucht, achtsam zu kommunizieren. Es fällt mir schwerer als gedacht, nicht sofort mit eigenen Geschichten, Gedanken und Ideen auf das Gesagte zu reagieren. Ich denke, dafür muss man immer wieder darauf achten und dann nach und nach die Eindrücke von Stimme und Körpersprache hinzunehmen.

Stimme und Lorpersprach hinunehmun. 
S. B.

\subsubsection{8}

\section{Wo: Meditationsraum im Paoso}

\section{Was: Achtsame Kommunikation}

Meine Kommunikationspartnerin beginnt zu erzählen, was Glück für sie ist. Ich merke, wie sie während des Gesprächs immer langsamer wird und eigentlich auf eine Antwort von mir wartet. Mir fällt es schwer, nicht $\mathrm{zu}$ antworten, ich finde das ist unhöflich. Aber andererseits ist es auch schön, keine Meinung zu einem Thema abgeben zu müssen. Durch die ununterbrochene Kommunikation fällt es mir leicht, ihre Gesichtszüge zu verfolgen, wenn sie etwas ausspricht, was sie begeistert oder eher weniger.

\section{S.Z.}

\section{Achtsames Zuhören}

Zeit: ca. 15 Min.

Mir ist bewusst, dass ich jemand bin, der, wenn mir jemand etwas erzählt, einen inneren Drang hat, sofort einen Beitrag dazu abzugeben, ohne vorher die Person wirklich zu Ende reden zu lassen und zu verarbeiten, was diese eigentlich gesagt hat.

$\mathrm{Da}$ ich manchmal dazu neige viel zu reden, habe ich Angst, dass ich Leute damit überfordern kann oder ihnen nicht genug die Möglichkeit biete, vor allem wenn jemand schüchtern ist, sich zu äußern. Da ich mich heute mit einer Freundin getroffen habe, die mir etwas zu erzählen hatte, wollte ich versuchen, achtsam zuzuhören und mich auf den Inhalt zu fokussieren, ohne gleich zurückzuspielen.

Ich muss wirklich zugeben, dass es mir zu Beginn schwerfiel, nichts zu sagen, da meine Freundin mir von dem negativen Verhalten ihrer Freundin erzählte, zu der ich kein gutes Verhältnis habe und daher meistens Abstand zu ihr halte. So fiel es mir schwer, keinen Kommentar dazu abzugeben, was ich auch geschafft habe, aber dafür wurden meine Gesichtszüge ausdrucksstärker als sonst. Mir ist aber auch aufgefallen, dass die Reaktion meiner Freundin auf mein Schweigen dazu geführt hat, dass sie viel umfangreicher davon erzählt hat. Dadurch ist es mir leichter gefallen, ihre Ansicht besser zu verstehen und zu interpretieren, da ich auch ihre Mimik besser analysiert habe als sonst.

Da meine Freundin nichts von meinem „Experiment" wusste, habe ich die Meditation nur ca. in den ersten 5 Minuten durchgeführt. Aber mir ist aufgefallen, dass ich auch danach intensiver zugehört habe und weniger geredet habe als sonst.

\section{Achtsames Zuhören}

\section{Zeit: ca. 8 Min.}

Heute war eine Freundin von mir sehr traurig, daher habe ich sie besucht. Ich habe oft bemerkt, dass viele Menschen in einer solchen Situation einfach verstanden werden wollen oder bestimmte Dinge von ihrer Seele reden möchten, weshalb ich mir vorgenommen hatte, einfach zuzuhören.

Anfangs fiel es mir schwer, keine Verbesserungsoder Lösungsvorschläge anzubringen und sie zu unterbrechen, aber als ich dann bemerkte, dass sie langsam zum Ende dessen kann, was sie sich von der Seele reden wollte, fragte sie mich selber, was ich denn vorschlagen würde und davon halte. Mir ist dadurch aufgefallen, wie viel Verständnis mir für die Situation fehlen würde, hätte ich nicht zu Ende gehört und sie direkt mit meinen Vorschlägen bombardiert. Es fiel mir auch auf, dass ein solches Verhalten den Eindruck erwecken könnte, dass man das Thema schnell abhaken möchte oder ich mich nicht für ihr Problem interessieren würde. 


\title{
Bodyscan
}

\author{
Wenn wir unsere Energie einmal darauf richten, \\ unseren Körper wirklich zu spüren, und uns davor hüten, \\ in das urteilende Denken über den Körper zu verfallen, \\ können sich unser ganzes Körpererleben und das Erleben \\ unserer selbst in radikaler Weise verwandeln.
}

Jon Kabat-Zinn

Der Bodyscan ist eine Übung, bei der wir unsere Aufmerksamkeit auf einzelne Körperbereiche richten und so schrittweise den ganzen Körper durchwandern. Wichtig dabei ist, die jeweiligen Körperteile achtsam zu spüren, ohne aufkommende Gedanken und Gefühle zu bewerten.

In der Regel macht man diese Übung im Liegen. Alternativ kann sie natürlich auch im Sitzen, beispielsweise am Schreibtisch im Büro oder als Entspannungsübung im Unterricht, durchgeführt werden.

Die Übung wurde durch den Medizinprofessor und Pionier der Achtsamkeitsbewegung Jon Kabat-Zinn bekannt, der sie im Rahmen seines MBSR-Programms am Medical Center der University of Massachusetts für Schmerzpatienten Ende der 1970er-Jahre entwickelte.

Es gibt viele Formen des Bodyscans, und zahlreiche Anleitungen finden sich in Büchern und Audiomedien sowie im Internet. Auch die Länge der Übung variiert bei den Angeboten stark.

Wir haben uns im Unterricht an den Übungsbeschreibungen von Jon Kabat-Zinn orientiert. Die Übungsdauer war bei uns allerdings kürzer und betrug gewöhnlich eine Viertelstunde.

Beim Übergang von einer Körperregion zur nächsten wurde zur Ankündigung eine Klangschale angeschlagen, und auch, um Teilnehmenden die eventuell dabei einschlafen, sanft zurückzuholen.

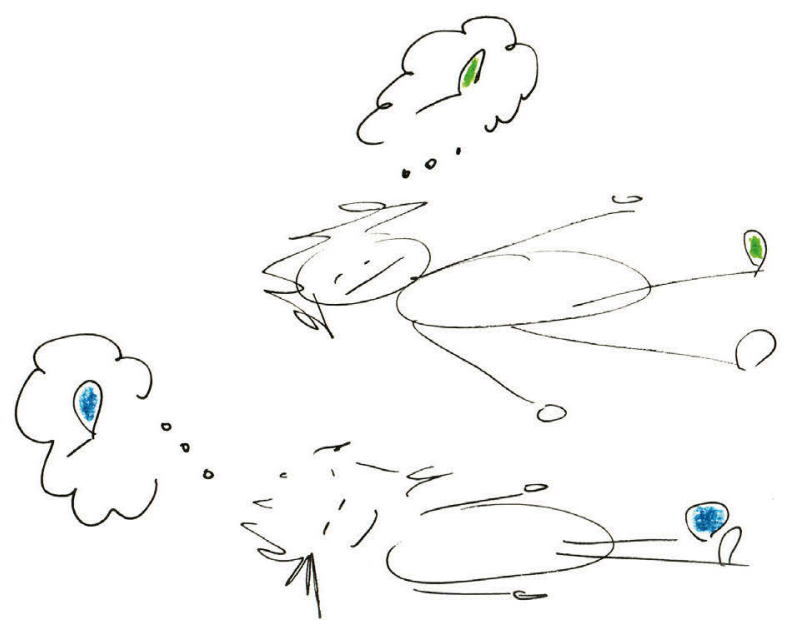

Bodyscan im Liegen kann auch helfen, wenn man Probleme beim Einschlafen hat. 


\section{Anleitung}

\section{Klangschale $1 \mathrm{x}$ anschlagen}

Wir legen uns entspannt auf eine Matte. Wir können spüren, wie der Körper den Boden bzw. die Oberfläche der Matte berührt. Wir können uns dabei auch vorstellen, dass wir im Sand am Meer liegen, auf einer Wiese, auf dem Sofa zu Hause oder einfach hier im Raum. Wir sind ganz entspannt. Nun schauen wir auf unseren Atem. Er kommt und geht von alleine. Ganz entspannt atmen wir - ein und aus ... ein ... aus ...

Jetzt gehen wir mit unserer Aufmerksamkeit zum linken Fuß und weiter zu den Zehen des linken Fußes. Wir spüren, wie sie sich anfühlen. Wir spüren einfach nur und beobachten, ohne das Gefühl zu beurteilen. Einfach abscannen, daher auch der Name Bodyscan. Wenn wir möchten, können wir die Zehen auch etwas bewegen. Dann gehen wir mit unserer Aufmerksamkeit zum mittleren Bereich des linken Fußes ...

Linker Fußknöchel

Unterschenkel links (Wade, Schienbein ...)

Linkes Knie

Oberschenkel links (wir können ihn auch kurz an- und entspannen)

Linkes Becken

\section{Klangschale $1 \mathrm{x}$ anschlagen}

Jetzt zum rechten Fuß. Wir spüren, wie sich die Zehen anfühlen. Wieder spüren wir nur und beobachten, ohne das Gefühl zu beurteilen. Einfach abscannen. Wenn wir möchten, können wir die Zehen auch etwas bewegen. Dann zum mittleren Bereich des rechten Fußes ...

Rechter Fußknöchel

Unterschenkel rechts (Wade, Schienbein ...)

Rechtes Knie

Oberschenkel rechts (wir können ihn auch kurz an- und entspannen)

Rechtes Becken

\section{Klangschale 1x anschlagen}

Jetzt das Steißbein und von dort langsam etwas nach oben den Rückenwirbel entlang. Vorne die Leisten und von dort langsam in Richtung Bauch.

Hüfte

Hinten weiter den Rückenwirbel nach oben.

Vorne entspannt in Richtung Brustbein.

Brustbereich

Hinterer Schulterbereich

Klangschale 1x anschlagen

Jetzt zur linken Hand und hier zunächst zu den Fingern.

Wir können auch unseren Atem dort hinlenken. Vielleicht sogar mit den 
Fingern atmen. Einfach mal ausprobieren.

Dann weiter zum mittleren Bereich der linken Hand. Auch hier spüren wir nur, beobachten.

Linkes Handgelenk

Unterarm links

Linker Ellbogen

Oberarm links ... Trizeps, Bizeps

Diesen können wir, wenn man möchte, auch kurz an- und entspannen.

Linke Schulter

Klangschale $1 \mathrm{x}$ anschlagen

Jetzt zur rechten Hand und hier zu den Fingern.

Wir können auch unseren Atem dort hinlenken. Vielleicht sogar mit den Fingern atmen. Einfach mal ausprobieren.

Dann weiter zum mittleren Bereich der rechten Hand. Auch hier spüren, beobachten.

\section{Rechtes Handgelenk}

Unterarm rechts

Rechter Ellbogen

Oberarm rechts ... Trizeps, Bizeps

Diesen können wir, wenn man möchte, auch kurz an- und entspannen.

Rechte Schulter

Klangschale $1 \mathrm{x}$ anschlagen

Jetzt den Nackenbereich, entspannt hineinspüren.

Vorne Hals- und Kehlbereich

Kinn

Mundbereich

Nase und Nebenhöhlen

Ohren

Augen - entspannt fallen lassen in Richtung Hinterkopf

Stirnbereich

Jetzt der Scheitel, hier können wir uns ein imaginäres Loch vorstellen, in das wir hineinatmen.

Oben am Scheitel hineinatmen und den Atmen durch den ganzen Körper fließen lassen.

Am Ende unten an den Fußsohlen wieder ausströmen lassen.

Und dies einfach wiederholen: Oben am Scheitel hineinatmen, durch den

Körper fließen lassen und unten an den Fußsohlen wieder heraus.

Dies einige Male weiter wiederholen, und wenn wir irgendwo im Körper eine Stelle spüren, die sich nicht so angenehm anfühlt, können wir den Atem dorthin lenken.

Wir können sogar in die Stelle hineinatmen.

Jetzt kommen wir langsam wieder zurück.

Klangschale 3x anschlagen 
Wir machen die Augen langsam auf. Nicht gleich aufrichten. Vielleicht erst noch die Zehen und Finger etwas bewegen. Wir können auch einfach noch liegen bleiben.

\section{Moderation: „Wie war's?"}

Wenn die Übung zum ersten Mal gemacht wird, kann zunächst ein Gespräch in Zweierteams und anschließend in der Gesamtgruppe folgen.

Meine bisherigen Erfahrungen zeigen, dass in der Regel eine große Anzahl der Teilnehmerinnen und Teilnehmer nicht die gesamte Zeit aufmerksam und fokussiert bleiben kann. Viele berichten, dass sie ein oder mehrere Passagen nicht (ganz) mitbekommen haben, sprich: kurz abgedriftet bzw. eingeschlafen sind. Das zeigt, dass viele Teilnehmenden relativ müde sind und durch diese Übung entspannen und zur Ruhe kommen können.

Aus den Meditationstagebüchern ist zu entnehmen, dass viele Teilnehmerinnen und Teilnehmer den Bodyscan abends zum Einschlafen nutzen. Zwar ist die Übung in erster Linie nicht dafür gedacht, aber viele von ihnen können dadurch rascher und besser einschlafen.

Für den Unterricht benutzte Primärliteratur

Kabat-Zinn, Jon (2019). Gesund durch Meditation. München: Knaur Verlag.

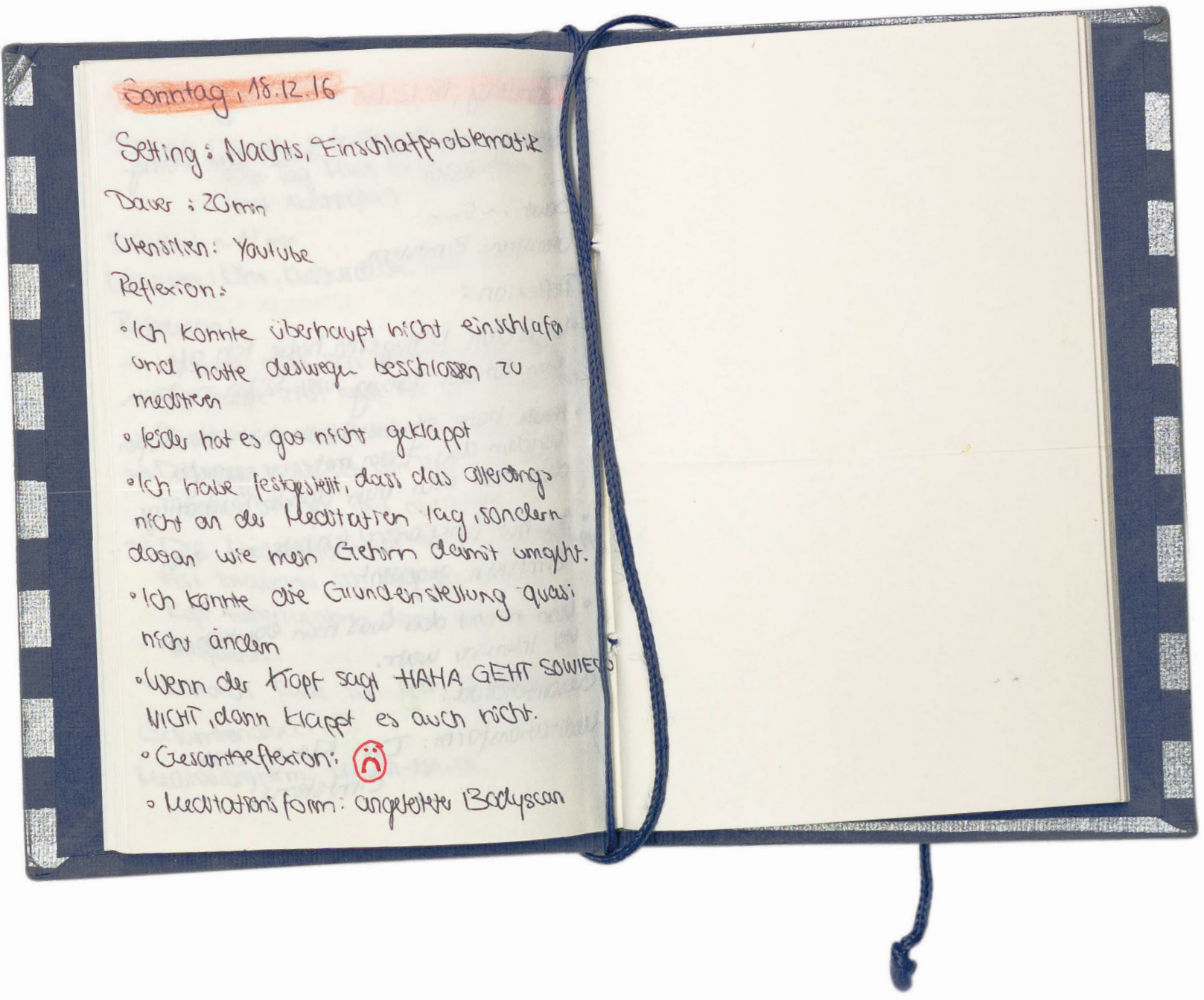




\section{Tagebuchnotizen Bodyscan}

F. S.

\subsubsection{9}

\section{Dauer: 15 Min.}

Heute begann der Tag für mich früh um $8 \mathrm{Uhr}$ an der Uni. Nach zwei Kursen hatte ich aus und fuhr nach Hause. Dort war ich sehr geschafft und antriebslos. Deshalb legte ich mich auf die Couch und suchte mir auf YouTube eine geführte Meditation - Bodyscan - aus. Bevor ich anfing, bereitete ich mich mit einer kurzen Atemmeditation vor, um gedanklich ganz abschalten zu können. Dies gelang mir sehr gut. Beim Bodyscan konnte ich die einzelnen Partien, auf die ich mich konzentrierte, intensiv spüren. Mein Füße und Beine durchflutete ich mit Energie, genauso meine Hüfte, Arme und zum Schluss meinen Kopf. Am Ende atmete ich einige Male tief ein und fühlte mich bereit für den zweiten Teil des Tages.

\section{H. V.}

Ich liege im Bett. Bin unruhig. Flower-Bauch. Wie kann ich mich ein bisschen beruhigen?

Ich fing an, ruhig und bewusst zu atmen. Meine Zehen zu spüren.

Der Luftstrom ging weiter das Schienbein hinauf. Dann die Kniescheibe und die Hüfte. Es fühlte sich alles schwer an. Ich wurde ruhiger, träger und müder.

Dem Magen hat der Bodyscan besonders gutgetan, er wurde mit Luft durchströmt und weitete sich und entspannte sich.

Die Brust weitete sich ebenfalls.

An dieser Stelle wurden die Augen so sehr schwer. Der Bodyscan verlor sich im Schlaf.

\section{J. Z.}

\subsubsection{4}

An einem Abend konnte ich nicht einschlafen, sodass ich mich für einen Bodyscan entschied. In meinem Kopf ging ich nach und nach die Körperteile durch [...]. Es tat mir gut, meine Gedanken auf meinen Körper zu lenken und ich merkte, wie ich müder wurde. Als ich ihn ganz durchgeführt hatte, war ich zufrieden und ruhig und konnte relativ schnell einschlafen.

Auch diese Woche konnte ich bei mir selbst wieder Fortschritte bemerken. Ich reagierte in Stresssitu-

\section{Bodyscann} ch liege im Bett. Bin unruhig. Flower Bauch. Wie kann ich
mich bisschen beruhigen?

mich bisschen ber und bewusst zu atmen.

Meine Zehen zu spüren.

Der Luftstrom ging weiter das Schiufte. Es fühlte sich alles Dann die Kniescheibe und die Häte. Es und müder. schwer an. Ich wurde ruhiger, trager unders gut getan, er Dem Magen hat der Bodyscan besond weitete sich und wurde mit Luft durchströmt und wurde mit Luft Die Brust weitete sich ebenfalls. An dieser Stelle wurden die Augen

Bodyscann verlor sich im Schlaf.

ationen gelassener und regte mich nicht über Personen bzw. Situationen auf, die mich sonst immer schnell genervt hatten. Ich hatte auch das Gefühl, dass ich meine Umgebung intensiver wahrnahm und mich besser auf eine Sache konzentrieren konnte.

M. K.

\section{Nachts, Einschlafproblematik}

\section{Dauer: 20 Min.}

\section{Utensilien: YouTube}

- Ich konnte überhaupt nicht einschlafen und hatte deswegen beschlossen zu meditieren.

- Leider hat es gar nicht geklappt.

- Ich habe festgestellt, dass das allerdings nicht an der Meditation lag, sondern daran, wie mein Gehirn damit umgeht.

- Ich konnte die Grundeinstellung quasi nicht ändern.

- Wenn der Kopf sagt: „Ha ha, geht sowieso nicht“, dann klappt es auch nicht.

- Gesamtreflexion: $:$

- Meditationsform: angestrebter Bodyscan

\section{M.}

\section{Wann: Montag, 10.11.2014, 16.25 Uhr}

\section{Wo: Im Schlafzimmer auf dem Holzboden, liegend} auf einer Decke

Warum: Bessere Konzentrationsfähigkeit; die Woche beginnt - ich muss Verschiedenes fürs Studium lernen, ängstliche Gedanken ziehen lassen, meinen Körper spüren 


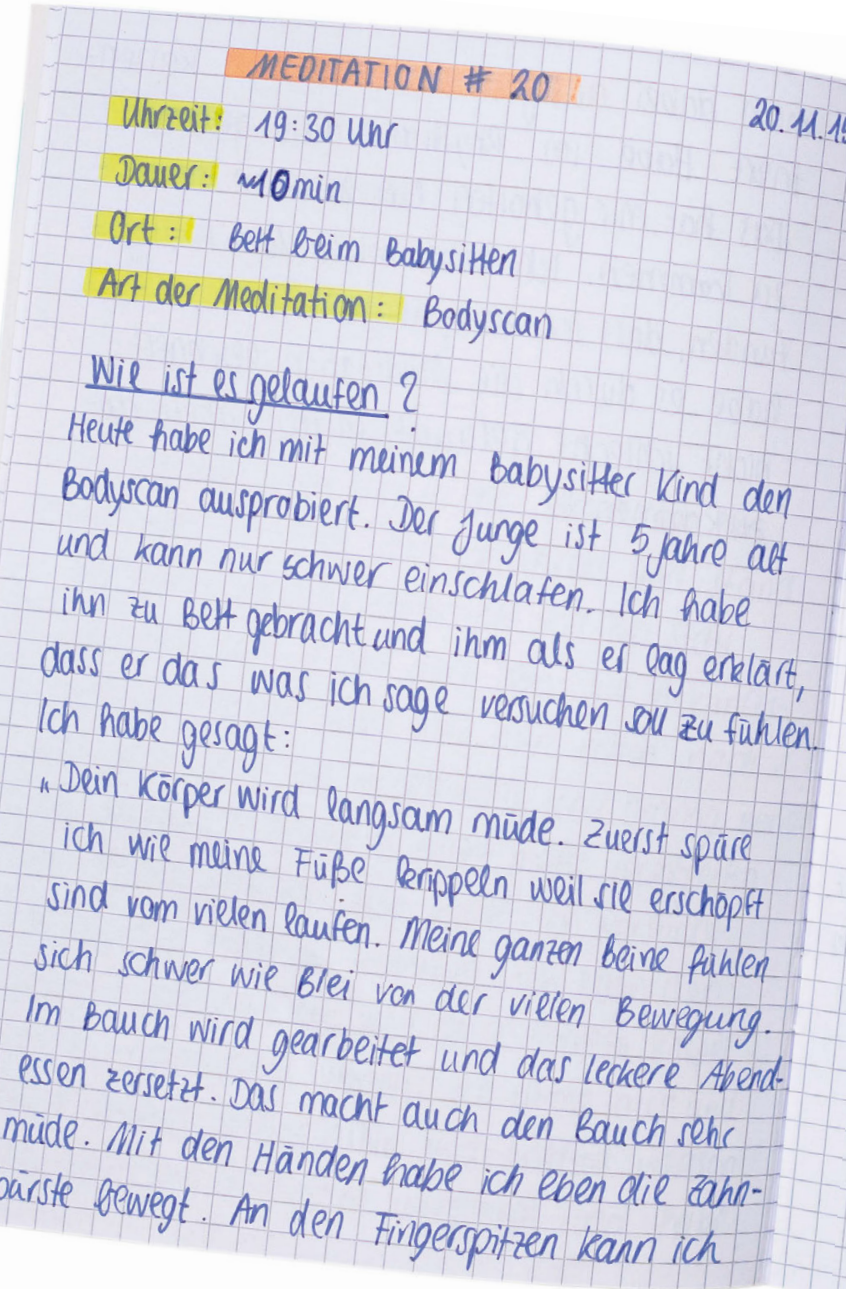

Recherche: Der Bodyscan ist eine Meditationsübung, die ihren Ursprung in der buddhistischen VipassanaTradition hat. Diese Übung stärkt die Körperwahrnehmung. Denn meist nehmen wir unseren Körper nur von außen wahr. Beim Bodyscan handelt es sich um eine Reise durch den Körper, ein gedankliches Abtasten (Scannen) des eigenen Körpers. Nacheinander wird der Fokus der Gedanken auf verschiedene Bereiche des Körpers gerichtet. So wird die Blickrichtung geändert, wir betrachten unseren Körper von innen. Der Bodyscan kann sehr herausfordernd sein, denn oft springt unser Geist ständig von Gedanke zu Gedanke und kommt nicht zur Ruhe. Bei der Übung geht es darum, eigene Körperempfindungen, Gedanken und Gefühle wahrzunehmen und sich dieser bewusst zu werden, ohne sie zu bewerten. Der Bodyscan ist auch gut geeignet, um die eigene Konzentration zu stärken, die eine Voraussetzung für die Achtsamkeitspraxis ist.

\section{Bodyscan-Übung:}

- Ich schaue mir verschiedene Bodyscan-Videos auf YouTube an, um herauszufinden, welche Stimme angenehm ist und mich anspricht.

- Ich entscheide mich, den Bodyscan mit folgender Anleitung durchzuführen: „15 Minuten Bodyscan unter Anleitung" (angenehme Stimme des Sprechers $\odot$ ). noch die kalten Wassertropten vem Händewasch spüren. In meinem Mund liegt die zunge erzanlt habe. Mein Gehirn ist mide o viel es den ganzen Tru Gehirn ist müde, weil Meine Augenlieder liegen geschlossen über meinen Augen und wünschen ihnen eine
gute Nacht..." während dem bodyscan ist mein Baby-
sitter Kind eingeschlaten. Ich habe mich dann leise aus dem timmer geschlichen.

- Wie im Meditationsseminar fühle ich mich beim Bodyscan relativ schnell "bei mir", ich komme schnell zur Ruhe.

- Die Sätze des Sprechers: „... Du hast keine Eile, lass los, so gut du kannst, ... nimm dir Zeit, es gibt nichts, was in dem Moment zu erledigen wäre, ... alles ist gut, ... es ist okay, wenn dein Geist dich ablenkt", beruhigen mich und machen es mir relativ leicht, mich auf mich selbst zu konzentrieren. Auch das Achten auf das Atmen in die Finger usw. tut mir gut.

- Ich merke, dass ich loslassen kann, und durch das Wahrnehmen verschiedener Körperteile kann ich meinen Körper gut spüren.

- Mein Körper fühlt sich leichter an, ich fühle mich befreiter von ängstlichen Gedanken.

- Ich stelle (trotz geschlossener Augen) fest, dass ich während des Bodyscans einen lächelnden Gesichtsausdruck habe. $;$

N. H.

20.11.2015, 19.15 Uhr

Dauer: 10 Min.

Ort: Bett beim Babysitten

Art der Meditation: Bodyscan

Heute habe ich mit meinem Babysitter-Kind den Bodyscan ausprobiert. Der Junge ist 5 Jahre alt und kann nur schwer einschlafen. Ich habe ihn zu Bett gebracht und ihm, als er lag, erklärt, dass er das, was ich sage, versuchen soll zu fühlen.

Ich habe gesagt: „Mein Körper wird langsam müde. Zuerst spüre ich, wie meine Füße kribbeln, weil sie erschöpft sind vom vielen Laufen. Meine Beine fühlen sich schwer wie Blei an von der vielen Bewegung. Im 
Bauch wird gearbeitet und das leckere Abendessen zersetzt. Das macht auch den Bauch sehr müde. Mit den Händen habe ich eben die Zahnbürste bewegt. An den Fingerspitzen kann ich noch die kalten Wassertropfen vom Händewaschen spüren. In meinem Mund liegt die Zunge schwer und müde, weil ich heute so viel erzählt habe. Mein Gehirn ist müde, weil es den ganzen Tag hart gearbeitet hat. Meine Augenlider liegen geschlossen über meinen Augen und wünschen ihnen eine gute Nacht ..."

Während des Bodyscans ist mein Babysitter-Kind eingeschlafen. Ich habe mich dann leise aus dem Zimmer geschlichen.
T. O.

Wann: Im Nachtdienst, ca. 2.30 Uhr

\section{Wo: In der Schlafkammer, im Bett}

Was/Wie: Der heutige Nachtdienst war sehr unruhig und arbeitsintensiv. Deshalb war es schwer für mich, zur Ruhe zu kommen, vor allem mit dem Gedanken daran, dass ich um 6 Uhr wieder für den Frühdienst aufstehen muss. Also versuchte ich es wieder mit dem Bodyscan. Der Einstieg fiel mir zunächst etwas schwer, doch schon bald gelang es mir, meine Gedanken zu ignorieren und mich auf meinen Körper zu konzentrieren. Ich kann gar nicht genau sagen, wie weit ich gekommen bin oder wann ich eingeschlafen bin. Auf jeden Fall konnte ich mich schnell entspannen und gut schlafen.

\subsubsection{9: Bodyscan}

Dauer: ca. $15 \mathrm{Min}$. Heute begann der Tag für mich früh um 8:00 Uhr in der Uni. Nach zwei Kursen hatte ich aus
und fuhr nach Hause. Dort war ich sehr geschafft und antriebslos. Deshalb legte ich mich auf anfing bereitete ich mich mit einer kurzen Ateührte Meditation - Bodyscan - aus. Bevor ich abschalten zu können. Dies gelang mir sehr gut. Bitation vor, um gedanklich ganz Partien, auf die ich mich konzentrierte intensiv Beim Bodyscan konnte ich die einzelnen ich mit Energie; genauso meine Hüfte, Arme und üren. Meine Füße und Beine durchflutete ich einige Male tief ein und fühlte mich bereit für zum Schluss meinen Kopf. Am Ende atmete

16.6.16 Bodyscan Wann im Nachidienst, ca $2^{30}$ th Wo in der Schlafthammer, im Beth Waslwe: Der heatige Nachrdienst war sehrunruhig und asbeitsintensix. Deshalb war es schwe fir mich zur Ruhe zu Llommen, v.a. mit dem Gedanhon dairen, dass ich un 6 "Whr weder fur den Frindienst Qufstchen muss. Also versuchte ich es wieder mit dem Bodyscan. Der Einstieg fiel mir zunächso etwas schwo, doch schor bald gelang es mir mine Gedonken zu ignoriern und mich ouf meinen Vörper zu hontentrieren. Joh hann gar niche genau sagen, wi weir ich gevommen bin oder wann ich eingeschlafen bir. Auf jeden Fall Vannte ich mich schnell entspanmen und gut schlafen.
19.6.16 Mantram Heranatha Wanni abends, ca. $23^{30}$ Whe Wo WG-Zimmer, am Boden im Schncidersitz

Waslwe: Nach einem Streit mit meinem Freund Vam ich zicmlich spar und gestresst in Mlinchen an. Da mich de Gedanken daran nicht loseirßen, versuchteich mit Meditation etwos zur Ruhe zu Uommen. Da ich noch sehr aufgewinlt was, fiel mis der Einstieg schwer und ich brauchte Stlinaten, bis ich folkassiest was. Dann fand ich aber relativ schned in meinen Rhythmus "Mara-natha". Jkh merute, wi meine Gedanken immer weiter weg waren wa ch mich immer besser ceuf die Meditation ein Cassen Vonnte. Den hatte ich aut 20 leinuten wroler hatte ich 
Liebende Güte"

11.5 .16

wie sie es so nett sagem,

Matte ich hente den ganzen Nachmittag in

Gedanken mit ainem /einer "Nerverlein"

zu kämpfen.

Ich habe mich derart über diese Persoto

anfyeregt, dass ich mix dachte a jetzt reik

dich zusammen, das bringt doch alles

nix, dass Du dich ayregit... wedev Du liastetwas davon noch das "Nervertein".

Also liabe ach einen Konter versucht: liebende Güte "..das andere Extrem: Also hale wh mich ay dew Boden gesetzt, und die Sätze der liebenden aite fur andere Personen langram, vorgetesen and jeden sats nocheinmal leise

in Gedarken wiederhalt.

"Hoge diese Persow sicher und gehorgew sein und frei vou innerer und äußerer Not. Moge sie glädclich and zufriedew sein. Mogge sie gesund und heil sein. Moge sie die Leichtighert $d$. Wohlbefindens erfahsen." Und tatsählich, ich selbst war glïhlicher darüber, nicht sdilecht über 


\section{Liebende Güte}

Die Meditation der Liebenden Güte ist eine Form der Metta-Meditation, bei der wir uns anstelle auf ein Objekt oder eine Handlung auf eine Eigenschaft fokussieren, und zwar die des Mitgefühls.

Es gibt viele verschiedene Varianten. Wir können beispielsweise innerlich Affirmationen (positiv bestätigende, bejahende Aussagen) sprechen, mit denen wir uns und anderen Menschen Sicherheit, Geborgenheit, Zufriedenheit, Gesundheit etc. wünschen. Im Unterricht haben wir dabei ein Beispiel aus dem Buch Persönlichkeit und Präsenz. Achtsamkeit im Lehrerberuf von der Achtsamkeitstrainerin Vera Kaltwasser genutzt:

Für mich persönlich

Möge ich sicher und geborgen sein und frei von innerer und äußerer Not.

Möge ich glücklich und zufrieden sein.

Möge ich gesund und heil sein.

Möge ich die Leichtigkeit des Wohlbefindens erfahren.

Für andere Personen

Möge diese Person sicher und geborgen sein und frei von innerer und äußerer Not. Möge sie glücklich und zufrieden sein.

Möge sie gesund und heil sein.

Möge sie die Leichtigkeit des Wohlbefindens erfahren. ${ }^{* 1}$

Wir können die Affirmationen innerlich sprechen, wir können aber auch nur einzelne Wörter wiederholen oder das Gefühl weitergeben, das die jeweiligen Wörter bei uns auslösen. Wichtig ist, dass wir uns auf die Affirmation einlassen.

\section{Wohlwollen und Mitgefühl visualisieren - für sich selbst und für andere}

Wenn wir die Metta-Meditation für andere machen, können wir uns entweder auf einige nahestehende Menschen beschränken oder auch einen größeren Personenkreis miteinbeziehen. Wir können an Menschen denken, die wir sehr gerne haben wie Partner, Eltern, Geschwister, Freunde etc. Aber auch Personen, die wir zwar kennen, doch zu denen wir keine direkte persönliche Beziehung haben, beispielsweise der Busfahrer, die Dame an der Kasse im Supermarkt, Menschen in der U-Bahn, an der Hochschule usw. Oder auch Personen, die wir nicht mögen, die uns manchmal nerven oder mit denen wir Streit haben.

Wir können in einer Metta-Meditation beispielsweise konkret visualisieren, dass wir einen ganzen Eimer gefüllt mit liebender Güte über uns selbst oder jemand anderen ausgießen. Diese Form der Liebenden Güte kann man auch gut anwenden, wenn man sich mit Menschen, die sich in räumlicher Entfernung von einem befinden, verbinden möchte. Zum Beispiel, wenn Angehörige oder Freunde sich in einer schwierigen Situationen befinden oder krank sind und man gerne für sie da sein möchte.

* Die Rechte liegen beim Beltz Verlag und der Autorin. Eine Weiterbearbeitung und Weiterverwendung darf nur mit deren Zustimmung erfolgen. 
Die Meditation der Liebenden Güte für Menschen durchzuführen, die man nicht so gerne mag, ist nicht unbedingt leicht. Um den Eimer ausgießen zu wollen, benötigt es vorab die Motivation, dass man der jeweiligen Person etwas Gutes wünschen möchte. Und das beinhaltet gleichzeitig eine Auseinandersetzung mit der vorgefassten Meinung, dass man diese Person nicht mag. Diesen Konflikt können wir lösen, indem wir unseren Blickwinkel auf die Person verändern: Vielleicht hat diejenige/derjenige es nicht so gemeint und ist derzeit eventuell gestresst? Vielleicht war auch die jeweilige Situation schuld, warum die Person sich so benommen hat? Vielleicht habe ich selbst überreagiert?

Die Fähigkeit, in einer Situation verschiedene Perspektiven einzunehmen, hilft uns, im Leben gelassener zu sein und uns weniger aufzuregen.

Die Variante, bei der man nervigen Menschen Verständnis und liebende Güte schenkt, birgt aber eine kleine Gefahr: Wenn Sie jemand sind, der naturgemäß ohnehin schon viel Mitgefühl für andere hat und sich selbst eher vernachlässigt in der Wertschätzung, beinhaltet diese Übung nicht, noch mehr geben zu müssen, noch mehr Verständnis zu zeigen. Sondern in diesem Fall ist es besser, sich selbst mehr schätzen und schützen zu lernen und öfter mal einen Eimer mit liebender Güte über sich selbst auszugießen.

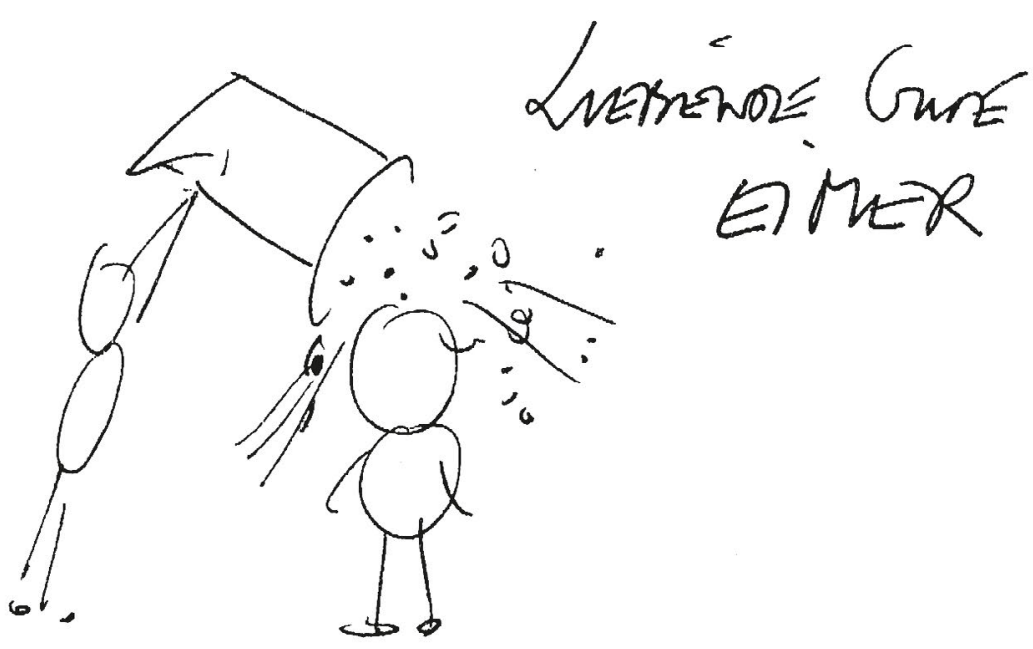

Ärger

Wenn man sich mal ärgert, aufregt oder sauer ist, ist das ganz normal. Man darf sich ruhig auch mal „Luft machen“ und sollte nicht alle Sorgen, Wut und Ärger unterdrücken und „herunterschlucken“.

Apropos sich ärgern: Ich erinnere mich an eine Studentin, die in ihrem Tagebuch berichtet hat, dass ihre Mutter einen leckeren Himbeerkuchen gebacken hatte und am nächsten Tag noch zwei Stücke davon übrig waren: eins für die Studentin und eins für ihre kleine Schwester. Als sie gegen Abend nach einem langen Tag an der Uni ziemlich erschöpft nach Hause kam und mit großer Vorfreude ihr Stück Himbeerkuchen 
genießen wollte, stellte sie fest, dass ihre Schwester beide Stücke gegessen hatte! „Hier hilft auch die Liebende Güte nicht mehr!" stand im Meditationstagebuch in Großbuchstaben. Abends allerdings hat die Studentin die Liebende Güte doch noch ausprobiert und auch der kleinen Schwester etwas davon gesendet. Das heißt nicht, dass sie ihrer kleinen Schwester „verzieh“, aber die Studentin versuchte darüberzustehen und konnte sogar einigermaßen entspannt einschlafen.

Die Geschichte ging am Ende noch gut aus. Zwei Tage später backte die kleine Schwester aus Mitleid selbst einen Himbeerkuchen, um ihn mit der großen Schwester und der Mutter zu teilen.
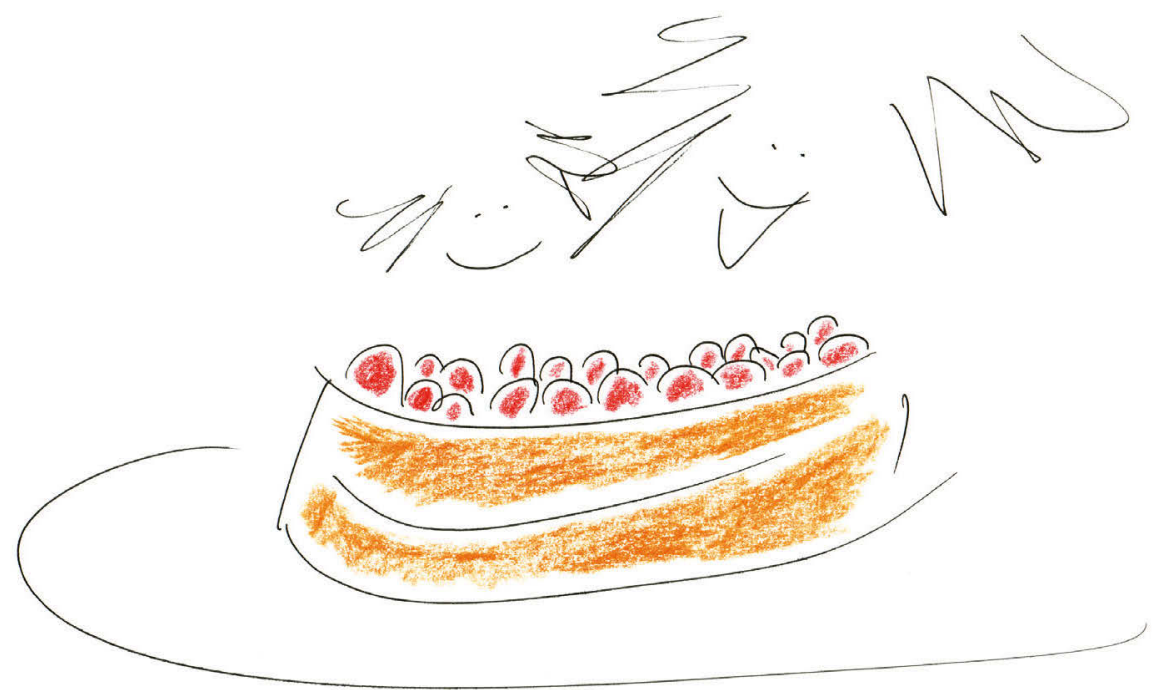

Himbeertorte,

Schwestern

und Happy End.

\section{Verbundenheit mit allem, was ist}

Eine andere Variante der Liebenden Güte betont nicht nur die Verbundenheit mit anderen Menschen, sondern auch mit Tieren, Pflanzen - mit allen fühlenden Lebewesen auf der Erde. Der Radius kann so weit ausgedehnt werden, dass auch Orte, Städte, Länder, Kontinente, die ganze Erde sowie Planeten und Sterne oder sogar das Universum eingebunden werden.

Eine Übung, die wir im Unterricht angewendet haben, geht folgendermaßen:

„Stellen Sie sich ein Gefühl eines tiefen inneren Wohlbefindens und eines Zufriedenseins vor. Vielleicht erinnern Sie sich dabei an eine besonders schöne Situation im Leben. Jetzt geht es darum, in dieses Gefühl einzutauchen. Wenn Sie eine gute Verbindung zu dem Gefühl aufgebaut haben, dehnen Sie dieses jetzt im Raum aus. Dabei visualisieren Sie, wie die Menschen und andere Lebewesen, die sich im gleichen Raum befinden, von diesem Gefühl umhüllt werden.“

Diese Übung des Ausdehnens des wohlwollenden Gefühls können Sie noch ausweiten: auf das Haus oder die Institution, wo sich der Raum befindet. Oder vielleicht sitzen Sie im Park und dehnen den Radius nach und nach so weit aus, dass er am Ende den gesamten Park umfasst. 


\title{
Wenn Liebe zu göttlicher Liebe wird, erfüllt auch Mitgefühl das Herz. Liebe ist die innere Empfindung, und Mitgefühl ist ihr Ausdruck.
}

\author{
Mata Amritanandamayi
}

\section{Einheit der Menschheit}

Eine weitere Variante ist, dass man sich Menschen in anderen Orten, Städten, Ländern, Kontinenten vorstellt, die genauso wie wir ihrem Alltag nachgehen. Vielleicht hat man Bekannte in einem anderen Land, die man innerlich vor Augen hat, wenn man einen bestimmten Ausdehnungsbereich visualisiert. Oder man visualisiert die Bilder, die man aus den Medien kennt. Die Menschen sind zwar unterschiedlich und individuell, aber uns verbinden auch viele Gemeinsamkeiten. Menschen kümmern sich um ihre Familien, spielen mit ihren Kindern, lieben Fußball oder begeistern sich für andere Sportarten, sind gerne mit Freunden zusammen, arbeiten, um ihren Lebensunterhalt zu verdienen. Alle Menschen wollen glücklich sein. Dabei haben manche gute Lebensbedingen, andere sehr schwere. Indem wir Verbundenheit visualisieren und Wohlbefinden und Liebe ausschütten, können wir die grundsätzliche Einheit der Menschheit spüren.

\section{Zellen, Sterne, Universum}

Bringen wir auch das Universum in die Übung ein, kann das einen tiefen Effekt der Ruhe und Geborgenheit auf uns haben. Wenn man darüber nachdenkt, dass es Milliarden Sterne in der Milchstraße gibt, ist das eine unfassbare Dimension. Und wenn man sich dann noch überlegt, dass der menschliche Körper aus bis zu Billionen Zellen besteht, erinnert uns das daran, dass wir Teil eines größeren Ganzen sind.

Es gibt viele Formen der Liebenden Güte oder Metta-Meditationen. In Büchern, auf CDs, im Internet - am besten, man probiert einfach aus, was zu einem passt. Oder man erfindet eine eigene Form. Das Wesentliche ist das Gefühl des Wohlwollens, das vermittelt wird.

\section{Für den Unterricht benutzte Primärliteratur}

Kornfield, Jack (2017). Frag den Buddha und geh den Weg des Herzens. Was uns bei der spirituellen Suche unterstützt. München: Kösel-Verlag. Kornfield, Jack (2010). Meditationen, die unser Herz öffnen. München: Arkana. Ricard, Matthieu (2009). Meditation. München: nymphenburger. 


\section{Tagebuchnotizen Liebende Güte}

A. B.

\section{Zeit: 15.45-15.52 Uhr (7 Min.) \\ Ort: Wartezimmer (sitzend) \\ Methode: Liebende Güte \\ Utensilien: LSF*-Text „Liebende Güte“}

Ich hatte einen Termin beim Arzt um 15.30 Uhr. Die Sprechstundenhilfe schickte mich zunächst noch ins ziemlich volle Wartezimmer. Neben mir saß ein älterer Mann, der ständig - ohne seine Hand vor den Mund zu halten - in meine Richtung hustete und nieste. Ich bekam ab und zu ein paar Tröpfchen seiner Spucke ins Gesicht. Da ich krank bin, war meine Laune heute eh schon nicht besonders gut. Ich drehte mich leicht weg von dem Mann, um in Ruhe mein Buch weiterzulesen.

Kurz nach mir betraten noch zwei weitere Patienten das Wartezimmer. Beide wurden vor mir in die Behandlungsräume gerufen, obwohl ich einen Termin hatte, der eindeutig vor den beiden lag. Durch die Lautstärke der Unterhaltung eines Pärchens in einer anderen Ecke des Wartezimmers konnte ich mich kaum auf das Lesen konzentrieren. Ich bemerkte, dass ich immer wütender und unzufriedener mit der Situation wurde. Der Mann, der mich anspuckte, die Patienten, die vor mir drankamen, und die lautstarke Unterhaltung des Pärchens waren zu viel für mich.

Ich war kurz davor, meinen Termin zu verschieben, da erinnerte ich mich an unsere heutige Seminarsitzung, in der wir die Technik der „Liebenden Güte“ besprochen hatten. Mit meinem Handy lud ich mir die Datei aus dem LSF herunter. Ich las mir die Sätze einmal einzeln vor, um sie dann mit geschlossenen $\mathrm{Au}$ gen nachzusprechen. So kippte ich den Eimer liebende Güte über meinen Sitznachbarn, über das Pärchen, die anwesenden Patienten und über die Sprechstundenhilfe. Ich merkte, wie sich nach den Übungen meine Wut verringerte und ich mich ausgeglichener und belastbarer fühlte. Ich schenkte neu ankommenden Patienten sogar ein Lächeln und ein fröhliches „Grüß Gott“.

Mir ist klargeworden, dass Aggression in der Situation absolut nicht zielführend gewesen wäre. Für mich hat die Übung sehr gut funktioniert und gleichzeitig die Wartezeit verkürzt.

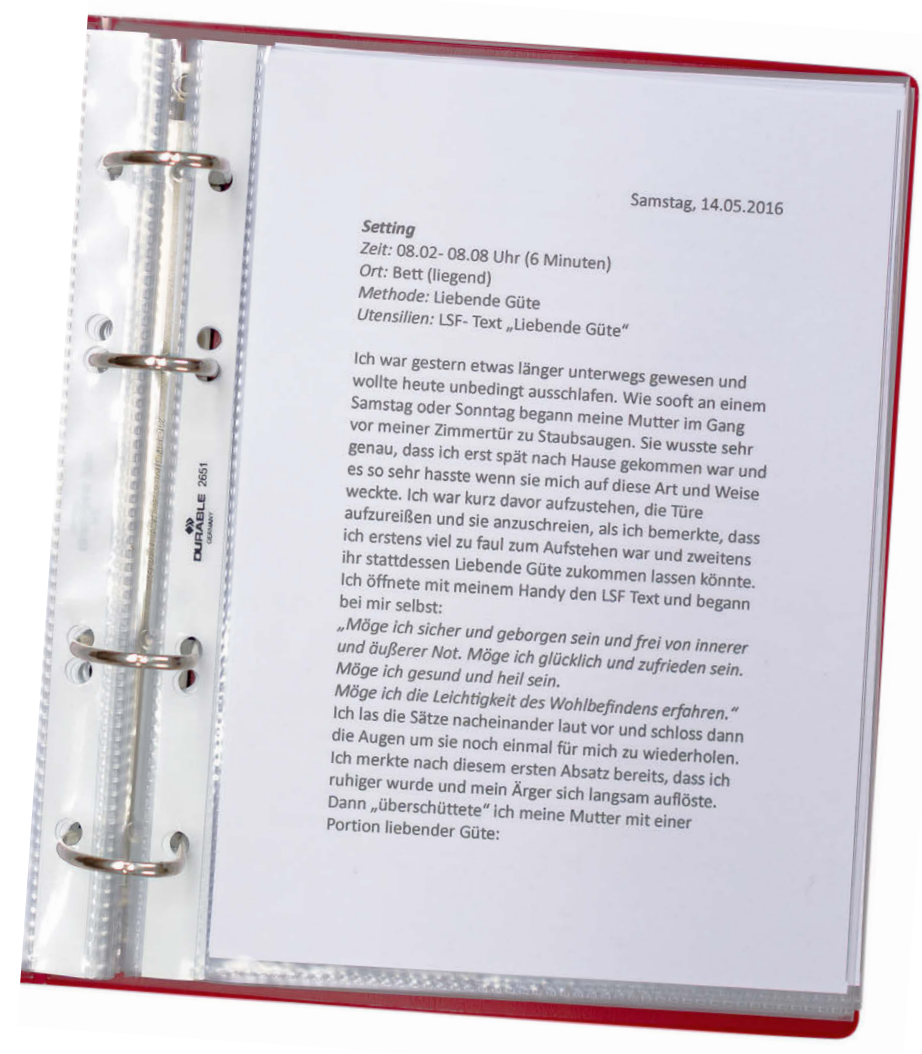

Sonntag, 14.5.2016

Zeit: 8.02-8.08 Uhr (6 Min.)

Ort: Bett (liegend)

\section{Methode: Liebende Güte}

Utensilien: LSF*-Text „Liebende Güte“

Ich war gestern etwas länger unterwegs gewesen und wollte heute unbedingt ausschlafen. Wie sooft an einem Samstag oder Sonntag begann meine Mutter im Gang vor meiner Zimmertür zu staubsaugen. Sie wusste sehr genau, dass ich erst spät nach Hause gekommen war und es so sehr hasste, wenn sie mich auf diese Art und Weise weckte. Ich war kurz davor, aufzustehen, die Türe aufzureißen und sie anzuschreien, als ich bemerkte, dass ich erstens viel zu faul zum Aufstehen war und zweitens ihr stattdessen liebende Güte zukommen lassen könnte.

Ich öffnete mit meinem Handy den LSF-Text und begann bei mir selbst:

„Möge ich sicher und geborgen sein und frei von innerer und äußerer Not. Möge ich glücklich und zufrieden sein. Möge ich gesund und heil sein. Möge ich die Leichtigkeit des Woblbefindens erfahren."

Ich las die Sätze nacheinander laut vor und schloss dann die Augen, um sie noch einmal für mich zu wiederholen. Ich merkte nach diesem ersten Absatz bereits, dass ich ruhiger wurde und mein Ärger sich langsam auflöste. Dann „überschüttete“ ich meine Mutter mit einer Portion liebende Güte. 
Metta Liebande Güte

22:40 uhr

Wo: im Bett, vor dem Einschlafen

Zunarchst habe ich die Übung beimir durchgeführt. Im Anschluss wand ich die Übung bei einer älteren Freundin, mit der ich keinen Kontaht mehr habe, an. Vor der übung empfand ich "negative" Gefühe gegenuber ihr, weil ich ihr vor zevei Jahnen 100 t gelieken habe da sie in Not war. Gedanken kamen auf und wollten sie "verfluchen" weil sie sie mir bis jekt nicht zurüidgegeben hat obuwch sie standig auf Reisen ist und diese 100 locher besitzt. Mir ist die Liebende Güte übung eingefallen und hattedas Bedürnis anders zu reagieren, als sonst bisher. Immerhin bringt es mir nur, negative" Gefühle für mich und das bringt nichts. Während der übung war es etwas komisch, ihr den Güteeimer über zus schütten, da ich das Geld schon Zurüdchaben möchte, jedoch auch sehr schön, da ihve Person selbst ous weitaus mehr besteht" als nur mir das Geld nicht za geben. Deswegen mochte ich nidit sie im Ganzen "verf luchen" oder hritisieren. Im Gegenteil, der Gedanke, ihr trotz den etwas gutes zu wünschen, ist sehr schän.

\section{W.}

\section{Metta, Liebende Güte \\ 22.40 Uhr}

\section{Wo: Im Bett, vor dem Einschlafen}

Zunächst habe ich die Übung bei mir durchgeführt. Im Anschluss wandte ich die Übung bei einer älteren Freundin, mit der ich keinen Kontakt mehr habe, an. Vor der Übung empfand ich „negative“ Gefühle ihr gegenüber, weil ich ihr vor zwei Jahren 100 Euro geliehen habe, da sie in Not war. Gedanken kamen auf und wollten sie „verfluchen“, weil sie sie mir bis jetzt nicht zurückgegeben hat, obwohl sie ständig auf Reisen ist und diese 100 Euro locker besitzt.

Mir ist die Liebende-Güte-Übung eingefallen, und ich hatte das Bedürfnis anders zu reagieren als bisher. Immerhin bringt es nur „negative“ Gefühle für mich, und das bringt nichts. Während der Übung war es etwas komisch, ihr den „Güte-Eimer“ überzuschütten, da ich das Geld schon zurückhaben möchte, [es war] jedoch auch sehr schön, da ihre Person selbst aus weitaus mehr besteht, als nur mir das Geld nicht zu geben. Deswegen möchte ich sie im Ganzen nicht „verfluchen“ oder kritisieren. Im Gegenteil, der Gedanke, ihr trotzdem etwas Gutes zu wünschen, ist sehr schön.
Donnerstag, 9.5.2019

Körper ruhig \& frieduall / Geist ruhig \& freduoll 17:30 Uhr ca. 10-15 Minuten beim Zahnaret

Da ich ouffgeregt und etwas angstlich beim. Zahnarzt bin und eine Untersuchung bzgl. enes Eingriffsmil spritze bevorsteht habe ich die zeit im Wartezimmer und auf dem Behandlungsstuhl genutzt um mich mit der Atemtechnik zuberuhigen Zunärhst habe ich mehvere Minuten meinen Körper beim Fin-und Ausatmen beruigt. Im Anschluss meinen Geist. Zu Beginn war es sehr schwierig, da ish inmer wieder Bilder von Spritzon etc vor mir hatte und wathrend dem Warten auf dem Behandlungsstwhldie Werkzeuge liegan gesehen habe. Mit der Zeit wurde es jedoch besser. Ich konnte mich mit viel mïhe ouf meinen Atem konzentrieven und den Gedankenstrudel verlassen. Unsicher warich frokdem, jedoch deutlich weniger. Zumal die eigentliche Behandlung enst menem lalben Jahr sein wird. Zuvor werde ich mit sicherbeit meditiera!

\section{E. B.}

Eine Lieblingsübung, wenn ich wütend bin. Gerade wenn ich das Gefühl habe, dass jemand richtig unfair zu mir war oder ich mich erniedrigt oder ausgeschlossen fühle, ist diese Übung bis jetzt auch die einzige Bewältigungsstrategie, die ich habe. Wenn ich dann mit mir selbst wieder „im Reinen“ bin, kann ich verzeihen und die Dinge haben nicht mehr so viel mit mir zu tun und ich kann mein Ego zurücknehmen.

\section{H. K.}

\section{Samstag, 29.6.2013, 7.30 Uhr}

Da ich schon so früh wach war, aber noch nicht aufstehen wollte, beschloss ich, die Liebende Güte durchzuführen. Ich konnte recht schnell meine negativen Gedanken, Selbstvorwürfe und -zweifel ablegen und den anderen Menschen und mir selbst so etwas Schönes geben sowie einen guten Tag wünschen. Danach konnte ich dann entspannt und ruhig noch einmal einschlafen.

\section{P. S.}

\section{Sonntag, 26.11.2016, bei mir zu Hause,} ca. 7 Min.

\section{Ergebnis: Liebende Güte}

Am Sonntag habe ich mich dazu entschlossen, die Woche mit der Meditationsform der Liebenden Güte aus- 


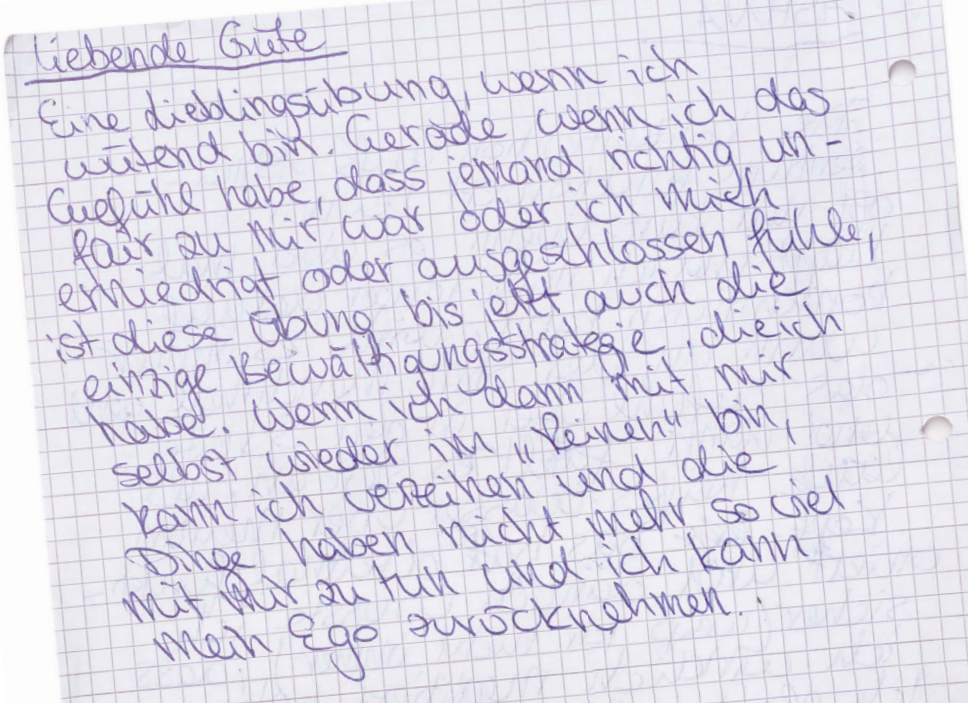

klingen zu lassen. Diese Form gefällt mir sehr gut und ich konnte mich auch gut entspannen.

Als liebende Person habe ich meine Mama ausgewählt. Da sie erst vor kurzem Geburtstag hatte und sie für unsere Familie (v.a. mich mit leckerem Essen verwöhnt, Wäsche wäscht, wenn ich am Wochenende mal wieder zu Hause bin) so viel Gutes leistet, hat sie die positiven Gedanken richtig verdient.

Als neutrale Person fiel mir ganz spontan der Mann vom Studentenwerk München ein,der mir einen Bungalow im Olympiazentrum vermittelt hat. Ich bin ihm echt sehr dankbar dafür.

Und als zweite liebende Person habe ich mich für meine Nachhilfeschülerin in Latein entschieden. Sie hatte diese Woche eine Schulaufgabe und ich habe deshalb sehr viel an sie gedacht. Hoffentlich halfen ihr meine warmen Worte, um entspannt und ohne große Angst die Prüfung zu meistern und ihr Bestes zu geben (hoffentlich kommt ein gutes Ergebnis dabei heraus).

\section{Mittwoch, 14.12.2016, ca. 10 Min., Wohnung München \\ Ergebnis: Liebende Güte}

Ich habe mich heute noch mal an die Liebende Güte gewagt, weil ich mich heute wirklich über eine Person sehr aufregen musste. Ich habe zwar in dem Moment nicht gleich an die Liebende Güte gedacht, doch als ich zu Hause den Tag Revue passieren ließ, musste ich es dann doch machen. Denn als ich heute auf dem Weg zur Uni war, hatte ich es wirklich eilig und musste vorher noch etwas im Supermarkt einkaufen. Weil an der Kasse eine ältere Frau vor mir war und dort sehr langsam ihre Einkäufe nach dem Zahlen in den Wagen gelegt hat, wobei sie sich auch noch sehr angeregt mit der Verkäuferin unterhalten hat, habe ich meine U-Bahn verpasst und musste 10 Minuten warten und kam dann wegen einer Verzögerung nur ganz knapp noch pünktlich in die Vorlesung und war da schon ganz gestresst.

$\mathrm{Zu}$ Hause, nachdem einige Zeit vergangen war, habe ich dann warme Gedanken an die Person geschickt, was mir wirklich nicht leicht gefallen ist. Vielleicht muss ich nächstes Mal einfach eher losgehen und solche „Zwischenfälle" einplanen.

\section{W. W.}

Normalerweise habe ich mit anderen Menschen nicht sehr viele Probleme, aber manche bringen einen einfach auf die Palme.

Eine meiner Arbeitskolleginnen scheint mich nicht großartig zu mögen. Doch man muss ja auskommen. Ich versuche immer sehr höflich und freundlich zu ihr zu sein. Doch das fällt manchmal extrem schwer, wenn von der anderen Seite sehr wenig Freundlichkeit zurückkommt. Gestern Abend musste ich an die Übung Liebende Güte denken. Ich habe also einfach versucht, daran zu denken, dass man den Menschen nicht wirklich böse sein kann, da sie doch meistens aus Problemen mit sich selbst, andere schlecht behandeln. Diese Menschen können nicht wirklich glücklich sein, weshalb man ihnen wünschen sollte, dass sie ihr Glück und ihre Zufriedenheit finden.

Also habe ich versucht, einen großen „Eimer" liebende Güte für sie übrig zu haben und ihr bildlich zu übergeben. Sowas fällt natürlich nicht immer leicht, aber die absolut beste Lösung, Konflikte zu lösen und anderen Menschen vielleicht sogar somit zu helfen und sie zu unterstützen. Ich werde mein Bestes geben, weiterhin so zu handeln und vielleicht auch die Übung Liebende Güte des Öfteren anzuwenden.

$$
\begin{aligned}
& \text { Woche } 6 \\
& 14.0520 .05 \\
& \text { MONTAG } \\
& \text { Normale } \\
& \text { manche bringe habe ich } \\
& \text { muss ja aus Arbeitsk einfach auf oni Mienschon nich sehn } \\
& \text { Doch das fält mmen. Ich veren scheint mich } \\
& \text { Freundichkeit manchmal extreche immer seh nicht grolßartig zu mo he } \\
& \text { denken. ich haburuckkommt. Gesterier, werin oflich und freund } \\
& \text { nicht wirkfich böse soino einfach versuch Abend musster anderen Soich zu ihr zu sein } \\
& \text { andere schlecht be sein kann, da sieht daran zuste ich an die Ubuner sehr wenig } \\
& \text { weshaib man Itinen wendein. Diese doch meistens aun, dass man ding Liebende Güt } \\
& \text { inden. Also habe er wünschen Menschen köns aus Problemen den Menschen } \\
& \text { Liebende Güte für ich versucht einen solte, dass sie ihr richit wirklich mit sich selbst, } \\
& \text { natürlich nicht imm übrig zu hagen großen ,Eimer" } \\
& \text { anderen Menschen r leicht, aber diend ihr bildlich } \\
& \text { liebendein Bestes vielleicht sogar somit lut beste lösübergeben. Sow } \\
& \text { liebenden Güte des } \text { Oeben weiterhin so zit zu helfen und } \\
& \text { SONNTAG } \\
& \text { Da diese wo } \\
& \text { Was sehr woche etwas streste } \\
& \text { bin seh schade ist, dastressig war ho } \\
& \text { verschafft, um dienstagsstunde nicht zu meiner Routitieren leider } \\
& \text { - anzableibe und mir auchkbar, da diese dazu gehört. Gerhälssigt. } \\
& \text { auch immer ein sehr imimer wiedera deshalb } \\
& \text { ein sehr gutes Gefüieder Anreiz }
\end{aligned}
$$



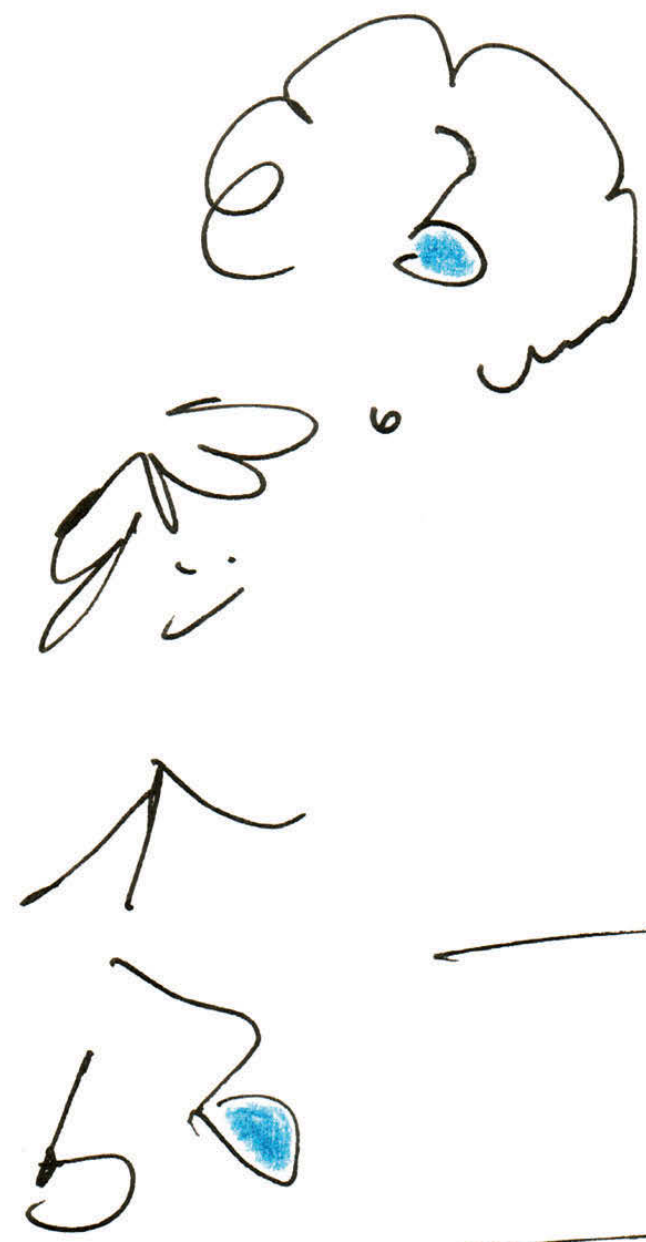

Aufmerksamkeit bei den Füßen: die Gehmeditation. 


\section{Gehmeditation}

Kennen Sie das? Wenn man es mal eilig hat, geht just vor Ihnen jemand viel zu langsam und bewegt sich dabei zudem so weit nach links und rechts, dass man unmöglich überholen kann.

Es gibt auch Situationen, in denen Menschen bewusst darauf achten, wie sie gehen: wenn sie im Kleinkindalter zum ersten Mal Laufen lernen; wenn sie nach einem Unfall wieder Gehen lernen müssen; und wer beruflich mit richtigem Gehen befasst ist, wie etwa Physio- und Ergotherapeutinnen und -therapeuten.

Zweck der Gehmeditation ist die Gehmeditation selbst.

Entscheidend ist das Gehen, nicht das Ankommen, denn Gehmeditation ist kein Mittel, es ist das Ziel selbst. Jeder Schritt ist Leben; jeder Schritt ist Frieden. Das ist der Grund, warum wir nicht zu eilen brauchen; darum verlangsamen wir unsere Schritte. Gehen Sie, aber gehen Sie nicht.

Gehen Sie, aber lassen Sie sich durch nichts antreiben, was immer es auch sei. So wird, wenn wir gehen, wie von selbst ein Halblächeln auf unserem Gesicht sein.

Thich Nhat Hanh

In der Zeit meiner Promotion an der LMU München habe ich regelmäßig in der Münchner Staatsbibliothek gemeinsam mit einem Studienfreund gelernt. Nicht immer war ich konzentriert. In solchen Momenten habe ich mich teils gedanklich durch die Geschehnisse in der Bibliothek ablenken lassen. Zum Beispiel durch ein sehr vergnügliches „Spiel“: Lassen sich von der Gangart eines Menschen möglicherweise Rückschlüsse auf den Studiengang ziehen? - Genau das haben wir versucht! Zunächst ohne sie anzusehen haben wir von der Art und Weise, wie die an uns vorübergehenden Kommilitoninnen und Kommilitonen aufgetreten sind, ihren Studiengang erraten. Und das ging so: Augen zu. Genau zuhören, wie die Vorübergehenden gehen und auftreten (schlurfend, hastig, langsam, schnell, polternd, leise etc.). Studiengang aufschreiben. Vorbeigehende anschauen. Fazit abgeben: Jura, ... BWL, ... Philosophie ... oder doch eher Politikwissenschaft?

Natürlich hakt dieses „Spiel“, und man darf nicht generalisieren und stereotypisieren; es gibt die unterschiedlichsten Gehweisen in jedem Studiengang. Das Beispiel soll lediglich darauf aufmerksam machen, dass die Art und Weise, wie wir gehen und uns bewegen, auch durch Dinge, Themen, mit denen wir uns beschäftigen und identifizieren, durch unsere Tagesform, durch unsere Emotionen usw. beeinflusst wird. Und da das Gehen automatisch abläuft, denken wir auch nicht bewusst darüber nach, wir tun es einfach. 
Bei der Gehmeditation wird die Aufmerksamkeit auf das eigene Gehen gelegt.

Moderation: „Wo setze ich ab, wenn ich meinen Fuß anhebe? Wann geht der andere Fuß in die Höhe? Wie bewegt sich das Knie? Welche Stelle meines Fußes berührt den Boden beim Aufsetzen als Erstes? Dies werden wir jetzt ausprobieren. Und wir nehmen uns Zeit dafür; wir sind ruhig und fokussiert."

Gehmeditation ausprobieren.

Moderation: „Was haben Sie dabei erfahren?"

Wortmeldung 1: „Gut, es hat mir geholfen, nur auf das Gehen zu achten.“

Wortmeldung 2: „Es war schwierig, mein eigenes Tempo zu finden, weil viele Personen im Raum waren."

Wortmeldung 3: „Ich hatte das Gefühl, aus dem Gleichgewicht zu geraten.“

Moderation: „Haben Sie dabei an etwas anderes gedacht?"

Wortmeldung 1: „Hin und wieder.“

Wortmeldung 2: „Nein, ich war sehr konzentriert.“

Wortmeldung 3: „Ich war bei meinen Füßen und bei den anderen Personen.“

Moderation: „Haben Sie auch geatmet? Und wissen Sie vielleicht, wie viele Schritte pro Atemzug?"

Ein zweiter Teil der Übung besteht aus dem Zählen der Atemzüge während des Gehens. Wie viele Schritte fallen auf einem Atemzug?

Moderation / Vormachen: „Zum Beispiel 2 Schritte auf einem Atemzug beim Einatmen und 2 Schritte auf einem Atemzug beim Ausatmen."

Dies kann allerdings bei jedem anders sein. Probieren wir das einmal aus, wir haben jetzt drei Punkte, worauf wir achten: Schritte, Atmen und Zählen.

Wenn Sie allerdings gleich merken, dass es Ihnen aus irgendwelchen Gründen nicht guttut oder gefällt, können Sie die Gehmeditation auch ohne das Zählen der Atemzüge machen, wie vorher.

Gehmeditation samt Atemzählen ausprobieren.

Moderation: „Wie sind Sie damit zurechtgekommen?"

Wortmeldung 1: „Schwierig, ich bin durcheinandergekommen.“

Wortmeldung 2: „Bei mir war es gut.

Wortmeldung 3: „Ich fand es ungewöhnlich, mehr kann ich noch nicht sagen.“

Wortmeldung 4: „Mir gefällt das nicht, ich mache das lieber ohne Zählen der Atemzüge.“

Moderation: „Wer hat dabei auch zählen können?"

Wortmeldung 1: „2 Schritte auf einem Atemzug beim Einatmen, beim Ausatmen ebenso."

Wortmeldung 2: „Ich habe 2 Schritte beim Einatmen und 3 beim Ausatmen gemacht." Wortmeldung 3: „Ich bin langsam gegangen: pro Schritt 1 Atemzug.“

Wortmeldung 4: „Ich schneller, 4 Schritte beim Einatmen, 3 Schritte beim Ausatmen.“

Moderation: „Warum die Kombination mit den Atemzügen?"

Wortmeldung 1: „Es ist leichter, sich zu konzentrieren.“

Wortmeldung 2: „Man denkt nur noch an das Gehen und Zählen.“ 
Die Gehmeditation kann auch im Alltag praktiziert werden. Zum Beispiel auf dem Weg nach Hause, bei einem Waldspaziergang, auf dem Weg zu einem Termin, zur Uni. Sogar kurz vor einer Prüfung: Bevor man den Raum betritt und die Prüfung anfängt, bietet sie eine Möglichkeit, für ein paar Minuten den Geist zu entspannen.

Man kann die Gehmeditation langsam oder etwas schneller machen, mit oder ohne Zählen des Atmens bzw. der Schritte - wie es einem gerade passt. Es geht um den Fokus, und der ist auf das Gehen gerichtet.

Für den Unterricht benutzte Primärliteratur

Thich Nhat Hanh / Nguyen Anh-Huong (2008). Geh-Meditation. München:

Arkana. [mit CD und DVD]

Thich Nhat Hanh (2007). Der Geruch von frisch geschnittenem Gras. Anleitung zur Gehmeditation. Stuttgart: Theseus Verlag.

Personem: Mama, Schwester, selbst

Technik: Geh-Heditation, bas pus

Sifuation: Kch 6lieb bis henk ouf dem

C. If.d bei meine Mama, sehlieplich war Feiertag und ich liebe und geniepe jede freie Minuth dort. neber einem chonderige inner die Geh dachte ich, Zugammen mit dem Hund was Meditation. es etwas scherierig. nianänge entagen hames, immer wieder spaziegainger enterer haber. Aluedings die Angot vor kundeneinsam ind sowohl reflektierten wir gemeinsam un solsohl es lgut

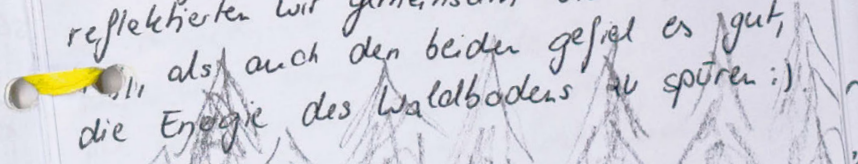



Fils ich gerade auf dem weg vom Bannhof
zu neiner Familie war, viel mir ein, dass ich doch die Gehmeditation macher lönnte. Also schaute ich ouf den boden su meinen Füben und versuchte ausschlieplich an diese und das behen tu dentuen. Ich merkte, dass ich mich auromatisch langsamer und getielter bewegte und liep es wieder normal gehend aussehen lassen. Dann viel mis ein, dass ich die Atrmung noch auzu tun konnte, was ich auch tat. Wieder ham mir ases Genen zu "gestellt" vor, dass ich mich zwang einfach our zu genen und bu atmen, gener undatimen, gehen und atmen... irgendwann wonnte ich aufhoren an diesen satz denken zu mirsen und ich dachte ausschlieflich an das Gehen. Nur evider, als es dann ganz gut lelappte i fing es an zu regren und der wind wurde stärker. Dann musste ich abbrechen.

Mir ist har geworden, dass es draupen viel nehr pinge gibt, die einen ablentuen. Im Raum, wo pedes das selbe getan hat, $\rightarrow$ ist es mir vill peichter gefallen, mich ouf das Gehen $\forall$ bea whantieren. Auch den Schülerinnen, lionte ich mir vorstellen 


\section{Tagebuchnotizen Gehmeditation}

\section{A. P. Z.}

\subsubsection{7, Gehmeditation}

Ein Spaziergang mit dem Hund ermöglicht oft eine kurze Meditation. Die Stille im Wald oder auf der Wiese lassen die Gedanken verschwinden. Die Betrachtung der Natur und die Fokussierung auf ein Objekt, z.B. einen Baum, lassen die Gedanken verschwinden.

\section{A. v. W.}

Als ich gerade auf dem Weg von Bahnhof zu meiner Familie war, fiel mir ein, dass ich doch die Gehmeditation machen könnte. Also schaute ich auf den Boden zu meinen Füßen und versuchte ausschließlich an diese und das Gehen zu denken. Ich merkte, dass ich mich automatisch langsamer und gezielter bewegte, und ließ es wieder normal gehend aussehen. Dann fiel mir ein, dass ich die Atmung noch dazu machen könnte, was ich auch tat. Wider kam mir das Gehen zu "gestellt“ vor, dass ich mich zwang, einfach nur zu gehen und zu atmen, gehen und atmen, gehen und atmen ... Irgendwann konnte ich aufhören, an diesen Satz denken zu müssen, und ich dachte ausschließlich an das Gehen. Nur leider, als es dann ganz gut klappte, fing es an zu regnen und der Wind wurde stärker. Dann musste ich abbrechen. Mir ist klargeworden, dass es draußen viel mehr Dinge gibt, die einen ablenken. Im Raum, wo jeder dasselbe getan hat, ist es mir viel leichter gefallen, mich auf das Gehen allein konzentrieren.

F. G.

Datum / Dauer: 5.6.2017 / 15.30 Uhr, ca. 30 Min. Material / Ort: Wald bei Mama

Personen: Mama, Schwester, selbst Technik: Gehmeditation, barfuß

Situation: Ich blieb bis heute auf dem Land bei meiner Mama, schließlich war Feiertag, und ich liebe und genieße jede freie Minute dort. Da meine Mama genau neben einem wunderschönen Wald wohnt, dachte ich, ich zeige ihnen die Gehmeditation. Zusammen mit dem Hund war es etwas schwierig, nicht zu sprechen, da immer wieder Spaziergänger entgegenkamen, die Angst vor Hunden haben Allerdings reflektierten wir gemeinsam und sowohl mir als auch den beiden gefiel es gut, die Energie des Waldbodens zu spüren.
J.Z.

\subsubsection{4}

Gehmeditation von der Uni nach Hause, 15.45 Uhr

Nachmittags auf dem Heimweg von der Uni probierte ich mal die Gehmeditation aus. Ich versuchte intensiv auf meinen Gang und meine Empfindungen an den Füßen zu achten. Ich spürte den Boden unter meinen Schuhsohlen und nahm die unterschiedlichen Untergründe intensiv wahr. Alles andere um mich herum blendete ich aus. Zuerst ging ich die Treppen im Treppenhaus des Unigebäudes hinab und merkte, wie ich mich konzentrieren musste, meine Füße genau auf den Stufen zu platzieren, um das Gleichgewicht zu halten. Der Boden war hart und zum Teil klebrig. Mein rechter Fuß kribbelte noch leicht, da er in der Seminarstunde eingeschlafen war. Mein linker großer Zeh drückte im Schuh. Ich achtete auf die Geräusche, die meine Schuhe auf den unterschiedlichen Böden machten. Die Treppen außerhalb des Gebäudes waren rutschiger und auch breiter, sodass ich immer 1,5 Schritte pro Stufe machen musste, was nicht meinem natürlichen „Gehrhythmus“ entsprach. Auf der Rolltreppe spürte ich leicht die gerillten Stufen. Meine Beine fühlten sich nach einem langen und anstrengenden Tag schwer und müde an. In der U-Bahn konnte ich mich hinsetzen, sodass meine Beine entlastet wurden. Ich streckte meine Beine aus und bewegte meine Zehen in meinen Schuhen. Sie drückten an den Seiten leicht. An meiner Haltestelle stieg ich aus und ging die Treppen zu Fuß nach oben. Ich nahm jede Stufe mit einem Schritt. Auf dem Gehweg zu meinem Haus versuchte ich, nicht auf die Linien der Quadrate am Boden zu treten, sondern mit meinen Füßen in den „Vierecken“ zu bleiben. Dann musste ich durch einen kleinen Park über deinen Kiesweg laufen und nahm das Rasseln der Kieselsteine unter meinen Schuhen wahr. Zum Schluss lief ich noch über ein Stück Wiese mit Laub und hörte auch dort das Rascheln des Laubes unter meinen Füßen bewusst. Die Stufen zu meiner Wohnung waren rutschig und quietschten unter meinen Schuhsohlen. In meiner Wohnung angekommen, konnte ich endlich meine Schuhe ausziehen und meine Füße aus der Enge befreien. Das tat gut. So genau habe ich mich bis jetzt noch nie auf meinen Nachhausweg konzentriert, ab sofort will ich diese Übung öfter durchführen. 
04.06.17 11:00 $\rightarrow$ ca.45 min: Gelomeditation

Endlich bin ich mal wiecler in Italien. Jdu habe mich die letten Wochen schon so richtig any den Toskana-Urlaub ausammen onit meiner Familie gofent. Unsere Unterhunyt ist gleich oun Meer. Ide ging hente Vormittag etwa 45 min. Es tat gut die Sanne, den Wind, die salrige Meermigt any der Haut zu

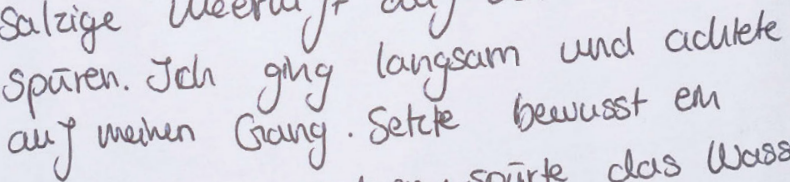
Fuß uor den anderen. spärte das wasser an meinen Fäßen. Es war ein tolles Gefanl am Meer entlang $2 u$ gehen. Nach dem Spaziengany frigh frei und exfischt.

\section{R.}

\subsubsection{7, 11 Uhr, ca. 45 Min., Gehmeditation}

Endlich bin ich mal wieder in Italien. Ich habe mich die letzten Wochen schon so richtig auf den ToskanaUrlaub zusammen mit meiner Familie gefreut. Unsere Unterkunft ist gleich am Meer. Ich ging heute Vormittag etwa 45 Min. am Strand von Cecina spazieren. Es tut gut, die Sonne, den Wind, die salzige Meerluft auf der Haut zu spüren. Ich ging langsam und achtete auf meinen Gang. Setzte bewusst einen Fuß vor den anderen. Spürte das Wasser an meinen Füßen. Es war ein tolles Gefühl, am Meer entlangzugehen. Nach dem Spazierengehen fühlte ich mich richtig frei und erfrischt.

\subsubsection{7, 18 Uhr, ca. 30 Min., Gehmeditation}

Nach dem Seminar beschloss ich, bei dem schönen Wetter die Leopoldstraße bis zum Odeonsplatz und dann bis zum Marienplatz entlangzugehen. Ich hatte meine Lieblingssommersandalen an und achtete auf einen bewussten Gang. Ich ging viel langsamer als sonst, und das bewusste Abrollen der Füße beim Gehen stabilisierte mich in meiner Haltung und mei-
$06.06 .1721 .00 \rightarrow$ ca. 5 min: Kenzen-Meditation

An diesem Aloend machten wir ein

Ulanes Lagerfeuer im Garten vor unserer Wohmung in Cecina. Jch schloss Jü Smin. die Atugen und meditierte. Jch nahm das Ulustern des Feners und die Warwe any meiner Hent wahr. Jch stelle mir innerlich deas Licht des Feuers vor. Daun öffuete ich die Augen. Schante noch eve Weile in die fladernden Fammen. Jch spurte den innesen Frieden in

mir.

nem Gang. Ich spürte zunehmend eine Sicherheit in mir. Der Gedanke "Ich gehe meinen Weg“ war sehr präsent während meiner Gehmeditation. Am Marienplatz angelangt, hätte ich noch ein Stück so weitergehen können. Doch ich machte mich gestärkt auf den Heimweg und fuhr mit dem Zug nach Hause. Nach dem langen Unitag freute ich mich auf ein schönes Abendessen mit meiner Familie.

M. M.

\section{Gehmeditation in der Natur}

Wann: Mittwoch, 3. Dezember 2014, 8.15-8.50 Uhr Wo: Gilching (Gehweg neben Wiesen und Bäumen) Warum: um gelassener in den Tag zu starten Wie:

- Bevor ich nach draußen gehe, höre ich mir auf der CD von Thich Nhat Hanh und Nguyen AnhHuong die "Gehmeditation in der Natur an".

- Im Freien stelle ich mir vor, wie ein Baum zu stehen und die Verbindung mit der Erde unter meinen Füßen zu spüren, was mir nicht leicht fällt.

- Ich gehe los und beginne bei 2 Schritten „einzuatmen“, bei 3 Schritten „auszuatmen“.

- Ich spüre die frische, kühle, feuchte Luft in meinem Gesicht, dadurch werde ich wacher.

- Ich sage beim „Einatmen - ein, ein, ein“, beim Ausatmen - aus, aus, aus", ich merke, dass ich damit nicht klarkomme und lasse das. 
- Mir fällt auf, dass sich meine Schritte weniger hektisch anfühlen als sonst, mein Bauch entspannt sich allmählich, ich werde ruhiger.

- Während des Einatmens sage ich nun vor mich hin, „ich bin angekommen“, beim Ausatmen „ich bin zu Hause" - in mir stellt sich ein beruhigendes, zufriedenes Gefühl ein.

- Während des Gehens fallen mir verschiedene Farben auf, auf der Wiese neben mir wächst viel Klee, auf den Blättern sind viele silberne Wassertropfen in unterschiedlichen Größen zu sehen.

- Ich denke daran, mal wieder meine Kamera rauszuholen und ein paar schöne Fotos zu machen.

N. H.

Uhrzeit: 18.30 Uhr

Dauer: ca. 30 Min.

Ort: draußen - Spaziergang im Wald

Art der Meditation: Gehmeditation

Wie ist es gelaufen?

Nachdem wir gestern im Seminar die Gehmeditation kennengelernt haben, habe ich es heute gleich ausprobiert. Ich gehe generell sehr oft abends spazieren, draußen an der frischen Luft, das ist für mich Entspannung und da kann ich abschalten. Also dachte ich mir schon, dass die Gehmeditation für mich bestimmt eine gute Methode ist. So war es auch! Ich bin durch ein kleines Wäldchen spaziert in der Nähe meines Zuhauses. Normalerweise lege ich diesen Weg in 15 Min. zurück. Ich habe heute die doppelte Zeit gebraucht. Schritt für Schritt habe ich jeden Fuß langsam abgerollt und habe meine Schritte an meine Atmung angepasst. Ich war überrascht, wie viele Details und Beschaffenheiten des Waldbodens mir bewusst aufgefallen sind.

\section{Meditation 23.11.2015}

Uhrzeit: 11.45 Uhr

Dauer: 3 Min.

\section{Ort: Weg zur Uni}

\section{Art der Meditation: Gehmeditation}

\section{Wie ist es gelaufen?}

Ich war auf dem Weg zu Uni und sehr nervös, da ich in meinem Seminar um 12.00 Uhr gleich Referat halten sollte. Ich habe mich dann auf dem Weg von der UBahn bis in meinen Seminarraum nur auf mich selbst und meine Füße und meine Atmung konzentriert. Habe mein Tempo etwas verlangsamt und einen Fuß vor den anderen gesetzt. Ich habe darauf geachtet, mich jetzt nur auf meinen Gang und die Atmung zu konzentrieren. Habe tief in die Brust und den Bauch eingeat- met und die Füße von der Ferse bis zu den Zehen langsam abgerollt. So konnte ich mich selber beruhigen.

P. S.

Donnerstag, 8.12.2016, bei mir zu Hause, ca. 7 Min. Ergebnis: Gehmeditation

Auf dem Weg zu meinem Nachhilfeschüler habe ich mir die Gehmeditation vorgenommen. Da es heute Vormittag bei mir ein bisschen stressig war (putzen, kochen für meine Schwester, waschen), wollte ich entspannter und gelassener bei meinem Nachhilfeschüler ankommen. Ich konnte zwar nicht so langsam gehen, wie bei mir zu Hause in einem Zimmer (sonst wäre ich den Leuten aufgefallen), aber ich habe dennoch versucht, möglichst langsam in einem Rhythmus zu gehen. Am Anfang fiel es mir schwer, einen guten Rhythmus zu finden und nach unten auf meine Füße zu sehen normalerweise achte ich da eher auf die Umgebung -, doch auf der Hälfte des Weges ging es immer besser. Habe dabei sogar die Schritte noch gezählt.

Bei der Gehmeditation insgesamt hat mir sehr gutgetan, sich mal nur auf das Gehen zu konzentrieren. und den Gedankenwirbel „im Kopf“ völlig auszublenden. Ich hab dabei wirklich an nichts anderes gedacht und auch total vergessen, dass meine Mama dabei war. Auch sie hat die Meditation ausprobiert, und es hat ihr für den Anfang sehr gut gefallen.

\section{T. K.}

\subsubsection{9}

\section{Achtsames Gehen / Gehmeditation}

Daheim im Ort beim Gassigehen

Heute Morgen durfte ich den Hund Gassi führen. Da dachte ich mir, verbinde ich es doch diesmal, bevor es im Anschuss wieder mit der BA weitergeht, mit achtsamem Gehen. Das hat auch wunderbar geklappt, trotz Hund. Liegt wohl daran, dass ich ihn nicht an der Leine hatte und er somit nicht ziehen konnte und seine Narrenfreiheit hatte. Genau wie ich, ich fand das achtsame Gehen schon in der Übung während der Vorlesung sehr interessant, habe es bis dato leider nicht mehr wiederholt. Dies wird sich aber in Zukunft ändern. Habe gemerkt, dass ich danach viel konzentrierter war als üblich nach der Meditation. Werde das achtsame Gehen nun öfter statt der regulären Meditation einbinden und sehen, was mir längerfristig mehr zusagt. 
T. O.

\section{Wo: im Wald}

\section{Wann: ca. 14.00 Uhr}

Was / Wie: Mein Freund und ich machten heute Nachmittag einen Spaziergang im Wald. Ich wollte dabei die Gehmeditation machen, und auch er interessierte sich dafür. Also erklärte ich ihm, wie die Übung funktioniert, und die Kombination mit der Atmung fiel mir an der frischen Luft noch viel leichter. Ich machte die Gehmeditation ca. 20 Minuten lang, dann konnte ich mich nicht mehr konzentrieren. Mein Freund hielt es nicht ganz so lange durch, aber grundsätzlich fand er die Übung gut.

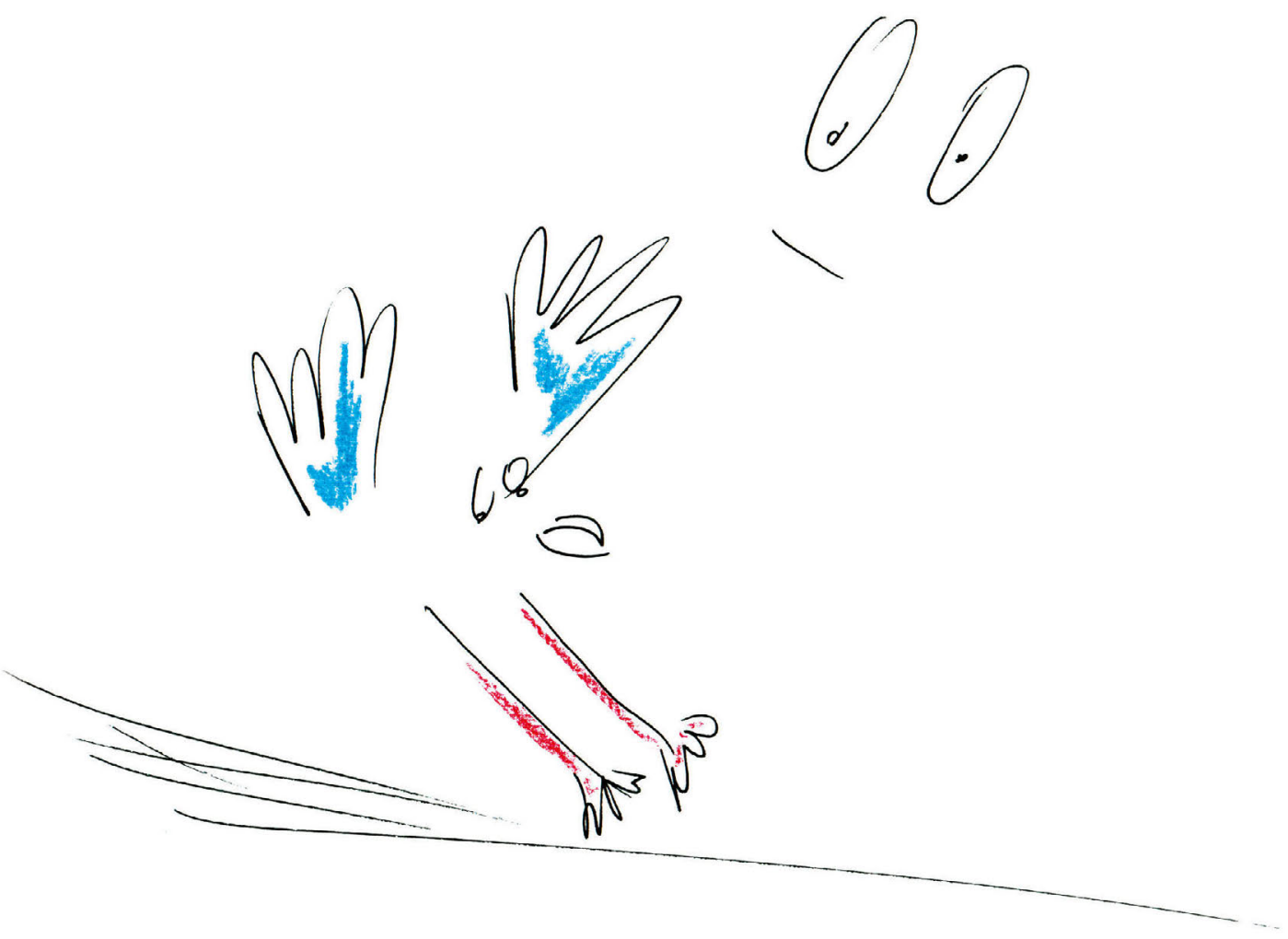

Tauben am Münchner Hauptbahnhof benötigen einen Bremsweg. 


\title{
Sutras
}

Sutras sind Lehrreden. Durch Sutras wird die Konzentrationsfähigkeit entwickelt und ein tiefes Schauen ermöglicht. ${ }^{1}$ Im Unterricht haben wir Übungssätze bezogen auf das Atem-Sutra aus dem Buch Das Wunder des bewussten Atmens von Thich Nhat Hanh benutzt. Er verknüpft sie mit dem eigenen Atem und beschreibt 16 an der Zahl. Sie werden im ruhigen Tempo rezitiert oder innerlich wiederholt.

Wir haben unter anderem folgende Übungssätze behandelt:

„Ich atme ein und lasse meinen Körper ruhig und friedvoll werden. Ich atme aus und lasse meinen Körper ruhig und friedvoll werden. “2

„Ich atme ein und lasse die Aktivitäten meines Geistes ruhig und friedvoll werden. Ich atme aus und lasse die Aktivitäten meines Geistes ruhig und friedvoll werden. ${ }^{\text {"3 }}$

Die Übungssätze sind leicht anwendbar. Man braucht nicht viel Zeit dazu, und sie sind so gut wie an jedem Ort praktizierbar.

\section{Konzentrieren und sammeln wir unseren Geist, so erwerben wir auf natürliche Weise die Fähigkeit zu sehen, Dinge zu erkennen.}

\author{
Thich Nhat Hanh
}

Als ich im Jahr 2018 vom Bayerischen Fernsehen frühmorgens um 7 Uhr am Hauptbahnhof München interviewt wurde, fragte man mich, ob ich auch in der Bahnhofshalle stehend zwischen den vorbeigehenden Menschen und einfahrenden Zügen meditieren könne. Die Szene würde ein schöner Anfang für das Interview sein. Man würde die Kamera laufen lassen und dann nach kurzer Zeit auf mich zulaufen und das Interview beginnen. Ich stimmte zu und überlegte mir kurz, welche Technik ich am besten anwenden könnte, um meine Fokussierung zu halten. Mir fielen die Übungssätze des Atem-Sutras ein. Ich wiederholte also leise für mich: „Ich atme ein und lasse die Aktivitäten meines Geistes ruhig und friedvoll werden. Ich atme aus und lasse die Aktivitäten meines Geistes ruhig und friedvoll werden." Es funktionierte.

Leider musste man die Aufnahme mehrfach wiederholen. Einmal ging der Ton nicht und zweimal liefen zu viele Passanten zwischen mir und der Kamera her. Nach dem letzten Versuch hatte sich meine Wahrnehmung verändert. Sie war intensiver und ich sah alles viel klarer und fokussierter. Plötzlich fielen mir die Tauben in der Halle auf. Insbesondere wie sie landeten, konnte ich genau beobachten. Ich bemerkte, dass sie beim Landen immer einen ziemlich langen Bremsweg benötigten, weil der Boden dort für sie sehr rutschig ist. Obwohl ich oft am Hauptbahnhof München bin, ist mir dies noch nie aufgefallen. Die Übungssätze des Atem-Sutras hatten meine Präsenz im gegenwärtigen Moment deutlich verstärkt. 
Im Unterricht haben wir manchmal auch folgende Übungssätze ausprobiert:

„Ich atme ein und betrachte das Loslassen. Ich atme aus und betrachte das Loslassen." ${ }^{4}$

Wiederholt man diesen Satz öfter, merkt man, dass die Bedeutung des Wortes intensiver wird. „Loslassen“ ist sehr vielschichtig. Ich kann überflüssigen Besitz loslassen, was einigermaßen leicht ist. Ich kann auch auf Dinge verzichten, die ich zunächst nicht gerne loslassen möchte, aber Umstände mich dazu zwingen. Oder es kann sogar sein, dass ich jemanden verliere, beispielsweise indem eine Beziehung endet oder jemand stirbt. Und nicht zuletzt können auch Gedankenmuster und Konditionierungen losgelassen werden.

Anschließend haben wir über die mit dem Atem-Sutra gemachten Erfahrungen im Unterricht gesprochen.

Meine Teilnehmerinnen und Teilnehmer haben darüber hinaus auch eigene Varianten entwickelt, beispielsweise: „Ich atme ein und weiß, dass ich wertvoll bin. Ich atme aus und weiß, dass ich wertvoll bin."

Für den Unterricht benutzte Primärliteratur

Thich Nhat Hanh (2000). Das Wunder des bewussten Atmens. Stuttgart: Theseus Verlag.

\footnotetext{
Vgl. Thich Nhat Hanh 2000, S. $97 f$.

Thich Nhat Hanh 2000, S. 9 und S. 57ff.

Thich Nhat Hanh 2000, S. 9 und S. 72 f.

4 Thich Nhat Hanh 2000, S. 10 und S. 94ff.
} 


\section{Tagebuchnotizen Sutras}

A. U.

Freitag, 18.12.2015

Wann: ca. 18.30 Uhr

Was: Sutra-Meditation

Wo: Im Auto und beim Einkaufen

An diesem Tag war es schon in der Früh unwahrscheinlich stressig und ich beeilte mich mit allem, damit ich rechtzeitig um 18 Uhr fertig bin, da wir zu einem Geburtstag eingeladen waren. Ich schaffte es auch pünktlich, nur war ich die Einzige, die fertig war. Somit musste ich warten, bis wir losfahren konnten. Nachdem es nun abzusehen war, dass wir wohl mindestens eine Stunde später loskommen als geplant, entschied ich mich, noch kurz einkaufen zu gehen. Obwohl ich noch etwas Sinnvolles machen konnte, war ich unwahrscheinlich wütend. Zum einen, weil ich mich den ganzen Tag so beeilt hatte, und zum anderen, weil ich es hasse, zu spät zu kommen. Nachdem alles nichts half, probierte ich das Sutra „Mein Geist ist ruhig und friedvoll“" aus und sagte es die ganze Zeit vor mich hin und versuchte dabei bewusst zu atmen. Ich brauchte zwar mindestens eine halbe Stunde, bis ich richtig ruhig wurde, aber ich hatte ja Zeit!

\section{W.}

\section{Donnerstag, 9.5.2019}

Körper ruhig und friedvoll, Geist ruhig und friedvoll 17.30 Uhr, ca. 10-15 Min., beim Zahnarzt

Da ich aufgeregt und etwas ängstlich beim Zahnarzt bin und eine Untersuchung bzgl. eines Eingriffs mit Spritze bevorsteht, habe ich die Zeit im Wartezimmer und auf dem Behandlungsstuhl genutzt, um mich mit der Atemtechnik zu beruhigen. Zunächst habe ich mehrere Minuten meinen Körper beim Ein- und Ausatmen beruhigt. Im Anschluss meinen Geist. Zu Beginn war es sehr schwierig, da ich immer wieder Bilder von Spritzen etc. vor mir hatte und während des Wartens auf dem Behandlungsstuhl die Werkzeuge liegen sehen habe. Mit der Zeit wurde es jedoch besser. Ich konnte mich mit viel Mühe auf meinem Atem konzentrieren und den Gedankenstrudel verlassen. Unsicher war ich trotzdem, jedoch deutlich weniger. Zumal die eigentliche Behandlung erst in einem halben Jahr sein wird. Zuvor werde ich mit Sicherheit meditieren!
J. H.

\section{Wo: Zu Hause \\ Was: Sutras}

Wie lange: 10 Min.

Ich wollte die Sutras noch einmal ausprobieren - diesmal hat mir eine Mitbewohnerin die Sätze vorgelesen und ich habe sie im Kopf wiederholt. Das war am Anfang für sie eher komisch, aber am Schluss meinte sie dann auch, dass sie merkte, dass sie etwas ruhiger ist. Man liest erst nur so über die Sätze drüber, aber wenn man sie mal wirklich aufmerksam liest, bekommen sie eine andere Bedeutung.

\section{Wann: 25.5.2017}

\section{Was: Sutras / Atmung}

Wie lange: insgesamt ca. 20 Min.

Heute habe ich mich an den Sutras versucht. Zuerst habe ich ca. 10-15 Minuten meditiert und dann habe ich die Nr. 3-6 jeweils ein paarmal laut vorgelesen. Ich fand es in der Stunde ehrlich gesagt etwas einfacher, weil uns die Sätze da vorgelesen wurden. So musste man immer wieder die Augen aufmachen, um zu lesen. Vielleicht ist es deshalb besser, wenn man sich die Sätze vorlesen lässt. Trotzdem fand ich die Art sehr gut, und kann mir auch vorstellen, es noch öfter zu wiederholen.

\section{L.}

\subsubsection{6}

Heute haben wir in der Lehrveranstaltung die Sutras besprochen. Bereits auf der Heimfahrt haben sich diese als ausgesprochen hilfreich erwiesen. Als mir ein anderer Autofahrer direkt vor die Schnauze zog, stieg es mir sofort ins Gedächtnis: „Ich atme ein und lasse meinen Geist ruhig und friedvoll werden. Ich atme aus und lasse meinen Geist ruhig und friedvoll werden." Und tatsächlich spürte ich, wie die Angst vor einem Unfall und die Wut auf den Autofahrer nachließen.

\subsubsection{6}

Zwischen all dem Stress für die anstehenden Leistungsnachweise gibt es eine Übung, die ich so ziemlich jeden Tag anwende: die Sutras! Ich fühle mich danach immer ein ganzes Stück ruhiger. Ich kann mich ganz auf mich und meine Atmung konzentrieren. 
N. H.

18.11.2015

Dauer: 2 Min.

Uhrzeit: $7.30 \mathrm{Uhr}$

\section{Ort: Auto}

Ich war auf dem Weg in die Arbeit. Ich bin heute mit dem Auto gefahren und stand im Berufsverkehr natürlich voll im Stau. Ich sollte um 8 Uhr dort sein. Die Ankunftszeit auf meinem Navi war nun schon nach 8! Ich spürte, wie ich ungeduldig und grantig wurde. Ich wusste, ich könnte es nicht mehr rechtzeitig bis $8 \mathrm{Uhr}$ schaffen! Also habe ich versucht, ein bisschen runterzukommen. Ich habe den Satz: „Ich atme ein und lasse meinen Körper ruhig und friedvoll werden" 3-mal laut und langsam aufgesagt [...] und habe im Rhythmus dazu geatmet. Das hat mir geholfen, ein bisschen runterzukommen. Ich habe mich damit abgefunden, dass ich zu spät sein werde. Aber habe es durch die Meditation geschafft, nicht schlecht gelaunt in der Arbeit anzukommen.

\section{Uhrzeit: 14 Uhr}

Dauer: 5 Min.

Ort: S-Bahn

Art der Meditation: Sutra

Wie ist es gelaufen?

Ich war auf dem Heimweg von der Uni und auf hundertachtzig, weil ich von Dozent zu Dozent gelaufen bin wegen meines Praktikums. Jeder hat etwas anderes gesagt und alle schienen mit der Situation überfordert. Ich glaube, ich selbst war am meisten überfordert, da ich einfach nicht weiß, wo ich jetzt hinkomme. In der S-Bahn habe ich die Augen geschlossen und an folgenden Satz gedacht: „Ich atme ein und lasse meinen Geist ruhig und friedvoll werden, ich atme aus ..."

Das hat mir wahnsinnig geholfen, mich zu beruhigen. Ich habe danach alles etwas entspannter gesehen. Jeder ist mit dieser Praktikumssituation überfordert, nicht nur ich!

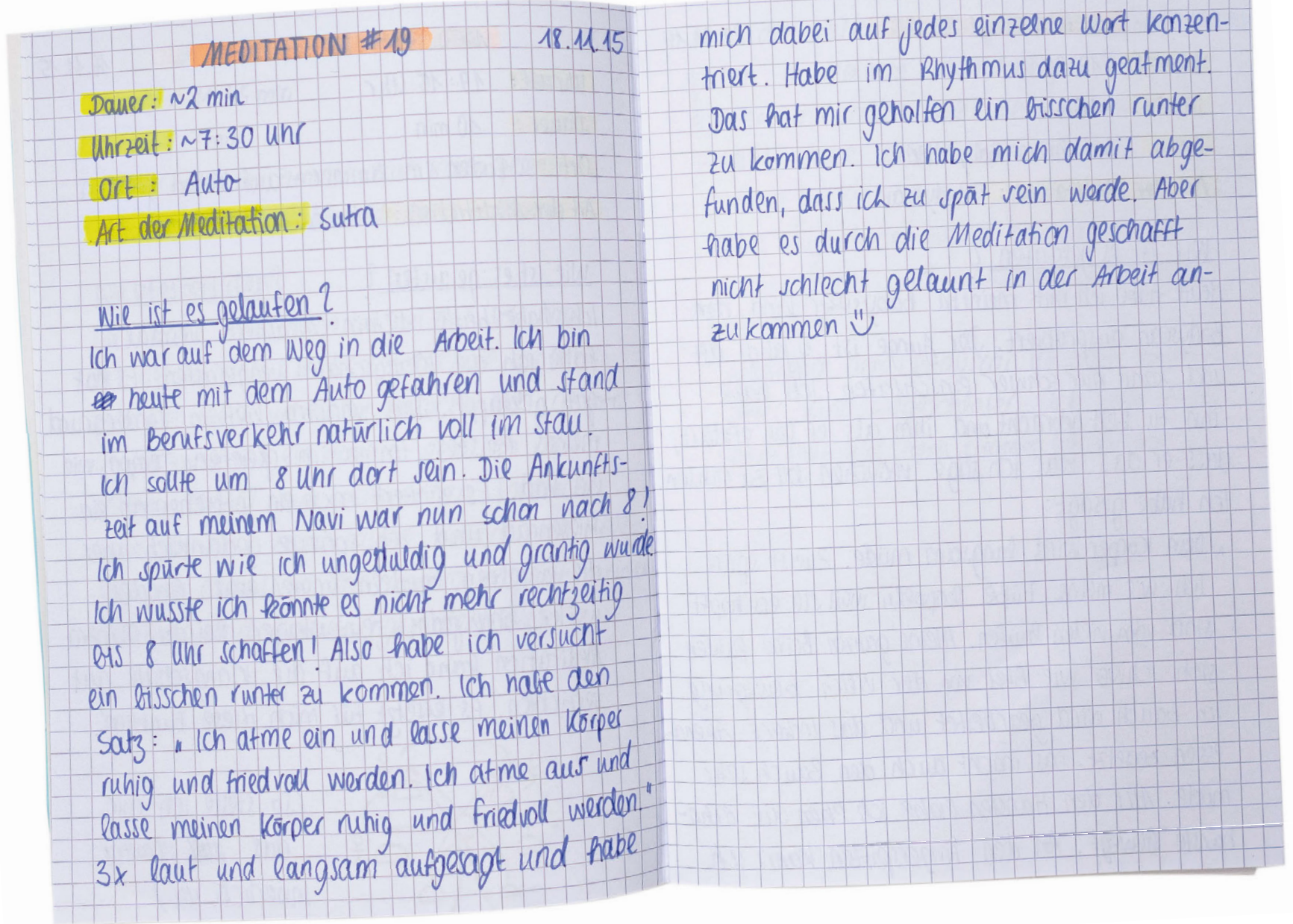




\title{
Geräuschmeditation
}

\author{
Das Hören bringt mehr herein, \\ aber das Sehen zeigt mehr hinaus. \\ Und darum werden wir im ewigen Leben \\ viel seliger sein in der Kraft des Hörens \\ als in der Kraft des Sehens.
}

Meister Eckhart (1260-1327)

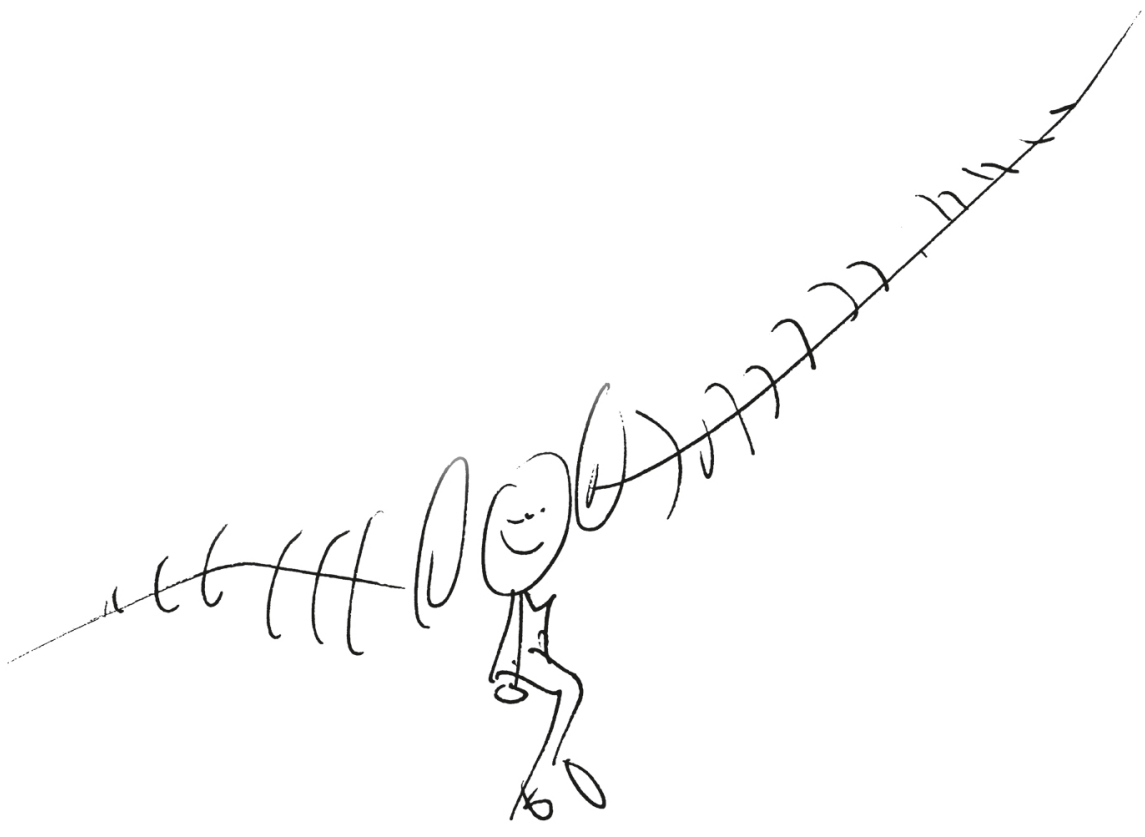

Unser Alltag, die Welt um uns herum ist voller Geräusche. Oft sind wir uns der Geräusche aber nicht bewusst, da wir die meisten davon automatisch filtern. Wir können uns aber auch vornehmen, sie wieder mehr wahrzunehmen. Zum einen, indem wir in einer bestimmten Umgebung bewusst auf die Vielfalt der Geräusche achten, zum andern, indem wir die Qualität der Geräusche vertiefen.

Bewusstes Hören: nah und weit. 
Im Unterricht habe ich oftmals die folgende Geräuschmeditation durchgeführt:

\section{Anleitung}

Wir schließen die Augen. Dadurch können die visuellen Reize ausgeblendet werden. Wenn wir uns gleich auf die Geräusche konzentrieren, wollen wir sie eins nach dem anderen bewusst wahrnehmen. Wir werden versuchen, die Geräusche einfach „nur“ zu hören, ohne sie subjektiv zu interpretieren und zu bezeichnen. Damit werden wir in eine eigene Welt eintauchen, in der sich viel Neues entdecken lässt.

Ist das Geräusch laut oder eher leise? Ist es hoch oder tief? Ist es weich oder eher hart? Wiederholt sich das Geräusch oder kommt es nur einmalig? Hat das Geräusch eine Melodie oder ist es eher monoton? Baut es sich langsam auf oder ist es gleich sehr präsent? Und gibt es auch Momente der absoluten Stille?

Als Erstes versuchen wir, uns jetzt auf Geräusche zu konzentrieren, die sehr weit weg sind. Weit außerhalb des Raumes, in dem wir uns gerade befinden. Beispiele: Autos, in der Ferne ein Flugzeug, jemand arbeitet im Garten mit einer Säge ...

Jetzt versuchen wir uns auf Geräusche zu konzentrieren, die noch immer außerhalb des Raumes, aber näher bei uns sind. Beispiele: Stimmen, vorbeigehende Menschen, Vogelgezwitscher ...

Jetzt achten wir auf die Geräusche im Raum selbst. Beispiele: Heizkörper, Teilnehmer, die sich bewegen, husten, jemand trinkt Wasser ...

Und jetzt konzentrieren wir uns auf die Geräusche im eigenen Körper.

Beispiele: Atemzüge, Körperbewegungen, Grummeln im Bauch, Schlucken ...

Und kann man die eigenen Gedanken auch hören?

Die Geräuschmeditation kann man an jedem Ort machen, und sie ist zeitlich nicht limitiert. Jeder Ort hat seine eigene Klangwelt. Ob im Wald, am Bahnhof, im Café, in der Einkaufstraße, am Meer. Übt man das achtsame Hören öfter, wird man automatisch empfindsamer für die diversen Geräusche. Ebenso für Stille und Lärm.

Man lernt dabei, selektiver zu hören, und diese Fähigkeit kann man in vielerlei Situationen anwenden. Die eigene Stimme besser wahrzunehmen, ist eine schönes Beispiel dafür. Sie kann viel über das eigene Befinden sagen. Ebenso verhält es sich mit dem bewussten Hören der Stimmen anderer. Akustische Merkmale der Stimme sagen etwas aus über die Persönlichkeit eines Menschen, wie beispielsweise über die emotionale Stabilität, über Introvertiertheit und Extrovertiertheit etc.

Achtsames Hören sollte am besten mit Neugierde und einem gewissen Entdeckergeist geübt werden.

Ein sehenswerter Film über Stille und Geräusche ist Zeit für Stille von Patrick Shen. Die Dokumentation erforscht auf meditative Weise unsere Beziehung zu Stille, Geräuschen und dem Einfluss von Lärm auf unser Leben.

Für den Unterricht benutzte Primärliteratur

Shen, Patrick (2018). Zeit für Stille. Köln: mindjazz pictures. [DVD] 


\section{Tagebuchnotizen Geräuschmeditation}

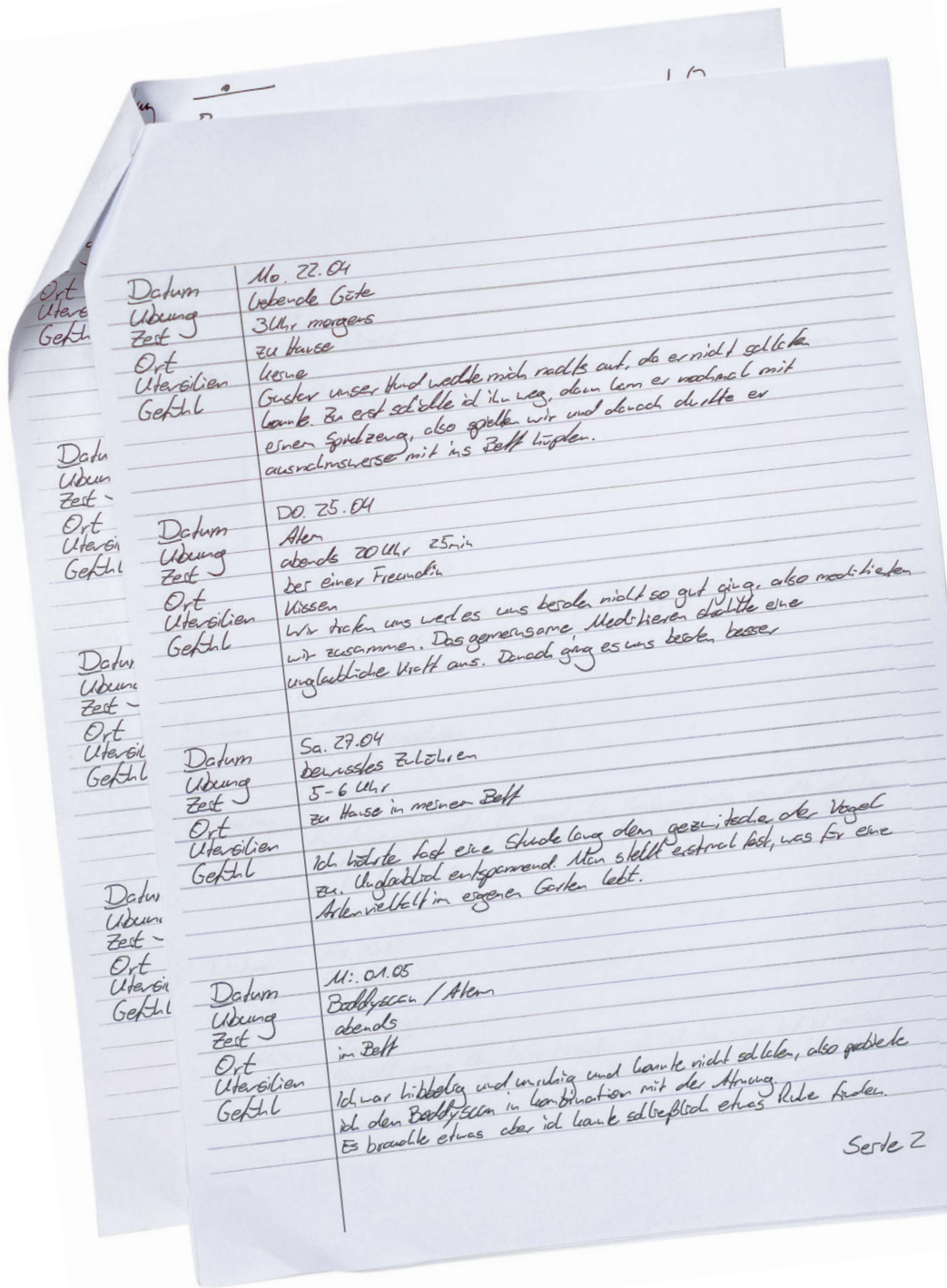

Name / Initialen nicht genannt

Datum: Sa. 27.04

Übung: Bewusstes Zuhören

Zeit: 5-6 Uhr

Ort: zu Hause in meinem Bett

Utensilien:

Gefühl: Ich hörte fast eine Stunde lang dem Gezwitscher der Vögel zu. Unglaublich entspannend. Man stellt erstmal fest, was für eine Artenvielfalt im eigenen Garten lebt.

\section{F. S.}

\subsubsection{9, Hör- und Atemmeditation}

Dauer: ca. 10-15 Min.

Ich habe einen bestimmten Platz, den ich nur alleine aufsuche. Er liegt auf einem kleinen Hügel mit vielen Bäumen und Büschen. Ich setze mich dann gerne auf die Erde und lehne mich an einen Baum an. Dort fühle ich mich, als wäre ich ein Teil der Natur.

Ich fange an, mich auf meine Atmung zu konzentrieren und auf das, was ich um mich herum wahrnehmen und hören kann. Alles passiert direkt vor meinen Augen. Bienen fliegen umher, schwermütige Hummeln fliegen langsam von Blatt zu Blatt. Blätter rascheln neben mir und über mir. Sonnenstrahlen bahnen sich ihren Weg durch die Blätter auf die Erde. Der Wind bläst mal stärker, mal schwächer, mal kommt er von rechts, dann von links.

Ich versuche oft auch Dinge zu hören, die nicht direkt im Vordergrund zu hören sind. So hörte ich weiter entfernte Fußgänger oder Radfahrer von dem kleinen Weg, der am Bach entlangführt. Mein Kopf ist frei, und keine Gedanken stören mich. Dieser Ort gibt mir Kraft.

J.W.

\subsubsection{9}

\section{Hör-Meditation \\ Wo: Auf meinem Balkon \\ Dauer: 15 Min.}

Vor der Meditation war ich etwas unkonzentriert. Deshalb konzentrierte ich mich umso mehr auf die ganzen Geräusche, die von meinem Balkon aus zu hören sind: die Vögel, Autos, sprechende Menschen, der Wind, Flugzeuge, bellende Hunde, das Auf- und Zumachen der Mülltonne usw. Wahnsinnig viele Geräusche und noch mehr ... Danach war ich plötzlich viel mehr bei der Sache und ich hörte auch noch Stunden nach der Meditation viel intensiver als sonst. Sogar auf meiner Tastatur nahm ich jedes Tippen (das Geräusch) wahr, es gab mir das Gefühl, ganz bewusst da zu sein. Jedes Geräusch passiert gerade JETZT, in der Gegenwart, lässt mich ganz da sein. 
M. N.

\subsubsection{9}

Dauer: ca. 5 Min.

Heute Morgen hatte ich meditiert, als das Tageskind meiner Freundin hier war. Dieses hat die ganze Zeit geweint und geschrien. Also dachte ich mir, ich versuche diese Geräuschkulisse durch meine Meditation auszublenden. Leider hat dies nur bedingt geholfen - das Geschrei war zu penetrant und so habe ich abgebrochen.

N. S.

\subsubsection{9}

\section{5-minütige Geräuschmeditation}

Ich habe probiert, innerhalb und außerhalb des Hauses Geräusche zu hören, zu identifizieren und einzuteilen.

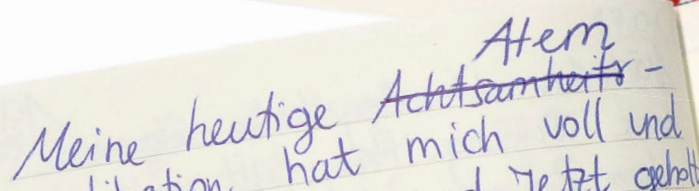
meditation Hier und Jetzt gehat gant inr habe setr starh meine Ich hägel beim Ein- ind Aur Naxsflüge watrgenommen und war

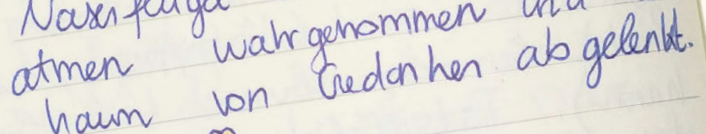
8

\section{$\rightarrow$ Hör-Meditation}

$\rightarrow$ Aut meinem Balhon

21.06

$\rightarrow$ Minuten Meditation war ich Vor der unhonzentriest. Derhalb etwar unhonzen mich umro mek honzentrierte ich mich wäurche, die out die ganzen Balhn aur zu von meinem die vogel, Autos, tóren Mind: Menrcher, der wind, spredende Merrder, der Wind dar
Feugzeuge, bellende tunde, und zumachen der Mullonke usw.
Wann sinnig viele Geraurche und noch
Danach war ich plotelch mehr... Danach war 1 bei der sache ind viel mehr herte auch hoch Stunden nach ich horte auch viel inknniver als der Meditation veiner Tastatur nahm ronst. Sogor aut (dar Gräusch) wahr, ich jeder lippen dar faphe ganz er gab mir da zu sein. Jedes bewusst Da Zu seinade JETZT: Goräusch pasniert guart; làsst mich

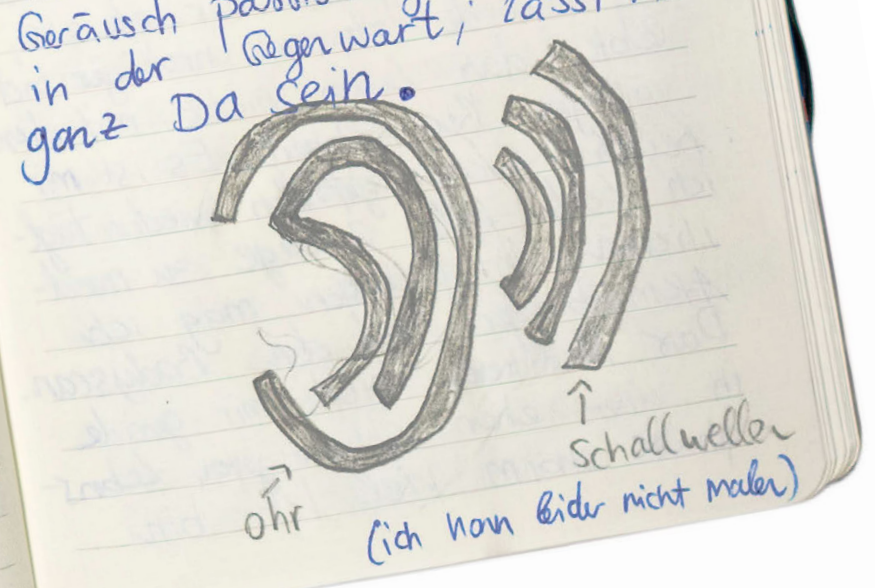




$$
\text { FAZIT }
$$


Seite 169 Allgemeines Fazit

Seite 173 Tagebuchnotizen

Erwartungen

Seite 175 Tagebuchnotizen

Lernen und Prüfungen

Seite 177 Tagebuchnotizen

Rückblick

Seite $184 \quad$ Statements

Seite 187 Feedback von Studierenden aus dem Film „Shanti"

Seite 189 Implementierungserfahrungen aus der Praxis 


\section{Allgemeines Fazit ${ }^{1}$}

Das Münchner Modell „Achtsamkeit und Meditation im Hochschulkontext“ ist seit seinem Beginn im Sommersemester 2010 kontinuierlich gewachsen. Insgesamt nehmen 150 Studierende pro Semester daran teil. Dass die Teilnehmerzahl allerdings viel höher sein könnte, zeigt die Menge der Bewerberinnen und Bewerber. Allein für die Lehrveranstaltung „Stressmanagement und Meditation“ an der Fakultät Studium Generale und Interdisziplinäre Studien (Hochschule München) bewerben sich für die 15 zur Verfügung stehenden Plätze pro Semester über 700 Studierende! Andere Lehrveranstaltungen im Rahmen des Münchner Modells sind ebenfalls überbucht und haben, bezogen auf die jeweils vorhandenen Plätze, zumeist zwei bis drei Mal so viele Bewerberinnen und Bewerber.

Meine persönlichen Beobachtungen als Dozent und die Lektüre von bislang etwa 2000 Meditationstagebüchern à 25-40 Seiten bestätigen, dass die Teilnehmenden nach etwa 4-monatiger Teilnahme an den Lehrveranstaltungen besser mit alltäglichen Herausforderungen, dem allgemeinen Leistungsdruck und der teils hohen Belastung im Studium umgehen können. Sie können Stresssituationen besser handhaben und sie neutraler, sachlicher bewerten.

Kleine Unstimmigkeiten, die zu Streit und Konflikten führen können, etwa im Straßenverkehr oder bei Meinungsverschiedenheiten in der Familie, können jetzt konstruktiver bewältigt werden. Durch die Achtsamkeits- und Meditationsübungen haben Sie gelernt, auf Impulse nicht sofort zu reagieren, sondern kurz innezuhalten, und diese Pause zu nutzen, um mit der Situation anders, überlegter umzugehen. So können sie aus der Emotion „aussteigen“.

Manche Studierende berichten auch, dass der Drang zu konsumieren nachgelassen hat, und nicht wenige können abends besser einschlafen. Mir ist auch aufgefallen, dass sie im Unterricht insgesamt entspannter sind und das gegenseitige Vertrauen zugenommen hat. Das ist auch deshalb wichtig, weil die Erfahrungen innerhalb der Gruppe geteilt werden.

Die Berichte und Feedbackgespräche im Unterricht zeigen zudem, dass die Übungen der Liebenden Güte und der Mitgefühlsmeditationen zu einem besseren Verständnis anderen gegenüber und zu mehr emotionaler Gelassenheit führen. Den Studierenden fällt es leichter, eine Metaebene einzunehmen, was auch für den Umgang mit wissenschaftlichen Fragen hilfreich ist.

Für die Implementierung eines Angebots wie das Münchner Modell an anderen Hochschulen ist eventuell damit zu rechnen, dass zunächst viel Überzeugungsarbeit geleistet werden muss, da für manche Entscheidungsträger der Sinn von Achtsamkeit und Meditation in einem solchen Kontext nicht deutlich ist. Profunde Kenntnisse über Forschungsergebnisse im Allgemeinen und solche, die sich auf Hochschulen beziehen, können helfen, das Vorhaben überzeugend zu präsentieren (siehe „Forschung“, S. $41 \mathrm{ff}$.).

Ein wichtiges Kriterium ist die didaktische Vermittlung. Weil Achtsamkeit und insbesondere Meditation eine so grundlegende Auswirkung auf die Psychohygiene haben, tragen die Lehrenden eine hohe Verantwortung. Für die Vermittlung von Meditationstechniken, insbesondere die der inneren Versenkung, braucht man eine lange und fundierte Meditationserfahrung sowie Kenntnisse über verschiedene Meditationsansätze, um ein vielfältiges Angebot für die Studierenden zu gewährleisten. Allgemeine Achtsamkeitspraktiken dagegen sind einfacher zu vermitteln, etwa als Lehrveranstaltungen und Zusatzangebote bis hin zu Kurzeinheiten in thematisch anderen regulären Lehr- 
veranstaltungen. Allerdings ist dabei für die Lehrenden eine achtsame innere Haltung ausschlaggebend, damit die jeweiligen Übungen nicht mechanisch angeleitet werden und ihre Wirkungskraft verfehlen (siehe S. 18ff., 32f., 87 sowie 122f.). Achtsamkeitsschulungen können dabei einen wichtigen Beitrag leisten. Die Zusammenarbeit mit dem Zentrum für Hochschuldidaktik (DiZ) in Bayern wird hierzu weitere Erkenntnisse liefern und ebenso der Austausch mit den Kooperationspartnern in Jena und Osnabrück, die bereits Ausbildungsprogramme des Thüringer Modells für Hochschullehrende durchführen.

Einige Studierende sind nach dem Abschluss des Studiums selbst Lehrbeauftragte im Rahmen des Münchner Modells geworden. Mit diesen Studierenden finden regelmäßig Abstimmungstreffen statt, sodass sie in Bezug auf Unterrichtsinhalte, didaktische Vermittlung sowie Beurteilung der Leistungsnachweise begleitet werden. Zwar sollen sie die Vorgaben bei der Vermittlung der Lehrinhalte beachten, gleichzeitig aber auch ihre eigene Art des Unterrichtens finden. Authentizität wird als eine wesentliche Voraussetzung für das Lehren angesehen (siehe „Über das Lehren“, S. 81).

Des Weiteren muss die passende Infrastruktur für die Implementierung der Achtsamkeits- und Meditationsangebote an Hochschulen geschaffen werden. Die üblichen Unterrichtsräume mit Tischen und Stühlen sind nur teilweise geeignet. Für die praktischen Übungen ist ein Raum wichtig, in dem man ungestört ist und der mit Kissen, Decken und Matten ausgestattet werden kann. Zudem sind Lagerschränke für diese Materialien sowie ausleihbare Fachliteratur und Filme sinnvoll. Man könnte darüber hinaus auch bestimmte Hochschulbereiche mehr auf „Achtsamkeit und Meditation“ ausrichten, beispielsweise in der Mensa einen Bereich für diejenigen reservieren, die gerne in Stille essen möchten.

Eine andere Frage ist, ob die Achtsamkeits- und Meditationsveranstaltungen auf freiwilliger Basis zusätzlich zum Studium angeboten oder als Lehrveranstaltungen fest in die Lehrpläne verankert werden sollen. Letztere sind entsprechend zu gestalten: Lernziele, Beurteilungskriterien, ggfs. Noten und ECTS. Dass dies grundsätzlich machbar ist, zeigt das Münchner Modell.

Um eine qualitativ hochwertige und nachhaltige Implementierung der Achtsamkeits- und Meditationsangebote zu gewährleisten, ist es wichtig, dass es Personen gibt, die dafür die Verantwortung übernehmen. Dies können Lehrende sein, die im eigenen Schwerpunktbereich diese Themen festigen. Oder eine Verankerung im Bereich „Gesunde Hochschule“. Oder wie im Fall der Hochschule Osnabrück: die Einrichtung einer Stelle zur Koordination und Durchführung der jeweiligen Angebote.

Es stellt sich auch die Frage nach einer Spezialisierung und Zertifizierung im Studium der Studierenden. Anvisiert ist im Studiengang Soziale Arbeit, dass die Studierenden, die an allen Lehrveranstaltungen zum Thema „Achtsamkeit und Meditation“ im Studiengang teilnehmen, zum regulären Bachelorzeugnis ein zusätzliches Zertifikat erhalten. Damit ist ersichtlich, dass sie sich intensiv mit dem Thema auseinandergesetzt haben und in diesem Bereich über eine extra Qualifizierung verfügen. Eine solche Schwerpunktsetzung ist auch in anderen Studiengängen, beispielsweise Pädagogik, denkbar.

In den Endreflexionen der Meditationstagebücher werden auch die Transfermöglichkeiten in den späteren Berufsfeldern ausgearbeitet. Daraus wird ersichtlich, dass es ein enormes Potenzial gibt, eine Implementierung von Achtsamkeits- und Meditationsansätzen in den verschiedenen Handlungsfeldern zu gestalten. Dies könnte durch entsprechende Hochschulzertifikatsausbildungen für Externe, beispielsweise Erzieherinnen/Erzieher, Lehrkräfte sowie Berufstätige aus der offenen Kinder- und Jugendarbeit oder dem Pflegebereich, realisiert werden.

Sollte sich die Nachfrage an „Achtsamkeit und Meditation“ gesellschaftlich weiter ausweiten, ist auch eine berufliche Spezialisierung in Form eines Masterstudien- 
gangs denkbar. An der Universität Wien hat man dies bereits umgesetzt in Form des Weiterbildungsmasterstudiengangs „Achtsamkeit in Bildung, Beratung und Gesundheitswesen".

Ausbildungsmöglichkeiten für Lehrpersonal wurden bereits angesprochen. Denkbar sind beispielsweise kurze Workshops sowie Seminare bis hin zu umfangreichen Zertifikatsausbildungen. Die Hochschule und Universität Jena sowie die Hochschule Osnabrück haben diese Idee bereits umgesetzt (siehe S. 65f.).

Inzwischen haben über 2000 Studierende an den Lehrveranstaltungen des Münchner Modells teilgenommen. Dabei hat sich zeigt, dass viele Teilnehmerinnen und Teilnehmer auch über die Lehrveranstaltungen hinaus Meditation gerne fortsetzen möchten, aber dies ohne eine passende Gruppe nur schwierig oder gar nicht schaffen. Manche machen zwar eigenständig weiter und besuchen Meditationsschulen, um sich die entsprechenden Meditationstechniken anzueignen. Aber diese Anzahl ist bislang relativ gering. Die bisherigen Teilnehmerinnen und Teilnehmer hätten gerne von Anfang des Studiums an in jedem Semester eine Folgelehrveranstaltung zu „Achtsamkeit und Meditation " und ebenso freiwillige Meditationszusatzangebote an der Universität bzw. Hochschule. Die Forschungsarbeit der Hochschule Coburg (siehe S. 64) zeigt, dass die Teilnehmerinnen und Teilnehmer des Münchner Modells diese Meditationsangebote sinnvoll finden und sich weitere Angebote dieser Art wünschen.

Das Bedürfnis, sich untereinander auszutauschen, ist groß unter den (ehemaligen) Teilnehmerinnen und Teilnehmern. Das ist vor allem der Fall bei Studierenden, die im Rahmen von Abschlussarbeiten eigenständig Achtsamkeits- und Meditationsprojekte durchführen oder diese nach dem Studium im Berufsfeld realisieren, wie beispielsweise in Kindergärten und Schulen. Vor diesem Hintergrund erscheint es wünschenswert, einen Ort innerhalb der Hochschule zu schaffen, wo Studierende gemeinsam meditieren und sich austauschen können. Für München wäre das ein Zentrum für Achtsamkeit und Meditation, das sowohl der Hochschule als auch der Universität angehören würde. Ich bin davon überzeugt, dass ein solcher Ort ein enormer Gewinn für die Studierenden sein könnte. Und da Achtsamkeit und Meditation in der Gesellschaft auf immer mehr Interesse und breitere Akzeptanz stoßen, könnte ein solches Zentrum für die Hochschulen auch ein Aushängeschild sein.

\section{QUELLEN}

de Bruin, Andreas (2019a). „Spiritualität im säkularen Raum am Beispiel des Münchner Modells - Achtsamkeit und Meditation im Hochschulkontext", in: Rötting, Martin / Hackbarth-Johnson, Christian (Hrsg.), Spiritualität der Zukunft. Suchbewegungen in einer multireligiösen Welt. Sankt Ottilien: EOS Editions, S. 341-350.

Zeitschrift

de Bruin, Andreas (2017). „Möglichkeiten der Geistesschulung: Meditation im universitären Kontext? - Das Münchener-Modell“, in: Zeitschrift für Bewusstseinswissenschaften. Transpersonale Psychologie und Psychotherapie, 23. Jahrgang 2. Petersberg: Verlag Via Nova, S. 68-84.

1 Der Text ist zum Teil entnommen aus: de Bruin 2017 sowie de Bruin 2019a. 


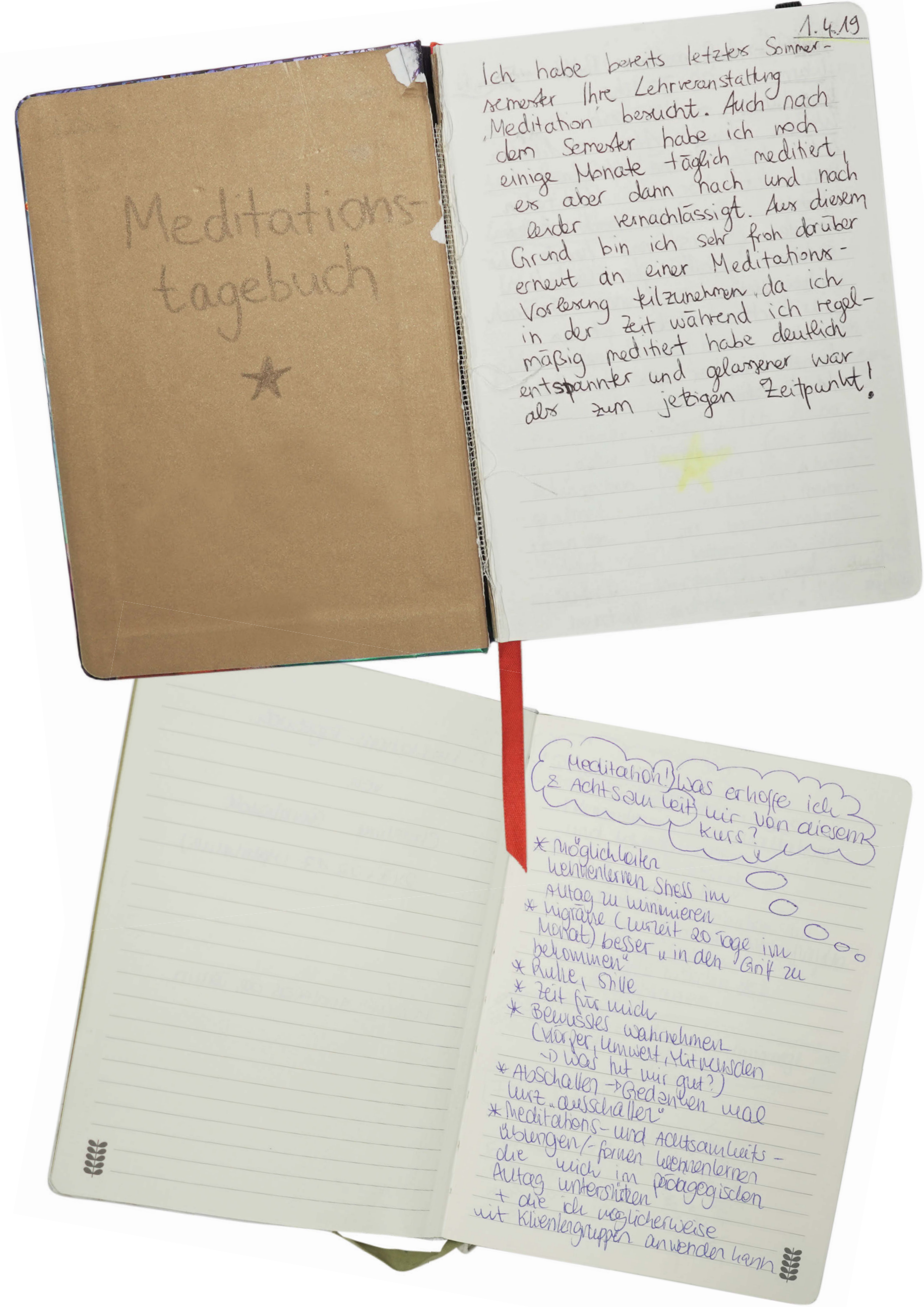




\section{Tagebuchnotizen Erwartungen}

B. K.

Ich meditiere schon seit vielen Jahren. Leider habe ich es bisher nicht geschafft, Meditieren zum Alltag zu machen; es gab immer wieder Phasen, in denen ich viel zum Meditieren gekommen bin, und dann wieder gar nicht.

Nach einer Zeit, wo ich nicht meditiert habe, fällt es schwer, wieder anzufangen, da ich oft von Gedankenfluten überrollt werde.

Deswegen wünsche ich mir für mich: Ich will Meditieren fest in mein Leben integrieren und keine Phasen mehr haben, in denen ich die Motivation zu meditieren nicht finde!

Der Uni-Kurs „Meditation“ von Andreas de Bruin wird mir bestimmt dabei helfen.

\section{B.}

Meditation \& Achtsamkeit! Was erhoffe ich mir von diesem Kurs?

- Möglichkeiten kennenlernen, Stress im Alltag zu minimieren

- Migräne (zurzeit 20 Tage im Monat) besser „in den Griff“ zu bekommen

- Ruhe, Stille

- Zeit für mich

- Bewusstes Wahrnehmen (Körper, Umwelt, Mitmenschen - was tut mir gut?)

- Gedanken mal kurz „abschalten“

- Meditations- und Achtsamkeitsübungen und -formen kennenlernen, die mich im pädagogischen Alltag unterstützen und die ich möglicherweise mit Klientengruppen anwenden kann

\section{J.W.}

\subsubsection{9}

Ich habe bereits letztes Sommersemester Ihre Lehrveranstaltung „Meditation“ besucht. Auch nach dem Semester habe ich noch einige Monate täglich meditiert, es aber dann nach und nach leider vernachlässigt. Aus diesem Grund bin ich sehr froh darüber, erneut an einer Meditationsvorlesung teilzunehmen, da ich in der Zeit während ich regelmäßig meditiert habe, deutlich entspannter und gelassener war als zum jetzigen Zeitpunkt!

\section{N.}

Heute haben wir im Unterricht zum ersten Mal meditiert. Zuvor habe ich nie dergleichen ausprobiert. War aber ein eigenartiges Gefühl, denn nie zuvor habe ich es geschafft, meine Gedanken abzustellen und habe das auch immer so hingenommen, dass es sowieso nicht gehen würde.

Und auch nach der ersten Meditation konnte ich es nicht. Stattdessen hatte ich mit Müdigkeit zu kämpfen. Aber ich denke, das wird besser.

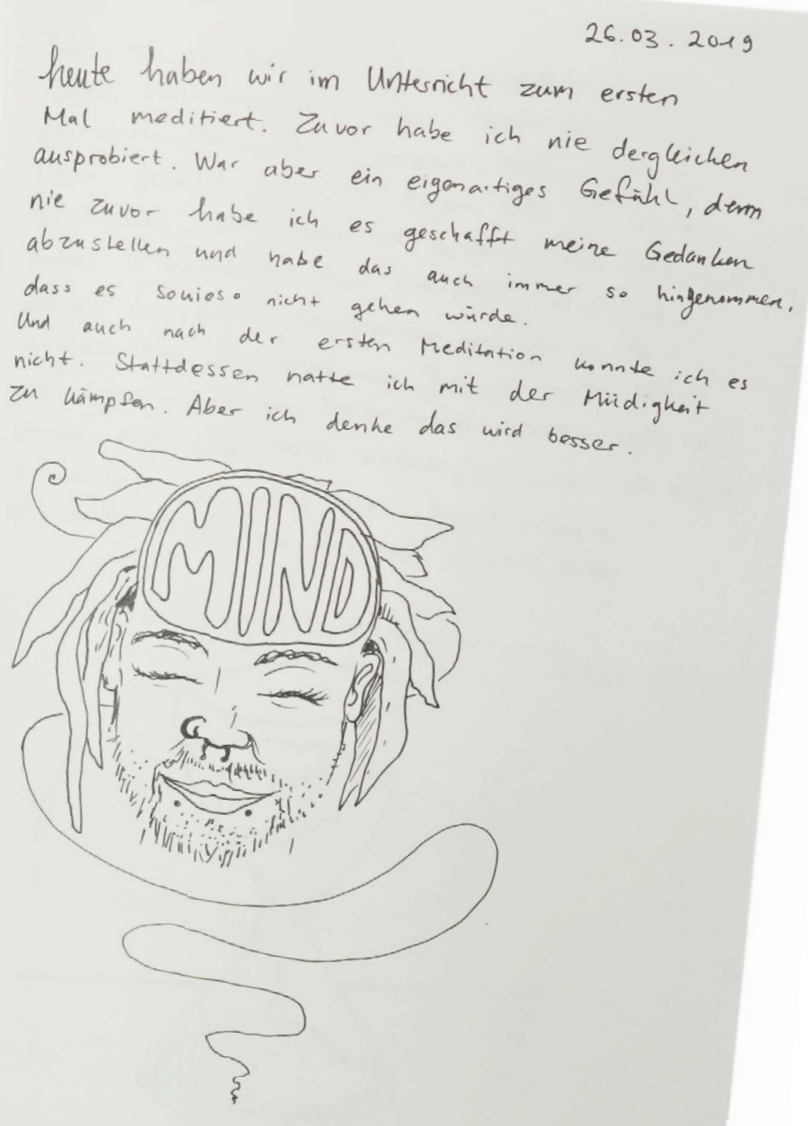




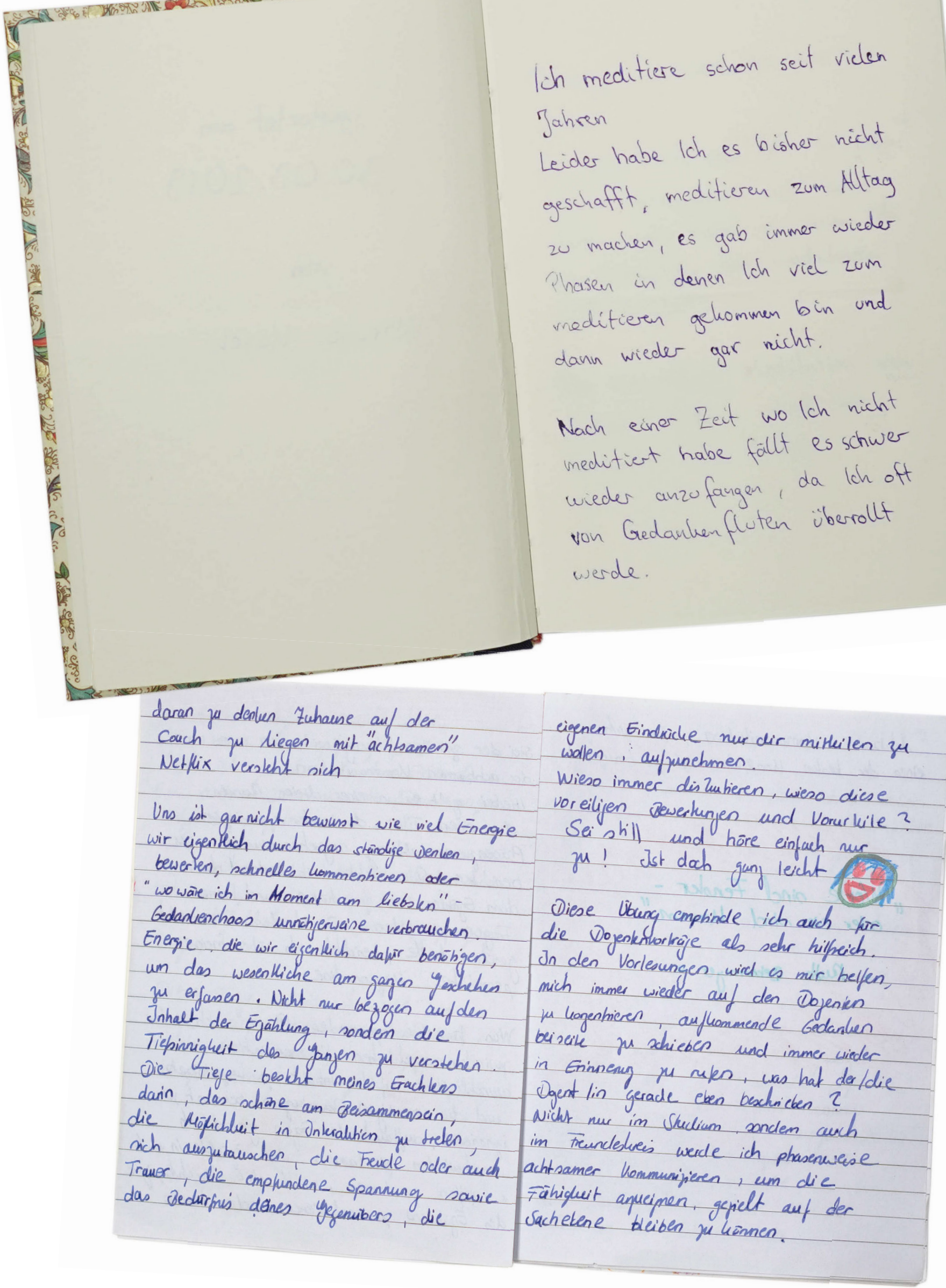




\section{Tagebuchnotizen Lernen und Prüfungen}

J.S.

\section{Donnerstag, 6.6.2019, ca. 15.20-15.25 Uhr}

Heute war ich in der Uni beim Lernen. Nachdem ich sehr lange Elektrotechnik gelernt habe, ist mir die Decke auf den Kopf gefallen. Ich habe dann kurz meine Augen zugemacht und mich einfach nur auf den Atem konzentriert, um einen freien Kopf zu bekommen. Irgendwann habe ich dann gemerkt, dass sich mein Kopf nicht mehr so „schwer" anfühlt. Ich habe dann die Augen wieder aufgemacht, einen Schluck getrunken und dann ein anderes Fach gelernt. Es hat mir in dieser Situation unheimlich viel geholfen.

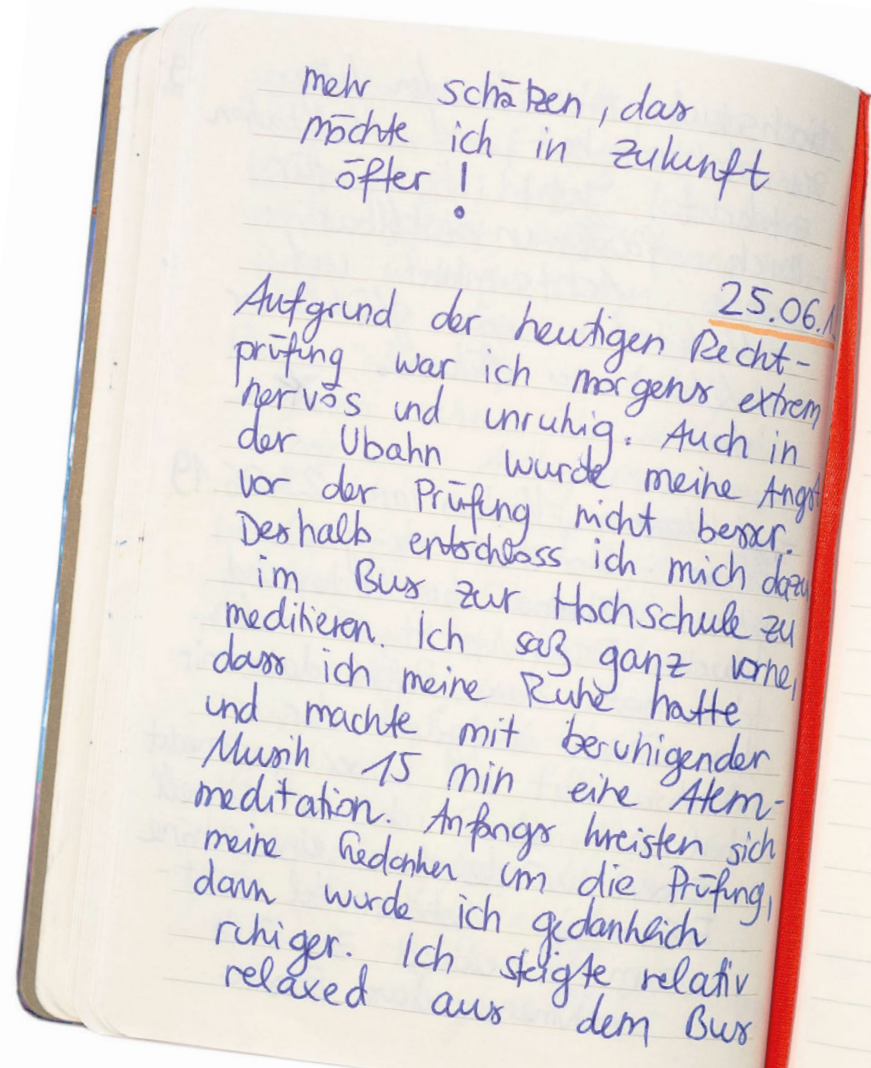

J. W.

\subsubsection{9}

Aufgrund der heutigen Rechtprüfung war ich morgens extrem nervös und unruhig. Auch in der U-Bahn wurd meine Angst vor der Prüfung nicht besser. Deshalb ent schloss ich mich dazu, im Bus zur Hochschule zu meditieren. Ich saß ganz vorne, sodass ich meine Ruhe hatte und machte mit beruhigender Musik 15 Minuten eine Atemmeditation. Anfangs kreisten meine Gedanken um die Prüfung, dann wurde ich gedanklich ruhiger.
Ich stieg relativ relaxt aus dem Bus aus und blieb auch während der Prüfung relativ gelassen. - Wahnsinn, was Meditation mit mir machen kann!

K. K.

\section{Wo: In meinem Zimmer}

Wie: Schneidersitz auf meinem Teppich, angelehnt an die Schrankwand

Wann: Montag, 12.5.2014, $12 \mathrm{Uhr}$

Dauer: 10 Min.

Meine erste Klausur in diesem Semester hat heute stattgefunden, in meinem Hauptfach Biologie. Das Thema umfasste Mensch- und Tierphysiologie und hat mich persönlich (endlich!) auch sehr interessiert - im Gegen-

$$
\begin{gathered}
\text { aus und blieb auch wär end } \\
\text { der früfing nelativ gelassen - } \\
\text { wahnsinn was Hedistion } \\
\text { mit mir machen hann } \\
\text { O- }
\end{gathered}
$$

satz zu Semester 1 und 2, in denen die Ökologie mich fast in den Wahnsinn trieb.

Die Noten haben leider mein mangelndes Interesse widergespiegelt, aber heute lief es gut und ich hoffe auf eine 2. Ich konnte mich wirklich gut konzentrieren und schreibe diese Tatsache auch meiner Idee zu, vor der Fahrt zur Klausur und nach dem letzten Mal Zetteldurchlesen 10 Minuten zu meditieren.

Ich hatte wirklich gut gelernt und konnte deshalb auch schnell meine Aufmerksamkeit auf mich und meinen Geist lenken. Ich habe zum ersten Mal versucht, einen fiktiven Punkt zwischen meinen Augenbrauen etwas oberhalb auf der Stirn als Fokussierungspunkt auszuwählen und durch das Denken von OM immer wieder darauf zurückzukommen, statt mich auf meine Nasenatmung zu konzentrieren.

Es hat wirklich erstaunlich gut funktioniert, und die von mir ausgewählten 10 Minuten habe ich erst wahrgenommen, als der Ton ertönte, dass sie jetzt schon vorbei sind. 
Ich habe weder das Fokussieren auf eine andere Stelle des Gesichts noch die Dauer als anstrengend empfunden und bin danach gestärkt zur Klausur.

\section{R.}

\subsubsection{7, $18 \mathrm{Uhr}$, ca. 20 Min.}

\section{Achtsames Klavierspielen}

Ich habe mir selbst mittlerweile 3 Lieder auf dem Klavier beigebracht. Ich liebe diese Melodien und spiele sie sehr gern auswendig auf dem Klavier. Heute machte ich ein Experiment, und zwar schloss ich meine Augen und verließ mich ganz auf meine Finger, die diese [Melodien] schon so oft gespielt haben. Meine Sinne richtete ich mit voller Aufmerksamkeit auf die Melodien in meinem Kopf, und entspannt und locker versuchte ich, diese Melodien auf dem Klavier zu spielen. Ich nahm die Musik dadurch noch intensiver wahr. Ich war sehr glücklich.

\subsubsection{7, 12 Uhr, ca. 10 Min.}

\section{Atemübung}

An diesem Samstag hatte ich zwei Klausuren. Nach der ersten Klausur ging ich eine halbe Stunde spazieren (achtsame Gehmeditation). Danach setzte ich mich im Luitpoldpark auf eine Parkbank, schloss die Augen und meditierte. Da spürte ich auch wieder das Kreisen auf meinen Handinnenflächen, die nach oben geöffnet waren.

Ich sog die frische Luft durch die Nasenflügel ein. Ich achtete bewusst auf meinen Atem. So gelang es mir, meine Kräfte wieder zu sammeln und klar zu denken. Ruhig und gestärkt ging ich in die zweite Klausur.

\section{S. B.}

Uns ist gar nicht bewusst, wie viel Energie wir eigentlich durch das ständige Denken, Bewerten, schnelles Kommentieren oder „Wo wäre ich im Moment am liebsten"-Gedankenchaos unnötigerweise verbrauchen. Energie, die wir eigentlich dafür benötigen, um das Wesentliche am ganzen Geschehen zu erfassen. Nicht nur bezogen auf den Inhalt der Erzählung, sondern die Tiefsinnigkeit des Ganzen zu verstehen. Die Tiefe besteht meines Erachtens darin, das Schöne am Beisammensein, die Möglichkeit in Interaktion zu treten, sich auszutauschen, die Freude oder auch Trauer, die empfundene Spannung sowie das Bedürfnis deines Gegenübers, die eigenen Eindrücke nur dir mitteilen zu wollen, aufzunehmen.
Wieso immer das Imitieren? Wieso diese voreiligen Bemerkungen und Vorurteile? Sei still und höre einfach nur zu! Ist doch ganz leicht.

Diese Übung empfinde ich auch für die Dozentenvorträge als sehr hilfreich. In den Vorlesungen wird es mir helfen, mich immer wieder auf den Dozenten zu konzentrieren, aufkommende Gedanken beiseitezuschieben und immer wieder in Erinnerung zu rufen, was hat der Dozent/die Dozentin gerade eben beschrieben?

Nicht nur im Studium, sondern auch im Freundeskreis werde ich phasenweise achtsamer kommunizieren, um die Fähigkeit anzueignen, gezielt auf der Sachebene bleiben zu können.

\section{S. Z.}

\section{Was: Ajnameditation}

Zeit: 10 Min.

Während einer ca. 5-stündigen Lernsession habe ich, nachdem ich so viele Leichtsinnsfehler gemacht habe und mich einfach nicht konzentrieren konnte, versucht, die Situation durch eine Ajnameditation zu verbessern.

$\mathrm{Zu}$ Beginn fand ich es komisch, mitten in der Bibliothek zu meditieren und die Augen zu schließen, deshalb habe ich mich auf einen Punkt etwas vor mir fixiert. Durch die Ruhe, die in der Bibliothek vorhanden ist, fiel es mir sehr leicht die Übung durchzuführen.

Ich habe, ohne die Zeit zu stoppen, mit der Meditation angefangen, weil ich wissen wollte, wie lange es dauert, bis ich einen Unterschied zu vorher feststelle. Obwohl ich das Gefühl hatte, nur vielleicht 4 bis $5 \mathrm{Mi}$ nuten meditiert zu haben, waren es am Ende fast 10 Minuten.

Im Nachhinein hatte ich das Gefühl, als ob ich einen Mittagsschlaf gemacht hätte, da ich viel mehr Konzentration als vorher hatte. 


\section{Tagebuchnotizen Rückblick}

\section{A. B.}

Als ich in LSF die Lehrveranstaltung „Meditation in der Schule" belegte, konnte ich mir noch nicht so besonders viel darunter vorstellen. Ich persönlich hatte mit Meditation noch kaum Erfahrungen gemacht, aber die Beschreibung sprach mich total an. Ich war gespannt darauf, neue Meditationsformen kennenzulernen und auch Kenntnisse über deren Anwendbarkeit mit Kindern in der Grundschule. Obwohl ich zu diesem Zeitpunkt noch keine Ahnung vom Meditieren hatte, beschloss ich, mich voll und ganz auf etwas Neues einzulassen.

Die erste Übung, die wir gemeinsam im Seminar gemacht haben, war das achtsame Essen einer Rosine. Mich hat die Wirkung der Geschichte auf meinen Geschmackssinn fasziniert. Ich habe diese Rosine sehr intensiv wahrgenommen, mit allen Sinnen, und sie letztendlich mit einem Genuss gegessen wie nie zuvor. Auch die Wirkung der Zitronengeschichte auf den Körper fand ich total spannend.

Das erste gemeinsame Meditieren fiel mir noch etwas schwer. Wir sollten uns auf unsere Nasenflügel fixieren und auf die Atmung achten. Es gelang mir, dieses erste Mal noch ganz und gar nicht, meine Gedanken wie Wolken vorbeiziehen $\mathrm{zu}$ lassen, und ehrlich gesagt, dachte ich mir danach: „Ich mach in Zukunft halt die Augen zu und tue so, als würde ich meditieren, merkt ja eh keiner!“

In unserer zweiten Seminarsitzung meditierten wir mit Fokus auf das Ajnazentrum zwischen den Augenbrauen. Auch mit dieser Form der Meditation konnte ich zunächst nichts anfangen und mir kamen Gedanken wie „ich bin nicht für das Meditieren gemacht“ oder ,ich kann das einfach nicht“.

Erst als wir das Mantra MA-RA-NA-THA eingeführt haben, konnte ich mich auf das Meditieren wirklich einlassen und bemerkte, dass es mir von Mal zu Mal leichter fiel, mich nur auf mich und meinen Körper zu konzentrieren. Von da an freute ich mich total auf das Seminar und war eifrig dabei, die Übungen zu Hause auszuprobieren. Ich versuchte, mich auch noch ein paarmal an den beiden anderen Meditationsformen, und es klappte zwar allemal besser als die ersten Male, trotzdem blieb das Mantra meine liebste Übung für zu Hause. Es war mitunter die Übung, die ich am häufigsten gewählt habe. Durch das innere Vorsprechen der Silben MA-RA-NA-THA hatten andere Gedanken kaum die Chance in mein Bewusstsein zu gelangen. Ich glaube, das ist auch einer der Gründe, warum mir diese Übung so gefallen hat, einfach weil sie die erste „richtige" Meditationsform war, die auch bei mir sofort gut funktionierte.

Das Mantra kam bei mir auch oft zum Einsatz, wenn ich beim Lernen einen totalen Blackout hatte und bemerkte, dass ich eine Pause brauchte. Es beruhigte mich extrem und brachte mich wieder runter. Ich fühlte mich danach oft viel leistungsfähiger und voller Energie.

Während des Bodyscans fühlte ich mich total entspannt und konzentriert zugleich. Ich hatte mich noch nie zuvor in die einzelnen Stellen meines Körpers so intensiv hineingedacht und fand die Erfahrung total spannend. Leider dachte ich mir viel zu oft, dass der Bodyscan zu viel Zeit in Anspruch nimmt und ich deshalb lieber eine andere Form der Meditation wählen sollte. Allerdings benutze ich den Bodyscan auch heute noch zum Abschalten und Runterkommen, wenn ich im Bett liege und mein Kopf so voller Gedanken ist, dass ich nicht zur Ruhe komme und Probleme mit dem Einschlafen habe.

Die Übung hilft mir sehr dabei, mich aus der Welt kurz mal auszuklinken und mich wirklich nur auf mich und meinen eigenen Körper zu konzentrieren. Ich bin wirklich sehr dankbar, diese Übung zu kennen, und habe sie auch schon an Freunde weitergegeben, die sie nun auch ab und an als Einschlafhilfe verwenden. :)

Das Meditieren hat mir wirklich sehr viel gegeben. Ich werde auf jeden Fall weitermachen und mich nach weiteren Lehrveranstaltungen zum Thema Meditation umsehen. Das Führen des Meditationstagebuchs hat mir geholfen, immer bei der Stange zu bleiben und meine Übungen auch wirklich regelmäßig auszuführen. Ich finde die Idee eines solchen Tagebuchs als Leistungsnachweis super. Jeder kann seine eigenen individuellen Gedanken in Worte fassen und ist dabei noch ein bisschen gezwungen, immer weiterzumachen, selbst wenn man mal eine Art Durchhänger hat.

Aus meiner erwartungsvollen, aber auch leicht kritischen Einstellung vom Anfang ist ein wahnsinniges Interesse für das Meditieren gewachsen. Ich weiß, dass 
das, was wir in der Lehrveranstaltung lernen durften, wirklich etwas ist, dass man sehr gut für sich selbst, aber auch in der Schule mit seinen Schülern und Schülerinnen einsetzen kann.

\section{A. U.}

\section{Was sich für mich durch die Meditation verändert hat}

$\mathrm{Zu}$ Beginn waren die einzelnen Meditationsübungen wie einzelne Elemente, die ich zu einer bestimmten Zeit übte.

Mittlerweile hat sich das verändert. Meditation (und die einzelnen Übungen) ist für mich jetzt etwas, das ich immer „dabeihabe“, etwas das mir (wenn es nötig ist, auch sehr schnell) im Alltag hilft, wie z.B. das bewusste Atmen, die Gehmeditation, Mantras etc.

Wenn ich mich komisch fühle oder ich einfach gerade ein paar Minuten Zeit habe, setze ich mich kurz hin und schließe die Augen und versuche, bewusster und achtsamer zu sein. Oft hat mir schon ein kurzer Augenblick geholfen, mich besser zu fühlen.

Alles in allem ist mir aufgefallen, dass ich durch die Meditation im Alltag ruhiger und gelassener geworden bin. Gerade in Situationen, die mich früher wahnsinnig gestresst und aus der Bahn geworfen haben, habe ich immer öfter das Gefühl, aus diesem „Hamsterrad“ aussteigen zu können und eine Art „Metasicht“ zu bekommen.

Dabei helfen mir vor allem die längeren Meditationen, die ich meist abends (vor dem Schlafengehen) mache. Gerade hierdurch versuche ich mich, meine Gefühle und Verhaltensweisen zu verstehen, indem ich die Ursache dessen suche.

Gerade in den letzten Tagen habe ich das Gefühl, dass durch die Meditation eine Türe aufgegangen ist, die mich dahingehend wieder ein Stück weitergebracht hat.

Vor allem durch das Vertrauen und den besseren Kontakt zu mir selbst, welchen ich durch die Meditation immer mehr aufbauen kann, habe ich das Gefühl immer tiefer in meine Themen einsteigen zu können und klarer zu werden. Das ist ein schönes Gefühl, denn so habe ich einen Weg gefunden, (besser) mit schweren Situationen umgehen zu können und mich weniger hilflos und allein zu fühlen. Gerade der Bodyscan ist für mich eine total schöne und wirkungsvolle Meditationsart.

Auf der anderen Seite habe ich das Gefühl, dass die Meditation dich auch auf Dinge aufmerksam machen kann. Zum Beispiel fällt mir das achtsame Essen unwahrscheinlich schwer. Des Öfteren habe ich versucht, diese Übung zu machen, aber ich bin immer wieder an meine Grenzen gestoßen.
Anfangs habe ich mich sehr geärgert und ich habe diese Übung schon fast verflucht, bis ich mich näher damit befasst habe und versucht habe, „hinter die Kulissen“ zu schauen. Über das Bewusstwerden meines sonst nicht so guten Essverhaltens, bin ich auf die Suche nach den Ursachen dafür gegangen und habe Erstaunliches festgestellt. Hierdurch hat sich ein großes Feld ergeben, es haben sich Bereiche aufgetan, mit denen ich mich nun beschäftigen kann und die mich ein großes Stück weiterbringen können. Ich bin zwar bei Weitem noch nicht fertig mit dieser Aufgabe. Aber ich arbeite dran und bin auf dem Weg!

Alles in allem kann ich sagen, dass ich unfassbar froh bin, die Meditation kennengelernt zu haben. Es ist wie ein Geschenk, und die Veränderungen, die ich erlebt habe, sind der Wahnsinn.

Meditation ist mittlerweile ein fester Bestandteil in meinem Leben geworden, den ich nicht mehr missen möchte!

B. K.

\section{Vorbei ... und weiter gehts}

Morgen werde ich dieses Tagebuch abgeben. Kaum zu glauben, dass das Semester schon wieder vorbei ist.

Vielen Dank für diesen tollen Kurs; es war sehr schön, und ich freue mich schon auf die weiterführenden Kurse mit Meditation.

Ich weiß jetzt schon, dass ich in Zukunft in dem gleichen Bereich wie Sie arbeiten will. Sie haben mir gezeigt, dass die Arbeitswelt offener wird und man in dem Feld Stressbewältigung, Meditation etc. Fuß fassen kann.

Das ist großartig für mich, ich habe jetzt ein Ziel im Berufsleben. Vielen Dank dafür!

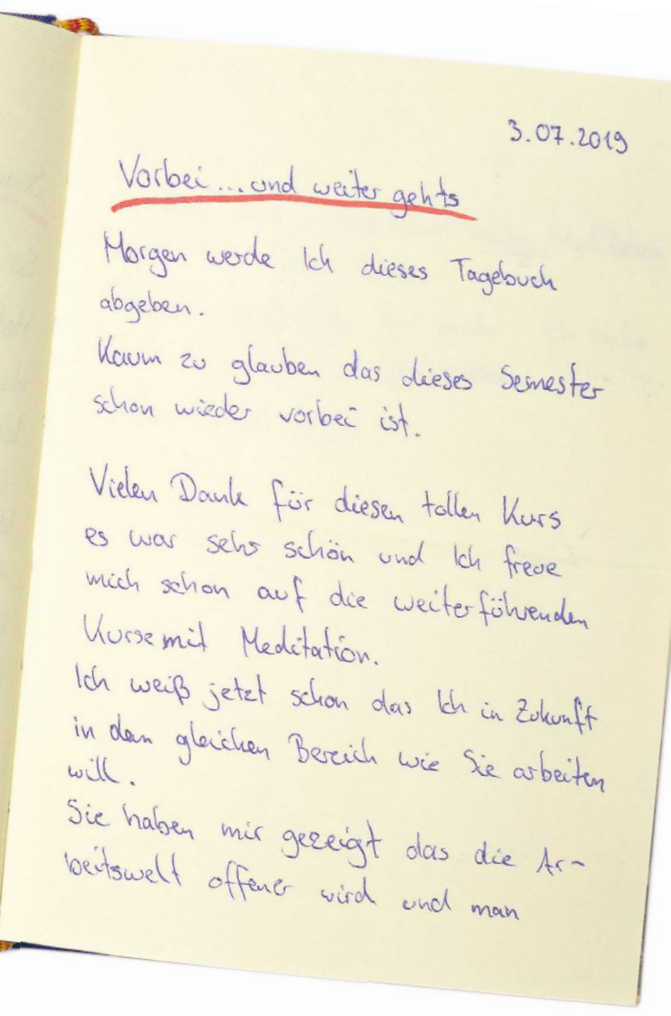




\section{W.}

In der Zeit zu Beginn des Kurses bis jetzt, würde ich sagen, hat sich viel verändert. Alleine die Übung Liebende Güte, die ich täglich automatisch in alle möglichen Situationen in meinen Alltag einbaue, öffnet mirimmer wieder neue Blickwinkel und lässt mich viel entspannter und friedlicher leben.

Ich habe mich jede Woche wieder auf die Meditationskurse gefreut, auf neue Übungen, neue Geschichten, die zum Weiterdenken anregen.

Ich bin sehr dankbar, dass viele Studierende die Möglichkeit haben, Meditation im Rahmen einer Vorlesung kennenzulernen!

Ich kann nur von mir sagen, dass die Seminare sehr bereichernd für mich waren/sind. Mittlerweile sehe ich Meditation ganz anders als zu Beginn, wo ich die Zeit oft im Kopf hatte oder Meditieren als etwas gesehen habe, wo ich etwas erreichen muss, wo es gut oder schlecht, richtig oder falsch gibt. Dabei spielt das alles keine Rolle!

Während den Meditationen kann ich Zeit mit mir verbringen, aus der Gesellschaft sozusagen aussteigen und zu mir/meinem Inneren finden.

Für mich hat sich schon alleine die Sicht auf die Welt verändert. Ich weiß nicht, ob das aufs Meditieren zurückzuführen ist, aber zuvor habe ich mir so viele Gedanken über Leid, Kriege und Ungerechtigkeiten gemacht und so viel gezweifelt und konnte überhaupt nicht verstehen, wenn es doch einen Gott gibt, warum es so viele unschuldige Menschen gibt, die hungern, sterben etc. Ich habe keine Antwort darauf, aber die Zweifel, Wut, Unverständnis sind einfach nicht mehr da. Ich kann das nur schwer erklären, aber ich vertraue dem Leben auf einmal. Ich kann alles viel entspannter sehen, mache mir keine Angst vor der Zukunft, sondern tue das Beste für mich und meine Mitmenschen.

In vielerlei Hinsicht gibt mir Meditation Kraft, Glaube und starke Entspannung und Freude!

Ich danke Ihnen für Ihre Zeit und alles, was Sie an die Welt weitergeben!

\section{E. M}

Vor dem Seminar habe ich viel über Meditation und dessen Wirkung gehört. Jedoch konnte ich mir nicht viel darunter vorstellen, vor allem nicht, wie man es im Kontext Schule umsetzen kann. Anfangs war ich mir auch nicht ganz sicher, ob ich das richtig mache. Als mir immer wieder Gedanken in das Bewusstsein kamen, dachte ich wirklich, ich mache es falsch. Doch als ich weitermachte, bemerkte ich, dass etwas anders ist.

\section{Reflexion}

In der zeit - zu Beginn des Kurses bis Alleine die Liebende Gän, hat sich viel verändert täglish automatisch Gute libung, die ish Situationen in meinen alle moggichen mir immer wieder neu Alitag einbaue, offnet mish viel entrog neue Bickwinkel und asst michannter und friedlicher leben.

Ish habe mich jede woche wieder auf die Meditationskurse gefrent, auf neue abuno neure Geschichten, die zum weiterdbungen, antegen.

Ish bin sehr dantbar do die Moglichkeit haben, Meditatiele Studierende siner vorlesury kennenzulemen!

$$
\begin{aligned}
& \text { ch kann } n \\
& \text { Selr beexchernd fon mir sagen, dass die Seminare } \\
& \text { weile sehe ich Meditation waren/sind. Mittler- } \\
& \text { begin, wo ich die Zeit oft janz anders als zu } \\
& \text { Meditieren al die Zeit oft in Kopf hatte oder } \\
& \text { erreichen muss, wo es gh habe, wo ich etwas } \\
& \text { richtig oder falischo es gut oder schlecht } \\
& \text { gibt. Dabei spielt das alles }
\end{aligned}
$$

Es fiel mir konkret auf, als ich beim Arzt zur Behandlung war. Der Arzt wollte mir eine Spritze verabreichen. Ich hab jedoch eine panische Phobie vor Spritzen und Nadeln, was bei mir häufig Tränen auslöst. Mir kam, trotz leichter Angst, der Gedanke, Maranatha anzuwenden, und so versuchte ich mich auf meine Atmung und auf Maranatha zu konzentrieren. Ich merkte zwar, dass mein Körper unruhig war und dass mein Herz schneller schlägt. Jedoch war die Reaktion meines Körpers sehr viel entspannter, als es sonst der Fall war. Obwohl ich im Nachhinein betrachtet, diese Behandlung als einer der schmerzhaftesten bewerten würde. Ich war selbst erstaunt über meine Standfestigkeit.

Des Weitern habe ich festgestellt, dass. meine Reaktion auf das Verhalten anderer rücksichtsvoller und hinterfragender geworden ist. Bei einem mir negativ auffallenden Verhalten ärgere ich mich nicht gleich bzw. versuche mich nicht aufzuregen. Ich denke darüber nach, warum er wohl grade so handelt und welche Ursachen es dafür geben könnte. Es hilft mir meine aufbrausenden Emotionen zu regulieren.

Auch bemerkte ich, dass ich weniger Stressempfinden vor den Prüfungen hatte. Ich hatte weniger Schlafstörungen, Angst oder panische Gedanken. Man könnte sogar sagen, ich ging entspannt in die Prüfung rein. 
Die Meditation half mir während des Lernens, meine Konzentration zu sammeln. Tatsächlich habe ich während der Lernphase mehr meditiert als in der restlichen Zeit. Es war mein Hilfsmittel, um bei Lernblockaden wieder Aufnahmebereit zu sein. Selbst kurze Zeiträume und ein großes Lernpensum konnten mich nicht beunruhigen.

Oft bemerke ich, wenn meine Gedanken um Meditation kreisen, dass mein Meditationspunkt an der Stirn zu kribbeln beginnt. Dies kommt auch vor, wenn ich die Maranatha-Meditation anwende. Während der Meditation ist das Kribbeln an der Stirn mein Kontrollmechanismus, der mir zeigt, ob ich noch konzentriert meditiere oder bereits abschweife. Je intensiver ich den Punkt an der Stirn spüre, umso fixierter bin ich in der Meditation.

Ich werde versuchen, trotz fehlenden Lernstresses viel zu meditieren. Die Meditation hat mir sehr geholfen, meine Emotionen zu regulieren. Ich möchte jedoch mein affektives Verhalten noch besser steuern können und so bewusster in emotionalen Situationen handeln.

\section{F. S.}

Die Meditation hilft nach wie vor, innerlich ruhiger zu werden und mehr ins Gleichgewicht zu kommen. Ich habe einiges über mich selbst erfahren können. Ich benötige öfter Ruhe. Ich sollte mir öfter selbst Ruhe gönnen, körperlich und geistig. Ich habe festgestellt, dass die Umwelt und die Menschen mich viel zu sehr stressen und mich in ein Ungleichgewicht bringen. Alles empfinde ich als stressig. Wenige Menschen kommen zur Ruhe. Das sehe ich täglich, wenn ich z.B. S-Bahn fahre. Alle schauen ins Handy, keiner ist bei sich. Ich versuche oftmals, das auszublenden oder die Augen zu schließen, um meine innere Ruhe zu finden. Doch alles um mich herum ist gehetzt und gestresst. Es gibt immer neue Termine oder Zeiten, an die man sich halten „muss“. Oft mache ich mir selbst den Stress, durch diverse Erwartungen an mich oder durch Erwartungen von anderen an mich. Ich versuche, all dem gerecht zu werden, doch oftmals höre ich nicht auf mich selbst. [...]

Meine Gedanken mir gegenüber haben sich geändert. Es ist OK, Fehler zu machen und Fehler zu haben - das zeichnet einen Menschen aus. Nur Maschinen laufen einwandfrei. Ich weiß, dass die Natur mir selbst viel Kraft geben kann. Deshalb gehe ich nun öfter in die Natur oder zu Orten, die still und harmonisch sind. Damit ich selbst wieder Harmonie in mein Inneres bringe. Ich habe nun eine Richtung und einen Weg ge- funden. Ich weiß, was mir guttut und was nicht. Dank der Meditation finde ich mehr und mehr zu mir selbst. Ich will dies unbedingt weiterführen.

H. K.

\section{Reflexion des vergangenen Semesters}

Dieses Seminar war für mich von ganz besonderer Bedeutung. Es beinhaltete sehr interessante Inhalte und ich konnte viele Ideen für meinen zukünftigen Beruf als Lehrkraft sammeln. Besonders wertvoll war dieser Kurs für mich, da ich mich auch privat mit diesem Thema beschäftige, hier aber einmal andere Standpunkte und neue Methoden kennenlernen konnte und diese vor allem für mich sehr gut angeleitet wurden .

Besonders schön fand ich es zu beobachten, wie ich mich nach und nach immer besser in die Methoden, vor allem in das „Aufmerksame Atmen“ hineinfühlen konnte, wie sich mein Körper und Geist immer besser darauf einstellten und ich mit der Zeit ein anderes, besseres Körpergefühl entwickeln konnte.

Wenn ich mein Tagebuch nochmal zurückverfolge, kann ich feststellen, dass ich anfangs noch Schwierigkeiten hatte, mich zu konzentrieren und in den richtigen Zustand zu finden sowie diesen beizubehalten. Auch das Ziehenlassen der Gedanken bereitete mir zu Beginn etwas Schwierigkeiten. Aber im Laufe des Semesters konnte ich mich immer besser einfühlen, ich fand die richtige Sitzposition für mich heraus und konnte mich voll und ganz auf die Meditationen konzentrieren. Natürlich gab es während des Semesters auch Situationen, wo ich mich aufraffen musste, die Übungen durchzuführen. Wenn ich es dann aber trotzdem tat, fühlte ich mich danach sehr gut.

Besonders bemerkenswert fand ich, wie sich mein Körper immer besser und schneller auf die Meditation einstellen konnte und ich mich immer besser entspannte.

Mein Alltag fällt mir seitdem auch etwas leichter, ich kann die Tage viel entspannter und gelassener genießen, außerdem fiel mir auf, dass ich einige Probleme auch viel lockerer anging und diese dann auch besser lösen konnte.

Durch diese Erfahrung habe ich für mich beschlossen zu versuchen, Meditationen fest in meinen Alltag zu integrieren. Besonders schön fand ich das „Aufmerksame Atmen“, den „Bodyscan“ und die „Gehmeditation“. Eine ebenfalls sehr wertvolle Erfahrung für mich war, dass mir die Übungen am Morgen bessertaten als am Abend, so konnte ich die Tage viel entspannter beginnen und bewältigen. 
Durch Meditation habe ich gelernt, meinen Körper

Ich bin sehr glücklich darüber, dass ich erneut Ihre Vorlesung besuchen konnte. Ich habe ja bereits im Sommersemester 2018 die Meditations-Vorlesung besucht und wirklich regelmäßig meditiert.

Auch danach meditierte ich, jedoch auch mal Wochen nicht. In diesen Wochen fehlte mir etwas, ich wurde etwas unruhig und lebte das Leben nicht mit dieser völligen Bewusstheit. Es ist mir relativ leicht gefallen, wieder täglich oder alle 2 Tage zu meditieren. Am liebsten mag ich Atemübungen und den Bodyscan. Das Meditieren hilft mir gerade in einer schwierigen Lebensphase enorm viel. Ich bin froh, Meditation und Achtsamkeit als ein Teil meines Lebens kennen und schätzen gelernt zu haben. Ich hätte vor einigen Jahren niemals gedacht, dass das Meditieren die Lebensqualität so stark verbessern kann.

Auch andere Menschen, wie eine Freundin und vor allem meine Mutter, konnte ich dafür begeistern. Dies hat mich sehr glücklich gemacht. Es gibt eigentlich keinen Tag mehr, an dem ich mich nicht mit Achtsamkeit und Meditation beschäftige. Sei es, selbst zu meditieren, Bücher über Achtsamkeit zu lesen oder zum Einschlafen Meditations-CDs zu hören - es ist einfach Teil meines Lebens geworden. zu schätzen und mehr auf Signale meines Körpers zu hören. Ich habe mich selbst näher kennengelernt und kann wirklich sagen, dass ich mit mir deutlich mehr im Reinen bin als vor 1-2 Jahren. Außerdem nehme ich die kleinen Dinge im Alltag intensiver wahr und ich bin spontaner geworden. Es muss nicht alles nach Plan laufen. Ich lasse die Dinge mehr auf mich zukommen, gehe alles entspannter an.

Ich kann mich auch nur bei Ihnen bedanken, dass Sie Studierenden die Möglichkeit geben, Meditation für sich zu entdecken. Vielleicht hätte ich ohne dieses Angebot der Hochschule niemals den Weg zu Achtsamkeit und Meditation entdeckt. Jetzt ist es für mich fast unvorstellbar, ohne Achtsamkeit und Meditation ein glückliches Leben zu führen.

\section{M. W.}

Ich muss sagen, dass ich mich nach Ihrem Fach „Meditation und Stressbewältigung" viel besser fühle. Ich habe gelernt, mit Erlebnissen, Tätigkeiten und Gefühlszuständen achtsamer und bewusster umzugehen. Bedauerlicherweise leide ich an ADS und muss täglich Tabletten zur Konzentration einnehmen. Seitdem ich 
bei Ihnen im Meditationskurs war, habe ich meine Tablettendosierung stark reduziert und bin größtenteils ohne Arzneimittel.

Ich bin superfroh, dass ich die Möglichkeit hatte, in Ihren Kurs zu kommen und solch positive Erfahrungen zu machen.

\section{R.}

Ich habe mich sehr gefreut, im Rahmen meines Studiums einmal ein Seminar zu Meditation belegen zu dürfen. Rückblickend hat mir dieses Seminar sehr gut gefallen. Jeden Montag bin ich immer mit Freude in die Universität gefahren, weil ich wusste, dass ich wieder meditieren und etwas Neues und Interessantes erfahren und lernen konnte. Kurz vor dem Seminar habe ich zufälligerweise die Autobiografie von Paramahansa Yogananda gelesen, durch die ich neue Einblicke in unser Leben gewann. Auch sind mir ein paar Yogatechniken (z.B. Sonnengruß) bekannt. Achtsamkeitsübungen habe ich davor auch schon unbewusst gemacht, mich vertieft und bewusst einer Sache gewidmet (z.B. Kochen, Spazierengehen, Putzen, ein Instrument spielen etc.). Sehr interessant fand ich auch den Film Mönche im Labor.

Das Thema Meditation wird bei uns in den westlichen Ländern immer präsenter. Sogar die Wissenschaft befasst sich mehr und mehr mit diesem Thema und es werden immer mehr Befunde entdeckt, die die positiven Auswirkungen von Meditation aufzeigen.

Von den Meditationstechniken, die ich in diesem Seminar gelernt habe, haben mir besonders das Fokussieren auf das Ajnazentrum und die Gehmeditation gefallen. Diese Übungen habe ich oft in meinen Alltag integrieren können. So haben mich diese Übungen dieses halbe Jahr intensiv begleitet und ich werde diese auch weiter praktizieren. Die Meditation ist für mich wie ein Schlüssel zu mir selbst. Dadurch finde ich Ruhe im oft stressigen und kurzlebigen Alltag. Das Praktizieren hat mir schon in einigen Situationen (v.a. auch in der Prüfungsphase) weitergeholfen. Ich freue mich darauf, diese Techniken auch in der Schule einzusetzen und mit den Kindern zu praktizieren.

\section{K.}

Ich persönlich habe mich vor dem Seminar noch nie mit Meditation näher beschäftigt und hatte auch nicht das Gefühl, dass ich es jemals schaffen könnte, einfach nur ruhig dazusitzen und meinen Atem zu beobachten. Ebenso konnte ich mir nicht vorstellen, dass ich daraus einen Mehrwert für meinen Stand im Alltag ziehen könnte.
Ich habe innerlich meine Meditation ständig bewertet und mich geärgert, wenn es nicht so geklappt hat, wie ich es mir vorgestellt habe. Im Laufe der Zeit würde ich nicht behaupten, dass das Meditieren einfacher geworden ist, sondern meine Einstellung gegenüber dem Meditieren sich verändert hat. Ich habe gelernt, dass es nicht immer gleich läuft und auch kein gradliniger Prozess ist. Ich habe gelernt, die ganze Sache entspannter zu sehen, da es ja auch um Entspannung geht. Mit der Zeit habe ich gemerkt, wie Meditation mich verändert.

Die Idee, ein Meditationstagebuch zu führen, finde ich sehr gut, da ich so "gezwungen" war zu meditieren und mich danach auch damit beschäftigen musste, was eigentlich durch Meditation mit mir geschieht.

\section{N.}

Angefangen habe ich den Kurs ohne große Erwartungshaltung. Mittlerweile bin ich mir aber über die Wirksamkeit von Meditation als Instrument der Selbstfindung, Stressbewältigung und Konzentrationsförderung im Klaren. Ich werde auch in Zukunft weiterhin meditieren.

Vielen Dank für die super Einweisung in dieses interessante Themengebiet!

07. 2119

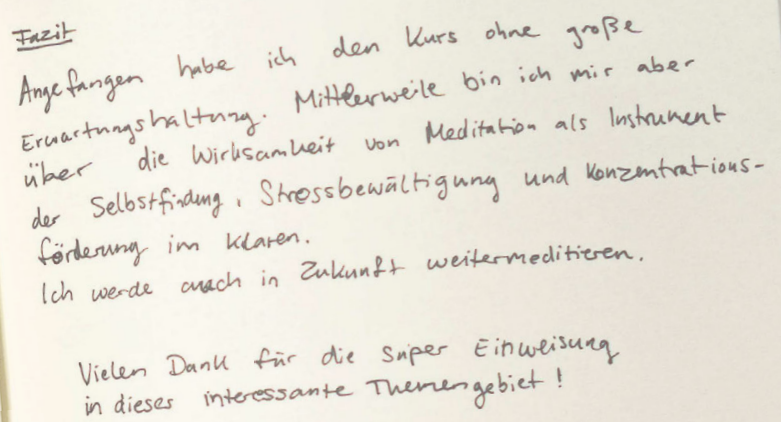




\section{T. F.}

Zum anderen finde ich den Gedanken, dass Meditation in den Grundschulunterricht eingebracht wird, total modern und sinnvoll - ich kann mir wirklich gut vorstellen, dass einzelne Sequenzen zwischen den Stunden vor allem Kindern, die Konzentrationsschwierigkeiten haben, sehr gut helfen können, sich wieder zu fokussieren oder einfach mal den Kopf freizukriegen.

Also, Daumen hoch für dieses Seminar und tausend Dank dafür!

T. K.

Als ich das Studium begonnen habe, dachte ich, ich werde mich dort genau so leicht tun wie ich mich während des Fachabiturs getan habe. Ohne großartigen Aufwand alles ziemlich gut abzuschließen. Jedoch habe ich bereits im ersten Semester (Wirtschaftsingenieurwesen) gemerkt, dass es diesmal nicht so laufen wird. Die Anzahl und Masse der Fächer war im Vergleich zur normalen Schule erschlagend.

Ich bin im ersten Semester in 1/6 Fächern durchgefallen, im zweiten in 4/8, im dritten Semester dann in $1 / 7$, was mir allerdings einen Drittversuch bescherte. Im vierten Semester bin ich dann das letzte Mal in 2/7 Prüfungen durchgefallen und seitdem habe ich alles mehr oder weniger gut auf Anhieb bestanden. Wie Sie sich sicherlich vorstellen können, haben die ganzen Zweitversuche und der eine Drittversuch (vor allem im Grundstudium) für einiges an zusätzlichem Druck gesorgt, den ich nicht abzubauen wusste. Dies führte zu kompletten Nervenzusammenbrüchen in der Prüfungsvorbereitung, die so schlimm waren, dass meine Mutter zwischenzeitlich wollte, dass ich das Studium abbreche. Nachdem ich die dritte Prüfungsphase meines Studiums dann einigermaßen überstanden hatte und diese vorbei war, war für mich klar, dass sich etwas ändern muss. Ich habe nach Möglichkeiten zum Stressabbau gesucht und sogar einige Seminare zum Umgang mit Stress besucht. Dort wurde des Öfteren davon gesprochen, dass Meditation für den einen oder anderen ein guter Zufluchtsort sei.

Da ich bereits in meinem ersten Semester von Bekannten, die schon weiter im Studium waren als ich, gehört hatte, wie begeistert sie von Ihrem Kurs waren, habe ich seit jeher bereits versucht, in Ihren Kurs zu gelangen. Leider jedoch ohne Erfolg.

Als mein letztes Semester bevorstand und ich immer noch ein AW-Fach offen hatte, dass ich mit aller Hoffnung für Ihren Kurs „aufgehoben“ hatte, dachte ich mir, ich schreibe Ihnen rechtzeitig vor Semesterstart eine E-Mail. Sie sind mir so freundlich entgegenge- kommen und haben es mir ermöglicht, trotz erneutem Fehlschlag im Losverfahren an Ihrem Kurs teilzunehmen. Dafür bin ich Ihnen wirklich sehr dankbar!

Ich habe so viel von Ihrem Kurs mitnehmen können, dass es vermutlich den Rahmen sprengen würde, hier nun alles davon genauer zu beschreiben. Daher möchte ich mich auf das für mich mit Abstand Wichtigste konzentrieren, die Achtsamkeit. Ich merke, wie mir die Regelmäßigkeit der Achtsamkeit zu einem viel intensiveren Lebensstil verholfen hat, und mit intensiv meine ich nicht aktionsgeladener, sondern vielmehr die viel bewusstere Wahrnehmung meiner Umgebung und meiner selbst. Ich habe bereits in der kurzen Zeit gelernt, viel mehr auf mein Inneres zu hören und mich viel mehr mit mir selbst zu beschäftigen.

Ich habe es dadurch sogar geschafft, alle Social-Media-Apps von meinem Smartphone zu löschen und die Zeit lieber für ruhige 5 Minuten mit mir selbst zu nutzen, anstatt andauernd auf das Handy zu starren und zu prüfen, was es denn so Neues gibt.

Ich weiß jetzt, dass ich nicht wissen muss, ob es irgendwo auf Social Media etwas Neues gibt, da ich das bereits Bekannte, nämlich meine nähere Umgebung und vor allem mich selbst, bisher noch nicht einmal richtig kennengelernt habe. Und das ist mein nächstes Ziel. Mich selbst und mein Inneres besser kennenzulernen und dort einen dauerhaften Zufluchtsort für die ein oder andere ruhige Minute zu gestalten.

Ich möchte Ihnen für Ihre aufgeschlossene, freundliche und sympathische Art danken und auch dafür, dass Sie mir die Möglichkeit geboten haben ein Teil Ihres Kurses zu sein.

\section{W. W.}

Vor jeder Stunde haben wir immer erst mal alltägliche Dinge besprochen oder über die letzte Stunde gesprochen. Dies hat mir sehr gut gefallen und gehört auch zu einem der Punkte, warum ich diese „Vorlesung“ besser als übliche finde, da auf einen persönlich eingegangen wird und es tatsächlich um uns Studenten als Menschen geht und nicht als „Arbeitsmaschinen“. Natürlich kommt es auch auf den Studiengang an, aber ich zum Beispiel studiere Betriebswirtschaftslehre und dabei wird nicht viel auf die Studenten eingegangen. Beziehungsweise geht es einfach nicht um menschliche Dinge, was natürlich klar ist, aber auch schade, da man trotzdem mit Menschen zu tun hat und sich selbst auch nicht vergessen darf. 


\section{Statements}

A. L. Das ist eine Sache, die ich aus dem Kurs mitgenommen habe. Ich habe die Kontrolle über meine Reaktionen und kann entscheiden, wie ich reagieren will.

\section{A. P. Z. Die Ruhe lässt dem Körper Zeit zur Erholung. Es ist wieder eine Phase, in der Meditation hilft - wie so oft.}

C. B. Wundervoll! Es ging sehr schnell, in die Meditation einzusteigen. Auch Gedanken konnte ich leicht "weiterschicken" und andere Geräusche gar nicht beachten.

Wie war es? - Leicht! Sogar die anderen Menschen konnte ich total vergessen, ausblenden. Ich war ganz bei mir.

C. M. Ich habe nach dem Aufstehen 10 Minuten eine Meditation im Sitzen durchgeführt. Es hat mir sehr gut geholfen, meine Gedanken zu ordnen und mit einem klaren Kopf in den Tag zu starten.

F. E. Sehr gut gefällt mir die Liebende Güte. Ich finde, so eine Übung sollten viel mehr Leute machen. F. S. Der Wind wurde stärker und schwächer -

J. Z. Bei einem Spaziergang am Wochenende bin gen meines Körpers und die verschiedenen Untergründe intensiv wahrgenommen und mich nur auf das Gehen konzentriert. Dabei konnte ich meine Gedanken sortieren und fühlte mich anschließend klar im Kopf.

Nach und nach zeigen die Meditationsübungen positive Auswirkungen auf mein Leben. 
Ich habe zwischen beiden Übungen meinen Atem sehr wahrgenommen. Er ist vorausgegangen und ich bin ihm gefolgt. Das hat mich sehr zufrieden gemacht.

Besonders Geräusche nicht zu beachten fällt mir schwer (Baulärm, Hund, Stimmen).

Ich fange sofort an beim Meditieren alles, was passiert, zu bewerten.

Ich konnte einen Zustand der Konzentration nur sehr kurz halten (mein Kopf plante lieber schon mal diesen Eintrag ...).

Beim Bodyscan bin ich teilweise abgedriftet in Richtung Schlaf ...

Die Natur ist eine tolle „Meditationslocation".

In meinem Alltag springen meine Gedanken oft von einem Thema zum nächsten. Ich fühle mich dann gestresst und setze mich häufig unter Druck. Die Ruhe in meinem Inneren fehlt. Nun freue ich mich, dass ich im Rahmen meines Studiums die Möglichkeit habe, am Seminar "Meditation" teilzunehmen. Über Meditation Verschiedenes zu erfahren und durch Meditationsübungen ein Stück weit meine Ängste loszulassen, um wieder mehr „bei mir zu sein".

Das Mantra ist für mich wie ein Rad, an dem ich mich entlanghangle, ohne abzusteigen. Innerlich kann ich mich fallen lassen. Vollkommen ungestört von Gedanken. Das Mantra versperrt meinen Gedanken und Ablenkungen den Weg.

Die heutige Meditation war wie ein Ankommen, Heimkommen, Zufriedenwerden. Ich habe ca. 20 Minuten eine Sitzmeditation gemacht, bei der ich mich nur auf meine Atmung konzentriert habe.

\section{B.}

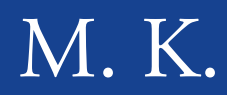

M. M.

N. H.

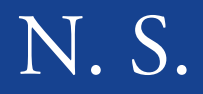




\section{SHANTI}
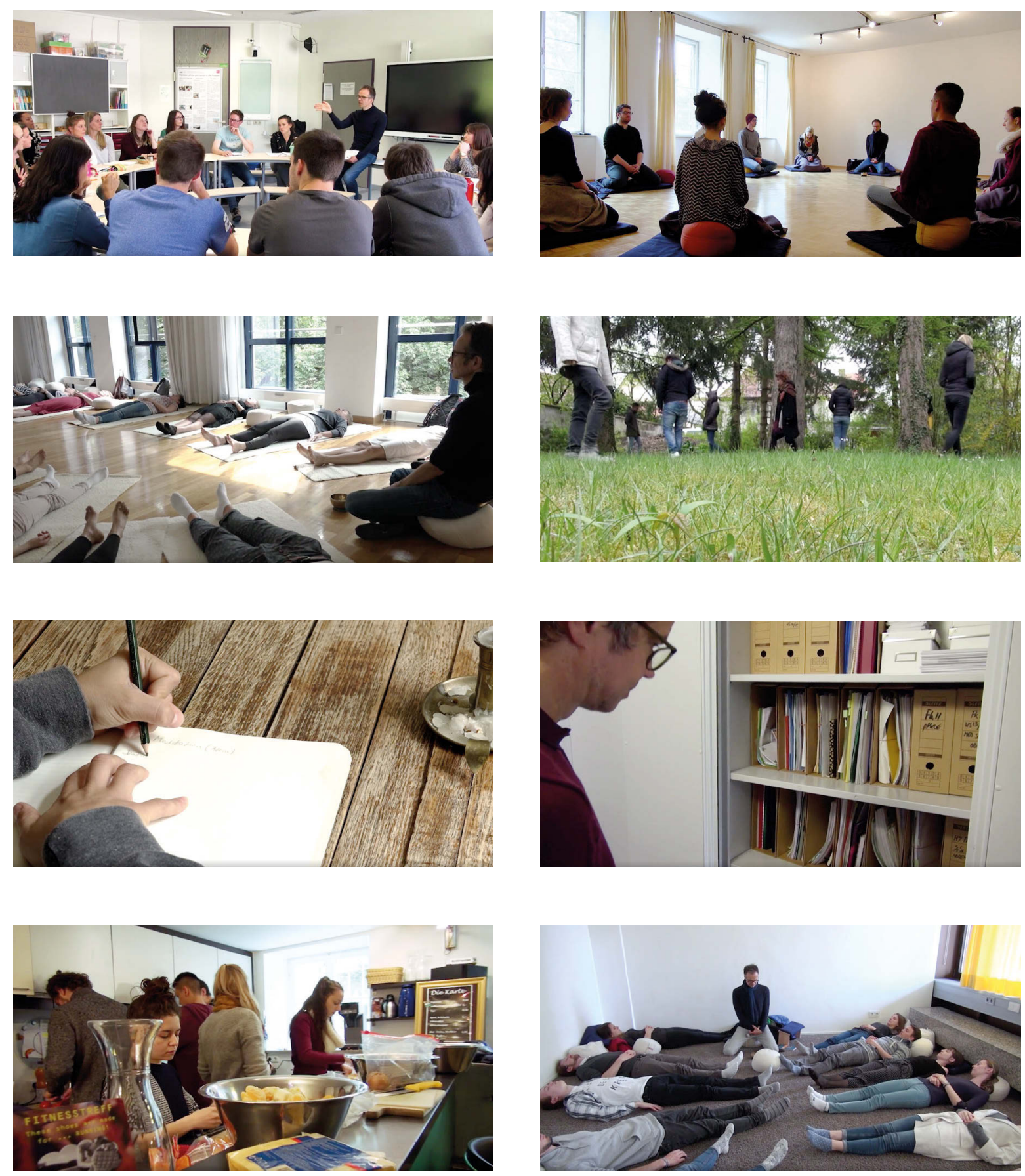

Der Film Shanti (Bachelorarbeit, siehe S. 70) ist abrufbar unter: www.hm.edu/meditationsmodell. 


\section{Feedback von Studierenden aus dem Film „Shanti“}

\section{Was passiert in den Lehrveranstaltungen?}

\section{A. S.}

\section{Soziale Arbeit}

Die Übung „Liebende Güte“ ist eine Mitgefühlsübung. Man denkt an einen Menschen, den man sehr gerne hat und stellt sich das auch wirklich vor; man schickt dann ganz bewusst Kraft zu diesem Menschen und stellt sich diese Verbindung vor, und alleine durch diese Vorstellung, durch diese Herzensöffnung, bewegt sich etwas. Eine weitere, schwierigere Übung ist, sich jemanden vorzustellen, zu dem man gar keine Beziehung hat, zum Beispiel irgendeinen Busfahrer, den man nur mal gesehen hat, und dann schickt man auch dort Energie hin, genau diese wärmende liebende Güte, die man ja auch für jemanden empfindet, den man sehr gerne mag.

Und als schwierigste Übung stellt man sich jemanden vor, den man einfach gar nicht leiden kann oder der einen nervt, also so eine Art Nerverli, und dort schickt man genauso diese Kraft hin, und es bewegt sich etwas; also es tut sich da unheimlich viel, auch wenn es am Anfang vielleicht eine Überwindung ist, weil da das Ego so ein bisschen im Weg steht. Aber im Endeffekt ist es auch sehr heilsam für einen selbst.

\section{J. H.}

\section{Soziale Arbeit}

Eine ganz spannende Sache war der Achtsamkeitstag, den wir gemacht haben. Einfach aus dem Grund, weil man sich dort die Zeit genommen hat, einmal länger als nur 1,5 Seminarstunden sich dem Meditationsthema hinzugeben. [...]

Bei der Gehmeditation, die wir gemacht haben, waren wir im Garten und sind eine Viertelstunde lang nur auf unsere Füße und unser Gehen fokussiert durch den Garten gelaufen. [...]

Das achtsame Kochen, das wir gemacht haben, bei dem wir ohne zu sprechen mit 15 verschiedenen Charakteren versucht haben, uns gegenseitig ein Essen zu kochen. Das war eine Erfahrung, die eindrucksvoll war, weil ich vorher nicht dachte, dass es so gut funktioniert, wie es dann am Ende des Tages auch geklappt hat. Das besondere war, dass es weniger Leute gab, die reinreden konnten, und man hat sich irgendwie arrangiert und geeinigt, ohne da große Absprachen zu treffen, und am Ende haben wir gemeinschaftlich gegessen, das war überraschend gut.

\section{Projekte - das Münchner Modell in der Praxis}

\section{J. H.}

\section{Soziale Arbeit}

Grundsätzlich kam mir der Gedanke, mit Meditation ein weiteres Werkzeug zu haben, um mit Jugendlichen in Kontakt zu kommen und mit Jugendlichen zu arbeiten. In meinem Fall ist es so, dass der Alltag von Jugendlichen im Leistungssport stark durch eine ganz klare Alltagsstruktur und auch viel Stress und viel Erwartungsdruck von verschiedensten Seiten verbunden ist und ich mir überlegt habe, aus den Erfahrungen, die ich selbst in dem Seminar gemacht habe, das mit Jugendlichen zur Anwendung zu bringen. Und hier den Jugendlichen im Rahmen einer Meditationsstunde - was dann auch effektiv vielleicht nur 10 Minuten waren - gemeinsam den Rahmen zu schaffen, um eben aus dieser Tenniswelt herauszukommen und aus dieser stetigen Drucksituation.

\section{H. B.}

\section{Soziale Arbeit}

Bei meinem Projekt ging es darum, eine Gruppe zu implementieren bestehend aus ehrenamtlichen Hospizbegleitern im stationären Hospiz, die regelmäßig Achtsamkeitsmeditation miteinander üben und dann auch natürlich im Tun, in der Begleitung auch anwenden können. Und ich hab ganz viel abgeguckt von Andreas de Bruin, von seinen Meditationsunterrichtseinheiten, wie er das bei uns aufgebaut hat, wie man an das Thema hinführt und Hintergrundwissen vermittelt. Im Endergebnis war es dann wirklich sehr positiv, und die Leute waren wirklich begeistert, ich würde sagen, es war ein Erfolg. Ich hab dieses Projekt 2014 durchgeführt als Bachelorarbeit, und seitdem bis heute - 2017 - gibt es diese Gruppe. 


\section{A. S.}

\section{Soziale Arbeit}

Und dann sind wir zusammen in eine Grundschule gegangen und haben ein Projekt zum Thema „Achtsamkeit in der Grundschule" entwickelt. Wir haben uns überlegt, welche Übungen infrage kommen. [...] Die Kinder haben das wirklich total gut angenommen, und es hat Spaß gemacht. Es war schön zu sehen, wie die Kinder mitwirken, wie sie sich auf die Übungen einlassen. Wir haben diese ganz klassischen Übungen gemacht: Rosinenübung, Zitronenübung, die Entspannungsreisen, die Bauchatmung ..., das ist so gut angekommen, dass die Schule jetzt auch vorhat, weiterhin mit der Hochschule zusammenzuarbeiten, und freut sich auf Studenten, die diesbezüglich weitere Ideen einbringen.

\section{Wie kann Meditation bewertet werden? Das Potenzial für unser Bildungssystem}

\section{B.}

\section{Evaluierte das Münchner Modell im Rahmen ihrer Bachelorarbeit}

Das hat so ein bisschen auch meinen Gedanken wiedergespiegelt, dass dieser Raum für Selbstreflexion und Nachinnengehen einfach gar nicht gegeben ist in unserem Bildungssystem. Ich glaube, dass dieses ganze Wissen, das wir tagtäglich bekommen und was uns ein Stückweit vielleicht auch überfordert manchmal, auch Zeit braucht und zusammenwachsen muss und mit einem inneren Reifeprozess einhergeht. Es geht einfach um dieses Sowohl-als-auch, dass man Dinge kombiniert und dass man Intuition und Persönlichkeit und Selbstentwicklung, Selbstreflexion fördert - gleichermaßen zusammen mit der Wissensvermittlung.

\section{Was weiß die Wissenschaft über Meditation?}

\section{P. K}

\section{Kommunikationsdesign}

Der Stress war schon wirklich enorm. Es waren teilweise sehr hohe Spannungszustände in mir, und die Meditation hat es mir ermöglicht, da einfach runterzufahren - irgendwie das als nicht so wichtig zu sehen.

Dann haben wir auch die Amygdala behandelt. Wenn Angstreaktionen ausgelöst werden, ist dieser Bereich [im Gehirn] aktiv. Wir sehen, dass bei Me- ditierern und Meditiererinnen dieser Bereich kleiner ist, also vermutet man auch, dass diese anders mit bestimmten Dingen umgehen, sodass es nicht zu Angstreaktionen kommt. Wir wissen vom Inselcortex, dass die Körperwahrnehmung und die Verbindung zu Emotionen sich verstärkt, dass man das schneller registriert, ob man aufgeregt ist und dass man auch die Impulse des Körpers für sich besser registrieren kann und nutzen kann. Die Emotionsregulation, also auch die Art, wie man mit Emotionen umgeht, und wenn vielleicht Wut hochkommt, dass man noch kurz nachdenkt oder schaut, gibt es noch andere Handlungsszenarien als nur aufzubrausen oder sauer zu sein, hat mir sehr geholfen, die Spannungszustände abzubauen.

L. O.

\section{Chemische Technik}

In Streitsituationen zum Beispiel bin ich gelassener, denk anders drüber nach, reagier auch anders und nicht so schnell, sondern denk meistens drüber nach, bevor ich etwas antworte, und ich versuche, mich auch in die Leute reinzuversetzen.

I. B.

\section{Evaluierte das Münchner Modell im Rahmen ihrer Bachelorarbeit}

Erwähnenswert ist, dass die Studierenden, die sich als achtsamer empfinden, auch gleichzeitig selbstwirksamer handeln oder sich als selbstwirksamer erleben, das heißt, dass deren subjektive Überzeugung, gewisse Situationen eigenständig oder aus eigener Kraft zu meistern, gestärkt ist. 


\section{Implementierungserfahrungen aus der Praxis}

Im Rahmen des Münchner Modells führen Absolventinnen und Absolventen nach dem Studium im Berufsfeld eigenständig Achtsamkeits- und Meditationsprojekte durch. Manche sind auch Lehrbeauftragte geworden. Im Folgenden berichten Absolventinnen und Absolventen von ihren Erfahrungen.

\section{A. U.}

\section{Absolventin der Hochschule München}

Wie das Münchner Modell „Achtsamkeit und Meditation im Hochschulkontext" mein Studium sowie meinen weiteren beruflichen Weg geprägt hat.

Zwar habe ich bereits vor meinem Bachelorstudium der Sozialen Arbeit an der Hochschule München Yoga praktiziert, jedoch war es mir zu diesem Zeitpunkt noch ein Rätsel, was es genau mit diesem „Meditieren" auf sich hat. Während meines Studiums hörte ich dann von den verschiedenen Lehrveranstaltungen zum Thema Meditation. Da ich unbedingt mehr zu diesem Thema erfahren wollte, ich jedoch nie einen Platz in den Lehrveranstaltungen zugelost bekommen habe, beschloss ich, Prof. de Bruin zu fragen, ob ich nicht auch freiwillig an seinen Vorlesungen teilnehmen dürfte.

Ich habe großes Glück gehabt, dass ich auf diesem Wege teilnehmen durfte. Ich habe in diesen Lehrveranstaltungen immer mehr verstanden, was Meditation wirklich bedeutet. Seitdem hat mich das Thema nicht mehr losgelassen und ich wollte so viel wie möglich darüber erfahren. Deshalb habe ich versucht, alle möglichen Leistungsnachweise mit dem Thema „Achtsamkeit und Meditation“ zu verbinden, um mich so auch auf wissenschaftlicher Ebene damit auseinandersetzten zu können. Auch in meinem Praxissemester hatte ich die Möglichkeit, zu erleben, welche positiven Auswirkungen die Haltung der Achtsamkeit in der Arbeit mit Kindern haben kann. Aus diesem Grund habe ich mich auch in meiner Bachelorarbeit mit diesem Thema beschäftigt, was mich in dem Entschluss bestärkt hat, dass „Achtsamkeit und Meditation“ auf jeden Fall ein fester Bestandteil meiner weiteren (pädagogischen) Arbeit sein soll. Als wir dann im Rahmen einer Lehrveranstaltung einen Businessplan zu einem eigenen Thema erstellen sollten, entstand der Grundstein für mein Kleinunternehmen „Meditation verleiht Flügel“, mit welchem ich mich Anfang 2017 selbstständig gemacht habe.

Ich bin sehr dankbar dafür, dass ich schon früh durch die Lehrveranstaltungen die Meditation kennenlernen durfte, die seitdem sowohl in meinem privaten, als auch meinem beruflichen Leben nicht mehr wegzudenkenden ist. Deshalb habe ich mich auch entschlossen, parallel zu meinem anschließenden Masterstudiengang (Beratung, Diagnostik und Intervention) an der Hochschule München die Ausbildung zur MBSR-Lehrerin nach Jon Kabat-Zinn (Stressreduktion durch Achtsamkeit) sowie die Weiterbildung zur MSC-Trainerin (Achtsames Selbstmitgefühl) zu machen, was mich intensiv und nachhaltig geprägt hat. Auch in meiner Masterarbeit beschäftige ich mich mit dem achtsamen Selbstmitgefühl und erforsche „Die Relevanz des achtsamen Selbstmitgefühls als Ressource für Studierende der Sozialen Arbeit". Ziel ist es, herauszufinden, welche Auswirkungen diese Praxisform auf Menschen insbesondere in helfenden Berufen etwa im Umgang mit Fürsorgemüdigkeit haben kann und welche Bereicherungen für die pädagogische Arbeit damit einhergehen können.

Im WiSe 2019/20 ist dann ein Traum von mir wahrgeworden und ich durfte eine der Lehrveranstaltungen von Prof. de Bruin übernehmen und das Thema "Achtsamkeit und Selbstmitgefühl im Studium“ an der Hochschule München anbieten. Es hat mir unwahrscheinlich viel Spaß gemacht, gemeinsam mit den Studierenden über ein ganzes Semester lang zu meditieren und über wichtige theoretische Themen und wissenschaftliche Befunde im Kontext von Achtsamkeit und (Selbst-)Mitgefühl zu sprechen und gemeinsam zu diskutieren, wie und wo diese Praxisformen im eigenen Leben, im Studium und insbesondere im späteren beruflichen Leben als Sozialpädagogin/Sozialpädagoge eingesetzt werden können. 
G. B.

\section{Absolvent der Technischen Universität München}

\section{Meditation in der Uni?}

Ein Kommilitone erzählte mir von einem Wahlfach, in dem man Meditieren lernt. Ich hielt für einen Moment inne. Meditieren lernen in der Uni - ein komischer Gedanke. Er meinte, dass das Seminar komplett überbucht sei, aber ich soll doch mal in die erste Stunde kommen und fragen, ob es noch einen Platz für mich gibt. Nun gut, dachte ich mir, ich habe ja nichts zu verlieren. Meditation im universitären Kontext. $\mathrm{Na}$ ja, wenn es nichts taugt, sind es wenigstens leicht verdiente ECTS-Punkte, schoss es mir durch den Kopf. Gesagt getan. Schon saß ich in einem Raum mit vielen anderen Mitstudenten, die auch an dem Seminar „Meditation in der Schule" für angehende Lehrer teilnehmen wollten. Und da kam auch schon der „Meditationsprofessor" Andreas de Bruin. Er wirkte wie ein ganz normaler Mensch. Keine Schlabberhosen, keine Räucherstäbchen und auch kein OM zur Begrüßung. Ohne viel Erklärung begann er einen fliegenden Elefanten auf eine bis dahin noch weiße Leinwand zu zeichnen und fragte uns, was das wohl bedeute? Umherschwirrende Gedanken, stellte sich später heraus. Kurz darauf bekam der Elefant einen Anker, damit dieser nicht ganz so uneingeschränkt alles niedertrampeln oder besser gesagt niederfliegen konnte.

Und das war der Anfang einer langen Reise für mich, einer Reise in die Welt der Achtsamkeit. Dieses Seminar sollte mein Leben verändern. Einmal die Woche trafen wir uns nun für gemeinsame Achtsamkeitsübungen. Meditationen im Gehen und Sitzen, Bodyscan im Liegen, bewusstes Essen. Die friedvolle Stille, die während dieser Übungen in mir reifte, hatte eine große Wirkung auf mich. Ich fühlte mich so erleichtert nach jeder Mediation und war unglaublich dankbar für diese Werkzeuge. Dann hatte ich auch noch das Glück, dass Andreas de Bruin mich zur jährlichen MBSR-Konferenz (Stressreduktion durch Achtsamkeit) in München als Assistenz mitgenommen hat. Nach dem Wochenende gab es für mich keine Zweifel mehr: Ich werde eine MBSR-Ausbildung machen!

Und nun, 7 Jahre und ca. 20 MBSR-Kurse später, sitze ich jetzt auf dem Mediationskissen und erzähle den nachkommenden Studenten etwas über umherschwirrende Gedanken, die sich mit ein wenig Disziplin und Leichtigkeit bändigen lassen und so den Stress reduzieren.
Ich bin sehr dankbar, dass die LMU ein solches innovatives Projekt unter der Leitung von Andreas de Bruin ermöglichte und damit eine Vorreiterposition in Europa einnimmt.

\section{K. Absolvent der Hochschule München}

Meine Mutter veränderte sich vor ihrem Tod sehr positiv durch das tägliche einstündige Meditieren, über fünf Jahre lang. Daher war ich ein paar Jahre später als junger Erwachsener sehr neugierig, als an unserer Hochschule Meditation tatsächlich als Fach angeboten wurde und man zudem auch noch mit Credits belohnt wurde und dadurch weiterkam.

Durch das Meditieren stellte ich fest, was es bedeutet, total präsent zu sein, lebendig zu sein und auch zu spüren, dass Gedanken lediglich Muster und Angebote sind, die wir ohne Achtsamkeit normalerweise einfach verinnerlichen und dementsprechend handeln und reagieren. Meditation wurde für mich der Weg, Freiheit von Gedanken zu erlangen. Nicht nur, Phasen der Gedankenstille zu erfahren, nein, auch eine völlige Distanzierung von gedachten Gedanken. So basierte letztlich auch das meiste Leid des Verlusts meiner Mutter auf schmerzlichen Gedankenkonstrukten. [...]

Den Wert von Meditation und Achtsamkeit erkennend wollte ich nach dem Studium begeistert ausziehen, um mehr Achtsamkeit in die Soziale Arbeit bringen, und entschied mich für einen Weg als Suchttherapeuten. Voller Erstaunen musste ich jedoch feststellen, dass Achtsamkeit hier schon lange implementiert ist und von Traumatherapie über Verhaltenstherapie sämtliche therapeutische Prozesse erobert hat. In den Gruppen, an denen ich teilnahm, war die Antwort auf die Frage, was therapieerprobte Klienten für das Wichtigste halten, etwa bei drohenden Rückfällen, oftmals: Achtsamkeit. Und so wird auch meine therapeutische Antwort Achtsamkeit bleiben, denn sie bringt uns uns selbst und dem Leben näher. Sie ist die Basis, dass Emotionen uns nicht überwältigen und wir auch toxische Sichtweisen über unsere Vergangenheit und Zukunft besser erkennen und loslassen können. Danke für das Münchner Modell und Danke an Andreas de Bruin! 
Drogenberatung im Gefängnis

In einer einführenden Drogenberatung hatte ich 20 Leute mit der Auflage vom Jugendgericht, an einer Suchtberatung teilzunehmen. Wir hatten den Superluxus, dass sie sich zwischen einer Achtsamkeitsstunde und der üblichen Drogenberatung entscheiden konnten, was alles andere als üblich ist. Wir haben einen buddhistischen Bodyscan und eine Atemmeditation (Anapana) gemacht, eine Filmsequenz über Meditation an einer Schule in einem Problemviertel in Baltimore angeschaut und generell über Achtsamkeit gesprochen. Wir hatten auch eine „Schneekugel“ dabei, die den Gedanken-Traffic symbolisierte, der in uns herrscht und unser Glücklichsein einschränkt denn die Seele oder die Seelenkräfte können sich erst zeigen, wenn Ruhe herrscht.

In der Einzelberatung führe ich Achtsamkeit unter anderem so ein, dass ein leerer Stuhl dafür reserviert ist. Im Gespräch versuche ich die Aufmerksamkeit immer wieder auf den Körper zu richten, der ja unser $\mathrm{Zu}-$ gang zum Hier und Jetzt ist. - Was tut sich? Der Stuhl ermöglicht es, dass man sich auf den Platz der Achtsamkeit setzt und so einen Schritt von sich selbst zurücktritt und mit ein bisschen Distanz auf sich schauen kann: Was nimmt die Achtsamkeit jetzt bei dir wahr?

\section{A. S.}

\section{Absolventin der LMU München}

Ich habe im Studium am Münchner Modell teilgenommen und führe es jetzt in Lehre und Forschung an der Ludwig-Maximilians-Universität München weiter.

Meditation und Achtsamkeit waren bereits Teil meines Lebens, bevor ich das Glück hatte, sie im Rahmen der Schulpädagogik "Meditation in der Schule“ im Lehramtsstudium zu vertiefen. Inspiriert davon, die von mir als überaus wertvoll empfundene Bereicherung des Lebens durch Meditation und Achtsamkeit weitergeben zu können, begann ich nach Staatsexamen und Magister die Promotion über „Achtsamkeit und Meditation im Schulbereich“.

\section{Meditation in der Schule}

Für meine Doktorarbeit entwickelte ich mit jugendlichen Schülern ein Meditations- und Achtsamkeitsprogramm, das innerhalb einer festen Schulstunde im Stundenplan verankert wurde. Die Jugendlichen erhalten dabei durch zahlreiche praktische Übungen einen Raum, in dem sie eine intensivere Wahrnehmung ihres Körpers sowie ihrer Gedanken- und Gefühlswelt entwi- ckeln können. Darüber hinaus beschäftigen sie sich mit Themen wie Konzentration und Selbstregulation und lernen Fakten aus Psychologie und Neurowissenschaften kennen. Am Programm sowie an der Begleitstudie, die mit meiner Dissertation veröffentlicht wird, nahmen über hundert Münchner Schülerinnen und Schüler zwischen 10 und 18 Jahren teil. „Besonders gut hat mir gefallen, dass wir die Augen schließen konnten und einfach mal bei uns bleiben durften", berichtete eine Fünftklässlerin. Ein Schüler der sechsten Klasse stellte fest: „Ich fühle mich jetzt viel ausgeglichener.“

\section{Meditation an der Universität}

Neben der Promotion habe ich außerdem die Gelegenheit, an der LMU München Studenten der Grundschulund Sonderschulpädagogik Meditation und Achtsamkeit näherzubringen. Im Rahmen regulärer Seminare erleben die angehenden Lehrkräfte die Effekte der Übungen, die sie für die Zukunft resilient machen können. Zudem haben sie Gelegenheit, selbst Übungen anzuleiten. Die Teilnehmer schätzen an diesem Seminarangebot „die abwechslungsreichen Übungen, angepasst an die Grundschule " sowie „den wissenschaftlichen Hintergrund und theoretische Einblicke“. Persönlich profitieren die jungen Menschen offenbar auch. Eine Studentin resümiert: „Das Seminar hat mir Wege gezeigt, wie ich mich gut fühlen und das Leben genießen kann."

Meiner Erfahrung nach ist es ein richtiger Schritt in die Zukunft, Meditation und Achtsamkeit einen Platz in Schule und Hochschule zu sichern. Nur wer sich selbst spürt, kann sein Potenzial entfalten und ein selbstbestimmtes, erfolgreiches und glückliches Leben führen.

\section{A. M. H. \\ Absolventin der Hochschule München}

Ich bin in Landshut, Niederbayern, geboren, wo ich mit 21 Jahren meine Ausbildung zur staatlich anerkannten Erzieherin abgeschlossen habe. Als ich nach einem berufstätigen Jahr als Gruppenleitung einer städtischen Kinderkrippe zunehmend den Eindruck bekam, dass wichtige Elemente in der Erziehung und Bildung, die bereits bei den Kleinsten ausschlaggebend für die weitere Entwicklung sind, im Alltag oft zu kurz kommen oder gar untergehen, entschied ich mich für eine persönliche und berufliche Weiterentwicklung durch den Studiengang Bildung und Erziehung im Kindesalter, mit dem Abschluss zur staatlich anerkannten Kindheitspädagogin an der Hochschule für angewandte Wissenschaften in München. 
Im Studium besuchte ich die Lehrveranstaltung „Methoden der Stressbewältigung “ bei Prof. Dr. Andreas de Bruin, welche im Rahmen des Münchner Modells stattfand. Als wir in den ersten Lehreinheiten verschiedene Methoden und Formen der Meditation und Achtsamkeit ausprobierten, nahm ich bei mir selbst bereits erste positive Veränderungen meiner Konzentrationsfähigkeit und meiner inneren Ausgeglichenheit wahr. Der Arbeitsauftrag, ein Meditationstagebuch über die Dauer eines Semesters zu führen, brachte mich dazu, gezielt Übungen in meinen Tagesablauf einbauen. Durch das regelmäßige Meditieren fühlte ich mich in meinen Gedanken klarer, war gelassener und fröhlicher. Die Wissenschaft bestätigt diese Wirkungen in einigen Studien, und ich fand gleichzeitig den Anknüpfpunkt an die Ausgangsfrage, mit der ich in das Studium hineingegangen war. Ich hatte nämlich nun herausgefunden, welche Elemente ich in der Bildung und Erziehung von Kindern vermisste, die gleichzeitig aber so bedeutsam für die Entwicklung von Kindern sind.

Während des gesamten Studiums baute ich meine Achtsamkeitsübungen immer weiter aus. Ich bemerkte dadurch viele positive Auswirkungen auf meine Familie und meine Freunde. Insgesamt wurde mein Alltag unbeschwerter und ich konnte mich leichter schwierigen Herausforderungen stellen. Das brachte mich auf die Verbindung von Achtsamkeit und Widerstandsfähigkeit gegenüber schwierigen Lebenssituationen. Schnell wuchs in mir der Wunsch, diese Zusammenhänge wissenschaftlich basiert begründen zu wollen. Ich entschied mich, meine Bachelorarbeit bei Prof. de Bruin zu schreiben, da er mich in den vergangenen Prozessen begleitete und mich durch seine Lehrveranstaltung auf diesen Weg brachte. Die Ausgangsfrage meiner Arbeit konzentrierte sich auf die Zusammenhänge von Achtsamkeit in Kindertageseinrichtungen und die Stärkung der personalen Resilienzfaktoren.

Da mich die Ergebnisse verschiedenster Studien dazu dermaßen beeindruckten, bildete ich mich gleichzeitig an der Akademie für freie Gesundheitsberufe "fitmedi“ zur Entspannungspädagogin für Kinder fort und wurde zur Multiplikatorin für Resilienzförderung in Kindertageseinrichtungen an der evangelischen Hochschule Freiburg, im Zusammenarbeit mit dem Zentrum für Kinder- und Jugendforschung, von Frau Rönnau-Böse und Herrn Fröhlich-Gildhoff angeleitet.

Im Anschluss daran baute ich mein Unternehmen „Heldenstärke“ auf, indem ich verschiedene Kurse zu Entspannungsverfahren für Kinder und Erwachsene anbot. Nach kurzer Zeit wurden meine Kurse von verschiedensten Krankenkassen als erfolgreiches Prä- ventionsangebot im Bereich Gesundheit und Stress anerkannt, und die Fachzeitschrift für Träger und Leitungen von Kindertageseinrichtungen Kita-Aktuell vom Wolters-Kluwer Verlag wurde auf mich aufmerksam. Als ich meine Bachelorarbeit erfolgreich abgeschlossen hatte, unterstützte mich Prof. de Bruin bei der Verfassung meines Artikels zum selbigen Thema „Achtsamkeit und Resilienz in der Kita" für Kita-Aktuell. Dieser wurde kürzlich veröffentlicht.

Mittlerweile bin ich 24 Jahre alt, gebe mehrere Fortbildungen und Seminare für Fachkräfte, Eltern und Themenbegeisterte, leite eine Kindertageseinrichtung und gebe regelmäßig Präventionskurse für Kinder und Erwachsene. Rückblickend auf die letzten drei Jahre war für mich der Besuch der Lehrveranstaltung „Methoden zur Stressbewältigung " ausschlaggebend für meine berufliche Entwicklung.

\section{A. S. \\ Absolventin der Hochschule München}

\section{Achtsamkeit in der Vorschul-Heilpädagogischen} Tagesstätte

Die acht Kinder meiner Gruppe sind zwischen 3 und 6 Jahre alt und werden von mir und einer weiteren Pädagogin betreut. Wir leben zwischen 8.30 Uhr und 14.30 Uhr wie in einer kleinen Familie zusammen. Eingebettet ist unsere Gruppe in ein Haus, das Werte wie Achtsamkeit und Menschlichkeit auch den Großen vorlebt.

Was meine Arbeit mit den Kindern so bereichert, ist die Achtsamkeit und der Entdeckergeist, den die Kinder von sich aus schon mitbringen.

Das Klangschalenritual bereitet unserer Gruppe einen sanften Einstieg in den Tag. Wir kommen im Morgenkreis zusammen, die Kinder reichen sich die Hände; ich halte die Klangschale in meiner Hand und lasse sie einmal erklingen. Die Kinder dürfen dem Klang bis zum Ende lauschen und sich dann, wenn sie ihn nicht mehr hören können, hinsetzen.

Wir üben uns auch in Achtsamkeit, wenn wir mit Sand spielen. Wir haben drei Zen-Gärten in der Gruppe, und so rechen die Kinder manchmal ganz versunken darin und gestalten ihren eigenen Garten. Sie dürfen ihn auch immer wieder neu gestalten.

Wir üben uns in Achtsamkeit, wenn wir Tiere beobachten und dabei ganz leise sind, um sie nicht zu verschrecken. Verbunden mit dem einen Moment, in dem wir innehalten. Dabei sehen wir vor unserer Balkontüre gerne den beiden Eichhörnchen zu, wenn 
sie sich Futter aus dem Futterkasten holen, den wir gemeinsam befüllt haben. Wir beobachten die Tiere dabei, wie sie in der Regenrinne trinken und auf den Bäumen turnen und klettern.

Die Kinder sind dabei ganz fokussiert und andächtig. Danach reflektieren wir, was wir gerade beobachtet haben.

Wir haben gemeinsam die Zitronenübung gemacht und gemerkt, dass es den meisten von uns sehr schwergefallen ist, sich eine Zitrone vorzustellen. So haben wir die Vorübung gemacht und den Kindern, eine echte Zitronenscheibe zum Reinbeißen angeboten. Dabei haben wir gemerkt, wie sauer so eine Zitrone ist. $\mathrm{Zu}$ meinem Erstaunen wurde daraufhin die Zitrone zu ihrer liebsten Frucht. Sie habe sich die ganze Woche Zitronen zum Nachtisch gewünscht.

Im Kinderyoga üben wir die ersten Figuren wie Katze, Kuh, Hund und Berg.

Letzten Frühling gab es einen Sinnesparcour mit verschiedenen Übungen. Die Füße sind ganz tolle Tastinstrumente, sie können auf verschiedenen Untergründen laufen, und manche Kinder trauen sich das auch schon mit geschlossenen Augen.

Nach dem gemeinsamen Mittagessen ist Ausruhzeit. In unserer Pause entspannen wir uns regelmäßig während einer zur Ruhe führenden Fantasiereise. Die Kinder mögen gerne mitbestimmen, welche Inhalte darin vorkommen. Es kommen viele Impulse von den Kindern, und wir möchten sie so gut es geht aufgreifen und darauf eingehen.

Gitarrespielen, Musik machen und Singen, all das sind kleine Achtsamkeitsübungen, wenn sie bewusst mit den Kindern gestaltet werden. Wir möchten hierzu einen Rahmen schaffen und ihnen stets vorleben, was für einen Umgang wir uns wünschen, denn wer selbst nicht ruhig und achtsam ist, kann es meiner Meinung nach auch nicht vermitteln.

Ich freue mich, wenn Achtsamkeit auch in anderen Einrichtungen einen festen Platz findet.

Besonders positiv aufgefallen ist mir, dass die Kinder freundlicher untereinander sind und auch herzlicher auf ihre Mitmenschen zugehen. Es kommt weniger zu Streit und das friedliche Spielen klappt besser. Die festen Rituale im Gruppenalltag helfen ihnen dabei.

\section{J. F. S.}

\section{Absolvent der LMU München}

Achtsamkeit bedeutet für mich, die tiefe Authentizität und Integrität, Autor des eigenen Lebens zu sein. Achtsamkeit zu kultivieren, heißt mich für mich: ein waches, bewusstes und gegenwärtiges Leben zu leben. Mit der Intention, ein solches Leben leben zu wollen, bin ich auf Meditation und Achtsamkeit gestoßen.

Mir fallen einige biografische Anknüpfungspunkte zu Achtsamkeit ein. Einen entscheidenden dieser Punkte mag es nicht geben, wohl aber hatten bei mir einige Erlebnisse und Erfahrungen einen bestimmenden Einfluss auf den Weg, den ich heute beschreite: Die Suche nach mentaler Balance und Selbstakzeptanz seit meiner Pubertät, eine schwere Sportverletzung mit Anfang 20 und der Umgang mit dieser körperlichen und geistigen Herausforderung, der Wunsch ein klares und aufgeklärtes Leben zu leben. Nach viel Ausprobieren praktiziere ich heute täglich vor allem Qigong und Achtsamkeitsmeditation.

Die Qualitäten WachSEIN, BewusstSEIN, GegenwärtigSEIN sind zwar an sich jedem Menschen inhärent. Jedoch in welchem Ausmaß und mit welcher Tiefe, ist wohl das entscheidende Moment. Es braucht schon eine gewisse Übung, eine Kultivierung der Haltung der Achtsamkeit, damit diese wirklich spür- und lebbar wird. Mir ist es ein Anliegen, dass Achtsamkeit insbesondere in den Bildungseinrichtungen unserer Gesellschaft kultiviert wird. So arbeite ich im Rahmen meiner Promotion an einem systematisierten Achtsamkeitskonzept für die Lehrerinnen- und Lehrerbildung. Darüber hinaus halte ich das Seminar „Achtsamkeit in der Pädagogik“ an der Universität Passau, gebe Workshops, etwa an der LMU München und am MZL München, veranstalte Trainings, etwa bei PROFiL der LMU München oder beim Elitenetzwerk Bayern, und halte Vorträge zum Thema Achtsamkeit vor allem im universitären und schulischen Bereich. 

APPENDIX 
Seite $197 \quad$ Nachwort

Seite 199 Quellenverzeichnis

Hauptzitate

Seite 203 Gesamtquellenverzeichnis

Seite $209 \quad$ Förderung

Seite $211 \quad$ Zum Autor 


\section{Nachwort}

Seit Beginn des Münchner Modells im März 2010 hat es sich stetig weiterentwickelt und ist zum festen Bestandteil des Hochschullehrplans geworden. Die daran teilnehmenden Studierenden schätzen die Achtsamkeits- und Meditationsübungen in vielerlei Hinsicht: als innovatives und sinnvolles Lehrangebot zur Vorbereitung auf ihr künftiges Berufsfeld, als Hilfe zur Bewältigung des Studienpensums, als Unterstützung des täglichen Stressabbaus und generell, um mehr zu sich selbst zu finden.

Mit dem weltweiten Ausbruch der neuen Atemwegserkrankung Covid-19 zum Jahresbeginn 2020 und den temporären Einschränkungen des öffentlichen Lebens zur Eindämmung der Pandemie musste sich auch das Münchner Modell ganz neuen Herausforderungen stellen. Präsensunterricht wurde mit einem Mal durch OnlineUnterricht ersetzt.

Die Erfahrungen der letzten Monate belegen, dass sich auch diese noch nie dagewesene Vorgehensweise für das Münchner Modell bewährt hat und die Online-Lehrveranstaltungen und die dazugehörigen praktischen Übungen den Studierenden in den Zeiten der Isolation innere Verbundenheit und Zusammengehörigkeit vermittelten.

Es wird sich zeigen, wie der Hochschulbetrieb in den kommenden Semestern weitergeht. Das Münchner Modell wird seinen Platz beibehalten, erbringt es doch den Beweis, dass die seit Urzeiten bekannten Unterweisungen in Achtsamkeit und Meditation, angepasst an unsere moderne Welt von heute, den Studierenden eine wertvolle Unterstützung für das Studium und das Leben im Allgemeinen bieten. 
198 Appendix
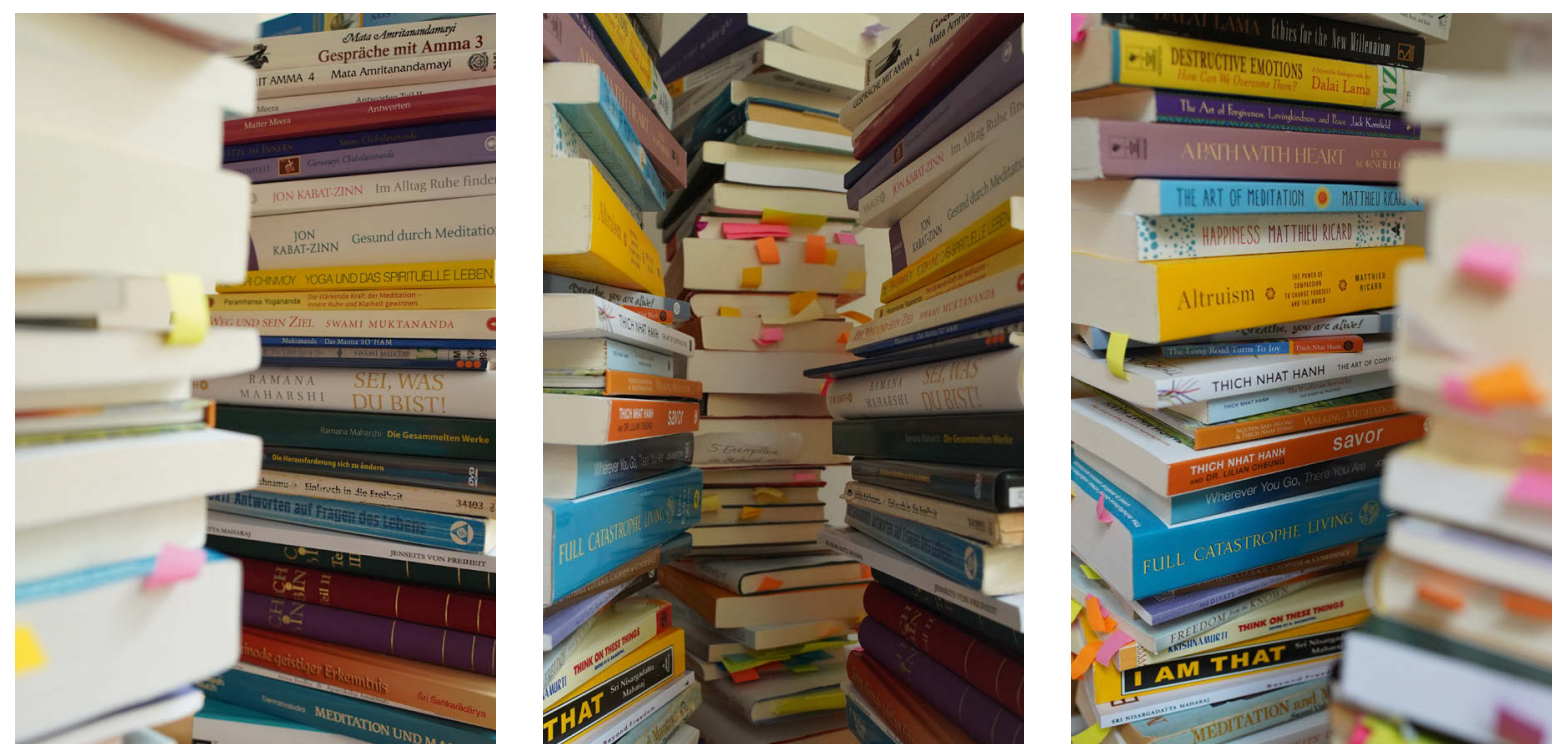


\section{Quellenverzeichnis Hauptzitate}

\section{Paradigmenwechsel}

„Dein spirituelles Selbst ruft jeden Tag nach dir. Du musst nur erkennen, dass du nicht dein Körper bist, sondern das unendliche Selbst, das in ihm wohnt."

Paramahansa Yogananda (1893-1952)

Yogananda, Paramahansa (2018). Die stärkende Kraft der Meditation - innere Ruhe und Klarheit gewinnen. Petersberg: Verlag Via Nova, S. 60.

„Um still zu sein, musst du den Geist völlig im Selbst versenken."

Ramana Maharshi (1879-1950)

Maharshi, Ramana (2011a). „Wer bin ich?" Der Übungsweg der Selbstergründung. Norderstedt: BoD, S. 19.

\section{Selbst, Seele und Geist}

„Das wesentliche Merkmal des Selbst-Bewusstseins ist daher die Kontinuität, die Dauerhaftigkeit. Die des bewussten Ich ist jedoch nur eine blasse Reflexion der ewigen, unsterblichen Essenz des spirituellen Ich, des Selbst."

\section{Roberto Assagioli (1888-1974)}

Assagioli, Roberto (2008). Psychosynthese und transpersonale Entwicklung. Rümlang / Zürich: Nawo Verlag GmbH, S. 32.

„Die Seele agiert als Beschützer während unserer Leben und ist immer bei uns. Sie hat keine eigenen Wünsche. Sie ist nicht nur Zeuge oder Lenker, sondern unterstützt auch unsere Entwicklung. Frei vom Einfluss unserer Handlungen, bleibt sie während unserer Leben ständig bei uns, bis wir mit dem Göttlichen eins werden."

\section{Mutter Meera}

Mutter Meera (1994). Antworten. Teil I. DornburgThalheim: Verlag \& Buchvertrieb Adilakshmi, S. 136.

\section{Herz, Liebe und Selbst}

„Aber vom Augenblick an, in dem Sie in Ihrem Herzen dieses Außergewöhnliche haben, das Liebe genannt wird, und Sie ihre Tiefe spüren, ihr Entzücken, ihre Ekstase, werden Sie entdecken, dass für Sie die Welt verwandelt ist."
Jiddu Krishnamurti (1895-1986)

Krishnamurti, Jiddu (1992). Antworten auf Fragen des Lebens. Verlag Hermann Bauer KG: Freiburg im Breisgau, S. 189.

"Menschen, welche die Schriften wirklich kennen und erfahren, gewinnen ihr Verständnis nicht nur aus Büchern, sondern aus ihrem Herzen. Das Herz ist die größte Bibliothek."

\section{Swami Chidvilasananda}

Chidvilasananda, Swami (Gurumayi) (2004). Mut und Zufriedenheit. Telgte: Siddha Yoga Verlag GmbH, S. 65.

\section{Intellekt und Intuition}

"Die Intuition ist ein göttliches Geschenk, der denkende Verstand ein treuer Diener. Es ist paradox, dass wir heutzutage angefangen haben, den Diener zu verehren und die göttliche Gabe zu entweihen. "

Albert Einstein (1879-1955)

Sinngemäß zitiert, Quelle nicht mehr auffindbar (A.d.B).

\section{Forschung}

"Wenn ihr den Geist verstehen wollt, dann meditiert."

Buddhistischer Mönch

Morel, Delphine (2016). Mönche im Labor, 17:37 Min.

"Das ist eine Erfahrung, die alle machen, die anfangen zu meditieren. [...] dass diese Praktiken viel mehr bewirken, dass sie das Leben auf unerklärliche Art bereichern und einem in Dingen helfen, bei denen man vorher gar nicht wusste, dass man Hilfe braucht."

\section{Sara Lazar}

Lazar, Sara (2015). „Die neurowissenschaftliche Erforschung der Meditation“, in: Zimmermann, Michael / Spitz, Christof / Schmidt, Stefan (Hrsg.), Achtsamkeit. Ein buddhistisches Konzept erobert die Wissenschaft. Bern: Verlag Hans Huber, S. 80. 
"Die Erde wäre ein anderer Ort, würden wir für die Pflege unseres Geistes auch nur die gleiche kurze Zeit wie fürs Zähneputzen aufwenden."

\section{Richard Davidson}

Davidson, Richard (2019). „Interview“, in: Moment by Moment, Ausgabe 01. Freiburg: Hammer Solutions Media, S. 49.

\section{Sitzmeditationen}

„Wenn der Mensch ein inwendiges Werk werden will, so muss er all seine Kräfte in sich ziehen, wie in einen Winkel seiner Seele, und muss sich verbergen vor allen Bildern und Formen, und da kann er dann wirken. Da muss er in ein Vergessen und in ein Nichtwissen kommen."

\section{Meister Eckhart (1260-1327)}

Meister Eckhart (2019). Mystische Schriften. Frankfurt am Main / Leipzig: Insel Verlag, S. 29f.

"Die Höchste Meditation ist der Zustand der völligen inneren Stille. In diesem Zustand taucht nicht ein einziger Gedanke im Geist auf. Allerdings können die meisten Menschen diese innere Stille nicht sofort erlangen. Aus diesem Grunde ist es für den Meditierenden von größter Wichtigkeit zu wissen, wie er mit dem Geist umgehen soll."

\section{Swami Muktananda (1908-1982)}

Muktananda, Swami (2001). Meditiere. Das Glück liegt in Dir. Telgte: Siddha Yoga Verlag GmbH, S. 19.

\section{Achtsamer Alltag}

"Um wirklich in Kontakt mit unserem Hier und Jetzt zu sein - wo auch immer das sein mag-, müssen wir so lange in unserem Wahrnehmen innehalten, bis der gegenwärtige Augenblick in uns einsinken kann - so lange, bis wir den gegenwärtigen Augenblick wirklich spüren, bis wir ihn in seinem ganzen Ausmaß sehen, bis wir seiner gewahr werden und ihn dadurch besser kennenlernen und verstehen. "

\section{Jon Kabat-Zinn}

Kabat-Zinn, Jon (2015). Im Alltag Ruhe finden. Meditationen für ein gelassenes Leben. München: Knaur Verlag, S. 9f.

\section{Achtsames Kochen und Essen}

„Der Schlüssel zu Gesundheit und innerem Frieden liegt darin, zu lernen, wie man achtsam isst und lebt."

\section{Thich Nhat Hanh / Dr. Lilian Cheung}

Thich Nhat Hanh / Cheung, Lilian (2016). achtsam essen-achtsam leben. Der buddhistische Weg zum gesunden Gewicht. München: O. W. Barth Verlag, S. 9.

\section{Achtsame Kommunikation}

„Am Anfang einer Kommunikation mit einem anderen Menschen ist es hilfreich, sich daran zu erinnern, dass es in jedem von uns einen Buddha gibt."

\section{Thich Nhat Hanh}

Thich Nhat Hanh (2019). achtsam sprechen - achtsam zuhören. Die Kunst der bewusten Kommunikation. München: Knaur Verlag, S. 39.

\section{Bodyscan}

„Wenn wir unsere Energie einmal darauf richten, unseren Körper wirklich zu spüren, und uns davor hüten, in das urteilende Denken über den Körper zu verfallen, können sich unser ganzes Körpererleben und das Erleben unserer selbst in radikaler Weise verwandeln."

\section{Jon Kabat-Zinn}

Kabat-Zinn, Jon (2019). Gesund durch Meditation. München: Knaur Verlag, S. 113.

\section{Liebende Güte}

„Wenn Liebe zu göttlicher Liebe wird, erfüllt auch Mitgefühl das Herz. Liebe ist die innere Empfindung, und Mitgefühl ist ihr Ausdruck."

\section{Mata Amritanandamayi}

Mata Amritanandamayi (1995). Gespräche mit Amma 2. Die Lehren der Heiligen Mutter. Interlaken: Ansata-Verlag, S. 91. 


\section{Gehmeditation}

„Zweck der Gehmeditation ist die Gehmeditation selbst. Entscheidend ist das Gehen, nicht das Ankommen, denn Gehmeditation ist kein Mittel, es ist das Ziel selbst.

Jeder Schritt ist Leben; jeder Schritt ist Frieden. Das ist der Grund, warum wir nicht zu eilen brauchen; darum verlangsamen wir unsere Schritte. Gehen Sie, aber gehen Sie nicht. Gehen Sie, aber lassen Sie sich durch nichts antreiben, was immer es auch sei. So wird, wenn wir gehen, wie von selbst ein Halblächeln auf unserem Gesicht sein." Thich Nhat Hanh

Thich Nhat Hanh (2007). Der Geruch von frisch geschnittenem Gras. Stuttgart: Theseus Verlag, S. 14.

\section{Sutras}

"Konzentrieren und sammeln wir unseren Geist, so erwerben wir auf natürliche Weise die Fähigkeit zu sehen, Dinge zu erkennen."

\section{Thich Nhat Hanh}

Thich Nhat Hanh (2000). Das Wunder des bewussten Atmens. Stuttgart: Theseus Verlag, S. 117.

\section{Geräuschmeditation}

"Das Hören bringt mehr herein, aber das Sehen zeigt mehr hinaus. Und darum werden wir im ewigen Leben viel seliger sein in der Kraft des Hörens als in der Kraft des Sehens."

\section{Meister Eckhart (1260-1327)}

Meister Eckhart (2019). Mystische Schriften. Frankfurt am Main / Leipzig: Insel Verlag, S. 31. 


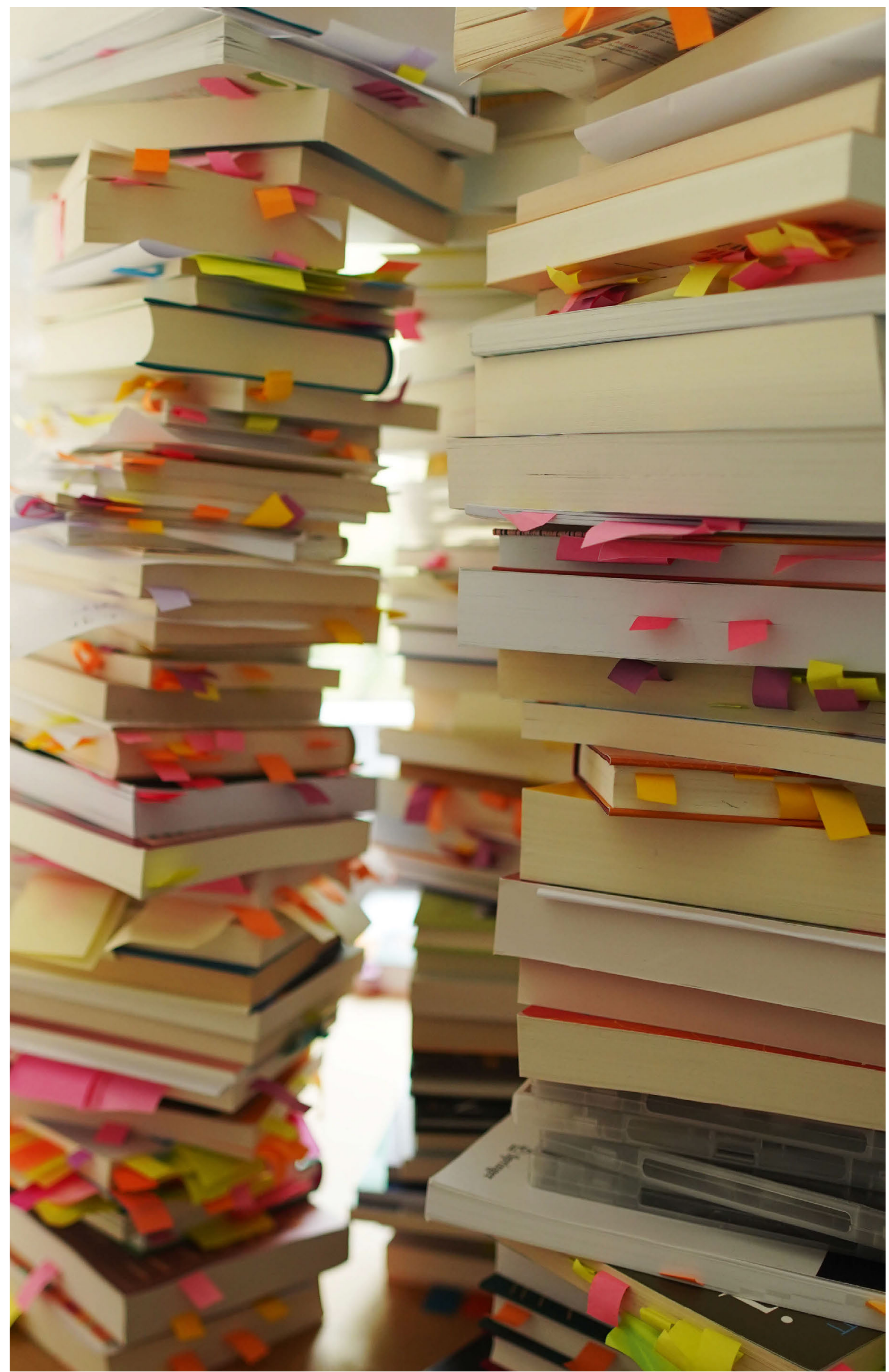




\section{Gesamtquellenverzeichnis}

\section{Fachliteratur}

Abell, Arthur M. (1981). Gespräche mit berühmten Komponisten. Über die Entstehung ihrer unsterblichen Meisterwerke, Inspiration und Genius. Kleinjörl bei Flensburg: G. E. Schroeder-Verlag.

Altner, Nils (2009). Achtsam mit Kindern leben. Wie wir uns die Freude am Lernen erhalten. Ein Entdeckungsbuch. München: Kösel-Verlag.

Anderssen-Reuster, Ulrike (2015). „Achtsamkeit in Psychosomatik und Psychotherapie“, in: Zimmermann, Michael / Spitz, Christof / Schmidt, Stefan (Hrsg.), Achtsamkeit. Ein buddhistisches Konzept erobert die Wissenschaft. Bern: Verlag Hans Huber, S. 103-114.

Assagioli, Roberto (2008). Psychosynthese und transpersonale Entwicklung. Rümlang / Zürich: Nawo Verlag $\mathrm{GmbH}$.

Blackburn, Elizabeth / Epel, Elissa (2019). Die Entschlüsselung des Alterns. Der Telomer-Effekt. München: Wilhelm Goldmann Verlag.

Braunger, Isabel (2016): Evaluation des Münchner Modells: Meditation an der Hochschule - ein zukunftsweisendes Konzept? Coburg / München.

Chidvilasananda, Swami (Gurumayi) (2004). Mut und Zufriedenheit. Telgte: Siddha Yoga Verlag GmbH.

Chidvilasananda, Swami (Gurumayi) (2003). Schätze im Innern. Telgte: Siddha Yoga Verlag GmbH.

Dalai Lama XIV. (2015a). „Achtsam sein heißt, den Geist bewusst zu gebrauchen", in: Zimmermann, Michael / Spitz, Christof / Schmidt, Stefan (Hrsg.), Achtsamkeit. Ein buddhistisches Konzept erobert die Wissenschaft. Bern: Verlag Hans Huber, S. 211-216

Dalai Lama XIV. (2015b). „Anstelle eines Schlussworts: Gespräch mit S. H. dem Dalai Lama“, in: Zimmermann, Michael / Spitz, Christof / Schmidt, Stefan (Hrsg.). Achtsamkeit. Ein buddhistisches Konzept erobert die Wissenschaft. Bern: Verlag Hans Huber, S. 337-345.

Dalai Lama XIV. (2000). Das Buch der Menschlichkeit. Eine neue Ethik für unsere Zeit. Köln: Bastei Lübbe AG.

Davidson, Richard J. / Goleman, Daniel (2017). Altered Traits. Science Reveals How Meditation Changes Your Mind, Brain, and Body. New York: Avery.
Davidson, Richard J. / Begley, Sharon (2012). Warum wir füblen, wie wir fühlen. Wie die Gehirnstruktur unsere Emotionen bestimmt - und wie wir darauf Einfluss nehmen können. München: Arkana.

de Bruin, Andreas (2019a). „Spiritualität im säkularen Raum am Beispiel des Münchner Modells - Achtsamkeit und Meditation im Hochschulkontext", in: Rötting, Martin / Hackbarth-Johnson, Christian (Hrsg.), Spiritualität der Zukunft. Suchbewegungen in einer multireligiösen Welt. Sankt Ottilien: EOS Editions, S. 341-350.

de Bruin, Andreas (2004). Jugendliche - ein fremder Stamm? Jugendarbeitslosigkeit aus aktionsethnologischer Sicht. Zur kritischen Reflexion von Lehrkräften und Unterrichtskonzepten im deutschen Schul-und Ausbildungssystem. Münster: LIT Verlag.

de Vries, Gerard (1985). De ontwikkeling van de wetenschap. Een inleiding in de wetenschapsfilosopfie. Groningen: Wolters-Noordhoff.

Dievernich, Frank, E. P. / Döben-Henisch, Gerd-Dietrich / Frey, Reiner (2019). Bildung 5.0: Wissenschaft, Hochschulen und Meditation. Das Selbstprojekt.

Weinheim / Basel: Beltz Juventa.

Frey, Reiner (Hrsg.) (2020). Meditation und die Zukunft der Bildung: Spiritualität und Wissenschaft. Weinheim / Basel: Beltz Juventa.

Fuchs, Brigitte (2011). „Sitzen in Stille, was kann das schon bewegen? Meditieren mit kranken Menschen “, in: Büssing, Arndt / Kohls, Niko (Hrsg.), Spiritualität transdisziplinär. Wissenschaftliche Grundlagen im Zusammenhang mit Gesundheit und Krankheit. Berlin / Heidelberg: Springer-Verlag, S. 223-229.

Fürst, Maria (1985). Philosophie - Band 1: Psychologie. Wien: Verlag Carl Ueberreuter.

Giovetti, Paola (2007). Roberto Assagioli. Leben und Werk des Begründers der Psychosynthese. Rümlang / Zürich: Nawo Verlag GmbH.

Goleman, Daniel (2003). Dialog mit dem Dalai Lama. Wie wir destruktive Emotionen überwinden können. München / Wien: Carl Hanser Verlag. Goleman, Daniel (1997). Emotionale Intelligenz. München: dtv Verlagsgesellschaft mbH \& Co. KG. 
Gunaratana, Mahathera Henepola (2010). Von der Achtsamkeit zur Sammlung. Eine Einführung in die tieferen Stadien der Meditation. Heidelberg: Werner Kristkeitz Verlag.

Gunaratana, Mahathera Henepola (1996). Die Praxis der Achtsamkeit. Eine Einführung in die VipassanaMeditation. Heidelberg: Werner Kristkeitz Verlag. Haug, Achim (2018). Das kleine Buch von der Seele. Ein Reiseführer durch unsere Psyche und ihre Erkrankungen. München: Verlag C. H. Beck oHG.

Hazrat Inayat Khan (2019). Heilung aus der Seele. Die Mystik der Sufis. Polling: Verlag Heilbronn.

Hazrat Inayat Khan (2018). Die Suf-Botschaft von Hazrat Inayat Khan. Jubiläumsausgabe Band 1. Das Innere Leben. Polling: Verlag Heilbronn.

Hölzel, Britta (2015). „Mechanismen der Achtsamkeit. Psychologisch-neurowissenschaftliche Perspektiven“, in: Hölzel, Britta / Brähler, Christine (Hrsg.), Achtsamkeit mitten im Leben. Anwendungsgebiete und wissenschaftliche Perspektiven. München: O. W. Barth, S. 43-77.

Hölzel, Britta / Brähler, Christine (2015). „Achtsamkeit mitten im Leben“, in: Hölzel, Britta / Brähler, Christine (Hrsg.), Achtsamkeit mitten im Leben. Anwendungsgebiete und wissenschaftliche Perspektiven. München: O. W. Barth Verlag, S. 7-19.

Hofmann, Liane / Heise, Patrizia (2017). „Spirituelle Krisen: allgemeine Kennzeichen, Auslöser und Gefährdungsfaktoren“, in: Hofmann, Liane / Heise, Patrizia (Hrsg.), Spiritualität und spirituelle Krisen. Handbuch zu Theorie, Forschung und Praxis. Stuttgart: Schattauer GmbH, S. 141-155

Jäger, Willigis (2017). Über die Liebe. München: Penguin Verlag.

Jäger, Willigis (2015). Kontemplation - ein spiritueller

Weg. Freiburg im Breisgau: Verlag Herder.

Kabat-Zinn, Jon (2019). Gesund durch Meditation.

München: Knaur Verlag.

Kabat-Zinn, Jon (2015). Im Alltag Ruhe finden. Meditationen für ein gelassenes Leben. München: Knaur Verlag.

Kahler, Thomas / Nitsch, Thomas (1994). Neues großes Wörterbuch. Fremdwörter. Karlsruhe: Trautwein. Kaltwasser, Vera (2010). Persönlichkeit und Präsenz. Achtsamkeit im Lehrerberuf. Weinheim / Basel: Beltz Verlag.

Kapleau, Philip (2012). Die drei Pfeiler des Zen. Lehre - Übung - Erleuchtung. München: O. W. Barth Verlag.
Kluge, Maria (2019). The Toolbox is You. 4. überarbeitete Auflage. Osterloh: Verein für Achtsamkeit in Osterloh e.V.

Kohls, Niko (2017). „Spiritualität und außergewöhnliche Erfahrungen im Kontext der akademischen Psychologie“, in: Hofmann, Liane / Heise, Patrizia (Hrsg.), Spiritualität und spirituelle Krisen. Handbuch zu Theorie, Forschung und Praxis. Stuttgart: Schattauer GmbH, S. 3-14.

Kornfield, Jack (2017). Frag den Buddha und geh den Weg des Herzens. Was uns bei der spirituellen Suche unterstützt. München: Kösel-Verlag.

Kornfield, Jack (2010). Meditationen, die unser Herz öfnen. München: Arkana.

Krishnamurti, Jiddu (1993). Einbruch in die Freiheit. Berlin / Frankfurt am Main: Ullstein.

Krishnamurti, Jiddu (1992). Antworten auf Fragen des Lebens. Freiburg im Breisgau: Verlag Hermann Bauer KG.

Krystal, Phyllis (2017). Monkey Mind. Den Verstand bändigen. Dietzenbach: Sathya Sai Vereinigung e.V.

Kuhn, Thomas, S. (1967). Die Struktur wissenschaftlicher Revolutionen. Frankfurt: Suhrkamp.

Langer, Ellen J. (1989). Mindfulness. Boston: AddisonWesley Publishing Company, Inc.

Lazar, Sara (2015). „Die neurowissenschaftliche Erforschung der Meditation“, in: Zimmermann, Michael / Spitz, Christof / Schmidt, Stefan (Hrsg.), Achtsamkeit. Ein buddhistisches Konzept erobert die Wissenschaft. Bern: Verlag Hans Huber, S. 71-81

Maharishi Mahesh Yogi (2010). Die Wissenschaft vom Sein und die Kunst des Lebens. Bielefeld: J. Kamphausen Verlag \& Distribution GmbH.

Maharshi, Ramana (2019). Die Gesammelten Werke. Norderstedt: BoD.

Maharshi, Ramana (2011a). „Wer bin ich?" Der Übungsweg der Selbstergründung. Norderstedt: BoD.

Maharshi, Ramana (2011b). Sei, was Du bist! Die wichtigsten Lehren des großen indischen Weisen. München: O.W. Barth Verlag.

Main, John (2015). Das Herz der Stille. Einführung ins Herzensgebet. München: Claudius Verlag.

Marti, Lorenz (2016). Mystik an der Leine des Alltäglichen. Freiburg im Breisgau: Verlag Herder GmbH.

Mata Amritanandamayi (1995). Gespräche mit Amma 2. Die Lehren der Heiligen Mutter. Interlaken: Ansata-Verlag.

Meister Eckhart (2019). Mystische Schriften. Frankfurt am Main / Leipzig: Insel Verlag.

Muktananda, Swami (2001). Meditiere. Das Glück liegt in Dir. Telgte: Siddha Yoga Verlag GmbH. 
Muktananda, Swami (1996). Der Weg und sein Ziel. Ein Handbuch für die spirituelle Reise. München: Siddha Yoga Verlag GmbH.

Mutter Meera (2007). Antworten. Teil II. DornburgThalheim: Verlag \& Buchvertrieb Adilakshmi.

Mutter Meera (1994). Antworten. Teil I. DornburgThalheim: Verlag \& Buchvertrieb Adilakshmi.

Ng, Peter (Hrsg.) (2008). Die Sehnsucht nach Tiefe und Sinn. Meditieren mit John Maine. Bielefeld: Aurum.

Nisargadatta Maharaj (2017). Ich Bin. Teil I. Gespräche mit Sri Nisargadatta Maharaj. Bielefeld: J. Kamphausen Mediengruppe $\mathrm{GmbH}$.

Nisargadatta Maharaj (2016). Ich Bin. Teil II. Gespräche mit Sri Nisargadatta Maharaj. Bielefeld: J. Kamphausen Mediengruppe GmbH.

Nisargadatta Maharaj (2014a). Ich Bin. Teil III. Gespräche mit Sri Nisargadatta Maharaj. Bielefeld: J. Kamphausen Mediengruppe GmbH.

Nisargadatta Maharaj (2014b). Jenseits von Freiheit. Gespräche mit Sri Nisargadatta Maharaj. Lohne: Lotus Press.

Ott, Ulrich (2015). Meditation für Skeptiker. Ein Neurowissenschaftler erklärt den Weg zum Selbst. München: Droemer Knaur.

Piron, Harald (2020). Meditationstiefe. Grundlagen, Forschung, Training, Psychotherapie. Berlin: Springer-Verlag.

Ricard, Matthieu (2020). Glück. München: Knaur Verlag.

Ricard, Matthieu (2018a). Allumfassende Nächstenliebe. Hamburg: Edition Blumenau.

Ricard, Matthieu (2015). „Achtsamkeit - ein Zustand vollkommener Einfachheit“, in: Zimmermann, Michael / Spitz, Christof / Schmidt, Stefan (Hrsg.), Achtsamkeit. Ein buddhistisches Konzept erobert die Wissenschaft. Bern: Verlag Hans Huber, S. 49-55.

Ricard, Matthieu (2009). Meditation. München: nymphenburger.

Sandbothe, Mike / Albrecht, Reyk (Hrsg.) (2021). Achtsame Hochschulen in der digitalen Gesellschaft. Bielefeld: transcript Verlag.

Sedlmeier, Peter (2016). Die Kraft der Meditation. Was die Wissenschaft darüber weiß. Reinbek bei Hamburg: Rowohlt Taschenbuch Verlag.

Segal, Zindel V. / Williams, J. Mark G. / Teasdale, John D. (2015). Die Achtsamkeitsbasierte Kognitive Therapie der Depression. Tübingen: dgvt-Verlag.

Shankaracharya (2016). Aparoksha Anubhuti. Die direkte Verwirklichung des Selbst. Horn-Bad Meinberg: Yoga Vidya Verlag.
Siegel, Daniel J. (2017). Das achtsame Gehirn. Freiburg im Breisgau: Arbor Verlag.

Singer, Michael A. (2019). Die Seele will frei sein. Eine Reise zu sich selbst. Berlin: Ullstein Buchverlage $\mathrm{GmbH}$.

Singer, Tania (2019b). „Perspektiven der kontemplativen Neurowissenschaften zu Macht und Fürsorge: wie man sich in Fürsorge und Mitgefühl übt", in: Ricard, Matthieu / Singer, Tania / Karius, Kate (Hrsg.), Die Macht der Fürsorge. Für eine gemeinsame Zukunft. Wissenschaft und Buddhismus im Dialog mit dem Dalai Lama. München: Knaur Verlag, S. 86-99.

Singer, Tania / Ricard, Matthieu (2015). Mitgefühl in der Wirtschaft. München: Albrecht Knaus Verlag.

Singer, Wolf / Ricard, Matthieu (2018). Jenseits des Selbst. Dialoge zwischen einem Hirnforscher und einem buddhistischen Mönch. Berlin: Suhrkamp Taschenbuch Verlag.

Singer, Wolf / Ricard, Matthieu (2008). Hirnforschung und Meditation. Ein Dialog. Frankfurt am Main: Suhrkamp Verlag.

Sri Chinmoy (2015). Answers, Volume I, Book 19. Oxford: Ganapati Press.

Sri Chinmoy, (2012). Beyond likes and dislikes, Illumining questions and answers. New York: Agni Press.

Sri Chinmoy (2004). Der Körper, die Festung der Menschheit. Nürnberg: The Golden Shore Verlagsges.mbH.

Sri Chinmoy (1974). Meditation: God's Duty and man's beauty. New York: Agni Press.

Steindl-Rast, David (2018). Dankbarkeit. Das Herz allen Betens. Freiburg im Breisgau: Verlag Herder $\mathrm{GmbH}$.

Steindl-Rast, David (2016). Auf dem Weg der Stille. Das Heilige im Alltag leben. Freiburg im Breisgau: Verlag Herder GmbH.

Tang, Yi-Yuan (2019). Die Wissenschaft der Achtsamkeit. Wie Meditation die Biologie von Körper und Geist verändert. Paderborn: Junfermann Verlag.

Thich Nhat Hanh (2019). achtsam sprechen - achtsam zuhören. Die Kunst der bewussten Kommunikation. München: Knaur Verlag.

Thich Nhat Hanh / Cheung, Lilian (2016). achtsam essen - achtsam leben: Der buddhistische Weg zum gesunden Gewicht. München: O. W. Barth Verlag Thich Nhat Hanh (2015). Achtsamkeits-Survival-Kit. Fünf grundlegende Übungen. München: O. W. Barth Verlag.

Thich Nhat Hanh (2011). Versöhnung mit dem inneren Kind. Von der heilenden Kraft der Achtsamkeit. München: O. W. Barth Verlag. 
Thich Nhat Hanh / Nguyen Anh-Huong (2008). GehMeditation. München: Arkana. [mit CD und DVD] Thich Nhat Hanh (2007). Der Geruch von frisch geschnittenem Gras. Anleitung zur Gehmeditation. Stuttgart: Theseus Verlag.

Thich Nhat Hanh (2005). Friedlich miteinander leben. Handbuch für harmonische Gemeinschaft. München: Lotos Verlag.

Thich Nhat Hanh (2000). Das Wunder des bewussten Atmens. Stuttgart: Theseus Verlag.

Treleaven, David (2019). Traumasensitive Achtsamkeit. Posttraumatischen Stress erkennen und vermindern. Freiburg im Breisgau: Arbor Verlag.

Tremmel, Michael / Ott, Ulrich (2017). „Negative Wirkungen von Meditation“, in: Hofmann, Liane / Heise, Patrizia (Hrsg.), Spiritualität und spirituelle Krisen. Handbuch zu Theorie, Forschung und Praxis. Stuttgart: Schattauer GmbH, S. 233-243.

Vishnu Devananda (1986). Meditation und Mantras. Eine Darstellung der großen Yoga-Systeme in Theorie und Praxis. München: Heinrich Hugendubel Verlag.

Ware, Bronnie (2015). 5 Dinge, die Sterbende am meisten bereuen. Einsichten, die Ihr Leben verändern werden. München: Wilhelm Goldmann Verlag. Ware, Kallistos / Jungclaussen, Emmanuel (2004). Hinführung zum Herzensgebet. Freiburg im Breisgau: Verlag Herder.

Wolinsky, Stephen H. (2002). Ich bin dieses Eine: Begegnungen mit Sri Nisargadatta Maharaj. Kirchzarten bei Freiburg: VAK Verlags GmbH.

Yogananda, Paramahansa (2018). Die stärkende Kraft der Meditation - innere Ruhe und Klarheit gewinnen. Petersberg: Verlag Via Nova.

\section{Zeitschrift}

Davidson, Richard J. (2019c). „Interview“, in: Moment by Moment, Ausgabe 01. Freiburg: Hammer Solutions Media, S. 48-52.

Davidson, Richard J. (2018). „Interview“, in: Moment by Moment, Ausgabe 03. Freiburg: Hammer Solutions Media, S. 56-63.

de Bruin, Andreas (2019b). „Meditation and Art Große Werke der Malerei bewusst wahrnehmen", in: Zeitschrift für Bewusstseinswissenschaften. Transpersonale Psychologie und Psychotherapie, 25. Jahrgang 1. Petersberg: Verlag Via Nova, S. 10-20. de Bruin, Andreas (2017). „Möglichkeiten der Geistesschulung: Meditation im universitären Kontext? Das Münchener-Modell“, in: Zeitschrift für Bewusstseinswissenschaften. Transpersonale Psychologie und Psychotherapie, 23. Jahrgang 2. Petersberg: Verlag Via Nova, S. 68-84.

Ott, Ulrich (2018). „Meditation auf dem Prüfstand“, in: Moment by Moment, Ausgabe 03, Freiburg: Hammer Solutions Media, S. 64-67.

Ricard, Matthieu (2018b). „Interview“, in: Moment by Moment, Ausgabe 03, Freiburg: Hammer Solutions Media, S. 36-42.

Singer, Tania (2019a). „Interview“, in: Moment by Moment, Ausgabe 04. Freiburg: Hammer Solutions Media, S. 58-64.

Sri Chinmoy (1966). „Questions and Answers“, in: $A U M$, Vol. 2, No. 4/5, Nov.- Dec. 27. Brooklyn / New York: Boro Park Printers, S. 12-15.

\section{DVD}

Ambo, Phie (2013). Free the Mind. Kann ein Atemzug dein Denken verändern. Köln: mindjazz pictures.

Engle, Dawn Gifford. (2019). The Dalai LamaScientist. Arvada / Colorado: PeaceJam Foundation. Gilman, Sylvie / Lestrade, Thierry de (2016). Die Revolution der Selbstlosen. Köln: mindjazz pictures.

Mendiza, Michael (2008). Jiddu Krishnamurti - Die Herausforderung sich zu ändern. Eine Dokumentation über Krishnamurtis Werdegang und einige seiner wichtigsten Aussagen. Sulzbach: Arbeitskreis für freie Erziehung e.V.

Shen, Patrick (2018). Zeit für Stille. Köln: mindjazz pictures.

Wolinsky, Stephen H. (2009). Ich bin das Ich bin. Erfahre die Lehren des Sri Nisargadatta MaharajTeil 1. Stuttgart: Mouna GmbH.

\section{Internet}

Brown University (USA). Meditation Safety Toolbox, https://www.brown.edu/research/labs/britton/meditation-safety-toolbox

(letzter Zugriff: 13.09.2020)

Center for Healthy Minds (2017). Kindness Curriculum, https://centerhealthyminds.org/science/ studies/kindness-curriculum-study-with-prekindergarten-students (letzter Zugriff: 13.09.2020) 
Davidson, Richard J. (2019a). Vortrag und Podiumsdiskussion: „We can change the brain, by changing the mind" am 19. März 2019 in München beim Forum für den Wandel der Edith-Haberland-Wagner Stiftung, www.ehw-stiftung.de (letzter Zugriff: 13.09.2020)

Davidson, Richard J. (2019b). „How mindfulness changes the emotional life of our brains. TEDxSanFrancisco", www.youtube.com/ watch?V=7CBfCW67xT8 (letzter Zugriff: 13.09.2020)

Davidson, Richard J. (2016). „The Four Constituents of Well-Being", www.youtube.com/watch?v=HeBpsiFQiTI (letzter Zugriff: 13.09.2020)

Killingsworth, Matthew A. / Gilbert, Daniel T. (2010). A Wandering Mind Is an Unhappy Mind, www.danielgilbert.com/KILLINGSWORTH\%2O \&\%20GILBERT\%20(2010).pdf (letzter Zugriff: 13.09.2020)

Kornfield, Jack (2014). „Jack Kornfleld on Nisargadatta Maharaj (2)“, www.youtube.com/

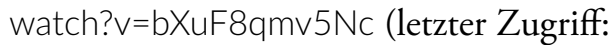
13.09.2020)

Kutschi, David / Nowack, Katharina / Wegele, Ramona (2017). Shanti - Meditation im Hochschulkontext am Beispiel des Münchener Modells, www.sw.hm.edu/ die_fakultaet/personen/professoren/bruin/ muenchner_modell/film.de.html (letzter Zugriff 13.09.2020)

Lazar, Sara (2011). „How Meditation Can Reshape Our Brains: Sara Lazar at TEDxCambridge“ www.youtube.com/watch?v=m8rRzTtP7Tc (letzter Zugriff: 13.09.2020)

Magiera, Birgit (2020). BR2 IQ - Wissenschaft und Forschung ,Achtsamkeitsforschung - Frieden für die Welt oder Wellness fürs Ich?“, https://www.br.de/mediathek/podcast/iq-wissenschaft-und-forschung/achtsamkeitsforschungfrieden-fuer-die-welt-oder-wellness-fuersich/1793281 (letzter Zugriff: 13.09.2020)

Morel, Delphine (2016). Mönche im Labor, www.youtube.com/watch? $v=3 q p L U p k d b K E$ (letzter Zugriff 13.09.2020)

Ott, Ulrich (2016). Vortrag: „Ist Achtsamkeit für jeden gut? Neue Forschungsergebnisse." im Rahmen der Vortragsreihe „Achtsamkeit - kritischer Blick auf einen Trend" der Universität Hamburg am 17. November 2016, https://lecture2go.uni-hamburg.de/
I2go/-/get/v/20619 (letzter Zugriff: 13.09.2020) Singer, Michael A. (2018). "What Do You Really Want?", www.youtube.com/watch?V=73-2PggJJWO (letzter Zugriff: 13.09.2020)

Singer, Tania / Bolz, Matthias (Hrsg.), (2013). eBook Mitgefühl. In Alltag und Forschung, www.compassion-training.org/?lang=de (letzter Zugriff: 13.09.2020)

Wolinsky, Stephen H. (2010). „Nisargadatta Maharaj / Experiential Meditation", www.youtube.com/watch?v=rE6kS7vVsXw (letzter Zugriff: 13.09.2020) 

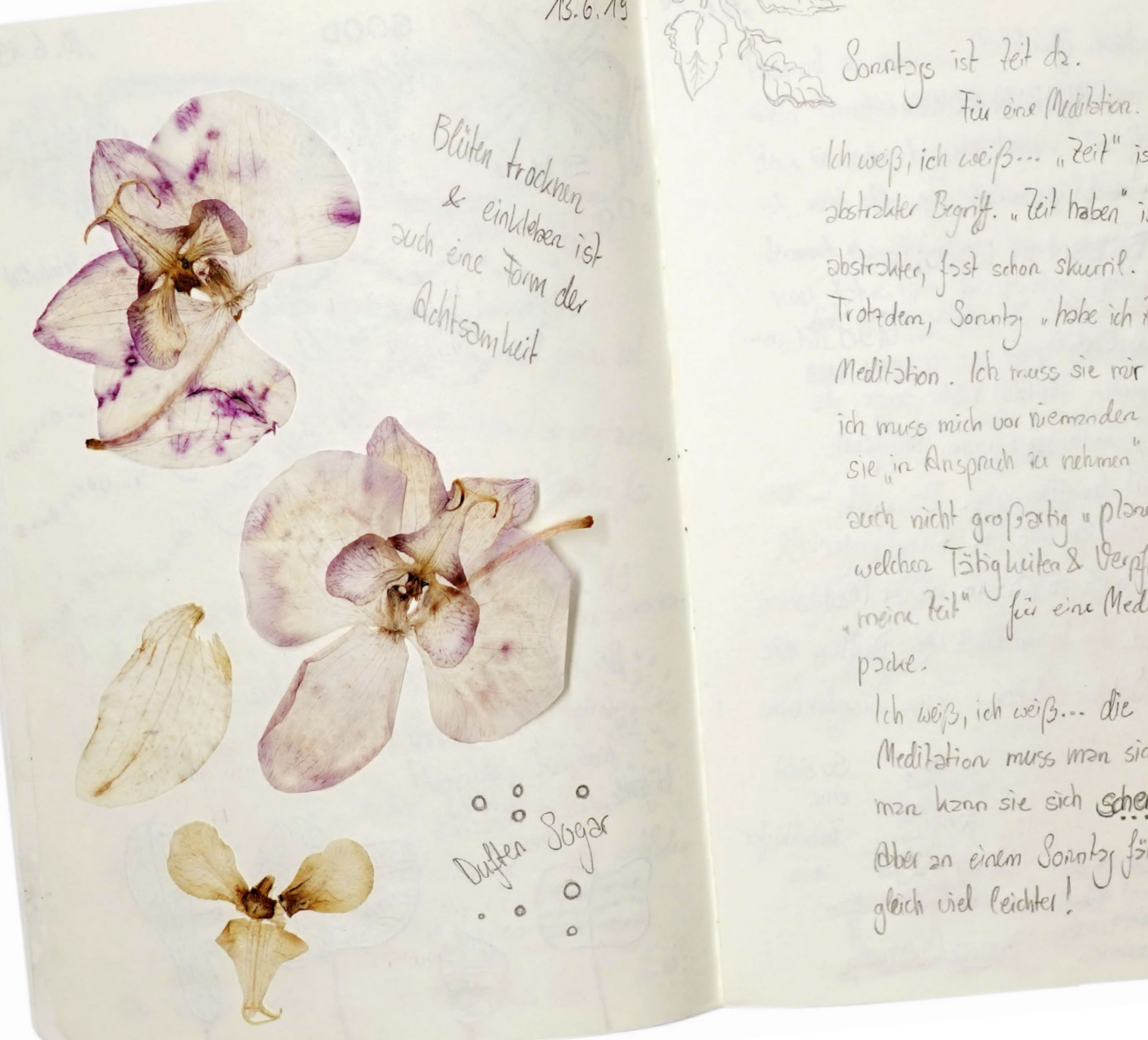
thwer, ich weip... "Zeit" ist ein "S.?. abstrakter Bronif. "Zit haben" ist noch. abstrouter, fast schon skuernil. Trotzdem, Sonnizy "habe ich teit "fü eine Meditstion. Ich muss sie mir nicht "nehmen", ich muss mich vor nemanden rechifertigen Sie in Ansprecth tee netumen, ich muss auch nicht gropartig "plosen" zuishen welcher Tätiqueitea\& Verplichtungen ioh meine tit" fis eine Meditation hinein padke.

The weiß, ich weiß... die Zeit für eine Medilation muss man sich widat netumen, man kann sie sich shenken! aber an einem Sonntary fallt das Schentien gleich viel leichter! 


\section{Förderung}

Dem Münchner Modell und dessen Initiator Andreas de Bruin begegnete ich 2018 bei einem Netzwerktreffen für (ehemalige) Studierende. Er lud mich anschließend ein, an einigen seiner Meditationslehrveranstaltungen teilzunehmen. Die gemeinsame Meditation mit ihm und seinen Studierenden berührte und begeisterte mich gleichermaßen. Das Besondere am Münchner Modell: Die Angebote ermöglichen die Freiheit der Selbsterfahrung und Selbstentfaltung, spontan, kreativ, annehmend. Die Teilnehmenden erhalten genügend Raum und erleben eine Atmosphäre liebevollen, nicht wertenden Miteinanders. Eine wichtige Erfahrung für junge Menschen, die im Bildungsbereich bislang sehr einseitig mit Wettbewerb und Leistung konfrontiert sind.

Die Vielfalt der niedergeschriebenen Eindrücke und Erfahrungen im vorliegenden Buch spiegelt die Buntheit und Einzigartigkeit der Teilnehmerinnen und Teilnehmer und ihrer Entwicklung wider. Und es zeigt die Offenheit des Münchner Modells: Es schränkt nicht ein, grenzt nicht aus. Es ermöglicht jedem Einzelnen, sich mit Freude zu entfalten und zu lernen - auf ganz natürliche Weise ohne äußere Pflicht und ohne Be-Urteilung durch den Lehrenden. Wie sollte so ein Urteil auch aussehen? Inneres Wachstum lässt sich weder durch Wollen erzwingen, noch mit Geld erkaufen oder durch Gebete erbitten. Wir können es nicht „machen“ oder den Prozess gar kontrollieren. Das ist auch gut so.

Verein für Achtsamkeit in Osterloh e.V. Maria Kluge

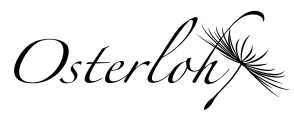

Eine grundlegende Aufgabe der Edith-Haberland-Wagner Stiftung ist es, das Leben für heutige und zukünftige Generationen lebenswerter zu machen. Mit ihrem Forum für den Wandel gibt sie inspirierende Impulse und eröffnet einen Dialog über die sinnstiftende Gestaltung unserer Gegenwart und Zukunft. In diesem Sinne hat die Edith-Haberland-Wagner Stiftung sich dazu entschieden, das Münchner Modell "Achtsamkeit und Meditation im Hochschulkontext" zu fördern. Die Angebote im Rahmen des Modells, insbesondere die Lehrveranstaltungen für Studierende, bieten einen fruchtbaren Boden für neue Erfahrungen und haben eine positive Auswirkung auf Psychohygiene, Konzentration, Selbstwertgefühl und Selbstwirksamkeit, Kreativität sowie Empathie und Mitgefühl. Viele Studierende wenden die Lehransätze nach dem Studium auch in ihrem späteren Berufsfeld, wie etwa in Kindertagesstätten und Schulen, an; so können auch die jeweiligen Zielgruppen vor Ort davon profitieren. Darüber hinaus inspiriert das Münchner Modell als erfolgreiches Beispiel andere Hochschulen und Bildungsinstitutionen, sich mit den Themen Achtsamkeit und Meditation auseinanderzusetzen und gegebenenfalls Ansätze vor Ort zu implementieren. Auf diese Weise trägt es in vielerlei Hinsicht zu einem positiven gesellschaftlichen Wandel bei.

Vorstand der Edith-Haberland-Wagner Stiftung Catherine Demeter, Martin Liebhäuser

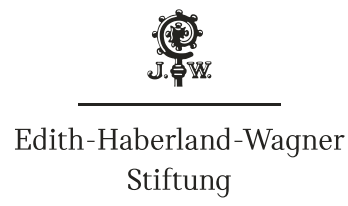




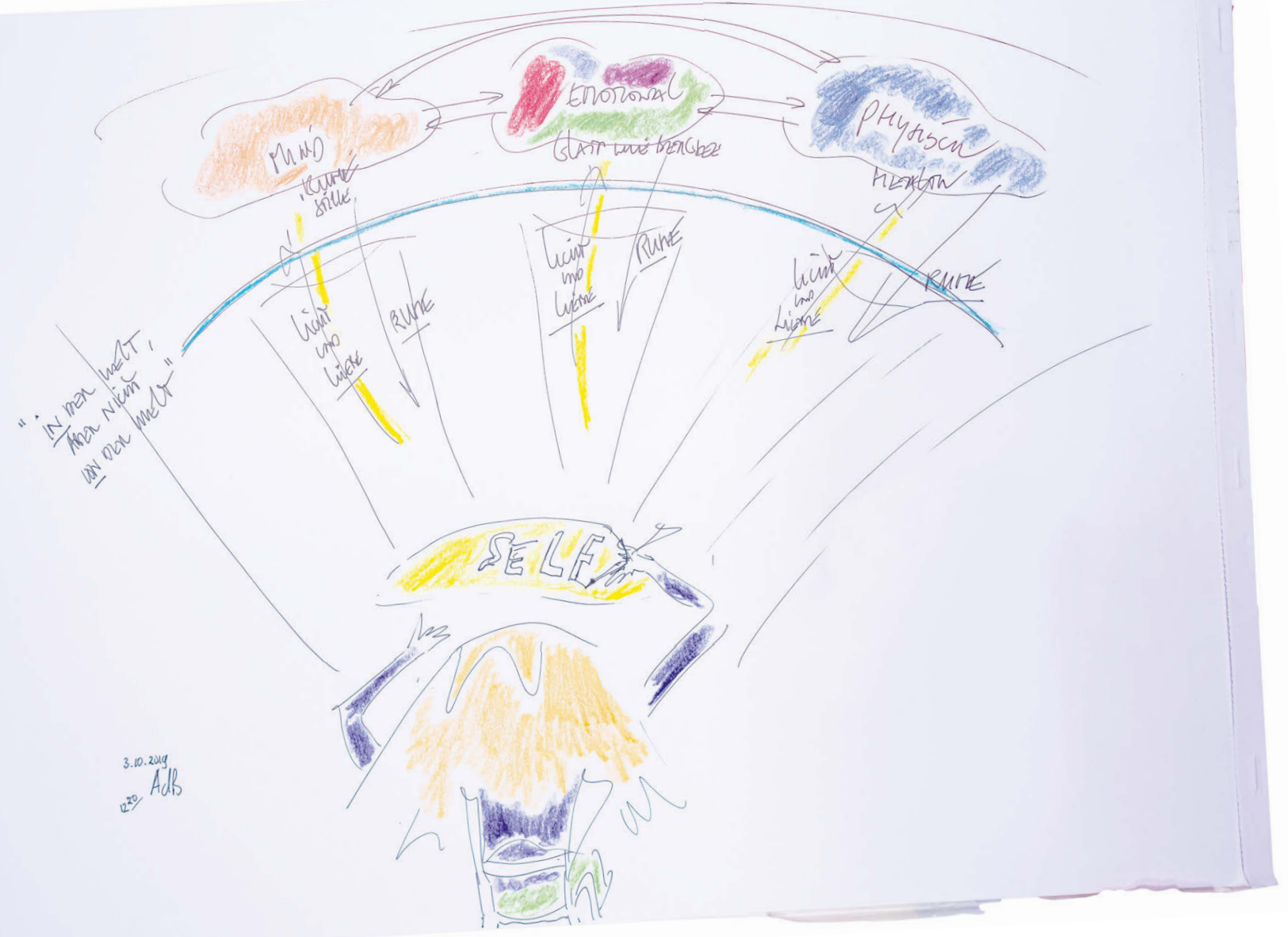




\section{Zum Autor}

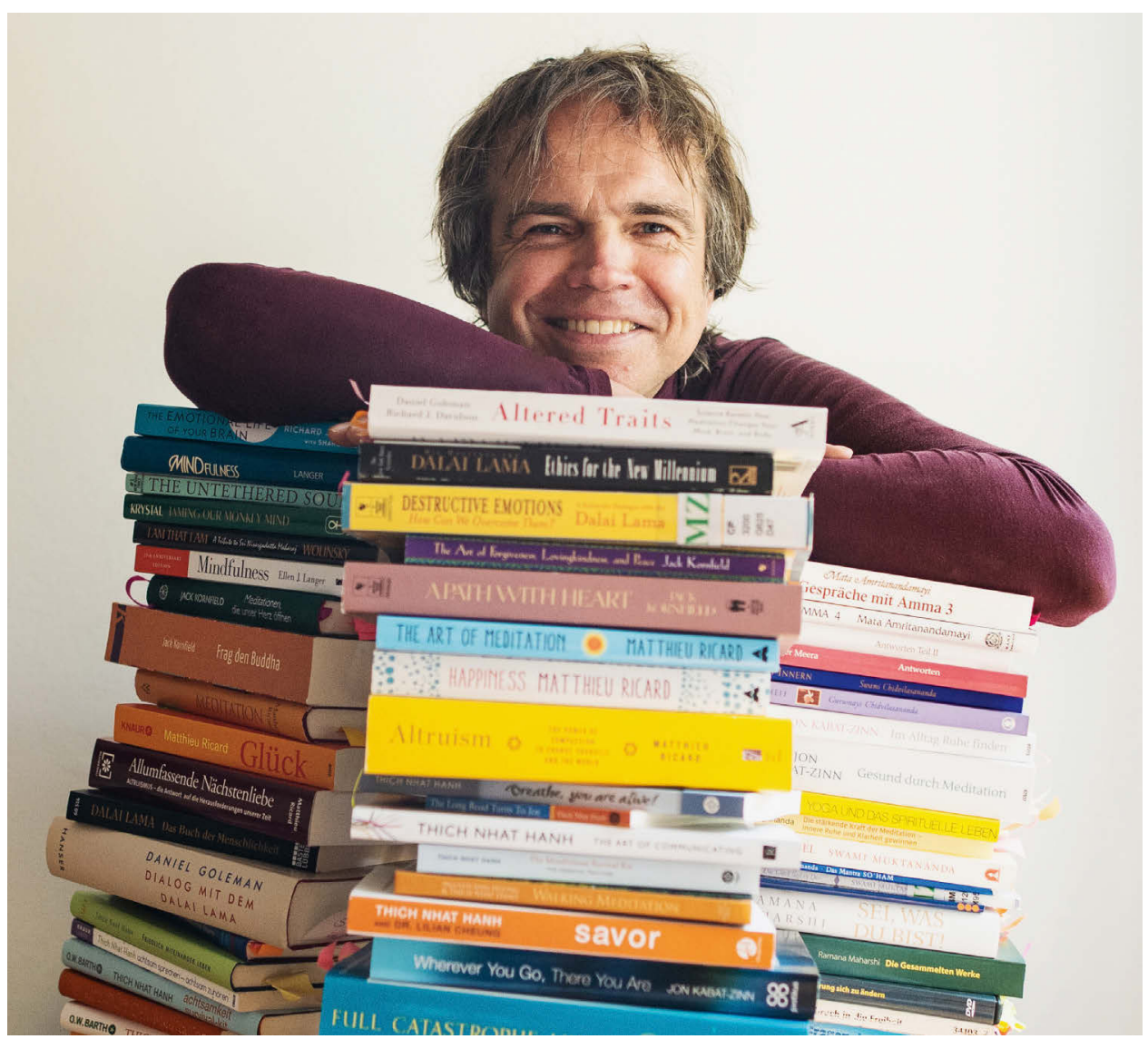

Andreas de Bruin wurde 1965 in Delft, Niederlande, geboren. Nach seinem Studium der Technischen Betriebswirtschaft in Rijswijk studierte er Psychologie in Leiden und Heidelberg sowie Ethnologie in München. Er promovierte 2003 in Ethnologie und wurde 2006 Professor für Ästhetische Medien an der Hochschule München (MUAS). Er unterrichtet im Rahmen einer Bildungs- und Forschungskooperation zusätzlich an der Ludwig-Maximilians-Universität (LMU) München.

De Bruins Hauptfelder in Forschung und Lehre sind Achtsamkeit und Meditation und deren Auswirkungen auf physischer, emotionaler, kognitiver und sozialer Ebene. Er begann seine eigene Meditationspraxis 1991. De Bruin unterrichtet zudem bewusstes Wahrnehmen in der Malerei und klassischen Musik; weitere Fächer sind Hochbegabung sowie Kreativität im Allgemeinen.

De Bruin ist Gründer und Leiter des Münchner Modells „Achtsamkeit und Meditation im Hochschulkontext" - eines der ersten Programme in Europa, das Lehrveranstaltungen in Achtsamkeit und Meditation anbietet und dafür auch Leistungspunkte vergibt. Neben regulären Kursen organisiert er auch Zusatzangebote für (ehemalige) Studenten, Lehrpersonal und Hochschulangestellte. Um seine Erfahrungen zu teilen, arbeitet de Bruin eng mit anderen Hochschulen im In- und Ausland zusammen. Er ist ein gefragter Redner zu diesem Thema; er wurde vielfach interviewt und sein Konzept in verschiedenen Medien und Publikationen vorgestellt.

Darüber hinaus veranstaltet de Bruin regelmäßig eine kostenlose Reihe öffentlicher Vorträge und Filmvorführungen mit dem Titel „Spirituelle Lehrer und ihre Unterweisungen“. Sein jüngstes Projekt „Meditation and Art“ lehrt die bewusste Beobachtung der Gemälde der alten Meister. 


\section{Achtsamkeit - Bildung - Medien}

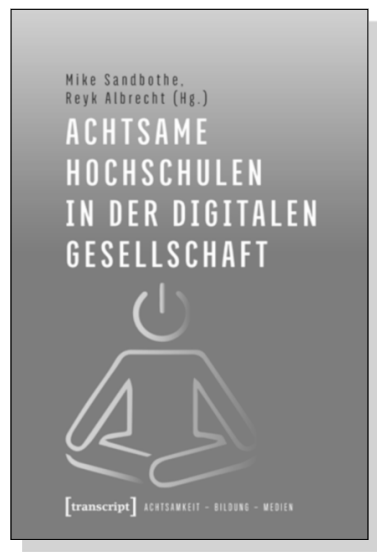

Mike Sandbothe, Reyk Albrecht (Hg.)

Achtsame Hochschulen

in der digitalen Gesellschaft

Januar 2021, ca. 250 S., kart.

ca. $25,00 €(D E), 978-3-8376-5188-1$

E-Book: kostenlos erhältlich als Open-Access-Publikation, ISBN 978-3-8394-5188-5

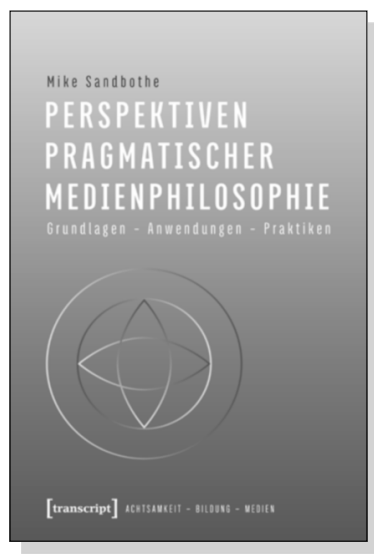

Mike Sandbothe

Perspektiven pragmatischer Medienphilosophie Grundlagen - Anwendungen - Praktiken

Oktober 2020, 230 S., kart.

ca. $34,00 €(D E), 978-3-8376-5205-5$

E-Book: kostenlos erhältlich als Open-Access-Publikation,

ISBN 978-3-8394-5205-9

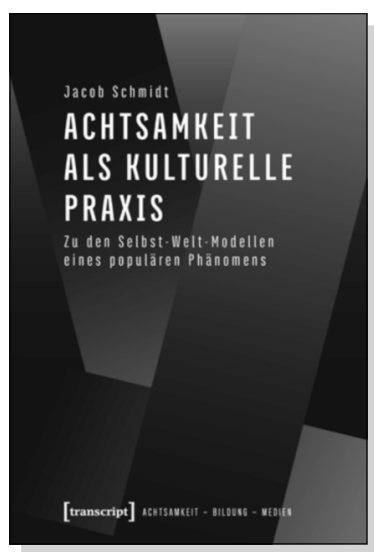

Jacob Schmidt

Achtsamkeit als kulturelle Praxis

Zu den Selbst-Welt-Modellen eines populären Phänomens

April 2020, 400 S., kart.

35,00€ (DE), 978-3-8376-5230-7

E-Book: kostenlos erhältlich als Open-Access-Publikation, ISBN 978-3-8394-5230-1 